\title{
Mathematical Modeling and Analysis of Epidemiological and Chemical Systems
}

\author{
Calistus N. Ngonghala \\ West Virginia University
}

Follow this and additional works at: https://researchrepository.wvu.edu/etd

\section{Recommended Citation}

Ngonghala, Calistus N., "Mathematical Modeling and Analysis of Epidemiological and Chemical Systems" (2011). Graduate Theses, Dissertations, and Problem Reports. 3405.

https://researchrepository.wvu.edu/etd/3405

This Dissertation is protected by copyright and/or related rights. It has been brought to you by the The Research Repository @ WVU with permission from the rights-holder(s). You are free to use this Dissertation in any way that is permitted by the copyright and related rights legislation that applies to your use. For other uses you must obtain permission from the rights-holder(s) directly, unless additional rights are indicated by a Creative Commons license in the record and/ or on the work itself. This Dissertation has been accepted for inclusion in WVU Graduate Theses, Dissertations, and Problem Reports collection by an authorized administrator of The Research Repository @ WVU.

For more information, please contact researchrepository@mail.wvu.edu. 


\title{
Mathematical Modeling and Analysis of Epidemiological and Chemical Systems
}

\author{
Calistus N. Ngonghala
}

\author{
Dissertation Submitted to the \\ Eberly College of Arts and Sciences \\ at West Virginia University \\ in Partial Fulfillment of the Requirements \\ for the Degree of
}

Doctor of Philosophy

in

Mathematics

Committee:

Kenneth Showalter, Ph.D., Chair

Mary Ann Clarke, Ph.D., Co-Chair

Harumi Hattori, Ph.D.

Ian Christie , Ph.D.

Harvey Diamond, Ph.D.

Morgantown, West Virginia

2011

Keywords: Epidemiology, Mosquito Demography, Malaria Control, Disease-free Equilibrium, Endemic Equilibrium, Hopf Bifurcation, Backward Bifurcation, Deterministic Model, Stochastic Model, Poverty Traps, Safety Nets, Public Health, Economic Development, Dissipative System, Conservative System, Multistability, Conserved Quantity, Synchronization, Basin of Attraction. 


\title{
Abstract
}

\author{
Mathematical Modeling and Analysis of \\ Epidemiological and Chemical Systems \\ Calistus N. Ngonghala
}

This dissertation focuses on three interdisciplinary areas of applied mathematics, mathematical biology/epidemiology, economic epidemiology and mathematical physics, interconnected by the concepts and applications of dynamical systems.

In mathematical biology/epidemiology, a new deterministic SIS modeling framework for the dynamics of malaria transmission in which the malaria vector population is accounted for at each of its developmental stages is proposed. Rigorous qualitative and quantitative techniques are applied to acquire insights into the dynamics of the model and to identify and study two epidemiological threshold parameters $\mathscr{R}^{*}$ and $R_{0}$ that characterize disease transmission and prevalence, and that can be used for disease control. It is shown that nontrivial disease-free and endemic equilibrium solutions, which can become unstable via a Hopf bifurcation exist. By incorporating vector demography; that is, by interpreting an aspect of the life cycle of the malaria vector, natural fluctuations known to exist in malaria prevalence are captured without recourse to external seasonal forcing and delays. Hence, an understanding of vector demography is necessary to explain the observed patterns in malaria prevalence. Additionally, the model exhibits a backward bifurcation. This implies that simply reducing $R_{0}$ below unity may not be enough to eradicate the malaria disease. Since, only the female adult mosquitoes involved in disease transmission are identified and fully accounted for, the basic reproduction number $\left(R_{0}\right)$ for this model is smaller than that for previous SIS models for malaria. This, and the occurrence of both oscillatory dynamics and a backward bifurcation provide a novel and plausible framework for developing and implementing optimal malaria control strategies, especially those strategies that are associated with vector control.

In economic epidemiology, a deterministic and a stochastic model are used to investigate the effects of determinism, stochasticity, and safety nets on disease-driven poverty traps; 
that is, traps of low per capita income and high infectious disease prevalence. It is shown that economic development in deterministic models require significant external changes to the initial economic and health care conditions or a change in the parametric structure of the system. Therefore, poverty traps arising from deterministic models lead to more limited policy options. In contrast, there is always some probability that a population will escape or fall into a poverty trap in stochastic models. It is demonstrated that in stochastic models, a safety net can guarantee ultimate escape from the poverty trap, even when it is set within the basin of attraction of the poverty trap or when it is implemented only as an economic or health care intervention. It is also shown that the benefits of safety nets for populations that are close to the poverty trap equilibrium are highest for the stochastic model and lowest for the deterministic model. Based on the analysis of the stochastic model, the following optimal economic development and public health intervention questions are answered:

(i) Is it preferable to provide health care, income/income generating resources, or both health care and income/income generating resources to enable populations to break cycles of poverty and disease; that is, escape from poverty traps? (ii) How long will it take a population that is caught in a poverty trap to attain economic development when the initial health and economic conditions are reinforced by safety nets?

In mathematical physics, an unusual form of multistability involving the coexistence of an infinite number of attractors that is exhibited by specially coupled chaotic systems is explored. It is shown that this behavior is associated with generalized synchronization and the emergence of a conserved quantity. The robustness of the phenomenon in relation to a mismatch of parameters of the coupled systems is studied, and it is shown that the special coupling scheme yields a new class of dynamical systems that manifests characteristics of dissipative and conservative systems. 


\section{Dedication}

This work is dedicated to the Almighty God for his constant love, protection and guidance,

and to my beloved mother, Ngwebu Magdalene for investing almost all her wealth and energy to ensure that I acquire the best education. 


\section{Acknowledgements}

First of all, I will like to bow down and offer thanks and praises to the Almighty God for providing me with the good health, wisdom and courage required to pursue this research. Next, I wish to express sincere gratitude to my advisor, Dr. Kenneth Showalter, for partially funding my Ph.D. program, for providing me with the conducive atmosphere where this work was carried out, for his patience, assistance and guidance through out this research, and from whom I acquired a great deal of skills, especially in scientific writing. I am also very thankful to Dr. Mary Ann Clarke, co-chair of my examining committee, for her constant motivation, friendly advice, and for taking time to proof-read some of the chapters of this dissertation.

Special thanks are due to Drs. Harvey Diamond and Eddie Fuller, Graduate Director and Chairman of the Mathematics Department, respectively, for doing all in their positions to ensure the success of this interdisciplinary-interdepartmental (Mathematical BiologyChemistry) research. I also wish to thank the Faculty of the Mathematics Department for demonstrating genuine interest and enthusiasm in their teaching and research and the staff of the Mathematics and Chemistry Departments for their relentless support.

I owe a debt of gratitude to Drs. Gideon A. Ngwa and Miranda I. Teboh-Ewungkem, with whom I had valuable and productive collaborations that contributed greatly towards the realization of the project on mathematical biology. My deepest appreciation is extended to Dr. Ulrike Feudel, whose constructive criticisms and intellectually stimulating arguments contributed enormously towards the realization of the project on mathematical physics as well as enabled me to appreciate research on multistability. Unreserved thanks are due to Dr. Mathew Bonds and Mathew Pluciński, whose contributions rendered the project on economic epidemiology possible and enjoyable. I am heavily indebted to Dr. Mark Tinsley, who furnished me with deep insight perspectives into the programming aspects of this work.

I am very grateful to my family, especially my mother, Ngwebu Magdalene, whose constant support has been invaluable to my education. I would also like to take this opportunity to express my profound appreciation to Shelby Wilson, Donald Showalter, my friends, colleagues, and members of the Showalter research group especially Desmond Yengi who created the fun, relaxing and enjoyable environment in which this work was carried out. 


\section{Table of Contents}

$\begin{array}{ll}\text { Title Page } & \text { i }\end{array}$

$\begin{array}{ll}\text { Abstract } & \text { ii }\end{array}$

Dedication $\quad$ iv

Acknowledgements $\quad$ v

Table of Contents vi

List of Figures xiii

$\begin{array}{ll}\text { List of Tables } & \text { xxi }\end{array}$

1 Introduction and General Review of Dynamical System Concepts 1

1.1 Introduction . . . . . . . . . . . . . . . . . . . . . . . 1

1.2 General Review of Dynamical System Concepts . . . . . . . . . . . . . . . 3

1.2 .1 Dynamical Systems . . . . . . . . . . . . . . . . . . . . . . 3

1.2.2 Equilibria, Stability, Attractors and Bifurcations . . . . . . . . . 5

1.2 .3 Chaos and Strange Attractors . . . . . . . . . . . . . . . . 10

1.2.4 Dissipative and Conservative Systems . . . . . . . . . . . . . . 11

1.2.4.1 Illustration of Conservative and Dissipative Systems . . . 12

1.2.4.2 Undamped Simple Pendulum (Undamped Oscillator) . . . . 13

1.2.4.3 Damped Simple Pendulum (Damped Oscillator) . . . . . . . 16 
1.3 Synchronization . . . . . . . . . . . . . . . . . . . . 18

References . . . . . . . . . . . . . . . . . . . . . . . 19

I A New Route to Periodic Oscillations in the Dynamics of $\begin{array}{ll}\text { Malaria Transmission } & 27\end{array}$

$\begin{array}{llr}2 & \text { Introduction } & 28\end{array}$

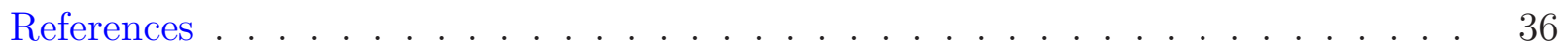

3 The Classical Ross-Macdonald Type SIS Model 44

3.1 Introduction . . . . . . . . . . . . . . . . . . . . . . . 44

3.2 Classical Model Derivation . . . . . . . . . . . . . . . . . . 45

3.3 Classical Model Analysis . . . . . . . . . . . . . . . . . . . . . 47

3.4 Numerical Simulations of the Classical SIS Model . . . . . . . . . . . . 57

3.5 Conclusion . . . . . . . . . . . . . . . . . . . . . . . . . . . 61

References . . . . . . . . . . . . . . . . . . . . . . 62

4 Derivation of the Basic ODE Model 65

4.1 Introduction . . . . . . . . . . . . . . . . . 65

4.2 Model Variables and Parameters . . . . . . . . . . . . . . . . . . 66

4.3 THE ODE MODEL . . . . . . . . . . . . . . . . . . . . . . . . . 69

4.3 .1 Model Equations . . . . . . . . . . . . . . . . . . 70

4.4 Simplified Model . . . . . . . . . . . . . . . . . . . 76

4.4.1 Well Posedness, Positivity and Boundedness _. . . . . . . . . 79

4.4 .2 Nondimensionalization and Reparametrization . . . . . . . . . . 80

4.5 Conclusion . . . . . . . . . . . . . . . . . . . . . 84

References . . . . . . . . . . . . . . . . . . 85

$\begin{array}{llr}5 & \text { Model Analysis } & 89\end{array}$

5.1 Introduction . . . . . . . . . . . . . . . . . . . . . . 89

5.2 Model in the Absence of the Malaria Disease . . . . . . . . . . . . . . . 90 
5.2.1 Existence of Realistic Equilibrium Solutions . . . . . . . . . . . . 90

5.2 .2 Stability of Equilibrium Solutions . . . . . . . . . . . . . . 90

5.2 .3 Numerical Simulations of the Disease-free Model . . . . . . . . . . . . 95

5.3 Model Analysis in the Presence of the Disease . . . . . . . . . . . . . 102

5.3.1 A Discussion on the Basic Reproduction Number, $R_{0} \ldots \ldots$. . . 102

5.3 .2 Existence of Equilibria . . . . . . . . . . . . . . . . 105

5.3 .3 Stability of Equilibria . . . . . . . . . . . . . . . . . . . . 113

5.3.3.1 Stability of the Trivial Equilibrium . . . . . . . . . 113

5.3.3.2 Stability of the Disease-free Equilibrium . . . . . . . . . 114

5.3.3.3 Stability of Endemic Equilibria . . . . . . . . . . 117

5.3 .4 Numerical Simulations . . . . . . . . . . . . . . . . . . 120

5.3.4.1 Exploration of the Unique Endemic Equilibrium Solution in Region (I) of Fig. $5.6 \ldots \ldots \ldots \ldots$

5.3.4.2 Exploration of the Unique Endemic Equilibrium that exists when $A_{1}=0, A_{2}>0$ and $\Delta>0 \ldots \ldots \ldots \ldots$

5.3.4.3 Characterization of the Unique Endemic Equilibrium Solution in Region (III) of Fig. 5.6 . . . . . . . . . . . . . 129

5.3.4.4 Exploration of the Unique Endemic Equilibrium that Exists when $A_{1}>0, A_{2}=0$ and $\Delta>0 \ldots \ldots \ldots \ldots$

5.3.4.5 Exploration of the Endemic Equilibrium that Exists when $A_{1}>0, A_{2}<0$ and $\Delta=0 \ldots \ldots \ldots \ldots$

5.4 Characterization of Backward Bifurcation . . . . . . . . . . . 137

5.5 Effects of Birth Rate Function . . . . . . . . . . . . . . . . 146

5.6 Conclusion . . . . . . . . . . . . . . . . . . . . . . . . . 150

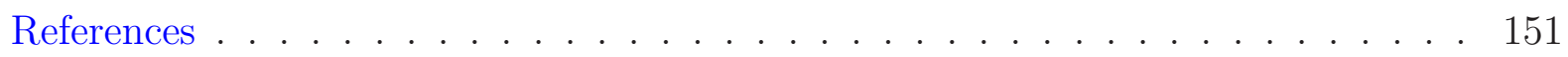

6 Part I Conclusion and Open Questions 155

6.1 Intoduction . . . . . . . . . . . . . . . . . . 155

6.2 Comparison of Model (4.22) to the Classical Ross SIS Model . . . . . . . . 155

6.3 Synopsis and Conclusion . . . . . . . . . . . . . . . . . 157 
6.4 Possible Extensions and Areas for Future Research . . . . . . . . . . . . 160

References . . . . . . . . . . . . . . . . . . . . . . 162

II Disease-driven Poverty Traps 163

7 Disease-driven Poverty Traps: The Effects of Determinism, Stochasticity, $\begin{array}{lr}\text { and Safety Nets } & 164\end{array}$

7.1 Introduction . . . . . . . . . . . . . . . . . . . . . . . . . . . 164

7.2 The Deterministic Approach . . . . . . . . . . . . . . . . . 167

7.2.1 Existence of Equilibrium Solutions and Linear Stability Analysis . . . 173

7.2 .2 Numerical Simulations of the Deterministic Model . . . . . . . . . . 175

7.3 Stochastic Model . . . . . . . . . . . . . . . . . . . . . . . . . . . 181

7.3.1 Partial Stochastic Model . . . . . . . . . . . . . . . . . . . . . . . 182

7.3.1.1 Numerical Simulations of the Partial Stochastic Model . . . 183

7.3 .2 Full Stochastic Model . . . . . . . . . . . . . . . . . . . . . . . . . . 188

7.4 Policy Interpretation $\ldots \ldots$

7.5 Conclusion . . . . . . . . . . . . . . . . . . . . . . . 199

References . . . . . . . . . . . . . . . . . . . . . 201

\section{Extreme Multistability in a Chemical Model System 209}

8 Introduction to Chemical Chaos $\quad 210$

8.1 Introduction to Chemical Chaos through the Autocatalator Model . . . . . . 210

8.2 The Two-variable Autocatalator Model . . . . . . . . . . . . . . . . . . . . . 211

8.3 The Three-variable Autocatalator Model . . . . . . . . . . . . . . . . . . 215

8.3 .1 The Model . . . . . . . . . . . . . . . . . . . . . . . . . . . . . 215

8.3.2 A Quantitative Study of the Three-variable Autocatalator Model . 217

8.3.3 Qualitative Study of the Three-variable Autocatalator Model . . . . . 225

8.4 Conclusion . . . . . . . . . . . . . . . . . . . . . . . . . . 231

References . . . . . . . . . . . . . . . . . . . . . 232 
9 Extreme Multistability in the Autocatalator Model 235

9.1 Introduction . . . . . . . . . . . . . . . . . . . 235

9.2 Coupled Autocatalator Model . . . . . . . . . . . . . . . . . . 238

9.3 Nondimensionalization . . . . . . . . . . . . . . . . . . . . . . . . 239

9.4 Model Analysis . . . . . . . . . . . . . . . . . . . . . . . . . 242

9.5 Conserved Quantity . . . . . . . . . . . . . . . . . . 252

9.6 Nonidentical coupled systems . . . . . . . . . . . . . . . . 261

9.7 Conclusion . . . . . . . . . . . . . . . . . . . . . . 268

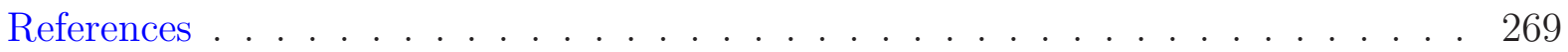

10 Robustness of Extreme Multistability $\quad 274$

10.1 Introduction . . . . . . . . . . . . . . . . . . . 274

10.2 Second Coupling Scheme . . . . . . . . . . . . . . . . . 275

10.2.1 The Model . . . . . . . . . . . . . . . . . . . 275

10.2.2 Model Analysis . . . . . . . . . . . . . . . . . . . . . . 277

10.2.2.1 Dynamical Behavior for Varying $\mu$. . . . . . . . . . . 277

10.2.2.2 Illustration of Extreme Multistability . . . . . . . . . . . . 280

10.2 .3 Reduced System . . . . . . . . . . . . . . . . . 284

10.3 Third Coupling Scheme . . . . . . . . . . . . . . . . . . . . 295

10.4 Fourth Coupling Scheme . . . . . . . . . . . . . . . . . . . . 297

10.5 Brief Synopsis and Conclusion . . . . . . . . . . . . . . . 301

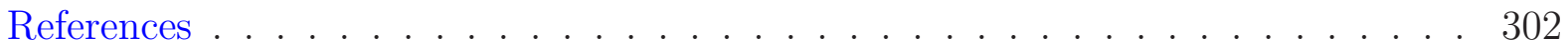

11 Generalization of Extreme Multistability 303

11.1 Introduction . . . . . . . . . . . . . . . . . 303

11.2 Overview and Generalization . . . . . . . . . . . . . . 303

11.3 Conclusion . . . . . . . . . . . . . . . . . . . . . 307

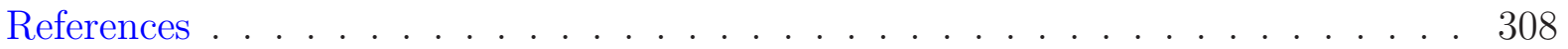

$\begin{array}{ll}\text { Appendices } & 309\end{array}$

$\begin{array}{ll}\text { Appendix A Principle of Linear Stability } & 310\end{array}$ 
Appendix B Libnitz Rule, Descartes' Rule of Signs, and Routh-Hurwitz Criterion

B.1 Libnitz Rule . . . . . . . . . . . . . . . . . . . . . . . . . . . . . . . . 313

B.2 Descartes' Rule of Signs . . . . . . . . . . . . . . . . . . . . . . 313

B.3 Routh-Hurwitz Criterion . . . . . . . . . . . . . . . . . . . . . . . 314

References . . . . . . . . . . . . . . . . . . . . . . 316

Appendix C Existence and Uniqueness Theorem for Solutions to Systems of $\begin{array}{ll}\text { First-order Ordinary Differential Equations } & 317\end{array}$ References . . . . . . . . . . . . . . . . . . . . . . . . . . . . 318

$\begin{array}{ll}\text { Appendix D Lyapunov Functions and Lyapunov Exponents } & 319\end{array}$

D.1 Lyapunov Functions ． . . . . . . . . . . . . . . . . . . . . . . . . 319

D.2 The Largest Lyapunov Exponent _ . . . . . . . . . . . . . . . . . . 321

References . . . . . . . . . . . . . . . . . . . . . . 323

$\begin{array}{lll}\text { Appendix E LaSalle's Invariance Principle } & 324\end{array}$

References . . . . . . . . . . . . . . . . . . . . 325

Appendix F Third Hands-on Research on Complex Systems ASI Hand-out 326

F.1 Session Description Summary . . . . . . . . . . . . . . . . . . 327

F.1.1 Theoretical and Experimental Part . . . . . . . . . . . . . . 328

F.1.2 Simulation Introduction $\ldots \ldots \ldots$

F.2 Detailed Session Description . . . . . . . . . . . . . . . . . . . . . . . 329

F.2.1 Deterministic SIR Model . . . . . . . . . . . . . . . . . . . . . 329

F.2.1.1 Exercise . . . . . . . . . . . . . . . . . 330

F.2.1.2 Simulating the Deterministic Model with no Vital Dynamics 330

F.2.1.3 Simulating the Deterministic Model with Vital Dynamics . . 331

F.2.2 SIR Model with Periodic Contact Rate . . . . . . . . . . . . . . 333

F.2.2.1 Background . . . . . . . . . . . . . . . . . . 333

F.2.2.2 Simulations . . . . . . . . . . . . . . . 333

F.2.2.3 Exercise . . . . . . . . . . . . . . . . 336 
F.2.3 Stochastic SIR Model . . . . . . . . . . . . . . . . . . . . . . 336

F.2.3.1 Model Description . . . . . . . . . . . . . . . 336

F.2.3.2 Simulation of the Stochastic Model . . . . . . . . . . 338

F.2.3.3 Exercise . . . . . . . . . . . . . . . . . . . . . 340

F.2.4 Spatiotemporal SEIRS Malaria Model . . . . . . . . . . . . . . . 340

F.2.4.1 Background . . . . . . . . . . . . . . . . . . 340

F.2.4.2 Simulations . . . . . . . . . . . . . . . . 341

F.2.4.3 Exercise . . . . . . . . . . . . . . . . . . . . 343

F.2.4.4 Some Results with the Parameter Values in Table F.2 . . . . 344

References . . . . . . . . . . . . . . . . . . . . . 347

Appendix G Latex Files, MATLAB Codes, and Figures 349

G.1 Latex Code . . . . . . . . . . . . . . . . . . . . . . . . . . . . . . . . . . . . 349

G.2 MATLAB Codes . . . . . . . . . . . . . . . . . . . . . . . . 349 


\section{List of Figures}

1.1 Undamped simple pendulum . . . . . . . . . . . . . . . . . . . . . 14

1.2 Damped simple pendulum, $b=0.5 \ldots \ldots \ldots \ldots \ldots \ldots$

1.3 Damped simple pendulum, $b=3.0 \ldots \ldots \ldots \ldots \ldots \ldots$

3.1 A conceptual framework of the classical SIS model for malaria . . . . . . . 45

3.2 Disease-free equilibrium of the classical SIS model . . . . . . . . . . . . . 59

3.3 Endemic equilibrium of the classical SIS model . . . . . . . . . . . . . 60

4.1 Conceptual framework for new SIS malaria model . . . . . . . . . . . . 69

5.1 Solutions of the disease-free model. . . . . . . . . . . . . . . . . . . 97

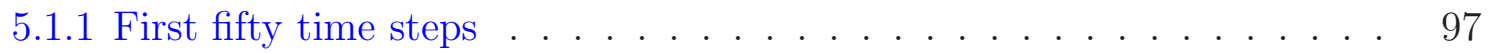

5.1 .2 Stable equilibrium solution. . . . . . . . . . . . . . . . . . 97

5.2 Time series and phase plots within the oscillatory region. . . . . . . . . 98

5.2 .1 Time series showing periodic oscillations. . . . . . . . . . . . . 98

5.2 .2 Phase plot showing a limit cycle. . . . . . . . . . . . . . . 98

5.3 Total mosquito population. . . . . . . . . . . . . . . . . . . . 99

5.4 Bifurcation diagrams for the disease-free model. . . . . . . . . . . . . 100

5.4.1 Bifurcation diagram with bifurcation parameter $\lambda_{0} \ldots \ldots \ldots$. . . 100

5.4.2 Bifurcation diagram with bifurcation parameter $\mathscr{R}^{*}$. . . . . . . 100

5.5 Bifurcation diagram for the disease-free model as a function of $\gamma . \ldots 101$

5.6 Endemic equilibrium diagram . . . . . . . . . . . . . . . . . . . . 112 
5.7 Threshold parameter plots . . . . . . . . . . . . . . . . . . . . 122

5.7.1 Plot of $\mathscr{R}^{*}$ against $\gamma \ldots \ldots \ldots \ldots$

5.7 .2 Plot of $R_{0}$ against $\gamma \ldots \ldots \ldots \ldots \ldots \ldots \ldots$

5.7 .3 Plot of $R_{0}$ and $\mathscr{R}^{*}$ against $\lambda_{0} \ldots \ldots \ldots \ldots \ldots \ldots$

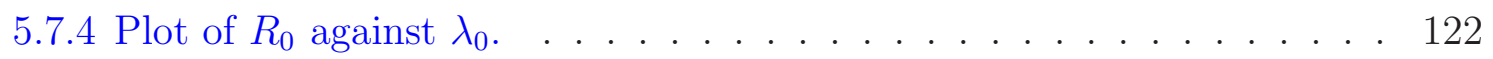

5.8 Endemic curves. . . . . . . . . . . . . . . . . . . . . . . . . . . . . 123

5.9 Plot of solutions to system (4.35) illustrating a stable disease-free equilibrium

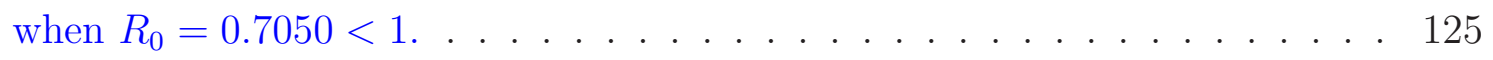

5.10 Plot of solutions to system (4.35) depicting oscillatory dynamics when $R_{0}=$

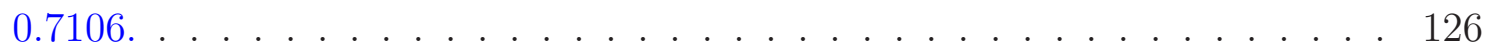

5.10 .1 Plot of proportion of infectious humans. . . . . . . . . . 126

5.10 .2 Plot of $u_{s}$ against time. . . . . . . . . . . . . . . 126

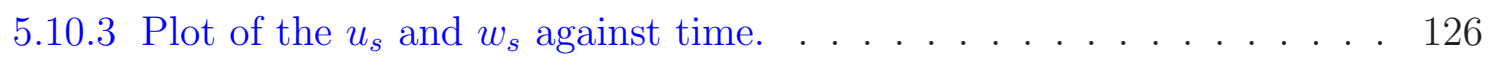

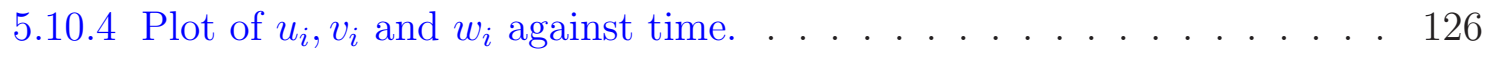

5.11 Unique endemic equilibrium solution in Region (I) . . . . . . . . . . 127

5.12 Plot of the unique endemic equilibrium solution that exists when $A_{1}=0, A_{2}>$

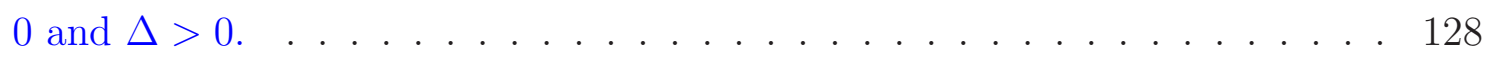

5.13 Plot of unique endemic equilibrium solution in Region (III) of Fig. 5.6. . . 129

5.14 Plot of unique endemic equilibrium solution in Region (III) of Fig. 5.6. . . . 130

5.15 Typical time series plots illustrating oscillatory dynamics in Region (III) of

Fig. 5.6. . . . . . . . . . . . . . . . . . . . . . . . . 132

5.15 .1 Plot of proportion of infectious humans against time. . . . . . . 132

5.15 .2 Plot of $u_{s}$ against time. . . . . . . . . . . . . . . . 132

5.15 .3 Plot of the $u_{s}$ and $w_{s}$ against time. . . . . . . . . . . 132

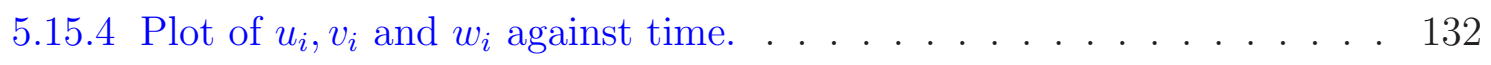

5.16 Limit cycle solutions of system $(4.35) \ldots \ldots \ldots \ldots$

5.16 .1 Plot of $I$ against $u_{s} \ldots \ldots \ldots \ldots \ldots \ldots \ldots \ldots$

5.16 .2 Plot of $I$ against $v_{s} \ldots \ldots \ldots \ldots \ldots \ldots \ldots \ldots$

5.16 .3 Plot of $I$ against $w_{s} \ldots \ldots \ldots \ldots \ldots \ldots \ldots \ldots$

5.16 .4 Plot of $I$ against $u_{i} \ldots \ldots \ldots \ldots \ldots \ldots \ldots \ldots$ 
5.16 .5 Limit cycle: Plot of $I$ against $v_{i} \ldots \ldots \ldots \ldots \ldots \ldots \ldots$

5.16 .6 Plot of $I$ against $w_{i} \ldots \ldots \ldots \ldots \ldots \ldots \ldots$

5.17 Total mosquito population in the presence of the disease. . . . . . . . . . . 134

5.18 Plot of system (4.35) when $A_{1}>0, A_{2}=0$ and $\Delta>0 \ldots \ldots \ldots$

5.19 Plot of system (4.35) when $A_{1}>0, A_{2}<0$ and $\Delta=0 \ldots \ldots 136$

5.20 Backward bifurcation plot . . . . . . . . . . . . . . . . 145

5.21 Oscillations with Verhulst-Pearl birth rate function. . . . . . . . . . . . 148

5.22 Oscillations with the Maynard-Smith-Slatkin birth rate functions. . . . . . . 149

7.1 A schematic framework illustrating the transition of individuals between classes.169

7.2 A sketch of the transmission and recovery rate functions. . . . . . . . . . 172

7.3 Path to high income-low disease prevalence stable equilibrium solution. . . . 176

7.4 Path to low income-high disease prevalence equilibrium solution. . . . . . . 177

7.5 Deterministic plot showing two different paths. . . . . . . . . . . . 178

7.6 Phase plot illustrating the effect of initial conditions on the deterministic model.180

7.7 Schematic framework for the stochastic model. . . . . . . . . . . . . . . . 181

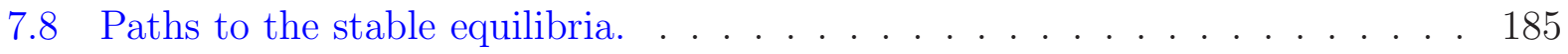

7.8.1 One possible path to the stable development equilibrium point. . . . . 185

7.8.2 Second path to the stable development equilibrium point. . . . . . . . 185

7.8 .3 One possible path to the poverty trap . . . . . . . . . 185

7.8.4 Second path to the poverty trap . . . . . . . . . . . . . 185

7.9 An illustration of the probability of attaining and exiting the poverty trap. . 186

7.10 Shortest path to the high income-low prevalence equilibrium from the poverty trap. . . . . . . . . . . . . . . . . . . . . 187

7.11 Stochastic simulations . . . . . . . . . . . . . . . . . . . . 189

7.12 Stochastic simulations . . . . . . . . . . . . . . . . . . 190

7.13 Probability of attaining the poverty trap equilibrium or development equilibrium. . . . . . . . . . . . . . . . . . . . . . 191

7.14 Probability of attaining the poverty trap equilibrium or development equilib-

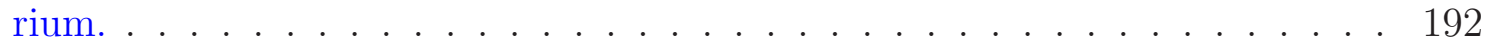


7.15 Income safety net. . . . . . . . . . . . . . . . . . . . 194

7.16 Health safety net. . . . . . . . . . . . . . . . . . . 195

7.17 Income and health safety net. . . . . . . . . . . . . . . 196

7.18 Time to attain the development equilibrium. . . . . . . . . . . 198

8.1 Bifurcation diagram for the two-variable autocatalator model . . . . . . . . . 213

8.2 Two-variable autocatalator plots . . . . . . . . . . . . . . . . . . . . . 214

8.2.1 Two-variable autocatalator time series plot . . . . . . . . . . . . 214

8.2 .2 Two-variable autocatalator phase plot . . . . . . . . . . . . . . . 214

8.3 Typical time series plots of the three-variable autocatalator model . . . . . 218

8.3.1 Time series for $\mu=0.016$ showing period-1 behavior. . . . . . . . . . . 218

8.3.2 Time series for $\mu=0.143$ showing period-2 behavior. . . . . . . . . 218

8.3.3 Time series for $\mu=0.153$ showing period-4 behavior. . . . . . . . . . 218

8.3.4 Time series for $\mu=0.154$ showing chaotic behavior. . . . . . . . . . . 218

8.4 Typical attractors of the three-variable autocatalator model . . . . . . . 219

8.4.1 Phase plot for $\mu=0.016$ showing period-1 behavior. . . . . . . . . . . 219

8.4.2 Phase plot for $\mu=0.143$ showing period-2 behavior. . . . . . . . . . . 219

8.4.3 Phase plot for $\mu=0.153$ showing period-4 behavior. . . . . . . . . . . 219

8.4.4 Phase plot for $\mu=0.154$ showing chaotic behavior. . . . . . . . . . 219

8.5 Bifurcation diagram of the three-variable autocatalator model . . . . . . . . 220

8.6 Lyapunov exponents . . . . . . . . . . . . . . . . . . . . . . . . . . 222

8.7 Illustration of sensitive dependence on initial conditions . . . . . . . . . . 223

8.7 .1 Small initial divergence . . . . . . . . . . . . . . . . . . . . 223

8.7 .2 Large divergence over time . . . . . . . . . . . . . . . . . . 223

8.8 First recurrence map . . . . . . . . . . . . . . . . . . . . . . . . . . . . . . 224

9.1 Time series plots of the coupled six-variable autocatalator model. . . . . . . 245

9.1.1 Time series for $y_{02}=4.0$ showing chaotic behavior. . . . . . . . . . 245

9.1.2 Time series for $y_{02}=5.2$ showing period-4 behavior. . . . . . . . . . 245

9.1.3 Time series for $y_{02}=6.5$ showing period-2 behavior. . . . . . . . . . . 245

9.1.4 Time series for $y_{02}=8.0$ showing period-1 behavior. . . . . . . . . . 245 
9.2 Attractors of the coupled six-variable autocatalator model. . . . . . . . . . 246

9.2.1 Phase plot for $y_{02}=4.0$ showing a chaotic attractor. . . . . . . . . . 246

9.2.2 Phase plot for $y_{02}=5.2$ showing a period-4 attractor. . . . . . . . . . . 246

9.2.3 Phase plot for $y_{02}=6.5$ showing a period-2 attractor. . . . . . . . . . . 246

9.2.4 Phase plot for $y_{02}=8.0$ showing a period-1 limit cycle. . . . . . . . . 246

9.3 Long term dynamics of the coupled six-variable autocatalator model. . . . . 247

9.4 Difference plots for the variables $x_{1}$ and $x_{2} \ldots \ldots \ldots \ldots$. . . . . . . 249

9.4.1 Difference in $x$ within the chaotic regime. . . . . . . . . . . . . . . . . 249

9.4.2 Difference in $x$ within the period-4 regime. . . . . . . . . . . . . . 249

9.4.3 Difference in $x$ within the period-2 regime. . . . . . . . . . . . . . . . . 249

9.4.4 Difference in $x$ within the period-1 regime. . . . . . . . . . . . . . 249

9.5 Difference plots for the variables $y_{1}$ and $y_{2} \ldots \ldots \ldots$. . . . . . . . . . 250

9.5.1 Difference in $y$ within the chaotic regime. . . . . . . . . . . . . . 250

9.5.2 Difference in $y$ within the period-4 regime. . . . . . . . . . . . . 250

9.5.3 Difference in $y$ within the period-2 regime. . . . . . . . . . . 250

9.5.4 Difference in $y$ within the period-1 regime. . . . . . . . . . . . . . . . . 250

9.6 Difference plots for the variables $z_{1}$ and $z_{2} \ldots \ldots \ldots$. . . . . . . . 251

9.6.1 Difference in $z$ within the chaotic regime. . . . . . . . . . . . . . . 251

9.6 .2 Difference in $z$ within the period-4 regime. . . . . . . . . . . . . . 251

9.6.3 Difference in $z$ within the period-2 regime. . . . . . . . . . . . . . . 251

9.6.4 Difference in $z$ within the period-1 regime. . . . . . . . . . . . . . . 251

9.7 A sampling of the synchronization manifolds as a function of the conserved quantity. . . . . . . . . . . . . . . . . . 253

9.8 A sampling of the synchronization manifolds as a function of the initial condition. 254

9.9 Reduced system bifurcation diagram . . . . . . . . . . . . . . . . 256

9.10 Dynamics of Lyapunov exponents of the coupled six-variable autocatalator model. . . . . . . . . . . . . . . . . . . . . . . 258

9.11 Dynamics of largest Lyapunov exponents. . . . . . . . . . . . . . . . . . 259

$9.120 .01 \%$ parameter mismatch . . . . . . . . . . . . . . . 263

$9.130 .05 \%$ parameter mismatch . . . . . . . . . . . . . . . 264 
9.14 Time series plots for a $0.5 \%$ parameter mismatch. . . . . . . . . . 265

9.14.1 Time series for a $0.5 \%$ parameter mismatch. . . . . . . . . . 265

9.14.2 Phase plot for a $0.5 \%$ parameter mismatch. . . . . . . . . . . . 265

9.15 Phase plots for a $0.5 \%$ parameter mismatch . . . . . . . . . . . 266

9.15.1 Phase plot for a $0.5 \%$ parameter mismatch. . . . . . . . . . . 266

9.15.2 Phase plot for a $0.5 \%$ parameter mismatch. . . . . . . . . . . . 266

9.16 Squeezing of dynamics for increasing mismatches. . . . . . . . . . . . . 267

10.1 Long-term dynamics of the coupled six-variable system as a function of $\mu$. 279

10.2 Long-term dynamics of the coupled six-variable model as a function of $y_{02}$. . 281

10.3 Second coupled autocatalator model time series plots . . . . . . . . . . . . 282

10.3.1 Time series for $y_{02}=0.5$ showing period-1 behavior. . . . . . . . . . 282

10.3.2 Time series for $y_{02}=2.0$ showing period-2 behavior. . . . . . . . . . 282

10.3.3 Time series for $y_{02}=6.7$ showing period-4 behavior. . . . . . . . . . . 282

10.3.4 Time series for $y_{02}=7.325$ showing chaotic behavior. . . . . . . . . . 282

10.4 Typical attractors of the second coupled autocatalator model . . . . . . . . 283

10.4.1 Phase plot for $y_{02}=0.5$ showing period-1 behavior. . . . . . . . . 283

10.4.2 Phase plot for $y_{02}=2.0$ showing period-2 behavior. . . . . . . . . 283

10.4.3 Phase plot for $y_{02}=6.7$ showing period-4 behavior. . . . . . . . . 283

10.4.4 Phase plot for $y_{02}=7.325$ showing chaotic behavior. . . . . . . . . . . 283

10.5 A sampling of the synchronization manifolds as a function of the conserved

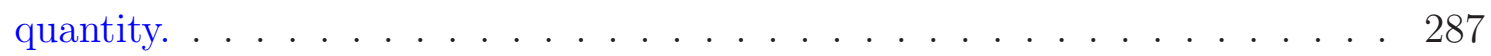

10.6 Typical time series and phase plots of the reduced system. . . . . . . . . . 289

10.6.1 Time series for $c=-1.5$ showing period-1 behavior. . . . . . . . . . . 289

10.6.2 Phase plot for $c=-1.5$ showing period-1 behavior. . . . . . . . . . 289

10.6.3 Time series for $c=0.0$ showing period-2 behavior. . . . . . . . . . . 289

10.6.4 Phase plot for $c=0.0$ showing period-2 behavior. . . . . . . . . 289

10.6.5 Time series for $c=5.2$ showing chaotic behavior. . . . . . . . . . . 289

10.6.6 Phase plot for $c=5.2$ showing chaotic behavior. . . . . . . . . . 289

10.7 Bifurcation diagram of the reduced autocatalator model. . . . . . . . . . . 290 
10.8 Difference plots for the variables $y_{1}$ and $y_{2} \ldots \ldots \ldots \ldots$

10.8.1 Difference in $y$ within the chaotic regime. . . . . . . . . . . . 292

10.8.2 Difference in $y$ within the period-2 regime. . . . . . . . . . . . 292

10.8.3 Difference in $y$ within the period-1 regime. . . . . . . . . . . . 292

10.8.4 Difference in $y$ within the stable steady state regime. . . . . . . . . 292

10.9 Difference plots for $x_{1}$ and $x_{2}$ and $z_{1}$ and $z_{2} \ldots \ldots \ldots 293$

10.9.1 Complete synchronization of $x_{1}$ and $x_{2}$ within the chaotic regime. . . 293

10.9.2 Complete synchronization of $z_{1}$ and $z_{2}$ within the chaotic regime. . . 293

10.9.3 Complete synchronization of $x_{1}$ and $x_{2}$ within the period-2 regime. . 293

10.9.4 Complete synchronization of $z_{1}$ and $z_{2}$ within the period-2 regime. . . 293

10.9.5 Complete synchronization of $x_{1}$ and $x_{2}$ within the period-1 regime. . 293

10.9.6 Complete synchronization of $z_{1}$ and $z_{2}$ within the period-1 regime. . . 293

10.10 The three largest Lyapunov exponents of the coupled system . . . . . . . 294

10.11 Long term dynamics of the third coupled autocatalator system. . . . . . . 297

10.12 Long term dynamics of the fourth coupled autocatalator system. . . . . . 300

F.1 Schematic framework of an SIR disease model. . . . . . . . . . . . . . . 329

F.2 Simulation results with $\mu=0 \ldots \ldots \ldots \ldots \ldots \ldots \ldots \ldots$

F.3 Solution of system with vital dynamics . . . . . . . . . . . . . . 332

F.3.1 Time series . . . . . . . . . . . . . . . . . . . . . . . 332

F.3.2 Phase plot . . . . . . . . . . . . . . . . . . . . . . . . . . 332

F.4 Stable spiral and period-1 behavior. . . . . . . . . . . . . . . . . . . 334

F.4.1 Stable equilibrium solution. . . . . . . . . . . . . . . . . . 334

F.4.2 Stable spiral. . . . . . . . . . . . . . . . . . . . . . . . . . . 334

F.4.3 Time series showing period-1. . . . . . . . . . . . . . . . 334

F.4.4 Limit cycle. . . . . . . . . . . . . . . . . . . . . . . . . 334

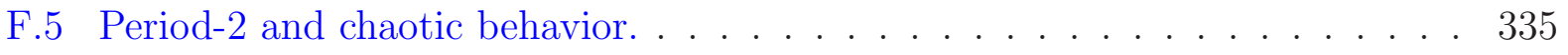

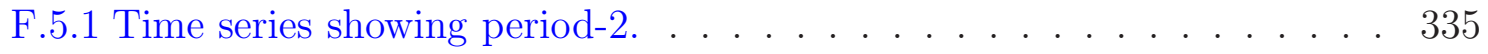

F.5.2 Phase plot showing Period-2. . . . . . . . . . . . . . . . 335

F.5.3 Time series showing chaos. . . . . . . . . . . . . . 335 
F.5.4 Chaotic attractor. . . . . . . . . . . . . . . . . . . . 335

F.6 Schematic framework for the stochastic model. . . . . . . . . . . . . . 337

F.7 Stochastic model plots for different runs. . . . . . . . . . . . . . . . 339

F.7.1 Results for run number $1 . \ldots \ldots 39$

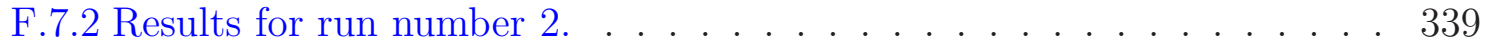

F.7.3 Results for run number $3 . \ldots$. . . . . . . . . . . . . . . . . . . . . 339

F.7.4 Results for run number $4 . \ldots \ldots 39$

F.8 Simulation results for the ode model using ODESEIRSMain.m. . . . . . . . . 344

F.9 Solution of the ode model in cell $(1,1) \ldots \ldots$. . . . . . . . . . . . . 345

F.10 Plot of solutions within different cells. . . . . . . . . . . . . . . . . 346 


\section{List of Tables}

4.1 Model variables and their definitions. . . . . . . . . . . . . . . . 67

4.2 System parameters . . . . . . . . . . . . . . . . . . . . 68

5.1 Summary of conditions for the existence of realistic equilibrium solutions and the number of realistic equilibrium solutions. . . . . . . . . . . . . 111

5.2 Physically meaningful ranges of parameter values for system (4.35) . . . . . . 120

5.3 Numerical exploration of the existence and stability of endemic equilibria . . 121

7.1 Model parameters . . . . . . . . . . . . . . . . . . . . . . . . 168

7.2 Model parameters . . . . . . . . . . . . . . . . . . . . . . . . . 168

8.1 Stability examples for the 3 -variable autocatalator model . . . . . . . . . . . 230

9.1 An illustration of the shift in dynamical behavior. . . . . . . . . . . . . 264

F.1 Summary of events and transition probabilities. . . . . . . . . . . . 338

F.2 Parameter values for the SEIRS malaria model. . . . . . . . . . . . . . . 342

G.1 Latex Code and Figures . . . . . . . . . . . . . . . . . . . . . . . . . . 349

G.2 MATLAB Codes and Figures . . . . . . . . . . . . . . . . . . . 349 
$\overline{\text { Chapter }} 1$

\section{Introduction and General Review of Dynamical System Concepts}

\subsection{Introduction}

This dissertation focuses on three interdisciplinary areas of applied mathematics, mathematical biology/epidemiology, economic epidemiology and mathematical physics, interconnected by the notions and applications of dynamical systems. In mathematical biology, we shall study the dynamics of malaria transmission through a new modeling framework in which the malaria vector population is accounted for at each of its developmental stages. In economic epidemiology, we shall explore the interplay between per capita income and infectious disease prevalence, and propose intervention strategies that can enable societies to escape from traps of disease and poverty. In mathematical physics we shall investigate an unusual form of multistability involving the coexistence of an infinite number of attractors that arises from a new class of dynamical systems obtained by coupling chaotic systems in a special way. We organize the dissertation as follows:

In Part I, we propose and study a new deterministic SIS (Susceptible-Infectious-Susceptible) model for the dynamics of malaria transmission that explicitly integrates mosquito demography together with its interaction with the human population and the disease dynamics. This model differs from standard SIS models in that the mosquito population is further subdivided into unfed and resting mosquitoes present at mosquito breeding sites, unfed and fertilized mosquitoes questing for human blood meals in human habitats, and fed and reproducing 
mosquitoes returning from human habitats to mosquito breeding sites. The mosquitoes involved and accounted for in disease transmission are only the female mosquitoes that are questing for human blood. Our main goals are to capture the natural oscillations that are known to exist in the prevalence of malaria without recourse to external seasonal forcing, compute a new basic reproduction number for malaria and compare it with that for the classical Ross' model [1], expose possible reasons why malaria eradication is difficult, and propose possible areas of malaria control based on our analysis.

The interplay between income and infectious disease prevalence is examined in Part ??. Here, we use standard SIS deterministic and stochastic models in which the contact and recovery rates depend on income and an additional equation that models income to provide a theoretical structure for understanding general feedback between income and disease prevalence. We show that both models possess three equilibrium solutions, a stable high income-low disease prevalence equilibrium (also called development equilibrium), a stable low income-high disease burden equilibrium (poverty trap), and an unstable intermediate income-intermediate disease equilibrium. We also illustrate that with stochasticity, the system approaches the development equilibrium for some simulations and the poverty trap for others for the same set of parameters and initial data. Hence, the stable equilibrium point to which the system relaxes in the long-term is not fully determined by the initial conditions as is the case with determinism. Furthermore, we demonstrate that deterministic models can result in poverty traps that can be broken only by substantial external changes to the initial epidemiological and economic conditions, whereas in stochastic models there is always some probability that a population will leave or enter a poverty trap. Finally, we use the stochastic model to show that a safety net, defined as an externally enforced minimum level of health care or economic condition below which a population is not allowed to fall, can guarantee ultimate escape from the poverty trap even if the safety net is set within the basin of attraction of the poverty trap or if the safety net is imposed only in the form of a public health or economic measure. We conclude this part with some policy interpretations, one of which answers the question, "which is the most effective method to enable populations escape from disease-driven poverty traps: provide the populations with money/money generating resources, health care, or both money/money generating resources and health care?" 
In Part III, we investigate two coupled chemical systems possessing an infinite number of coexisting attractors. We show how four different coupling schemes for the same dynamical system yield the dynamics of extreme multistability. In all four cases, this phenomenon is related to generalized synchronization of the two coupled systems as well as the emergence of a conserved quantity. Using the first coupling scheme, we show that the conserved quantity is connected with a conservation law in which all intermediate chemical reactants are weighted by their reaction time constants and is therefore given by the initial concentrations of the intermediates. This is also true for the fourth coupling scheme. For the second and third coupling schemes, this conserved quantity appears dynamically only in the long-term limit as time approaches infinity. Hence, the dependence on the initial conditions is more complex. We show that due to the emergence of this conserved quantity the state space is divided into submanifolds, each of them characterized by a certain value of the conserved quantity as well as a particular attractor. Since the conserved quantity can take any real value we obtain infinitely many attractors of different periods.

We now present a brief overview of some basic concepts of dynamical systems.

\subsection{General Review of Dynamical System Concepts}

Here, we briefly review some basic concepts of dynamical systems that will be essential in our studies. These include the notions of equilibria, attractors, multistability, bifurcations, chaos, dissipative systems, conservative systems and synchronization.

\subsubsection{Dynamical Systems}

Living and most physical systems interact with other systems and evolve with time. These are examples of dynamical systems. Dynamical systems are generally concerned with the time evolution of processes in response to specified rules. Such systems can either be discrete, evolving in discrete time, or continuous, evolving in continuous time [2]. Discrete dynamical systems are defined over the set of integers and they usually assume the form

$$
x_{n+1}=f\left(x_{n}\right)
$$


where $f=\left(f_{1}, f_{2}, f_{3}, \ldots, f_{n}\right), x_{i}=\left(x_{i}^{(1)}, x_{i}^{(2)}, x_{i}^{(3)}, \ldots, x_{i}^{(N)}\right),(i=n, n+1), n$ is the current time and $n+1$ is the future time. Notice that time here is measured in terms of discrete intervals such as days, years, generations, etc. and that equation (1.1) represents a system of $N$ difference equations. On the other hand, continuous dynamical systems are defined over the set of real numbers and they are usually described by differential equations of the form

$$
\dot{x}=f(x(t))
$$

where $x, f \in \mathbb{R}^{n}$ and the dot on the variables indicates differentiation with respect to time [3]. This is an $n$-dimensional system of ordinary differential equations. Continuous dynamical systems also include systems that change with both time and space. In fact, many interesting physical phenomena change with both time and space. For example, to model the transmission of a vector-borne disease, we might not be interested only at the evolution of the number or proportion of the vector or host with time but, more importantly, the evolution in time and distribution in space of the vector and/or host. This gives the system both a temporal and spatial structure.

In both equations (1.1) and (1.2), $x \in \mathbb{R}^{n}$ represent the state variables of the system while $f \in \mathbb{R}^{n}$ measure how fast the state variables change. Our studies shall be based entirely on continuous dynamical systems. The variables in the right-hand-side functions for each equation in (1.2) may depend on each other in a linear or nonlinear fashion. The nonlinear case is more interesting and leads to the theory of nonlinear dynamics, which has far reaching applications to real-world phenomena. With the passage of time, the theory of dynamical systems has gained strength in enhancing the understanding of many phenomena in disciplines such as engineering and the physical, biological, medical and social sciences. Examples of dynamical systems include population models such as the food chain and epidemiological models, chemical systems such as the Belousov-Zhabotinsky (BZ) reaction, motion of planets under gravitational forces, market prices, interest on bank loans, etc. [4]. See Refs. [3-6] for more information on dynamical systems.

Unfortunately, most dynamical systems that describe real-world phenomena are strongly nonlinear and analytical solutions can not easily be found in order to access their time evolution. Consequently, researchers resort to qualitative methods in order to acquire insights into 
the dynamical behaviors of such systems. Numerical techniques are also employed to access the different qualitative and quantitative behaviors of nonlinear dynamical systems. General qualitative methods in the theory of nonlinear dynamics include the determination and classification of equilibrium points, the study of the stability, appearance and disappearance of these equilibria, and the transitions between them as a system parameter is varied.

\subsubsection{Equilibria, Stability, Attractors and Bifurcations}

Due to nonlinearities, many dynamical systems cannot be solved analytically. To acquire insight into the long-term behavior of such systems, it is essential to begin by determining rudimentary solutions that can enable us to examine the behavior of all other solutions.

Definition 1.2.1 (Equilibrium Solution). Let $x^{*}, f \in \mathbb{R}^{n}$, then $x^{*}$ is an equilibrium or steady-state solution of the dynamical system defined by the autonomous system of first order ordinary differential equations (1.2) if $f\left(x^{*}\right)=0$ [7].

That is, an equilibrium solution or point of a dynamical system is a solution or point that does not evolve with time. Equilibrium solutions are also called fixed, steady-state, or stationary solutions. Dynamical systems can possess one or more equilibria. An equilibrium point is called hyperbolic if none of the eigenvalues of the corresponding linear system has a zero real part. Nodes, saddles and spirals are hyperbolic equilibria while centers are nonhyperbolic equilibria. For more on equilibria, see [8,9]. Trajectories that originate close to an equilibrium solution of a dynamical system can either stay close to the solution forever, stay close to the solution and eventually converge to it in the long run or, move away from the solution. This leads us to the concept of stability of solutions to dynamical systems. The concept of stability plays a crucial role in characterizing the long-term behavior of solutions to dynamical systems without necessarily solving the systems.

Definition 1.2.2 (Stable Solution). A solution $x^{*}(t) \in \mathbb{R}^{n}$ of the dynamical system (1.2) is said to be stable if given $\epsilon>0, \exists \delta\left(\epsilon, t_{0}\right)>0$ such that for $t \geq t_{0}$ and for any neighboring solution $x(t) \in \mathbb{R}^{n}$,

$$
\left\|x(t)-x^{*}(t)\right\|<\epsilon \text { whenever }\left\|x\left(t_{0}\right)-x^{*}\left(t_{0}\right)\right\|<\delta[7] \text {. }
$$


The solution $x^{*}(t) \in \mathbb{R}^{n}$ of a dynamical system is said to be locally stable if neighboring trajectories or solutions that originate close to $x^{*}(t)$ stay close it over time. The solution $x^{*}(t) \in \mathbb{R}^{n}$ is locally asymptotically stable if it is locally stable and slight or infinitesimal small perturbations from it decay to zero over time. Additionally, the solution $x^{*}(t) \in \mathbb{R}^{n}$ is globally asymptotically stable if it is stable and any perturbations from the solution decay to zero or if trajectories originating from any initial data eventually relax to the solution. The stability of hyperbolic equilibria of both linear and nonlinear dynamical systems can be completely determined from the eigenvalues of the corresponding linearized system. Such equilibria are asymptotically stable if the real components of all eigenvalues of the linearized system are negative and unstable if at least one of the eigenvalues of the linearized system has a negative real part. The process of investigating the stability of equilibria of a nonlinear dynamical system through the corresponding linear system is termed linear stability analysis. See Appendix A for the principle of linearized stability. This process turns out not to be very useful in the case of non-hyperbolic equilibria. Lyapunov functions are more useful in accessing the stability of non-hyperbolic equilibria. It is worth noting that Lyapunov functions can also be used for the case of hyperbolic equilibria. Moreover, when Lyapunov functions be found, they can easily be used to establish global stability of equilibria as opposed to linear stability analysis that deals more with local stability.

Definition 1.2.3 (Lyapunov Function). A positive definite scalar-valued function $V$ is a Lyapunov function for the dynamical system (1.2), if $\dot{V}$ is negative semi-definite or negative definite with respect to the dynamical system (1.2) [7].

Lyapunov functions provide a sufficient but not necessary condition for assessing the stability of equilibria without necessarily solving the system explicitly. As mentioned above, Lyapunov functions are also used for establishing global stability. Even though Lyapunov functions play a crucial role in establishing the stability of equilibria, there is, however, no general approach for constructing them [10]. Generally, one has to approach this through trial-an-error.

Definition 1.2.4 (Attractor). An attractor is defined as the smallest invariant ${ }^{1}$ closed set

\footnotetext{
${ }^{1} \mathrm{~A}$ set is invariant if solution curves that originate from the set remain in it [11]. Common examples of invariant sets include periodic orbits.
} 
$A \subseteq \mathbb{R}^{n}$ contained in an open set $\mathfrak{U}$ such that $\forall x_{0} \in \mathfrak{U}, d\left(A, x^{*}(t)\right) \rightarrow 0$ as $t \rightarrow \infty$ where $x^{*}(t)$ is the solution of the initial value value problem

$$
\dot{x}=f(x(t)), x(0)=x_{0} .
$$

From the above definition, it is clear that an attractor is a set in a dynamical system's phase space $^{2}$ where trajectories or points that get close to the set eventually approach it asymptotically in the long run irrespective of the initial conditions. Hence, equilibria that are asymptotically stable are attractors. An attractor can be a single point, for instance a single fixed point in one-dimensional space, a stable node or focus, a limit cycle ${ }^{3}$, a combination of two or more of the above or even a strange attractor (see Sec. 1.2.3). Some dynamical systems, especially coupled systems and systems in neuroscience, biology and physics may have two or more coexisting attractors $[12,13]$. Dynamical systems with two or more co-existing attractors for a specified set of parameters are said to exhibit multistability. The special case in which we have two co-existing attractors is referred to as bistability. Multistability can arise as a result of the coexistence of two or more stable fixed points, stable fixed points and a limit cycles, etc. Attractors cannot be discussed in isolation without invoking the notion of basin of attraction. By a basin of attraction or domain of attraction we are referring to the collection of all possible initial conditions of a dynamical system that converge to a particular attractor of the system over time. Basins of attraction become more complex for the case of multistable systems. In such systems, the basins of attraction may be riddled or even intermingled in extreme cases [14-16].

One other important aspect of dynamical systems that is worth mentioning is the notion of bifurcations. A variation in the value of a parameter in a dynamical system can trigger a change in the stability of an equilibrium point or a change in the number of equilibria. That is, as a system's parameter is varied, a dynamical system can witness a change in which a stable solution becomes unstable, an unstable solution becomes stable, previous solutions disappear or solutions with new qualitative properties emerge. Parameters that give rise to

\footnotetext{
${ }^{2}$ By phase space of a system, we are referring to the space of all possible state variables of the system.

${ }^{3} \mathrm{~A}$ limit cycle is an isolated closed orbit (or curve) in phase space that is not a member of a family of continuous curves. Many practical systems in chemistry and population dynamics exhibit periodic behavior. Note that the orbits of periodic solutions constitute limit cycles.
} 
changes in the qualitative behaviors of dynamical systems are called bifurcation parameters.

Definition 1.2.5 (Bifurcation). A bifurcation refers to a change in the qualitative properties of a dynamical system in response to changes in specific system parameters [17].

Generally, bifurcations can be classified as local or global. A local bifurcation refers to a change in the stability properties of equilibrium solutions of a dynamical system as the threshold value of a parameter in the system is traversed. A gain or loss of stability of equilibrium solutions of dynamical systems usually occurs at the same time at which equilibrium solutions either emerge or disappear. Hence, the above definition ties with the more general use of the word bifurcation to denote the emergence and departure of equilibria to dynamical systems. Many types of local bifurcations have been identified and explored. These include, transcritical bifurcations, period-doubling bifurcations, pitchfork bifurcations, saddle-node bifurcations, Hopf bifurcations, etc.

Definition 1.2.6 (Transcritical Bifurcation). A transcritical bifurcation occurs when an equilibrium solution of a dynamical system switches stability (from stable to unstable or vice versa) as the threshold value of a parameter in the system is traversed.

It is worth mentioning that at any point in time, there is always a stable and an unstable equilibrium point on either side of the threshold parameter mentioned in Definition 1.2.6.

Definition 1.2.7 (Saddle-node Bifurcation). A saddle-node bifurcation occurs when a stable node and an unstable saddle come together and annihilate one another.

For example, the one-dimensional equation $\dot{x}=r+x^{2}$ exhibits a saddle-node bifurcation.

Unlike a transcritical bifurcation, a saddle-node bifurcation witnesses a disappearance of equilibria. The single species logistic growth equation $\dot{x}=r x-x^{2}$ is a simple example of an equation that can be used to demonstrate the occurrence of a transcritical bifurcation.

Definition 1.2.8 (Period-doubling Bifurcation). A period-doubling bifurcation is one that result in the creation of new orbits of periodic solutions whose periods are two times those of the previous limit cycles from each bifurcation point. 
Period-1 oscillations have a single and regular maximum, period-2 oscillations have two alternating high and low or low and high maxima and so forth.

Another type of bifurcation that is witnessed mostly in symmetric systems is a pitchfork bifurcation. A consequence of pitchfork bifurcations is the emergence and disappearance of pairs of equilibria. Pitchfork bifurcations can be either supercritical or subcritical. In a supercritical pitchfork bifurcation, a stable equilibrium solution gives birth to two stable branches of equilibrium solutions that are symmetrical about the original equilibrium solution which is now unstable. In a subcritical pitchfork bifurcation, an unstable equilibrium solution gains stability accompanied by the emergence of a symmetric pair of unstable equilibria. Supercritical and subcritical pitchfork bifurcations are generally described in the literature through the prototype one-dimensional equations $\dot{x}=\mu x-x^{3}$ and $\dot{x}=\mu x+x^{3}$, respectively. See Refs. [7, 11] for an extensive exploration of these local bifurcations.

Definition 1.2.9 (Hopf Bifurcation). A Hopf bifurcation occurs when the real part of a pair of imaginary eigenvalues of the linearized version of a nonlinear system switches from negative to positive (or moves from the left half plane to the right half plane).

A Hopf bifurcation results to the emergence of periodic solutions. A Hopf bifurcation can either be supercritical, in which case a stable spiral switches to an unstable spiral that is enclosed within a stable limit cycle or subcritical, in which case the ensuing limit cycle is unstable. See, for example, Refs. [11, 18] for a formal definition of the Hopf bifurcation and a detailed discussion on bifurcations. Bifurcation diagrams depicting pitchfork bifurcations are analogous to those depicting Hopf and period-doubling bifurcations.

A global bifurcation arises as a result of a collision between equilibrium solutions of a dynamical system and larger invariant sets of the system. Examples of global bifurcations include homoclinic bifurcations, in which there is a collision between a saddle and a limit cycle, heteroclinic bifurcations, in which there is a collision between at least two saddles and a limit cycle, infinite-period bifurcations, which involve the appearance of both a saddle and a stable node on a limit cycle, and chaotic attractors [19]. Compared to local bifurcations, global bifurcations are complex and can not easily be characterized through stability analysis.

Points at which bifurcations occur are called bifurcation points. Bifurcations are com- 
monly illustrated on diagrams referred to as bifurcation diagrams. On bifurcation diagrams, the long-term maximum value, minimum value, average value, frequency, etc., of a variable of interest is plotted on the ordinate against the bifurcation parameter, on the abscissa. Solid lines are typically utilized to denote stable equilibria while broken lines are used to denote unstable equilibria. See Refs. [8, 9, 11, 20] for more information on bifurcations.

\subsubsection{Chaos and Strange Attractors}

The wide range of dynamical behavior arising from nonlinear dynamical systems has attracted much attention in the scientific world. One such behavior is chaos.

Definition 1.2.10 (Chaos). Chaos is deterministic aperiodic motion that is complex but ordered, unpredictable in the long run and manifests sensitivity to initial conditions [11, 21].

Each of the key words in the definition 1.2.10 has a specific meaning. The word deterministic denotes the existence of a governing rule to the motion. Even though chaotic systems show irregularity in order or pattern, they are not random. Aperiodicity indicates that there is no repetition in the motion or behavior as is the case for periodic behavior. Effects of slight disturbances persist in aperiodic systems. Sensitive dependence on initial conditions indicates that solution curves or trajectories that originate close to each other move further and further away from each other over time. This aspect of chaos can be quantified through the largest Lyapunov exponent. A positive largest Lyapunov exponent indicates that nearby trajectories grow further apart in an exponential fashion as time progresses, while zero and negative largest Lyapunov exponents denote limit cycles and fixed points respectively [22]. Sensitive dependence on initial conditions indicates that motion can be predicted initially and within a short time but not in the long-term [23].

Systems that exhibit chaos are said to be chaotic. Chaos surfaces in almost every discipline, mathematics, physics, chemistry, ecology, finance, etc. Some examples of well-studied chaotic systems include the Lorenz system [24], the Rössler model [25], the three-variable food chain model [26], the Hindmarsh-Rose neuron model [27], the BZ reaction in chemistry [11], etc. Chaotic systems generally attract questions with regard to the emergence of the chaos. This has given rise to many studies on the various routes to chaos. Identification and 
description of the route to chaos has been shown to play a crucial role in the interpretation of experimental and numerical observations. For instance, it may be quite difficult to decide whether an experimental system is chaotic as a result of errors or noise in the system. This difficulty can be resolved by understanding the route to chaos and by carrying out time series analysis [28]. A number of routes to chaos have been identified and investigated [3, 28, 29]. The simplest of these routes is period-doubling. Period-doubling usually culminates in chaos, whereby the period is infinite. In some systems, chaos is followed by reverse period-doubling.

The limit set of chaotic trajectories is termed a strange attractor $[11,30]$.

\subsubsection{Dissipative and Conservative Systems}

Many systems, such as open systems, interact with their environments. Interaction between systems and their environments may result in dissipation, loss, gain or conservation of certain properties of the system such as matter, energy, area, volume, etc. Systems in which such properties are dissipated or phase space volume shrinks over time are called dissipative systems [11]. A dissipative system supplies less energy or information to its surroundings than it absorbs $[31,32]$. We now quote, Willem's mathematical definition of a dissipative system [31]. The original variables in the definition have been changed in order to ensure consistency in the variables used in this chapter.

Definition 1.2.11 (Dissipative Systems). Let $(x, y, z) \in \mathbb{R}^{m} \times \mathbb{R}^{n} \times \mathbb{R}^{p}$ where $x, y$, and $z$ are, respectively, the state, output, and input values. Then the dynamical system

$$
\dot{x}=f(x, z), y=g(x, z)
$$

is said to be dissipative with respect to the function

$$
h: \mathbb{R}^{m} \times \mathbb{R}^{n} \rightarrow \mathbb{R}
$$

if there exists a function

$$
V: \mathbb{R}^{m} \rightarrow \mathbb{R}
$$

such that

$$
\dot{V}(x(.)) \leq h(z(.), y(.))
$$

for all $x(),. y(),. z($.$) in the set of all solutions (x, y, z): \mathbb{R} \rightarrow \mathbb{R}^{m} \times \mathbb{R}^{n} \times \mathbb{R}^{p}[31,33]$. 
Notice that the concept of dissipative systems in open systems generalizes Lyapunov theory in closed systems, with dissipation in the open systems corresponding to the notion of stability in the closed systems [33]. The long-term behavior of a dissipative system is characterized by an attractor that is the target of trajectories in the basin of attraction in phase space. Small perturbations of the system from these attractors decay to zero over time, and therefore, the long-term dynamics within the basin of attraction relax to the attractor. [21]. Dissipative systems generally emerge in physical contexts associated with energy dissipation, viscosity, friction, etc. [11]. Examples of dissipative dynamical systems include electrical circuits, mechanical systems, thermodynamic systems, the Belousov-Zhabotinsky (BZ) reaction, the autocatalator model, and the three-variable food chain model. Many studies have been carried out on dissipative systems, for example, see Refs. [31-38].

Although many physical or real-world systems are naturally dissipative, some are conservative or nearly conservative, for example, the solar system. In conservative systems, there is no dissipation or loss of energy or shrinking of phase space volume. Rather, these properties are conserved. Hence, properties of the system such as total energy are constant over time and, generally, Hamiltonian systems are conservative. In contrast to dissipative systems, the long-term behavior of conservative systems depends on initial conditions.

\subsubsection{Illustration of Conservative and Dissipative Systems: The Simple Pendulum}

Periodic motion is common in the real world, which may be due to an initial push or the effect of an external force, for example, swinging of tree leaves and branches under the influence of the wind, mechanical motion such as the motion of a mass-spring system, or the pendulum. The simple pendulum is one of the most fundamental nonlinear dynamical systems, which consists of a point mass attached to the lower end of a massless rod with its upper end fixed to a point. The simple pendulum exhibits periodic phenomena and its motion can be either conservative or dissipative depending on whether the system is undamped or damped. The motion of the simple pendulum is generally described by the following second-order ordinary differential equation:

$$
\ddot{\theta}+b \dot{\theta}+\frac{g}{l} \sin \theta=0 .
$$


Here, $\theta$ represents the angle (measured in radians) or the angular displacement, $l$ represents the length of the pendulum rod, $b>0$ is the ratio of the damping coefficient (or damping strength) to the mass of the pendulum bob, $g$ is the acceleration due to gravity and the dot on $\theta$ denotes differentiation with respect to time, $t$. An essential assumption made in the derivation of equation (1.4) is that the weight of the rod is negligible compared to the mass

of the point object. In preliminary studies of the dynamics of the simple pendulum, the approximation $\sin \theta \simeq \theta$, for small $\theta$ is used to simplify the nonlinear equation (1.4) into a linear equation whose solution can easily be determined analytically.

\subsubsection{Undamped Simple Pendulum (Undamped Oscillator)}

In the absence of friction and/or other damping forces, $b=0$ and Eq.. (1.4) reduces to

$$
\ddot{\theta}+\frac{g}{l} \sin \theta=0
$$

By setting $\dot{\theta}=\nu$ where $\nu$ represents the angular velocity of the simple pendulum, we can rewrite equation (1.5) as the following system of first order ordinary differential equations:

$$
\begin{aligned}
\dot{\theta} & =\nu, \\
\dot{\nu} & =-\frac{g}{l} \sin \theta .
\end{aligned}
$$

The equilibrium solutions of this system are $\left(\theta^{*}, \nu^{*}\right)=(0,0)$ and $\left(\theta^{*}, \nu^{*}\right)=(n \pi, 0)$, where $n \in \mathbb{Z}$ and the Jacobian matrix of the system evaluated at $\left(\theta^{*}, \nu^{*}\right)$ is

$$
J\left(\theta^{*}, \nu^{*}\right)=\left(\begin{array}{cc}
0 & 1 \\
-\frac{g}{l} \cos \theta^{*} & 0
\end{array}\right) .
$$

If $\lambda$ is an eigenvalue of $J$, then we have the following characteristic equation:

$$
\lambda^{2}+\frac{g}{l} \cos \theta^{*}=0
$$

At $\left(\theta^{*}, \nu^{*}\right)=(0,0), \lambda_{1,2}= \pm i \sqrt{g / l}$. Hence, the origin is a center, which is neutrally stable.

For the equilibrium solution $\left(\theta^{*}, \nu^{*}\right)=(n \pi, 0)$, we consider the case in which $n$ is even and the case in which $n$ is odd. The characteristic equation and, hence, the eigenvalues reduce to 
those obtained above when $n$ is even. Therefore, the equilibrium solution $(n \pi, 0)$ is a center for even $n$. For odd $n, \lambda_{1,2}= \pm \sqrt{g / l}$, and therefore, the equilibrium solution $(n \pi, 0)$ is a saddle point. Simulations confirming these characteristics are presented in Fig. 1.1.

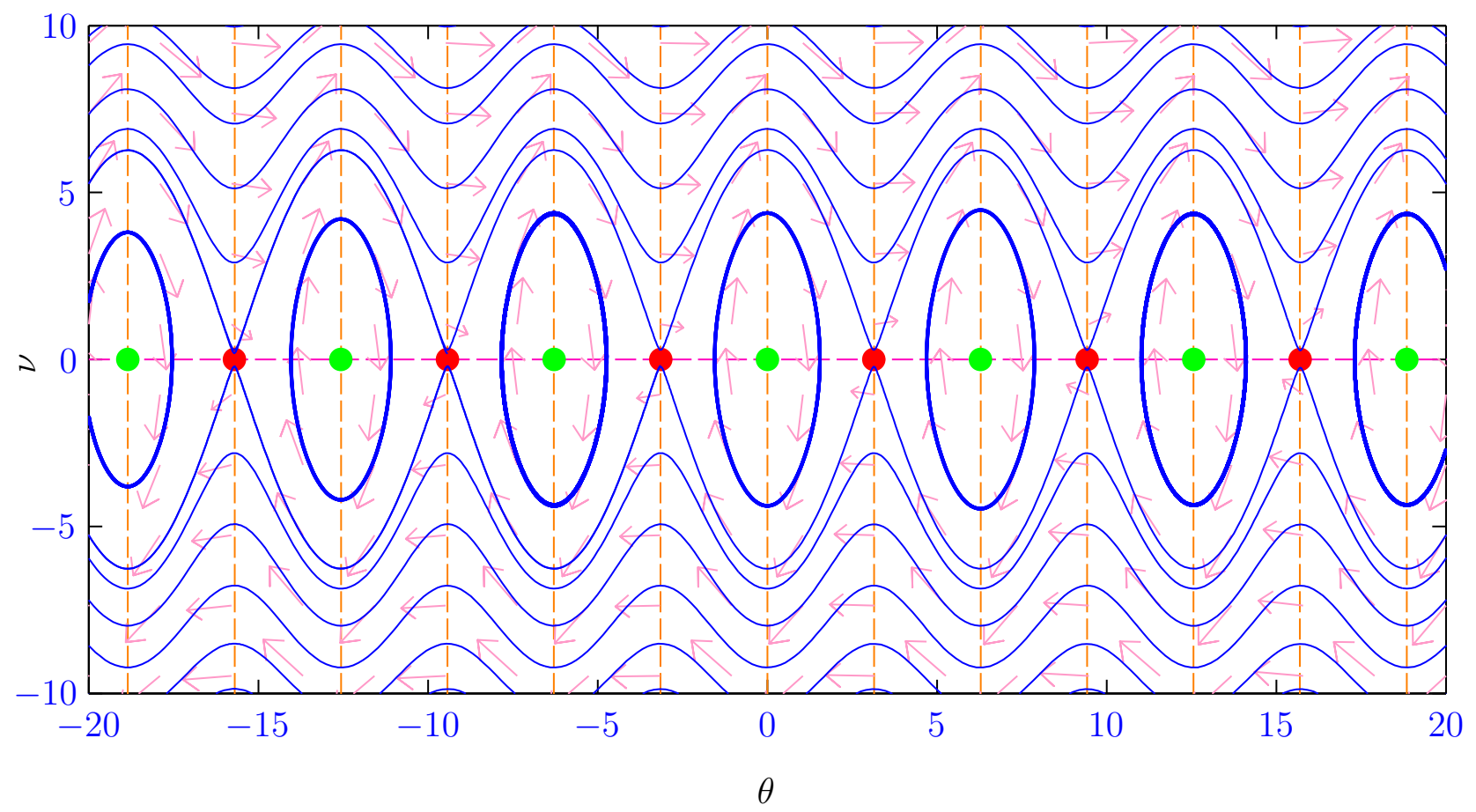

Figure 1.1. Simulation results for the undamped simple pendulum system with $g=9.81$ and $l=1$. The equilibrium solution $\left(\theta^{*}, \nu^{*}\right)=(n \pi, 0)$ is a center (denoted by green filled circles) for even $n$ and a saddle point (denoted by red filled circles) for odd $n$. Notice that the horizontal line that passes through the origin and the vertical lines are nullclines. Equilibria occur at the intersection of these nullclines. The arrowed lines represent the direction fields. 
The system (1.6)-(1.7) is conservative with a conserved quantity, the total energy $E$. One way to determine the equation for the phase paths is to divide equation (1.6) by equation (1.7) and solve the ensuing differential equation, which is separable in this particular case. The division yields

$$
\frac{d \theta}{d \nu}=\frac{\nu}{-\frac{g}{l} \sin \theta} .
$$

Separating the variables and integrating yields,

$$
\frac{1}{2} \nu^{2}=\frac{g}{l} \cos \theta+\text { Constant } \Leftrightarrow \frac{1}{2} \nu^{2}-\frac{g}{l} \cos \theta=\text { Constant }
$$

where $\frac{1}{2} \nu^{2}$ is the kinetic energy of the system and $\frac{g}{l} \cos \theta$ is the potential energy. Hence,

$$
E=\frac{1}{2} \nu^{2}-\frac{g}{l} \cos \theta=\text { Constant }
$$

The fact that all equilibrium solutions are only centers and saddles is consistent with the system being conservative or Hamiltonian since such systems do not possess nodes and foci.

Alternatively, we can use the standard approach of Jordan and Smith [7] to show that the system of equations (1.6)-(1.7) is Hamiltonian. To this effect, we need to find a function $E$ that satisfies the conditions

$$
\begin{aligned}
& \frac{\partial E}{\partial \nu}=\nu, \\
& \frac{\partial E}{\partial \theta}=\frac{g}{l} \sin \theta .
\end{aligned}
$$

Since $\frac{\partial}{\partial \theta}(\nu)+\frac{\partial}{\partial \nu}\left(-\frac{g}{l} \sin \theta\right)=0$, as each of the partial derivatives is clearly zero, $E$ is the Hamiltonian function of the system. Hence, if $\theta(t)$ and $\nu(t)$ are specific solutions, using the chain rule, we obtain the following governing equation for the associated phase path:

$$
\frac{d E}{d t}=\frac{\partial E}{\partial \theta} \frac{d \theta}{d t}+\frac{\partial E}{\partial \nu} \frac{d \nu}{d t}=\left(\frac{g}{l} \sin \theta\right) \nu+\nu\left(-\frac{g}{l} \sin \theta\right)=0
$$

Now,

$$
\frac{d E}{d t}=0 \Rightarrow E(\theta, \nu)=\text { Constant }
$$

Integrating (1.11) with respect to $\nu$ and (1.12) with respect to $\theta$ gives

$$
\begin{aligned}
& E(\theta, \nu)=\frac{1}{2} \nu^{2}+A(\theta) \\
& E(\theta, \nu)=\frac{g}{l} \cos \theta+B(\nu)
\end{aligned}
$$


where $A$ and $B$ are arbitrary functions of single variables. The only time the above two equations will be equal is when $A(\theta)=\frac{g}{l} \cos \theta-C$ and $B(\nu)=\frac{1}{2} \nu^{2}-C$, where $C$ is a constant. Hence, the phase paths of the system are characterized by the energy equation

$$
E(\theta, \nu)=\frac{1}{2} \nu^{2}-\frac{g}{l} \cos \theta=C
$$

\subsubsection{Damped Simple Pendulum (Damped Oscillator)}

This is the case in which the damping coefficient $b$ is non-zero. Again, by setting $\dot{\theta}=\nu$, we rewrite (1.4) as the following system of ordinary differential equations:

$$
\begin{aligned}
\dot{\theta} & =\nu, \\
\dot{\nu} & =-\frac{g}{l} \sin \theta-b \nu .
\end{aligned}
$$

Again, the equilibrium solutions of this system are $\left(\theta^{*}, \nu^{*}\right)=(0,0)$ and $\left(\theta^{*}, \nu^{*}\right)=(n \pi, 0)$, where $n \in \mathbb{Z}$. Linearizing equations (1.15)-(1.16) about $\left(\theta^{*}, \nu^{*}\right)$ yields the Jacobian matrix

$$
J\left(\theta^{*}, \nu^{*}\right)=\left(\begin{array}{cc}
0 & 1 \\
-\frac{g}{l} \cos \theta^{*} & -b
\end{array}\right) .
$$

If $\lambda$ is an eigenvalue of $J$, then we have the following characteristic equation:

$$
\lambda^{2}+b \lambda+\frac{g}{l} \cos \theta^{*}=0
$$

At the equilibrium solution $\left(\theta^{*}, \nu^{*}\right)=(0,0), \lambda_{1,2}=\left(-b \pm \sqrt{b^{2}-4 g / l}\right) / 2$, and $\lambda_{1}<0$ and $\lambda_{2}<0$ irrespective of whether $b^{2}-4 g / l<0$ or $b^{2}-4 g / l>0$. However, when $b^{2}-4 g / l<0$, the origin is a stable spiral, and when $b^{2}-4 g / l>0$ the origin is a stable node. Hence, the origin is asymptotically stable. Note that the origin is still stable when $b^{2}-4 g / l=0$.

For the equilibrium solution $\left(\theta^{*}, \nu^{*}\right)=(n \pi, 0)$, the case of even $n$ yields the same stability results as that of the origin. However, for the case of odd $n$, we have $\lambda_{1,2}=$ $\left(-b \pm \sqrt{b^{2}+4 g / l}\right) / 2$, and both eigenvalues are real with one positive and the other negative. Therefore, the equilibrium solution $(n \pi, 0)$, where $n$ is odd is a saddle. As opposed to the undamped system, we now have only spirals or nodes and saddles, and no centers. Simulation results are presented in Figs. 1.2-1.3. 


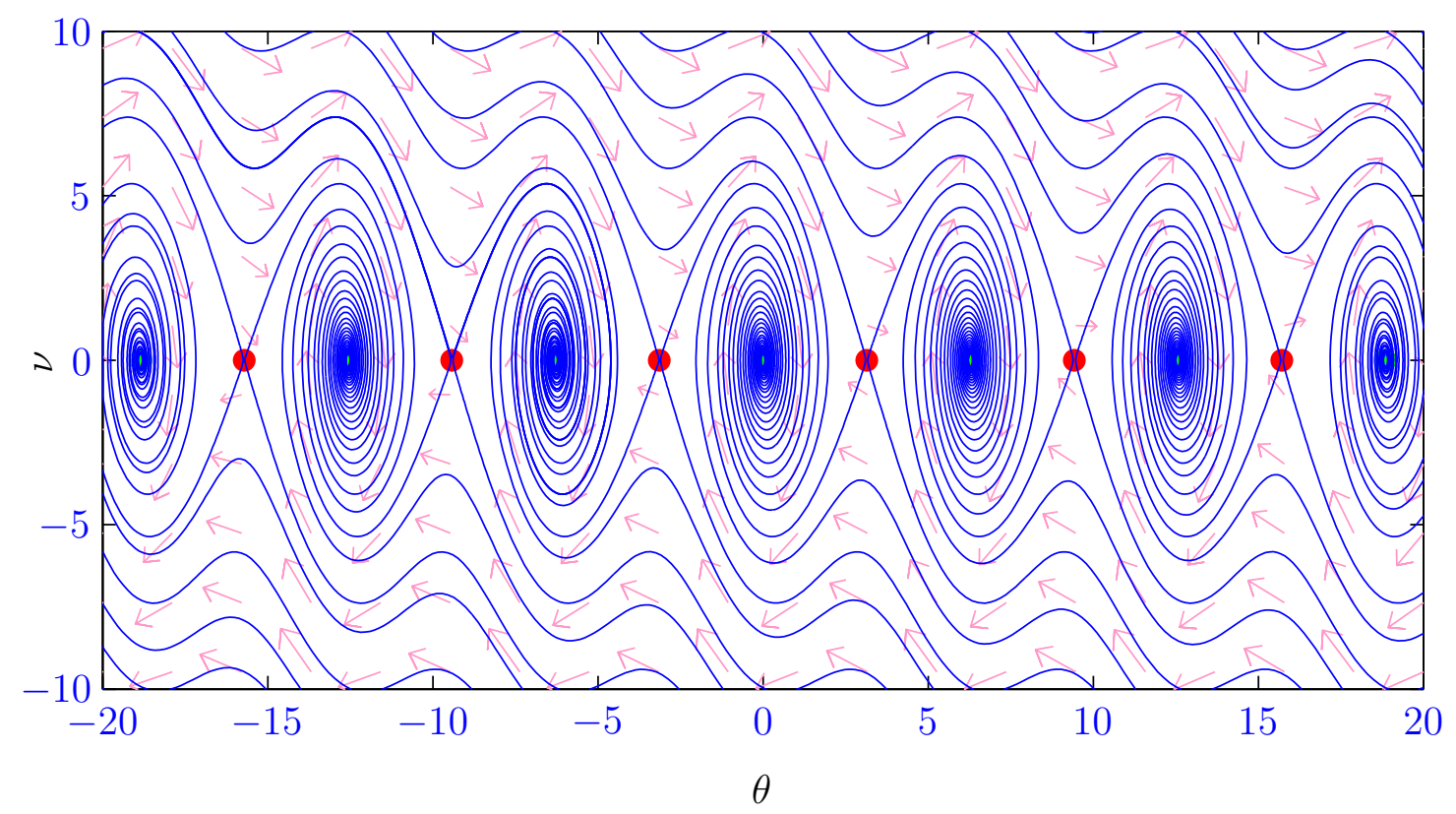

Figure 1.2. Simulation results for $g=9.81, l=1$ and $b=0.5$. Note that we have saddles (denoted by red filled circles) and stable foci (denoted by green filled circles), corresponding to the case in which $b^{2}-4 g / l<0$.

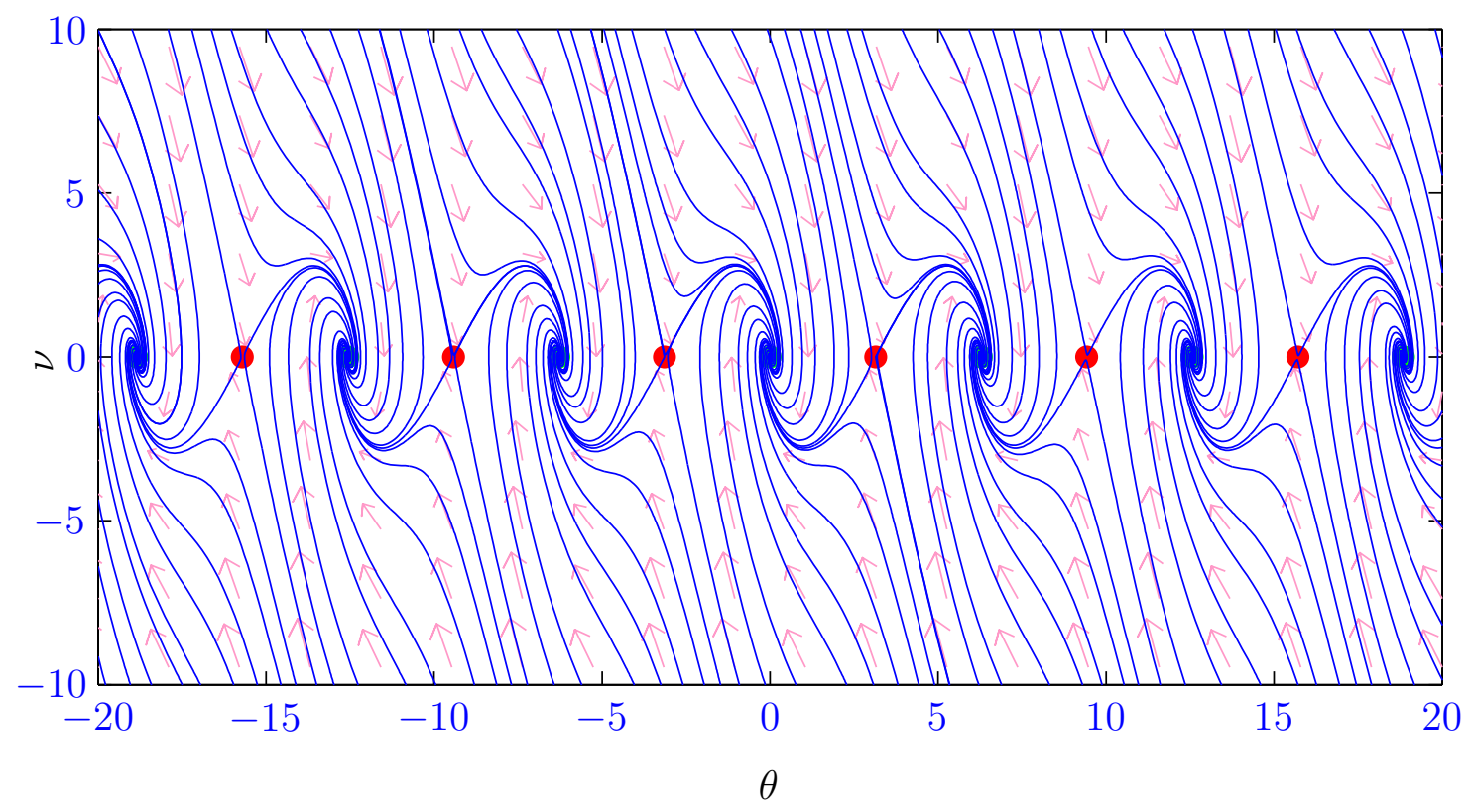

Figure 1.3. Simulation results for the damped simple pendulum system for $g=9.81, l=1$ and $b=3.0$. In this case, we have stable foci (denoted by green filled circles) and saddle points (denoted by red filled circles). 
The system (1.15)-(1.16) is dissipative, and we show that there is no conserved quantity [11]. Rewriting (1.4) as

$$
\ddot{\theta}+\frac{g}{l} \sin \theta=-b \dot{\theta}
$$

and multiplying through by $\dot{\theta}$ yields

$$
\dot{\theta}\left(\ddot{\theta}+\frac{g}{l} \sin \theta\right)=\dot{\theta}(-b \dot{\theta}) \Leftrightarrow \frac{d}{d t}\left(\frac{1}{2}\left(\frac{d \theta}{d t}\right)^{2}-\frac{g}{l} \cos \theta\right)=-b\left(\frac{d \theta}{d t}\right)^{2} .
$$

That is,

$$
\dot{E}=-b \dot{\theta}^{2} \leq 0
$$

Hence, the energy function $E$ is monotonically decreasing. Therefore, the total energy of the system is no longer constant, due to it being consumed in overcoming friction.

The analysis in Sections 1.2.4.2 and 1.2.4.3 indicates that the undamped simple pendulum is conservative. However, on introducing damping or friction, the system becomes dissipative.

\subsection{Synchronization}

Many natural phenomena, including human activities, occur in synchrony. Synchronization easily arises in coupled systems, and the notion is not restricted to physical motion in coupled dynamical systems. Synchronization also involves the dynamics of coupled systems that coincide in time or in which the motion of one subsystem seems to precede that of the other. Studies on synchronization have flourished since the days of Christiaan Hugens, the first scientist to perceive and communicate the phenomenon in $1665[39,40]$.

Generally, synchronization is a phenomenon in which the asymptotic behaviors of two or more coupled dynamical systems are in synchrony for coupling strength values beyond a certain threshold [41]. See Refs. [42, 43] for a mathematical definition of synchronization. Synchronization can be achieved in a number of ways, including varying the coupling strength and entrainment generated by external forcing. Introducing the notion of synchronization in chaotic systems turns out to be interesting and surprising since slight differences in initial conditions of chaotic systems are amplified over time. Chaos synchronization occurs when identical dynamical behavior is exhibited by two or more coupled 
chaotic dynamical systems [43-46]. Chaos synchronization has useful applications in engineering and it can also be helpfully in understanding some complex real-world systems. Like other types of synchronization, chaos synchronization can be triggered by external forcing or a variation in the coupling strength between the systems. Apart from chaos synchronization, other classes of synchronization such as complete or identical synchronization [47-49], general synchronization [50], phase synchronization [48, 51-53], lag synchronization [51, 54], and amplitude envelope synchronization [55] have been identified and studied. 


\section{References}

[1] R. Ross, The Prevention of Malaria (John Murray, London, 1911). 2

[2] R. C. Robinson, An Introduction to Dynamical Systems: Continuous and Discrete (Prentice Hall, New York, 2004). 3

[3] E. Ott, Chaos in Dynamical Systems (Cambridge University Press, Cambridge, UK, 2002), 2nd edition. 4, 11

[4] A. Katok and B. Hasselbaltt, Introduction to the Modern Theory of Dynamical Systems (Cambridge University Press, Cambridge, UK, 1995). 4

[5] K. T. Alligood, T. Sauer, and J. A. Yorke, Chaos: An Introduction to Dynamical Systems (Springer-Verlag New York, Inc., USA, 1996).

[6] D. Li and X. Zhang, "On Dynamical Properties of General Dynamical Systems and Differential Inclusions," Journal of Mathematical Analysis and Applications 274, 705724 (2002).

Online Version 4

[7] D. W. Jordan and P. Smith, Nonlinear Ordinary Differential Equations. An Introduction to Dynamical Systems (Oxford University Press Inc., New York, USA, 1999). 5, 6, 9, 15

[8] Y. A. Kuznetsov, Elements of Applied Bifurcation Theory, volume 112 of Applied Mathematical Sciences (Springer-Verlag, New York, USA, 2004), 3rd edition. 5, 10 
[9] J. Guckenheimer and P. Holmes, Nonlinear Oscillations, Dynamical Systems and Bifurcations of Vector Fields, volume 42 of Applied Mathematical Sciences (Springer-Verlag, New York Inc., USA, 1983). 5, 10

[10] H. Khalil, Nonlinear Systems (Prentice Hall, Englewood Cliffs, NJ, 2002), 3rd edition. 6

[11] S. Strogatz, Nonlinear Dynamics and Chaos with Applications to Physics, Biology, Chemistry and Engineering (Persus Books Publishsing LLC, USA, 1994). 6, 9, 10, $11,12,18$

[12] U. Feudel, "Complex Dynamics in Multistable Systems," International Journal of Bifurcation and Chaos 18 (6), 1607-1626 (2008).

Online Version 7

[13] B. Goswami and A. Pisarchik, "Controlling Multistability by Small Perturbations," International Journal of Bifurcation and Chaos 18, 1645-1673 (2008).

Online Version 7

[14] E. Ott, J. C. Sommerer, J. C. Alexander, I. Kan, and J. A. Yorke, "Scaling Behavior of Chaotic Systems with Riddled Basins," Physical Review Letters 71, 4134-4137 (1993). Online Version 7

[15] Y. -C. Lai and R. L. Winslow, "Riddled Parameter Space in Spatiotemporal Chaotic Dynamic Systems," Physical Review Letters 72 (11), 1640-1644 (1994). Online Version

[16] M. D. Shrimali, A. Prasad, and R. Ramaswamy, "The Nature of Attractor Basins in Multistable Systems," International Journal of Bifurcation and Chaos 18 (6), 16751688 (2008).

Online Version 7

[17] P. Blanchard, R. L. Devaney, and G. R. Hall, Differential Equations (Thompson, London, 2006). 8 
[18] B. D. Hassard, N. D. Kazarinoff, and Y. N. Wan, Theory and Application of Hopf Bifurcation, London Mathematical Society Lecture Notes Series (Cambridge University Press, Cambridge, 1981). 9

[19] E. J. Doedel, B. Krauskopf, and H. M. Osinga, "Global Bifurcations of the Lorenz Manifold," Nonlinearity 19, 2947-2972 (2006).

Online Version 9

[20] J. D. Crawford, "Introduction to Bifurcation Theory," Reviews of Modern Physics 63, 991-1037 (1991).

Online Version 10

[21] T. Tél and M. Gruiz, Chaotic Dynamics. An Itroduction Based on Classical Mechanics (Cambridge University Press, New York, USA, 2006). 10, 12

[22] S. C. Manrubia, A. S. Mikhailov, and D. H. Zanette, Emergence of Dynamical Order. Synchronization and Phenomena in Complex Systems, volume 2 of World Lecture Notes in Complex Systems (World Scientific Publishing Co. Pte. Ltd., Singapore, 2004). 10

[23] D. Kaplan and L. Glass, Understanding Nonlinear Dynamics (Springer-Verlag New York Inc., USA, 1995). 10

[24] C. Sparrow, The Lorenz Equations: Bifurcations, Chaos, and Strange Attractors (Springer, New York, 1982). 10

[25] O. E. Rössler, "An Equation for Continuous Chaos," Physics Letters A 57, 397-398 (1976).

Online Version 10

[26] A. Hastings and T. Powell, "Chaos in a Three-species Food Chain," Ecology 72 (3), 896-903 (1991).

Online Version 10

[27] J. L. Hindmarsh and R. M. Rose, "A Model for Neural Bursting Using Three Coupled First Order Differential Equations," Proceedings of the Royal Society London, Series B 
221 (1222), 87-102 (1984).

Online Version 10

[28] P. Glendinning, Routes to Chaos. In: A. Scott, (eds). Encyclopedia of Nonlinear Science (Routldge, New york and London, 2005). 11

[29] J. B. H. Marcus, F. O. Lars, V. B. Tatiana, and M. S. William, "Routes to Chaos in the Peroxidase-Oxidase Reaction: Period-doubling and Period-adding," The Journal of Physical Chemistry B 101, 5075-5083 (1997).

Online Version 11

[30] D. Ruelle, "What is a Strange Attractor?" Notices of the American Mathematical Society 53 (7), 764-765 (2006).

Online Version 11

[31] J. C. Willem, "Dissipative Dynamical Systems Part I: General Theory," Archive for Rational Mechanics and Analysis 45 (5), 321-351 (1972).

Online Version 11, 12

[32] R. Lozano, B. Brogliato, O. Egeland, and B. Maschke, Dissipative Systems Analysis and Control: Theory and Applications (Springer-Verlag, London Limited, 2007), 2nd edition. 11

[33] J. Willems, "Dissipative Dynamical Systems," European Journal of Control 13, 134151 (2007).

Online Version 11, 12

[34] J. P. LaSalle, Dissipative Systems, in Ordinary Differential Equaions (Academic Press, New York, 1972).

[35] D. Hill and P. Moylan, "The Stability of Nonlinear Dissipative Systems," IEEE Transactions on Automatic Control 21 (5), 708-711 (1976).

Online Version 
[36] J. K. Hale, Asymptotic Behavior of Dissipative Systems (American Mathematical Society, USA, 1988).

Online Version

[37] O. Kaneko, P. Rapisarda, and K. Takaba, "Totally Dissipative Systems," Systems \& Control Letters 54 (7), 705-711 (2005).

Online Version

[38] W. Bia and M. Xiaowu, "Stability for Uncertain Nonlinear Dissipative Systems," Applied Mathematical Modelling 32 (12), 2768-2774 (2008).

Online Version 12

[39] C. H. Huygens, Horologium Oscillatorium (Paris, France, 1673). 18

[40] A. Pikovsky, M. Rosenblum, and J. Kurths, Synchronization: A Universal Concept in Nonlinear Sciences (Cambridge University Press, Cambridge, 2001). 18

[41] S. N. Chow, R. Johnson, R. Nussbaum, R. Conti, and J. Mallet-Paret, Dynamical Systems, Lecture Notes in Mathematics (Springer-Verlag, Heidelberg, 2003). 18

[42] R. Brown and L. Kocarev, "A Unifying Definition of Synchronization for Dynamical Systems," Chaos: An Interdisciplinary Journal of Nonlinear Science 10, 344-349 (2000). Online Version 18

[43] S. Boccaletti, J. Kurths, G. Osipovd, D. L. Valladares, and C. S. Zhou, "The Synchronization of Chaotic Systems," Physics Reports 366, 1-101 (2002).

Online Version 18, 19

[44] E. Barreto, K. Josić, C. J. Morales, E. Sander, and P. So, "The Geometry of Chaos Synchronization," Chaos: An Interdisciplinary Journal of Nonlinear Science 13 (1), 151-164 (2003).

Online Version

[45] J. Lü, X. Yu, and G. Chen, "Chaos synchronization of General Complex Dynamical Networks," Physica A: Statistical Mechanics and its Applications 334, 281-302 (2004). Online Version 
[46] J. M. González-Miranda, Synchronization and Control of Chaos. An Introduction for Scientists and Engineers (Imperial College Press, London, 2004). 19

[47] M. L. Pecora and L. T. Carroll, "Synchronization in Chaotic Systems," Physical Review Letters 64 (8), 821-824 (1990).

Online Version 19

[48] S. Rim, I. Kim, P. Kang, Y.-J. Park, and C.-M. Kim, "Routes to Complete Synchronization via Phase Synchronization in Coupled Nonidentical Chaotic Oscillators," Physical Review E 66, 015205-1-4 (2002).

Online Version 19

[49] M. Zhan, X. Wang, X. Gong, G. W. Wei, , and C. Lai, "Complete Synchronization and Generalized Synchronization of One-way Coupled Time-delay Systems," Physical Review E 68, 036208-1-5 (2003).

Online Version 19

[50] L. Kocarev and U. Parlitz, "Generalized Synchronization, Predictability, and Equivalence of Unidirectionally Coupled Dynamical Systems," Physical Review Letters 76, 1816-1819 (1996).

Online Version 19

[51] M. Rosenblum, A. Pikovsky, and J. Kurths, "From Phase to Lag Synchronization in Coupled Chaotic Oscillators," Physical Review Letters 78, 4193-4196 (1997).

Online Version 19

[52] V. N. Belykh, G. V. Osipov, N. Kuckländer, B. Blasius, and J. Kurths, "Automatic Control of Phase Synchronization in Coupled Complex Oscillators," Physica D: Nonlinear Phenomena 200, 81-104 (2005).

Online Version

[53] D. V. Senthilkumar, M. Lakshmanan, and J. Kurths, "Phase Synchronization in Unidirectionally Coupled Ikeda Time-delay Systems," European Physical Journal Special 
Topics 164, 35-44 (2008).

Online Version 19

[54] S. Taherion and Y.-C. Lai, "Observability of Lag Synchronization of Coupled Chaotic Oscillators," Physical Review E 59(6), 6247-6250 (1999).

Online Version 19

[55] J. M. González-Miranda, "Amplitude Envelope Synchronization in Coupled Chaotic Oscillators," Physical Review E 65, 036232-1-10 (2002).

Online Version 19 


\section{Part I}

\section{A New Route to Periodic Oscillations in the Dynamics of Malaria Transmission}


Chapter 2

\section{Introduction}

Malaria, a mass killer communicable disease, is one of the leading causes of morbidity and death to the human race in many parts of the world. Its devastating impact has persisted for decades. A February 2010 News Week article [1] states that Malaria is the likely killer of the boy Pharaoh in about 1324 BC. For more technical details, see the article by Hawass et al. [2]. Despite the longevity of the disease, malaria, which has been brought under control in some developed countries, still constitutes a major health menace in many developing countries, where most areas of high endemicity reside. Some African countries, especially countries within sub-Saharan Africa, still feature among the leading areas of high malaria endemicity in the world [3]. According to the latest version of the World Health Organization report [4], an estimated 225 million malaria clinical cases occurred in 2009, with an estimated 781,000 malaria mortalities. Although these statistics reflect a reduction compared to an estimated 243 million malaria cases, with an estimated 863,000 malaria deaths, $89 \%$ of which occurred in Africa in 2008 [5], the reduction is not sufficient. Generally, susceptibility to malaria is universal, that is, any person residing in or traveling to a country where malaria is prevalent is at risk of contracting the disease. However, the impact of malaria is greatest amongst children below five [6], where one in every five childhood deaths is due to the effects of the disease, among pregnant women, and among people from non-malarious regions. Due to easy worldwide transportation and other factors, non-malarious regions of the world risk experiencing malaria resurgence $[7,8]$. In addition to being the paramount source of morbidity and mortality in malaria endemic regions, malaria also weakens the active and potential workforce, thereby negatively impacting economic growth in these areas [9]. Gallup 
et al. [10] in their 2001 paper stated, "malaria and poverty are intimately connected." Hence, continuous research to find ways to effectively control the disease and bring down the number of malaria deaths will not only save lives and a future generation, but improve the economic conditions of the most affected nations, which tend to be very poor.

Recently published research by Rich et al. [11] traces the origin of malaria to chimpanzees. This breakthrough eliminates the uncertainty in answering questions related to the origin of malaria that have lingered for years, and opens new avenues for research that may lead to the discovery of more effective drugs and/or vaccines for malaria.

Malaria is caused by a micro-parasitic organism of the genius Plasmodium and indirectly conveyed from one human to another ${ }^{1}$ through female Anopheles mosquito bites. Both male and female Anopheles mosquitoes feed mostly on nectar, fruit juices, and other plant liquids. However, the female Anopheline also requires blood for the development of its eggs. In the course of acquiring a blood meal, a female Anopheles mosquito may pick up the form of the parasite that is transmissible from humans to mosquitoes (called gametocytes) from an infectious human or deposit the form of the parasite that is transmissible from mosquitoes to humans (salivary glands sporozoites) to a human (who may be susceptible or already infected). A bite from an infectious female Anopheles mosquito initiates the human phase of the parasite's life cycle and culminates with the production of gametocytes (the sexual and transmissible form of the parasite from humans to mosquitoes) in the human blood system. Four species of human Plasmodium, Plasmodium falciparum, Plasmodium vivax, Plasmodium ovale and Plasmodium malariae have been identified. Plasmodium falciparum is the most pernicious species and it is transmitted by the Anopheles gambiae complex. It accounts for about $80 \%$ of malaria related cases annually and this percentage rose to 93 in 2008 [5]. Coexistence of more than a single species of the human malaria parasite in the same malaria endemic community may be possible. In such cases, humans may harbor multiple species of the parasite concurrently. Other species of Plasmodium are also found in animals;

\footnotetext{
${ }^{1}$ Unlike directly transmissible diseases such as Influenza, TB, HIV, sexually transmitted diseases, etc., indirectly transmitted diseases such as dengue fever and malaria are transmitted from one human to another by an intermediate transmission agent, the disease vector. This leads to a vicious cycle in which humans are infected by vectors and vectors are infected by humans in turn. However, except in the rare case of blood transfusion, humans do not infect other humans directly and vectors do not infect other vectors directly.
} 
for example, Plasmodium reichenowi in the chimpanzee.

Plasmodium has an intricate life process, with part invested within the human host and the other part within the vector host. The human phase begins with an infectious bite from a female Anopheles mosquito, which introduces sporozoites (the asexual form of the parasite) from its salivary glands into the human victim's bloodstream. These sporozoites make their way into the victim's liver through the blood circulatory system and encroach on liver cells where they undergo asexual replication. At this stage the human is clinically sick but with no symptoms. After a few days, merozoites are unleashed into the human blood stream. These merozoites parasitize erythrocytes, where they eventually enlarge into trophozoites. The human phase culminates with further production of merizoites or gametocytes (sexual and infectious forms of the parasite) in the human blood system. At this point, there is a clear manifestation of malaria symptoms ${ }^{2}$ and the malaria vector can pick up these parasites during a blood meal. The incubation period ${ }^{3}$ of the parasite within the human host varies between 8 and 30 days and it depends on the species of the parasite and the human's immune response. The vector phase of the life cycle begins with the female Anopheles mosquito picking up male and female gametocyte forms of the parasite from the human host during a blood meal, and culminates with the release of sporozoites into the mosquito's salivary glands. It is worth noting that sporozoites constitute the infectious form of the parasite within the vector host. Generally, completion of the sporogonic cycle of the parasite within the vector depends on both intrinsic and extrinsic factors, such as the human Plasmodium species, ambient temperature, humidity, etc. The incubation period of the parasite within the vector ranges from 10 to 18 days. The life cycles of the four plasmodium species are similar with only slight differences, for example, in the incubation period. See [12-16] for an extensive discussion on malaria epidemiology.

\footnotetext{
${ }^{2}$ Malaria symptoms include bouts of fever, headache, vomiting, fatigue, pains in the joints and flu-like symptoms. Manifestation of symptoms ceases once the parasites are destroyed, e.g. through the use of drugs.

${ }^{3}$ By incubation period, we are referring to the time that elapses between infection and infectiousness. For humans, this is the time between the introduction of sporozoites into the blood system and the emergence of gametocytes in the blood system. For the vectors, this is the time between the introduction of gametocytes in the vector's gut and the appearance of sporozoites in the salivary glands.
} 
Temperature is known to affect the developmental stages of the Plasmodium falciparum parasite $[17,18]$ and also the metamorphic stages of the mosquito [19]. There is a general consensus that future changes in climate may alter the prevalence and incidence of malaria; however, there are conflicting views among authors as to what the role of global warming is [20-23]. Additionally, some authors have argued that climate and ecology are the main determinants in the severity of malaria and the difficulty in controlling it [10]. Other factors that have led to difficulties in controlling the disease in the most affected malaria countries are shortcomings in socioeconomic conditions, population growth, urbanization, drug resistance, deficiencies in health care systems, poor sanitation, lack of information and education, water storage, garbage disposal, unpaved roads, and drainage systems that generate excellent breeding grounds for disease transmission agents close to human settlements [19, 24-27]. Thus, research in malaria that integrates the disease dynamics with breeding sites/demographic properties of the vector and the different developmental stages of the parasite may provide novel insights toward disease control and eradication.

Collective interdisciplinary research efforts involving the medical field, computer science, biological and mathematical sciences have been formed, all in an attempt to develop strategies of containing malaria. Multidisciplinary approaches will be necessary in order to determine the best methods for combatting this virulent disease. However, some policy makers in developing countries, as well as in the biological and medical sciences, fail to appreciate the role of mathematical models in epidemiology. On the contrary, mathematical models have much to contribute to the design, evaluation and implementation of optimal health policies. Mathematical models provide a means for better understanding the disease dynamics and transmission in terms of disease variables and underlying parameters, and provide a platform for new containment strategies as well as health policies. The year 1911 witnessed the dawn of a new era in studies of malaria with the development of the first mathematical model for malaria by R. Ross [28]. Ross' model featured two ordinary differential equations: one for the total human population and the other for the total mosquito population. This model has evolved through time, with variations and extensions to incorporate different characteristic features of malaria. Some of these extensions include splitting the human and vector populations into different compartments, thereby giving rise to SI, SIS, SIR, SIRS, SEI, SEIS, 
SEIR, and SEIRS models, where S represents susceptible individuals, E represents latent or exposed individuals, I represents infectious individuals, and $\mathrm{R}$ represents recovered individuals. Other extensions include the notions of superinfection [29] and acquired immunity [30-32]. A comprehensive survey of classical mathematical models for malaria can be found in Refs. [33-35]. More recent mathematical models for malaria include Ngwa and Shu's SEIRS model [24], which features a class of partially immune individuals, Ngwa et al. proposed a SEIS [36] model that incorporates a time delay to capture the time lapse between egg laying and adult mosquito eclosion and also accounts for vector deaths in life stages prior to the adult stage. In the former model, an explicit expression was derived for the contact rates based on the fact that mosquitoes have a human biting rate, and for both models threshold parameters that determine the stability and instability of equilibria were calculated. An extension of the Ngwa and Shu's model to incorporate a generalization of the human biting rate of the mosquitoes and human immigrations was developed and studied by Chitnis et al. [37, 38]. Other models for malaria have focused on the basic unit of study, the malaria parasite, for example, Teboh-Ewungkem et al. [39, 40] developed models that examine the contribution of gametocytes in the spread of malaria and the within-vector dynamics of the malaria parasite. For a few other recent malaria models see Refs. [41-48].

Although malaria is deadly, it can be cured by administering anti-malaria drugs. However, in endemic regions, the malaria parasite develops resistance to such drugs [49] and there is no effective vaccine for malaria. Consequently, prevention is the only other option. Prevention can be achieved through the use of prophylactic drugs and vector control strategies. To advance, plan, design, and implement effective or better vector control measures, a clear understanding of mosquito population dynamics, the disease dynamics, and mosquito interaction with the human population is necessary.

We introduce a new approach to the development of models for malaria transmission, wherein the mosquito vector is placed at the centre of the transmission process. Our objective is to develop a mathematical model for the dynamics of malaria transmission that takes into consideration the population dynamics of the malaria vector and how these vectors interact with the human population. To do that, an understanding of the vector population demography and dynamics is needed. 
The malaria vector undergoes a complete metamorphosis, as it passes through four different life stages: egg, larva, pupa and adult. The egg, larva and pupa stages are aquatic, while the adult stage is terrestrial. The entire cycle from egg laying to the emergence of the adult mosquito takes approximately 7-20 days, with 2-3 days spent in the egg stage, 4-10 days spent in the larva stage, and 2-4 days spent in the pupa stage [19]. While the average life span of the adult female mosquito ranges from 2-3 weeks, that of the males is approximately one week. As for the first three life stages, the life span of the adult mosquito depends on the species and ambient temperature. In addition to natural factors, survival of the adult female Anopheles mosquito also depends on its success in acquiring blood meals from humans. The flight range of an unnourished adult female Anopheles mosquitoes is about $2 \mathrm{Km}$ [50]. However, transportation by car, aircraft or ship can enable mosquitoes to go much further. Like other disease vectors, the malaria vector is capable of locating humans $[19,51]$ and attempting as many times as possible to feed. The vectors exhibit varied behavioral habits: some will nourish inside human homes (endophagic), while others nourish outside (exophagic); some bite humans at night (nocturnal), while others bite humans at dawn or dusk (crepuscular); some share a common resting area with humans (endophilic), while others favor resting on different sites from human dwellings (exophilic) [52, 53]. In some areas where there is constant use of insecticide impregnated mosquito nets and application of other strategies to avoid mosquito bites, mosquitoes may seek alternative sources of blood meals from animals, such as cattle [54-56]. Nourishment is usually followed by a choice of breeding site, and this is where the mosquito eventually lays its eggs. Proximity to the human habitat from which the mosquito acquired its blood meal and safety from predation are major factors $[57,58]$. It is clear that the feeding and resting habits of the malaria vector are major factors to be considered when designing vector intervention strategies. For instance, female mosquitoes that prefer human blood to animal blood have a higher chance of assisting in spreading the parasite within the human community than those that prefer animal blood. Intervention strategies may entail destroying or effecting changes to potential mosquito breeding sites, such as application of insecticides through indoor spraying or introduction of biological agents such as predators [59]. Common mosquito breeding sites include openings in trees, shallow slow running streams, swampy and humid areas, stagnant 
water reservoirs such as bad drainage systems, depressions in the soil, pot-holes filled with water, and improperly disposed hollow material like cans, bowls, wheels, etc.

The mosquito population density fluctuates both seasonally and by virtue of its life cycle and reproductive pattern, which gives rise to oscillations in the vector population dynamics. Some modeling attempts aimed at quantifying the dynamics of infectious disease vectors have been proposed [60-62]. However, none of the models distinguish between reproducing and non-reproducing vectors. Ngwa [52] recently developed and studied a mathematical model for the dynamics of the malaria vector. The model splits the total adult female vector population into three compartments, comprising fed and reproducing vectors, unfed and questing vectors, and unfed and resting vectors. A delay to model vector deaths in prior life stages and the time lapse between egg laying and the emergence of the adult vector is also included in the model. A detailed analysis of the model for the case in which the delay is set to zero [52] and of the full delay model [63] produced results that included the appearance of a Hopf bifurcation. The model also captured the naturally occurring oscillatory dynamics that govern the mosquito population without the usual external seasonal forcing that is incorporated in many insect population dynamics models. However, the model considered only the interaction between the human population and questing vectors that are recruited from resting vectors from a single breeding site. The model was later extended to study the vector population dynamics when more than one breeding site is involved [25]. Since the focus of the models in Refs. $[25,52,63]$ is on the population dynamics of the malaria vector, none of them include malaria dynamics and interactions between humans and the vector.

Our objective here is to update the model in Ref. [52] by developing and analyzing a deterministic SIS model for the dynamics of malaria transmission that includes the dynamics of the malaria vector. The vector dynamics and demography together with their interaction with the human host will be included as well as the transmission of the malaria parasite from one human host to another. It is well known that the most general form of a compartmental model for the dynamics of malaria transmission is an SEIRS model [24, 38]. However, in order to highlight the novelty in our modeling approach, we develop and analyze (using rigorous analytical and numerical techniques) a simple SIS model with the following objectives:

1. to capture the natural oscillations that are known to exist in the prevalence of malaria 
without recourse to external seasonal forcing,

2. to redefine and calculate a new value of the basic reproduction number for malaria,

3. to present a possible interpretation of why it is difficult to eradicate malaria,

4. to compare our SIS model with the original Ross SIS malaria model and, hence, reveal the novelty in our approach,

5. to propose possible areas of control based on the model results.

The rest of this part is organized as follows: In Chapter 3, we derive and analyze the classical SIS model. We derive and nondimensionalize the basic ordinary differential equation model in Chapter 4. A detailed analysis of the model in the absence and in the presence of the disease is presented in Chapter 5. Finally, we present a comparison of our model and the classical SIS model and some concluding remarks in Chapter 6. 


\section{References}

[1] J. N. Wilford, "Malaria is a Likely Killer in King Tuts Post-mortem," Technical Report 16, The New York Times, accessed March 2011.

Online Version 28

[2] Z. Hawass, Y. Z. Gad, S. Ismail, R. Khairat, D. Fathalla, N. Hasan, A. Ahmed, H. Elleithy, M. Ball, F. Gaballah, S. Wasef, M. Fateen, H. Amer, P. Gostner, A. Selim, A. Zink, and C. M. Pusch, "Ancestry and Pathology in King Tutankhamun's Family," The Journal of the American Medical Association 303, 638-647 (2010).

Online Version 28

[3] K. Marsh, "Malaria Disaster in Africa," Lancet 352, 924-925. (1998).

Online Version 28

[4] World Health Organisation, "The World Malaria Report," WHO Press, acessed March 2011 (2010).

Online Version 28

[5] World Health Organisation, "The World Health Report," WHO Press (2009). Online Version 28, 29

[6] World Health Organization, "10 Facts on Malaria," WHO Press (2009). Online Version 28

[7] C. Faraj, S. Ouahbi, E. Adlaoui, D. Boccolini, R. Romi, and R. E. Aouad, "Risque de Reemergence du Paludisme au Maroc. Etude de la Capacite Vectorielle d'Anopheles 
Labranchiae dans une Zone Rizicole au Nord du Pays," Parasite 15, 605-610 (2008).

Online Version 28

[8] S. Sainz-Elipe, J. Latorre, R. Escosa, M. Masia, M. Fuentes, S. Mas-Coma, and M. Bargues, "Malaria Resurgence Risk in Southern Europe: Climate Assessment in an HistoricallyEendemic Area of Rice Fields at the Mediterranean Shore of Spain," Malaria Journal 9, 221-236 (2010).

Online Version 28

[9] J. Sachs and P. Malaney, "The Economic and Social Burden of Malaria," Nature 415, 680-685 (2002).

Online Version 28

[10] J. L. Gallup and J. D. Sachs, "The Economic Burden of Malaria," American Journal of Tropical Medicine and Hygiene 64, 85-96 (2001).

Online Version 29, 31

[11] S. M. Rich, F. H. Leendertz, G. Xu, M. LeBreton, C. F. Djoko, M. N. Aminake, E. E. Takang, J. L. D. Diffo, B. L. Pike, B. M. Rosenthal, P. Formenty, C. Boesch, F. J. Ayala, and N. D. Wolfe, "The Origin of Malignant Malaria," Proceedings of the National Academy of Sciences 106 (35), 14902-14907 (2009).

Online Version 29

[12] I. W. Sherman, Malaria: Parasite Biology, Pathogenesis, and Protection (ASM Press, 1998). 30

[13] S. Wyborny, Parasites. The Malaria Parasite (KidHaven Press, 2005), 1st edition.

[14] T. Hayakawa, R. Culleton, H. Otani, T. Horii, and K. Tanabe, "Big Bang in the Evolution of Extant Malaria Parasites," Molecular Biology and Evolution 25(10), 2233-2239 (2008).

Online Version

[15] C. C. Carlos, Malaria: In Infectious Diseases (J. B. Lipincolt Company, Philadelphia, 1989). 
[16] G. Macdonald, The Epidemiology and Control of Malaria (Oxford University Press, London, 1957). 30

[17] L. A. Baton and L. C. Ranford-Cartwright, "Spreading the Seeds of Million-murdering Death: Metamorphoses of Malaria in the Mosquito," Trends in Parasitology 21, 573$580(2005)$.

Online Version 31

[18] M. I. Teboh-Ewungkem and T. Yuster, "A Within-vector Mathematical Model of Plasmodium Falciparum and Implications of Incomplete Fertilization on Optimal Gametocyte Sex Ratio." Journal of Theoretical Biology 264, 273-86 (2010).

Online Version 31

[19] H. M. Giles and D. A. Warrel, Bruce-Chwatt's Essential Malariology (Hodder Arnold Publication, London, 2002), 4th edition. 31, 33

[20] P. Martens, R. S. Kovats, S. Nijhof, P. de Vries, M. J. T. Levermore, D. J. Bradley, J. Cox, and A. J. McMichael, "Climate Change and Future Populations at Risk of Malaria," Global Environmental Change 9, S89-S107 (1999).

Online Version 31

[21] G. Zhou, N. Minakawa, A. K. Githeko, and G. Yan, "Association Between Climate Variability and Malaria Epidemics in the East African Highlands," Proceedings of the National Academy of Sciences 101, 2375-2380 (2004).

Online Version

[22] J. A. Patz and S. H. Olson, "Malaria Risk and Temperature: Influences from Global Climate Change and Local Land use Practices," Proceedings of the National Academy of Sciences 103, 5635-5636 (2006).

Online Version

[23] K. L. Gage, T. R. Burkot, R. J. Eisen, and E. B. Hayes, "Climate and Vectorborne Diseases," American Journal of Preventive Medicine 35 (5), 436-450 (2008). Online Version 31 
[24] G. A. Ngwa and W. S. Shu, "A mathematical Model for Endemic Malaria with Variable Human and Mosquito Populations," Mathematical and Computer Modelling 32, 747763 (2000).

Online Version 31, 32, 34

[25] S. Nourridine, M. I. Teboh-Ewungkem, and G. A. Ngwa, "A Mathematical Model of the Population Dynamics of Disease Transmitting Vectors with Spatial Consideration," Journal of Biological Dynamics 1751-3758 (2011).

Online Version 34

[26] S. G. Staedke, E. W. Nottingham, J. Cox, M. R. Kamya, P. J. Rosenthal, and G. Dorsey, "Proximity to Mosquito Breeding Sites as a Risk Factor for Clinical Malaria Episodes in an Urban Cohort of Ugandan Children," American Journal of Tropical Medicine and Hygiene 69 (3), 244-246 (2003).

Online Version

[27] M. I. Teboh-Ewungkem, Malaria Control: The Role of Local Communities as Seen through a Mathematical Model in a Changing Population-Cameroon, Chapter 4, 103140, in Advances in Disease Epidemiology (Nova Science Publishers, 2009). 31

[28] R. Ross, The Prevention of Malaria (John Murray, London, 1911). 31

[29] G. Macdonald, "The Analysis of Infection Rates in Diseases in Which Superinfection Occurs," Tropical Diseases Bulletin 47, 907-915 (1950). 32

[30] K. Dietz, L. Molineaux, and A. Thomas, "A Malaria Model Tested in the Africa Savanna," Bull of World Hearlth Organization 50, 347-357 (1974). 32

[31] T. J. N. Bailey, The Mathematical Theory of Infectious Diseases and its Application (Griffin, London, 1975), 2nd edition.

[32] J. L. Aron, "Mathematical Modeling of Immunity to Malaria," Mathematical Biosciences 90, 385-396 (1988). 32 
[33] J. L. Aron and R. M. May, The Population Dynamics of Malaria, In: Population Dynamics of Infectious Diseases (R. M. Ed) (Chapman and Hall, London, 1982). 32

[34] R. M. Anderson and R. M. May, Infectious Diseases of Humans: Dynamics and Control (Oxford University Press, Oxford, 1991).

[35] J. C. Koella, "On the Use of Mathematical Models of Malaria Transmission," Acta Tropica 49, 1-25 (1991).

Online Version 32

[36] G. A. Ngwa, C. N. Ngonghala, and N. B. S. Wilson, "A Model for Endemic Malaria with Delay and Variable Populations," Jounal of the Cameroon Academy of Sciences 1(3), 169-186 (2001). 32

[37] N. Chitnis, J. M. Cushing, and J. M. Hyman, "Bifurcation Analysis of a Mathematical Model for Malaria Transmission," SIAM Journal of Applied Mathematics 67 (1), 24-45 (2006).

Online Version 32

[38] N. Chitnis, J. M. Hyman, and J. M. Cushing, "Determining Important Parameters in the Spread of Malaria through the Sensitivity Analysis of a Mathematical Model," Bulletin of Mathematical Biology 70, 1272-1296 (2008).

Online Version 32, 34

[39] M. I. Teboh-Ewungkem, C. N. Podder, and A. B. Gumel, "Mathematical Study of the Role of Gametocytes and an Imperfect Vaccine on Malaria Transmission Dynamics," Bulletin of Mathematical Biology 72 (1), 63-93 (2009).

Online Version 32

[40] Teboh-Ewungkem, M. I. Yuster, and T. Newman, A Mathematical Model of the Withinvector Dynamics of the Plasmodium Falciparum Protozoan Parasite, In Infectious Disease Modelling Research Progress (Tchuenche J. M. and Chiyaka C., eds) (Nova Science Publishers, New York, 2009). 32 
[41] J. Labadin, C. M. L. Kon, and S. F. S. Juan, "Deterministic Malaria Transmission Model with Acquired Immunity," Proceedings of the World Congress on Engineering and Computer Science 2, 779-784 (2009).

Online Version 32

[42] C. Chiyaka, W. Garira, and S. Dube, "Mathematical Modelling of the Impact of Vaccination on Malaria Epidemiology," International Journal of Qualitative Theory of Differential Equations and Applications 1 (1), 28-58 (2007a).

Online Version

[43] C. Chiyaka, J. M. Tchuenche, W. Garira, and S. Dube, "A Mathematical Analysis of the Effects of Control Strategies on the Transmission Dynamics of Malaria," Applied Mathematics and Computation 46 (5-6), 806-822 (2007b).

Online Version

[44] P. C. McQueen and F. E. McKenzie, "Age-structured Red Blood Cell Susceptibility and the Dynamics of Malaria Infections," Proceedings of the National Academy of Sciences 101, 9161-9166 (2004).

Online Version

[45] A. P. Wyse, L. Bevilacqua, and M. Rafikovd, "Simulating Malaria Model for Different Treatment Intensities in a Variable Environment," Ecological Modelling 206, 322-330 (2007).

[46] S. H. Saker, "Stability and Hopf Bifurcations of Nonlinear Delay Malaria Epidemic Model," Nonlinear Analysis: Real World Applications 11, 784 - 799 (2010).

Online Version

[47] H. Diaz, A. Ramirez, A. Olarte, and C. Clavijo, "A Model for the Control of Malaria Using Genetically Modified Vectors," Journal of Theoretical Biology 276, 57-66 (2011). Online Version

[48] O. Makinde and K. Okosun, "Impact of Chemo-therapy on Optimal Control of Malaria 
Disease with Infected Immigrants," Biosystems 104, 32-41 (2011).

Online Version 32

[49] Y. Artzy-Randrup, D. Alonso, and M. Pascual, "Transmission Intensity and Drug Resistance in Malaria Population Dynamics: Implications for Climate Change," PLoS ONE 5, e13588 (2010).

Online Version 32

[50] C. Kaufmann and H. Briegel, "Flight performance of the malaria vectors Anopheles gambiae and Anopheles atroparvus," Journal of Vector Ecology 29 (1), 140-153 (2004). Online Version 33

[51] P. J. McCall and D. W. Kelly, "Learning and Memory in Disease Vectors," Research Update. Trends Parasitology 18 (10), 429-433 (2002).

Online Version 33

[52] G. A. Ngwa, "On the Population Dynamics of the Malaria Vector," Bulletin of Mathematical Biology 68 (8), 2161-2189 (2006).

Online Version 33, 34

[53] CDC, "Malaria: Anopheles Mosquito," Division of Parasitic Diseases National Center for Zoonotic, Vector-Borne, and Enteric Diseases (ZVED) (2008).

Online Version 33

[54] S. Muriu, E. Muturi, J. Shililu, C. Mbogo, J. Mwangangi, B. Jacob, L. Irungu, R. Mukabana, J. Githure, and R. Novak, "Host Choice and Multiple Blood Feeding Behaviour of Malaria Vectors and Other Anophelines in Mwea Rice Scheme, Kenya," Malaria Journal 7, 43-50 (2008).

Online Version 33

[55] J. Ijumba, F. Mosha, and S. W. Lindsay, "Malaria Transmission Risk Variations Derived from Different Agricultural Practices in an Irrigated Area of Northern Tanzania," Medical and Veterinary Entomology 16, 28-38 (2002).

Online Version 
[56] G. Dolo, O. J. T. Brit, A. Dao, S. F. Traor, M. Bouar, N. Sogoba, O. Niar, M. Bagayogo, D. Sangar, T. Teuscher, and Y. T. Tour, "Malaria transmission in relation to rice cultivation in the irrigated Sahel of Mali," Acta Tropica 89, 147-159 (2004).

Online Version 33

[57] F. Wrona and J. Dixon, "Group Size and Predation Risk: A Field Analysis of the Encounter and Dilution Effects," The American Society of Naturalists 137 (2), 186201 (1991).

Online Version 33

[58] B. Yuval, J. W. Wekesa, and R. K. Washino, "Effect of Body Size on Swarming Behaviour and Mating Success of Male Anopheles Freeboni (Diptera: Culicidae)," Journal of Insect Behavior 6 (3), 333-342 (1993). 33

[59] J. D. Smyth, An Introduction to Animal Parasitology. (Cambridge University Press, Cambridge, 1996). 33

[60] R. F. Costantino, R. A. Desharnais, J. M. Cushing, and D. Brian, "Chaotic Dynamics in an Insect Population," Science 275 (5298), 389-391 (1998).

Online Version 34

[61] T. Porphyre, D. J. Bicout, and P. Sabatier, "Modelling the Abundance of Mosquito Vectors Versus Flooding Dynamics," Ecological Modelling 183, 173-181 (2005). Online Version

[62] M. Raffy and A. Tran, "On the Dynamics of Flying Insects Populations Controlled by Large Scale Information," Theoretical Population Biology 68, 91-104 (2005). Online Version 34

[63] G. A. Ngwa, A. M. Niger, and A. B. Gumel, "Mathematical Assessment of the Role of Non-linear Birth and Maturation Delay in the Population Dynamics of the Malaria Vector," Applied Mathematics and Computation 217, 3286-3313 (2010).

Online Version 34 
Chapter 3

\section{The Classical Ross-Macdonald Type SIS Model}

\subsection{Introduction}

To highlight the novelty of the new deterministic SIS model for the dynamics of malaria transmission that we derive in Chapter 4, it is necessary to begin by deriving and studying a simple classical SIS model for malaria [1-3]. This will enable us to identify the limitations of this simple model and, hence, substantiate the need to develop and study a new framework for modeling malaria transmission dynamics. We derive and analyze the Ross-Macdonald's malaria model. Depending on whether the basic reproduction number is less than unity or larger than unity, this model can exhibit a stable disease-free equilibrium solution, denoting the situation in which the malaria disease is absent from the population, or a stable endemic equilibrium solution, denoting the situation in which the malaria disease perpetuates itself within the population. Oscillatory dynamics may be possible only when a delay or a nonlinear external forcing term is introduced in the model.

This chapter is organized as follows: We derive the classical SIS Ross-Macdonald's malaria model in Section 3.2 and establish the existence, local stability, and global stability of equilibrium solutions to the model in Section 3.3. We present numerical simulation results and a summary of the Chapter in Sections 3.4 and 3.5, respectively. 


\subsection{Classical Model Derivation}

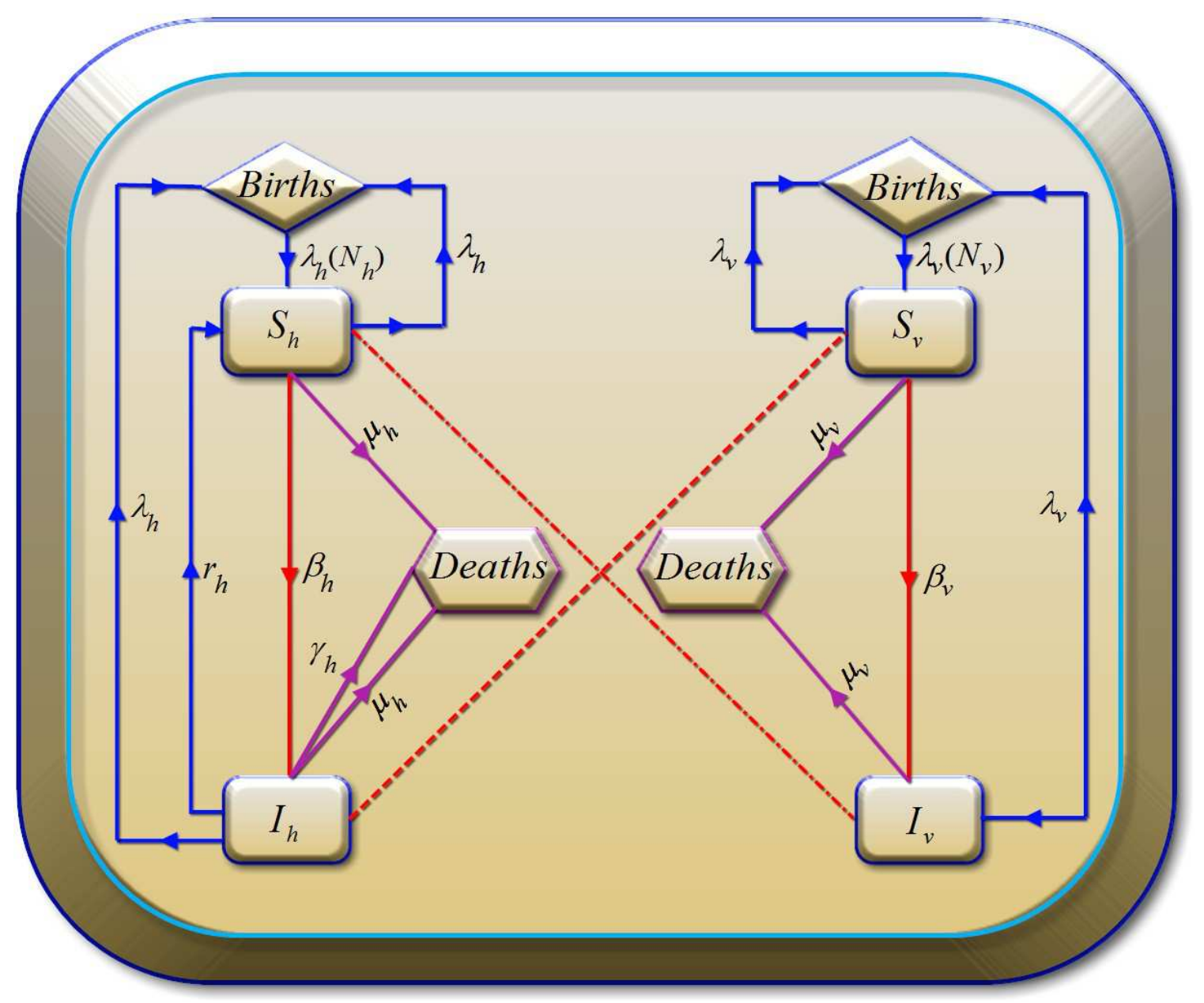

Figure 3.1. A conceptual framework of the classical SIS model for malaria. Mosquitoes interact with humans and transfer the malaria parasite from human to human and within the mosquito population. Broken red lines represent interactions between humans and questing mosquitoes, solid blue lines denote links that result in an increase in the susceptible populations, solid red lines denote links that result in growth in the infectious populations, while purple lines represent links that result in deaths in both the human and mosquito populations. At any time $t$, the human and mosquito populations are subdivided into susceptible and infectious individuals so that $N_{h}(t)=S_{h}(t)+I_{h}(t)$ and $N_{v}(t)=S_{v}(t)+I_{v}(t)$. 
In the classical model derivation, both the human and mosquito populations are broken down into two disjoint groups consisting of susceptible and infectious individuals so that at any time $t$, there are $S_{h}(t)$ susceptible humans, $S_{v}(t)$ susceptible mosquitoes, $I_{h}(t)$ infectious humans and $I_{v}(t)$ infectious mosquitoes. The total human and mosquito populations are denoted by $N_{h}(t)$ and $N_{v}(t)$, respectively, where $N_{h}(t)=S_{h}(t)+I_{h}(t)$ and $N_{v}(t)=S_{v}(t)+$ $I_{v}(t)$, and $N_{h}$ and $N_{v}$ are assumed to be large enough so that the variables $S_{h}, S_{v}, I_{h}$ and $I_{v}$ are treated as continuous variables. It is also assumed that all new human and mosquito births are susceptible and occur at non-increasing density dependent rates $\lambda_{h}$ and $\lambda_{v}$, respectively. On the other hand, natural deaths in the human and mosquito classes occur at density dependent rates $\mu_{h}$ and $\mu_{v}$, respectively, where $\mu_{h}$ and $\mu_{v}$ are assumed to be monotone nondecreasing. The average human and mosquito life spans are, respectively, $1 / \mu_{h}$ and $1 / \mu_{v}$. In addition, humans die as a result of the malaria disease at rate $\gamma$. Humans recover and join the susceptible class at rate $r_{h}$ and therefore the average duration of infection is $1 / r_{h}$. Mosquitoes do not recover from the infection. Therefore, the human portion of the model is SIS while the mosquito portion of the model is SI. Uniform and homogeneously mixing populations are considered and $\beta_{h}$ and $\beta_{v}$ are, respectively, the contact rates between susceptible humans and infectious mosquitoes and susceptible mosquitoes and infectious humans. Contacts at the above rates bring about transitions between the susceptible and infectious classes.

Using the flow chart in Figure 3.1 and the assumptions above, we write the following system of differential equations for the SIS model:

$$
\begin{aligned}
\dot{S}_{h} & =\lambda_{h}\left(N_{h}\right) N_{h}+r_{h} I_{h}-\beta_{h}\left(N_{h}\right) I_{v} S_{h}-\mu_{h}\left(N_{h}\right) S_{h} \\
\dot{I}_{h} & =\beta_{h}\left(N_{h}\right) I_{v} S_{h}-\left(r_{h}+\gamma_{h}+\mu_{h}\left(N_{h}\right)\right) I_{h} \\
\dot{S}_{v} & =\lambda_{v}\left(N_{v}\right) N_{v}-\beta_{v}\left(N_{h}\right) I_{h} S_{v}-\mu_{v}\left(N_{v}\right) S_{v} \\
\dot{I}_{v} & =\beta_{v}\left(N_{h}\right) I_{h} S_{v}-\mu_{v}\left(N_{v}\right) I_{v}
\end{aligned}
$$

and the total human and mosquito populations are modeled by the equations,

$$
\begin{aligned}
& \dot{N}_{h}=\lambda_{h}\left(N_{h}\right) N_{h}-\mu_{h}\left(N_{h}\right) N_{h}-\gamma I_{h} \\
& \dot{N}_{v}=\lambda_{v}\left(N_{v}\right) N_{v}-\mu_{v}\left(N_{v}\right) N_{v}
\end{aligned}
$$

where the functional forms for $\lambda_{h}, \mu_{h}, \lambda_{v}$ and $\mu_{v}$ are chosen accordingly. For instance, if 
$\lambda_{h}$ and $\lambda_{v}$ are non-increasing functions and $\mu_{h}$ and $\mu_{v}$ are monotone non-decreasing, then equilibrium total populations will exist. By substituting $S_{h}=N_{h}-I_{h}$ and $S_{v}=N_{v}-I_{v}$ into system (3.1), the equations for $S_{h}$ and $S_{v}$ can be dropped and we end up with two equations; one modeling the infectious human population $I_{h}$ and the other modeling the infectious mosquito population $I_{v}$.

$$
\begin{aligned}
& \dot{I}_{h}=\beta_{h}\left(N_{h}\right)\left(N_{h}-I_{h}\right) I_{v}-\left(r_{h}+\gamma_{h}+\mu_{h}\left(N_{h}\right)\right) I_{h} \\
& \dot{I}_{v}=\beta_{v}\left(N_{h}\right)\left(N_{v}-I_{v}\right) I_{h}-\mu_{v}\left(N_{v}\right) I_{v} .
\end{aligned}
$$

\subsection{Classical Model Analysis}

The total human and mosquito populations are constant in the classical Ross-Macdonald's model. That is, $\lambda_{h}=\mu_{h}=$ constant, $\lambda_{v}=\mu_{v}=$ constant and $\gamma_{h}=0$. In this case, $\beta_{h}\left(N_{h}\right)=\beta_{h}=$ constant and $\beta_{v}\left(N_{h}\right)=\beta_{v}=$ constant. We use these assumptions to further simplify systems (3.1) and (3.3) to systems (3.4) and (3.5), respectively.

$$
\begin{aligned}
\dot{S}_{h} & =\mu_{h} N_{h}+r_{h} I_{h}-\beta_{h} I_{v} S_{h}-\mu_{h} S_{h}, \\
\dot{I}_{h} & =\beta_{h} I_{v} S_{h}-\left(r_{h}+\mu_{h}\right) I_{h}, \\
\dot{S}_{v} & =\mu_{v} N_{v}-\beta_{v} I_{h} S_{v}-\mu_{v} S_{v} \\
\dot{I}_{v} & =\beta_{v} I_{h} S_{v}-\mu_{v} I_{v} \\
\dot{I}_{h} & =\beta_{h}\left(N_{h}-I_{h}\right) I_{v}-\left(r_{h}+\mu_{h}\right) I_{h}, \\
\dot{I}_{v} & =\beta_{v}\left(N_{v}-I_{v}\right) I_{h}-\mu_{v} I_{v} .
\end{aligned}
$$

Note that the assumptions of constant population sizes and no disease-induced mortalities are valid for diseases of short duration. To complete the formulation of system (3.4), appropriate initial conditions must be defined. The simplest type of initial conditions are of the form

$$
\left(S_{h}(0), I_{h}(0), S_{v}(0), I_{v}(0)\right)=\left(S_{h}^{0}, I_{h}^{0}, S_{v}^{0}, I_{v}^{0}\right)
$$

Since the variables represent human and mosquito populations, they are all positive and so we can explore system (3.4) in the following positively invariant feasible region:

$$
\Psi_{1}=\left\{\left(S_{h}, I_{h}, S_{v}, I_{v}\right) \in \mathbb{R}_{+}^{4}: S_{h}, I_{h}, S_{v}, I_{v}>0, S_{h}+I_{h}=N_{h}, S_{v}+I_{v}=N_{v}\right\}
$$


Similarly, system (3.5) can be studied in the following realistic region

$$
\Psi_{2}=\left\{\left(I_{h}, I_{v}\right) \in \mathbb{R}_{+}^{2}: 0<I_{h}<N_{h}, 0<I_{v}<N_{v}\right\}
$$

We use the next generation matrix approach [4] to calculate the basic reproduction number for the simplified classical SIS model. This gives

$$
R_{0}^{c}=\frac{\beta_{v} \beta_{h} N_{v} N_{h}}{\mu_{v}\left(r_{h}+\mu_{h}\right)} .
$$

See Section 5.3.1 for details on the basic reproduction number of epidemiological models.

Definition 3.3.1 (Disease-free equilibrium solution). A disease-free or infection-free equilibrium solution is a solution that exists when there is no disease in the population.

Stability of a disease-free equilibrium represents a situation in which the disease is completely eradicated from the population. In epidemiological modeling, this is possible when $R_{0} \leq 1$.

Definition 3.3.2 (Endemic equilibrium solution). An endemic or infection equilibrium solution is an equilibrium solution that exists when there is disease in the population.

Stability of an endemic equilibrium solution represents a situation in which a disease establishes itself within a community. In epidemiological modeling, this is possible when $R_{0}>1$.

Theorem 3.3.3. System (3.5) has a disease-free equilibrium solution when $R_{0} \leq 1$, and a unique endemic equilibrium solution when $R_{0}>1$, where $R_{0}$ is given by (3.9).

Proof. Let $E_{0}^{*}$ and $E_{e}^{*}$ be the disease-free and endemic equilibrium solutions of system (3.5), respectively. Then these equilibrium solutions are given by the solutions of the system

$$
\begin{aligned}
& 0=\beta_{h}\left(N_{h}\right)\left(N_{h}-I_{h}\right) I_{v}-\left(r_{h}+\gamma_{h}+\mu_{h}\left(N_{h}\right)\right) I_{h}, \\
& 0=\beta_{v}\left(N_{h}\right)\left(N_{v}-I_{v}\right) I_{h}-\mu_{v}\left(N_{v}\right) I_{v} .
\end{aligned}
$$

Solving this system of algebraic equations yields,

$$
\begin{aligned}
& E_{0}^{*}=\left(I_{h}^{*}, I_{v}^{*}\right)=(0,0), \\
& E_{e}^{*}=\left(I_{h}^{*}, I_{v}^{*}\right)=\left(\frac{\mu_{v}\left(r_{h}+\mu_{h}\right)\left(R_{0}-1\right)}{\beta_{v}\left(\beta_{h} N_{v}+r_{h}+\mu_{h}\right)}, \frac{\mu_{v}\left(r_{h}+\mu_{h}\right)\left(R_{0}-1\right)}{\beta_{h}\left(\beta_{v} N_{h}+\mu_{v}\right)}\right) .
\end{aligned}
$$

The trivial equilibrium solution $E_{0}^{*}$ exists and is the only equilibrium solution for $R_{0} \leq 1$, while the endemic equilibrium solution $E_{e}^{*}$ exists for $R_{0}>1$. Notice that $E_{e}^{*}$ reduces to $E_{0}^{*}$ when $R_{0}=1$. This proves the theorem. 
Remark 3.3.4. (i) In terms of the full system (3.4), the equilibrium solutions are

$$
\begin{aligned}
& E_{0}=\left(S_{h}^{*}, I_{h}^{*}, S_{v}^{*}, I_{v}^{*}\right)=\left(N_{h}, 0, N_{v}, 0\right), \\
& E_{e}=\left(S_{h}^{*}, I_{h}^{*}, S_{v}^{*}, I_{v}^{*}\right)
\end{aligned}
$$

where

$$
\begin{aligned}
S_{h}^{*} & =\frac{\left(r_{h}+\mu_{h}\right)\left(\beta_{v} N_{h}+\mu_{v}\right)}{\beta_{v}\left(\beta_{h} N_{v}+r_{h}+\mu_{h}\right)}=\frac{\left(\beta_{h} N_{v}+R_{0}\left(r_{h}+\mu_{h}\right)\right) N_{h}}{R_{0}\left(\beta_{h} N_{v}+r_{h}+\mu_{h}\right)}>0, \\
I_{h}^{*} & =\frac{\mu_{v}\left(r_{h}+\mu_{h}\right)\left(R_{0}-1\right)}{\beta_{v}\left(\beta_{h} N_{v}+r_{h}+\mu_{h}\right)}>0 \text { when } R_{0}>1, \\
S_{v}^{*} & =\frac{\mu_{v}\left(\beta_{h} N_{v}+r_{h}+\mu_{h}\right)}{\beta_{h}\left(\beta_{v} N_{h}+\mu_{v}\right)}=\frac{\left(\beta_{h} N_{v}+r_{h}+\mu_{h}\right) N_{v}}{\beta_{h} N_{v}+R_{0}\left(r_{h}+\mu_{h}\right)}>0, \\
I_{v}^{*} & =\frac{\mu_{v}\left(r_{h}+\mu_{h}\right)\left(R_{0}-1\right)}{\beta_{h}\left(\beta_{v} N_{h}+\mu_{v}\right)} \text { when } R_{0}>1 .
\end{aligned}
$$

(ii) From the equilibrium values given by (3.10)-(3.12), $\beta_{v} \beta_{h} N_{v} N_{h}=\mu_{v}\left(r_{h}+\mu_{h}\right)$ when $R_{0}=1$. In this case, the endemic equilibrium $E_{e}$ reduces to the disease-free equilibrium $E_{0}$. On the other hand, when $R_{0}<1$, the only realistic equilibrium solution is $E_{0}$.

Theorem 3.3.5 (Local stability of equilibrium solutions). Let $E_{0}^{*}$ and $E_{e}^{*}$ be the disease-free and endemic equilibrium solutions of system (3.5). Then,

(i) $E_{0}^{*}$ is locally asymptotically stable when $R_{0}<1$, and unstable when $R_{0}>1$;

(ii) $E_{e}^{*}$ is locally asymptotically stable when $R_{0}>1$.

Proof. To investigate the local stability of equilibrium solutions to the simplified version of system (3.5), we linearize the system about $\left(I_{h}^{*}, I_{v}^{*}\right)$. This yields the Jacobian matrix

$$
J\left(I_{h}^{*}, I_{v}^{*}\right)=\left(\begin{array}{cc}
-\left(\beta_{h} I_{v}^{*}+r_{h}+\mu_{h}\right) & \beta_{h}\left(N_{h}-I_{h}^{*}\right) \\
\beta_{v}\left(N_{v}-I_{v}^{*}\right) & -\left(\beta_{v} I_{h}^{*}+\mu_{v}\right)
\end{array}\right),
$$

which gives rise to the characteristic equation

$\xi^{2}+\left(\beta_{h} I_{v}^{*}+\beta_{v} I_{h}^{*}+r_{h}+\mu_{h}+\mu_{v}\right) \xi+\left(\beta_{h} I_{v}^{*}+r_{h}+\mu_{h}\right)\left(\beta_{v} I_{h}^{*}+\mu_{v}\right)-\beta_{v} \beta_{h}\left(N_{v}-I_{v}^{*}\right)\left(N_{h}-I_{h}^{*}\right)=0$

where $\xi$ is an eigenvalue of $J$. 
(i) At $E_{0}^{*}$, equation $(3.13)$ reduces to

$$
\xi^{2}+\left(r_{h}+\mu_{h}+\mu_{v}\right) \xi+\mu_{v}\left(r_{h}+\mu_{h}\right)\left(1-R_{0}\right)=0
$$

From the quadratic formula,

$$
\xi_{1,2}=\frac{-\left(r_{h}+\mu_{h}+\mu_{v}\right) \pm \sqrt{\left(r_{h}+\mu_{h}+\mu_{v}\right)^{2}+4 \mu_{v}\left(r_{h}+\mu_{h}\right)\left(R_{0}-1\right)}}{2} .
$$

When $R_{0}<1$, the two values of $\xi$ are negative if they are real, or have negative real parts if they are complex. In this case, $E_{0}^{*}$ is locally asymptotically stable. On the other hand, if $R_{0}>1$, one value of $\xi$ is positive while the other value is negative. Hence, $E_{0}^{*}$ is a saddle when $R_{0}>1$, which is linearly unstable.

(ii) At $E_{e}^{*}$, the characteristic equation (3.13) reduces to

$$
\theta_{2} \xi^{2}+\theta_{1} \xi+\theta_{0}=0
$$

where

$$
\begin{aligned}
\theta_{2}= & \left(\beta_{h} N_{v}+r_{h}+\mu_{h}\right)\left(\beta_{v} N_{h}+\mu_{v}\right), \\
\theta_{1}= & \beta_{v} N_{h}\left(\beta_{h} N_{v}\left(r_{h}+\mu_{h}+\mu_{v}\right)+\left(r_{h}+\mu_{h}\right)^{2}\right)+\beta_{h} \mu_{v}^{2} N_{v} \\
& +\mu_{v}\left(r_{h}+\mu_{h}\right)\left(\beta_{h} N_{v}+\beta_{v} N_{h}+r_{h}+\mu_{h}+\mu_{v}\right) R_{0}, \\
\theta_{0}= & \mu_{v}\left(r_{h}+\mu_{h}\right)\left(\beta_{h} N_{v}+\mu_{v}\right)\left(\beta_{v} N_{h}+r_{h}+\mu_{h}\right)\left(R_{0}-1\right) .
\end{aligned}
$$

Using the quadratic formula once more, we obtain

$$
\xi_{1,2}=\frac{-\theta_{1} \pm \sqrt{\theta_{1}^{2}-4 \theta_{2} \theta_{0}}}{2 \theta_{2}}
$$

Since the other two coefficients $\theta_{1}$ and $\theta_{2}$ of the characteristic equation (3.15) are both positive, the sign of the constant term $\theta_{0}$ is essential in determining the stability of the endemic equilibrium solution $E_{e}^{*}$. Notice that when $R_{0}>1, \theta_{0}>0$. Therefore, when $R_{0}>1$, both $\xi_{1}$ and $\xi_{2}$ are negative, if they are real or have negative real parts, if they are complex. Hence, $E_{e}^{*}$ is locally asymptotically stable when $R_{0}>1$.

Corollary 3.3.6. The disease-free equilibrium solution $E_{0}^{*}$ is stable when $R_{0}=1$. 
Proof. Follows directly from the proof of Theorem 3.3 .5 by setting $R_{0}=1$ in (3.14).

Theorem 3.3.7 (Global stability of equilibrium solutions). Let $E_{0}$ and $E_{e}$ be the disease-free and endemic equilibrium solutions of system (3.4), respectively. Then,

(i) $E_{0}$ is globally and asymptotically stable when $R_{0}<1$,

(ii) $E_{e}$ is globally asymptotically stable when $R_{0}>1$.

Proof. In the proofs of both parts of the Theorem, we use LaSalle's invariance principle [5] and Lyapunov functions of the form

$$
V=\sum_{i=1}^{n} a_{i}\left(W_{i}-W_{i}^{*}-W_{i}^{*} \ln \frac{W_{i}}{W_{i}^{*}}\right),
$$

where $W_{i}, W_{i}^{*}$ and $a_{i}, i=1,2,3, \ldots, n$ are, respectively, state variables, the equilibrium values of $W_{i}$ and positive constants. See, for example, Refs. [6-14] for a detailed exploration of Lyapunov functions and global stability analysis for compartmental epidemiological models.

(i) As mentioned above, to show that the disease-free equilibrium solution $E_{0}$ of system (3.4) is globally asymptotically stable, we construct a Lyapunov function for the system. To this effect, consider the function $U$ defined as follows:

$$
\begin{aligned}
U:\left\{\left(S_{h}, I_{h}, S_{v}, I_{v}\right) \in \Psi_{1}: S_{h}, S_{v}>0\right\} \rightarrow \mathbb{R}, \\
U\left(S_{h}, I_{h}, S_{v}, I_{v}\right)=a_{0}\left(S_{h}-N_{h}-N_{h} \ln \frac{S_{h}}{N_{h}}\right)+b_{0} I_{h}+ \\
\\
c_{0}\left(S_{v}-N_{v}-N_{v} \ln \frac{S_{v}}{N_{v}}\right)+d_{0} I_{v},
\end{aligned}
$$

where $b_{0}=a_{0}, d_{0}=c_{0}$ and $a_{0}$ and $c_{0}$ are positive constants to be determined. Then $U$ is continuously differentiable in $\Psi_{1}$. Also, $U\left(S_{h}, I_{h}, S_{v}, I_{v}\right)>0$ and $U\left(S_{h}, I_{h}, S_{v}, I_{v}\right)=0$ if and only if $\left(S_{h}, I_{h}, S_{v}, I_{v}\right)=\left(S_{h}^{*}, I_{h}^{*}, S_{v}^{*}, I_{v}^{*}\right)=\left(N_{h}, 0, N_{v}, 0\right)$.

Next, if $U_{x}$, where $x=S_{h}, I_{h}, S_{v}, I_{v}$ denotes the partial derivative of $U$ with respect to $x$, while a dot on $U$ and the other variables denote time derivatives, then we have

$$
\dot{U}=U_{S_{h}} \dot{S}_{h}+U_{I_{h}} \dot{I}_{h}+U_{S_{v}} \dot{S}_{v}+U_{I_{v}} \dot{I}_{v}
$$




$$
\begin{aligned}
= & a_{0}\left(1-\frac{N_{h}}{S_{h}}\right) \dot{S}_{h}+a_{0} \dot{I}_{h}+c_{0}\left(1-\frac{N_{v}}{S_{v}}\right) \dot{S}_{v}+c_{0} \dot{I}_{v} \\
= & a_{0}\left(1-\frac{N_{h}}{S_{h}}\right)\left(\mu_{h} N_{h}+r_{h} I_{h}-\beta_{h} I_{v} S_{h}-\mu_{h} S_{h}\right)+a_{0}\left(\beta_{h} I_{v} S_{h}-\left(r_{h}+\mu_{h}\right) I_{h}\right)+ \\
& c_{0}\left(1-\frac{N_{v}}{S_{v}}\right)\left(\mu_{v} N_{v}-\beta_{v} I_{h} S_{v}-\mu_{v} S_{v}\right)+c_{0}\left(\beta_{v} I_{h} S_{v}-\mu_{v} I_{v}\right) .
\end{aligned}
$$

From $(3.10), E_{0}=\left(S_{h}^{*}, I_{h}^{*}, S_{v}^{*}, I_{v}^{*}\right)=\left(N_{h}, 0, N_{v}, 0\right)$. Since $S_{h}+I_{h}=N_{h}, I_{h}=N_{h}-S_{h}$ and $r_{h} I_{h}=r_{h}\left(N_{h}-S_{h}\right)=r_{h} N_{h}-r_{h} S_{h}$. This leads to

$$
\begin{aligned}
& \dot{U}=a_{0}\left(1-\frac{N_{h}}{S_{h}}\right)\left(\left(r_{h}+\mu_{h}\right) N_{h}-\beta_{h} I_{v} S_{h}-\left(r_{h}+\mu_{h}\right) S_{h}\right)+a_{0}\left(\beta_{h} I_{v} S_{h}-\left(r_{h}+\mu_{h}\right) I_{h}\right) \\
& +c_{0}\left(1-\frac{N_{v}}{S_{v}}\right)\left(\mu_{v} N_{v}-\beta_{v} I_{h} S_{v}-\mu_{v} S_{v}\right)+c_{0}\left(\beta_{v} I_{h} S_{v}-\mu_{v} I_{v}\right) \\
& =a_{0}\left(1-\frac{N_{h}}{S_{h}}\right)\left(-\beta_{h} I_{v} S_{h}-\left(r_{h}+\mu_{h}\right)\left(S_{h}-N_{h}\right)\right)+a_{0}\left(\beta_{h} I_{v} S_{h}-\left(r_{h}+\mu_{h}\right) I_{h}\right)+ \\
& c_{0}\left(1-\frac{N_{v}}{S_{v}}\right)\left(-\beta_{v} I_{h} S_{v}-\mu_{v}\left(S_{v}-N_{v}\right)\right)+c_{0}\left(\beta_{v} I_{h} S_{v}-\mu_{v} I_{v}\right) \\
& =-a_{0}\left(r_{h}+\mu_{h}\right) \frac{\left(S_{h}-N_{h}\right)^{2}}{S_{h}}+a_{0}\left(-\beta_{h} I_{v} S_{h}+\beta_{h} N_{h} I_{v}\right)+a_{0}\left(\beta_{h} I_{v} S_{h}-\left(r_{h}+\mu_{h}\right) I_{h}\right) \\
& -c_{0} \mu_{v} \frac{\left(S_{v}-N_{v}\right)^{2}}{S_{v}}+c_{0}\left(-\beta_{v} S_{v} I_{h}+\beta_{v} N_{v} I_{h}\right)+c_{0}\left(\beta_{v} I_{h} S_{v}-\mu_{v} I_{v}\right) \\
& =-a_{0}\left(r_{h}+\mu_{h}\right) \frac{\left(S_{h}-N_{h}\right)^{2}}{S_{h}}-c_{0} \mu_{v} \frac{\left(S_{v}-N_{v}\right)^{2}}{S_{v}}+a_{0}\left(\beta_{h} N_{h} I_{v}-\left(r_{h}+\mu_{h}\right) I_{h}\right)+ \\
& c_{0}\left(\beta_{v} N_{v} I_{h}-\mu_{v} I_{v}\right) \\
& =-a_{0}\left(r_{h}+\mu_{h}\right) \frac{\left(S_{h}-N_{h}\right)^{2}}{S_{h}}-c_{0} \mu_{v} \frac{\left(S_{v}-N_{v}\right)^{2}}{S_{v}}-a_{0}\left(r_{h}+\mu_{h}\right)\left(-\frac{\beta_{h} N_{h} I_{v}}{r_{h}+\mu_{h}}+I_{h}\right) \\
& -c_{0} \beta_{v} N_{v}\left(-I_{h}+\frac{\mu_{v}}{\beta_{v} N_{v}} I_{v}\right) .
\end{aligned}
$$


By setting $a_{0}=1$ and $c_{0}=\left(r_{h}+\mu_{h}\right) /\left(\beta_{v} N_{v}\right), \dot{U}$ becomes

$$
\begin{aligned}
\dot{U}= & -\left(r_{h}+\mu_{h}\right) \frac{\left(S_{h}-N_{h}\right)^{2}}{S_{h}}-\frac{\mu_{v}\left(r_{h}+\mu_{h}\right)}{\beta_{v} N_{v}} \frac{\left(S_{v}-N_{v}\right)^{2}}{S_{v}} \\
& -\left(r_{h}+\mu_{h}\right)\left(-\frac{\beta_{h} N_{h}}{r_{h}+\mu_{h}}+\frac{\mu_{v}}{\beta_{v} N_{v}}\right) I_{v} \\
= & -\left(r_{h}+\mu_{h}\right) \frac{\left(S_{h}-N_{h}\right)^{2}}{S_{h}}-\frac{\mu_{v}\left(r_{h}+\mu_{h}\right)}{\beta_{v} N_{v}} \frac{\left(S_{v}-N_{v}\right)^{2}}{S_{v}} \\
& -\frac{\mu_{v}\left(r_{h}+\mu_{h}\right)}{\beta_{v} N_{v}}\left(-\frac{\beta_{v} \beta_{h} N_{v} N_{h}}{\mu_{v}\left(r_{h}+\mu_{h}\right)}+1\right) I_{v} \\
= & -\left(r_{h}+\mu_{h}\right) \frac{\left(S_{h}-N_{h}\right)^{2}}{S_{h}}-\frac{\mu_{v}\left(r_{h}+\mu_{h}\right)}{\beta_{v} N_{v}} \frac{\left(S_{v}-N_{v}\right)^{2}}{S_{v}}-\frac{\mu_{v}\left(r_{h}+\mu_{h}\right)}{\beta_{v} N_{v}}\left(1-R_{0}\right) I_{v} \\
\leq & 0, \text { when } R_{0} \leq 1 .
\end{aligned}
$$

Now, by setting $\left(S_{h}, I_{h}, S_{v}, I_{v}\right)=\left(N_{h}, 0, N_{v}, 0\right)$, we have $\dot{U}=0$. Hence, $\dot{U}=0$ if and only if $\left(S_{h}, I_{h}, S_{v}, I_{v}\right)=\left(N_{h}, 0, N_{v}, 0\right)$. Thus, the singleton $\left\{E_{0}\right\}$ constitutes the largest compact invariant set in $\left\{\left(S_{h}, I_{h}, S_{v}, I_{v}\right) \in \Psi_{1}: \dot{U}=0\right\}$. Therefore, LaSalle's invariance principle assures us that the disease-free equilibrium solution $E_{0}$ is globally asymptotically stable in $\Psi_{1}$. This completes the proof of the first part of the Theorem.

(ii) Note that the endemic equilibrium solution $E_{e}=\left(S_{h}^{*}, I_{h}^{*}, S_{v}^{*}, I_{v}^{*}\right)$ that is given by (3.11)(3.12) exists only when $R_{0}>1$. Now, consider the function $V$ defined as follows:

$$
\begin{array}{r}
V:\left\{\left(S_{h}, I_{h}, S_{v}, I_{v}\right) \in \Psi_{1}: S_{h}, I_{h}, S_{v}, I_{v}>0\right\} \rightarrow \mathbb{R}, \\
V\left(S_{h}, I_{h}, S_{v}, I_{v}\right)=a\left(S_{h}-S_{h}^{*}-S_{h}^{*} \ln \frac{S_{h}}{S_{h}^{*}}\right)+b\left(I_{h}-I_{h}^{*}-I_{h}^{*} \ln \frac{I_{h}}{I_{h}^{*}}\right) \\
+c\left(S_{v}-S_{v}^{*}-S_{v}^{*} \ln \frac{S_{v}}{S_{v}^{*}}\right)+d\left(I_{v}-I_{v}^{*}-I_{v}^{*} \ln \frac{I_{v}}{I_{v}^{*}}\right),
\end{array}
$$

where $b=a, d=c$ and $a$ and $b$ are positive constants to be determined. We establish that $V$ is a Lyapunov function for system (3.4). 
First we note that $V$ is continuously differentiable in $\Psi_{1}, V\left(S_{h}, I_{h}, S_{v}, I_{v}\right)>0$ for all $\left(S_{h}, I_{h}, S_{v}, I_{v}\right) \neq\left(S_{h}^{*}, I_{h}^{*}, S_{v}^{*}, I_{v}^{*}\right)$, and $V\left(S_{h}^{*}, I_{h}^{*}, S_{v}^{*}, I_{v}^{*}\right)=0$. That is, $V\left(S_{h}, I_{h}, S_{v}, I_{v}\right)=0$ if and only if $\left(S_{h}, I_{h}, S_{v}, I_{v}\right)=\left(S_{h}^{*}, I_{h}^{*}, S_{v}^{*}, I_{v}^{*}\right)$, where the equilibrium values $S_{h}^{*}, I_{h}^{*}, S_{v}^{*}$ and $I_{v}^{*}$ are given by equation $(3.12)$.

Next, we show that $\dot{V}<0$ and that $\dot{V}=0$ if and only if $\left(S_{h}, I_{h}, S_{v}, I_{v}\right)=$ $\left(S_{h}^{*}, I_{h}^{*}, S_{v}^{*}, I_{v}^{*}\right)$. To this effect, if $V_{x}$, where $x=S_{h}, I_{h}, S_{v}, I_{v}$ denotes the partial derivative of $V$ with respect to $x$, while a dot on $V$ and the other variables denote time derivatives, then we have

$$
\begin{aligned}
\dot{V}= & V_{S_{h}} \dot{S}_{h}+V_{I_{h}} \dot{I}_{h}+V_{S_{v}} \dot{S}_{v}+V_{I_{v}} \dot{I}_{v} \\
= & a\left(1-\frac{S_{h}^{*}}{S_{h}}\right) \dot{S}_{h}+a\left(1-\frac{I_{h}^{*}}{I_{h}}\right) \dot{I}_{h}+c\left(1-\frac{S_{v}^{*}}{S_{v}}\right) \dot{S}_{v}+c\left(1-\frac{I_{v}^{*}}{I_{v}}\right) \dot{I}_{v} \\
= & a\left(1-\frac{S_{h}^{*}}{S_{h}}\right)\left(\mu_{h} N_{h}+r_{h} I_{h}-\beta_{h} I_{v} S_{h}-\mu_{h} S_{h}\right)+ \\
& a\left(1-\frac{I_{h}^{*}}{I_{h}}\right)\left(\beta_{h} I_{v} S_{h}-\left(r_{h}+\mu_{h}\right) I_{h}\right)+c\left(1-\frac{S_{v}^{*}}{S_{v}}\right)\left(\mu_{v} N_{v}-\beta_{v} I_{h} S_{v}-\mu_{v} S_{v}\right)+ \\
& c\left(1-\frac{I_{v}^{*}}{I_{v}}\right)\left(\beta_{v} I_{h} S_{v}-\mu_{v} I_{v}\right) .
\end{aligned}
$$

Since $S_{h}+I_{h}=N_{h}, I_{h}=N_{h}-S_{h}$ and $r_{h} I_{h}=r_{h} N_{h}-r_{h} S_{h}$. This leads to

$$
\begin{aligned}
\dot{V}= & a\left(1-\frac{S_{h}^{*}}{S_{h}}\right)\left(\left(r_{h}+\mu_{h}\right) N_{h}-\beta_{h} I_{v} S_{h}-\left(r_{h}+\mu_{h}\right) S_{h}\right)+ \\
& a\left(1-\frac{I_{h}^{*}}{I_{h}}\right)\left(\beta_{h} I_{v} S_{h}-\left(r_{h}+\mu_{h}\right) I_{h}\right)+c\left(1-\frac{S_{v}^{*}}{S_{v}}\right)\left(\mu_{v} N_{v}-\beta_{v} I_{h} S_{v}-\mu_{v} S_{v}\right)+ \\
& c\left(1-\frac{I_{v}^{*}}{I_{v}}\right)\left(\beta_{v} I_{h} S_{v}-\mu_{v} I_{v}\right) .
\end{aligned}
$$


Now, the equilibrium values of system (3.4) are given by the following system:

$$
\begin{aligned}
& \mu_{h} N_{h}+r_{h}\left(N_{h}-S_{h}^{*}\right)-\beta_{h} I_{v}^{*} S_{h}^{*}-\mu_{h} S_{h}^{*}=0, \\
& \beta_{h} I_{v}^{*} S_{h}^{*}-\left(r_{h}+\mu_{h}\right) I_{h} *=0, \\
& \mu_{v} N_{v}-\beta_{v} I_{h}^{*} S_{v}^{*}-\mu_{v} S_{v}^{*}=0, \\
& \beta_{v} I_{h}^{*} S_{v}^{*}-\mu_{v} I_{v}^{*}=0 .
\end{aligned}
$$

This can be expressed as follows:

$$
\begin{aligned}
\left(r_{h}+\mu_{h}\right) N_{h} & =\beta_{h} I_{v}^{*} S_{h}^{*}+\left(r_{h}+\mu_{h}\right) S_{h}^{*}, \\
r_{h}+\mu_{h} & =\frac{\beta_{h} I_{v}^{*} S_{h}^{*}}{I_{h}^{*}}, \\
\mu_{v} N_{v} & =\beta_{v} I_{h}^{*} S_{v}^{*}+\mu_{v} S_{v}^{*}, \\
\mu_{v} & =\frac{\beta_{v} I_{h}^{*} S_{v}^{*}}{I_{v}^{*}} .
\end{aligned}
$$

Substituting equations (3.17)-(3.20) in system (3.16) yields

$$
\begin{aligned}
\dot{V}= & a\left(1-\frac{S_{h}^{*}}{S_{h}}\right)\left(\beta_{h} I_{v}^{*} S_{h}^{*}+\left(r_{h}+\mu_{h}\right) S_{h}^{*}-\beta_{h} I_{v} S_{h}-\left(r_{h}+\mu_{h}\right) S_{h}\right)+ \\
& a\left(1-\frac{I_{h}^{*}}{I_{h}}\right)\left(\beta_{h} I_{v} S_{h}-\beta_{h} I_{v}^{*} S_{h}^{*} \frac{I_{h}}{I_{h}^{*}}\right)+ \\
& c\left(1-\frac{S_{v}^{*}}{S_{v}}\right)\left(\beta_{v} I_{h}^{*} S_{v}^{*}+\mu_{v} S_{v}^{*}-\beta_{v} I_{h} S_{v}-\mu_{v} S_{v}\right)+ \\
& c\left(1-\frac{I_{v}^{*}}{I_{v}}\right)\left(\beta_{v} I_{h} S_{v}-\beta_{v} I_{h}^{*} S_{v}^{*} \frac{I_{v}}{I_{v}^{*}}\right)
\end{aligned}
$$




$$
\begin{gathered}
=a\left(1-\frac{S_{h}^{*}}{S_{h}}\right)\left(\beta_{h} I_{v}^{*} S_{h}^{*}\left(1-\frac{I_{v} S_{h}}{I_{v}^{*} S_{h}^{*}}\right)-\left(r_{h}+\mu_{h}\right)\left(S_{h}-S_{h}^{*}\right)\right)+ \\
\quad a \beta_{h} I_{v}^{*} S_{h}^{*}\left(1-\frac{I_{h}^{*}}{I_{h}}\right)\left(\frac{I_{v} S_{h}}{I_{v}^{*} S_{h}^{*}}-\frac{I_{h}}{I_{h}^{*}}\right)+ \\
c\left(1-\frac{S_{v}^{*}}{S_{v}}\right)\left(\beta_{v} I_{h}^{*} S_{v}^{*}\left(1-\frac{I_{h} S_{v}}{I_{h}^{*} S_{v}^{*}}\right)-\mu_{v}\left(S_{v}-S_{v}^{*}\right)\right)+ \\
c \beta_{v} I_{h}^{*} S_{v}^{*}\left(1-\frac{I_{v}^{*}}{I_{v}}\right)\left(\frac{I_{h} S_{v}}{I_{h}^{*} S_{v}^{*}}-\frac{I_{v}}{I_{v}^{*}}\right) \\
a \beta_{h} I_{v}^{*} S_{h}^{*}\left(1-\frac{S_{h}^{*}}{S_{h}}\right)\left(1-\frac{I_{v} S_{h}}{I_{v}^{*} S_{h}^{*}}\right)-a\left(r_{h}+\mu_{h}\right)\left(1-\frac{S_{h}^{*}}{S_{h}}\right)\left(S_{h}-S_{h}^{*}\right)+ \\
a \beta_{v} I_{h}^{*} S_{v}^{*}\left(2-\frac{S_{v}^{*}}{S_{v}}-\frac{I_{v}}{I_{v}^{*}}+\frac{I_{h}}{I_{h}^{*}}-\frac{I_{h} S_{v} I_{v}^{*}}{I_{h}^{*} S_{v}^{*} I_{v}}\right) \cdot \\
a \beta_{h} I_{v}^{*} S_{h}^{*}\left(1-\frac{I_{h}^{*}}{I_{h}}\right)\left(\frac{I_{v} S_{h}}{I_{v}^{*} S_{h}^{*}}-\frac{I_{h}}{I_{h}^{*}}\right)+c \beta_{v} I_{h}^{*} S_{v}^{*}\left(1-\frac{S_{v}^{*}}{S_{v}}\right)\left(1-\frac{I_{h} S_{v}}{I_{h}^{*} S_{v}^{*}}\right)- \\
c \mu_{v}\left(1-\frac{S_{v}^{*}}{S_{v}}\right)\left(\frac{I_{h}^{*}}{I_{h}}+\frac{I_{v}}{I_{v}^{*}}-\frac{I_{h} S_{h}}{I_{h} I_{v}^{*} S_{h}^{*}}\right)+ \\
=a\left(r_{h}+\mu_{h}^{*}\right)+c \beta_{v} I_{h}^{*} S_{v}^{*}\left(1-\frac{I_{h}^{*} S_{v}}{I_{v}}-\frac{I_{v}}{I_{v}^{*}}\right)
\end{gathered}
$$

By setting $a=\beta_{v} I_{h}^{*} S_{v}^{*}$ and $c=\beta_{h} I_{v}^{*} S_{h}^{*}, \dot{V}$ reduces to

$$
\begin{aligned}
\dot{V}= & -\beta_{v} I_{h}^{*} S_{v}^{*}\left(r_{h}+\mu_{h}\right) \frac{\left(S_{h}-S_{h}^{*}\right)^{2}}{S_{h}}-\mu_{v} \beta_{h} I_{v}^{*} S_{h}^{*} \frac{\left(S_{v}-S_{v}^{*}\right)^{2}}{S_{v}}+ \\
& \beta_{v} \beta_{h} I_{h}^{*} S_{v}^{*} I_{v}^{*} S_{h}^{*}\left(4-\left(\frac{S_{h}^{*}}{S_{h}}+\frac{S_{v}^{*}}{S_{v}}+\frac{I_{h}^{*} I_{v} S_{h}}{I_{h} I_{v}^{*} S_{h}^{*}}+\frac{I_{h} S_{v} I_{v}^{*}}{I_{h}^{*} S_{v}^{*} I_{v}}\right)\right) .
\end{aligned}
$$

Clearly,

$$
4 \leq \frac{S_{h}^{*}}{S_{h}}+\frac{S_{v}^{*}}{S_{v}}+\frac{I_{h}^{*} I_{v} S_{h}}{I_{h} I_{v}^{*} S_{h}^{*}}+\frac{I_{h} S_{v} I_{v}^{*}}{I_{h}^{*} S_{v}^{*} I_{v}}
$$


for all values of $S_{h}, I_{h}, S_{v}, I_{v} \geq 0$, since the geometric mean is less than or equal to the arithmetic mean. Therefore,

$$
\begin{aligned}
\dot{V}= & -\left(\beta_{v} I_{h}^{*} S_{v}^{*}\left(r_{h}+\mu_{h}\right) \frac{\left(S_{h}-S_{h}^{*}\right)^{2}}{S_{h}}+\mu_{v} \beta_{h} I_{v}^{*} S_{h}^{*} \frac{\left(S_{v}-S_{v}^{*}\right)^{2}}{S_{v}}\right) \\
& -\beta_{v} \beta_{h} I_{h}^{*} S_{v}^{*} I_{v}^{*} S_{h}^{*}\left(\frac{S_{h}^{*}}{S_{h}}+\frac{S_{v}^{*}}{S_{v}}+\frac{I_{h}^{*} I_{v} S_{h}}{I_{h} I_{v}^{*} S_{h}^{*}}+\frac{I_{h} S_{v} I_{v}^{*}}{I_{h}^{*} S_{v}^{*} I_{v}}-4\right) \leq 0 .
\end{aligned}
$$

Now, by setting $\left(S_{h}, I_{h}, S_{v}, I_{v}\right)=\left(S_{h}^{*}, I_{h}^{*}, S_{v}^{*}, I_{v}^{*}\right)$ in $(3.21)$, we obtain $\dot{V}=0$. Hence, $\dot{V}<0$ for $\left(S_{h}, I_{h}, S_{v}, I_{v}\right) \neq\left(S_{h}^{*}, I_{h}^{*}, S_{v}^{*}, I_{v}^{*}\right)$ and $\dot{V}=0$ if and only if $\left(S_{h}, I_{h}, S_{v}, I_{v}\right)=$ $\left(S_{h}^{*}, I_{h}^{*}, S_{v}^{*}, I_{v}^{*}\right)$ for all $S_{h}^{*}, I_{h}^{*}, S_{v}^{*}, I_{v}^{*}>0$. Hence, the singleton $E_{e}$ constitutes the largest compact invariant set in $\left\{\left(S_{h}, I_{h}, S_{v}, I_{v}\right) \in \Psi_{1}: \dot{V}=0\right\}$ and LaSalle's invariance principle assures us that the unique endemic equilibrium solution $E_{e}$ of system (3.4) is globally asymptotically stable in $\Psi_{1}$. This concludes the proof of the Theorem.

\subsection{Numerical Simulations of the Classical SIS Model}

We now present numerical simulations that confirm the above results. We use a human natural death rate of $\mu_{h}=4.6 \times 10^{-5}$ per day, which corresponds to an average life expectancy of 60 years, a vector natural death rate of $\mu_{v}=4.8 \times 10^{-2}$ per day, which corresponds to an average vector life span of 21 days, a human disease recovery rate of $r_{h}=8.3 \times 10^{-3}$ per day, which corresponds to a duration of infection of 120 days, a total human population of $N_{h}=5.0 \times 10^{3}$, and a total vector population $N_{v}=1.0 \times 10^{4}$. We vary the contact rates $\beta_{h}$ and $\beta_{v}$ accordingly for the disease-free and endemic cases. Integrating system (3.5) using a regular fourth-order Runge-Kutta scheme with variable step-size control [15, 16] yields the results plotted in Figs. 3.2-3.3.

Figure 3.2 depicts a stable disease-free equilibrium. It thus represents the situation in which the disease is completely eradicated from the population. Notice that although there were 2,000 infectious humans from the onset, some of them recovered from the disease to join the susceptible class while some died. Since we assumed constant population sizes, the number of new births is exactly the same as the number of deaths, and so the total human 
population and vector populations, which become entirely susceptible, stabilize at 5,000 and 10,000, respectively, after some time.

Figure 3.3 depicts a stable endemic equilibrium solution. This is the situation in which the disease establishes itself within the community. Notice that although there was no infectious human and only one infectious vector from the onset, the disease eventually affects most of the human population, resulting in 4,525 infectious humans and 8,403 infectious vectors. 


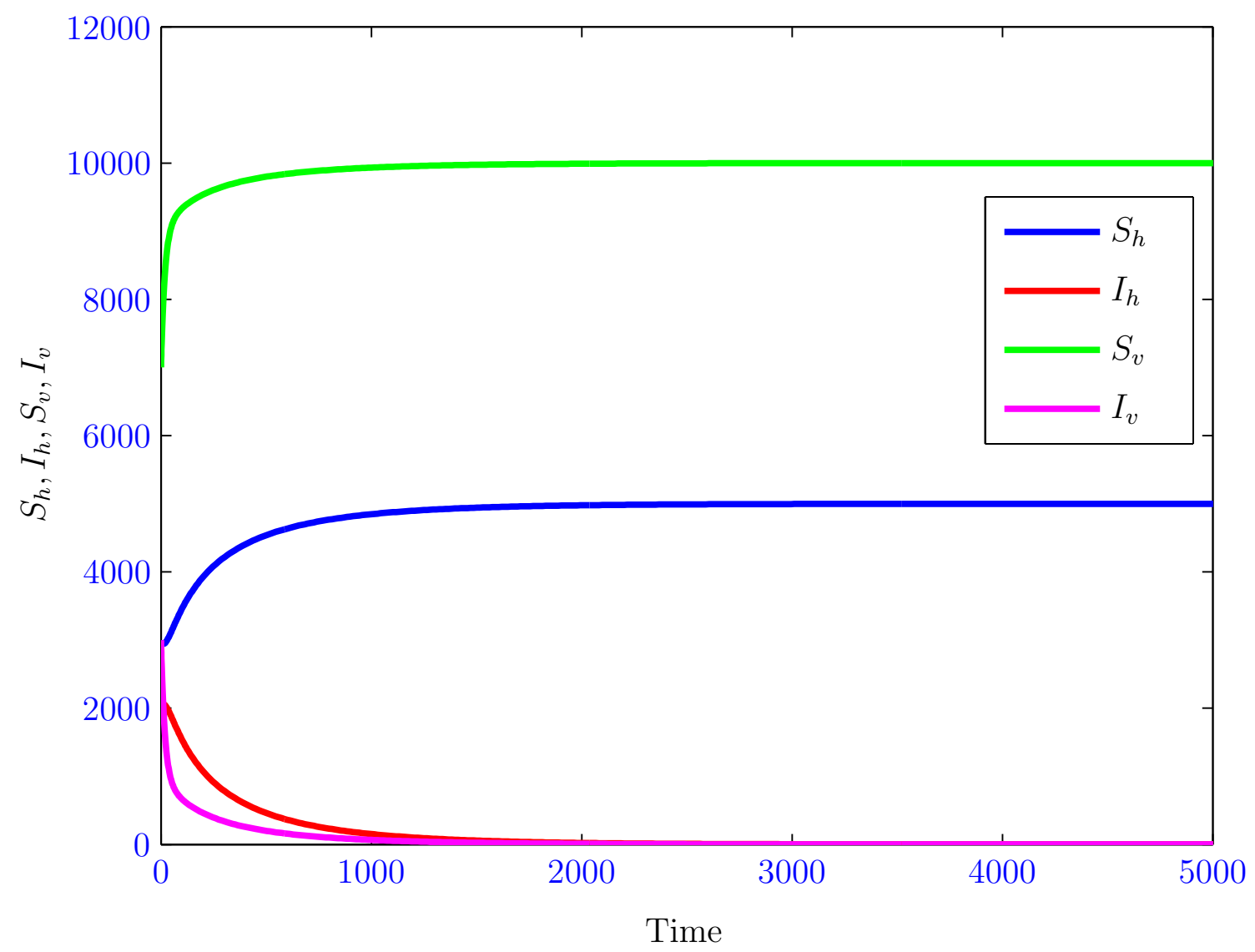

Figure 3.2. Time series plot of solutions to the classical SIS model depicting a stable disease-free equilibrium solution for the parameter regime $\mu_{h}=4.6 \times$ $10^{-5}, \mu_{v}=4.8 \times 10^{-2}, r_{h}=8.3 \times 10^{-3}, N_{h}=5.0 \times 10^{3}, N_{v}=1.0 \times 10^{4}, \beta_{h}=$ $5.0 \times 10^{-6}$, and $\beta_{v}=2.0 \times 10^{-6}$. The initial conditions are $\left(S_{h}^{0}, I_{h}^{0}, S_{v}^{0}, I_{v}^{0}\right)=$ $(3000,2000,7000,3000)$. The basic reproduction number is $R_{0}=0.7519$ and the equilibrium solution is $\left(S_{h}^{*}, I_{h}^{*}, S_{v}^{*}, I_{v}^{*}\right)=(5000,0,10000,0)$. 


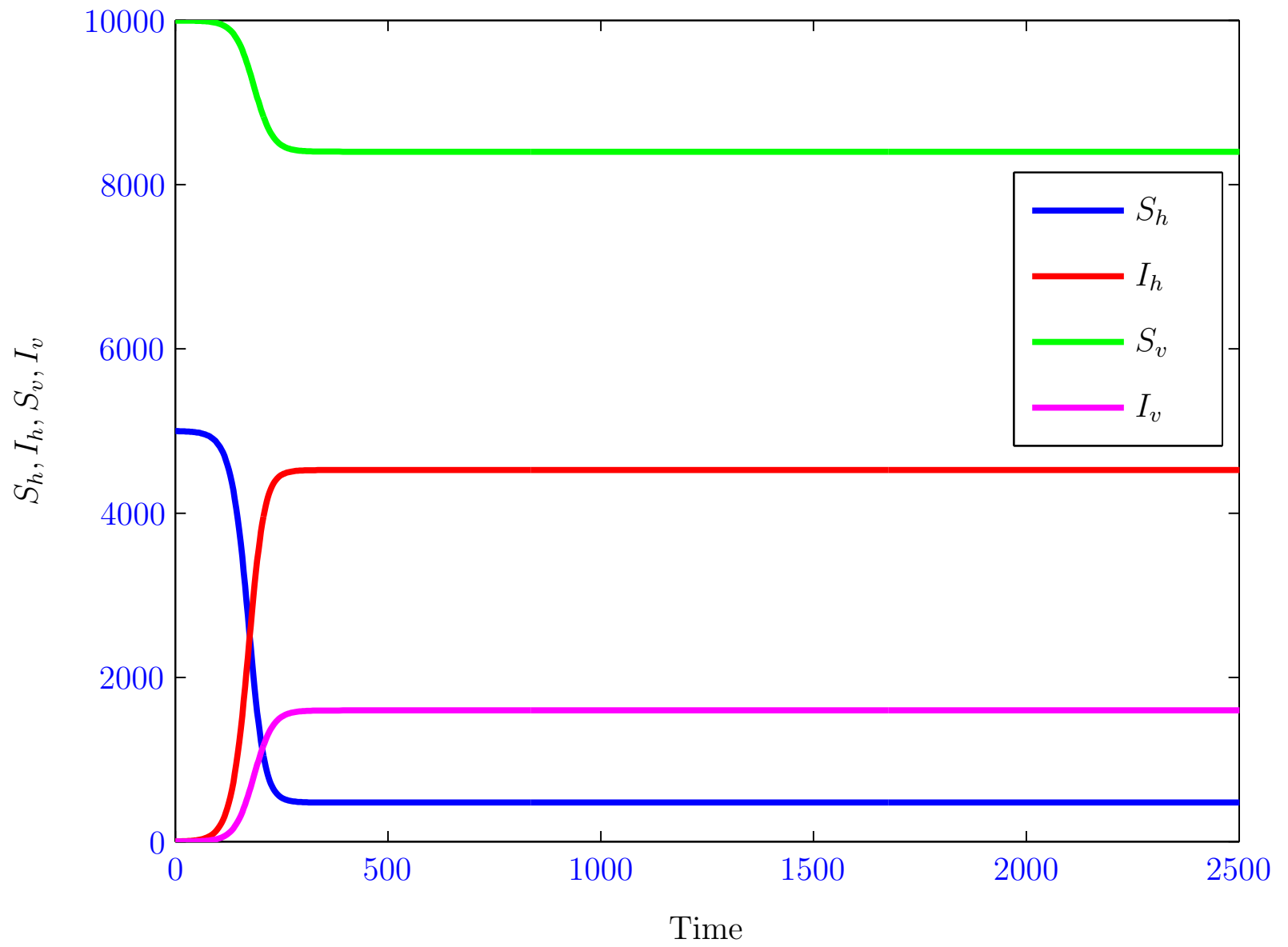

Figure 3.3. Time series plot of solutions to the classical SIS model depicting a stable endemic equilibrium solution for the parameter regime $\mu_{h}=4.6 \times 10^{-5}, \mu_{v}=$ $4.8 \times 10^{-2}, r_{h}=8.3 \times 10^{-3}, N_{h}=5.0 \times 10^{3}, N_{v}=1.0 \times 10^{4}, \beta_{h}=5.0 \times 10^{-5}$, and $\beta_{v}=2.0 \times 10^{-6}$. The initial conditions are $\left(S_{h}^{0}, I_{h}^{0}, S_{v}^{0}, I_{v}^{0}\right)=(5000,0,9999,1)$. The basic reproduction number is $R_{0}=12.53$ and the equilibrium solution is $\left(S_{h}^{*}, I_{h}^{*}, S_{v}^{*}, I_{v}^{*}\right)=(475,4525,8403,1597)$. 


\subsection{Conclusion}

We have derived and analyzed the classical SIS model for malaria. The model has two equilibrium solutions, a disease-free equilibrium solution that exists and is stable when the basic reproduction number is smaller than or equal to unity and an endemic equilibrium solution that exists and is stable when the basic reproduction number is larger than unity. A closer observation of the characteristic polynomial (3.13) shows, as has been established in the literature, that this classical SIS model for malaria transmission cannot generate oscillatory dynamics, a clear limitation of the model. This limitation can be attributed to the assumptions made in deriving the model. 


\section{References}

[1] R. Ross, The Prevention of Malaria (John Murray, London, 1911). 44

[2] G. Macdonald, The Epidemiology and Control of Malaria (Oxford university, Oxford, 1957).

[3] J. C. Koella, "On the use of Mathematical Models of Malaria Transmission," Acta Tropica 49, 1- 25 (1991).

Online Version 44

[4] O. Diekmann, J. A. P. Heesterbeek, and J. A. J. Metz, "On the Definition and the Computation of the Basic Reproduction Ratio $R_{0}$ in Rodels for Infectious Diseases in Heterogeneous Populations," Journal of Mathematical Biology 28, 365-382 (1990). Online Version 48

[5] J. P. LaSalle, The Stability of Dynamical Systems (SIAM, Philadelphia, PA, 1976). 51

[6] E. Beretta, T. Hara, W. Ma, and Y. Takeuchi, "Global Asymptotic Stability of an SIR Epidemic Model with Distributed Time Delay," Nonlinear Analysis 47, 4107-4115 (2001). 51

[7] A. Korobeinikov and G. C. Wake, "Lyapunov Functions and Global Stability for SIR, SIRS and SIS Epidemiological Models," Applied Mathematics Letters 15 (8), 955-960 (2002).

Online Version 
[8] A. Korobeinikov, "Lyapunov Functions and Global Stability for SIR and SIRS Epidemiological Models with Non-Linear Transmission," Bulletin of Mathematical Biology 68, 615-626 (2006).

Online Version

[9] Z. Jin, M. Zhien, and H. Maoam, "Global Stability of an SIRS Epidemic Model with Delays," Acta Mathematica Scientia 26B (2), 291-306 (2006).

[10] C. V. de León and J. A. C. Hernández, "Local and Global Stability of Host-Vector Disease Models," Foro-Red-Mat: Revista Electrónica de Contenido Matemático 25 (3), 1405-1745 (2008).

Online Version

[11] C. V. de León, "Constructions of Lyapunov Functions for Classics SIS, SIR and SIRS Epidemic Model with Variable Population Size," Foro-Red-Mat: Revista Electrónica de Contenido Matemático 26 (5), 1405-1745 (2009).

Online Version

[12] R. Xu and Z. Ma, "Global Stability of a SIR Epidemic Model with Nonlinear Incidence Rate and Time Delay," Nonlinear Analysis: Real World Applications 10, 3175-3189 (2009).

Online Version

[13] C. C. McCluskey, "Complete Global Stability for an SIR Epidemic Model with DelayDistributed or Discrete," Nonlinear Analysis: Real World Applications 11, 55-59 (2010).

Online Version

[14] M. Y. Li, Z. Shuai, and C. Wang, "Global Stability of Multi-group Epidemic Models with Distributed Delays," Journal of Mathematical Analysis and Applications 361, 3847 (2010).

Online Version 51 
[15] J. R. Cash and A. H. Karp, "A Variable Order Runge-Kutta Method for Initial Value Problems with Rapidly Varying Right-hand Sides," ACM Transactions on Mathematical Software 16 (3), 2001-222 (1990).

Online Version 57

[16] E. Hairer and G. Wanner, Solving Ordinary Differential Equations II: Stiff and Differential-Algebraic Problems. (Springer-Verlag, 1996), 2nd edition. 57 


\section{$\overline{\text { Chapter }} 4$}

\section{Derivation of the Basic ODE Model}

\subsection{Introduction}

In this chapter, we derive and nondimensionalize a new deterministic SIS (susceptibleinfectious-susceptible) mathematical model for the dynamics of malaria transmission. Since malaria does not confer permanent immunity, it makes sense to consider an SIS model, whereby infectious humans regain susceptibility after recovering from the disease. The new model shall pay greater attention to the population dynamics of the disease transmission agent, the mosquito, a factor that has hitherto been omitted in most malaria models. Contrary to traditional SIS models in the biological and epidemiological literature (see, for example, $[1-5]$ ), the new model further subdivides the vector population into three mutually disjoint classes based on the resting, fertility, nourishment, and reproductive status of the vector. Mosquito population dynamics was first modeled in this manner by Ngwa [6] and has been extended in Refs. [7, 8]. However, these models focused only on the dynamics of the vector population in the absence of the malaria disease. Here, we derive a full SIS malaria model consisting of a system of eight coupled ordinary differential equations - two for the human population dynamics and six describing the dynamics of the vector population.

This chapter is organized as follows: In Section 4.2, we briefly describe the variables and parameters to be used in the new model. The general SIS non-linear ordinary differential equation model is derived Section 4.3. A simplified SIS model is presented and nondimensionalized in Section 4.4, and we conclude the chapter in Section 4.5. 


\subsection{Model Variables and Parameters}

The full general SIS mathematical model will take the form of a non-linear delayed differ-

ential system of ordinary differential equations involving both the human and vector populations. Only populations involved in disease transmission are represented in the model formulation, and these include female Anopheles mosquitoes and humans of all ages and sexes. The equations are derived based on the additional fact that, in the presence of the malaria disease in the populations, both mosquitoes and humans can infect each other upon contact. While infected humans can recover from the malaria infection, it is assumed that once a mosquito is infected, it remains infected until death. To capture the life cycle of the mosquito vector in the model, the vector population is divided into three biologically realistic compartmental classes representing physiological status. These classes are: The class of fed and reproducing vectors returning from human habitats to vector breeding sites represented by the variable $U$; the class of unfed and resting vectors present at vector breeding sites represented by the variable $V$; and the class of unfed vectors questing (or foraging) for food (blood meal) in human habitats represented by the variable $W$. The human population and the vector population in each compartmental class are again classified into two epidemiologically realistic compartmental classes representing disease status: the susceptible class, consisting of individuals with the potential to contract the Plasmodium parasite but who have not yet contracted it, and the infectious class, consisting of individuals who have already contracted the Plasmodium parasite and can transmitted it. Therefore, there are eight dependent variables that continuously depend on time $t$. All variables and parameters in the human system will carry the subscript $h$, while those in the vector subsystem will carry one of the subscripts $v, u$ or $w$ depending on the physiological status of the vector. Thus, at any time $t$ we have humans of type $S_{h}(t)$ and $I_{h}(t)$, and vectors of type $S_{v}(t)$, $S_{w}(t), S_{u}(t)$, and type $I_{v}(t), I_{w}(t)$ and $I_{u}(t)$, as explained in Table 4.1. The total human and vector populations, denoted by $N_{h}(t)$ and $N_{m v}(t)$, respectively, are then given as

$$
\begin{aligned}
N_{h}(t) & =S_{h}(t)+I_{h}(t), \\
N_{m v}(t) & =S_{v}(t)+I_{v}(t)+S_{w}(t)+I_{w}(t)+S_{u}(t)+I_{u}(t) .
\end{aligned}
$$

See Tables 4.1 and 4.2 for descriptions model variables and parameters, respectively. 
Table 4.1. Model variables and their definitions.

\begin{tabular}{|c|c|}
\hline Variable & Definition \\
\hline$S_{h}(t)$ & $\begin{array}{l}\text { Susceptible humans (humans with the potential of being infected but who do } \\
\text { not harbor malaria parasites in their liver or blood stream) at time } t \text {. }\end{array}$ \\
\hline$I_{h}(t)$ & $\begin{array}{l}\text { Infectious humans (humans carrying either the liver or blood stage forms of } \\
\text { the malaria parasite) at time } t \text {. }\end{array}$ \\
\hline$N_{h}(t)$ & Total human population at time $t$. \\
\hline$S_{u}(t)$ & $\begin{array}{l}\text { Susceptible vectors of class } U \text {. These are the fertilized, fed and reproducing } \\
\text { susceptible female vectors that do not have any form of the malaria parasite. }\end{array}$ \\
\hline$S_{v}(t)$ & $\begin{array}{l}\text { Susceptible vectors of class } V \text {. This comprises of previously fertilized suscep- } \\
\text { tible female vectors at breeding site that have just laid their eggs but are still } \\
\text { resting at the breeding site together with all unfertilized females that have not } \\
\text { yet fed on blood from humans and are not questing for blood but are swarming } \\
\text { at the breeding site (some of these are the newly emerging vectors). }\end{array}$ \\
\hline$S_{w}(t)$ & $\begin{array}{l}\text { Susceptible vectors of class } W \text {. These are all the fertilized but non-reproducing } \\
\text { susceptible vectors that have left the breeding site and are questing for a } \\
\text { blood meal. They comprise of the newly fertilized vectors together with the } \\
\text { previously fertilized susceptible vectors; that is, those } S_{v} \text { vectors recruited from } \\
\text { the } S_{u}(t) \text { class of vectors that fed on susceptible humans and successfully made } \\
\text { it back to the breeding site. }\end{array}$ \\
\hline$I_{u}(t)$ & $\begin{array}{l}\text { Infectious vectors of class } U \text {. These are the fertilized, well nourished and } \\
\text { reproducing vectors that fed on infectious humans. }\end{array}$ \\
\hline$I_{v}(t)$ & $\begin{array}{l}\text { Infectious vectors of class } V \text { (previously fertilized infectious female vectors at } \\
\text { breeding site that have just laid eggs but are still resting at the breeding site). }\end{array}$ \\
\hline$I_{w}(t)$ & $\begin{array}{l}\text { Infectious vectors of class } W \text {. These are the fertilized but non-reproducing } \\
\text { infectious vectors that have left the breeding site and are questing for their } \\
\text { second or subsequent blood meal. }\end{array}$ \\
\hline$N_{m v}(t)$ & Total vector population at time $t$. \\
\hline
\end{tabular}


Table 4.2. System parameters representing the transition rates from one class to another together with recruitment and removal rates for humans and mosquitoes.

\begin{tabular}{|c|c|}
\hline Parameter & Description \\
\hline$a_{v}$ & The rate at which successfully fed vectors return to breeding site. \\
\hline$\alpha_{v}$ & The rate at which vectors are attracted to human habitats. \\
\hline$\mu_{h}$ & Natural human death rate. \\
\hline$\mu_{u, v, w}$ & Natural mosquito death rate from each mosquito population class. \\
\hline$\lambda_{h}$ & Human birth rate. \\
\hline$\tilde{\lambda}_{v}$ & Mosquito birth rate. \\
\hline$r_{h}$ & $\begin{array}{l}\text { Recovery rate among humans. Once infected, vectors are assumed to remain } \\
\text { infectious for the remainder of their lives. }\end{array}$ \\
\hline$\beta_{v}$ & $\begin{array}{l}\text { Flow rate from susceptible questing mosquito to either a susceptible human } \\
\text { or an infectious human. Contact between a susceptible questing mosquito } \\
\text { and an infectious human, denoted by } \beta_{v_{s i}} \text {, leads to an infectious mosquito or } \\
\text { new infection in the mosquito population, while contact between a susceptible } \\
\text { mosquito and a susceptible human, which we denote by } \beta_{v_{s s}} \text {, does not. }\end{array}$ \\
\hline$\beta_{h}$ & $\begin{array}{l}\text { Flow rate from infectious questing mosquitoes to susceptible or infectious } \\
\text { humans. Contact between an infectious mosquito and a susceptible human, } \\
\text { denoted by } \beta_{h_{i s}} \text {, leads to a new infectious human, while contact between an } \\
\text { infectious mosquito and an infectious human, denoted by } \beta_{h_{i i}} \text {, does not. }\end{array}$ \\
\hline$p$ & $\begin{array}{l}\text { Probability that a susceptible questing vector successfully takes a blood meal } \\
\text { from a susceptible human. }\end{array}$ \\
\hline$q$ & $\begin{array}{l}\text { Probability that a susceptible questing vector successfully takes a blood meal } \\
\text { from an infectious human. }\end{array}$ \\
\hline$p_{1}$ & $\begin{array}{l}\text { Probability that an infectious questing vector successfully takes a blood meal } \\
\text { from an infectious human. }\end{array}$ \\
\hline$q_{1}$ & $\begin{array}{l}\text { Probability that an infectious questing vector successfully takes a blood meal } \\
\text { from a susceptible human. }\end{array}$ \\
\hline$\eta$ & Proportion of emerging adult mosquitoes at the breeding site that are female. \\
\hline
\end{tabular}




\subsection{THE ODE MODEL}

A conceptual framework for the model is shown in Fig. 4.1.

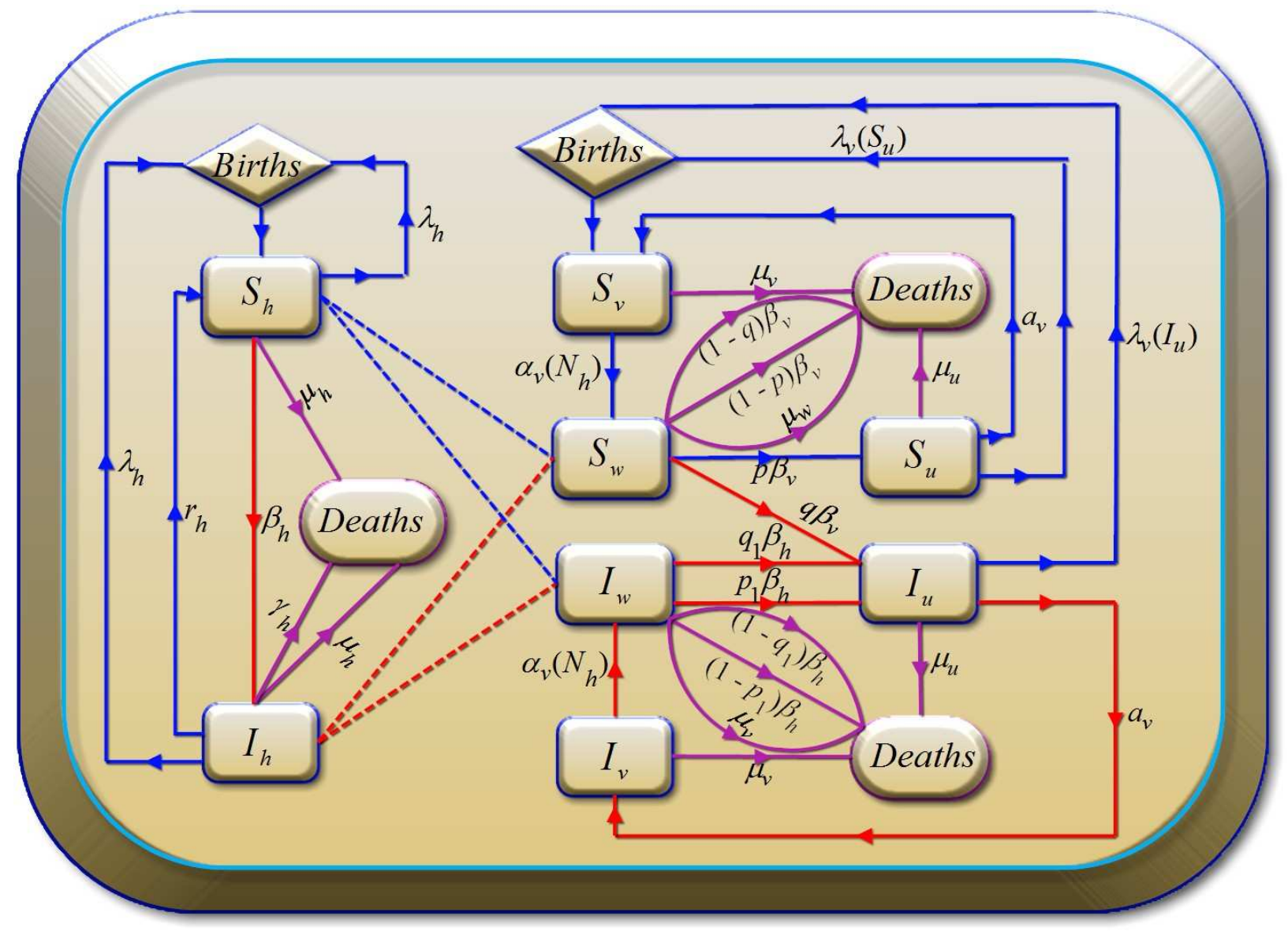

Figure 4.1. Conceptual framework for new SIS malaria model. Mosquitoes interact with humans and transfer the malaria parasite from human to human and within the mosquito population. Interactions between humans and mosquitoes are denoted by broken lines, with red broken lines representing interactions in which at least one of the parties is infectious and blue broken lines representing interactions in which both parties are susceptible. For all successful interactions, the mosquito population grows as a result of transfer of biomass/energy through blood meals taken from humans. Susceptible transitions are denoted by blue solid lines, infectious transitions are denoted by red solid lines, and death links are denoted by purple solid lines. The different flow rates are explained in Table 4.2 . 


\subsubsection{Model Equations}

The model derivation uses a restricted form of homogeneous mixing based on the idea that the mosquito has a human-biting rate. In our model, we assume that all new born humans and newly emerged mosquitoes are susceptible; that is, there is no vertical transmission.

Flow from susceptible humans to infectious humans is a result of contact between the susceptible humans, $S_{h}$, and an infectious questing vector, $I_{w}$. Recruitment of new susceptible humans is from births by both susceptible and infectious humans at rate $\lambda_{h}$. Deaths can occur among susceptible humans naturally at rate $\mu_{h}$. In the presence of the malaria disease in the human population, infectious humans can either die naturally at rate $\mu_{h}$ or due to malaria infection at rate $\gamma_{h}$ or recover and join the susceptible class at rate $r_{h}$. The transmission rate of the parasite from mosquitoes to humans depends on the interaction between the susceptible humans and infectious questing mosquitoes with contact rate $\beta_{h_{i s}}$. When an infectious questing mosquito successfully takes a blood meal from a susceptible human with contact rate $\beta_{h_{i s}}$ and success probability $p_{1}$, this mosquito becomes an infectious fed vector of type $U,\left(I_{u}\right)$, or it fails with probability $1-p_{1}$ and is assumed killed. Hence the equations governing the human populations are

$$
\begin{aligned}
\dot{S}_{h} & =\lambda_{h}\left(N_{h}\right) N_{h}+r_{h} I_{h}-\beta_{h_{i s}}\left(N_{h}\right) S_{h} I_{w}-\mu_{h}\left(N_{h}\right) S_{h}, \\
\dot{I}_{h} & =\beta_{h_{i s}}\left(N_{h}\right) S_{h} I_{w}-\left(\mu_{h}\left(N_{h}\right)+r_{h}+\gamma_{h}\right) I_{h}
\end{aligned}
$$

and the equation for the total human population is:

$$
\dot{N}_{h}=\left(\lambda_{h}\left(N_{h}\right)-\mu_{h}\left(N_{h}\right)\right) N_{h}-\gamma_{h} I_{h}
$$

Next, we derive the equations that describe the population dynamics in each of the vector classes. Resting susceptible vectors are recruited when susceptible or infectious fed vectors (respectively, types $S_{u}$ and $I_{u}$ vectors) reproduce at rate $\tilde{\lambda}_{v}$ per fed and reproducing vector. Note that $\tilde{\lambda}_{v}:[0, \infty] \rightarrow \mathbb{R}$ is a suitable continuously differentiable monotone decreasing vector birth rate function. We account only for the vector populations involved in disease transmission, the female vectors, by setting $\lambda_{v}=\eta \tilde{\lambda}_{v}$ where $\eta \in(0,1)$ is a constant. Now, reproduction by a female mosquito is possible only when the mosquito visits a human being at the human habitat, takes a blood meal, rests, and then lays eggs at a chosen breeding 
site. When eggs are laid, they hatch into larvae that further metamorphose into pupae and eventually develop into full adult vectors of class $S_{v}$. Hence, new adult mosquitoes only emerge after a delayed time period $T>0$, accounting for the elapsed time between egg laying and emergence of the new vectors. We assume there are no additional mortalities in any of the four life stages apart from natural deaths. Starting with the first stage, the egg, we suppose $T_{e}>0$ is the maturation period of $\operatorname{eggs}^{1}$ and that $\mu_{v_{e}}>0$ is the natural death rate constant of eggs. We also assume that the rate of egg laying depends only on the current size of the adult fed and reproducing vectors $S_{u}$ and $I_{u}$. Furthermore, let $a_{v} \lambda_{v}\left(S_{u}(t)\right)$ and $a_{v} \lambda_{v}\left(I_{u}(t)\right)$ be the respective rates at which susceptible and infectious fed and reproducing female vectors that return to the breeding site lay eggs; then at any time $t, a_{v} \lambda_{v}\left(S_{u}(t)\right) S_{u}(t)+a_{v} \lambda_{v}\left(I_{u}(t)\right) I_{u}$ eggs are produced by susceptible and infectious fed and reproducing vectors. Now, if $E(t)$ is the expected number (or density) of eggs from time $t-T_{e}$ to time $t$, then

$$
E(t)=\int_{t-T_{e}}^{t}\left(a_{v} \lambda_{v}\left(S_{u}(s)\right) S_{u}(s)+a_{v} \lambda_{v}\left(I_{u}(s)\right) I_{u}(s)\right) e^{-\mu_{v_{e}}(t-s)} d s,
$$

where $e^{-\mu_{v_{e}} T_{e}}$ is the probability that an egg laid at an earlier time $t-T_{e}$ survives to time t. See Refs. [6, 9-11] for details on how equation (4.6) is derived. Using Leibnitz rule (see Appendix B.1) to differentiate equation (4.6) with respect to $t$, we obtain the following equation describing the rate of change in the egg stage:

$$
\begin{aligned}
\dot{E}(t)= & a_{v} \lambda_{v}\left(S_{u}(t)\right) S_{u}(t)+a_{v} \lambda_{v}\left(I_{u}(t)\right) I_{u}(t)-a_{v} \lambda_{v}\left(S_{u}\left(t-T_{e}\right)\right) S_{u}\left(t-T_{e}\right) e^{-\mu_{v_{e}} T_{e}} \\
& -a_{v} \lambda_{v}\left(I_{u}\left(t-T_{e}\right)\right) I_{u}\left(t-T_{e}\right) e^{-\mu_{v_{e}} T_{e}}-\mu_{v_{e}} E(t),
\end{aligned}
$$

where $a_{v} \lambda_{v}\left(S_{u}(t)\right) S_{u}(t)+a_{v} \lambda_{v}\left(I_{u}(t)\right) I_{u}(t)$ is the density of unhatched eggs at the breeding site at time $t,-a_{v}\left[\lambda_{v}\left(S_{u}\left(t-T_{e}\right)\right) S_{u}\left(t-T_{e}\right)+\lambda_{v}\left(I_{u}\left(t-T_{e}\right)\right) I_{u}\left(t-T_{e}\right)\right] e^{-\mu_{v_{e}} T_{e}}$ is the density of eggs that hatch into larvae at time $t$, and $\mu_{v_{e}} E(t)$ is the density of eggs that die at time t. Note that the eggs that hatch into larvae at time $t$ are those eggs that were laid at an earlier time $t-T_{e}$ and that have survived through the maturation period of $T_{e}$ time units.

Next, let $T_{l}>0$ and $\mu_{v_{l}}>0$ be the maturation period and natural death rate constant of larvae, respectively. Then proceeding in a similar way, we obtain the following expression

\footnotetext{
${ }^{1}$ The maturation period of eggs is the length of time that elapses before laid eggs hatch into larvae.
} 
depicting the entry rate into the pupa stage at time $t$ :

$-a_{v}\left[\lambda_{v}\left(S_{u}\left(t-\left(T_{e}+T_{l}\right)\right) S_{u}\left(t-\left(T_{e}+T_{l}\right)\right)+\lambda_{v}\left(I_{u}\left(t-\left(T_{e}+T_{l}\right)\right)\right) I_{u}\left(t-\left(T_{e}+T_{l}\right)\right)\right] e^{-\mu_{v_{e}} T_{e}+\mu_{v_{l}} T_{l}}\right.$.

Notice that the total maturation period for the first two life stages is $T_{e}+T_{l}$ time units, and that the larvae that progress to the pupal stage are those that emerged from eggs laid at an earlier time and that have survived through this total maturation period.

Finally, let $T_{p}>0$ and $\mu_{v_{p}}$ be the maturation period and natural death rate constants of pupae. Then assuming that all deaths in earlier life stages occur at a uniform constant rate $\mu_{v}>0$, i.e., $0<\mu_{v}=\mu_{v_{e}}=\mu_{v_{l}}=\mu_{v_{p}}$, and continuing as in the first two stages, we obtain the following expression for newly emerging adult mosquitoes from the pupal stage:

$$
-a_{v}\left[\lambda_{v}\left(S_{u}(t-T)\right) S_{u}(t-T)+\lambda_{v}\left(I_{u}(t-T)\right) I_{u}(t-T)\right] e^{-\mu_{v} T}
$$

where $0<T=T_{e}+T_{l}+T_{p}$ is the total maturation period for the first three life stages.

We now use the above adult vector emergence rate to derive the equations describing the rates of change in the $S_{v}$ and $I_{v}$ classes. Additional recruitment of susceptible resting vectors is obtained via the susceptible fed vectors $\left(S_{u}\right)$ that return at rate $a_{v}$ from human habitats to the breeding sites to lay eggs. Thus, population growth of vectors of type $S_{v}$ is brought about by the newly emerging adult vectors as computed above and by old egg laying female vectors of type $S_{u}$ that returned from human habitats to the breeding site at rate $a_{v}$. If the fed vectors that returned to the breeding site are infected, i.e., they fed on type $I_{h}$ humans and thus are type $I_{u}$ vectors, upon arrival at the breeding site they become vectors of type $I_{v}$, and thus serve as recruiters of type $I_{v}$ vectors.

After acquiring some rest and after swarming, newly emerged adult female vectors and all previously fertilized adult susceptible $\left(S_{u}\right)$ and infected $\left(I_{u}\right)$ vectors can then return to human habitats at rate $\alpha_{v}\left(N_{h}\right)$ to seek blood meals. Additionally, individuals leave the susceptible and infected resting classes via natural death at rate $\mu_{v}$. Taking into consideration the descriptions above and all assumptions made, the time rates of change of the $S_{v}$ and $I_{v}$ classes are, respectively,

$$
\begin{aligned}
\dot{S}_{v}= & a_{v}\left[\lambda_{v}\left(S_{u}(t-T)\right) S_{u}(t-T)+\lambda_{v}\left(I_{u}(t-T)\right) I_{u}(t-T)\right] e^{-\mu_{e} T}+a_{v} S_{u} \\
& -\left(\mu_{v}+\alpha_{v}\left(N_{h}\right)\right) S_{v}
\end{aligned}
$$


and

$$
\dot{I}_{v}=a_{v} I_{u}-\left(\mu_{v}+\alpha_{v}\left(N_{h}\right)\right) I_{v}
$$

In equation (4.8), the birth rate function $\lambda_{v}$, which is assumed to be continuously differentiable and strictly monotonic decreasing, can be modeled using any of the common birth functions in the biological and ecological literature. Common birth functions include the Verhulst-Pearl logistic birth rate function [12-14], the Beverton-Holt birth rate function [15], the Ricker birth rate function [16], the Hassell birth rate function [17], and the Maynard-Smith-Slatkin birth rate function [18, 19]. See Section 5.5 and Refs. [11, 20] for more birth functions. The specific form of the birth rate function used here will be described in Section 4.4. Additionally, from equations (4.8) and (4.9), type $V$ vectors from the breeding site are attracted to humans at a rate $\alpha_{v}$ that is dependent on the total human population. To complete the description of these equations, we derive an appropriate functional form for $\alpha$. Let $B_{h}$ and $B_{a}$ be the blood indices of vectors that prefer human and animal blood, respectively. Then $B_{h} S_{v}$ susceptible unfed vectors feed on humans, while $B_{a} S_{v}$ susceptible unfed vectors feed on animals. The fractions of susceptible unfed vectors $S_{v}$ that feed on humans and animals are given by the expressions

$$
\frac{B_{h} S_{v}}{B_{h} S_{v}+B_{a} S_{v}} N_{h}=\frac{B_{h}}{B_{h}+B_{a}} N_{h} \text { and } \frac{B_{a} S_{v}}{B_{h} S_{v}+B_{a} S_{v}} N_{a}=\frac{B_{a}}{B_{h}+B_{a}} N_{a}
$$

respectively, where $N_{h}$ is the total human population and $N_{a}$ is the total animal population. Hence, the proportion of susceptible unfed vectors that prefer human blood to animal blood is given by

$$
\frac{\frac{B_{h}}{B_{h}+B_{a}} N_{h}}{\frac{B_{h}}{B_{h}+B_{a}} N_{h}+\frac{B_{a}}{B_{h}+B_{a}} N_{a}}=\frac{N_{h}}{N_{h}+k},
$$

where $k=B_{a} N_{a} / B_{h}$ is a positive constant accounting for the existence of an alternative blood source for the vector. Therefore, susceptible unfed vectors at the breeding site are attracted to humans at rate

$$
\alpha_{v}\left(N_{h}\right)=b N_{h} /\left(N_{h}+k\right)
$$

where $b N_{h} /\left(N_{h}+k\right)$ is the proportion of susceptible unfed vectors that prefer human blood meals to animal blood meals and $b$ is a positive constant to measure the flow rate of vectors 
from breeding sites to human habitats. We are assuming that the mosquito's preference for human or other blood is not altered when the mosquito is infected. Continuing as above, we can easily show that type $I_{v}$ vectors are also attracted to humans at the same rate.

We now model the rate of change for the type $W$ vectors $S_{w}$ and $I_{w}$. Once a vector, susceptible $\left(S_{v}\right)$ or infected $\left(I_{v}\right)$ from the vector breeding site, arrives at a human habitat, it becomes a questing vector of type $W$ (respectively, susceptible $\left(S_{w}\right)$ or infected $\left.\left(I_{w}\right)\right)$. A questing susceptible or infectious mosquito can either feed on a susceptible or infectious human. When a susceptible questing mosquito successfully takes a blood meal from an infectious human with contact rate $\beta_{v_{s i}}$ and success probability $q$, this mosquito becomes an infectious fed vector of type $U$, represented as $\left(I_{u}\right)$, or else it fails with probability $1-q$ and is assumed killed. These contacts may lead to the production of new infectious vectors. If a questing susceptible vector instead successfully feeds on a susceptible human with contact rate $\beta_{v_{s s}}$ and with success probability $p$, it becomes a susceptible fed vector of type $U$, represented as $\left(S_{u}\right)$, or else it fails with probability $1-p$ and is assumed killed. These contacts do not lead to any new infections. When an infectious questing mosquito successfully takes a blood meal from an infectious human with contact rate $\beta_{h_{i i}}$ and success probability $p_{1}$, this mosquito becomes an infectious fed vector of type $U,\left(I_{u}\right)$, or else it fails with probability $1-p_{1}$ and is assumed killed. No new infections ensue from these contacts. If an infectious questing vector instead successfully feeds on a susceptible human, with contact rate $\beta_{h_{i s}}$ and probability $q_{1}$, it becomes an infectious fed vector of type $U,\left(I_{u}\right)$, or else it fails with probability $1-q_{1}$ and is assumed killed. These contacts may lead to new infections in the human population but not in the vector population. Natural deaths in the $S_{w}$ and $I_{w}$ classes occur at rate $\mu_{w}$ which is considered to be constant at all times. From the above description, the respective governing equations for the population densities of the susceptible and infectious questing vectors assume the forms:

$$
\begin{aligned}
\dot{S}_{w} & =\alpha_{v}\left(N_{h}\right) S_{v}-\left(\mu_{w}+\beta_{v_{s s}} S_{h}+\beta_{v_{s i}} I_{h}\right) S_{w} \\
\dot{I}_{w} & =\alpha_{v}\left(N_{h}\right) I_{v}-\left(\mu_{w}+\beta_{h_{i s}} S_{h}+\beta_{h_{i i}} I_{h}\right) I_{w} .
\end{aligned}
$$

When susceptible questing vectors of type $S_{w}$ successfully feed on humans, they either become fed and reproducing vectors of type $S_{u}$ or of type $I_{u}$, depending on whether the 
human they fed on was susceptible or infectious, at the rates described above. However, when infectious questing vectors $\left(I_{w}\right)$ successfully feed on humans, they become infectious fed vectors of type $I_{u}$. Exits from the questing vector classes occur via natural deaths at constant rate $\mu_{u}$ and through vectors returning to the breeding site at rate $a_{v}$. Thus, the equations for the time rate of change of $S_{u}$ and $I_{u}$ are, respectively,

$$
\begin{aligned}
\dot{S}_{u} & =p \beta_{v_{s s}} S_{h} S_{w}-\left(a_{v}+\mu_{u}\right) S_{u} \\
\dot{I}_{u} & =p_{1} \beta_{h_{i s}} S_{h} I_{w}+q_{1} \beta_{h_{i i}} I_{h} I_{w}+q \beta_{v_{s i}} I_{h} S_{w}-\left(a_{v}+\mu_{u}\right) I_{u} .
\end{aligned}
$$

Combining equations (4.8) through (4.13), we obtain the following system of equations that govern the time rate of change of the vector population:

$$
\begin{aligned}
\dot{S}_{v} & =a_{v}\left(\lambda_{v}\left(S_{u}(t-T)\right) S_{u}(t-T)+\lambda_{v}\left(I_{u}(t-T)\right) I_{u}(t-T) e^{-\mu_{e} T}+a_{v} S_{u}-\left(\mu_{v}+\alpha_{v}\left(N_{h}\right)\right) S_{v},\right. \\
\dot{S}_{w} & =\alpha_{v}\left(N_{h}\right) S_{v}-\left(\mu_{w}+\beta_{v_{s s}} S_{h}+\beta_{v_{s i}} I_{h}\right) S_{w}, \\
\dot{S}_{u} & =p \beta_{v_{s s}} S_{h} S_{w}-\left(a_{v}+\mu_{u}\right) S_{u}, \\
\dot{I}_{v} & =a_{v} I_{u}-\left(\mu_{v}+\alpha_{v}\left(N_{h}\right)\right) I_{v}, \\
\dot{I}_{w} & =\alpha_{v}\left(N_{h}\right) I_{v}-\left(\mu_{w}+\beta_{h_{i s}} S_{h}+\beta_{h_{i i}} I_{h}\right) I_{w}, \\
\dot{I}_{u} & =p_{1} \beta_{h_{i s}} S_{h} I_{w}+q_{1} \beta_{h_{i i}} I_{h} I_{w}+q \beta_{v_{s i}} I_{h} S_{w}-\left(a_{v}+\mu_{u}\right) I_{u},
\end{aligned}
$$

where

$$
\alpha_{v}\left(N_{h}\right)=b N_{h} /\left(N_{h}+k\right) .
$$

The following equation governs the density of the total vector population:

$$
\begin{aligned}
\dot{N}_{v}= & a_{v} \lambda_{v}\left(S_{u}(t-T)\right) S_{u}(t-T) e^{-\mu_{e} T}+a_{v} \lambda_{v}\left(I_{u}(t-T)\right) I_{u}(t-T) e^{-\mu_{e} T} \\
& -\left(\mu_{v} S_{v}+\mu_{w} S_{w}+\mu_{u} S_{u}+\mu_{v} I_{v}+\mu_{w} I_{w}+\mu_{u} I_{u}\right)-(1-p) \beta_{v_{s s}} S_{h} S_{w} \\
& -(1-q) \beta_{v_{s i}} I_{h} S_{w}-\left(\beta_{h_{i s}} S_{h}+\beta_{h_{i i}} I_{h}\right) I_{w}+p_{1} \beta_{h_{i s}} S_{h} I_{w}+q_{1} \beta_{h_{i i}} I_{h} I_{w} .
\end{aligned}
$$

Combining equations (4.3)-(4.4) and system (4.14) yields the system 


$$
\begin{aligned}
\dot{S}_{h} & =\lambda_{h}\left(N_{h}\right) N_{h}+\gamma_{h} I_{h}-\beta_{h_{i s}}\left(N_{h}\right) S_{h} I_{w}-\mu_{h}\left(N_{h}\right) S_{h}, \\
\dot{I}_{h} & =\beta_{v_{i s}}\left(N_{h}\right) S_{h} I_{w}-\left(\mu_{h}\left(N_{h}\right)+\gamma_{h}+\nu_{h}\right) I_{h}, \\
\dot{S}_{v} & =a_{v} \lambda_{v}\left(S_{u}(t-T)\right) S_{u}(t-T) e^{-\mu_{e} T}+a_{v} \lambda_{v}\left(I_{u}\right) I_{u}+a_{v} S_{u}-\left(\mu_{v}+\alpha_{v}\left(N_{h}\right)\right) S_{v}, \\
\dot{S}_{w} & =\alpha_{v}\left(N_{h}\right) S_{v}-\left(\mu_{w}+\beta_{v_{s s}} S_{h}+\beta_{v_{s i}} I_{h}\right) S_{w}, \\
\dot{S}_{u} & =p \beta_{v_{s s}} S_{h} S_{w}-\left(a_{v}+\mu_{u}\right) S_{u}, \\
\dot{I}_{v} & =a_{v} I_{u}-\left(\mu_{v}+\alpha_{v}\left(N_{h}\right)\right) I_{v}, \\
\dot{I}_{w} & =\alpha_{v}\left(N_{h}\right) I_{v}-\left(\mu_{w}+\beta_{h_{i s}} S_{h}+\beta_{h_{i i}} I_{h}\right) I_{w}, \\
\dot{I}_{u} & =p_{1} \beta_{h_{i s}} S_{h} I_{w}+q_{1} \beta_{h_{i i}} I_{h} I_{w}+q \beta_{v_{s i}} I_{h} S_{w}-\left(a_{v}+\mu_{u}\right) I_{u},
\end{aligned}
$$

with the following equations for the total populations:

$$
\begin{aligned}
\dot{N}_{h}= & \left(\lambda_{h}\left(N_{h}\right)-\mu_{h}\left(N_{h}\right)\right) N_{h}-\gamma_{h} I_{h} \\
\dot{N}_{v}= & a_{v} \lambda_{v}\left(S_{u}(t-T)\right) S_{u}(t-T) e^{-\mu_{e} T}+a_{v} \lambda_{v}\left(I_{u}(t-T)\right) I_{u}(t-T) e^{-\mu_{e} T} \\
& -\left(\mu_{v} S_{v}+\mu_{w} S_{w}+\mu_{u} S_{u}+\mu_{v} I_{v}+\mu_{w} I_{w}+\mu_{u} I_{u}\right)-(1-p) \beta_{v_{s s}} S_{h} S_{w} \\
& -(1-q) \beta_{v_{s i}} I_{h} S_{w}-\left(\beta_{h_{i s}} S_{h}+\beta_{h_{i i}} I_{h}\right) I_{w}+p_{1} \beta_{h_{i s}} S_{h} I_{w}+q_{1} \beta_{h_{i i}} I_{h} I_{w}
\end{aligned}
$$

where $\alpha_{v}$ is given by equation (4.15).

To complete the formulation, system (4.17) must be solved with appropriate initial conditions. The simplest type of initial conditions would take the form

$$
\left(S_{h}(0), I_{h}(0), S_{v}(0), S_{w}(0), S_{u}(0), I_{v}(0), I_{w}(0), I_{u}(0)\right)=\left(S_{h}^{0}, I_{h}^{0}, S_{v}^{0}, S_{w}^{0}, S_{u}^{0}, I_{v}^{0}, I_{w}^{0}, I_{u}^{0}\right)
$$

\subsection{Simplified Model}

As a first step we study a simplified model, where we assume that $p_{1}=q_{1}$, and that deaths in each of the mosquito classes occur at a uniform constant rate $\mu_{v}$, that is, $\mu_{w}=\mu_{u}=\mu_{v}=$

constant. Since our objective is to demonstrate a new route to periodic oscillations in the dynamics of malaria transmission, we make yet another approximation by assuming instantaneous mosquito births and set the maturation delay parameter $T$ to zero. 
The malaria transmission process is essentially driven by the human biting habit of the mosquito and hence the contact rates. In classical epidemic models, contact rates can be assumed to be directly proportional to the population numbers, as in Ref. [21], or constant as in Ref. [22], or some intermediate form as in Ref. [23]. Therefore, the fractional incidence rates which we have computed as $\beta_{v_{s s}}, \beta_{v_{s i}}, \beta_{h_{i s}}$ and $\beta_{h_{i i}}$ can take several forms, especially if we attempt to model the rate as a probability distribution with parameter $C_{w h}\left(N_{m v}, N_{h}\right) / N_{h}$ or $C_{h w}\left(N_{m v}, N_{h}\right) / N_{h}$ as in Refs. [24-26]. Such a distribution can be binomial, Poisson, censored Poisson, exponential, negative exponential, uniform, etc. Assuming mass action contact rates in all cases leads to uniform distributions at rates $\beta_{v_{s s}}, \beta_{v_{s i}}, \beta_{h_{i s}}$ and $\beta_{h_{i i}}$. Hence, in the context of these simplifications, we assume uniform mass action contact rates, in which case the rates $\beta_{v_{s s}}, \beta_{v_{s i}}, \beta_{h_{i s}}$ and $\beta_{h_{i i}}$ are constants and then set $\beta_{v_{s s}}=\beta_{v_{s i}}=\beta_{v}\left(N_{h}\right)$ $=\beta_{v}=$ constant and $\beta_{h_{i s}}=\beta_{h_{i i}}=\beta_{h}\left(N_{h}\right)=\beta_{h}=$ constant .

Next, we choose a specific functional form for the birth rate function $\lambda_{v}$. To this effect, we consider the logistic function,

$$
\lambda_{v}(\vartheta)=\lambda_{0}\left(1-\frac{\vartheta}{L}\right)
$$

where $\lambda_{0}$ is a positive growth constant, $L>0$ is the environmental carrying capacity ${ }^{2}$, and $\vartheta=S_{u}$ or $\vartheta=I_{u}$. Notice that this is a positive real-valued continuously differentiable and strictly monotonic decreasing function of its argument provided $\vartheta<L$. The motivation for using the Verhulst-Pearl logistic birth rate function for the mosquito lies in the fact that it is linear, and in fact, is a general form for the first linear approximation to any monotone decreasing continuously differentiable function. More nonlinear forms, such as the MaynardSmith-Slatkin function, have been used to study the effect of nonlinear birth in the dynamics of mosquito populations [7].

Furthermore, we assume that human recruitment and deaths both occur at an equal constant rate $\mu_{h}$ (that is, $\lambda_{h}\left(N_{h}\right)=\mu_{h}\left(N_{h}\right)=\mu_{h}$, a constant) and that there are no diseaseinduced human deaths, that is, $\gamma_{h}=0$. These assumptions permit us to safely consider a

\footnotetext{
${ }^{2}$ By environmental carrying capacity, we are referring to the constant maximum population size of a specific species that a designated habitat or breeding site can accommodate over an indefinite period of time without any deleterious repercussions.
} 
constant human population, especially if there is always a human for a mosquito to attempt to feed on, in which case the total human population can be regarded essentially as constant.

These simplifications together with some rearrangement reduce our model to

$$
\begin{aligned}
\dot{S}_{h} & =\mu_{h} N_{h}+r_{h} I_{h}-\beta_{h} S_{h} I_{w}-\mu_{h} S_{h} \\
\dot{I}_{h} & =\beta_{h} S_{h} I_{w}-\left(\mu_{h}+r_{h}\right) I_{h} \\
\dot{S}_{u} & =p \beta_{v} S_{h} S_{w}-\left(a_{v}+\mu_{v}\right) S_{u} \\
\dot{S}_{v} & =a_{v} \lambda_{v}\left(S_{u}\right) S_{u}+a_{v} \lambda_{v}\left(I_{u}\right) I_{u}+a_{v} S_{u}-\left(\mu_{v}+\alpha_{v}\left(N_{h}\right)\right) S_{v} \\
\dot{S}_{w} & =\alpha_{v}\left(N_{h}\right) S_{v}-\left(\mu_{v}+\beta_{v} N_{h}\right) S_{w} \\
\dot{I}_{u} & =p_{1} \beta_{h} N_{h} I_{w}+q \beta_{v} I_{h} S_{w}-\left(a_{v}+\mu_{v}\right) I_{u}, \\
\dot{I}_{v} & =a_{v} I_{u}-\left(\mu_{v}+\alpha_{v}\left(N_{h}\right)\right) I_{v}, \\
\dot{I}_{w} & =\alpha_{v}\left(N_{h}\right) I_{v}-\left(\mu_{v}+\beta_{h} N_{h}\right) I_{w},
\end{aligned}
$$

together with the following equations for the total populations:

$$
\begin{aligned}
\dot{N_{h}=} & 0, \\
\dot{N_{m v}=} & a_{v} \lambda_{v}\left(S_{u}\right) S_{u}+a_{v} \lambda_{v}\left(I_{u}\right) I_{u}-\mu_{v} N_{m v}-(1-p) \beta_{v} S_{h} S_{w} \\
& -(1-q) \beta_{v} I_{h} S_{w}-\left(1-p_{1}\right) \beta_{h} N_{h} I_{w},
\end{aligned}
$$

where $\alpha_{v}\left(N_{h}\right)$ and $\lambda_{v}(\vartheta), \vartheta=S_{u}, I_{u}$ are given by (4.15) and (4.21), respectively. The simplified equation (4.23) immediately shows that $N_{h}$, the total human population, is constant and thus may appear as a parameter in the model. We note, in this case, that the mass action contact term is actually $\beta_{h}\left(N_{h}\right) S_{h} I_{w}$, where, to bring out the fact that it is really the proportion of susceptible humans that get infected, we can have $\beta_{h}\left(N_{h}\right)=\beta / N_{h}$, and the constant $\beta$ may be viewed as a mass action coefficient. Since $N_{h}$ is constant, we simply write $\beta_{h}\left(N_{h}\right)=\beta_{h}$, a constant, and the above simplification becomes reasonable. The same applies to $\beta_{v}=\beta_{v}\left(N_{h}\right)$. Now, for a constant human population, if we write $S_{h}=N_{h}-I_{h}$, we can compute $S_{h}$ once we know $I_{h}$, which can be computed from the $\dot{I}_{h}$ equation in (4.22).

In the absence of the malaria disease, $I_{h}=I_{v}=I_{w}=I_{u}=0$ and $S_{h}=N_{h}$. When these are substituted in system (4.22), the system reduces to 


$$
\begin{aligned}
& \dot{S}_{u}=p \beta_{v} N_{h} S_{w}-\left(a_{v}+\mu_{v}\right) S_{u} \\
& \dot{S}_{v}=a_{v} \lambda_{v}\left(S_{u}\right) S_{u}+a_{v} S_{u}-\left(\mu_{v}+\alpha_{v}\left(N_{h}\right)\right) S_{v} \\
& \dot{S}_{w}=\alpha_{v}\left(N_{h}\right) S_{v}-\left(\mu_{v}+\beta_{v} N_{h}\right) S_{w}
\end{aligned}
$$

so that when $\beta_{v}=\tau, N_{h}=H, S_{u}=U, S_{w}=W$ and $S_{v}=V$, we recover the demographics model for the population dynamics of the malaria vector developed and studied in Ref. [6] when $\eta=1$, and later extended in Ref. [8]. This model exhibits periodic behavior.

\subsubsection{Well Posedness, Positivity and Boundedness}

In this section, we discuss the existence and uniqueness of solutions, positivity of solutions when they exist and boundedness of solutions. Since the system (4.22) models human and mosquito populations, it is assumed that all human and mosquito population variables are non-negative. This assumption yields the epidemiologically reasonable domain

$$
\begin{gathered}
\mathfrak{D}=\left\{\left(S_{h}, I_{h}, N_{h}, S_{u}, S_{v}, S_{w}, I_{u}, I_{v}, I_{w}, N_{m v}\right) \in \mathbb{R}^{10}: S_{h} \geq 0, I_{h} \geq 0,0<S_{h}+I_{h} \leq N_{h},\right. \\
\left.S_{u} \geq 0, S_{v} \geq 0, S_{w} \geq 0, I_{u} \geq 0, I_{v} \geq 0, I_{w} \geq 0,0<S_{u}+S_{v}+S_{w}+I_{u}+I_{v}+I_{w} \leq N_{m v}\right\} .
\end{gathered}
$$

It can be shown using standard techniques described in Ref. [27, 28] that if initial conditions are specified for each of the state variables at time $t=0$, with $S_{v}(0)+S_{w}(0)+S_{u}(0)+I_{v}(0)+$ $I_{w}(0)+I_{u}(0)=N_{m v}(0)$, then there exist a unique solution satisfying these initial conditions for all $t \geq 0$, with $S_{v}(t)+S_{w}(t)+S_{u}(t)+I_{v}(t)+I_{w}(t)+I_{u}(t)=N_{m v}(t)$ for all $t \geq 0$. It can also be verified that if $N_{m v}(0)>0$, then $N_{m v}(t)>0$ for all $t$, whereas if $N_{m v}(0)=0$, then $N_{m v}(t)=0$ for all $t$. Of course similar arguments apply to the human equations with corresponding expressions. Thus the system (4.22) is well posed from a mathematical and physical standpoint. Additionally, our focus will be on the total mosquito population (equation (4.24)) since the total human population (equation (4.23)) is constant. Studying the system (4.24) yields the following theorem.

Theorem 4.4.1. The closed set

$$
\Psi=\left\{\left(S_{u}, S_{v}, S_{w}, I_{u}, I_{v}, I_{w}\right) \in \mathbb{R}_{+}^{6}: N_{m v}=S_{u}+S_{v}+S_{w}+I_{u}+I_{v}+I_{w} \leq \frac{2 a_{v} \lambda_{0} L}{\mu_{v}}\right\}
$$


is positively-invariant and attracting with respect to the system (4.22).

Proof. From equation (4.24) we see that

$$
\dot{N}_{m v} \leq a_{v} \lambda_{v}\left(S_{u}\right) S_{u}+a_{v} \lambda_{v}\left(I_{u}\right) I_{u}-\mu_{v} N_{m v},
$$

since all human and mosquito population variables are non-negative and $\beta_{v} \geq 0, \beta_{h} \geq 0$ and $0 \leq p, q, p_{1} \leq 1$. From equation (4.21), if $\vartheta \leq L$ then $\lambda_{v}(\vartheta) \leq \lambda_{0}$. Thus, if $S_{u} \leq L$ and/or $I_{u} \leq L$ then

$$
\dot{N}_{m v} \leq 2 a_{v} \lambda_{0} L-\mu_{v} N_{m v} .
$$

Separating variables and solving for $N_{m v}$, yields the following inequatity:

$$
N_{m v}(t) \leq \frac{2 a_{v} \lambda_{0} L}{\mu_{v}}+e^{-\mu_{v} t}\left(N_{m v}(0)-\frac{2 a_{v} \lambda_{0} L}{\mu_{v}}\right), \forall t \geq 0,
$$

where $N_{m v}(0)$ is the initial total mosquito population. Hence, if $N_{m v}(0) \leq 2 a_{v} \lambda_{0} L / \mu_{v}$, then from the last inequality, $N_{m v}(t) \leq 2 a_{v} \lambda_{0} L / \mu_{v}$ implying that $\Psi$ is a positively-invariant set. Notice that positivity is preserved if both $S_{u}>L$ and $I_{u}>L$, since in this case $\dot{N}_{m v}(t)<0$

for $t \geq 0$ and so the mosquito population is decreasing. Hence $N_{m v}(t) \leq N_{m v}(0)$. Next, if $N_{m v}(t)>2 a_{v} \lambda_{0} L / \mu_{v}$ then from equation (4.27) we see that $\dot{N}_{m v}(t)<0$ and so the mosquito population is decreasing. From (4.28), $N_{m v}(t)$ approaches $2 a_{v} \lambda_{0} L / \mu_{v}$ as $t$ approaches infinity. Thus, solutions either enter $\Psi$ in finite time or $N_{m v}(t)$ approaches $2 a_{v} \lambda_{0} L / \mu_{v}$. Hence, $\Psi$ is an attracting set. Therefore, the solutions of the model are considered epidemiologically and mathematically well-posed in $\Psi$.

\subsubsection{Nondimensionalization and Reparametrization}

We set $S_{h}=N_{h}-I_{h}$ and introduce the following new variables:

$$
\begin{aligned}
\tau & =\frac{t}{t^{0}}, I=\frac{I_{h}}{I_{h}^{0}}, v_{s}=\frac{S_{v}}{S_{v}^{0}}, w_{s}=\frac{S_{w}}{S_{w}^{0}}, \\
u_{s} & =\frac{S_{u}}{S_{u}^{0}}, v_{i}=\frac{I_{v}}{I_{v}^{0}}, w_{i}=\frac{I_{w}}{I_{w}^{0}}, u_{i}=\frac{I_{u}}{I_{u}^{0}},
\end{aligned}
$$

where $t^{0}, I_{h}^{0}, S_{v}^{0}, S_{w}^{0}, S_{u}^{0}, I_{v}^{0}, I_{w}^{0}$ and $I_{u}^{0}$ are reference values. Substituting in system (4.22) yields 


$$
\begin{aligned}
\frac{I_{h}^{0}}{t^{0}} \dot{I} & =\beta_{h} I_{w}^{0} I_{h}^{0}\left(\frac{N_{h}}{I_{h}^{0}}-I\right) w_{i}-\left(\mu_{h}+\gamma_{h}\right) I_{h}^{0} I, \\
\frac{S_{u}^{0}}{t^{0}} \dot{u}_{s} & =p \beta_{v} I_{h}^{0} S_{w}^{0}\left(\frac{N_{h}}{I_{h}^{0}}-I\right) w_{s}-\left(a_{v}+\mu_{v}\right) S_{u}^{0} u_{s}, \\
\frac{S_{v}^{0}}{t^{0}} \dot{v}_{s} & =a_{v} \lambda_{0}\left(1-\frac{S_{u}^{0} u_{s}}{L}\right) S_{u}^{0} u_{s}+a_{v} \lambda_{0}\left(1-\frac{I_{u}^{0} u_{i}}{L}\right) I_{u}^{0} u_{i}+a_{v} S_{u}^{0} u_{s}-\left(\mu_{v}+\frac{b N_{h}}{N_{h}+k}\right) S_{v}^{0} v_{s}, \\
\frac{S_{w}^{0}}{t^{0}} \dot{w}_{s} & =\frac{b N_{h} S_{v}^{0}}{N_{h}+k} v_{s}-\left(\mu_{v}+\beta_{v} N_{h}\right) S_{w}^{0} w_{s}, \\
\frac{I_{u}^{0}}{t^{0}} \dot{u}_{i} & =p_{1} \beta_{h} N_{h} I_{w}^{0} w_{i}+q \beta_{v} I_{h}^{0} S_{w}^{0} I w_{s}-\left(a_{v}+\mu_{v}\right) I_{u}^{0} u_{i}, \\
\frac{I_{v}^{0}}{t^{0}} \dot{v}_{i} & =a_{v} I_{u}^{0} u_{i}-\left(\mu_{v}+\frac{b N_{h}}{N_{h}+k}\right) I_{v}^{0} v_{i}, \\
\frac{I_{w}^{0}}{t^{0}} \dot{w}_{i} & =\frac{b N_{h} I_{v}^{0}}{N_{h}+k} v_{i}-\left(\mu_{v}+\beta_{h} N_{h}\right) I_{w}^{0} w_{i},
\end{aligned}
$$

which upon clearing the coefficients of the differentials on the left hand sides and carrying out some preliminary simplifications becomes

$$
\begin{aligned}
\dot{I} & =\beta_{h} I_{w}^{0} t^{0}\left(\frac{N_{h}}{I_{h}^{0}}-I\right) w_{i}-\left(\mu_{h}+\gamma_{h}\right) t^{0} I, \\
\dot{u}_{s} & =\frac{p \beta_{v} I_{h}^{0} S_{w}^{0} t^{0}}{S_{u}^{0}}\left(\frac{N_{h}}{I_{h}^{0}}-I\right) w_{s}-\left(a_{v}+\mu_{v}\right) t^{0} u_{s}, \\
\dot{v}_{s} & =\frac{a_{v} \lambda_{0} t^{0}}{S_{v}^{0}}\left(\left(1-\frac{S_{u}^{0} u_{s}}{L}\right) S_{u}^{0} u_{s}+\left(1-\frac{I_{u}^{0} u_{i}}{L}\right) I_{u}^{0} u_{i}\right)+\frac{a_{v} S_{u}^{0} t^{0}}{S_{v}^{0}} u_{s}-\left(\mu_{v}+\frac{b N_{h}}{N_{h}+k}\right) t^{0} v_{s}, \\
\dot{w}_{s} & =\frac{b N_{h} S_{v}^{0} t^{0}}{S_{w}^{0}\left(N_{h}+k\right)} v_{s}-\left(\mu_{v}+\beta_{v} N_{h}\right) t^{0} w_{s}, \\
\dot{u}_{i} & =\frac{p_{1} \beta_{h} N_{h} I_{w}^{0} t^{0}}{I_{u}^{0}} w_{i}+\frac{q \beta_{v} I_{h}^{0} S_{w}^{0} t^{0}}{I_{u}^{0}} I w_{s}-\left(a+\mu_{v}\right) t^{0} u_{i},
\end{aligned}
$$




$$
\begin{aligned}
\dot{v}_{i} & =\frac{a_{v} I_{u}^{0} t^{0}}{I_{v}^{0}} u_{i}-\left(\mu_{v}+\frac{b N_{h}}{N_{h}+k}\right) t^{0} v_{i}, \\
\dot{w}_{i} & =\frac{b N_{h} I_{v}^{0} t^{0}}{I_{w}^{0}\left(N_{h}+k\right)} v_{i}-\left(\mu_{v}+\beta_{h} N_{h}\right) t^{0} w_{i} .
\end{aligned}
$$

We now set

$$
\begin{aligned}
I_{h}^{0} & =N_{h}, S_{u}^{0}=L=I_{u}^{0}, S_{v}^{0}=\frac{S_{u}^{0}\left(a_{v}+\mu_{v}\right)\left(\mu_{v}+\beta_{v} N_{h}\right)}{p \beta_{v} N_{h} \alpha_{v}\left(N_{h}\right)}, \\
S_{w}^{0} & =\frac{\alpha_{v}\left(N_{h}\right) S_{v}^{0}}{\mu_{v}+\beta_{v} N_{h}}, I_{v}^{0}=\frac{a_{v} I_{u}^{0}}{\mu_{v}+\alpha_{v}\left(N_{h}\right)}, I_{w}^{0}=\frac{\alpha_{v}\left(N_{h}\right) I_{v}^{0}}{\mu_{v}+\beta_{h} N_{h}}, t^{0}=\frac{1}{a_{v}+\mu_{v}},
\end{aligned}
$$

and

$$
\begin{aligned}
\delta & =\frac{p_{1} \beta_{h} N_{h} I_{w}^{0} t^{0}}{I_{u}^{0}}=\frac{a_{v}}{\left(a_{v}+\mu_{v}\right)} \frac{\alpha_{v}\left(N_{h}\right)}{\left(\mu_{v}+\alpha_{v}\left(N_{h}\right)\right)} \frac{\beta_{h} N_{h}}{\left(\mu_{v}+\beta_{h} N_{h}\right)} p_{1}, \rho=\frac{\mu_{v}+\alpha_{v}\left(N_{h}\right)}{a_{v}+\mu_{v}}, \\
\beta & =\beta_{h} I_{w}^{0} t_{0}=\left(\frac{a_{v}}{\mu_{v}+a_{v}}\right)\left(\frac{\alpha_{v}\left(N_{h}\right)}{\mu_{v}+\alpha_{v}\left(N_{h}\right)}\right)\left(\frac{\beta_{h}}{\mu_{v}+\beta_{h} N_{h}}\right) L, \gamma=\frac{\mu_{v}+\beta_{v} N_{h}}{a_{v}+\mu_{v}}, \\
\alpha & =\frac{a_{v} t^{0} S_{u}^{0}}{S_{v}^{0}}=p\left(\frac{a_{v}}{\mu_{v}+a_{v}}\right)\left(\frac{\beta_{v} N_{h}}{\mu_{v}+\beta_{v} N_{h}}\right)\left(\frac{\alpha_{v}\left(N_{h}\right)}{\mu_{v}+a_{v}}\right), \alpha_{v}\left(N_{h}\right)=\frac{b N_{h}}{N_{h}+k}, \\
\sigma & =\frac{q \beta_{v} I_{h}^{0} S_{w}^{0} t^{0}}{I_{u}^{0}}=q / p, \epsilon=\frac{\mu_{v}+\beta_{h} N_{h}}{a_{v}+\mu_{v}}, \mu=\left(\mu_{h}+r_{h}\right) t^{0} .
\end{aligned}
$$

Using the new dimensionless variable and parameter groupings defined in (4.29) and (4.34), respectively, system (4.22) can be re-written as follows: 


$$
\begin{aligned}
\dot{I} & =\beta(1-I) w_{i}-\mu I, \\
\dot{u}_{s} & =(1-I) w_{s}-u_{s}, \\
\dot{v}_{s} & =\alpha \lambda_{o}\left(u_{s}\left(1-u_{s}\right)+u_{i}\left(1-u_{i}\right)\right)+\alpha u_{s}-\rho v_{s}, \\
\dot{w}_{s} & =\gamma\left(v_{s}-w_{s}\right), \\
\dot{u}_{i} & =\delta w_{i}+\sigma I w_{s}-u_{i}, \\
\dot{v}_{i} & =\rho\left(u_{i}-v_{i}\right), \\
\dot{w}_{i} & =\epsilon\left(v_{i}-w_{i}\right),
\end{aligned}
$$

where the dots on the dimensionless variables represent differentiation with respect to $\tau$ and the subscripts $s$ and $i$, respectively, denote dimensionless variables in the susceptible and infectious vector compartments.

For realistic dynamics of the density of susceptible fed vectors, $0 \leq I<1$, or else the susceptible fed vectors decays continuously to zero. Additionally, the dimensionless parameters can be expresses in terms of the original model parameters follows:

$$
\delta=\frac{p_{1} \beta_{h} N_{h} I_{w}^{0}}{L\left(a_{v}+\mu_{v}\right)}=\frac{p_{1} \beta_{h} N_{h} b^{*} I_{v}^{0}}{L\left(a_{v}+\mu_{v}\right)\left(a_{v}+\beta_{h} N_{h}\right)}=\frac{a_{v}}{\left(a_{v}+\mu_{v}\right)} \frac{b^{*}}{\left(\mu_{v}+b^{*}\right)} \frac{\beta_{h} N_{h}}{\left(\mu_{v}+\beta_{h} N_{h}\right)} p_{1},
$$

since $I_{v}^{0}=\left(a_{v} L\right) /\left(\mu_{v}+b^{*}\right)$. Clearly, each fractional term in equation (4.36) is less than one and since $0<p_{1}<1$ we see that $0 \leq \delta<1$.

Next, in terms of the original parameters

$$
\rho=\frac{\mu_{v}+b^{*}}{\mu_{v}+a_{v}}=\frac{\mu_{v}}{\mu_{v}+a_{v}}+\frac{b N_{h}}{\left(\mu_{v}+a_{v}\right)\left(N_{h}+k\right)}
$$

and

$$
\alpha=p\left(\frac{a_{v}}{\mu_{v}+a_{v}}\right)\left(\frac{\beta_{v} N_{h}}{\mu_{v}+\beta_{v} N_{h}}\right)\left(\frac{b N_{h}}{\left(\mu_{v}+a_{v}\right)\left(N_{h}+k\right)}\right) .
$$

Since $0<p<1,0<\frac{a_{v}}{\mu_{v}+a_{v}}<1,0<\frac{\beta_{v} N_{h}}{\mu_{v}+\beta_{v} N_{h}}<1,0<p\left(\frac{a_{v}}{\mu_{v}+a_{v}}\right)\left(\frac{\beta_{v} N_{h}}{\mu_{v}+\beta_{v} N_{h}}\right)<1$. Therefore,

$$
\alpha=p\left(\frac{a_{v}}{\mu_{v}+a_{v}}\right)\left(\frac{\beta_{v} N_{h}}{\mu_{v}+\beta_{v} N_{h}}\right)\left(\frac{b^{*}}{\mu_{v}+a_{v}}\right)<\frac{b^{*}}{\mu_{v}+a_{v}}<\frac{\mu_{v}}{\mu_{v}+a_{v}}+\frac{b^{*}}{\mu_{v}+a_{v}}=\rho .
$$


From the above verification, it is clear that $0 \leq \delta<1$ and $\alpha<\rho$ or $\rho-\alpha>0$. Assuming that $b=a_{v}$ (that is, the flow rates to and from human habitats are the same) implies $b^{*} \leq a_{v}$. Thus, $0<\rho<1$. Finally,

$$
\begin{aligned}
\beta & =\left(\frac{a_{v}}{\mu_{v}+a_{v}}\right)\left(\frac{b^{*}}{\mu_{v}+b^{*}}\right)\left(\frac{\beta_{h}}{\mu_{v}+\beta_{h} N_{h}}\right) L<\frac{L}{N_{h}}, \\
\sigma & =q / p \\
\alpha \sigma & <\rho<1 .
\end{aligned}
$$

Thus a feasible region for the parameter space is

$$
\Omega=\left\{\beta \geq 0, \mu>0,0<\alpha<\rho<1,0 \leq \delta<1, \epsilon>0, \sigma>0, \gamma>0, \lambda_{0}>0\right\} .
$$

\subsection{Conclusion}

We have derived and nondimensionalized a new deterministic SIS model for the dynamics of malaria transmission that explicitly integrates the demography of the vector that transmits malaria together with its interaction with the human population. The model incorporates disease dynamics and it is different from standard SIS models in that only female adult vectors that are questing for human blood are involved in disease transmission. We identified and accounted for these vectors. 


\section{References}

[1] H. W. Hethcote, "Asymptotic Behavior in a Deterministic Epidemic Model," Bulletin of Mathematical Biology 35, 607-614 (1973).

Online Version 65

[2] R. L. May and R. M. Anderson, Infectious Diseases of Humans: Dynamics and Control (Oxford University Press, Oxford, 1991).

[3] M. Iannelli, M.-Y. Kim, and E.-J. Park, "Asymptotic Behavior for an SIS Epidemic Model and its Approximation," Nonlinear Analysis 35, 797-814 (1999).

Online Version

[4] Y. Liu, Y. Du, and J. Wu, "Backward/Hopf Bifurcations in SIS Models with Delayed Nonlinear Incidence Rates," Frontiers of Mathematics in China 3 (4), 535-553 (2008). Online Version

[5] Y. Pei, S. Liu, C. Li, and L. Chen, "The Dynamics of an Impulsive Delay SI Model with Variable Coefficients," Applied Mathematical Modelling 33 (6), 2766-2776 (2009). Online Version 65

[6] G. Ngwa, "On the Population Dynamics of the Malaria Vector," Bulletin of Mathematical Biology 68, 2161-2189 (2006).

Online Version 65, 71, 79

[7] G. A. Ngwa, A. M. Niger, and A. B. Gumel, "Mathematical Assessment of the Role of Non-linear Birth and Maturation Delay in the Population Dynamics of the Malaria 
Vector," Applied Mathematics and Computation 217, 3286-3313 (2010).

Online Version 65, 77

[8] S. Nourridine, M. I. Teboh-Ewungkem, and G. A. Ngwa, "A Mathematical Model of the Population Dynamics of Disease Transmitting Vectors with Spatial Consideration," Journal of Biological Dynamics 1751-3758 (2011).

Online Version 65, 79

[9] G. A. Ngwa, C. N. Ngonghala, and N. B. W. Sama, "A Model for Endemic Malaria with Delay and Variable Populations," Jounal of the Cameroon Academy of Sciences 1(3), 169-186 (2001). 71

[10] W. H. Hethcote and P. van den Driessche, "Two SIS Epidemiologic Models with Delays," Journal of Mathematical Biology 40, 3-26 (2000).

Online Version

[11] K. Cook, P. van den Driessche, and X. Zou, "Interaction of Maturation Delay and Nonlinear Birth in Population and Epidemic Models," Journal of Mathematical Biology 39, 332-352 (1999).

Online Version 71, 73

[12] R. Pearl and L. J. Reed, "On the Rate of Growth of the Population of the United States since 1970 and its Mathematical Interpretation," Proceedings of the National academy of Sciences 6, 275-288 (1920). 73

[13] P. F. Verhulst, "Notice sur la Loi que la Population suit dans son Accroissement," Correspondence Mathématique et Physique 10, 113-121 (1938).

[14] K. Johst, A. Berryman, and M. Lima, "From Individual Interactions to Population Dynamics: Individual Resource Partitioning Simulation Exposes the Causes of Nonlinear Intra-specific Competition," Population Ecology 50, 79-90 (2008).

Online Version 73

[15] R. J. H. Beverton and S. J. Holt, "On the Dynamics of Exploited Fish Populations," 
Reviews in Fish Biology and Fisheries 4, 259-260 (1994).

Online Version 73

[16] W. E. Ricker, "Stock and Recruitment," Journal of the Fisheries Research board of canada 11, 559-623 (1954). 73

[17] M. P. Hassell, "Desity-dependence in Single Species Populations," Journal of Animal Ecology 44, 283-295 (1975). 73

[18] J. Maynard-Smith and M. Slatkin, "The Stability of Predator-prey Systems," Ecology 54, 384-391 (1973).

Online Version 73

[19] J. Maynard-Smith, Models in Ecology (Cambridge University Press, Cambridge, England, 1974). 73

[20] Å. Brännström and D. J. T. Sumpter, "The Role of Competition and Clustering in Population Dynamics," Proceedings of the Royal Society B 272, 2065-2072 (2005). Online Version 73

[21] R. M. Anderson and R. M. May, "Population Biology of Infectious Diseases: Part I," Nature 280, 361-367 (1979).

Online Version 77

[22] R. M. Anderson, "Mathematical and Statistical Study of the Epidemiology of HIV," AIDS 3 (6), 333-346 (1989).

Online Version 77

[23] H. R. Thieme, "Epidemic and Demorgraphic Interaction in the Spread of a Potentially Fatal Disease in a Growing Population," Mathematical Biosciences 111, 99-130 (1992). Online Version 77

[24] G. A. Ngwa and W. S. Shu, "A Mathematical Model for Endemic Malaria with Variable Human and Mosquito Populations," Mathematical and Computer Modelling 32, 747 - 
$763(2000)$.

Online Version 77

[25] M. I. Teboh-Ewungkem, Malaria Control: The Role of Local Communities as seen Through a Mathematical Model in a Changing Population-Cameroon, Chapter 4, 103140, in Advances in Disease Epidemiology (Nova Science Publishers, 2009).

[26] M. Teboh-Ewungkem, C. Podder, and A. Gumel, "Mathematical Study of the Role of Gametocytes and an Imperfect Vaccine on Malaria Transmission Dynamics," Bulletin of Mathematical Biology 72, 63-93 (2010).

Online Version 77

[27] H. K. Hale, Ordinary Differential Equations (John Wiley \& Sons, New York, 1969). 79

[28] J. K. Hale, Ordinary Differential Equations (Dover Publications, USA, 2009). 79 
Chapter 5

\section{Model Analysis}

\subsection{Introduction}

In this chapter, we analyze the model derived in Chapter 4. Rigorous qualitative and quantitative techniques shall be applied to acquire insights into the dynamical behavior of the model and to identify essential epidemiological threshold parameters that characterize disease transmission and prevalence in the presence and in the absence of the disease. In the presence of the disease, we show that there exist parameter spaces in which the model exhibits Hopf and backward bifurcations and compare the effects of two different birth rate functions on the dynamical behavior of the model.

The Chapter is organized as follows: In Section 5.2, we study the model in the absence of the disease. We calculate the equilibrium solutions of the disease-free model in Section 5.2.1, study the stability of these equilibria in Section 5.2.2, and present simulation results in Section 5.2.3. In Section 5.3, we analyze the full disease model. We compute a new basic reproduction number for malaria in Section 5.3.1, compute the equilibria of the disease model and explore their stability properties in Sections 5.3.2 and 5.3.3, respectively, and present simulation results to the disease model in Section 5.3.4. We investigate the existence of a backward bifurcation in Section 5.4 and the effects of birth rate functions on the dynamics of the disease model in Section 5.5. We end the Chapter with a conclusion in Section 5.6. 


\subsection{Model in the Absence of the Malaria Disease}

To be able to quantify the effect of the disease in the population, we first study the dynamics of the model in the absence of the disease. In the absence of the malaria disease, $I=0, u_{i}=0, v_{i}=0, w_{i}=0$ and system (4.35) reduces to the following three-variable system that models the population dynamics of the malaria vector:

$$
\begin{aligned}
& \dot{u}_{s}=w_{s}-u_{s} \\
& \dot{v}_{s}=\alpha \lambda_{0} u_{s}\left(1-u_{s}\right)+\alpha u_{s}-\rho v_{s} \\
& \dot{w}_{s}=\gamma\left(v_{s}-w_{s}\right) .
\end{aligned}
$$

We highlight important results on the existence and stability of equilibria of system 5.1.

\subsubsection{Existence of Realistic Equilibrium Solutions}

Theorem 5.2.1. There exists a threshold parameter, $\mathscr{R}^{*}=\alpha \lambda_{0} /(\rho-\alpha)$ such that when $\mathscr{R}^{*} \leq 1$, system (5.1) has a unique equilibrium solution, the trivial equilibrium solution, and when $\mathscr{R}^{*}>1$, a non-trivial equilibrium solution coexists with the trivial equilibrium solution.

Proof. By setting the left-hand sides of system (5.1) to zero and solving the ensuing system of algebraic equations simultaneously, we obtain the following two equilibrium solutions:

$$
\begin{aligned}
& E_{0}=\left(u_{s}^{*}, v_{s}^{*}, w_{s}^{*}\right)=(0,0,0), \\
& E_{1}=\left(1-\frac{1}{\mathscr{R}^{*}}, 1-\frac{1}{\mathscr{R}^{*}}, 1-\frac{1}{\mathscr{R}^{*}}\right),
\end{aligned}
$$

where

$$
\mathscr{R}^{*}=\alpha \lambda_{0} /(\rho-\alpha)
$$

is a threshold parameter directly linked to the demography of the vector. A realistic positive non-zero steady $E_{1}$ exists when $\mathscr{R}^{*}>1$. When $\mathscr{R}^{*}=1, E_{1}$ reduces to the trivial equilibrium $E_{0}$ and when $\mathscr{R}^{*}<1, E_{1}$ does not exist.

\subsubsection{Stability of Equilibrium Solutions}

Theorem 5.2.2. In the absence of the malaria disease, there exists a threshold parameter $\mathscr{R}^{*}$ satisfying equation (5.2) such that 
1. If $\mathscr{R}^{*} \leq 1$, there exists a unique equilibrium $E_{0}$, which is stable to small perturbations.

2. If $\mathscr{R}^{*}>1$, there are two equilibrium solutions, the trivial equilibrium $E_{0}$, which is linearly unstable to small perturbations, and a realistic non-trivial equilibrium $E_{1}$, whose stability is determined by the relationship between $\lambda_{0}(\gamma)$ and its critical value

$$
\lambda_{0}^{c}\left(\gamma_{c}\right)=\frac{(\gamma+\rho+1)(\gamma+\rho+\gamma \rho)+\gamma(\rho-\alpha)}{\alpha \gamma},
$$

or

$$
\zeta=\frac{P\left(\mathscr{R}^{*}-1\right)}{Q R}
$$

and its critical value $\zeta_{c}=1$.

(a) The non-trivial equilibrium $E_{1}$ is linearly stable to small perturbations when the coefficients of the characteristic equation (5.9) satisfy $Q R-P\left(\mathscr{R}^{*}-1\right)>0$, which is equivalent to $0<\lambda_{0}<\lambda_{0}^{c}$ or $0<\zeta<1$.

(b) The non-trivial equilibrium $E_{1}$ is linearly unstable to small perturbations when the coefficients of the characteristic equation (5.9) satisfy $Q R-P\left(\mathscr{R}^{*}-1\right)<0$, which is equivalent to $\lambda_{0}>\lambda_{0}^{c}$ or $\zeta>1$.

(c) When $Q R-P\left(\mathscr{R}^{*}-1\right)=0$, which is equivalent to $0<\lambda_{0}=\lambda_{0}^{c}$ or $0<\zeta=\zeta_{c}=$ 1, the characteristic equation (5.9) admits three roots: a real and negative root $\lambda=-Q$ and a pair of purely imaginary roots $\lambda= \pm \sqrt{R} i$, indicating that there is a parameter space where a Hopf bifurcation occurs.

Proof. The proof of the existence portion of this Theorem was already established in Theorem 5.2.1. Hence, we focus on the proof of stability here. To establish the stability of equilibrium solutions, we perturb system (5.1) about the equilibrium solution $\left(u^{*}, v^{*}, w^{*}\right)$ by setting $u_{s}=u+u^{*}, v_{s}=v+v^{*}$ and $w_{s}=w+w^{*}$ where $|u| \ll 1,|v| \ll 1$ and $|w| \ll 1$. Expanding the resulting system in a Taylor series about $\left(u^{*}, v^{*}, w^{*}\right)$ and retaining only linear terms in $u, v$ and $w$ gives rise to the following linear system: 


$$
\left(\begin{array}{c}
\dot{u} \\
\dot{v} \\
\dot{w}
\end{array}\right)=\underbrace{\left(\begin{array}{ccc}
-1 & 0 & 1 \\
\alpha\left(\lambda_{0}+1\right)-2 \alpha \lambda_{0} u^{*} & -\rho & 0 \\
0 & \gamma & -\gamma
\end{array}\right)}_{J\left(u_{s}^{*}, u_{s}^{*}, w_{s}^{*}\right)}\left(\begin{array}{l}
u \\
v \\
w
\end{array}\right),
$$

where $J\left(u_{s}^{*}, v_{s}^{*}, w_{s}^{*}\right)$ is the Jacobian matrix of the system evaluated at the equilibrium point $\left(u_{s}^{*}, v_{s}^{*}, w_{s}^{*}\right)$. At each of the equilibrium solutions, we seek solutions to the linear system of the form $\vec{X}=\vec{X}_{0} e^{\lambda t}$, where $\vec{X}=(u, v, w)^{T}, \vec{X}_{0}=\left(u_{0}, v_{0}, w_{0}\right)^{T}$ is a non-zero vector of constants, and $\lambda$ is an eigenvalue that measures the growth or decay rate of solutions to the linearized system. This gives rise to the linear system of algebraic equations $(J-\lambda I) \overrightarrow{X_{0}}=\overrightarrow{0}$, which has non-zero solutions when

$$
|J-\lambda I|=0 .
$$

Now, at the trivial equilibrium point $E_{0}$,

$$
J(0,0,0)=\left(\begin{array}{ccc}
-1 & 0 & 1 \\
\alpha\left(\lambda_{0}+1\right) & -\rho & 0 \\
0 & \gamma & -\gamma
\end{array}\right) .
$$

The eigenvalues of $J(0,0,0)$ are computed from the characteristic polynomial equation

$$
\lambda^{3}+(\gamma+\rho+1) \lambda^{2}+(\gamma+\rho+\gamma \rho) \lambda+\gamma \rho-\alpha \gamma\left(\lambda_{0}+1\right)=0,
$$

which is obtained by expanding the equation (5.5). Equation (5.6) can be rewritten as

$$
\lambda^{3}+Q \lambda^{2}+R \lambda+P\left(1-\mathscr{R}^{*}\right)=0,
$$

where

$$
\begin{aligned}
& Q=\gamma+\rho+1>0 \\
& R=\gamma+\rho+\gamma \rho>0, \\
& P=\gamma(\rho-\alpha)>0, \text { since } \rho>\alpha,
\end{aligned}
$$

and $\mathscr{R}^{*}$ is as defined in equation (5.2). Using Descartes" rule of signs and the Routh-Hurwitz conditions, we determine the following stability properties for the trivial equilibrium solution: 
1. If $\mathscr{R}^{*} \leq 1$, there is no sign change in the sequence of coefficients of equation (5.7) indicating that there is no positive real value of $\lambda$. Now, by the Routh-Hurwitz condition all roots of the characteristic equation (5.7) will have negative real parts since $Q>0, R>0$ and $P\left(1-\mathscr{R}^{*}\right)>0$ when $\mathscr{R}^{*} \leq 1$. Furthermore, $Q R-P\left(1-\mathscr{R}^{*}\right)=$ $(\gamma+\rho+\gamma \rho+1)(\gamma+\rho)+\gamma \rho \mathscr{R}^{*}+\gamma \alpha\left(1-\mathscr{R}^{*}\right)=(\gamma+\rho+\gamma \rho+1)(\gamma+\rho)+\gamma \alpha+\gamma(\rho-\alpha) \mathscr{R}^{*}>0$ for all parameters in the parameter space $\Omega$. Hence, when $\mathscr{R}^{*} \leq 1$, the trivial equilibrium is linearly stable, and it is the only existing equilibrium solution.

2. If $\mathscr{R}^{*}>1$, the constant term in equation (5.7) is negative and there is exactly one sign change in the sequence of coefficients. This indicates that equation (5.7) can have at most one positive real value of $\lambda$. We easily verify that the Routh-Hurwitz conditions fail and thus the trivial equilibrium $E_{0}$ is linearly unstable to small perturbations whenever $\mathscr{R}^{*}>1$.

Next, we examine the linear stability of the non-trivial equilibrium solution, $E_{1}=$ $\left(u_{s}^{*}, v_{s}^{*}, w_{s}^{*}\right)$. The Jacobian matrix evaluated at this equilibrium solution is

$$
J\left(u_{s}^{*}, v_{s}^{*}, w_{s}^{*}\right)=\left(\begin{array}{ccc}
-1 & 0 & 1 \\
2 \rho-\alpha\left(\lambda_{0}+1\right) & -\rho & 0 \\
0 & \gamma & -\gamma
\end{array}\right)
$$

and the eigenvalues of $J$ are given by the following characteristic polynomial equation:

$$
\lambda^{3}+Q \lambda^{2}+R \lambda+P\left(\mathscr{R}^{*}-1\right)=0
$$

where $Q>0, R>0, P>0$ are defined in (5.8). Since $\mathscr{R}^{*}>1, P\left(\mathscr{R}^{*}-1\right)>0$.

(a) By the Routh-Hurwitz necessary and sufficient conditions, all solutions of equation (5.9) have negative real parts if $Q R-P\left(\mathscr{R}^{*}-1\right)>0$. That is, if $0<\lambda<\lambda_{0}^{c}$ or $0<\zeta<1$. This establishes the linear stability of the non-trivial equilibrium, $E_{1}$.

(b) For a parameter space where $Q R-P\left(\mathscr{R}^{*}-1\right)<0$, that is, $\lambda>\lambda_{0}^{c}$ or $\zeta>1$, the Routh-Hurwitz criterion fails and so a pair of complex eigenvalues have positive real parts. This indicates that the nontrivial equilibrium solution $E_{1}$ is linearly unstable to small perturbations when $Q R-P\left(\mathscr{R}^{*}-1\right)<0$ or $\lambda_{0}>\lambda_{0}^{c}$. That is, 
$E_{1}$ loses stability at $Q R-P\left(\mathscr{R}^{*}-1\right)=0$ or at $\lambda_{0}=\lambda_{0}^{c}$, and the instability is accompanied by the emergence of periodic solutions.

(c) If $Q R=P\left(\mathscr{R}^{*}-1\right)$, equation (5.9) has a pair of purely imaginary roots. In fact, if $Q R=P\left(\mathscr{R}^{*}-1\right)$, which is equivalent to $\lambda_{0}=\lambda_{0}^{c}$ or $\zeta=\zeta_{c}=1$, equation (5.9) can be rewritten as

$$
\lambda^{3}+Q \lambda^{2}+R \lambda+Q R=0
$$

which factors to $(\lambda+P)\left(\lambda^{2}+Q\right)=0$ and thus admits three roots: a real and negative root $\lambda=-Q$ and a pair of purely imaginary roots $\lambda= \pm \sqrt{R} i$, indicating that there is a parameter space where a Hopf bifurcation occurs when $Q R=$ $P\left(\mathscr{R}^{*}-1\right)$. To confirm the occurrence of a Hopf bifurcation at $\lambda_{0}=\lambda_{0}^{c}$ or $\zeta=\zeta_{c}=1$, and to further explore the nature of the instability that arises when $\lambda_{0}>\lambda_{0}^{c}$ or $\zeta>\zeta_{c}=1$, we assume that the eigenvalue $\lambda$ depends continuously on $\zeta$. That is, $\lambda=\lambda(\zeta)$. Note that equation (5.9) can be rewritten as

$$
\lambda^{3}+Q \lambda^{2}+R \lambda+\zeta Q R=0
$$

We perturb $\zeta$ about $\zeta_{c}$ by setting $\zeta=\zeta_{c}+\varrho^{2} \nu$ where $|\varrho| \ll 1$ and $\nu= \pm 1$. This yields $\lambda(\zeta)=\lambda\left(\zeta_{c}+\varrho^{2} \nu\right)$. Expanding $\lambda$ in a Taylor series about $\zeta_{c}$ and keeping linear terms in $\varrho^{2} \nu$ only, we obtain

$$
\lambda(\zeta)=\lambda\left(\zeta_{c}\right)+\lambda^{\prime}\left(\zeta_{c}\right) \varrho^{2} \nu
$$

where the prime denotes differentiation with respect to $\zeta$. Differentiating (5.10) implicitly with respect to $\zeta$ and solving for $\lambda^{\prime}$ gives

$$
\lambda^{\prime}(\zeta)=-\frac{Q R}{3 \lambda(\zeta)^{2}+2 Q \lambda(\zeta)+R} .
$$

Now, at $\zeta=\zeta_{c}, \lambda\left(\zeta_{c}\right)= \pm \sqrt{R} i$. Evaluating $\lambda^{\prime}$ at $\zeta_{c}$ yields

$$
\lambda^{\prime}\left(\zeta_{c}\right)=\frac{Q R}{2\left(Q^{2}+R\right)} \pm i \frac{Q^{2} \sqrt{R}}{2\left(Q^{2}+R\right)} .
$$

Clearly, $\operatorname{Re} \lambda\left(\zeta_{c}\right)=0$ and $\operatorname{Re} \lambda^{\prime}\left(\zeta_{c}\right)=\frac{Q R}{2\left(Q^{2}+R\right)}>0$. Next, from equation (5.11),

$$
\lambda(\zeta)=\lambda\left(\zeta_{c}\right)+\lambda^{\prime}\left(\zeta_{c}\right) \varrho^{2} \nu=\frac{Q R}{2\left(Q^{2}+R\right)} \varrho^{2} \nu \pm i \sqrt{R}\left(1+\frac{Q^{2} \sqrt{R}}{2\left(Q^{2}+R\right)} \varrho^{2} \nu\right) .
$$


We have therefore demonstrated that for $0<\zeta<1$, all solutions of (5.1) have negative real parts. At $\zeta=\zeta_{c}=1$, a purely imaginary pair of eigenvalues arises, and for $\zeta>1$, the real parts of the imaginary eigenvalues become positive. Thus, as $\zeta$ traverses $\zeta_{c}=1$, stable solutions of system (5.1) become unstable. Associated with this loss of stability is the emergence of periodic oscillations with initial period and amplitude approximately given by

$$
\frac{2 \pi}{\sqrt{R}\left(1+\frac{Q^{2} \sqrt{R}}{2\left(Q^{2}+R\right)} \varrho \nu\right)} \text { and } \exp \left(\frac{Q R}{2\left(Q^{2}+R\right)} \varrho^{2} \nu \tau\right) \text {, respectively, where } \varrho=\sqrt{\frac{\left|\zeta-\zeta_{c}\right|}{|\nu|}} \text {. }
$$

This completes the proof of Theorem 5.2.2.

Remark 5.2.3. Instability of $E_{0}$ is reasonable from a physical stand point and rules out any possibility of easily eradicating the mosquito population permanently whenever $\mathscr{R}^{*}>1$.

Remark 5.2.4. Note that if in the absence of the malaria disease, $S_{u}, S_{v}$ and $S_{w}$ are scaled by $S_{u}^{0}=u^{*}, S_{v}^{0}=v^{*}, S_{w}^{0}=w^{*}$, where $u^{*}, v^{*}, w^{*}$ are the equilibrium solutions of the disease-free model, the associated dimensionless system will have a trivial equilibrium solution $(0,0,0)$ and a non-trivial equilibrium solution $(1,1,1)$. This will lead to the equivalent reparameterization of the reduced model (5.1) as studied in [1].

\subsubsection{Numerical Simulations of the Disease-free Model}

As demonstrated in Sections 5.2.1 and 5.2.2, in the absence of the disease, system (5.1) can exhibit either one or two equilibrium solutions depending on whether $\mathscr{R}^{*} \leq 1$ or $\mathscr{R}^{*}>1$. We also showed that when $\mathscr{R}^{*}>1$, the equilibrium solution $E_{1}$ is asymptotically stable if $\lambda_{0}<\lambda_{0}^{c}$, and unstable with the emergence of period oscillations if $\lambda_{0}>\lambda_{0}^{c}$. We now present numerical simulations to further confirm these results. Here, we use the parameter regime $\alpha=0.4636, \gamma=0.7849,0.01 \leq \lambda_{0} \leq 16$, and $\rho=0.6182$ (see Table 5.2 for details) and a fourth-order Runge Kutta method with variable step size to simulate system (5.1) for 10,000 time steps. Time series plots depicting stable solutions are shown in Fig. 5.1, while a time series and a phase plot depicting periodic oscillatory solutions are shown in Fig. 5.2. A bifurcation diagram illustrating the switch from a stable trivial equilibrium solution that exists when $0<\lambda_{0} \leq 0.33$ or $0<\mathscr{R}^{*} \leq 1$ to a stable non-trivial equilibrium solution that 
exists when $0.33 \leq \lambda_{0}<\lambda_{0}^{c}=12.79$ or $1<\mathscr{R}^{*}<38.37$, and then to periodic solutions when $\lambda_{0}>\lambda_{0}^{c}=12.79$ or $\mathscr{R}^{*}>38.37$ is presented in Fig. 5.4. Notice that a Hopf bifurcation occurs at $\lambda_{0}=\lambda_{0}^{c}=12.79$ or $\mathscr{R}^{*}=38.37$. Here, we plot the maximum and minimum values of $u_{s}$ against $\lambda_{0}$ and $\mathscr{R}^{*}$. Analogous plots can be obtained for $v_{s}$ and $w_{s}$. 


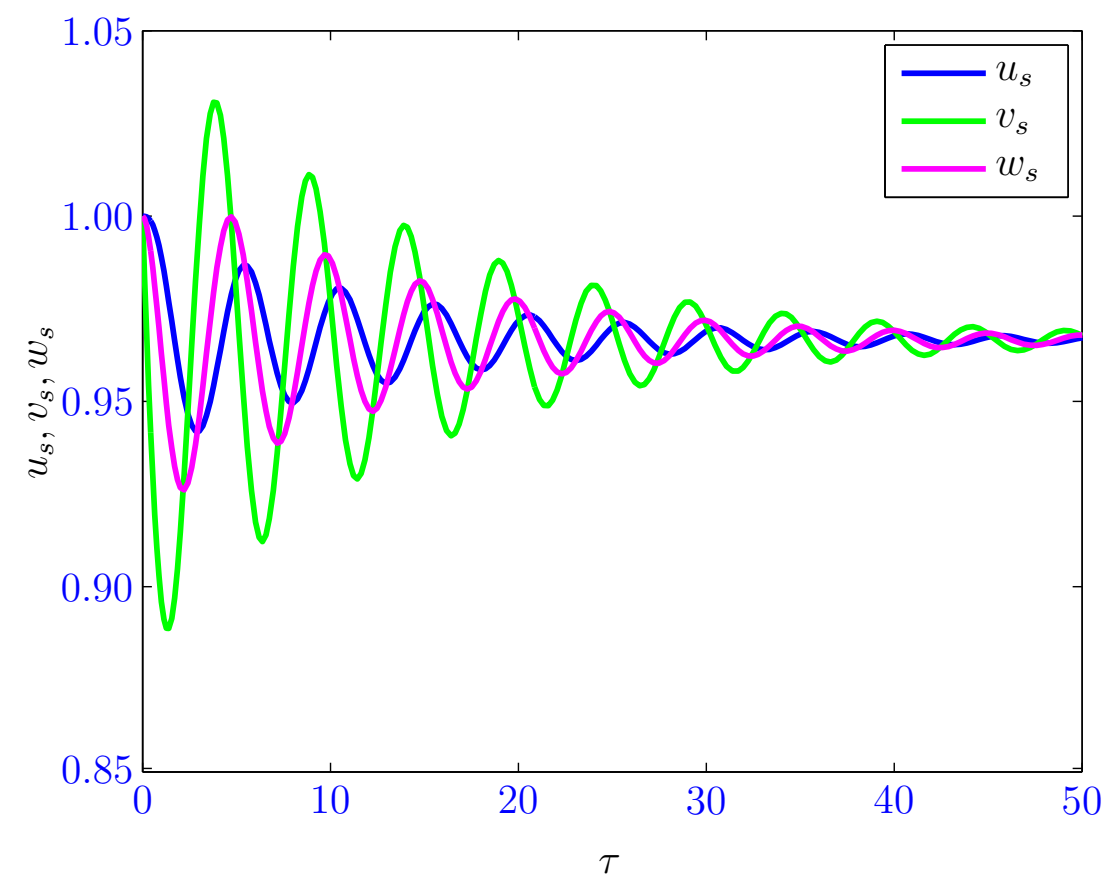

5.1.1. Solution for the first 50 time steps.

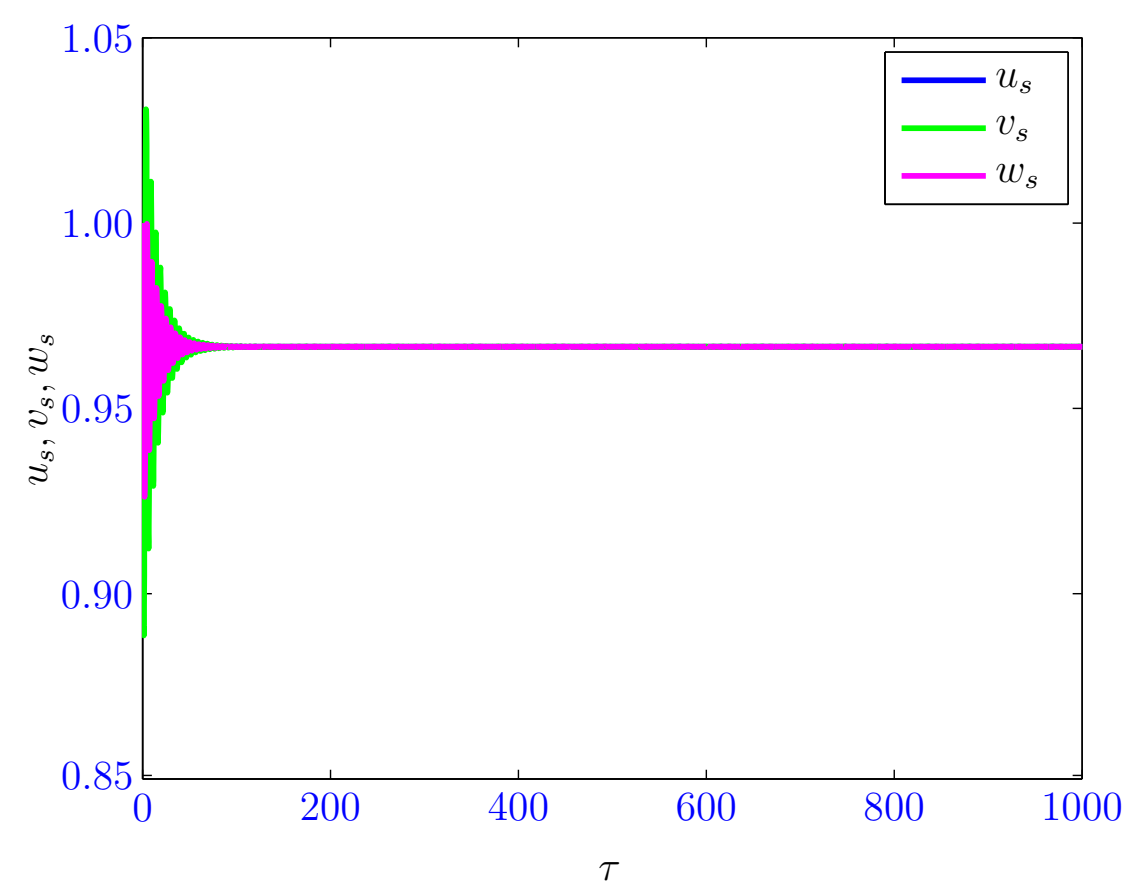

5.1.2. Solution for the first 1,000 time steps.

Figure 5.1. Solutions of the disease-free model (5.1). The system converges to the stable non-trivial equilibrium solution $E_{1}=\left(u_{s}^{*}, v_{S}^{*}, w_{s}^{*}\right)=(0.9667,0.9667,0.9667)$ for $\alpha=0.4636, \gamma=0.7849, \lambda_{0}=10$, and $\rho=0.6182$. Note that $\mathscr{R}^{*}=30$. 


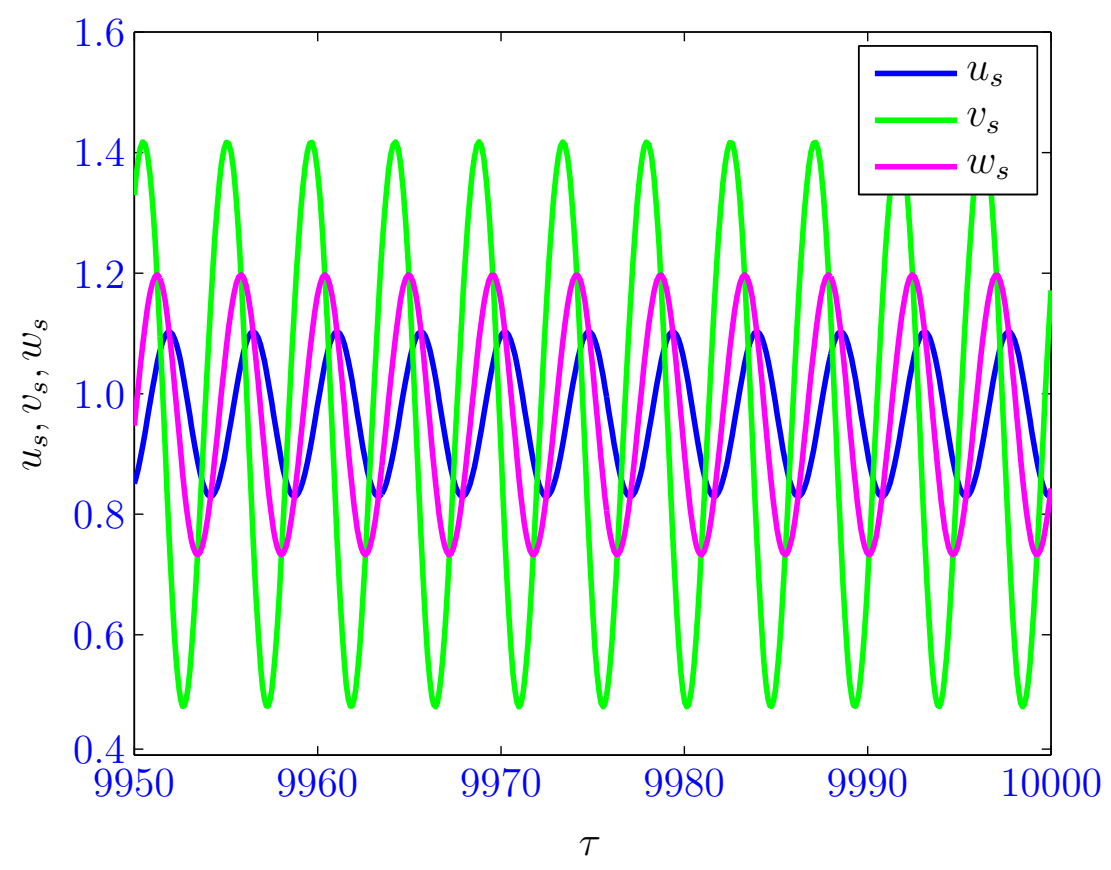

5.2.1. Time series showing periodic oscillations.

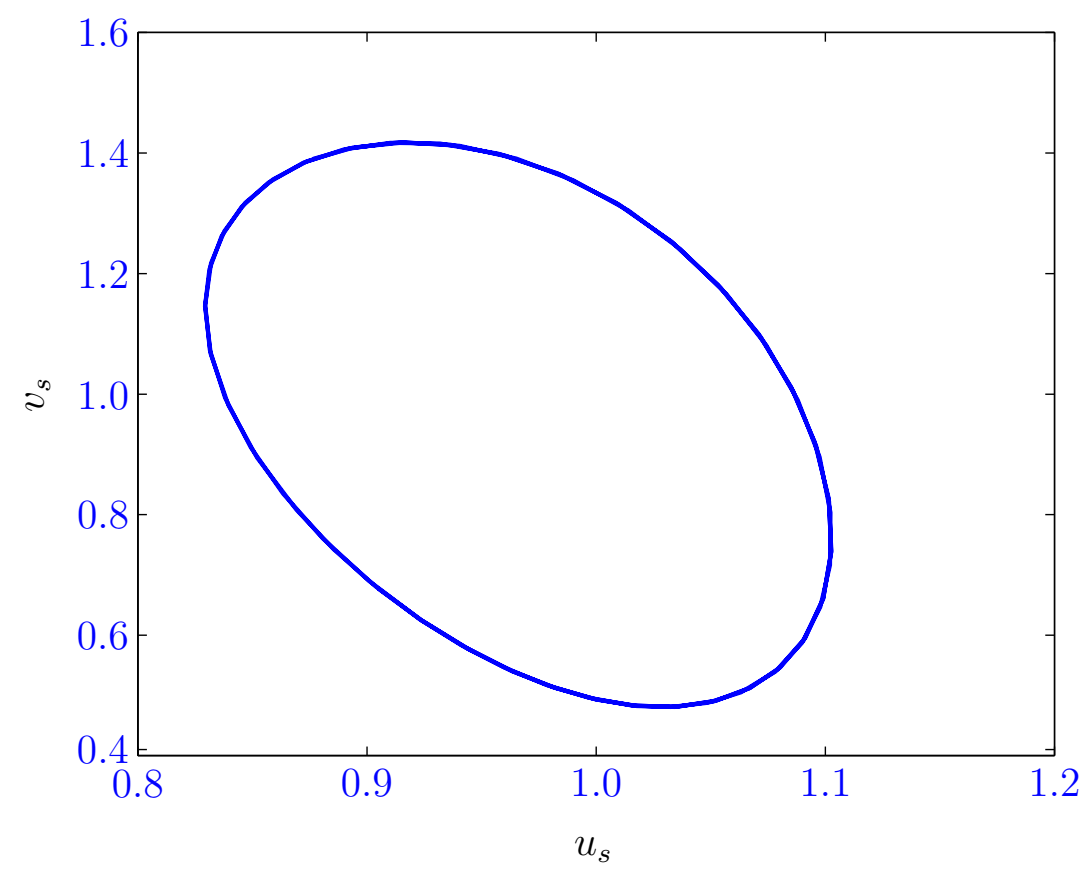

5.2.2. Phase plot showing the limit cycle.

Figure 5.2. Time series and phase plot of system (5.1) for $\alpha=0.4636, \gamma=0.7849, \lambda_{0}=$ 13 , and $\rho=0.6182$ showing periodic oscillations. 


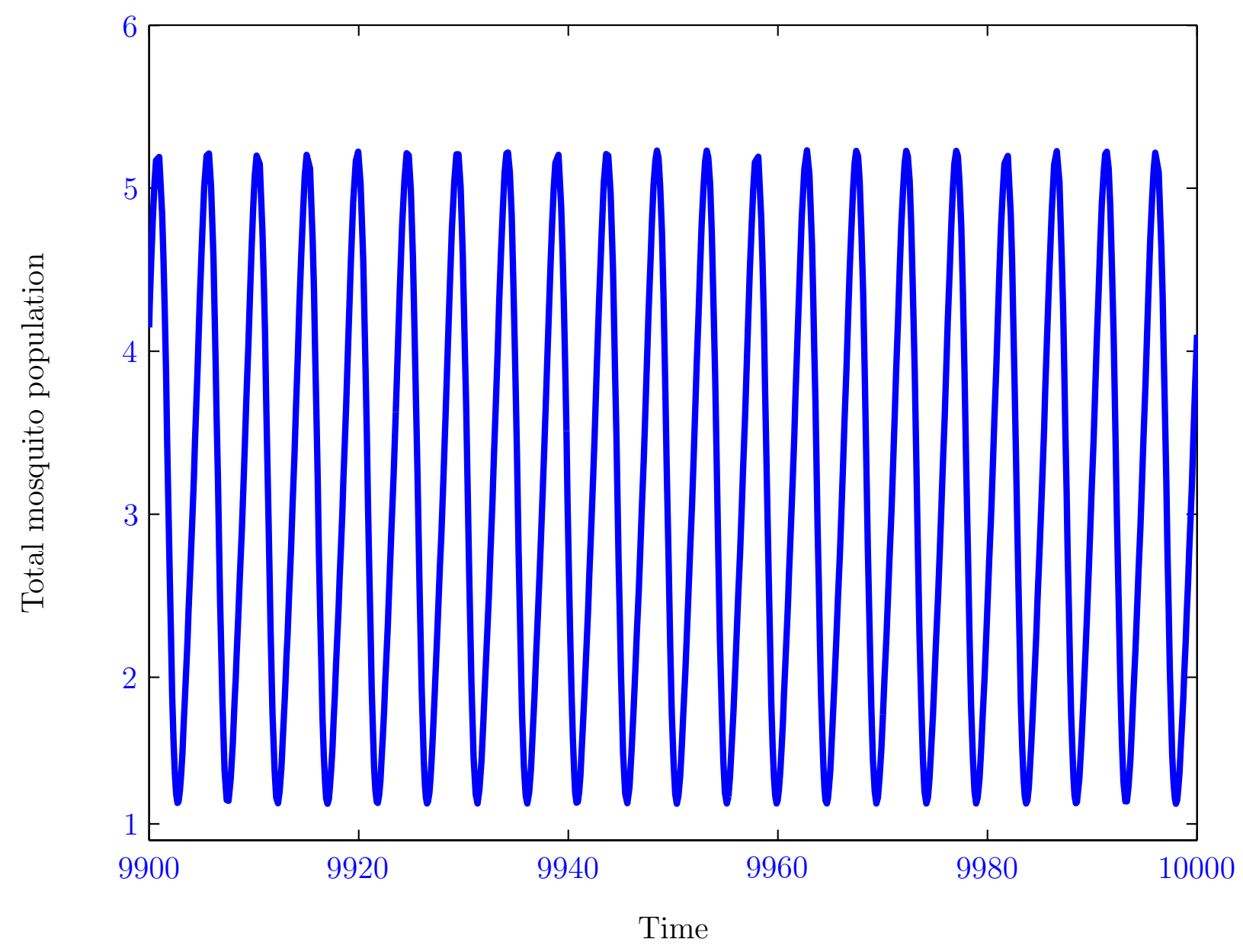

Figure 5.3. Plot of the total mosquito population showing sustained bounded oscillations. 


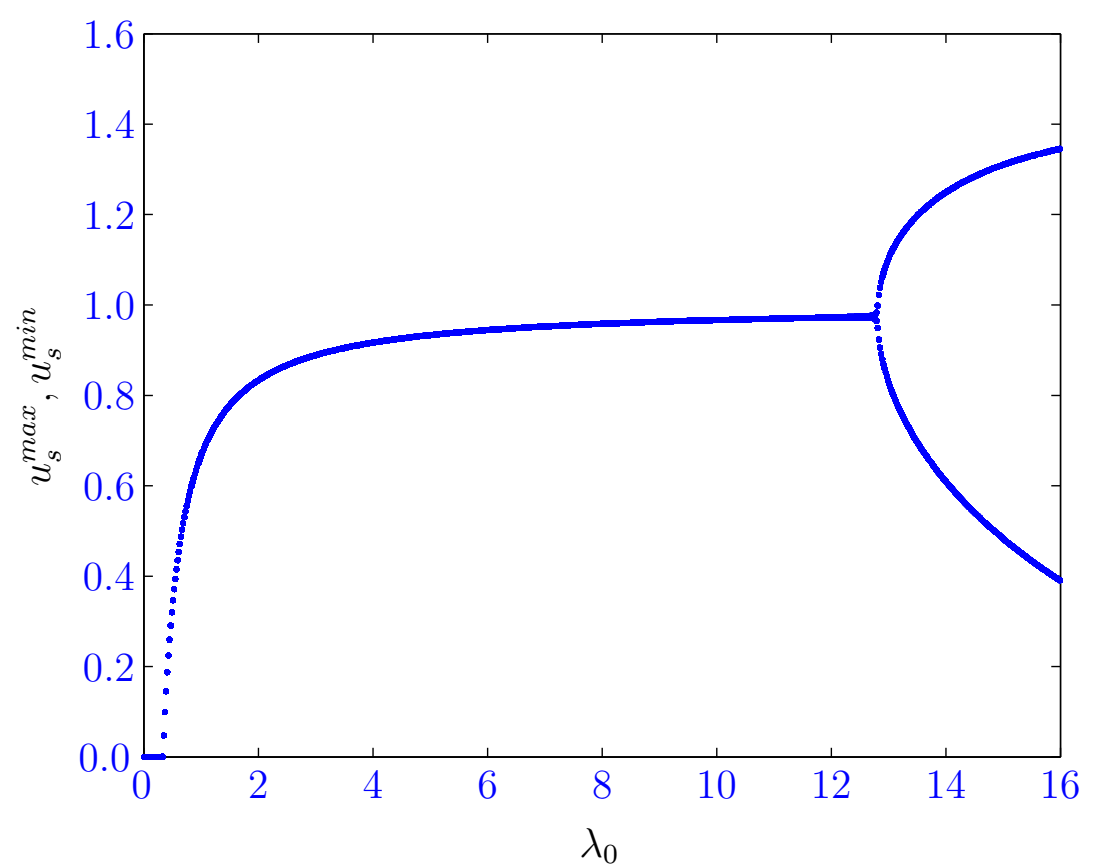

5.4.1. Maximum and minimum values of $u_{s}$ against $\lambda_{0}$.

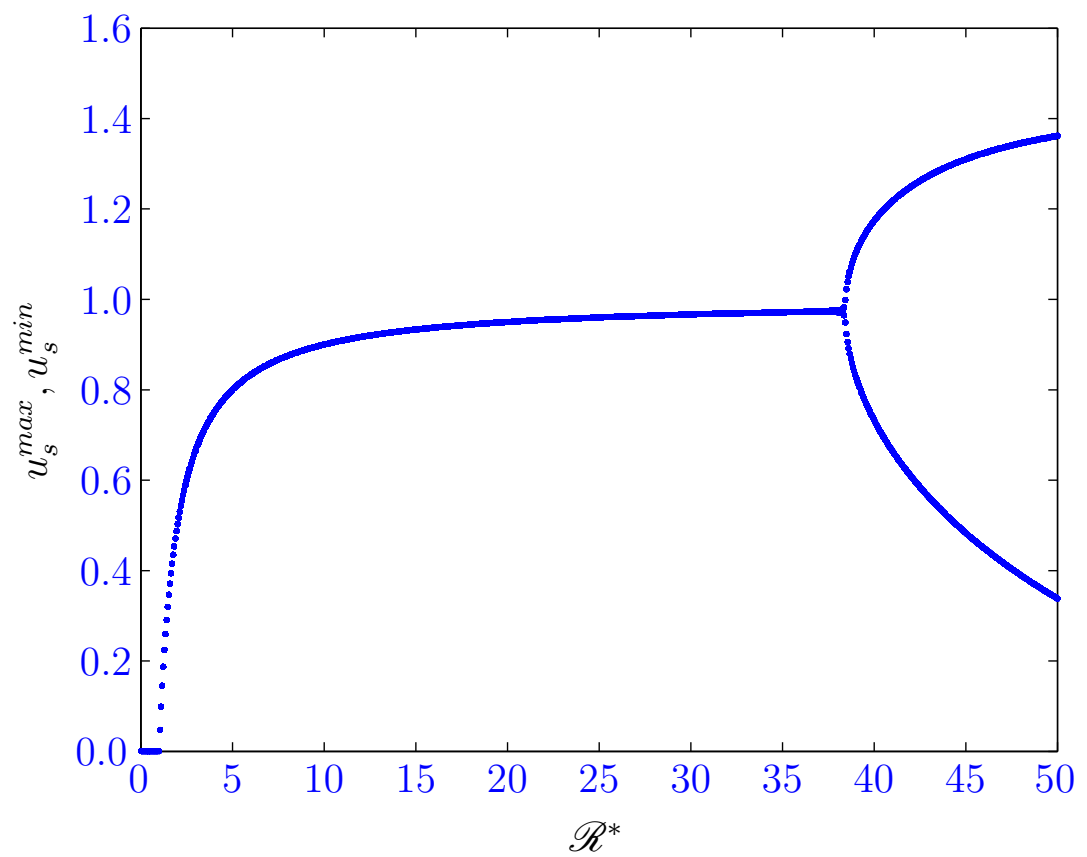

5.4.2. Maximum and minimum values of $u_{s}$ against $\mathscr{R}^{*}$.

Figure 5.4. Bifurcation diagrams showing the transition from a stable trivial equilibrium for $0<\lambda_{0} \leq 0.33\left(0<\mathscr{R}^{*} \leq 1\right)$ to a stable non-trivial equilibrium for $0.33 \leq$ $\lambda_{0}<12.79\left(1<\mathscr{R}^{*}<38.37\right)$, and then to periodic solutions for $\lambda_{0}>12.79$ $\left(\mathscr{R}^{*}>38.37\right)$. A Hopf bifurcation occurs at $\lambda_{0}=12.79\left(\mathscr{R}^{*}=38.37\right)$. 


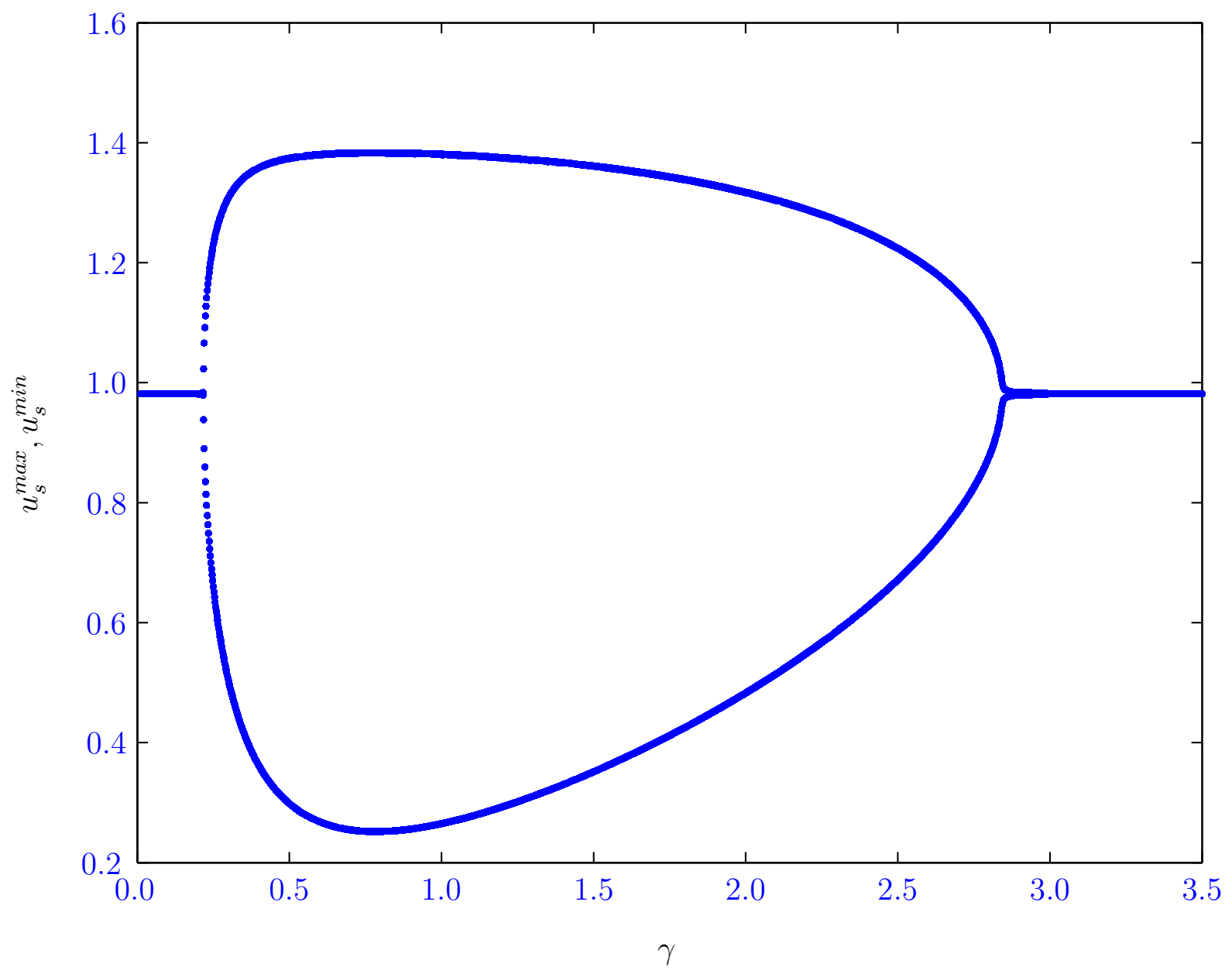

Figure 5.5. Bifurcation diagram illustrating the transition from a stable equilibrium solution to bounded periodic oscillations through a Hopf bifurcation and then back to a stable equilibrium solution through a second Hopf bifurcation as the parameter $\gamma$ is increased from 0 to 3.5 . 


\subsection{Model Analysis in the Presence of the Disease}

\subsubsection{A Discussion on the Basic Reproduction Number, $R_{0}$}

A threshold parameter that is of essential importance to infectious disease transmission is the basic reproduction number denoted by $R_{0}$. $R_{0}$ measures the average number of secondary clinical cases of infection generated in an absolutely susceptible population by a single infectious individual throughout the period within which the individual is infectious [2-7]. Generally, the disease disappears from the community if $R_{0}<1$ and may establish itself if $R_{0}>1$. Thus, $R_{0}$ serves as a direct quantitative measure of the intervention effort needed to eradicate the disease. Disease pathogens strive to maximize $R_{0}$. The dynamics of some diseases exhibit backward bifurcation involving the existence of two endemic equilibria when $R_{0}<1$. In such diseases, simply reducing $R_{0}$ below unity may not be enough for disease elimination. The critical case $R_{0}=1$ represents the situation in which the disease reproduces itself thereby leaving the community with a similar number of infection cases at any time. The definition of $R_{0}$ specifically requires that initially everybody but the infectious individual in the population be susceptible. Thus, this definition breaks down within a population in which some of the individuals are already infected or immune to the disease under consideration. In such a case, the notion of reproduction number $\mathfrak{R}$ becomes useful. Unlike $R_{0}$ which is fixed, $\mathfrak{R}$ may vary considerably with disease progression. However, $\mathfrak{R}$ is bounded from above by $R_{0}$ and it is computed at different points depending on the number of infected or immune cases in the population.

One way of calculating $R_{0}$ is to determine a threshold condition for which endemic equilibrium solutions to the system under study exists or for which the disease-free equilibrium is unstable. Another method is the next generation matrix approach, where $R_{0}$ is the spectral radius of the next generation matrix [2]. Smith et al. [8] applied a novel approach to compute $R_{0}$ for malaria within 121 African populations. They illustrated that $1 \leq R_{0} \leq 3000$. Large values for $R_{0}$ indicate that it may be impossible to eradicate malaria.

Using the next generation matrix approach, we identify all state variables for the infection, $I_{h}, I_{u}, I_{v}$ and $I_{w}$, and their corresponding equations to be: 


$$
\begin{aligned}
& \dot{I}_{h}=\beta_{h} S_{h} I_{w}-\left(\mu_{h}+r_{h}\right) I_{h}, \\
& \dot{I}_{u}=p_{1} \beta_{h} N_{h} I_{w}+q \beta_{v} I_{h} S_{w}-\left(a_{v}+\mu_{v}\right) I_{u}, \\
& \dot{I}_{v}=a_{v} I_{u}-\left(\mu_{v}+\alpha_{v}\left(N_{h}\right)\right) I_{v}, \\
& \dot{I}_{w}=\alpha_{v}\left(N_{h}\right) I_{v}-\left(\mu_{v}+\beta_{h} N_{h}\right) I_{w} .
\end{aligned}
$$

We then identify terms representing new infections from the above equations and rewrite the system as the difference of two vectors $\tilde{\mathfrak{F}}$ and $\tilde{\mathcal{V}}$ where $\tilde{\mathfrak{F}}$ consists of all new infections and $\tilde{\mathcal{V}}$ consists of the remaining terms or transitions between states. That is, we set $\dot{I}_{i}=\tilde{\mathfrak{F}}-\tilde{\mathcal{V}}$ where the subscript $i=(h, u, v, w)$. This gives rise to

$$
\tilde{\mathfrak{F}}=\left(\begin{array}{c}
\beta_{h} S_{h} I_{w} \\
q \beta_{v} I_{h} S_{w} \\
0 \\
0
\end{array}\right) \text { and } \tilde{\mathcal{V}}=\left(\begin{array}{c}
\left(\mu_{h}+r_{h}\right) I_{h} \\
-p_{1} \beta_{h} N_{h} I_{w}+\left(a_{v}+\mu_{v}\right) I_{u} \\
-a_{v} I_{u}+\left(\mu_{v}+\alpha_{v}\left(N_{h}\right)\right) I_{v} \\
-\alpha_{v}\left(N_{h}\right) I_{v}+\left(\mu_{v}+\beta_{h} N_{h}\right) I_{w}
\end{array}\right)
$$

Evaluating $\frac{d \tilde{\mathfrak{F}}}{d I_{i}}$ and $\frac{d \tilde{\mathcal{V}}}{d I_{i}}$, where $i=(h, u, v, w)$ at the disease-free equilibrium, $\left(0, S_{u}^{*}, S_{v}^{*}, S_{w}^{*}, 0,0,0\right)$ gives

$$
\mathfrak{F}=\frac{d \tilde{\mathfrak{F}}}{d I_{i}}=\left(\begin{array}{cccc}
0 & 0 & 0 & \beta_{h} N_{h} \\
q \beta_{v} S_{w}^{*} & 0 & 0 & 0 \\
0 & 0 & 0 & 0 \\
0 & 0 & 0 & 0
\end{array}\right)
$$

and

$$
\mathcal{V}=\frac{d \tilde{\mathcal{V}}}{d I_{i}}=\left(\begin{array}{cccc}
\mu_{h}+r_{h} & 0 & 0 & 0 \\
0 & a_{v}+\mu_{v} & 0 & -p_{1} \beta_{h} N_{h} \\
0 & -a_{v} & \mu_{v}+\alpha_{v}\left(N_{h}\right) & 0 \\
0 & 0 & -\alpha_{v}\left(N_{h}\right) & \mu_{v}+\beta_{h} N_{h}
\end{array}\right) .
$$

Note that $\mathfrak{F}$ and $\mathcal{V}$ are the rates of new infections and the transitions near the disease-free equilibrium, respectively. Next, we compute $\mathcal{V}^{-1}$ : 


$$
\mathcal{V}^{-1}=\left(\begin{array}{cccc}
\frac{1}{\mu_{h}+r_{h}} & 0 & 0 & 0 \\
0 & \frac{B_{2} B_{3}}{B_{0}} & \frac{p_{1} \alpha_{v}\left(N_{h}\right) \beta_{h} N_{h}}{B_{0}} & \frac{p_{1} \beta_{h} B_{2} N_{h}}{B_{0}} \\
0 & \frac{a_{v} B_{3}}{B_{0}} & \frac{B_{1} B_{3}}{B_{0}} & \frac{p_{1} a_{v} \beta_{h} N_{h}}{B_{0}} \\
0 & \frac{a_{v} \alpha_{v}\left(N_{h}\right)}{B_{0}} & \frac{\alpha_{v}\left(N_{h}\right) B_{1}}{B_{0}} & \frac{B_{1} B_{2}}{B_{0}}
\end{array}\right),
$$

where

$$
\begin{aligned}
B_{0} & =B_{1} B_{2} B_{3}-p_{1} a_{v} \alpha_{v}\left(N_{v}\right) \beta_{h} N_{h}, \\
B_{1} & =a_{v}+\mu_{v}, \\
B_{2} & =\mu_{v}+\alpha_{v}\left(N_{h}\right), \\
B_{3} & =\mu_{v}+\beta_{h} N_{h} .
\end{aligned}
$$

Note that $B_{0}>0$ since $0<p_{1}<1$. This still holds even if we set $p_{1}=1$. Also note that $\mathcal{V}^{-1}$ is proportional to the amount of time spent in each class. We find $\mathfrak{F} \mathcal{V}^{-1}$, the total rate at which new infections arise in the population within the entire duration of the infection:

$$
\mathfrak{F V}^{-1}=\left(\begin{array}{cccc}
0 & \frac{a_{v} \alpha_{v}\left(N_{h}\right) \beta_{h} N_{h}}{B_{0}} & \frac{\alpha_{v}\left(N_{h}\right) \beta_{h} B_{1} N_{h}}{B_{0}} & \frac{\beta_{h} B_{1} B_{2} N_{h}}{B_{0}} \\
\frac{q \beta_{v} S_{w}^{*}}{\mu_{h}+r_{h}} & 0 & 0 & 0 \\
0 & 0 & 0 & 0 \\
0 & 0 & 0 & 0
\end{array}\right) .
$$

We can then calculate $R_{0}$ to be the largest eigenvalue of the next generation Matrix $\mathscr{M}=$ $\mathscr{F} \mathscr{V}^{-1}$. Since the eigenvalues of $\mathscr{M}=\mathscr{F} \mathscr{V}^{-1}$ are

$$
\begin{gathered}
-\sqrt{\frac{q a_{v} \alpha_{v}\left(N_{h}\right) \beta_{v} \beta_{h} N_{h} S_{w}^{*}}{\left(\mu_{h}+r_{h}\right)\left(B_{0}\right)}}, 0,0, \sqrt{\frac{q a_{v} \alpha_{v}\left(N_{h}\right) \beta_{v} \beta_{h} N_{h} S_{w}^{*}}{\left(\mu_{h}+r_{h}\right)\left(B_{0}\right)}}, \\
\tilde{R}_{0}=\max \left(-\sqrt{\frac{q a_{v} \alpha_{v}\left(N_{h}\right) \beta_{v} \beta_{h} N_{h} S_{w}^{*}}{\mathscr{B}\left(\mu_{h}+r_{h}\right)}}, 0,0, \sqrt{\frac{q a_{v} \alpha_{v}\left(N_{h}\right) \beta_{v} \beta_{h} N_{h} S_{w}^{*}}{\mathscr{B}\left(\mu_{h}+r_{h}\right)}}\right)
\end{gathered}
$$




$$
=\sqrt{\frac{q a_{v} \alpha_{v}\left(N_{h}\right) \beta_{v} \beta_{h} N_{h} S_{w}^{*}}{\mathscr{B}\left(\mu_{h}+r_{h}\right)}}
$$

where

$$
\mathscr{B}=\mu_{v}\left(a_{v} \alpha_{v}\left(N_{h}\right)\left(1+\frac{\left(1-p_{1}\right) \beta_{h} N_{h}}{\mu_{v}}\right)+\left(\mu_{v}+\beta_{h} N_{h}\right)\left(a_{v}+\alpha_{v}\left(N_{h}\right)+\mu_{v}\right)\right) .
$$

Similar calculations can be carried out for the scaled model (4.35). In dimensionless parameters, $\tilde{R}_{0}$ takes the form

$$
\tilde{R}_{0}=\sqrt{\frac{\beta \sigma w_{s}^{*}}{\mu(1-\delta)}}=\sqrt{\frac{\beta \sigma\left(\mathscr{R}^{*}-1\right)}{\mu(1-\delta) \mathscr{R}^{*}}} .
$$

However, since $\tilde{R}_{0}>0$, if $\tilde{R}_{0} \leq 1$, then $\tilde{R}_{0}^{2} \leq 1$ and if $\tilde{R}_{0}>1, \tilde{R}_{0}^{2}>1$, we use the values $R_{0}=\tilde{R}_{0}^{2}$ which coincides with the value of the basic reproduction number obtained by seeking conditions for the existence of an endemic equilibrium or instability of the diseasefree equilibrium. That is,

$$
R_{0}=\frac{\beta \sigma\left(\mathscr{R}^{*}-1\right)}{\mu(1-\delta) \mathscr{R}^{*}}
$$

for the scaled model and

$$
R_{0}=\frac{\beta_{v} \beta_{h} N_{h} S_{w}^{*}}{\mu_{v}\left(r_{h}+\mu_{h}\right)} \cdot q \cdot \frac{a_{v} \alpha_{v}\left(N_{h}\right)}{a_{v} \alpha_{v}\left(N_{h}\right)\left(1+\frac{\left(1-p_{1}\right) \beta_{h} N_{h}}{\mu_{v}}\right)+\left(\mu_{v}+\beta_{h} N_{h}\right)\left(a_{v}+\alpha_{v}\left(N_{h}\right)+\mu_{v}\right)}
$$

for the unscaled model. Note that since

$$
0<q<1 \text { and } 0<\frac{a_{v} \alpha_{v}\left(N_{h}\right)}{a_{v} \alpha_{v}\left(N_{h}\right)\left(1+\frac{\left(1-p_{1}\right) \beta_{h} N_{h}}{\mu_{v}}\right)+\left(\mu_{v}+\beta_{h} N_{h}\right)\left(a_{v}+\alpha_{v}\left(N_{h}\right)+\mu_{v}\right)}<1
$$

it is evident that $R_{0}<\frac{\beta_{v} \beta_{h} N_{h} S_{w}^{*}}{\mu_{v}\left(r_{h}+\mu_{h}\right)}$.

\subsubsection{Existence of Equilibria}

Theorem 5.3.1. In the presence of the disease, there exists a non-disease threshold parameter $\mathscr{R}^{*}$ given by (5.2) and a disease threshold parameter $R_{0}$ given by (5.13) which exists when $\mathscr{R}^{*}>1$, such that 
1. When $\mathscr{R}^{*} \leq 1$, system (4.35) has a unique equilibrium, the trivial equilibrium $E_{0}$.

2. When $\mathscr{R}^{*}>1$, system (4.35) also has a non-trivial disease-free equilibrium $E_{d f e}$.

3. In addition to the threshold parameters $\mathscr{R}^{*}$ and $R_{0}$, there exist parameter groupings

$$
A_{1}=1-\frac{\beta \rho u_{s}^{*}}{\alpha \mu \lambda_{0}} \text { and } A_{2}=u_{s}^{*^{2}}\left(R_{0}-1\right),
$$

where $u_{s}^{*}$ is the equilibrium value of $u_{s}$ such that system (4.35) has

(a) two endemic equilibrium solutions when $R_{0}<1, A_{1}>0$, and $A_{1}^{2}>4 A_{2}$,

(b) a single endemic equilibrium solution when $R_{0}>1, A_{1}>0$, and $A_{1}^{2}>4 A_{2}$,

(c) a single endemic equilibrium solution when $R_{0}>1, A_{1}<0$ and $A_{1}^{2}>4 A_{2}$,

(d) a single endemic equilibrium solution when $R_{0}>1, A_{1}=0$, and $A_{1}^{2}>4 A_{2}$,

(e) a single endemic equilibrium solution when $R_{0}=1, A_{1}>0$, and $A_{1}^{2}>4 A_{2}$,

(f) a single endemic equilibrium solution when $R_{0}<1, A_{1}>0$ and $A_{1}^{2}=4 A_{2}$.

Proof. We compute the equilibrium solutions of system (4.35) by setting the left-hand sides of the system to zero and solving the resulting system of algebraic equations. This gives rise to the following equilibrium solutions:

$$
E_{0}=\left(I_{0}^{*}, u_{s_{0}}^{*}, v_{s_{0}}^{*}, w_{s_{0}}^{*}, u_{i_{0}}^{*}, v_{i_{0}}^{*}, w_{i_{0}}^{*}\right)=(0,0,0,0,0,0,0),
$$

a disease-free equilibrium,

$$
\begin{aligned}
E_{d f e} & =\left(I^{*}\left(\mathscr{R}^{*}\right), u^{*}\left(\mathscr{R}^{*}\right), v^{*}\left(\mathscr{R}^{*}\right), w^{*}\left(\mathscr{R}^{*}\right), u^{*}\left(\mathscr{R}^{*}\right), v^{*}\left(\mathscr{R}^{*}\right), w^{*}\left(\mathscr{R}^{*}\right)\right) \\
& =\left(0,1-\frac{1}{\mathscr{R}^{*}}, 1-\frac{1}{\mathscr{R}^{*}}, 1-\frac{1}{\mathscr{R}^{*}}, 0,0,0\right),
\end{aligned}
$$

where $\mathscr{R}^{*}$ is a disease-free threshold parameter as defined in (5.2). Additionally, there is an endemic equilibrium

$$
E_{e}=\left(I^{*}, u_{s}^{*}, v_{s}^{*}, w_{s}^{*}, u_{i}^{*}, v_{i}^{*}, w_{i}^{*}\right)=\left(I^{*}\left(w_{i}^{*}\right), u_{s}^{*}, w_{s}\left(w_{i}^{*}\right), w_{s}\left(w_{i}^{*}\right), w_{i}^{*}, w_{i}^{*}, w_{i}^{*}\right),
$$

where

$$
\begin{aligned}
I^{*}\left(w_{i}^{*}\right) & =\frac{\beta w_{i}^{*}}{\beta w_{i}^{*}+\mu}, u_{s}^{*}=\frac{\mu(1-\delta)}{\beta \sigma}, \\
v_{s}^{*}\left(w_{i}^{*}\right) & =w_{s}^{*}\left(w_{i}^{*}\right)=\frac{(1-\delta) \beta w_{i}^{*}+\beta \sigma u_{s}^{*}}{\beta \sigma}=u_{s}^{*}+\frac{\beta u_{s}^{*}}{\mu} w_{i}^{*}, \\
u_{i}^{*} & =v_{i}^{*}=w_{i}^{*},
\end{aligned}
$$


and $w_{i}^{*}$ is the positive solution of the quadratic equation

$$
w_{i}^{* 2}-A_{1} w_{i}^{*}-A_{2}=0
$$

where $A_{1}$ and $A_{2}$ are given by (5.15) and $R_{0}$ is given by (5.13). Solving the quadratic equation (5.20) for positive values of $w_{i}^{*}$ yields the following two solutions:

$$
w_{i 1,2}^{*}=\frac{A_{1} \pm \sqrt{A_{1}^{2}+4 A_{2}}}{2} .
$$

That is,

$$
w_{i 1,2}^{*}=\frac{\beta\left(\alpha \lambda_{0} \sigma-\rho(1-\delta)\right) \pm \sqrt{\left(\beta\left(\alpha \lambda_{0} \sigma-\rho(1-\delta)\right)^{2}+\left(2 \alpha \lambda_{0} \mu(1-\delta)\right)^{2}\left(R_{0}-1\right)\right.}}{2 \alpha \beta \lambda_{0} \sigma} .
$$

1. It is clear from (5.16) and (5.17) that when $\mathscr{R}^{*} \leq 1, E_{0}=(0,0,0,0,0,0,0)$ is the only equilibrium solution of system (4.35).

2. From (5.17), $E_{d f e}$ exists and is nontrivial when $\mathscr{R}^{*}>1$.

3. With the values of $w_{i}^{*}$ given by equation (5.21), we can write the various realistic endemic equilibria in closed form. However, the existence of these realistic equilibrium solutions are governed by the signs of $A_{1}, A_{2}$ and $\Delta$, where

$$
\Delta=A_{1}^{2}+4 A_{2}=\frac{\left(\beta\left(\alpha \lambda_{0} \sigma-\rho(1-\delta)\right)^{2}+\left(2 \alpha \lambda_{0} \mu(1-\delta)\right)^{2}\left(R_{0}-1\right)\right.}{\left(\alpha \beta \lambda_{0} \sigma\right)^{2}} .
$$

The following are worth noting:

- $A_{2}>0$ when $R_{0}>1, A_{2}<0$ when $R_{0}<1$, and $A_{2}=0$ when $R_{0}=1$; thus, a dependence on $R_{0}$;

- $A_{1}>0$ when $\lambda_{0}>\frac{\rho(1-\delta)}{\alpha \sigma}, A_{1}<0$ when $\lambda_{0}<\frac{\rho(1-\delta)}{\alpha \sigma}$, and $A_{1}=0$ when $\lambda_{0}=$ $\frac{\rho(1-\delta)}{\alpha \sigma}$, where $\sigma=q / p$ is the ratio of the probability that a susceptible questing mosquito feeds on an infectious human to the probability that a susceptible questing mosquito feeds on a susceptible human; thus, $A_{1}$ the depends on $\lambda_{0}$.

A detailed analysis of equation (5.21) yields the following non-negative, non-trivial realistic endemic equilibrium solutions in closed form: 
(a) When $A_{2}<0$ or $R_{0}<1$ and $A_{1}>0$ or $\lambda_{0}>\frac{\rho(1-\delta)}{\alpha \sigma}$ and $\Delta>0$, we have the following two realistic endemic equilibria:

$$
\begin{aligned}
I_{1,2}^{*} & =\frac{\beta\left(\alpha \lambda_{0} \sigma-\rho(1-\delta)\right) \pm \sqrt{\left(\alpha \beta \lambda_{0} \sigma\right)^{2} \Delta}}{2 \alpha \lambda_{0} \mu \sigma+\beta\left(\alpha \lambda_{0} \sigma-\rho(1-\delta)\right) \pm \sqrt{\left(\alpha \beta \lambda_{0} \sigma\right)^{2} \Delta}} \\
u_{s 1,2}^{*} & =\frac{\mu(1-\delta)}{\beta \sigma}, \\
v_{s 1,2}^{*} & =w_{s 1,2}^{*}=(1-\delta) \frac{2 \alpha \lambda_{0} \mu \sigma+\beta\left(\alpha \lambda_{0} \sigma-\rho(1-\delta)\right) \pm \sqrt{\left(\alpha \beta \lambda_{0} \sigma\right)^{2} \Delta}}{2 \alpha \beta \lambda_{0} \sigma^{2}} \\
u_{i 1,2}^{*} & =v_{i 1,2}^{*}=w_{i 1,2}^{*}=\frac{\beta\left(\alpha \lambda_{0} \sigma-\rho(1-\delta)\right) \pm \sqrt{\left(\alpha \beta \lambda_{0} \sigma\right)^{2} \Delta}}{2 \alpha \beta \lambda_{0} \sigma} .
\end{aligned}
$$

(b) When $A_{2}>0$ or $R_{0}>1$ and $A_{1}>0$ or $\lambda_{0}>\frac{\rho(1-\delta)}{\alpha \sigma}$ and $\Delta>0$, the unique endemic steady state is

$$
\begin{aligned}
& I^{*}=\frac{\beta\left(\alpha \lambda_{0} \sigma-\rho(1-\delta)\right)+\sqrt{\left(\alpha \beta \lambda_{0} \sigma\right)^{2} \Delta}}{2 \alpha \lambda_{0} \mu \sigma+\beta\left(\alpha \lambda_{0} \sigma-\rho(1-\delta)\right)+\sqrt{\left(\alpha \beta \lambda_{0} \sigma\right)^{2} \Delta}} \\
& u_{s}^{*}=\frac{\mu(1-\delta)}{\beta \sigma} \\
& v_{s}^{*}=w_{s}^{*}=(1-\delta) \frac{2 \alpha \lambda_{0} \mu \sigma+\beta\left(\alpha \lambda_{0} \sigma-\rho(1-\delta)\right)+\sqrt{\left(\alpha \beta \lambda_{0} \sigma\right)^{2} \Delta}}{2 \alpha \beta \lambda_{0} \sigma^{2}} \\
& u_{i}^{*}=v_{i}^{*}=w_{i}^{*}=\frac{\beta\left(\alpha \lambda_{0} \sigma-\rho(1-\delta)\right)+\sqrt{\left(\alpha \beta \lambda_{0} \sigma\right)^{2} \Delta}}{2 \alpha \beta \lambda_{0} \sigma}
\end{aligned}
$$

(c) When $A_{2}>0$ or $R_{0}>1$ and $A_{1}<0$ or $\lambda_{0}<\frac{\rho(1-\delta)}{\alpha \sigma}$ and $\Delta>0$, the only realistic equilibrium has the same representation as in equation (5.24) above. However, since $A_{1}$ is positive in (5.24) while here, $A_{1}<0$, that is, $\alpha \sigma \lambda_{0}-\rho(1-\delta)<0$, the two equilibrium values will differ numerically.

(d) When $A_{2}>0$ or $R_{0}>1$ and $A_{1}=0$ or $\lambda_{0}=\frac{\rho(1-\delta)}{\alpha \sigma}$ and $\Delta>0$, we have $w_{i}^{*}=\frac{\mu(1-\delta)\left(\sqrt{R_{0}-1}\right)}{\beta \sigma}$ and the corresponding equilibrium solution is 


$$
\begin{aligned}
& I^{*}=\frac{(1-\delta) \sqrt{R_{0}-1}}{\sigma+(1-\delta) \sqrt{R_{0}-1}}, \\
& u_{s}^{*}=\frac{\mu(1-\delta)}{\beta \sigma}, \\
& v_{s}^{*}=w_{s}^{*}=\frac{\mu(1-\delta)}{\beta \sigma}\left(1+\frac{(1-\delta)\left(\sqrt{R_{0}-1}\right)}{\sigma}\right), \\
& u_{i}^{*}=v_{i}^{*}=w_{i}^{*}=\frac{\mu(1-\delta)\left(\sqrt{R_{0}-1}\right)}{\beta \sigma} .
\end{aligned}
$$

(e) When $A_{2}=0$ or $R_{0}=1$ and $A_{1}>0$ or $\lambda_{0}>\frac{\rho(1-\delta)}{\alpha \sigma}$ and $\Delta>0$, we have $w_{i}^{*}=A_{1}=\frac{\alpha \lambda_{0} \sigma-\rho(1-\delta)}{\alpha \lambda_{0} \sigma}$ and the corresponding equilibrium solution is

$$
\begin{aligned}
& I^{*}=\frac{\beta\left(\alpha \lambda_{0} \sigma-\rho(1-\delta)\right)}{\beta\left(\alpha \lambda_{0} \sigma-\rho(1-\delta)\right)+\alpha \lambda_{0} \mu \sigma}, \\
& u_{s}^{*}=\frac{\mu(1-\delta)}{\beta \sigma}, \\
& v_{s}^{*}=w_{s}^{*}=(1-\delta) \frac{\alpha \lambda_{0} \mu \sigma+\beta\left(\alpha \lambda_{0} \sigma-\rho(1-\delta)\right)}{\alpha \beta \lambda_{0} \sigma^{2}}, \\
& u_{i}^{*}=v_{i}^{*}=w_{i}^{*}=\frac{\alpha \lambda_{0} \sigma-\rho(1-\delta)}{\alpha \lambda_{0} \sigma} .
\end{aligned}
$$

(f) When $A_{2}<0$ or $R_{0}<1, A_{1}>0$ or $\lambda_{0}>\frac{\rho(1-\delta)}{\alpha \sigma}$ and $\Delta=0$, we have $w_{i}^{*}=$ $\frac{A_{1}}{2}=\frac{\alpha \lambda_{0} \sigma-\rho(1-\delta)}{2 \alpha \lambda_{0} \sigma}$, and the corresponding equilibrium solution is

$$
\begin{aligned}
I^{*} & =\frac{\beta\left(\alpha \lambda_{0} \sigma-\rho(1-\delta)\right)}{\beta\left(\alpha \lambda_{0} \sigma-\rho(1-\delta)\right)+2 \alpha \lambda_{0} \mu \sigma}, \\
u_{s}^{*} & =\frac{\mu(1-\delta)}{\beta \sigma},
\end{aligned}
$$




$$
\begin{aligned}
& v_{s}^{*}=w_{s}^{*}=(1-\delta) \frac{2 \alpha \lambda_{0} \mu \sigma+\beta\left(\alpha \lambda_{0} \sigma-\rho(1-\delta)\right)}{2 \alpha \beta \lambda_{0} \sigma^{2}}, \\
& u_{i}^{*}=v_{i}^{*}=w_{i}^{*}=\frac{\alpha \lambda_{0} \sigma-\rho(1-\delta)}{2 \alpha \lambda_{0} \sigma} .
\end{aligned}
$$

This concludes the proof of the Theorem.

Various conditions for the existence of realistic equilibrium solutions and the number of equilibriums as outlined in Theorem 5.3.1 are summarized in Table 5.1 and illustrated in Fig. 5.6. As indicated both in Table 5.1 and illustrated in Fig. 5.6, it is possible to have realistic solutions even when $A_{1}<0 \Leftrightarrow \lambda_{0}<\frac{\rho(1-\delta)}{\alpha \sigma}$. Additionally, there is a bifurcation point in the model, when the number of realistic endemic equilibrium solutions switch from zero to one to two equilibria. The fact that, for $A_{2}<0$ when $R_{0}<1$, there are two non-trivial endemic equilibria is a prerequisite for the occurrence of backward bifurcation. 
Table 5.1. Summary of conditions for the existence of realistic equilibrium solutions and the number of realistic equilibrium solutions.

\begin{tabular}{|c|c|c|c|c|}
\hline$A_{2}$ & $A_{1}$ & $\Delta$ & Number of realistic equilibria & Region \\
\hline \multirow{3}{*}{$\begin{array}{c}A_{2}>0 \\
\left(R_{0}>1\right)\end{array}$} & $A_{1}<0$ & $\Delta>0$ & $\begin{array}{l}\text { One realistic equilibrium with } w_{i 1}^{*}=\frac{A_{1}+\sqrt{\Delta}}{2} \\
\left(\sqrt{\Delta}>\left|A_{1}\right|\right)\end{array}$ & (I) \\
\hline & $A_{1}=0$ & $\Delta>0$ & One realistic equilibrium with $w_{i 1}^{*}=\sqrt{A_{2}}$ & (II) \\
\hline & $A_{1}>0$ & $\Delta>0$ & $\begin{array}{l}\text { One realistic equilibrium with } w_{i 1}^{*}=\frac{A_{1}+\sqrt{\Delta}}{2} \\
\left(\sqrt{\Delta}>A_{1}\right)\end{array}$ & $(\mathrm{III})$ \\
\hline $\begin{array}{c}A_{2}=0 \\
\left(R_{0}=1\right)\end{array}$ & $A_{1}>0$ & $\Delta>0$ & One realistic equilibrium with $w_{i 1}^{*}=A_{1}$ & $(\mathrm{IV})$ \\
\hline \multirow{7}{*}{$\begin{array}{c}A_{2}<0 \\
\left(R_{0}<1\right)\end{array}$} & $A_{1}>0$ & $\Delta>0$ & $\begin{array}{l}\text { Two realistic equilibria with } w_{i 1,2}^{*}=\frac{A_{1} \pm \sqrt{\Delta}}{2} \\
\left(\sqrt{\Delta}<A_{1}\right)\end{array}$ & $(\mathrm{V})$ \\
\hline & $A_{1}>0$ & $\Delta=0$ & One realistic equilibrium with $w_{i}^{*}=A_{1} / 2$ & $(\mathrm{VI})$ \\
\hline & $A_{1}>0$ & $\Delta<0$ & No realistic equilibrium & $(\mathrm{VII})$ \\
\hline & $A_{1}=0$ & $\Delta<0$ & No realistic equilibrium & (VIII) \\
\hline & $A_{1}<0$ & $\Delta=0$ & No realistic equilibrium & $(\mathrm{IX})$ \\
\hline & $A_{1}<0$ & $\Delta<0$ & No realistic equilibrium & $(\mathrm{X})$ \\
\hline & $A_{1}<0$ & $\Delta>0$ & No realistic equilibrium & $(\mathrm{XI})$ \\
\hline $\begin{array}{c}A_{2}=0 \\
\left(R_{0}=1\right)\end{array}$ & $A_{1}>0$ & $\Delta>0$ & One realistic equilibrium with $w_{i 1}^{*}=A_{1}$ & $(\mathrm{XII})$ \\
\hline
\end{tabular}




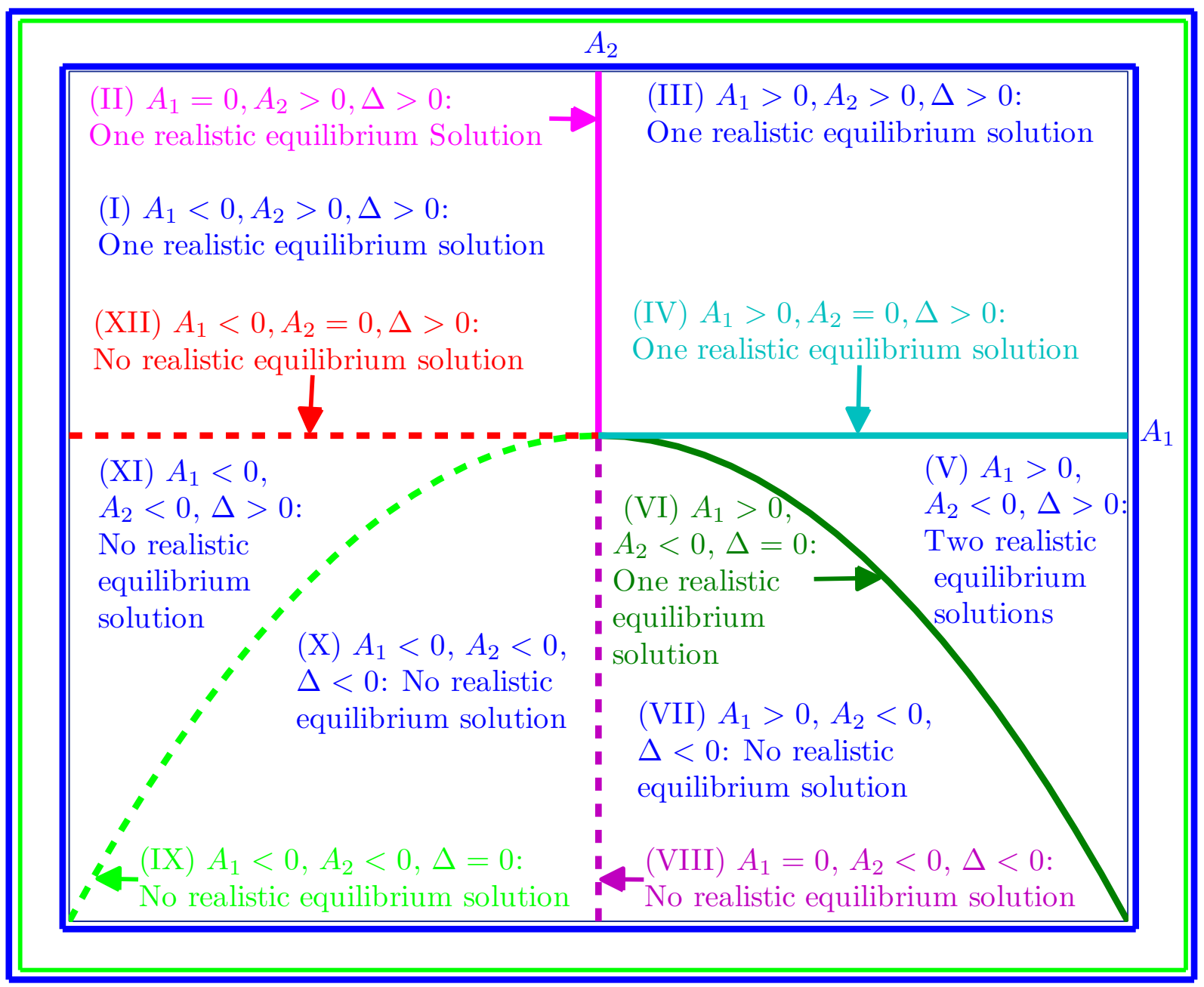

Figure 5.6. A diagram showing various regions in the $A_{1}-A_{2}$ plane within which zero, one or two endemic equilibrium solutions exist. $\Delta=A_{1}^{2}+4 A_{2}$. Endemic equilibrium solutions exist on the solid magenta line, light green line and dark green curve within the graph, while there are no endemic equilibrium solutions on the dashed lines. Endemic equilibrium solutions also exist in the first quadrant, the second quadrant and the upper part of the fourth quadrant (above or to the right of the curve $\Delta=0$ ). 


\subsubsection{Stability of Equilibria}

To investigate the linear stability of the equilibrium solutions to system (4.35), we begin by linearizing the system about the equilibrium point $\left(I^{*}, u_{s}^{*}, v_{s}^{*}, w_{s}^{*}, u_{i}^{*}, v_{i}^{*}, w_{i}^{*}\right)$. To this effect, we set $\vec{X}=\vec{X}^{*}+\vec{x}$, where $\vec{X}=\left(I, u_{s}, v_{s}, w_{s}, u_{i}, v_{i}, w_{i}\right)^{T}, \vec{X}^{*}=\left(I^{*}, u_{s}^{*}, v_{s}^{*}, w_{s}^{*}, u_{i}^{*}, v_{i}^{*}, w_{i}^{*}\right)^{T}, \vec{x}=$ $\left(I_{1}, u, v, w, x, y, z\right)^{T},\left|I_{1}\right| \ll 1,|u| \ll 1,|v| \ll 1,|w| \ll 1,|x| \ll 1,|y| \ll 1$, and $|z| \ll 1$ in system (4.35), and expand the resulting system in a Taylor series about $\left(I^{*}, u_{s}^{*}, v_{s}^{*}, w_{s}^{*}, u_{i}^{*}, v_{i}^{*}, w_{i}^{*}\right)$. This leads to the following linearized system:

$$
\left(\dot{I}_{1}, \dot{u}, \dot{v}, \dot{w}, \dot{x}, \dot{y}, \dot{z}\right)^{T}=J\left(I_{1}, u, v, w, x, y, z\right)^{T}
$$

where

$$
J=\left(\begin{array}{ccccccc}
-\left(\beta w_{i}^{*}+\mu\right) & 0 & 0 & 0 & 0 & 0 & \beta\left(1-I^{*}\right) \\
-w_{s}^{*} & -1 & 0 & 1-I^{*} & 0 & 0 & 0 \\
0 & \alpha \lambda_{0}\left(1-2 u_{s}^{*}\right)+\alpha & -\rho & 0 & \alpha \lambda_{0}\left(1-2 u_{i}^{*}\right) & 0 & 0 \\
0 & 0 & \gamma & -\gamma & 0 & 0 & 0 \\
\sigma w_{s}^{*} & 0 & 0 & \sigma I^{*} & -1 & 0 & \delta \\
0 & 0 & 0 & 0 & \rho & -\rho & 0 \\
0 & 0 & 0 & 0 & 0 & \epsilon & -\epsilon
\end{array}\right)
$$

is the Jacobian matrix of the linearized system evaluated at $\left(I^{*}, u_{s}^{*}, v_{s}^{*}, w_{s}^{*}, u_{i}^{*}, v_{i}^{*}, w_{i}^{*}\right)$.

\subsubsection{Stability of the Trivial Equilibrium}

Theorem 5.3.2. The trivial equilibrium solution $E_{0}$ is linearly and asymptotically stable when $\mathscr{R}^{*} \leq 1$ and linearly unstable otherwise.

Proof. The Jacobian matrix evaluated at the trivial equilibrium, $E_{0}=(0,0,0,0,0,0,0)$ is 


$$
J(0,0,0,0,0,0,0)=\left(\begin{array}{ccccccc}
-\mu & 0 & 0 & 0 & 0 & 0 & \beta \\
0 & -1 & 0 & 1 & 0 & 0 & 0 \\
0 & \alpha\left(\lambda_{0}+1\right) & -\rho & 0 & \alpha \lambda_{0} & 0 & 0 \\
0 & 0 & \gamma & -\gamma & 0 & 0 & 0 \\
0 & 0 & 0 & 0 & -1 & 0 & \delta \\
0 & 0 & 0 & 0 & \rho & -\rho & 0 \\
0 & 0 & 0 & 0 & 0 & \epsilon & -\epsilon
\end{array}\right) .
$$

We seek solutions of the form $\vec{x}=\vec{x}_{0} e^{\lambda t}$, where $\lambda$ is an eigenvalue of $J$ and $\vec{x}_{0}$ is a vector of constants. This gives the following characteristic equation:

$$
(\lambda+\mu)\left[\lambda^{3}+Q \lambda^{2}+R \lambda+P\left(1-\mathscr{R}^{*}\right)\right]\left[\lambda^{3}+Q_{1} \lambda^{2}+R_{1} \lambda+P_{1}\right]=0,
$$

where $P, Q$ and $R$ are as defined in (5.8) and

$$
Q_{1}=\epsilon+\rho+1>0 ; \quad R_{1}=\epsilon+\rho+\epsilon \rho>0 ; \quad P_{1}=\epsilon \rho(1-\delta)>0 \text { since } 0 \leq \delta<1
$$

Using Descartes' rule of signs and the Routh-Hurwitz conditions, we can easily show that the trivial equilibrium is linearly stable to small perturbations whenever $\mathscr{R}^{*} \leq 1$ and linearly unstable whenever $\mathscr{R}^{*}>1$. Since the coefficients of the first cubic polynomial on the lefthand side of $(5.28)$ are all positive and $Q R-P\left(1-\mathscr{R}^{*}\right)>0$ when $\mathscr{R}^{*} \leq 1$, and since all the coefficients of the second cubic polynomial on the left-hand side of equation (5.28) are all positive and $Q_{1} R_{1}-P_{1}=(\epsilon+\rho)(1+\epsilon+\rho+\epsilon \rho)+\delta \epsilon \rho>0$, the Routh-Hurwitz conditions assure us that all solutions of equation (5.28) have negative real parts when $\mathscr{R}^{*} \leq 1$.

\subsubsection{Stability of the Disease-free Equilibrium}

Theorem 5.3.3. When $\mathscr{R}^{*}>1$, the non-trivial disease-free equilibrium solution $E_{d f e}$ is

1. linearly stable to small perturbations when $R_{0} \leq 1$ and $Q R-P\left(\mathscr{R}^{*}-1\right)>0$,

2. linearly unstable to small perturbations, accompanied by the emergence of periodic oscillations, when $R_{0} \leq 1$ and $Q R-P\left(\mathscr{R}^{*}-1\right)<0$,

3. linearly unstable to small perturbations when $R_{0}>1$. 
Proof. At the disease-free equilibrium, $E_{d f e}$ given by (5.17),

$$
J\left(E_{d f e}\right)=\left(\begin{array}{ccccccc}
-\mu & 0 & 0 & 0 & 0 & 0 & \beta \\
-u_{s}^{*} & -1 & 0 & 1 & 0 & 0 & 0 \\
0 & \alpha \lambda_{0}\left(1-2 u_{s}^{*}\right)+\alpha & -\rho & 0 & \alpha \lambda_{0} & 0 & 0 \\
0 & 0 & \gamma & -\gamma & 0 & 0 & 0 \\
\sigma u_{s}^{*} & 0 & 0 & 0 & -1 & 0 & \delta \\
0 & 0 & 0 & 0 & \rho & -\rho & 0 \\
0 & 0 & 0 & 0 & 0 & \epsilon & -\epsilon
\end{array}\right) .
$$

We search for solutions of the linear system (5.3.3) with $J$ evaluated at $E_{d f e}$ in the form $\vec{x}=\vec{x}_{0} e^{\lambda t}$, where $\lambda$ is an eigenvalue of $J$ and $\vec{x}_{0}$ is a vector of constants. This leads to the system $\left(J-\lambda I_{7}\right) \vec{x}_{0}$ where $I_{7}$ is the $7 \times 7$ identity matrix. Non-zero solutions exist when

$\left|\begin{array}{ccccccc}-\mu-\lambda & 0 & 0 & 0 & 0 & 0 & \beta \\ -u_{s}^{*} & -1-\lambda & 0 & 1 & 0 & 0 & 0 \\ 0 & \alpha \lambda_{0}\left(1-2 u_{s}^{*}\right)+\alpha & -\rho-\lambda & 0 & \alpha \lambda_{0} & 0 & 0 \\ 0 & 0 & \gamma & -\gamma-\lambda & 0 & 0 & 0 \\ \sigma u_{s}^{*} & 0 & 0 & 0 & -1-\lambda & 0 & \delta \\ 0 & 0 & 0 & 0 & \rho & -\rho-\lambda & 0 \\ 0 & 0 & 0 & 0 & 0 & \epsilon & -\epsilon-\lambda\end{array}\right|=0$,

which can be rewritten as

$\left[(\lambda+1)(\lambda+\gamma)(\lambda+\rho)-\gamma\left(\alpha \lambda_{0}\left(1-2 u_{s}^{*}\right)+\alpha\right)\right]\left[(\lambda+\mu)(-(\lambda+1)(\lambda+\epsilon)(\lambda+\rho)+\delta \epsilon \rho)+\beta \epsilon \rho \sigma u_{s}^{*}\right]=0$, or the product of a cubic equation and a quartic polynomial equations as follows:

$\left(\lambda^{3}+Q \lambda^{2}+R \lambda+P\left(\mathscr{R}^{*}-1\right)\right)\left(\lambda^{4}+\left(Q_{1}+\mu\right) \lambda^{3}+\left(\mu Q_{1}+R_{1}\right) \lambda^{2}+\left(\mu R_{1}+P_{1}\right) \lambda+P_{1} \mu\left(1-R_{0}\right)\right)=0$.

That is,

$$
\lambda^{3}+Q \lambda^{2}+R \lambda+P\left(\mathscr{R}^{*}-1\right)=0
$$

or

$$
\lambda^{4}+\left(Q_{1}+\mu\right) \lambda^{3}+\left(\mu Q_{1}+R_{1}\right) \lambda^{2}+\left(\mu R_{1}+P_{1}\right) \lambda+P_{1} \mu\left(1-R_{0}\right)=0,
$$


where $P, Q$, and $R$ are as defined in (5.8), $P_{1}, Q_{1}$, and $R_{1}$ are as defined in (5.29), and $E_{d f e}$ is as given in (5.17). Notice that equation (5.30) is exactly the characteristic equation (5.9) of the model in the absence of the disease. The condition $\mathscr{R}^{*}>1$ is fulfilled trivially since the disease-free equilibrium only exists when $\mathscr{R}^{*}>1$. As demonstrated in Theorem 5.2.2, when $\mathscr{R}^{*}>1$, all solutions of this equation are either negative or have negative real parts if $Q R-P\left(\mathscr{R}^{*}-1\right)>0$, a Hopf bifurcation occurs when $Q R-P\left(\mathscr{R}^{*}-1\right)=0$, and solutions with positive real parts exist if $Q R-P\left(\mathscr{R}^{*}-1\right)<0$. Hence, to complete the proof of the first two parts of the Theorem, all we need here is equation (5.31).

Now, when $R_{0} \leq 1$, all coefficients of equation (5.31) are positive and thus by Descartes' rule of signs, there are no positive real solutions $\lambda$. Furthermore, from Lemma 5.3.4 below, the Routh-Hurwitz criterion assure us that when $R_{0} \leq 1$ all the roots of equation (5.31) have negative real parts. Therefore, the non-trivial disease-free equilibrium $E_{d f e}$ is linearly stable to small perturbations when $Q R-P\left(\mathscr{R}^{*}-1\right)>0$ and becomes unstable with the emergence of periodic oscillations when $Q R-P\left(\mathscr{R}^{*}-1\right)>0$. The instability is via a Hopf bifurcation as shown in Theorem 5.2.2. This proves the first two parts of the Theorem.

When $R_{0}>1, \mu P_{1}\left(1-R_{0}\right)<0$ and, hence, there is one sign change in the sequence of coefficients of equation (5.31). Thus, there exist at least one positive real value of $\lambda$ and so the disease-free equilibrium $E_{d f e}$ is linearly unstable to small perturbations. This proves the third part of the Theorem and, hence, completes the proof.

Lemma 5.3.4. All solutions of equation (5.31) have negative real parts when $R_{0}<1$.

Proof. Notice that $Q_{1}+\mu>0, \mu R_{1}+P_{1}>0$, and $\mu P_{1}\left(1-R_{0}\right)>0$ when $R_{0}<1$. All we need to show is that

$$
\left(Q_{1}+\mu\right)\left(\mu Q_{1}+R_{1}\right)\left(\mu R_{1}+P_{1}\right)>\left(\mu R_{1}+P_{1}\right)^{2}+\left(Q_{1}+\mu\right)^{2} \mu P_{1}\left(1-R_{0}\right) .
$$

Now, subtracting the expression on the left-hand side of the inequality from the expression on the right-hand side and simplifying yields

$$
\begin{aligned}
& \left(Q_{1}+\mu\right)\left(\mu Q_{1}+R_{1}\right)\left(\mu R_{1}+P_{1}\right)-\left[\left(\mu R_{1}+P_{1}\right)^{2}+\left(Q_{1}+\mu\right)^{2} \mu P_{1}\left(1-R_{0}\right)\right] \\
= & \left(\mu R_{1}+P_{1}\right)\left(\left(Q_{1}+\mu\right) \mu Q_{1}+Q_{1} R_{1}-P_{1}\right)-\left(Q_{1}+\mu\right)^{2} \mu P_{1}\left(1-R_{0}\right)
\end{aligned}
$$




$$
\begin{aligned}
= & \left(\mu R_{1}+P_{1}\right)\left(Q_{1} R_{1}-P_{1}\right)+\mu^{2} R_{1}\left(Q_{1}+\mu\right) Q_{1}+P_{1} \mu Q_{1}\left(Q_{1}+\mu\right) \\
& +\left(Q_{1}+\mu\right)^{2} \mu P_{1} R_{0}-\left(Q_{1}+\mu\right)^{2} \mu P_{1} \\
= & \left(\mu R_{1}+P_{1}\right)\left(Q_{1} R_{1}-P_{1}\right)+P_{1} \mu Q_{1}\left(Q_{1}+\mu\right)+\left(Q_{1}+\mu\right)^{2} \mu P_{1} R_{0} \\
& +\left(Q_{1}+\mu\right) \mu\left(\mu R_{1} Q_{1}-\left(Q_{1}+\mu\right) P_{1}\right) \\
= & \left(\mu R_{1}+P_{1}\right)\left(Q_{1} R_{1}-P_{1}\right)+P_{1} \mu Q_{1}\left(Q_{1}+\mu\right)+\left(Q_{1}+\mu\right)^{2} \mu P_{1} R_{0} \\
& +\left(Q_{1}+\mu\right) \mu\left(\mu\left(R_{1} Q_{1}-P_{1}\right)-Q_{1} P_{1}\right) \\
= & \left(\mu R_{1}+P_{1}\right)\left(Q_{1} R_{1}-P_{1}\right)+\left(Q_{1}+\mu\right)^{2} \mu P_{1} R_{0}+\left(Q_{1}+\mu\right) \mu^{2}\left(Q_{1} R_{1}-P_{1}\right)>0,
\end{aligned}
$$

since

$$
Q_{1} R_{1}-P_{1}=(1+\epsilon+\rho)(\epsilon \rho+\epsilon+\rho)-\epsilon \rho(1-\delta)=(\epsilon+\rho)(\epsilon \rho+\epsilon+\rho)+\epsilon+\rho+\epsilon \rho \delta \geq 0
$$

Hence, by the Routh-Hurwitz criterion, all solutions of equation (5.31) have negative real parts when $R_{0}<1$.

Remark 5.3.5. From Lemma 5.3.4, it is clear that when $R_{0}<1$, $E_{d f e}$ is linearly and asymptotically stable to small perturbations. When $\frac{\mu(1-\delta)}{\beta \sigma}=1-\frac{1}{\mathscr{R}^{*}}$ and $A_{2}=0, R_{0}=1$. There are no sign changes. Using the Routh-Hurwitz conditions, we can easily verify that, except for one eigenvalue which is zero, all other eigenvalues have negative real parts.

\subsubsection{Stability of Endemic Equilibria}

We illustrated in Section 5.3.2 that in the presence of malaria, system (4.35) can have zero, one or two equilibrium solutions when certain conditions are fulfilled. We explore the stability of these endemic equilibria in this section. At $\left(I^{*}, u_{s}^{*}, v_{s}^{*}, w_{s}^{*}, u_{i}^{*}, v_{i}^{*}, w_{i}^{*}\right)$, where

$$
\begin{aligned}
I^{*}\left(w_{i}^{*}\right) & =\frac{\beta w_{i}^{*}}{\beta w_{i}^{*}+\mu}<1, \\
u_{s}^{*} & =\frac{\mu(1-\delta)}{\beta \sigma}, \\
v_{s}^{*}\left(w_{i}^{*}\right) & =w_{s}^{*}\left(w_{i}^{*}\right)=\frac{(1-\delta) \beta w_{i}^{*}+\beta \sigma u_{s}^{*}}{\beta \sigma}=u_{s}^{*} \frac{1}{\mu}\left(\beta w_{i}^{*}+\mu\right), \\
u_{i}^{*} & =v_{i}^{*}=w_{i}^{*},
\end{aligned}
$$

the eigenvalues $\lambda$ of $J$ are given by the characteristic equation $\left|J-\lambda I_{7}\right|=0$, where $I_{7}$ is the $7 \times 7$ identity matrix and the determinant $\left|J-\lambda I_{7}\right|$ is 


\begin{tabular}{|ccccccc}
$-\left(\beta w_{i}^{*}+\mu\right)-\lambda$ & 0 & 0 & 0 & 0 & 0 & $\beta\left(1-I^{*}\right)$ \\
$-w_{s}^{*}$ & $-1-\lambda$ & 0 & $1-I^{*}$ & 0 & 0 & 0 \\
0 & $\alpha \lambda_{0}\left(1-2 u_{s}^{*}\right)+\alpha$ & $-\rho-\lambda$ & 0 & $\alpha \lambda_{0}\left(1-2 u_{i}^{*}\right)$ & 0 & 0 \\
0 & 0 & $\gamma$ & $-\gamma-\lambda$ & 0 & 0 & 0 \\
$\sigma w_{s}^{*}$ & 0 & 0 & $\sigma I^{*}$ & $-1-\lambda$ & 0 & $\delta$ \\
0 & 0 & 0 & 0 & $\rho$ & $-\rho-\lambda$ & 0 \\
0 & 0 & 0 & 0 & 0 & $\epsilon$ & $-\epsilon-\lambda$
\end{tabular} \mid.

By expanding $\left|J-\lambda I_{7}\right|=0$, we obtain the following polynomial equation:

$$
\begin{aligned}
{\left[\lambda+\left(\beta w_{i}^{*}+\mu\right)\right][} & -(\lambda+1)(\lambda+\epsilon)(\lambda+\rho)+\delta \epsilon \rho]\left[(\lambda+1)(\lambda+\gamma)(\lambda+\rho)-\gamma\left(\alpha \lambda_{0}\left(1-2 u_{s}^{*}\right)+\alpha\right)\left(1-I^{*}\right)\right] \\
& +\alpha \lambda_{0}\left(1-2 w_{i}^{*}\right) \gamma \sigma I^{*}\left(\lambda+\left(\beta w_{i}^{*}+\mu\right)\right)(\lambda+1)(\lambda+\epsilon)(\lambda+\rho) \\
+ & \beta \epsilon \rho \sigma\left(1-I^{*}\right) w_{s}^{*}\left[(\lambda+1)(\lambda+\gamma)(\lambda+\rho)-\gamma\left(\alpha \lambda_{0}\left(1-2 u_{s}^{*}\right)+\alpha\right)\right]=0, \quad(5.32)
\end{aligned}
$$

which can be be rewritten in the following expanded and simplified form:

$$
A_{7} \lambda^{7}+A_{6} \lambda^{6}+A_{5} \lambda^{5}+A_{4} \lambda^{4}+A_{3} \lambda^{3}+A_{3} \lambda^{3}+A_{2} \lambda^{2}+A_{1} \lambda+A_{0}=0
$$

where

$$
\begin{aligned}
A_{7}= & 1 \\
A_{6}= & Q_{1}+Q+\beta w_{i}^{*}+\mu \\
A_{5}= & \left(Q_{1}+Q\right)\left(\beta w_{i}^{*}+\mu\right)+Q Q_{1}+R+R_{1}, \\
A_{4}= & \left(Q_{1} Q+R+R_{1}\right)\left(\beta w_{i}^{*}+\mu\right)+P_{1}+Q_{1} R+R_{1} Q+\phi+\alpha \lambda_{0} \gamma \sigma\left(2 w_{i}^{*}-1\right) I^{*}, \\
A_{3}= & \left(Q_{1} R+R_{1} Q+\phi\right)\left(\beta w_{i}^{*}+\mu\right)+\alpha \gamma \lambda_{0} \sigma\left(2 w_{i}^{*}-1\right) I^{*}\left(\left(\beta w_{i}^{*}+\mu\right)+Q_{1}\right) \\
& +P_{1}\left(\beta w_{i}^{*}+Q\right)+\phi Q_{1}+R_{1} R \\
A_{2}= & \left(\phi Q_{1}+R_{1} R\right)\left(\beta w_{i}^{*}+\mu\right)+\alpha \gamma \lambda_{0} \sigma\left(2 w_{i}^{*}-1\right) I^{*}\left(Q_{1}\left(\beta w_{i}^{*}+\mu\right)+R_{1}\right) \\
& +P_{1}\left(\beta Q w_{i}^{*}+R\right)+\phi R_{1}, \\
A_{1}= & \phi R_{1}\left(\beta w_{i}^{*}+\mu\right)+\alpha \gamma \lambda_{0} \sigma\left(2 w_{i}^{*}-1\right) I^{*}\left(P_{1}\left(\beta w_{i}^{*}+\mu\right)+\epsilon \rho\right)+\beta P_{1} R w_{i}^{*}+\phi P_{1}, \\
A_{0}= & \phi P_{1}\left(\beta w_{i}^{*}+\mu\right)+\beta \epsilon \rho \sigma\left(\alpha \gamma \lambda_{0}\left(2 w_{i}^{*}-1\right) w_{i}^{*}+P\left(u_{s}^{*}\right)^{2} \mathscr{R}^{*}\left(R_{0}-2\right)\right), \\
\phi= & P+\gamma \alpha I^{*}-\gamma \alpha \lambda_{0}\left(1-2 u_{s}^{*}\right)\left(1-I^{*}\right)=P+\alpha \gamma\left[\left(I^{*}-\lambda_{0}\left(1-I^{*}\right)\right)+2 \lambda_{0} u_{s}^{*}\left(1-I^{*}\right)\right] .
\end{aligned}
$$


Note that

$$
\begin{aligned}
1-I^{*}\left(w_{i}^{*}\right) & =\frac{\mu}{\beta w_{i}^{*}+\mu}, \\
\left(\beta w_{i}^{*}+\mu\right) I^{*} & =\beta w_{i}^{*}, \\
\left(1-I^{*}\right) w_{s}^{*} & =u_{s}^{*}, \\
\beta \epsilon \rho \sigma u_{s}^{*} & =\mu(\epsilon \rho(1-\delta))=\mu P_{1}, \\
\beta \epsilon \rho \sigma Q u_{s}^{*} & =\mu P_{1} Q, \\
\beta \epsilon \rho \sigma R u_{s}^{*} & =\mu P_{1} R .
\end{aligned}
$$

Remark 5.3.6. All coefficients of the characteristic polynomial are positive if $\phi \geq 0, w_{i}^{*} \geq$ $1 / 2$ and $R_{0} \geq 2$. Notice that $\phi>0$ if $I^{*}-\lambda_{0}\left(1-I^{*}\right) \geq 0$ or if $\lambda_{0} \leq \frac{I^{*}}{1-I^{*}}$.

Whenever the conditions of Remark 5.3.6 hold, all the coefficients of the characteristic equation (5.33) are positive and, hence, Descartes' rule of signs assures us that there are no positive real values for $\lambda$ that are zeros of (5.33). This indicates that any instabilities that can arise as a result of perturbations in parameters are oscillatory instabilities. At this stage we can apply the Routh-Hurwitz criterion, as before, to obtain information on the signs of the real parts of the roots of the characteristic polynomial (5.33). Now, if we consider the endemic equilinrium solution presented in equation (5.25), which arises when $A_{2}>0$ or $R_{0}>1$ and $A_{1}=0$ or $\lambda_{0}=\frac{\rho(1-\delta)}{\alpha \sigma}$ and $\Delta>0$, we have

$$
I^{*}=\frac{(1-\delta) \sqrt{R_{0}-1}}{\sigma+(1-\delta) \sqrt{R_{0}-1}}, u_{s}^{*}=\frac{\mu(1-\delta)}{\beta \sigma} \text { and } w_{i}^{*}=\frac{\mu(1-\delta)\left(\sqrt{R_{0}-1}\right)}{\beta \sigma} .
$$

At this equilibrium,

$$
\begin{aligned}
& w_{i}^{*} \geq 1 / 2 \Leftrightarrow \frac{\mu(1-\delta)\left(\sqrt{R_{0}-1}\right)}{\beta \sigma} \geq \frac{1}{2} \Leftrightarrow R_{0} \geq 1+\left(\frac{\beta \sigma}{2 \mu(1-\delta)}\right)^{2}=1+\left(\frac{1}{2 u_{s}^{*}}\right)^{2} \text { and } \\
& \text { if } \lambda_{0} \leq \frac{I^{*}}{1-I^{*}}=\frac{(1-\delta) \sqrt{R_{0}-1}}{\sigma}, R_{0} \geq 1+\left(\frac{\lambda_{0} \sigma}{1-\delta}\right)^{2}=1+\left(\frac{\rho}{\alpha}\right)^{2} \text { in which case } \phi>0 .
\end{aligned}
$$

It is thus evident that there is richer dynamics in the region in parameter space where the conditions of Remark 5.3.6 fail for the equilibrium in (5.25) or even in the region where $0 \leq R_{0} \leq 2$. This can equivalently be shown for the other endemic equilibria. Thus, we are considering the fact that from a mathematical and physical stand point, all we wish to 
establish is whether there exists parameter regimes wherein the polynomial (5.33) has zeros with positive real parts. Such zeros will indicate the existence of growing oscillating solutions in the linear regime, which, should become bounded by the nonlinearity in the model to give (bounded) limit cycle solutions in phase space. Given the size of the system and the complex nature of the coefficients of (5.33), for the purposes of illustrating the potential of the model, we use MATLAB and the symbolic manipulation package MATHEMATICA to numerically evaluate and search the parameter space in view of exploring the nature of the solutions of the characteristic polynomial, and also having an insight into the possible solutions that exist for the full model. As illustrated in Section 5.3.4, there exist parameter regimes whereby the equilibrium solutions can be driven to instability via a Hopf Bifurcation and parameter regimes whereby a backward bifurcation occurs.

\subsubsection{Numerical Simulations}

We use the physically reasonable parameter values shown in Table 5.2 to explore the dynamics of system (4.35). All the parameters in the model have been explained in Table 4.2. See Table 5.3 for sample numerical values of equilibrium solutions of system (4.35) and their stability properties for some of the parameters in Table 5.2.

Table 5.2. Physically meaningful ranges of parameter values for system (4.35).

\begin{tabular}{||l|c|l|l||}
\hline \hline Parameter & Range of Values & Value Used & Reference \\
\hline$\mu_{h},($ per day $)$ & {$[1 /(72 \times 365), 1 /(45 \times 365)]$} & $1 /(60 \times 365)$ & {$[9]$} \\
\hline$r_{h},($ per day) & {$[1 / 120,1 / 80]$} & $1 / 120$ & {$[4]$} \\
\hline$\mu_{v},($ per day) & {$[1 / 21,1 / 14]$} & $1 / 21$ & {$[4,10]$} \\
\hline$a_{v}$ & $0.5 \leq a_{v} \leq 1$ & 1.0 & {$[4]$} \\
\hline$\alpha_{v}\left(N_{h}\right)$ & $0.5 \leq \alpha_{v}\left(N_{h}\right)<a_{v}$ & 0.8 & {$[1]$} \\
\hline$\lambda_{0}$ & variable & variable & \\
\hline$\epsilon$ & variable & 0.9 & \\
\hline$L$ & variable & variable & \\
\hline$N_{h}$ & {$\left[10^{3}, 10^{7}\right]$} & variable & \\
\hline \hline
\end{tabular}


Table 5.3. Sample simulation results for the existence and stability of endemic equilibria showing the possibility of a Hopf bifurcation when $A_{2}>0$ or $R_{0}>1$ and the possibility of a backward bifurcation when $A_{2}<0$ or $R_{0}<1$.

\begin{tabular}{|c|c|c|c|c|c|c|c|}
\hline$L$ & $N_{h}$ & $A_{1}$ & $A_{2}$ & $\Delta$ & $w_{i}^{*}$ & $\lambda$ & Stability \\
\hline $10^{4}$ & $10^{3}$ & 0.9822 & 0. 0002 & 0.9653 & 0.9823 & $\begin{array}{c}-7.8667,-2.4876,-1.0147 \\
-0.8904,-0.6215 \\
0.0487 \pm 1.4520 i\end{array}$ & unstable \\
\hline $10^{4}$ & $5 \times 10^{6}$ & 0.9822 & 0.0971 & 1.3532 & 1.0727 & $\begin{array}{c}-2.3158,-0.0062,-0.1401 \\
-0.0406 \pm 1.2995 i \\
-1.1916 \pm 0.5758 i\end{array}$ & stable \\
\hline \multirow[b]{2}{*}{$10^{4}$} & \multirow[b]{2}{*}{$6 \times 10^{6}$} & \multirow[b]{2}{*}{0.9822} & \multirow[b]{2}{*}{-0.0614} & \multirow[b]{2}{*}{0.7190} & 0.9150 & $\begin{array}{c}-2.4617,-0.0035,-0.0950 \\
-1.2140 \pm 0.6184 i \\
0.0310 \pm 1.4263 i\end{array}$ & unstable \\
\hline & & & & & 0.0671 & $\begin{array}{c}-2.4582,-0.0651,0.0014 \\
-1.2302 \pm 0.6486 i \\
0.0286 \pm 1.4228 i\end{array}$ & unstable \\
\hline
\end{tabular}

We now present numerical simulation results that illustrate the relationship between the two threshold parameters $\mathscr{R}^{*}$ and $R_{0}$ as the parameters $\gamma$ and $\lambda_{0}$ are varied. Let us note that in terms of the original system parameters, $\gamma$ depends on $\beta_{v}$, that is the contact rate between a susceptible questing mosquito and a susceptible or infectious human and $\lambda_{0}$ is the growth rate of newly emerging mosquitoes. By varying $\gamma$ and computing the corresponding values of $\mathscr{R}^{*}$ and $R_{0}$, we can determine the relationship between these threshold parameters and $\gamma$. On the other hand, by varying $\lambda_{0}$ and computing the corresponding values of the two threshold parameters, we can also determine a relationship between these threshold parameters and $\lambda_{0}$. See Fig. 5.7 for a graphical illustrations of this. Fig. 5.7.3 illustrates that there are potentially four regions of interest for $\mathscr{R}^{*}$ and $R_{0}$ that could be of great importance and could inform policy makers on control strategies. 


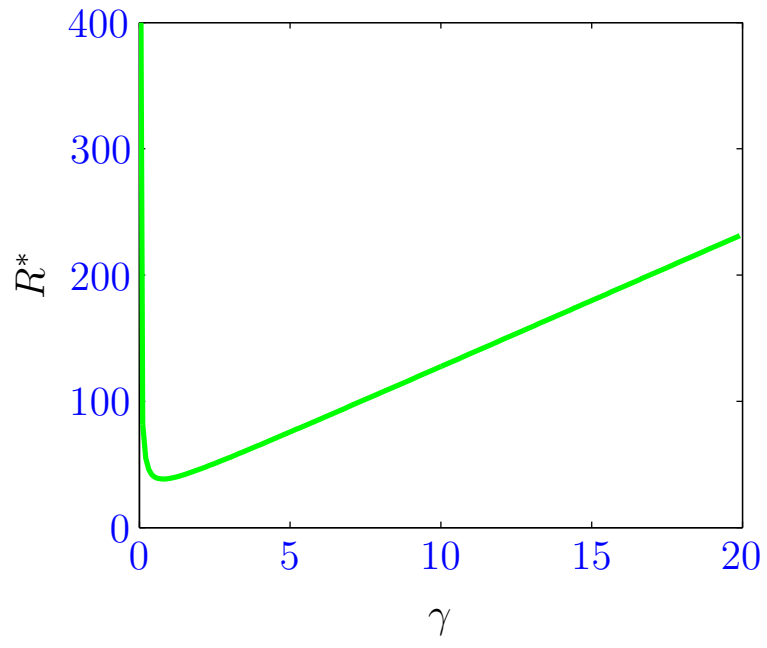

5.7.1. Plot of $\mathscr{R}^{*}$ against $\gamma$.

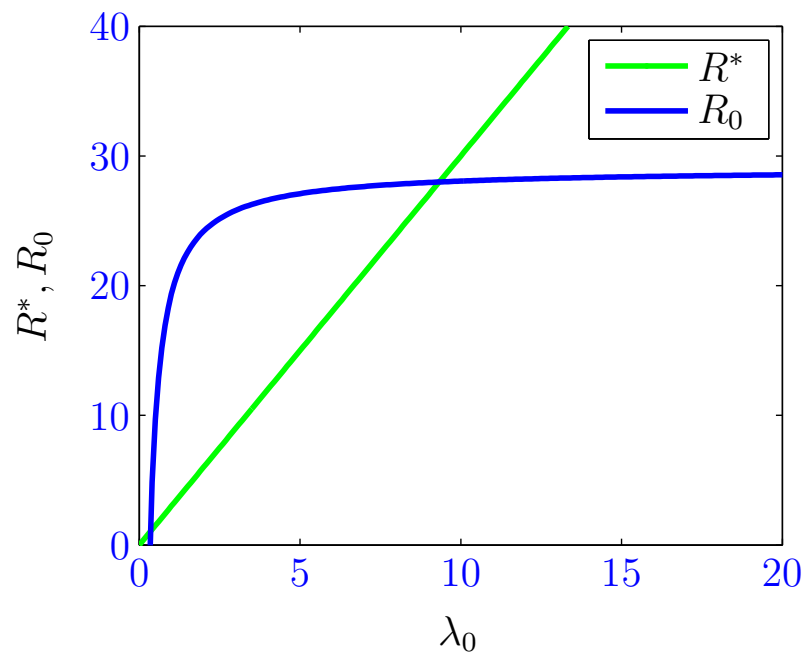

5.7.3. Plot of $R_{0}$ and $\mathscr{R}^{*}$ against $\lambda_{0}$.

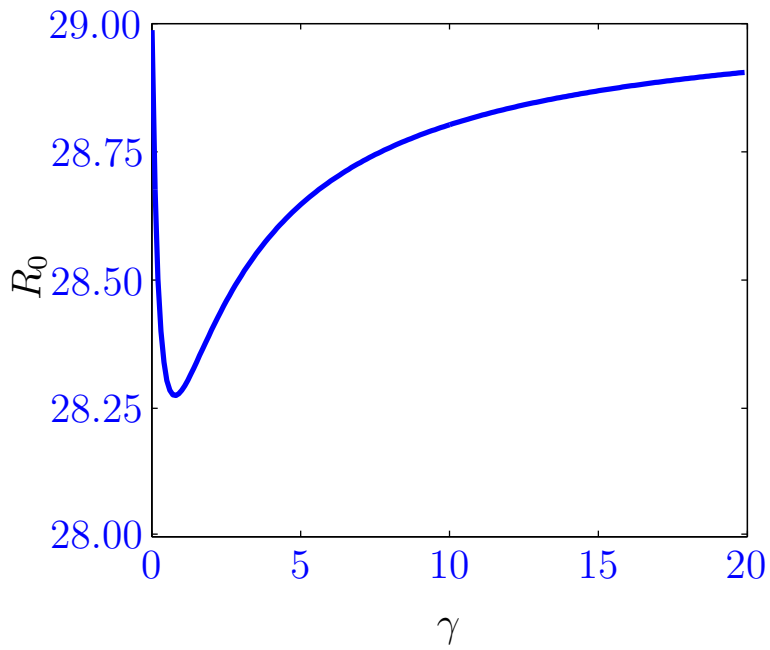

5.7.2. Plot of $R_{0}$ against $\gamma$.

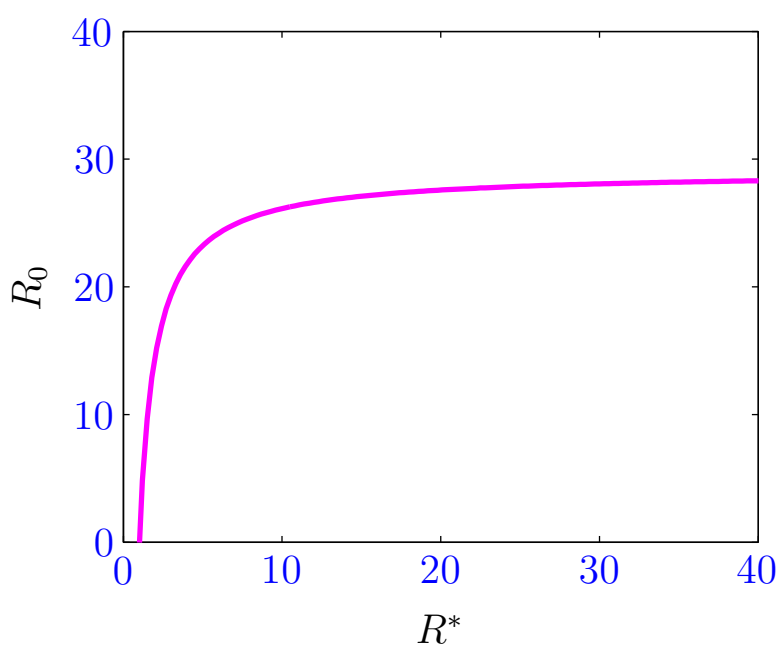

5.7.4. Plot of $R_{0}$ against $\lambda_{0}$.

Figure 5.7. Plots of the threshold parameters $\mathscr{R}^{*}$ and $R_{0}$ against the parameters $\gamma$ and $\lambda_{0}$. Notice that $R_{0}$ becomes unrealistic or becomes negative when $\mathscr{R}^{*}<1$ and that $R_{0}$ attains a maximum of $\frac{1}{u_{s}^{*}}=\frac{\beta \sigma}{\mu(1-\delta)}=29.03$. 
Next, we explore the endemicity of the disease by studying the relationship between the equilibrium solutions and the basic reproduction number, $R_{0}$. To do this, we set the other parameters as in Table 5.2 and vary the total human population $N_{h}$ from $10^{3}$ to $10^{6}$ and compute the corresponding values of $R_{0}, w_{i}^{*}$ and $I^{*}$. The results of this study confirm the analytical results presented in Section 5.3.2, which predicted the possibility of having two co-existing realistic endemic equilibria when $R_{0}<1$. This is a prerequisite for backward bifurcation. For $R_{0}>1$, we have a single realistic endemic equilibrium that is also stable.

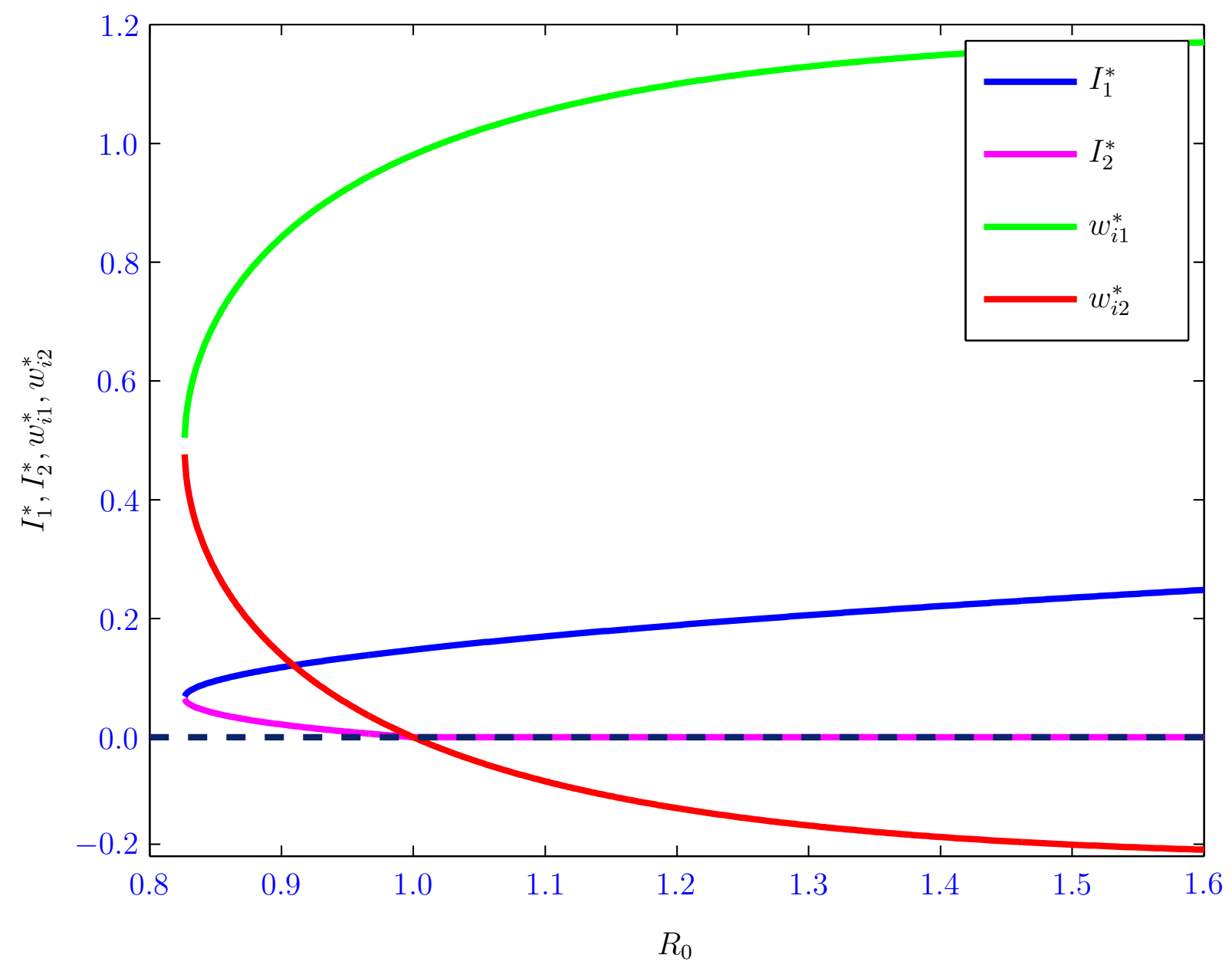

Figure 5.8. Endemic curves. Plot of the equilibrium number of infectious humans and vectors against the basic reproduction number $R_{0}$. The curves illustrate the possibility of having two realistic endemic equilibrium solutions when $R_{0}<1$ and a single realistic endemic equilibrium solution when $R_{0}>1$. 
We now apply a fourth-order Runge Kutta method with variable step size to simulate and characterize the disease-free equilibrium solution and each of the endemic equilibria depicted in Fig. 5.6. To explore, we set $\gamma=0.7849, \epsilon=0.9, L=5 \times 10^{3}$ and $N_{h}=4 \times 10^{6}$, where $N_{h}$ is specifically selected such that $R_{0}<1$. By choosing $\lambda_{0}$ appropriately and maintaining the other parameters as provided in Table 5.2, we can either have a stable disease-free equilibrium solution or oscillatory solutions. For instance, when $\lambda_{0}=10$, the above parameter regime yields $\mathscr{R}^{*}=30$ and $R_{0}=0.7016$ and our system relaxes to a stable disease-free equilibrium solution in the long time limit. This behavior is illustrated in Fig. 5.9. This is the case in which $\lambda_{0}$ is smaller than some critical value $\lambda_{0}^{c}=12.79$. For $\lambda_{0}>12.79$, we obtain oscillatory behavior around the disease-free equilibrium. Hence, there is a critical value of $\lambda_{0}$ below which the disease-free equilibrium is stable and above which it is unstable, with the instability giving rise to oscillatory phenomena. See Fig. 5.10 for a graphical demonstration of the oscillatory behavior. 


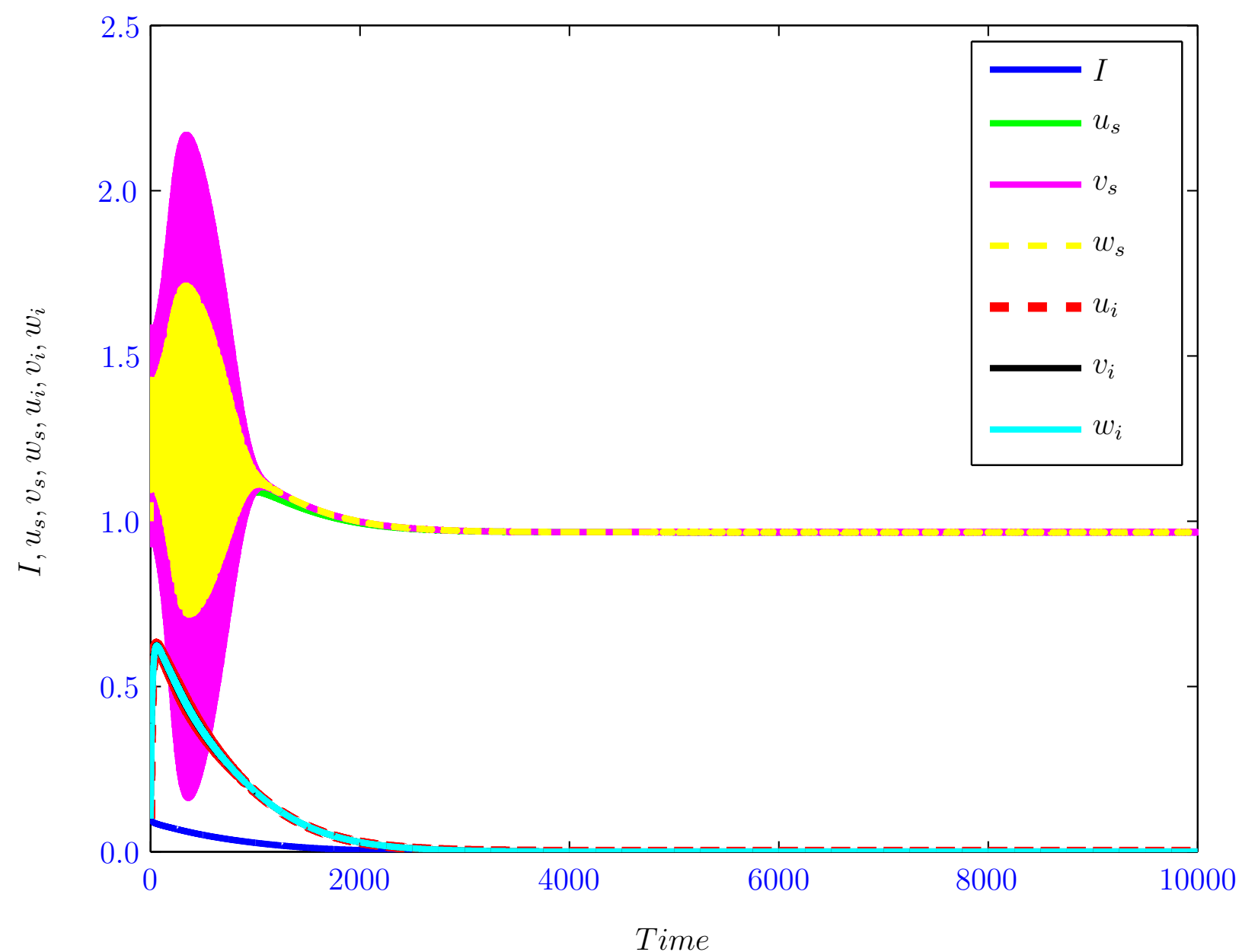

Figure 5.9. Time series plot of solutions to the full model (4.35) for $\gamma=0.7849, \lambda_{0}=$ $10, \epsilon=0.9, L=5 \times 10^{3}, N_{h}=4 \times 10^{6}$ depicting a stable disease-free equilibrium. The corresponding values of the two threshold parameters are $\mathscr{R}^{*}=30$ and $R_{0}=0.7050 . E_{d f e}=(0,0.9667,0.9667,0.9667,0,0,0)$ while the equilibrium value of each of the other variables is zero. 


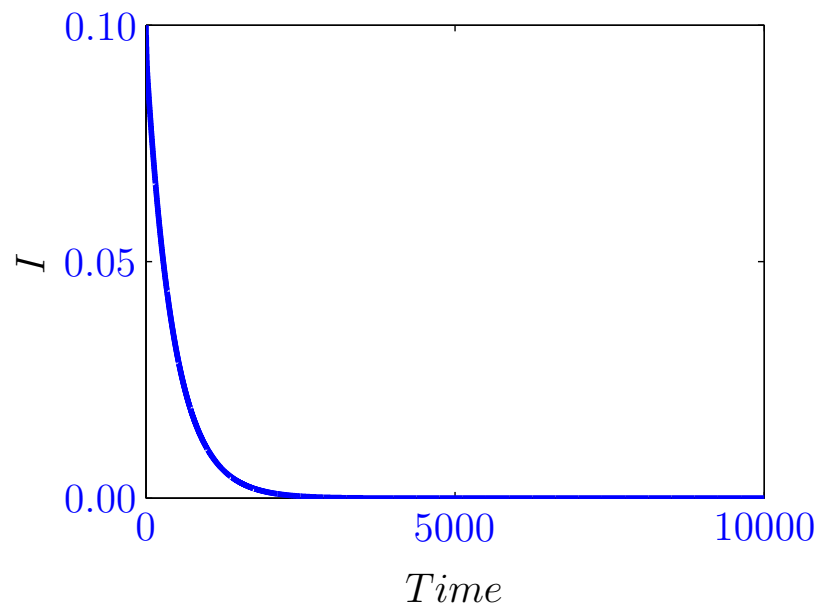

5.10.1. Plot of the proportion of infectious humans against time.

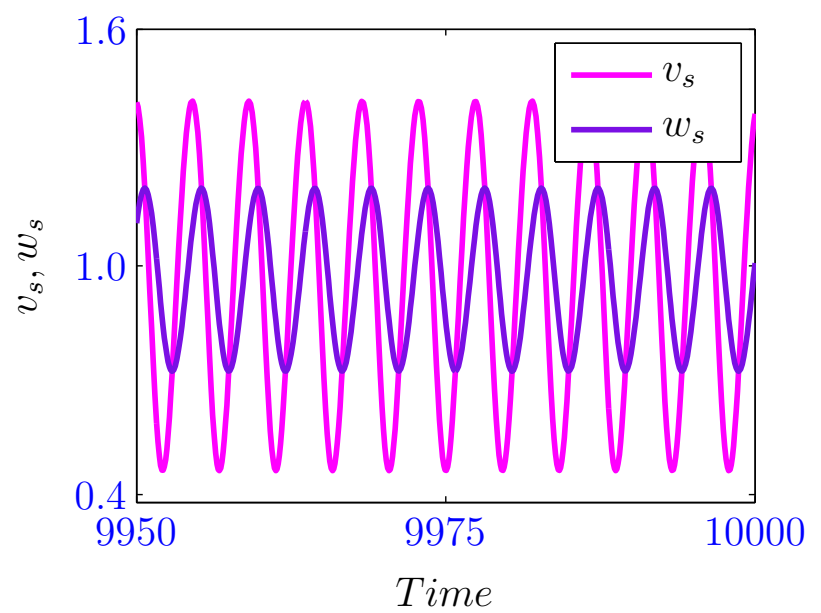

5.10.3. Plot of the dimensionless variables $v_{s}$ and $w_{s}$ against time.

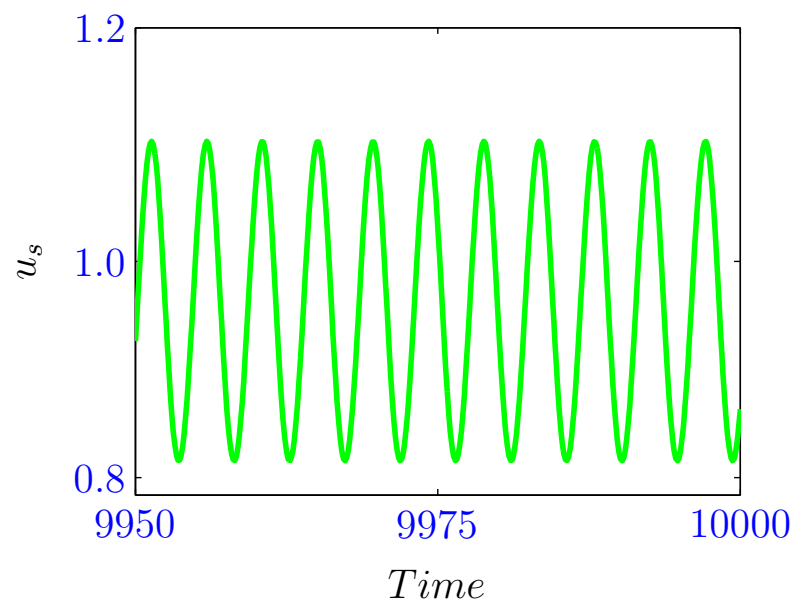

5.10.2. Plot of the dimensionless variable $u_{s}$ against time.

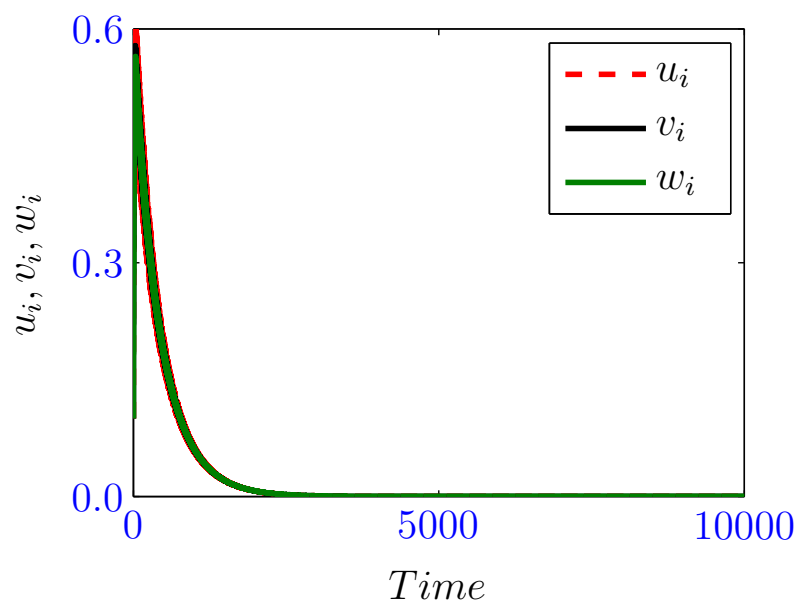

5.10.4. Plot of the dimensionless variables $u_{i}, v_{i}$ and $w_{i}$ against time.

Figure 5.10. Time series plot of solutions to our full model for $\gamma=0.7849, \lambda_{0}=13, \epsilon=$ $0.9, L=5 \times 10^{3}, N_{h}=4 \times 10^{6}$ showing oscillatory behavior for the variables $u_{s}, v_{s}$ and $w_{s}$. The corresponding values of $\mathscr{R}^{*}$ and $R_{0}$ are 39 and 0.7106 , respectively. Notice that each of the other variables relaxes to zero and that even though $R_{0}<1$, we still have oscillatory solutions since $\lambda_{0}>\lambda_{0}^{c}$. The approximate period of oscillation is 5 days. 


\subsubsection{Exploration of the Unique Endemic Equilibrium Solution in Region (I) of Fig. $\mathbf{5 . 6}$}

A single endemic equilibrium solution exists within Region (I) of Fig. 5.6. The conditions that guarantee the existence of this equilibrium solution are $A_{1}<0$ and $A_{2}>0$ or equivalently $R_{0}>1$. By retaining the parameter values in Table 5.2 and setting $\lambda_{0}=0.5$ and $\delta=0.4$, we obtain $R_{0}=3.2415, A_{1}=-0.3714, A_{2}=0.0237$ and $\Delta=0.2327$. This parameter regime gives rise to the asymptotically stable equilibrium solution

$$
\left(I^{*}, u_{s}^{*}, v_{s}^{*}, w_{s}^{*}, u_{i}^{*}, v_{i}^{*}, w_{i}^{*}\right)=(0.2173,0.1028,0.1314,0.1314,0.05552,0.05552,0.05552) .
$$

The long-term behavior of solutions to the system within Region (I) is presented in Fig. 5.11.

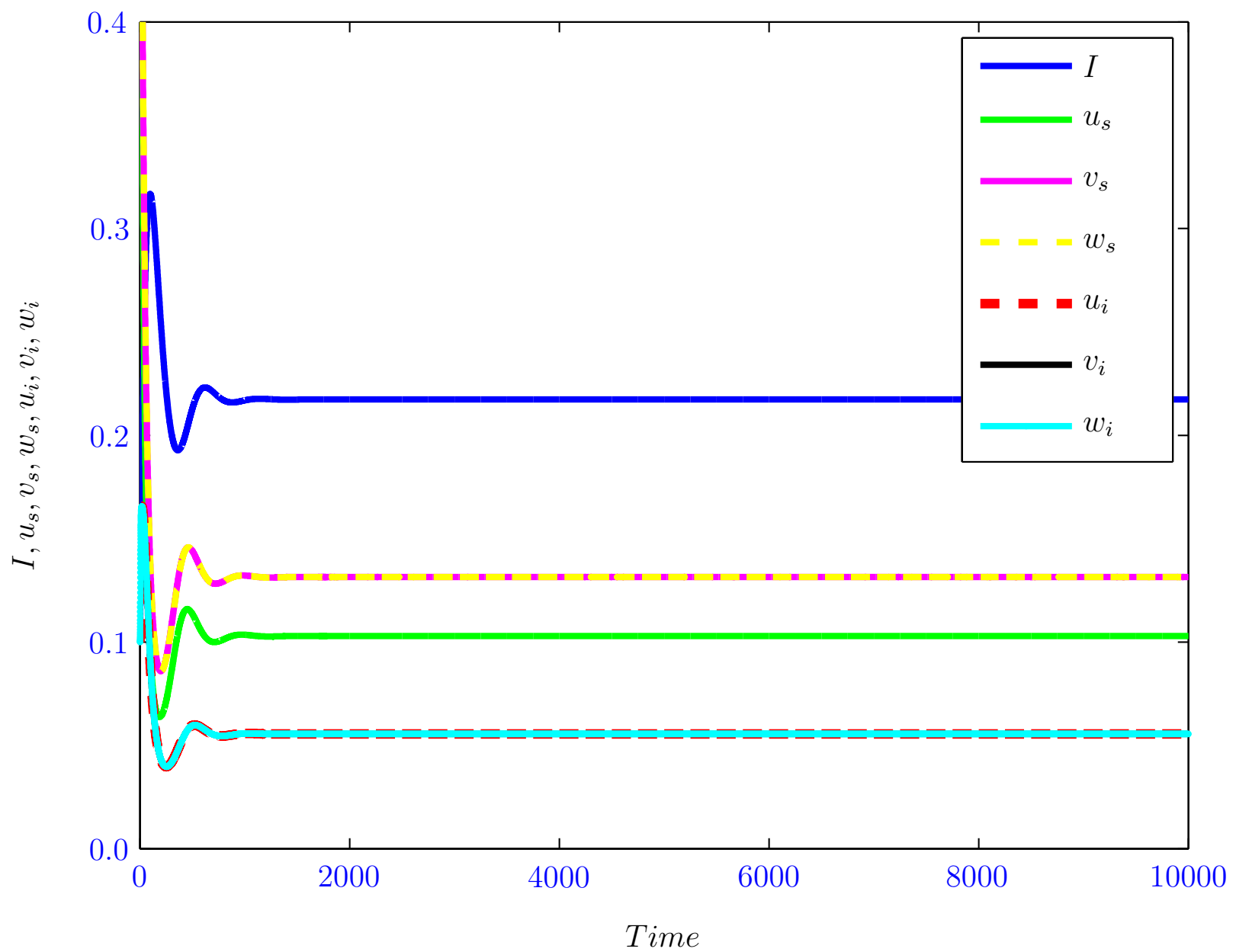

Figure 5.11. Long-term behavior of solutions of system (4.35) in Region (I) for $\lambda_{0}=0.5$ and $\delta=0.4$ depicting a stable endemic equilibrium. 


\subsubsection{Exploration of the Unique Endemic Equilibrium that exists when $A_{1}=0, A_{2}>0$ and $\Delta>0$}

We establish the stability of the equilibrium solution on the line labeled (II) in Fig. 5.6. For $\lambda_{0}=0.9143, \delta=0.2$, and the other parameters given in Table 5.2 , we obtain $R_{0}=$ 4.6343, $A_{1}=0.0, A_{2}=0.0683$ and $\Delta=0.2733$, which satisfy the existence condition for the endemic equilibrium solution on the line labeled (II).

$$
\left(I^{*}, u_{s}^{*}, v_{s}^{*}, w_{s}^{*}, u_{i}^{*}, v_{i}^{*}, w_{i}^{*}\right)=(0.5666,0.1371,0.3163,0.3163,0.2614,0.2614,0.2614) .
$$

As illustrated in Fig. 5.12, the equilibrium solution is asymptotically stable.

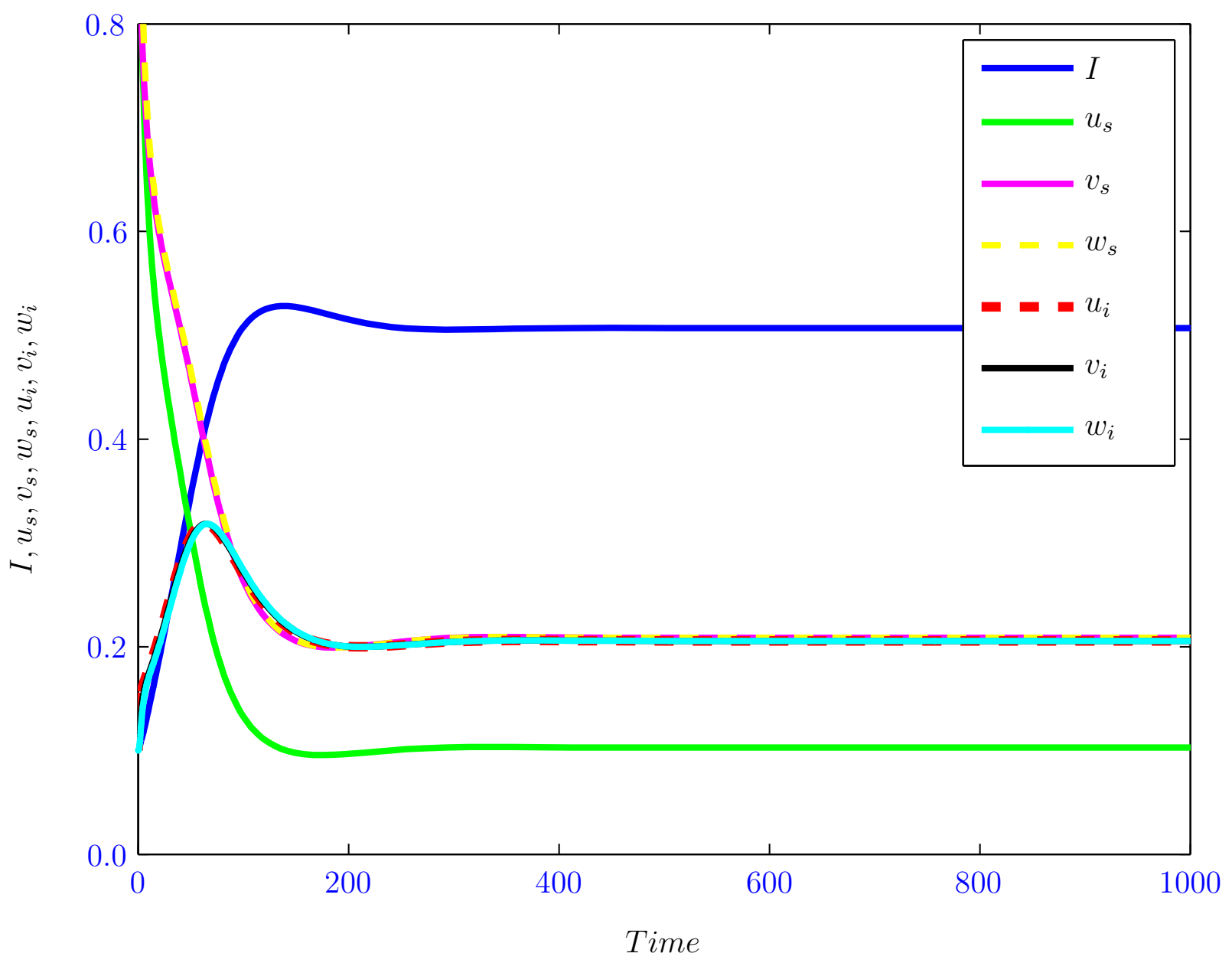

Figure 5.12. Time series plot of solutions to system (4.35) for $\lambda_{0}=0.9143$ or $R_{0}=4.6343$ showing a stable endemic equilibrium solution. Only the first 1,000 out of a total of 10,000 time steps are shown. 


\subsubsection{Characterization of the Unique Endemic Equilibrium Solution in Region (III) of Fig. 5.6}

We select $N_{h}$ such that $R_{0}>1$. For $N_{h}=10^{5}$ and $\lambda_{0}=12, \mathscr{R}^{*}=36$ and $R_{0}=28.2239$. This parameter set gives rise to a stable endemic equilibrium solution, representing the situation in which malaria establishes itself within the community. See Figs. 5.13 and 5.14.

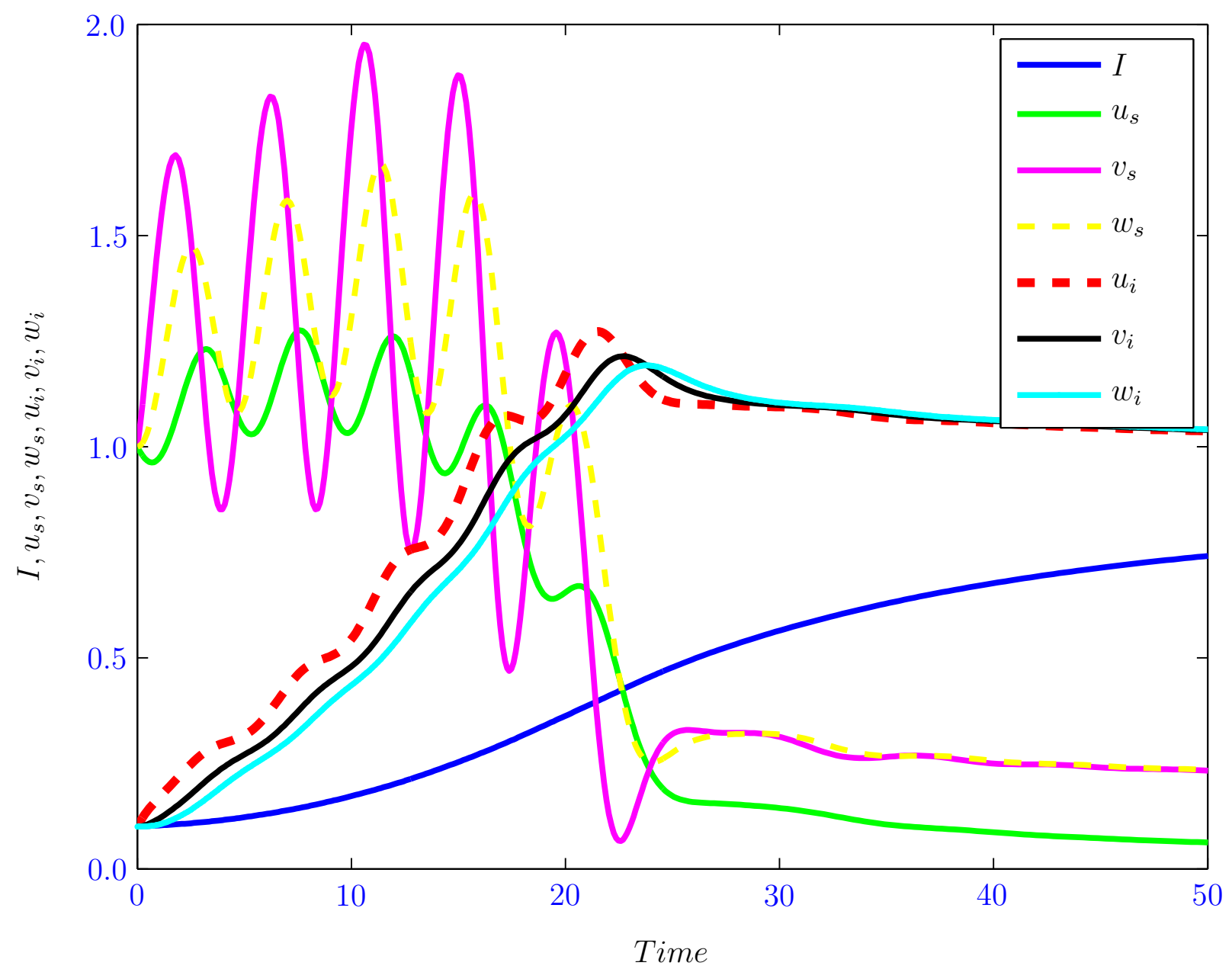

Figure 5.13. Time series plot of solutions to system (4.35) for $\gamma=0.7849, \lambda_{0}=12, \epsilon=$ $0.9, L=5 \times 10^{3}, N_{h}=10^{5}$. These and the rest of the parameters in Table 5.2 yield $\mathscr{R}^{*}=36, R_{0}=28.3631$, and the stable endemic equilibrium solution $\left(I^{*}, u_{s}^{*}, v_{s}^{*}, w_{s}^{*}, u_{i}^{*}, v_{i}^{*}, w_{i}^{*}\right)=(0.8351,0.0343,0.2079,0.2079,1.0127,1.0127$, 1.0127). Notice that as predicted by our analysis, $v_{s}^{*}=w_{s}^{*}$ and $u_{i}^{*}=v_{i}^{*}=$ $w_{i}^{*}$. Only the first 50 out of 10,000 time steps are shown. 


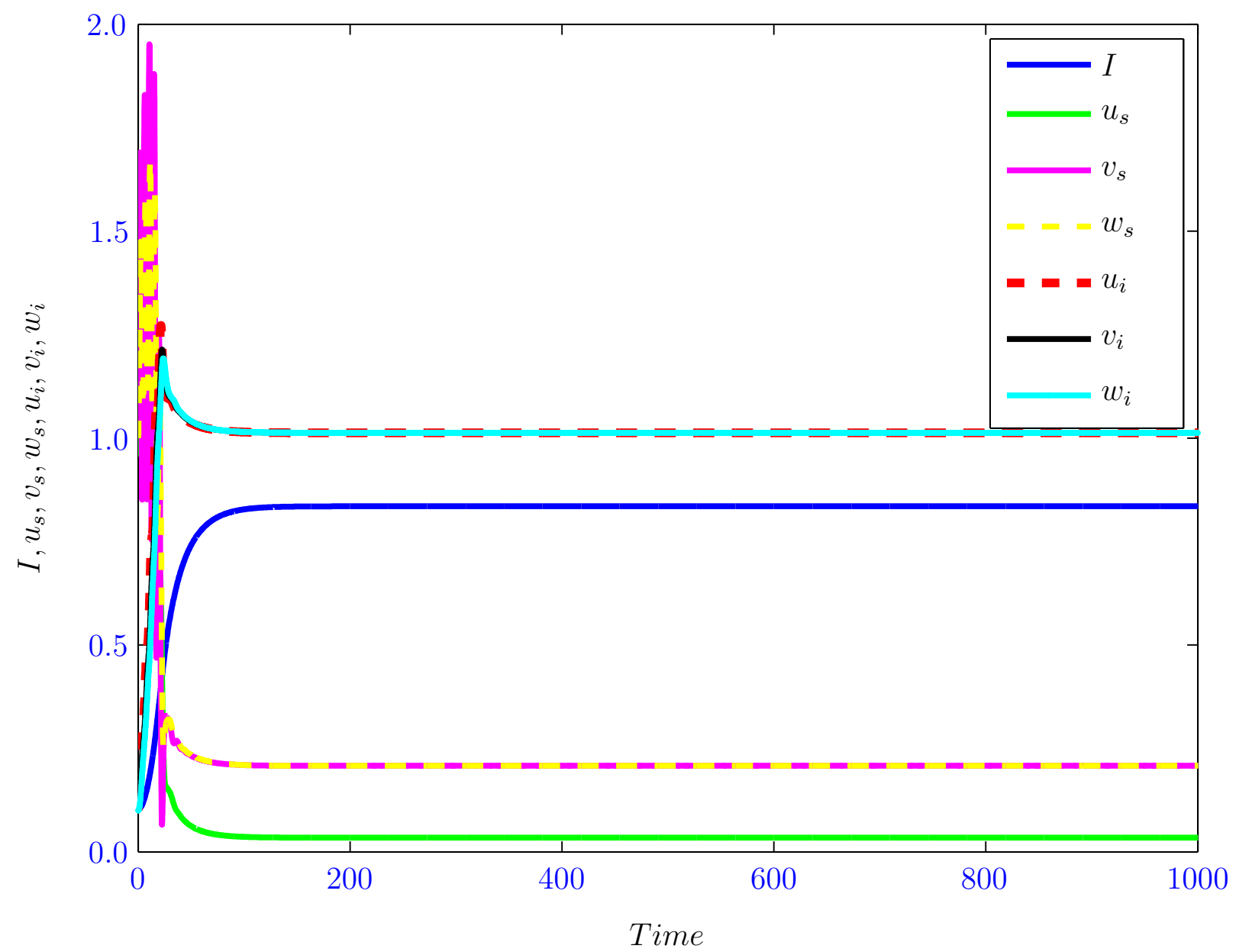

Figure 5.14. Time series plot of solutions to system (4.35) for $\gamma=0.7849, \lambda_{0}=12, \epsilon=$ $0.9, L=5 \times 10^{3}, N_{h}=10^{5}$. These and the rest of the parameters in Table 5.2 yield $\mathscr{R}^{*}=36, R_{0}=28.3631$, and the stable endemic equilibrium solution $\left(I^{*}, u_{s}^{*}, v_{s}^{*}, w_{s}^{*}, u_{i}^{*}, v_{i}^{*}, w_{i}^{*}\right)=(0.8351,0.0343,0.2079,0.2079,1.0127,1.0127$, 1.0127). Notice that as predicted by our analysis, $v_{s}^{*}=w_{s}^{*}$ and $u_{i}^{*}=v_{i}^{*}=$ $w_{i}^{*}$. Only the first 1,000 out of 10,000 time steps are shown. 
On the other hand, for $N_{h}=10^{5}$ and $\lambda_{0}=14.775, \mathscr{R}^{*}=44.325$ and $R_{0}=28.5153$. This gives rise to an unstable endemic equilibrium solution that is accompanied by the emergence of oscillatory solutions. See, for example, Fig. 5.15 for time series plots, Fig. 5.16 for phase plots, and Fig. 5.17 for a plot of the total mosquito population. The figures show sustained bounded oscillations which arise as the result of a Hopf bifurcation as the parameter $\lambda_{0}$ is varied. These indicate that natural occurring oscillations in the mosquito population introduce oscillations in the dynamics of malaria transmission. Until now, the way in which oscillations in malaria dynamics have been captured was by the inclusion of a seasonal forcing term or via delays. However, here we have demonstrated that it is possible to capture oscillations in the dynamics of malaria transmission by appropriately interpreting the life style and behavior of the transmitting vector, and by incorporating the relevant aspects into the malaria model without the consideration of delay or the inclusion of a seasonal forcing term. Note that from equations (5.12) and (5.13), $R_{0}=\tilde{R}_{0}^{2}$. Thus, though the values of $R_{0}$ computed here for the particular parameter regime may seem large $(<29)$, a focus should be placed on the value of $\tilde{R}_{0}$, which is less than 6 . 


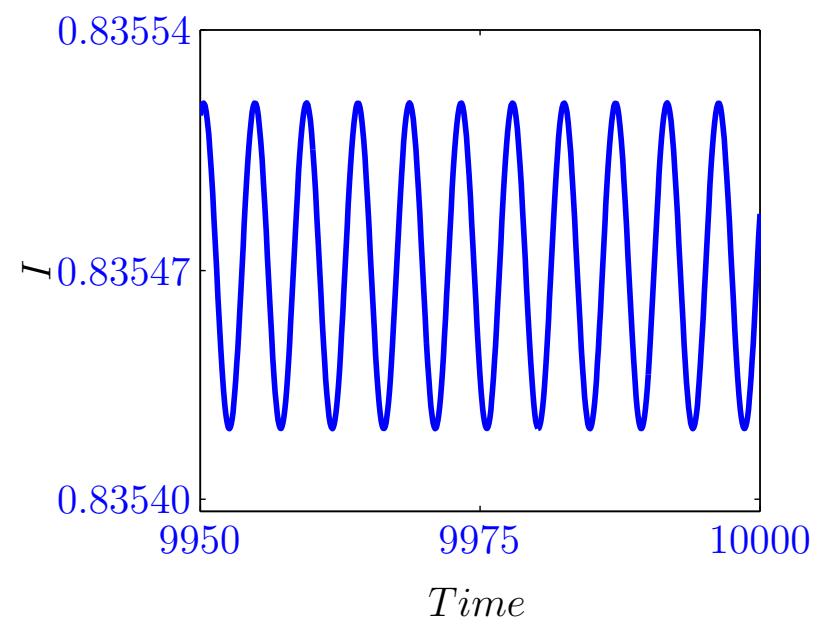

5.15.1. Plot of the proportion of infectious humans against time.

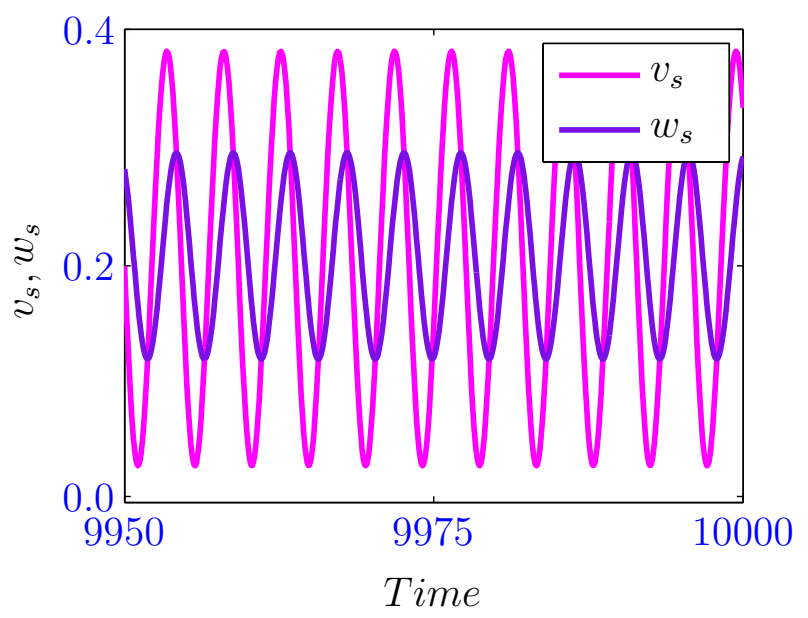

5.15.3. Plot of the dimensionless variables $v_{s}$ and $w_{s}$ against time.

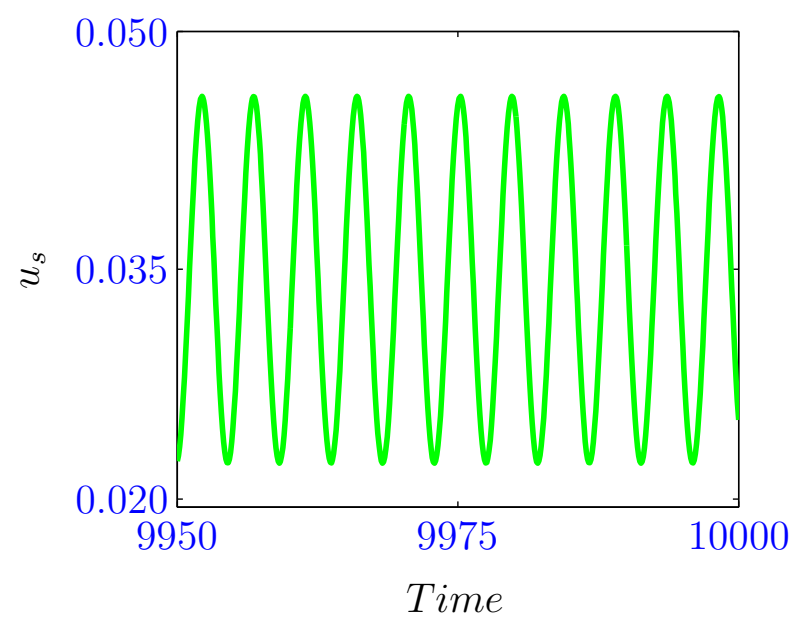

5.15.2. Plot of the dimensionless variable $u_{s}$ against time.

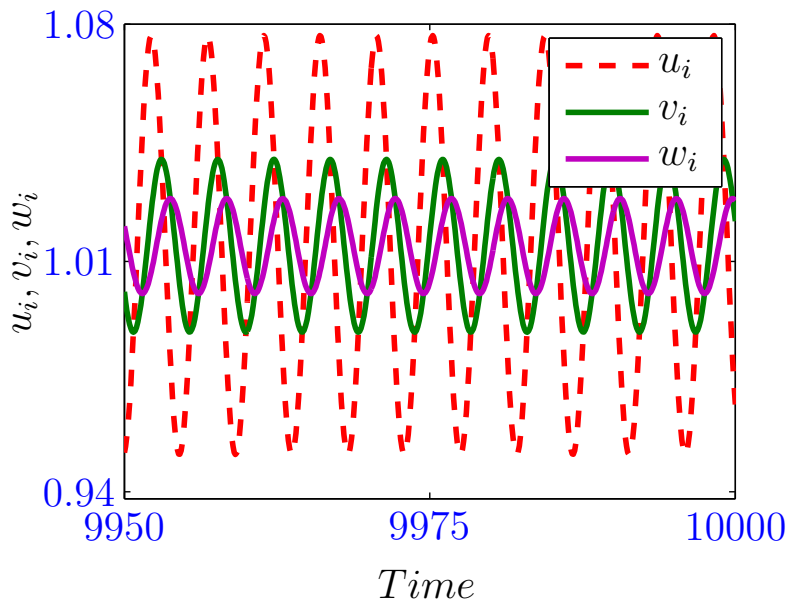

5.15.4. Plot of the dimensionless variables $u_{i}, v_{i}$ and $w_{i}$ against time.

Figure 5.15. Typical time series plots of solutions to system (4.35), showing oscillatory phenomena for $\gamma=0.7849, \lambda_{0}=14.775, \epsilon=0.9, L=5 \times 10^{3}$, and $N_{h}=10^{5}$. These and the rest of the parameters in Table 5.2 yield $\mathscr{R}^{*}=44.325$ and $R_{0}=28.5153$. The approximate period of oscillation is 5 days. 


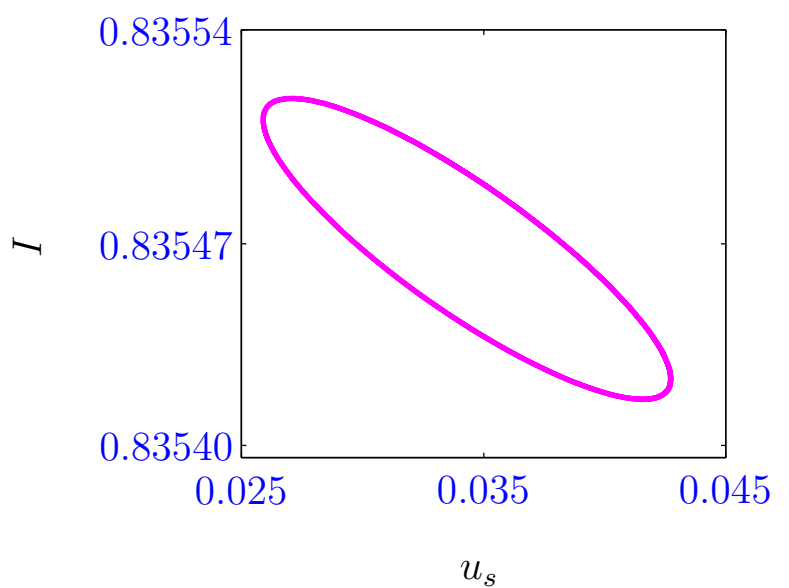

5.16.1. Limit cycle: Plot of $I$ against $u_{s}$.

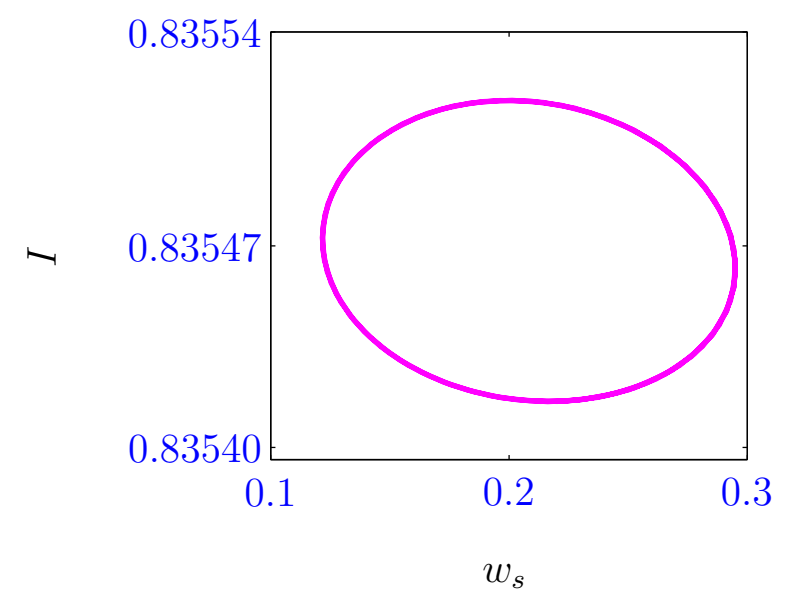

5.16.3. Limit cycle: Plot of $I$ against $w_{s}$.

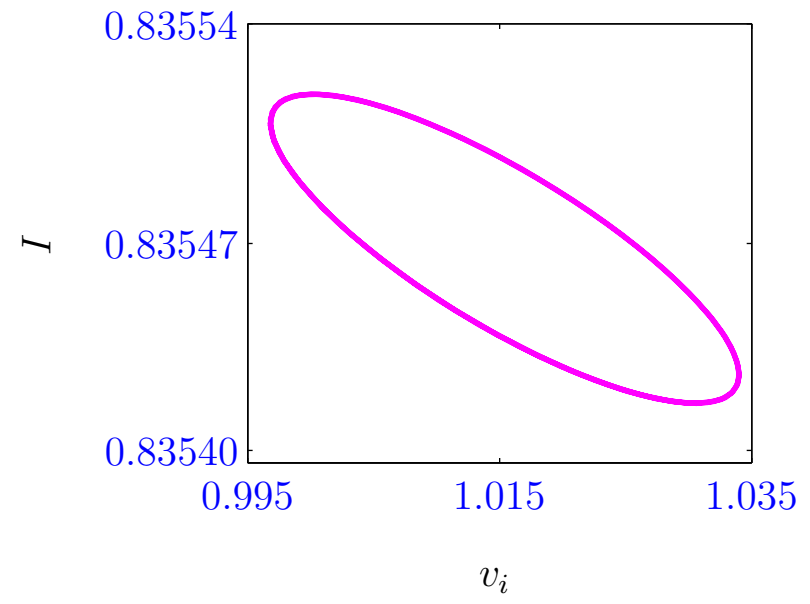

5.16.5. Limit cycle: Plot of $I$ against $v_{i}$.

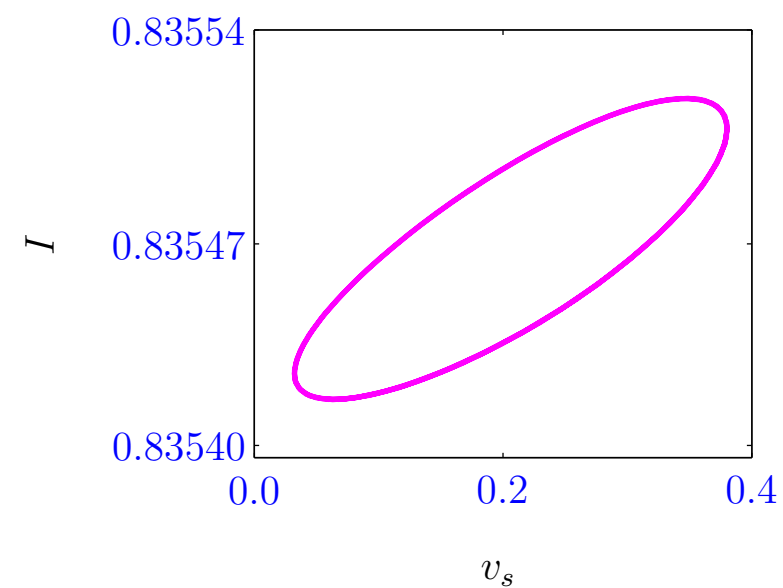

5.16.2. Limit cycle: Plot of $I$ against $v_{s}$.

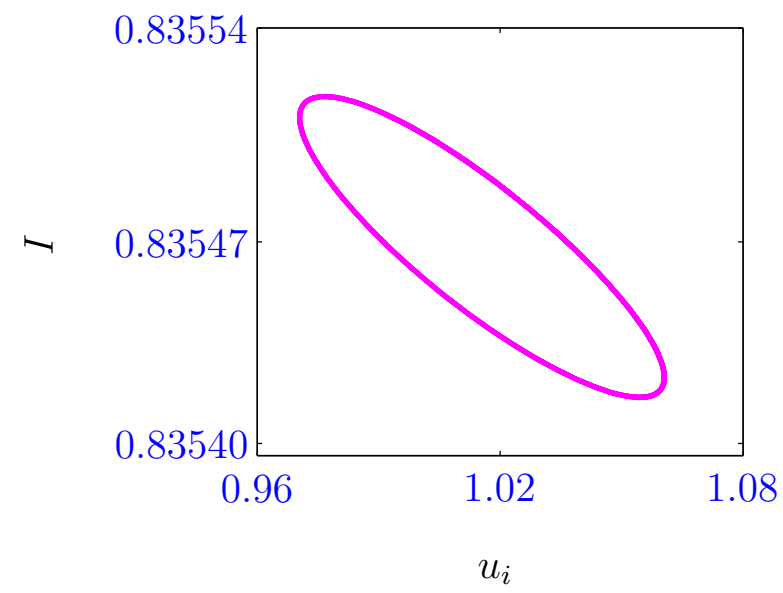

5.16.4. Limit cycle: Plot of $I$ against $u_{i}$.

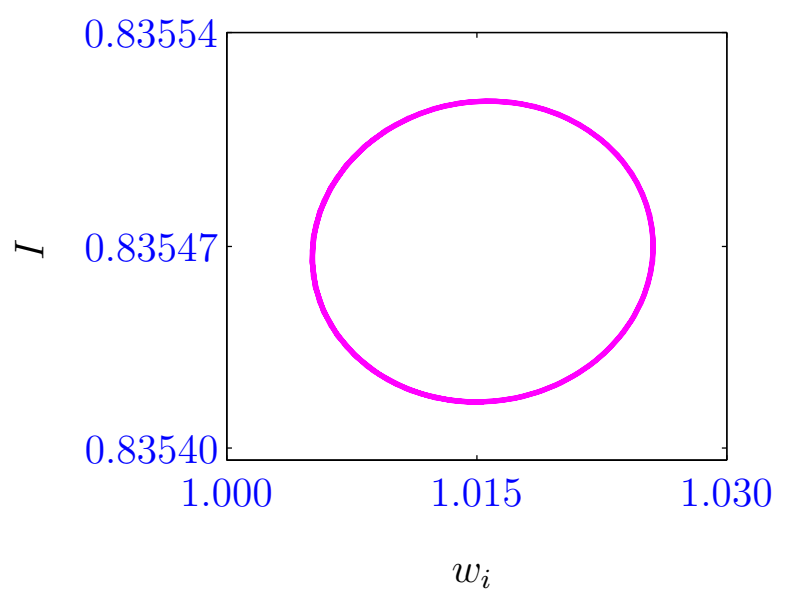

5.16.6. Limit cycle: Plot of $I$ against $w_{i}$.

Figure 5.16. Limit cycles of system (4.35) for $\gamma=0.7849, \lambda_{0}=14.775, \epsilon=0.9, L=5 \times$ $10^{3}, N_{h}=10^{5}$. For this parameter regime, $\mathscr{R}^{*}=44.325$ and $R_{0}=28.5153$. 


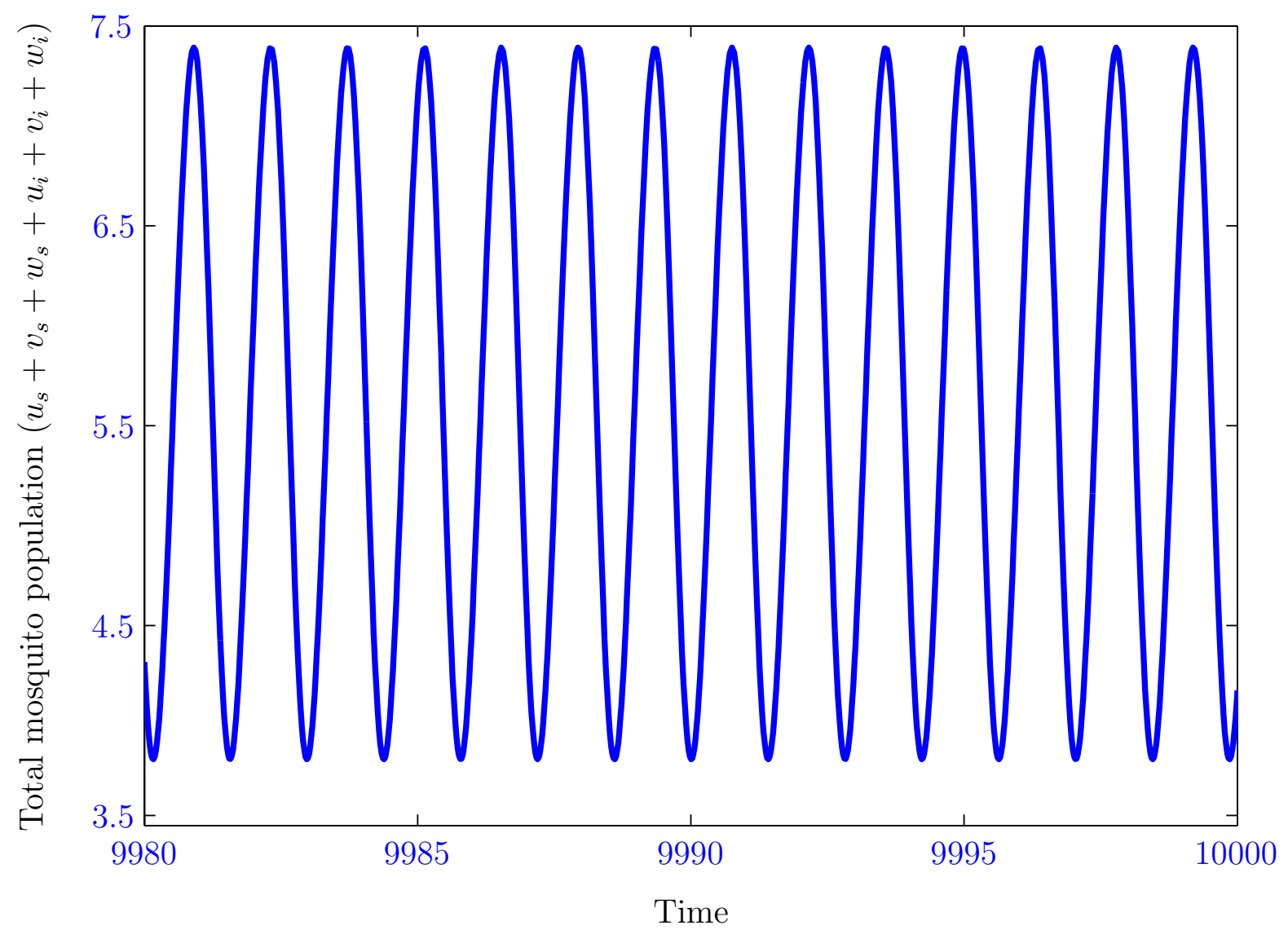

Figure 5.17. Pot of the total mosquito population in the presence of the disease showing bounded periodic oscillations. 


\subsubsection{Exploration of the Unique Endemic Equilibrium that Exists when $A_{1}>0, A_{2}=0$ and $\Delta>0$}

A realistic nontrivial endemic equilibrium exists when $A_{1}>0, \Delta>0$ and $A_{2}=0$ or equivalently $R_{0}=1$. Here, $\lambda_{0}=\lambda_{0}^{c}=0.3452, R_{0}=1, A_{1}=0.3378, A_{2}=0.0, \Delta=0.1141$ and the numerical value of the endemic equilibrium solution is

$$
\left(I^{*}, u_{s}^{*}, v_{s}^{*}, w_{s}^{*}, u_{i}^{*}, v_{i}^{*}, w_{i}^{*}\right)=(0.6282,0.0343,0.0922,0.0922,0.3378,0.3378,0.3378) .
$$

As illustrated in Fig. 5.18, the equilibrium is asymptotically stable.

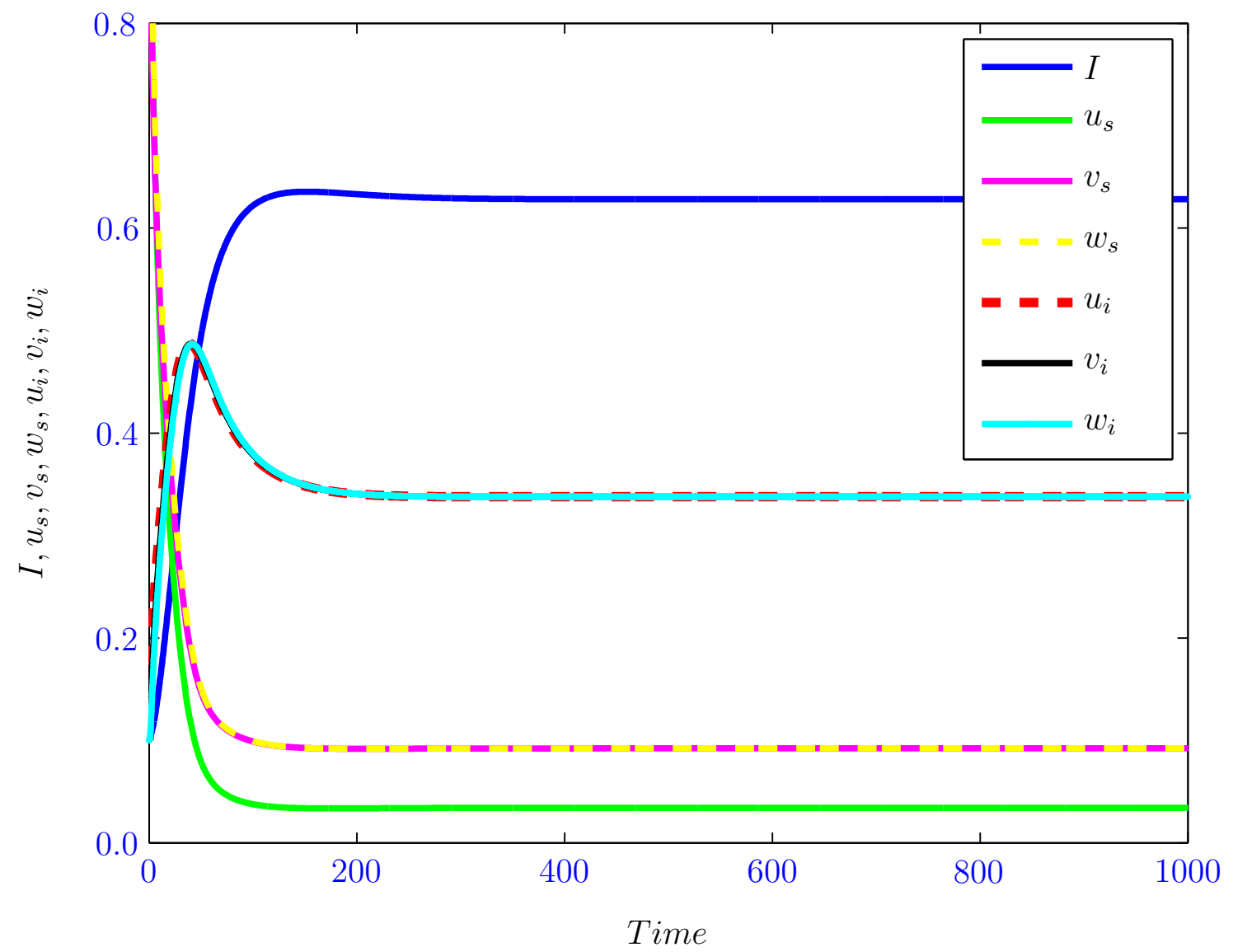

Figure 5.18. Dynamical behavior of solutions to system (4.35) for $\lambda_{0}=\lambda_{0}^{c}=0.3452$ or $R_{0}=1$. A stable endemic equilibrium exists for this parameter regime. 


\subsubsection{Exploration of the Endemic Equilibrium that Exists when $A_{1}>0, A_{2}<0$ and $\Delta=0$}

In contrast to many epidemiological models in which endemic equilibria exist only when $R_{0}>1$, depending on whether $\Delta=0$ or $\Delta>0$, system (4.35) can exhibit one or two endemic equilibrium solutions when $R_{0}<1$. Here, we focus on the a single endemic equilibrium solution that exists when $A_{1}>0, A_{2}<0$ and $\Delta=0$. The parameter regime, $\lambda_{0}=0.23, R_{0}=0.9918, A_{1}=0.0062, A_{2}=-1 \times 10^{-5}$ and $\Delta=0$ satisfies conditions for the existence of the endemic equilibrium solution $\left(I^{*}, u_{s}^{*}, v_{s}^{*}, w_{s}^{*}, u_{i}^{*}, v_{i}^{*}, w_{i}^{*}\right)=$ $(0.0153,0.0343,0.0348,0.0348,0.0031,0.0031)$; however, as illustrated in Fig. 5.19, the equilibrium solution is unstable.

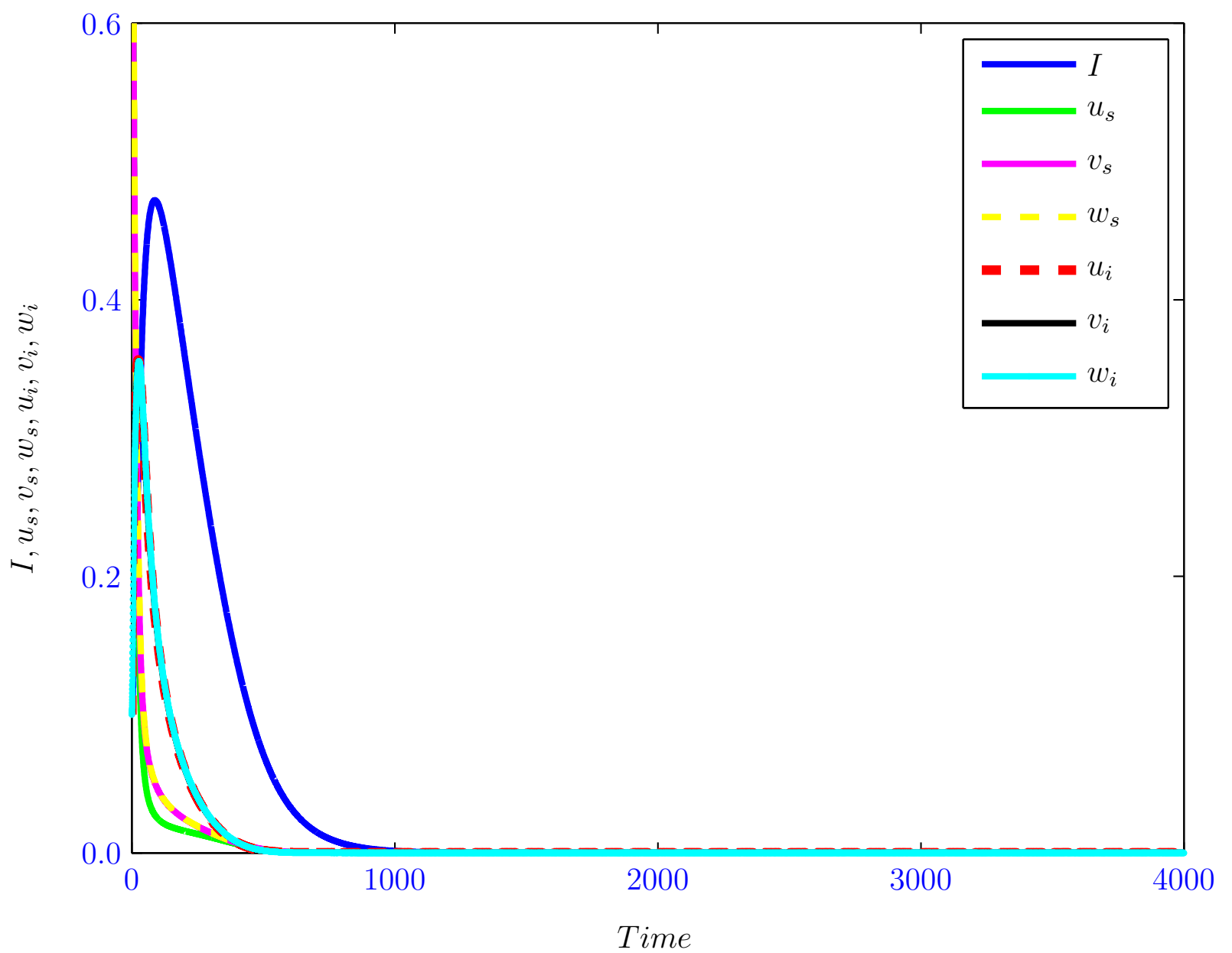

Figure 5.19. Dynamical behavior of solutions to system (4.35) when $A_{1}>0, A_{2}<0$ and $\Delta=0$. An unstable endemic equilibrium exists for this parameter regime. 
We completely characterize the two endemic equilibrium solutions in region (V) of Fig. 5.6 that occur when $A_{1}>0, A_{2}<0$ and $\Delta>0$ in the next section.

\subsection{Characterization of Backward Bifurcation}

Epidemiological models can exhibit some interesting dynamical behavior near $R_{0}=1$. In most cases, there is a supercritical or forward bifurcation at $R_{0}=1$. However, situations do arise in epidemiological modeling where we run into a double bifurcation at $R_{0}=1$. In this case, we have both a supercritical or forward bifurcation and a subcritical or backward bifurcation. The occurrence of a forward bifurcation is accompanied by the emergence of an asymptotically stable endemic equilibrium and loss of stability of the disease-free equilibrium. A major prerequisite for the occurrence of a backward bifurcation is the existence of two endemic equilibria when $R_{0}<1$. The other requirement is that one of the endemic equilibria together with the disease-free equilibrium have to be stable while the second endemic equilibrium has to be unstable. The phenomenon of backward bifurcation was first established by Huang et al. [11] and further exploited in Refs. [3, 12-17] among others. It is very essential in disease control in that it enlightens us to the fact that the basic reproduction number may not be the only possible threshold parameter to focus on in formulating disease control and eradication strategies. In fact, it tells us that simply reducing $R_{0}$ below unity might not be enough to eradicate certain diseases. We explore the existence of backward bifurcation in our model using the approach proposed by Castillo-Chavez et al. in Ref. [15] and then use our results to propose better malaria control strategies. We use the following Theorem due to Castillo-Chavez et al., which for convenience we quote in its entirety.

Theorem 5.4.1 (Theorem 4.1 in [15]). Consider a general system of ordinary differential equations with a parameter $\phi$ :

$$
\frac{d x}{d t}=f(x, \phi), f: \mathbb{R}^{n} \times \mathbb{R} \rightarrow \mathbb{R}^{n}, \text { and } f \in \mathbb{C}^{2}\left(\mathbb{R}^{n} \times \mathbb{R}\right) .
$$

Without loss of generality, it is assumed that 0 is an equilibrium for System (5.34) for all values of the parameter $\phi$; that is, $f(0, \phi)=0$ for all $\phi$. 
A1: $A=D_{x} f(0,0)=\left(\frac{\partial f_{i}}{\partial x_{j}}(0,0)\right)$ is the linearization matrix of a System (5.34) around the equilibrium 0 with $\phi$ evaluated at 0 . Zero is a simple eigenvalue of $A$ and all other eigenvalues of $A$ have negative real parts;

A2: Matrix A has a nonnegative right eigenvector $w$ and a left eigenvector $v$ corresponding to the zero eigenvalue.

Let $f_{k}$ be the kth component of $f$ and

$$
\begin{aligned}
a & =\sum_{k, i, j=1}^{n} v_{k} w_{i} w_{j} \frac{\partial^{2} f_{k}}{\partial x_{i} \partial x_{j}}(0,0), \\
b & =\sum_{k, i=1}^{n} v_{k} w_{i} \frac{\partial^{2} f_{k}}{\partial x_{i} \partial \phi}(0,0) .
\end{aligned}
$$

The local dynamics of the system around 0 are totally determined by a and $b$.

1. $a>0, b>0$. When $\phi<0$ with $|\phi| \ll 1,0$ is locally asymptotically stable, and there exists a positive unstable equilibrium; when $0<\phi \ll 1$, 0 is unstable and there exists a negative and locally asymptotically stable equilibrium;

2. $a<0, b<0$. When $\phi<0$ with $|\phi| \ll 1$, 0 is unstable; when $0<\phi \ll 1$, 0 is locally asymptotically stable, and there exists a positive unstable equilibrium;

3. $a>0, b<0$. When $\phi<0$ with $|\phi| \ll 1,0$ is unstable, and there exists a locally asymptotically stable negative equilibrium; when $0<\phi \ll 1$, 0 is stable and a positive unstable equilibrium appears;

4. $a<0, b>0$. When $\phi$ changes from negative to positive, 0 changes its stability from stable to unstable. Correspondingly a negative unstable equilibrium becomes positive and locally asymptotically stable.

For the system (4.35) we have the following result: 
Theorem 5.4.2. Let $0<\lambda_{0}(\gamma)<\frac{(\gamma+\rho+1)(\gamma+\rho+\gamma \rho)+\gamma(\rho-\alpha)}{\alpha \gamma}$. Then model (4.35) undergoes a backward bifurcation at $R_{0}=1$. In addition, there exists a threshold value of $R_{0}$, namely, $R_{0}^{c}=1-\xi$ where $\xi=\left(\frac{\beta\left(\alpha \lambda_{0} \sigma-\rho(1-\delta)\right)}{2 \alpha \lambda_{0} \mu(1-\delta)}\right)^{2}$ such that for $R_{0}<R_{0}^{c}$, there exists a unique stable disease-free equilibrium and for $R_{0}^{c}<R_{0}<1$, there exists a stable disease-free equilibrium, a stable endemic equilibrium and an unstable endemic equilibrium.

Proof. We follow through and establish that the conditions of Theorem 5.4.1 are satisfied and then conclude that there is a backward bifurcation in the model. The matrix A of the linearized system about the disease-free equilibrium is

$$
A=\left(\begin{array}{ccccccc}
-\mu & 0 & 0 & 0 & 0 & 0 & \beta \\
-u_{s}^{*} & -1 & 0 & 1 & 0 & 0 & 0 \\
0 & \alpha \lambda_{0}^{c}\left(1-2 u_{s}^{*}\right)+\alpha & -\rho & 0 & \alpha \lambda_{0}^{c} & 0 & 0 \\
0 & 0 & \gamma & -\gamma & 0 & 0 & 0 \\
\sigma u_{s}^{*} & 0 & 0 & 0 & -1 & 0 & \delta \\
0 & 0 & 0 & 0 & \rho & -\rho & 0 \\
0 & 0 & 0 & 0 & 0 & \epsilon & -\epsilon
\end{array}\right)
$$

and the characteristic equation of $\mathrm{A}$ is given by $\left[\lambda^{3}+Q \lambda^{2}+R \lambda+P\left(\mathscr{R}^{*}-1\right)\right]\left[\lambda^{4}+\left(Q_{1}+\mu\right) \lambda^{3}+\left(\mu Q_{1}+R_{1}\right) \lambda^{2}+\left(\mu R_{1}+P_{1}\right) \lambda+P_{1} \mu\left(1-R_{0}\right)\right]=0$

where

$$
\begin{aligned}
P & =\gamma(\rho-\alpha)>0, \\
Q & =1+\gamma+\rho>0, \\
R & =\gamma+\rho+\gamma \rho>0, \\
P_{1} & =\epsilon \rho(1-\delta)>0, \\
Q_{1} & =1+\epsilon+\rho>0, \\
R_{1} & =\epsilon+\rho+\epsilon \rho>0 .
\end{aligned}
$$

When $R_{0}=1$, we have

$$
\lambda\left[\lambda^{3}+Q \lambda^{2}+R \lambda+P\left(\mathscr{R}^{*}-1\right)\right]\left[\lambda^{3}+\left(Q_{1}+\mu\right) \lambda^{2}+\left(\mu Q_{1}+R_{1}\right) \lambda+\left(\mu R_{1}+P_{1}\right)\right]=0 .
$$


Hence, there is a simple zero eigenvalue when $R_{0}=1$. Also, when $R_{0}=1$,

$$
\begin{aligned}
\frac{\beta \sigma\left(\mathscr{R}^{* c}-1\right)}{\mu\left(1-\mathscr{R}^{* c}\right)} & =1 \\
& \Leftrightarrow \quad \mathscr{R}^{* c}=\frac{\beta \sigma}{\beta \sigma-\mu(1-\delta)}=\frac{1}{1-\frac{\mu(1-\delta)}{\beta \sigma}}, \\
& \Leftrightarrow \quad \lambda_{0}^{c}=\frac{\beta \sigma(\rho-\alpha)}{\alpha[\beta \sigma-\mu(1-\delta)]}=\frac{\rho-\alpha}{\alpha\left[1-\frac{\mu(1-\delta)}{\beta \sigma}\right]} .
\end{aligned}
$$

Since $\mathscr{R}^{*}=\mathscr{R}^{* c}>1$ when $R_{0}=1$ and

$$
\left(Q_{1}+\mu\right)\left(\mu Q_{1}+R_{1}\right)-\left(\mu R_{1}+P_{1}\right)=\mu Q_{1}\left(\mu+Q_{1}\right)+(\epsilon+\rho)\left(1+R_{1}\right)>0
$$

the Routh-Hurwitz conditions assure us that all other eigenvalues of A have negative real parts when $0<\lambda_{0}(\gamma)<\frac{(\gamma+\rho+1)(\gamma+\rho+\gamma \rho)+\gamma(\rho-\alpha)}{\alpha \gamma}$.

Now, if we rewrite system (4.35) as

$$
\begin{aligned}
\dot{I} & =\beta(1-I) w_{i}-\mu I=f_{1}\left(I, u_{s}, v_{s}, w_{s}, u_{i}, v_{i}, w_{i}\right), \\
\dot{u}_{s} & =(1-I) w_{s}-u_{s}=f_{2}\left(I, u_{s}, v_{s}, w_{s}, u_{i}, v_{i}, w_{i}\right), \\
\dot{v}_{s} & =\alpha \lambda_{o}\left(u_{s}\left(1-u_{s}\right)+u_{i}\left(1-u_{i}\right)\right)+\alpha u_{s}-\rho v_{s}=f_{3}\left(I, u_{s}, v_{s}, w_{s}, u_{i}, v_{i}, w_{i}\right), \\
\dot{w}_{s} & =\gamma\left(v_{s}-w_{s}\right)=f_{4}\left(I, u_{s}, v_{s}, w_{s}, u_{i}, v_{i}, w_{i}\right), \\
\dot{u}_{i} & =\delta w_{i}+\sigma I w_{s}-u_{i}=f_{5}\left(I, u_{s}, v_{s}, w_{s}, u_{i}, v_{i}, w_{i}\right), \\
\dot{v}_{i} & =\rho\left(u_{i}-v_{i}\right)=f_{6}\left(I, u_{s}, v_{s}, w_{s}, u_{i}, v_{i}, w_{i}\right), \\
\dot{w}_{i} & =\epsilon\left(v_{i}-w_{i}\right)=f_{7}\left(I, u_{s}, v_{s}, w_{s}, u_{i}, v_{i}, w_{i}\right),
\end{aligned}
$$

then we have the following derivatives evaluated at $\left(0, u_{s}^{*}, v_{s}^{*}, w_{s}^{*}, 0,0,0, \lambda_{0}^{c}\right)$ : 


$$
\begin{aligned}
\frac{\partial^{2} f_{1}}{\partial I \partial w_{i}}\left(0, u_{s}^{*}, v_{s}^{*}, w_{s}^{*}, 0,0,0, \lambda_{0}^{c}\right) & =\frac{\partial^{2} f_{1}}{\partial w_{i} \partial I}\left(0, u_{s}^{*}, v_{s}^{*}, w_{s}^{*}, 0,0,0, \lambda_{0}^{c}\right)=-\beta, \\
\frac{\partial^{2} f_{2}}{\partial I \partial w_{s}}\left(0, u_{s}^{*}, v_{s}^{*}, w_{s}^{*}, 0,0,0, \lambda_{0}^{c}\right) & =\frac{\partial^{2} f_{2}}{\partial w_{s} \partial I}\left(0, u_{s}^{*}, v_{s}^{*}, w_{s}^{*}, 0,0,0, \lambda_{0}^{c}\right)=-1, \\
\frac{\partial^{2} f_{3}}{\partial u_{s}^{2}}\left(0, u_{s}^{*}, v_{s}^{*}, w_{s}^{*}, 0,0,0, \lambda_{0}^{c}\right) & =-2 \alpha \lambda_{0}^{c}, \\
\frac{\partial^{2} f_{3}}{\partial u_{i}^{2}}\left(0, u_{s}^{*}, v_{s}^{*}, w_{s}^{*}, 0,0,0, \lambda_{0}^{c}\right) & =-2 \alpha \lambda_{0}^{c}, \\
\frac{\partial^{2} f_{5}}{\partial I \partial w_{s}}\left(0, u_{s}^{*}, v_{s}^{*}, w_{s}^{*}, 0,0,0, \lambda_{0}^{c}\right) & =\frac{\partial^{2} f_{5}}{\partial w_{s} \partial I}\left(0, u_{s}^{*}, v_{s}^{*}, w_{s}^{*}, 0,0,0, \lambda_{0}^{c}\right)=\sigma, \\
\frac{\partial^{2} f_{3}}{\partial u_{s} \partial \lambda_{0}}\left(0, u_{s}^{*}, v_{s}^{*}, w_{s}^{*}, 0,0,0, \lambda_{0}^{c}\right) & =\alpha\left(1-2 u_{s}^{*}\right), \\
\frac{\partial^{2} f_{5}}{\partial u_{i} \partial \lambda_{0}}\left(0, u_{s}^{*}, v_{s}^{*}, w_{s}^{*}, 0,0,0, \lambda_{0}^{c}\right) & =\alpha,
\end{aligned}
$$

where

$$
\begin{aligned}
& u_{s}^{*}=v_{s}^{*}=w_{s}^{*}=\frac{\alpha\left(1+\lambda_{0}^{c}\right)-\rho}{\alpha \lambda_{0}^{c}}=1-\frac{1}{\mathscr{R}^{* c}}=\frac{\mu(1-\delta)}{\beta \sigma}, \\
& \lambda_{0}^{c}=\frac{\beta \sigma(\rho-\alpha)}{\alpha[\beta \sigma-\mu(1-\delta)]}=\frac{\rho-\alpha}{\alpha\left[1-\frac{\mu(1-\delta)}{\beta \sigma}\right]} .
\end{aligned}
$$

Now,

$$
\begin{aligned}
a & =\sum_{k, i, j=1}^{n} v_{k} w_{i} w_{j} \frac{\partial^{2} f_{k}}{\partial x_{i} \partial x_{j}}\left(x^{*}, \lambda_{0}^{c}\right)=-2 \beta v_{1} w_{1} w_{7}-2 v_{2} w_{1} w_{4}-2 \alpha \lambda_{0}^{c} v_{3}\left(w_{2}^{2}+w_{5}^{2}\right)+2 \sigma v_{5} w_{1} w_{4}, \\
b & =\sum_{k, i=1}^{n} v_{k} w_{i} \frac{\partial^{2} f_{k}}{\partial x_{i} \partial \lambda_{0}}\left(x^{*}, \lambda_{0}^{c}\right)=\alpha v_{3} w_{2}\left(1-2 u_{s}^{*}\right)+\alpha v_{3} w_{5} .
\end{aligned}
$$

Next, we determine a nonnegative right eigenvector $w=\left(w_{1}, w_{2}, w_{3}, w_{4}, w_{5}, w_{6}, w_{7}\right)^{T}$ and a left eigenvector $v=\left(v_{1}, v_{2}, v_{3}, v_{4}, v_{5}, v_{6}, v_{7}\right)^{T}$ that correspond to the simple eigenvalue of the matrix $A$. For the right eigenvector, we solve the system

$$
A w=0
$$


This system is the same as

$$
\begin{aligned}
-\mu w_{1}+\beta w_{7} & =0, \\
-u_{s}^{*} w_{1}-w_{2}+w_{4} & =0, \\
\left(\alpha \lambda_{0}^{c}\left(1-2 u_{s}^{*}\right)+\alpha\right) w_{2}-\rho w_{3}+\alpha \lambda_{0}^{c} w_{5} & =0, \\
w_{3}-w_{4} & =0, \\
\sigma u_{s}^{*} w_{1}-w_{5}+\delta w_{7} & =0, \\
w_{5}-w_{6} & =0, \\
w_{6}-w_{7} & =0 .
\end{aligned}
$$

By solving this system, we obtain the following form for the right eigenvector $\boldsymbol{w}=\left(w_{1}, w_{2}\right.$, $\left.w_{3}, w_{4}, w_{5}, w_{6}, w_{7}\right)^{T}:$

$$
\left(\begin{array}{l}
w_{1} \\
w_{2} \\
w_{3} \\
w_{4} \\
w_{5} \\
w_{6} \\
w_{7}
\end{array}\right)=\left(\begin{array}{c}
\frac{\beta}{\mu} w_{7} \\
W_{2}^{*} w_{7} \\
W_{3}^{*} w_{7} \\
W_{4}^{*} w_{7} \\
W_{5}^{*} w_{7} \\
W_{6}^{*} w_{7} \\
W_{7}^{*} w_{7}
\end{array}\right),
$$

where

$$
\begin{aligned}
& W_{2}^{*}=\frac{u_{s}^{*} \frac{\beta}{\mu}\left(\alpha \lambda_{0}^{c}\left(1-2 u_{s}^{*}\right)+\alpha\right)-\alpha \lambda_{0}^{c}}{\alpha \lambda_{0}^{c}\left(1-2 u_{s}^{*}\right)+\alpha-\rho}-u_{s}^{*} \frac{\beta}{\mu}, \\
& W_{3}^{*}=\frac{u_{s}^{*} \frac{\beta}{\mu}\left(\alpha \lambda_{0}^{c}\left(1-2 u_{s}^{*}\right)+\alpha\right)-\alpha \lambda_{0}^{c}}{\alpha \lambda_{0}^{c}\left(1-2 u_{s}^{*}\right)+\alpha-\rho}, \\
& W_{4}^{*}=W_{3}^{*}, \\
& W_{5}^{*}=W_{6}^{*}, \\
& W_{6}^{*}=W_{7}^{*}, \\
& W_{7}^{*}=1 .
\end{aligned}
$$


Note that

$\lambda_{0}^{c}=\frac{\rho-\alpha}{\alpha\left(1-\frac{\mu(1-\delta)}{\beta \sigma}\right)}=\frac{\rho-\alpha}{\alpha\left(1-u_{s}^{*}\right)} \Rightarrow \alpha \lambda_{0}^{c}=\frac{\rho-\alpha}{1-u_{s}^{*}}$ and $\alpha \lambda_{0}^{c}\left(1-2 u_{s}^{*}\right)+\alpha=\rho-(\rho-\alpha) \frac{u_{s}^{*}}{1-u_{s}^{*}}$.

Also note that $0<1-u_{s}^{*}<1$, since when $R_{0}=1$, $u_{s}^{*}=1-\frac{1}{\mathscr{R}^{*}} \Rightarrow 1-u_{s}^{*}=\frac{1}{\mathscr{R}^{*}}<1$. It is also worth noting that since we are in the right half plane of the bifurcation diagram, $A_{1}>0$.

Using these simplifications we obtain

$$
w=\left(\begin{array}{c}
w_{1} \\
w_{2} \\
w_{3} \\
w_{4} \\
w_{5} \\
w_{6} \\
w_{7}
\end{array}\right)=\left(\begin{array}{c}
\beta / \mu \\
A_{1} / u_{s}^{*} \\
\left(\beta u_{s}^{* 2}+A_{1} \mu\right) /\left(\mu u_{s}^{*}\right) \\
\left(\beta u_{s}^{* 2}+A_{1} \mu\right) /\left(\mu u_{s}^{*}\right) \\
1 \\
1 \\
1
\end{array}\right)
$$

We then compute $v$ such that $v \cdot w=1$. To this end, we obtain

$$
v=\left(\begin{array}{c}
v_{1} \\
v_{2} \\
v_{3} \\
v_{4} \\
v_{5} \\
v_{6} \\
v_{7}
\end{array}\right)=\left(\begin{array}{c}
0 \\
0 \\
v_{3} \\
v_{3} \\
v_{5} \\
0 \\
0
\end{array}\right)=\left(\begin{array}{c}
0 \\
0 \\
\left(\beta \sigma w_{3}\right) /\left(2\left(2 \beta \sigma w_{3}^{2}+\alpha \lambda_{0}^{c} \mu\left(w_{2}^{2}+1\right)\right)\right) \\
\left(\beta \sigma w_{3}\right) /\left(2\left(2 \beta \sigma w_{3}^{2}+\alpha \lambda_{0}^{c} \mu\left(w_{2}^{2}+1\right)\right)\right) \\
\left(\beta \sigma w_{3}^{2}+\alpha \lambda_{0}^{c} \mu\left(w_{2}^{2}+1\right)\right) /\left(2 \beta \sigma w_{3}^{2}+\alpha \lambda_{0}^{c} \mu\left(w_{2}^{2}+1\right)\right) \\
0 \\
0
\end{array}\right)
$$

With the vectors $w$ and $v$, we obtain

$$
\begin{aligned}
a & =-2 \alpha \lambda_{0}^{c}\left(\frac{A_{1}^{2}}{u_{s}^{* 2}}+1\right) v_{3}+2 \frac{\sigma}{\mu} w_{3} v_{5}=\frac{\beta \sigma\left(\beta u_{s}^{* 2}+\mu A_{1}\right)}{2 \mu^{2} u_{s}^{*}}, \\
b & =\alpha\left(\left(1-2 u_{s}^{*}\right) w_{2}+w_{5}\right) v_{3}=\alpha\left(\frac{A_{1}}{u_{s}^{*}}-2 \frac{A_{1}}{u_{s}^{*}} u_{s}^{*}+1\right) v_{3} \\
& =\alpha\left(\frac{A_{1}}{u_{s}^{*}}-A_{1}-A_{1}+1\right) v_{3}=\frac{\alpha}{u_{s}^{*}}\left(A_{1}\left(1-u_{s}^{*}\right)+\left(1-A_{1}\right) u_{s}^{*}\right) v_{3} .
\end{aligned}
$$


Clearly, $a>0$. Since $0<u_{s}^{*}<1$ and $1-A_{1}>0, b>0$. Hence, the model exhibits a backward bifurcation since both $a$ and $b$ are strictly positive.

Finally, by using the existence condition for endemic equilibrium solutions to our system, we derive the critical value of $R_{0}$ below which only a stable disease-free equilibrium solution exists and above which three equilibrium solutions, a locally stable disease-free equilibrium, a locally stable endemic equilibrium, and an unstable second endemic equilibrium exist. Considering the third and fourth quadrants of the bifurcation diagram, $A_{2}<0$ and $A_{1}$ can either be positive, zero or negative and we can either have no endemic equilibrium solution, one equilibrium solution or two endemic equilibrium solutions. For instance, for $A_{1}>0$, when $\Delta<0$, there is no endemic equilibrium solution, when $\Delta=0$, there is a single endemic equilibrium solution, and when $\Delta>0$, there are two endemic equilibrium solutions. Therefore by setting $\Delta=0$, we can obtain the critical value of $R_{0}$, namely, $R_{0}^{c}<1$ below which we have no endemic equilibrium solution and above which we have two endemic equilibrium solutions.

Now,

$$
\Delta=0 \Leftrightarrow \frac{\left[\beta\left(\alpha \lambda_{0} \sigma-\rho(1-\delta)\right)\right]^{2}+\left[2 \alpha \lambda_{0} \mu(1-\delta)\right]^{2}\left(R_{0}^{c}-1\right)}{\left(\alpha \beta \lambda_{0} \sigma\right)^{2}}=0 .
$$

This yields

$$
R_{0}^{c}=1-\xi
$$

where

$$
\xi=\left(\frac{\beta\left(\alpha \lambda_{0} \sigma-\rho(1-\delta)\right)}{2 \alpha \lambda_{0} \mu(1-\delta)}\right)^{2}
$$

Clearly, $\xi \geq 0$. Note that $\xi<1$ if

$$
\lambda_{0}<\frac{\beta \rho(1-\delta)}{\alpha[\beta \sigma-2 \mu(1-\delta)]}=\frac{\rho(1-\delta)}{\alpha \sigma\left[1-2 \frac{\mu(1-\delta)}{\beta \sigma}\right]}=\frac{\beta \rho\left[\frac{\mu(1-\delta)}{\beta \sigma}\right]}{\alpha \mu\left[1-2 \frac{\mu(1-\delta)}{\beta \sigma}\right]} .
$$

When $0<\xi<1, R_{0}^{c}<1$ and

1. when $R_{0}<R_{0}^{c}$, there exists a stable disease-free equilibrium;

2. when $R_{0}^{c}<R_{0}<1$, there exists two endemic equilibrium solutions, one of which is stable and a locally stable disease-free equilibrium solution. 


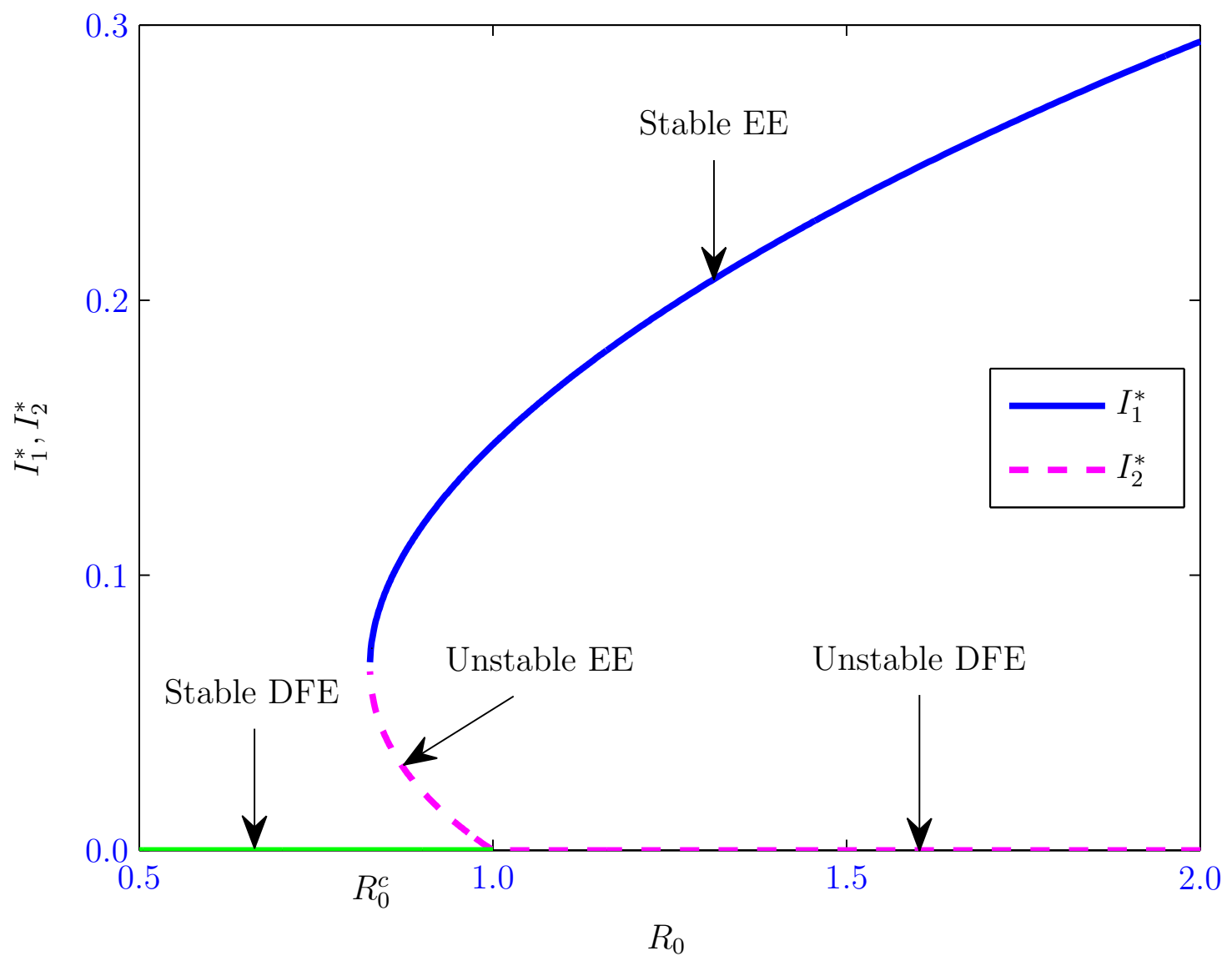

Figure 5.20. Plot depicting the existence of both a backward (subcritical) bifurcation and a forward (supercritical) bifurcation. There is a critical value $R_{0}^{c}=$ 0.83 of $R_{0}$ such that for $R_{0}<R_{0}^{c}$, there exists a unique stable disease-free equilibrium (solid green line to the left of $R_{0}=R_{0}^{c}$ ). For $R_{0}^{c}<R_{0}<$ 1 , there exists a stable disease-free equilibrium (solid green line segment between $R_{0}=R_{0}^{c}$ and $R_{0}=1$ ), a stable endemic equilibrium (solid blue line between $R_{0}=R_{0}^{c}$ and $R_{0}=1$ ), and an unstable endemic equilibrium (dashed magenta line between $R_{0}=R_{0}^{c}$ and $R_{0}=1$ ). Finally, for $R_{0}>1$, there exists a stable endemic equilibrium (solid blue line segment to the right of $R_{0}=1$ and an unstable disease-free equilibrium denoted by the dashed magenta line segment to the right of $R_{0}=1$ ). DFE represents disease-free equilibrium and EE represents endemic equilibrium. 


\subsection{Effects of Birth Rate Function}

Different recruitment or birth rate functions have been used in the biological and ecological literature to quantify birth processes. These include simple constant birth rates, linear birth rates, the Verhulst-Pearl logistic birth rate function [18-20], the Beverton-Holt birth rate function [21], the Ricker birth rate function [22], the Hassell birth rate function [23], and the Maynard-Smith-Slatkin birth rate function [24, 25]. The birth rate function used to model a specific biological or ecological process has a profound effect on the dynamics of the model. Some birth rate functions can lead to oscillatory dynamics. In this case, the size of the amplitude and perhaps period of oscillations may be different for different birth rate functions used to model the same process. In ecological processes, competition for sustainable resources among inhabitants of a community can lead to nonlinearities in the dynamics of the process. Such competition can be referred to as scramble or contest when individuals within the same species compete for the same resource [26, 27]. Scramble competition involves a situation in which the available resources are apportioned equally between the individuals involved. Generally, the resources are not sufficient to satisfy individual needs of the entire population, and in extreme situations, all individuals or species may become extinct. In contrast, contest competition is a "survival of the strongest" situation, in which the species or individuals that emerge victorious in the encounter can acquire the required amount of resources, while the losers may acquire little or nothing. By assuming that female Anopheles mosquitoes can always find humans to feed on, it is reasonable to consider the competition between female Anopheles mosquitoes for human blood as contest, whereby those that succeed to acquire human blood proceed to reproduce, whereas those that do not succeed to acquire blood meals are assumed to be killed. While the Verhulst-Pearl logistic birth rate function is suitable to modeling contest competition, the Maynard-Smith-Slatkin birth rate function is suitable for modeling both scramble and contest competition.

The analysis presented in this Part uses the Verhulst-Pearl logistic birth rate function (4.21) to model mosquito birth. Here, we compare the size of the amplitudes of the oscillations for two different birth rate functions, the Verhulst-Pearl logistic birth rate function and the Maynard-Smith-Slatkin birth rate function. The Maynard-Smith-Slatkin birth rate 
function is given by

$$
\lambda_{v}(\vartheta)=\frac{\vartheta}{1+\left(\frac{\vartheta}{L}\right)^{n}},
$$

where $\vartheta=S_{u}, I_{u}$ and $n$ is a constant. Note that the Verhulst-Pearl logistic birth rate function is an approximation to the Maynard-Smith-Slatkin birth rate function. Replacing the Verhulst-Pearl logistic birth rate function in system (4.22) by equation (5.44) and scaling the system appropriately, we obtain the following system:

$$
\begin{aligned}
\dot{I} & =\beta(1-I) w_{i}-\mu I \\
\dot{u}_{s} & =(1-I) w_{s}-u_{s} \\
\dot{v}_{s} & =\alpha \lambda_{0}\left(\frac{u_{s}}{1+u_{s}^{n}}+\frac{u_{i}}{1+u_{i}^{n}}\right)+\alpha u_{s}-\rho v_{s} \\
\dot{w}_{s} & =\gamma\left(v_{s}-w_{s}\right) \\
\dot{u}_{i} & =\delta w_{i}+\sigma I w_{s}-u_{i} \\
\dot{v}_{i} & =\rho\left(u_{i}-v_{i}\right) \\
\dot{w}_{i} & =\epsilon\left(v_{i}-w_{i}\right) .
\end{aligned}
$$

For the same parameter regime, system (5.45) produces periodic oscillations with higher amplitudes than system (4.35). For example, for $\gamma=0.7849, \lambda_{0}=14.775, \epsilon=0.9, L=5 \times$ $10^{3}, N_{h}=10^{5}, n=50$, and the other parameters as in Table 5.2, system (5.45) yields periodic solutions with an approximate amplitude of 0.15 while system (4.35) yields periodic solutions with an approximate amplitude of 0.0001. See Figs. 5.21 and 5.22 for illustrations. In dimensional terms, these numbers correspond to 1,500 and 10 infectious humans, respectively. The fact that system (5.45) produces more sustained higher amplitude periodic oscillations than system (5.45) implies that it is more appropriate for modeling biological, epidemiological and ecological birth processes. Hence, the Maynard-Smith-Slatkin birth rate function seems to be a better function for quantifying birth processes. With the Maynard-Smith-Slatkin function, $n$ also plays an important role in disease dynamics. System (5.45) exhibits sustained periodic oscillations for $n \geq 4$ and non-oscillatory stable equilibrium solutions for $n<4$. 


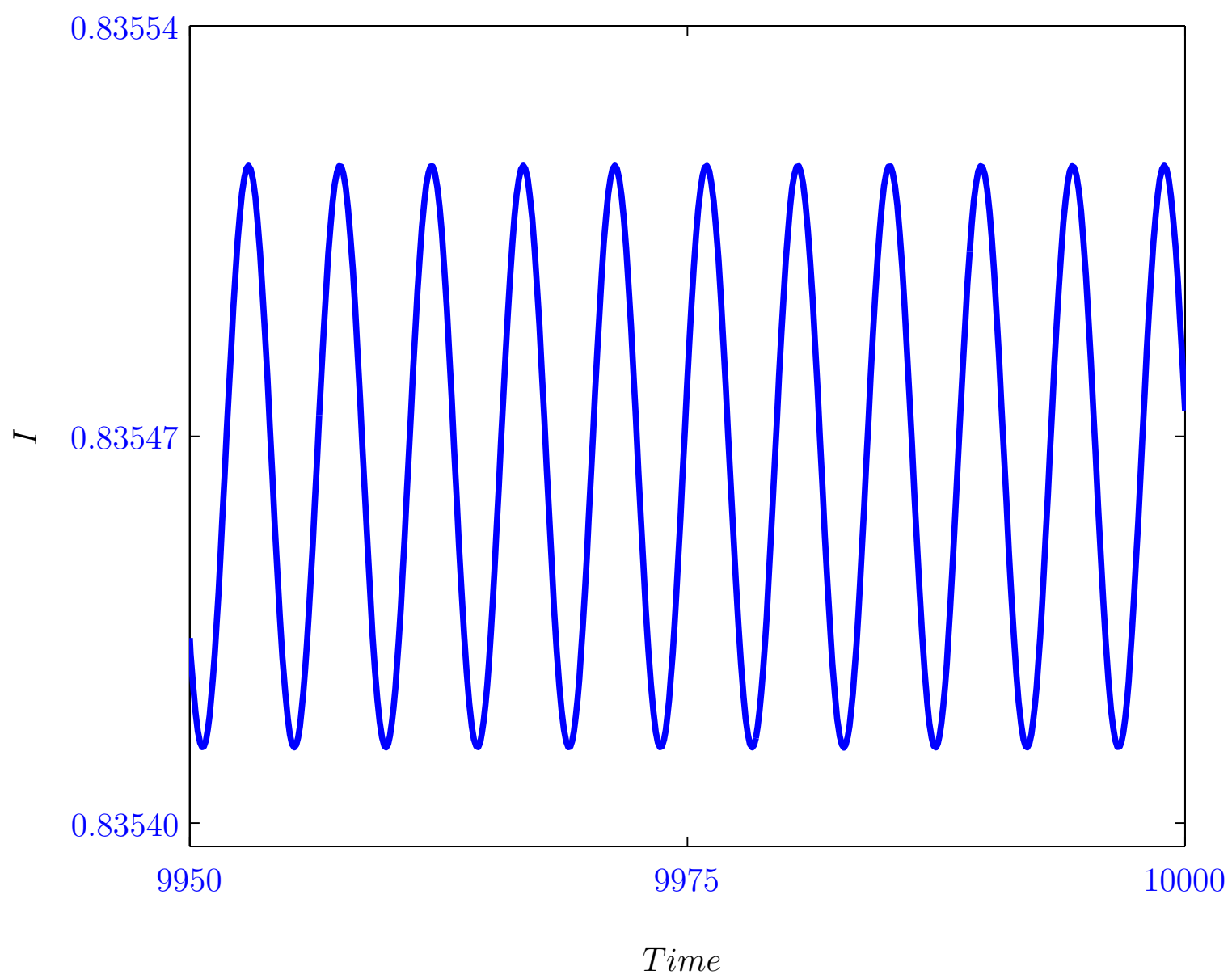

Figure 5.21. Illustration of the effect of the Verhulst-Pearl birth rate functions on the dynamics of system (4.35). The amplitude of the oscillations is 0.0001. But since $N_{h}=100,000$, the amplitude corresponds to 10 infectious human. The period is approximately 5 days. 


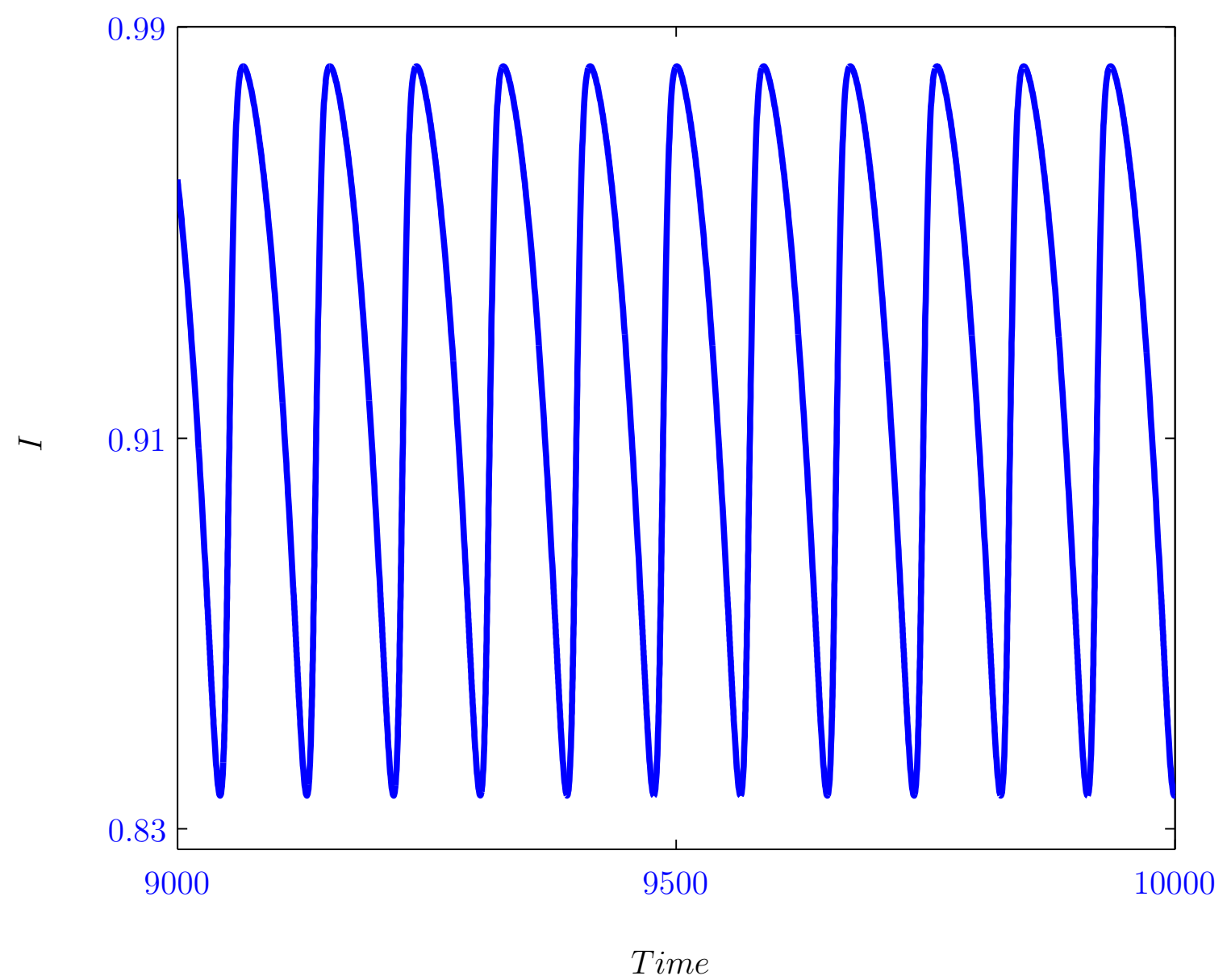

Figure 5.22. Illustration of the effect of the Maynard-Smith-Slatkin birth rate function on the dynamics of system (5.45). The amplitude of the oscillations is 0.15 . For $N_{h}=100,000$, the amplitude corresponds to 1,500 infectious humans. 


\subsection{Conclusion}

We have applied analytical and numerical techniques to study the SIS model for the dynamics of malaria transmission described in Chapter 4. In the absence of the disease, we identified a threshold parameter $\mathscr{R}^{*}$ that is linked to mosquito demography. The diseasefree model always has a trivial equilibrium solution which is linearly stable when $\mathscr{R}^{*} \leq 1$ and unstable otherwise. When $\mathscr{R}^{*}>1$, a non-trivial equilibrium solution co-exists with the trivial equilibrium solution. We identified a critical value $\lambda_{0}^{c}$ of $\lambda_{0}$ and showed that the non-trivial equilibrium solution is stable when $0<\lambda_{0}<\lambda_{0}^{c}$ and unstable with the emergence of periodic solutions when $\lambda_{0}>\lambda_{0}^{c}$. A Hopf bifurcation occurs at $0<\lambda_{0}=\lambda_{0}^{c}$.

In the presence of the disease, we calculated a new basic reproduction number for malaria and used it to explore the existence and stability of disease-free and endemic equilibria. We showed that the system has an unstable trivial equilibrium solution and a non-trivial diseasefree equilibrium solution. Conditions for the stability of the disease-free equilibrium solution were derived and, as in the disease-free model, we showed that the full model also exhibits a Hopf bifurcation. We derived conditions under which the model can have two, one or no endemic equilibrium solutions and also showed that there is a parameter space within which the model exhibits a backward bifurcation. We also demonstrated that the MaynardSmith-Slatkin birth rate function can lead to oscillatory dynamics with higher periods than the Verhulst-Pearl logistic function. Hence, the Maynard-Smith-Slatkin function is more suitable to modeling the population dynamics of the malaria vector under our framework.

The discovery of oscillatory dynamics in the analysis of the model indicates that we have been able to capture natural oscillations known to exist in mosquito dynamics and hence malaria prevalence without recourse to external seasonal forcing. This oscillatory dynamics provides a plausible framework for designing and implementing malaria control strategies. For example, an efficient strategy will be to apply control measures when the mosquito population is at its minimum amplitude. Applying control strategies when the mosquito population is at its peak amplitude will require substantial effort, otherwise only a portion

of the mosquito population will be suppressed and the population will eventually recover when conditions become favorable. 


\section{References}

[1] G. Ngwa, "On the Population Dynamics of the Malaria Vector," Bulletin of Mathematical Biology 68, 2161-2189 (2006).

Online Version 95, 120

[2] O. Diekmann, J. A. P. Heesterbeek, and J. A. J. Metz, "On the Definition and the Computation of the Basic Reproduction Ratio $R_{0}$ in Rodels for Infectious Diseases in Heterogeneous Populations," Journal of Mathematical Biology 28, 365-382 (1990).

Online Version 102

[3] P. van den Driessche and J. Watmough, "Reproduction Numbers and Sub-threshold Endemic Equilibria for Compartmental Models of Disease Transmission," Mathematical Biosciences 180, 29-48 (2002).

Online Version 137

[4] G. A. Ngwa and W. S. Shu, "A Mathematical Model for Endemic Malaria with Variable Human and Mosquito Populations," Mathematical and Computer Modelling 32, 747 $763(2000)$.

Online Version 120

[5] J. A. P. Heesterbeek, "A Brief History of $R_{0}$ and a Recipe for its Calculation," Acta Biotheoretica 50, 189-204 (2002).

Online Version

[6] J. M. Heffernan, R. J. Smith, and L. M. Wahl, "Perspectives on the Basic Reproductive 
Ratio," Journal of Royal Society Interface 2, 281-293 (2005).

Online Version

[7] M. Teboh-Ewungkem, C. Podder, and A. Gumel, "Mathematical Study of the Role of Gametocytes and an Imperfect Vaccine on Malaria Transmission Dynamics," Bulletin of Mathematical Biology 72, 63-93 (2010).

Online Version 102

[8] D. L. Smith, F. E. McKenzie, R. W. Snow, and S. I. Hay, "Revisiting the Basic Reproductive Number for Malaria and its Implications for Malaria Control," PLoS Biology 5 (3), e42: 0531-0542 (2007).

Online Version 102

[9] Central Intelligence Agency, "Country Comparison: Life Expectancy at Birth," The world fact book (Assessed April 2011).

Online Version 120

[10] M. I. Teboh-Ewungkem and T. Yuster, "A Within-vector Mathematical Model of Plasmodium Falciparum and Implications of Incomplete Fertilization on Optimal Sametocyte Sex Ratio." Journal of Theoretical Biology 264, 273-286 (2010).

Online Version 120

[11] W. Huang, K. L. Cooke, and C. Castillo-Chavez, "Stability and Bifurcation for a Multiple-group Model for the Dynamics of HIV/AIDS Transmission," SIAM Journal on Applied Mathematics 52, 835-854 (1992).

Online Version 137

[12] J. Dushoff, W. Huang, and C. Castillo-Chavez, "Backward Bifurcations and Catastrophe in Simple Models of Fatal Diseases," Journal of Mathematical Biology 36, 36:227-248 (1998).

Online Version 137

[13] C. M. Kribs-Zaleta and J. X. Velasco-Hernndez, "A Simple Vaccination Model with 
Multiple Endemic States," Mathematical Biosciences 164, 183- 201 (2000).

Online Version

[14] M. Martcheva and H. R. Thieme, "Progression Age Enhanced Backward Bifurcation in an Epidemic Model with Super-infection," Journal of Mathematical Biology 46, 385424 (2003).

Online Version

[15] C. Castillo-Chavez and B. Song, "Dynamical Models of Tuberculosis and their Applications," Mathematical Biosciences and Engineering 1 (2), 361-404 (2004).

Online Version 137

[16] H. Gómez-Acevedo and M. Y. Li, "Backward Bifurcation in a Model for HTLV-I Infection of CD4+ T Cells," Bulletin of Mathematical Biology 67, 101-114 (2005).

Online Version

[17] T. C. Reluga, J. Medlock, and A. S. Perelson, "Backward Bifurcations and Multiple Equilibria in Epidemic Models with Structured Immunity," Journal of Theoretical Biology 252 (1), 155-165 (2008).

Online Version 137

[18] R. Pearl and L. J. Reed, "On the Rate of Growth of the Population of the United States since 1970 and its Mathematical Interpretation," Proceedings of the National academy of Sciences 6, 275-288 (1920). 146

[19] P. F. Verhulst, "Notice sur la Loi que la Population suit dans son Accroissement," Correspondence Mathématique et Physique 10, 113-121 (1938).

[20] K. Johst, A. Berryman, and M. Lima, "From Individual Interactions to Population Dynamics: Individual Resource Partitioning Simulation Exposes the Causes of Nonlinear Intra-specific Competition," Population Ecology 50, 79-90 (2008).

Online Version 146

[21] R. J. H. Beverton and S. J. Holt, "On the Dynamics of Exploited Fish Populations," 
Reviews in Fish Biology and Fisheries 4, 259-260 (1994).

Online Version 146

[22] W. E. Ricker, "Stock and Recruitment," Journal of the Fisheries Research Board of Canada 11, 559-623 (1954). 146

[23] M. P. Hassell, "Desity-dependence in Single Species Populations," Journal of Animal Ecology 44, 283-295 (1975). 146

[24] J. Maynard-Smith and M. Slatkin, "The Stability of Predator-prey Systems," Ecology 54, 384-391 (1973).

Online Version 146

[25] J. Maynard-Smith, Models in Ecology (Cambridge University Press, Cambridge, England, 1974). 146

[26] S. R.-J. Jang, "Contest and Scramble Competition with a Dynamic Resource," Nonlinear Analysis 63, e109-e118 (2005), Invited Talks from the Fourth World Congress of Nonlinear Analysts (WCNA 2004).

Online Version 146

[27] Å. Brännström and D. J. T. Sumpter, "The Role of Competition and Clustering in Population Dynamics," Proceedings of the Royal Society B 272, 2065-2072 (2005). Online Version 146 
$\overline{\text { Chapter }} 6$

\section{Comparison of the New SIS Model with the Classical Ross-Macdonald Malaria Model, General Conclusions and Open Questions}

\subsection{Intoduction}

In this Chapter, we compare the classical SIS Ross-Macdonald model derived and studied in Chapter 3 with the SIS malaria model derived in Chapter 4 and analyzed in Chapter 5. We show that the new model is an improvement on the classical model, provide a general conclusion to Part I, and present some open questions and possible areas for future research.

\subsection{Comparison of Model (4.22) to the Classical Ross SIS Model}

We now examine how the simple Ross-type malaria model compares to the new simplified model (4.22) and how the Ross-type model can be recovered from the simplified model (4.22). To this effect, we set $p_{1}=1$ and $q=1$, or $p_{1} \beta_{h}=\beta_{h}$ and $q \beta_{v}=\beta_{v}$, which accounts for the proportion of successful contacts. Also, we set $I_{m}=I_{u}+I_{v}+I_{w}($ fed + questing + breeding site infectious mosquitoes) and $S_{m}=S_{u}+S_{v}+S_{w}$ (fed + questing + breeding site susceptible mosquitoes) where $I_{m}$ and $S_{m}$ denote infectious and susceptible mosquitoes, respectively. Additionally, we take $\lambda_{v}(\vartheta) \vartheta$, where $\lambda_{v}$ is as defined in (4.21) to be a constant, $\lambda_{0}$. This simplifies system (4.22) to the following system: 


$$
\begin{aligned}
\dot{S}_{h} & =\mu_{h} N_{h}+r_{h} I_{h}-\beta_{h} S_{h} I_{w}-\mu_{h} S_{h}, \\
\dot{I_{h}} & =\beta_{h} S_{h} I_{w}-\left(r_{h}+\mu_{h}\right) I_{h}, \\
\dot{S_{m}} & =a_{v} \lambda_{0}-\beta_{v} S_{w} I_{h}-\mu_{v} S_{m}, \\
\dot{I_{m}} & =\beta_{v} S_{w} I_{h}-\mu_{v} I_{m} .
\end{aligned}
$$

Let $N_{h}$ and $N_{w}$ be the total human and questing mosquito populations, respectively. Using $S_{h}=N_{h}-I_{h}$ and $S_{w}=N_{w}-I_{w}$, we can drop the equations for $S_{h}$ and $S_{m}$ from system (6.1) and have

$$
\begin{aligned}
\dot{I_{h}} & =\beta_{h}\left(N_{h}-I_{h}\right) I_{w}-\left(r_{h}+\mu_{h}\right) I_{h}, \\
\dot{I_{m}} & =\beta_{v}\left(N_{w}-I_{w}\right) I_{h}-\mu_{v} I_{m} .
\end{aligned}
$$

In our model, $\beta_{h}\left(N_{h}-I_{h}\right) I_{w}$ models the contact between infectious questing female mosquitoes and susceptible humans, while in the Ross-type model, such a term is the contact made between mosquitoes and susceptible humans. Likewise, $\beta_{v}\left(N_{w}-I_{w}\right) I_{h}$ models the contact between susceptible questing female mosquitoes and infectious humans, while such a term is the contact made between mosquitoes and infectious humans in the Ross-type model. Note that because the total human population in both models is constant, the incidence functions in both our model and the classical Ross-Macdonald model are comparable.

In Ross-type models, $S_{u}+I_{u}$ is called $N_{v}$, and $S_{w}$ is $S_{v}$. In model (4.22), the demography of the mosquito is important. Only those female mosquitoes that need to feed and are questing are involved in disease transmission, and they are appropriately accounted for. Additionally, only breeding vectors populate the susceptible class. This is true for fed vectors and breeding site vectors. The novelty is especially visible when control is taken into consideration. This is because with model (4.22), we can say how it will impact the model dynamics when the questing, breeding site, or even the fed mosquitoes are reduced.

The basic reproduction number provides another comparison between the Ross's model and model (4.22). The basic reproduction number for the Ross's model is

$$
R_{0}^{\text {classical }}=\frac{\beta_{h} \beta_{v} N_{h} N_{v}}{\mu_{v}\left(r_{h}+\mu_{h}\right)}
$$


while the basic reproduction number for model (4.22) is

$$
R_{0}=\frac{\beta_{v} \beta_{h} N_{h} S_{w}^{*}}{\left(r_{h}+\mu_{h}\right)} \cdot \frac{q a_{v} \alpha_{v}\left(N_{h}\right)}{\left(\left(a_{v}+\mu_{v}\right)\left(\mu_{v}+\alpha_{v}\left(N_{h}\right)\right)\left(\mu_{v}+\beta_{h} N_{h}\right)-p_{1} a_{v} \alpha_{v}\left(N_{h}\right) \beta_{h} N_{h}\right)},
$$

which can be rewritten as

$$
R_{0}=\frac{\beta_{v} \beta_{h} N_{h} S_{w}^{*}}{\mu_{v}\left(r_{h}+\mu_{h}\right)} \cdot q \cdot \frac{a_{v} \alpha_{v}\left(N_{h}\right)}{a_{v} \alpha_{v}\left(N_{h}\right)\left(1+\frac{\left(1-p_{1}\right) \beta_{h} N_{h}}{\mu_{v}}\right)+\left(\mu_{v}+\beta_{h} N_{h}\right)\left(a_{v}+\alpha_{v}\left(N_{h}\right)+\mu_{v}\right)} .
$$

It is evident that $R_{0}<R_{0}^{\text {classical }}$ since

$$
0<q<1 \text { and } 0<\frac{a_{v} \alpha_{v}\left(N_{h}\right)}{a_{v} \alpha_{v}\left(N_{h}\right)\left(1+\frac{\left(1-p_{1}\right) \beta_{h} N_{h}}{\mu_{v}}\right)+\left(\mu_{v}+\beta_{h} N_{h}\right)\left(a_{v}+\alpha_{v}\left(N_{h}\right)+\mu_{v}\right)}<1 .
$$

Additionally, since $S_{w}^{*} \leq N_{m v}$, the basic reproduction number of model (4.22) is less than or equal to the basic reproduction number of the classical SIS (Ross-type) model. Hence, our model brings out an important scaling factor for the $R_{0}$ obtained from the classical SIS model so that $R_{0} \leq \varrho R_{0}^{\text {classical }}$, where $\varrho<1$. Note that unlike the Ross-type model, whose basic reproduction number involves the total vector population, the basic reproduction number of model (4.22) involves the equilibrium value of the susceptible questing vectors, which are the only proportion of vectors that can become infected. Hence, $R_{0}$ can be reduced by regulating the rate at which susceptible female vectors visit human habitats to quest for blood meals.

The analysis of model (4.22) indicates that the model captures the natural oscillations known to occur in mosquito populations. These oscillations lead to oscillations in the malaria dynamics and thus the entire system oscillates, which is not captured by Ross-type models without recourse to external forcing by means of periodic contact rates, delays, etc. These oscillations provide a plausible framework for designing and implementing malaria intervention and control measures, especially measures that are related to mosquito control.

\subsection{Synopsis and Conclusion}

In this Part, we have developed a new SIS model for endemic malaria that explicitly integrates the demography of the mosquito vector that transmits the disease together with its interaction with the human population - and incorporates the disease dynamics. The 
general model is an improvement on the classical Ross-Macdonald SIS model and provides a new framework for modeling malaria transmission. A simplification of the derived model, which is comparable to but still an improvement on the classical Ross-Macdonald SIS model is used to study the dynamics of the malaria disease within a closed human population. In summary, the main differences and improvement of our model over the Ross-type model are:

1. Mosquitoes involved and accounted for in disease transmission are only the female mosquitoes that need to feed and thus are questing for a human blood meal.

2. The vector demography is emphasized and, therefore, the impact of malaria control on the vector populations, fed and questing, resting and breeding, or newly emerging can be studied appropriately and accounted for.

3. The basic reproduction number, $R_{0}$, computed for the new model is smaller than that computed for the Ross-Macdonald SIS model, since not all mosquitoes take part in the transmission process, a fact that has not been quantified in previous models.

4. Two threshold parameters, both of which can be used for control purposes, have been identified. One of these parameters is directly linked to the demography of the vector and the other is linked to the disease dynamics. The difficulty in eradicating malaria may therefore lie in the complicated life style of the malaria parasite; that is, in its ability to share its life cycle in the mosquito and in the human. Thus, model (4.22) is an improvement on the Ross model and provides important areas for malaria control.

5. The analysis of model (4.22) captures natural fluctuations as well as presents a new route to periodic oscillations in the dynamics of malaria transmission without recourse to external seasonal forcing and/or delays, a feature which the Ross-type model fails to capture unless some external forcing term is introduced.

In addition to the results highlighted in the discussion of the differences between model (4.22) and the Ross-type models, a detailed analytical and numerical exploration of model (4.22) yields the following results in the absence and in the presence of the malaria disease: 
In the absence of the malaria disease, we presented and discussed conditions for the existence and stability of a realistic non-trivial equilibrium solution and also conditions for the existence of a Hopf bifurcation and hence oscillatory solutions.

In the presence of the malaria disease, we have carried out the following analysis:

1. Demonstrated the existence and stability of both a trivial and a disease free steady state, as well as derived conditions for the existence of a Hopf bifurcation. Instability of the trivial steady state $E_{0}$ is reasonable from a physical stand point, which rules out the possibility of permanently eradicating the mosquito population.

2. Captured natural fluctuations known to occur in the mosquito population dynamics without external seasonal forcing or delays. These fluctuations led to fluctuations in the malaria disease dynamics and thus presented a new route to periodic oscillations and a plausible framework for developing and implementing control strategies.

3. Derived conditions that guarantee the existence and stability of endemic equilibria. When $R_{0}>1$, we have one endemic equilibrium. However, when $R_{0}<1$, there are conditions for which there exist no endemic equilibrium, one endemic equilibrium, or two endemic equilibria, which may lead to a backward bifurcation.

4. Applied techniques described in Refs. [1-3] to completely characterize the backward bifurcation that arises when $R_{0}<1$. The occurrence of a backward bifurcation indicates that simply reducing $R_{0}$ below unity is not enough to eradicate the disease. That is, the disease can still establish itself within the population even when $R_{0}<1$. We thus derived a critical value $R_{0}^{c}$ of $R_{0}$ that is also less than unity. The disease can be eradicated when $R_{0} \leq R_{0}^{c}$ and can persist when $R_{0}>R_{0}^{c}$.

The most striking result of our analysis is the highly multidimensional facets that seem to characterize the lives of the malaria mosquito population, the agents involved in transmitting the malaria parasite that causes malaria. This presents a significant challenge for malaria control and an opportunity for research in order to understand what this might mean for malaria control. In particular, control schemes need to be well thought out to capture all three phases of the mosquito population. Consequently, a desirable control scheme should be 
one that targets each of the three stages of the mosquito population. It should seek to limit contact between questing mosquitoes and humans and disrupt breeding sites or move them further from human habitats. In fact, from the re-interpreted basic reproduction number, as the carrying capacity of the breeding sites is halved, the basic reproduction number is also halved and thus can drive $R_{0}$ from a value greater than one to a value less than one.

We have thus accomplished the task of addressing the five goals stated in the introduction. We captured the natural oscillations that are known to exist in the prevalence of malaria without recourse to external seasonal forcing, redefined and calculated a new value for the basic reproduction number for malaria, presented a possible interpretation of why it is difficult to eradicate malaria, and have compared the new SIS model with the original Ross SIS type malaria model. Additionally, we identified a parameter regime within which our model exhibits a Hopf bifurcation and another regime where a backward bifurcation occurs.

Our model indicates that to fully understand the dynamics of indirectly transmitted diseases such as malaria fever, dengue fever, yellow fever, Leishmaniasis, etc., mathematical models should not be developed in isolation, but should incorporate the demography of the vectors or arthropods that transmit the agent that cause these diseases. We have therefore presented a framework that researchers can build upon and apply to other indirectly transmitted diseases. We hope that it provides information that might lead to eradicating malaria and other vector-borne diseases, or significantly reducing their burden on humans.

\subsection{Possible Extensions and Areas for Future Research}

From the analysis of model (4.22), it is evident that there are many areas that require further development and areas of possible extensions. Additionally, there are some open questions that can be investigated. We now present some of these.

1. The mathematical analysis of the stability results of the endemic steady states needs further development. In our brief exploration of the endemic steady states, a number of issues became evident. It is evident that there is a richer dynamics in the region in parameter space where the conditions of remark 5.3.6 hold or fail and understanding 
what this means mathematically is of importance. Additionally, exploring the dynamical behavior of the disease in the limit as $R_{0} \rightarrow 1$ may lead to interesting results.

2. Figure 5.7.3 shows four regions for the threshold parameters $\mathscr{R}^{*}$ and $R_{0}$ as the parameter $\lambda_{0}$ is varied. The dynamics of malaria in each of these regions may expose interesting features about malaria dynamics and offer potentially useful information towards malaria eradication. Therefore, this is worth investigating.

3. We have modeled the fact that a mosquito takes a bite from a human as a Bernoulli random variable - success or failure. However, it is possible that a mosquito may fail to take a blood meal and then try again and again. It will be interesting to see how this aspect affects the disease dynamics.

4. Another open question is the exploration of the possibility of chaotic dynamics. Does it occur, and if so, under what conditions?

5. It is well known that the most general form of a compartmental model for the dynamics of malaria transmission is an SEIRS-type model $[4,5]$. However, we used a simple SIS model. This was because we wanted to illustrate the novelty in our approach as well as acquire a deeper understanding of the associated dynamics and biological significance to malaria. Now that we have a sound understanding of this new framework and the role it can play in malaria control, it is necessary to extend it to capture and address more realistic features of the biology and dynamics of malaria transmission. Hence, it is necessary to develop and analyze deterministic and stochastic SEIRS models for malaria and other indirectly transmissible diseases that incorporate the following:

(a) disease-induced mortalities, since they are significant in endemic regions,

(b) vector demography and all the aquatic life stages of the malaria vector,

(c) multiple human habitats and multiple vector breeding sites. 


\section{References}

[1] W. Huang, K. L. Cooke, and C. Castillo-Chavez, "Stability and Bifurcation for a Multiple-group Model for the Dynamics of HIV/AIDS Transmission," SIAM Journal on Applied Mathematics 52, 835-854 (1992).

Online Version 159

[2] P. van den Driessche and J. Watmough, "Reproduction Numbers and Sub-threshold Endemic Equilibria for Compartmental Models of Disease Transmission," Mathematical Biosciences 180, 29-48 (2002).

Online Version

[3] C. Castillo-Chavez and B. Song, "Dynamical Models of Tuberculosis and their Applications," Mathematical Biosciences and Engineering 1 (2), 361-404 (2004).

Online Version 159

[4] G. A. Ngwa and W. S. Shu, "A Mathematical Model for Endemic Malaria with Variable Human and Mosquito Populations," Mathematical and Computer Modelling 32, 747 763 (2000).

Online Version 161

[5] N. Chitnis, J. Hyman, and J. Cushing, "Determining Important Parameters in the Spread of Malaria through the Sensitivity Analysis of a Mathematical Model," Bulletin of Mathematical Biology 70, 1272-1296 (2008).

Online Version 161 


\section{Part II}

\section{Disease-driven Poverty Traps}


$\overline{\text { Chapter }} 7$

\section{Disease-driven Poverty Traps: The Effects of Determinism, Stochasticity, and Safety Nets}

\subsection{Introduction}

About one sixth of the world's population is poor, living on less than one U.S. dollar per day [1]. The poor are located mostly in developing countries, where poverty has lingered for ages. Unfortunately, most of the chronically poor hardly experience real economic growth at any stage of their lives. The reasons for such extreme poverty are varied and include factors such as: bad governance, corruption, poor health care systems, lack of education and social infrastructure, and biophysical factors such as agricultural productivity and environmental degradation [2-7]. Extreme poverty is common among socially marginalized people, people who are discriminated against, unhealthy people, etc. Based on poverty and infectious disease prevalence levels, populations can be grouped into a number of classes that include:

1. under-developed and high disease-burden populations that are stuck in a vicious cycle of poverty and disease,

2. developed and low disease-burden populations with growing economies and good health,

3. populations with intermediate levels of income and infectious disease prevalence, etc.

The gap between the extremely rich and the extremely poor has attracted much attention from the economics and development communities [8-11]. This has given rise to recent 
research that is focused on identifying optimal intervention strategies for poverty alleviation and improvement of the health conditions of those leaving in extreme poverty. See, for example, Refs. [12-21] for an extensive coverage of this subject.

Any self-reinforcing system that prevents economic growth can be regarded as a poverty trap. One such system involves the interaction between disease and poverty. This interaction can lead to a vicious cycle where poor health and poverty reinforce each other in a positive feedback system, locking a country in a trap of poverty and poor health. Individuals and countries that are stuck in a poverty trap tend also to have high burdens of disease, while in contrast, rich individuals and developed countries have a relatively low disease burden. Health and nutrition are important for the acquisition of the education and training necessary for income generation, while disease can cause death and inhibit labor productivity, thereby limiting economic growth [22-24]. Disease weakens the workforce of a country and hence impacts economic growth negatively. On the other hand, disease prevalence tends to high in poor countries since such countries lack the necessary health care resources to contain and/or combat diseases. The reasons for the perpetuation of poverty traps are many but the role of per capita income and other economic variables cannot be discounted [9]. Other economic variables may include conflict [25, 26], household income, environmental factors [27] and infectious disease burdens [5, 23, 28].

Disease-driven poverty traps have been qualitatively studied and documented extensively [29]. While formal models of poverty traps have existed for decades, they have not been integrated with the natural sciences and epidemiology [9, 30-34]. Bonds et al. [35] recently proposed a theoretical framework for poverty traps based on an infectious disease model. They translated the generalized poverty trap cycle into a mathematical framework, in which the interaction between income and infectious disease prevalence is studied through a deterministic SIS (susceptible-infectious-susceptible) compartmental model with per capita income-dependent parameters. Although this framework formally demonstrates how poverty traps caused by infectious diseases can emerge in a very simple model, it is incomplete in several aspects. The framework fails to capture and/or explain the mechanism by which countries fall into disease-induced poverty traps and how countries can leave the poverty trap. Most importantly, which equilibrium the country relaxes to is determined entirely by 
its initial level of income and burden of infectious disease. Moreover, once a country steps into the high income and low disease state, or the low income and high disease state, the country has no way of leaving that state. This is unrealistic in real-life. These deficiencies in the framework are attributed to the fact that their system is deterministic.

Stochastic models, that is, models that include random variation, can lead to qualitatively different results than deterministic models in a range of systems and scales of analysis [36, 37]. Stochastic processes are also an important part of the economic growth literature [30, 38], and they have played a central role in the development of population ecological theory [39-41]. A substantial amount of empirical work, from laboratory studies of marine microorganisms to field studies of large mammals, has contributed to a growing consensus that stochastic processes are often as important as their underlying deterministic structure in explaining patterns of population dynamics [41-46]. For example, stochastic models have been essential in explaining the temporal behavior of infectious diseases of humans, such as measles, whooping cough, cholera, dengue fever, etc. [47-51]. Stochasticity can also play an important role in generating poverty traps, and in deciding whether or not a population or a country falls into, or leaves a poverty trap.

The question at issues is: what can be done about perpetual cycles of poverty and disease? There are those who suggest that what is needed is broad economic development, and everything else (such as mortality rates) will follow. Such goals have not only been elusive, they are not necessarily based on an understanding of the underlying mechanics of persistent poverty. Health conditions through a system of feedback mechanisms can be an important determinant of long-term economic growth and improving health care can play a role in breaking cycles of poverty and disease. Here, we explore deterministic and stochastic models of disease-driven poverty traps in view of determining the best ways of escaping disease-driven poverty traps. We show that deterministic models can give rise to poverty traps that can only be overcome by substantial external changes to the initial levels of income and health care, whereas there is always a possibility of breaking poverty traps arising from stochastic models. We also show that a "safety net" ${ }^{1}$, which can be implemented as an economic measure, a health measure or both an economic and health measure is a

\footnotetext{
${ }^{1} \mathrm{~A}$ safety net is an externally enforced minimum level of health or economic conditions.
} 
guaranteed measure of escaping from a poverty trap. This measure remains effective even when it is implemented within the basin of attraction of the poverty trap. Therefore, this research addresses structural versus stochastic poverty, and provides theoretical mechanisms for understanding the role of health safety nets in the context of stochastic poverty.

This study is motivated by the following findings developed from disparate literatures:

1. health, which depends largely on disease ecology, can influence economic productivity,

2. the effectiveness of policy is sensitive to the nature of poverty dynamics, and

3. the dynamics of income and disease have both deterministic and stochastic components.

The chapter is organized as follows: We explore the effects of determinism and stochasticity on disease-driven poverty traps in Sections 7.2 and 7.3, respectively, advance policy interpretations of our investigations in Section 7.4, and conclude the chapter in Section 7.5.

\subsection{The Deterministic Approach}

The parameters and variables used in this Section are described in Tables 7.1 and 7.2, respectively, while a schematic framework illustrating the flow of individuals between the susceptible and infectious classes is presented in Fig. 7.1. 
Table 7.1. Model parameters and their descriptions.

\begin{tabular}{||c|l||}
\hline \hline Parameter & \multicolumn{1}{|c||}{ Description } \\
\hline$\lambda$ & Birth rate of susceptible and infectious individuals \\
\hline$\beta$ & Transmission rate \\
\hline$\beta_{1}$ & Maximum transmission rate \\
\hline$r$ & Rate at which infectious individuals recover from the disease \\
\hline$r_{1}$ & Maximum rate at which infectious individuals recover from the disease \\
\hline$\mu$ & Natural death rate of susceptible and infectious individuals \\
\hline$\gamma$ & Disease-induced death rate \\
\hline$M_{0}$ & Maximum income \\
\hline$\rho$ & Growth rate of income \\
\hline \hline$\sigma$ & Volatility of per capita income \\
\hline \hline
\end{tabular}

Table 7.2. Model variables and their descriptions.

\begin{tabular}{||c|l||}
\hline \hline Variable & \multicolumn{1}{|c||}{ Description } \\
\hline$\tilde{S}(t)$ & $\begin{array}{l}\text { Susceptible individuals at time } t \text {. These are individuals with the } \\
\text { potential of contracting the disease but who has not yet contracted } \\
\text { the disease. }\end{array}$ \\
\hline$\tilde{I}(t)$ & $\begin{array}{l}\text { Infectious individuals at time } t \text {. These are individuals who have } \\
\text { already contracted the disease and can transmit it. }\end{array}$ \\
\hline$M(t)$ & Per capita income at time $t$. \\
\hline \hline
\end{tabular}




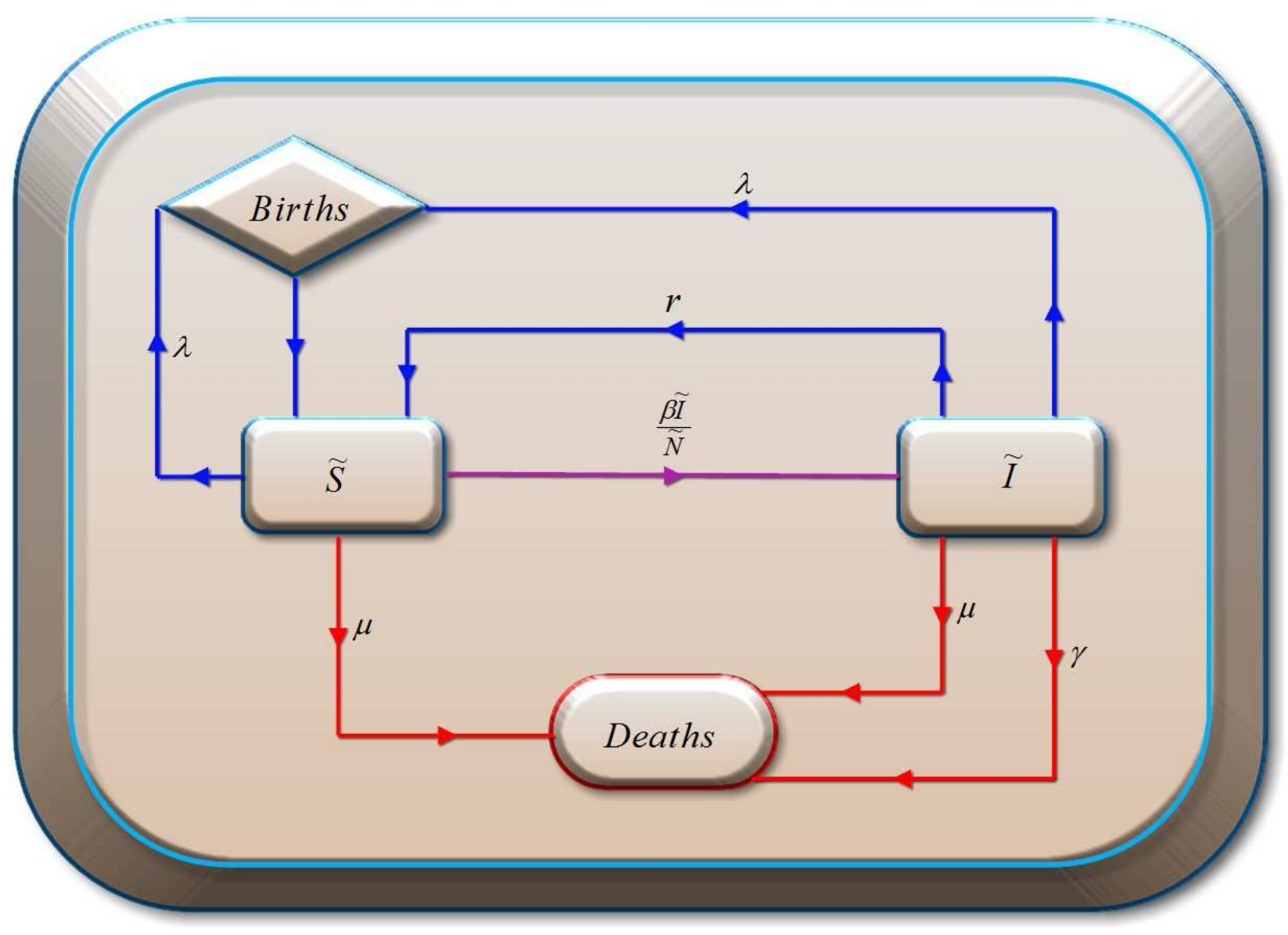

Figure 7.1. A schematic framework illustrating the movement of individuals into and out of the susceptible $(\tilde{S})$ and infectious $(\tilde{I})$ classes. The various transition rates are described in Table 7.1. At any time, the total population $\tilde{N}$, is given by $\tilde{N}=\tilde{S}+\tilde{I}$. 
We derive and analyze a SISM (Susceptible-Infectious-Susceptible-Per capita income) deterministic ordinary differential equation model for a class of infectious diseases of humans that can serially reinfect their hosts. Such diseases may include bacterial infections, sexually transmitted diseases, vector-borne diseases such as malaria and dengue fever. Literature indicates that such diseases have been accused of inhibiting economic growth $[23,52,53]$. The generic $\tilde{S} \tilde{I} \tilde{S}$ part of the model has been widely studied in the literature [54-57]. See, for example, Chapter 2 for the derivation of this part of the model. Since disease-driven poverty traps arise as a result of positive feedback between income and disease, we adopt the framework proposed by Bonds et al. and model the contact and recovery rates as functions of per capita income. Using standard mass action laws and and the schematic in Fig. 7.1, we write the following system of differential equations for the disease model:

$$
\begin{aligned}
\frac{d \tilde{S}}{d t} & =\lambda \tilde{N}+r(M) \tilde{I}-\left(\frac{\beta(M) \tilde{I}}{\tilde{N}}+\mu\right) \tilde{S}, \\
\frac{d \tilde{I}}{d t} & =\frac{\beta(M) \tilde{I}}{\tilde{N}} \tilde{S}-(r(M)+\mu+\gamma) \tilde{I},
\end{aligned}
$$

where the total population is modeled by the equation

$$
\frac{d \tilde{N}}{d t}=(\lambda-\mu) \tilde{N}-\gamma \tilde{I}
$$

For convenience, we rewrite the disease model in terms of proportions of susceptible and infectious individuals. To achieve this variable transformation, we set $S=\tilde{S} / \tilde{N}$ and $I=\tilde{I} / \tilde{N}$ in system (7.1). The derivatives on the left-hand sides are now given by:

$$
\begin{aligned}
\frac{d S}{d t} & =\frac{d(\tilde{S} / \tilde{N})}{d t}=\frac{1}{\tilde{N}}\left(\frac{d \tilde{S}}{d t}-\frac{\tilde{S}}{\tilde{N}} \frac{d \tilde{N}}{d t}\right), \\
\frac{d I}{d t} & =\frac{d(\tilde{I} / \tilde{N})}{d t}=\frac{1}{\tilde{N}}\left(\frac{d \tilde{I}}{d t}-\frac{\tilde{I}}{\tilde{N}} \frac{d \tilde{N}}{d t}\right) .
\end{aligned}
$$

Further simplification yields the following system:

$$
\begin{aligned}
\frac{d S}{d t} & =\lambda(1-S)-\beta(M) I S+r(M) I+\gamma I S, \\
\frac{d I}{d t} & =\beta(M) I S-(r(M)+\gamma+\lambda) I+\gamma I^{2},
\end{aligned}
$$


where $S$ and $I$ are, respectively, the proportions of susceptible and infectious individuals. Since $S+I=1, S=1-I$. We use this fact to reduce system (7.2) into the single equation

$$
\frac{d I}{d t}=\beta(M) I(1-I)-(r(M)+\gamma+\lambda) I+\gamma I^{2}
$$

In contrast to previous frameworks used in modeling income [28, 35, 58], we regard per capita income as a state variable and model it explicitly through the following ordinary differential equation that is chosen in such a way that per capita income increases with decreasing disease prevalence and decreases with increasing disease prevalence:

$$
\frac{d M}{d t}=-\rho M\left(M-M_{0}(1-I)\right)
$$

where $\rho$ is the growth rate of income and $M_{0}$ is the maximum income that would be earned if there is no disease in the population. To complete the description of the model, we select appropriate functions for the income-dependent rates. The transmission rate can be modeled through a decreasing function of income, while the recovery rate can be modeled through an increasing function of income. Note that the rich can easily afford treatment, cure and measures to avoid contacts with infectious individuals than the poor. We adopt the following functions from Ref. [35]:

$$
\begin{aligned}
& \beta(M)=\frac{\beta_{1} \epsilon}{M+\epsilon}, \\
& r(M)=\frac{r_{1} M}{M+\kappa},
\end{aligned}
$$

where $\epsilon$ and $\kappa$ are positive constants and $\beta_{1}$ and $r_{1}$ are defined in Table 7.1. The selected transmission rate function $\beta$, has a maximum value of $\beta_{1}$, which arises when income falls to zero. The recovery rate function $r$, attains a maximum of $r_{1}$. Note that $\beta$ approaches zero asymptotically, while $r$ approaches $r_{1}$ asymptotically as $M$ increases. See Fig. 7.2.

Rewriting equations (7.3) and (7.4), we obtain the following disease-income system:

$$
\begin{aligned}
\frac{d I}{d t} & =\beta(M) I(1-I)-(r(M)+\gamma+\lambda) I+\gamma I^{2} \\
\frac{d M}{d t} & =-\rho M\left(M-M_{0}(1-I)\right) .
\end{aligned}
$$




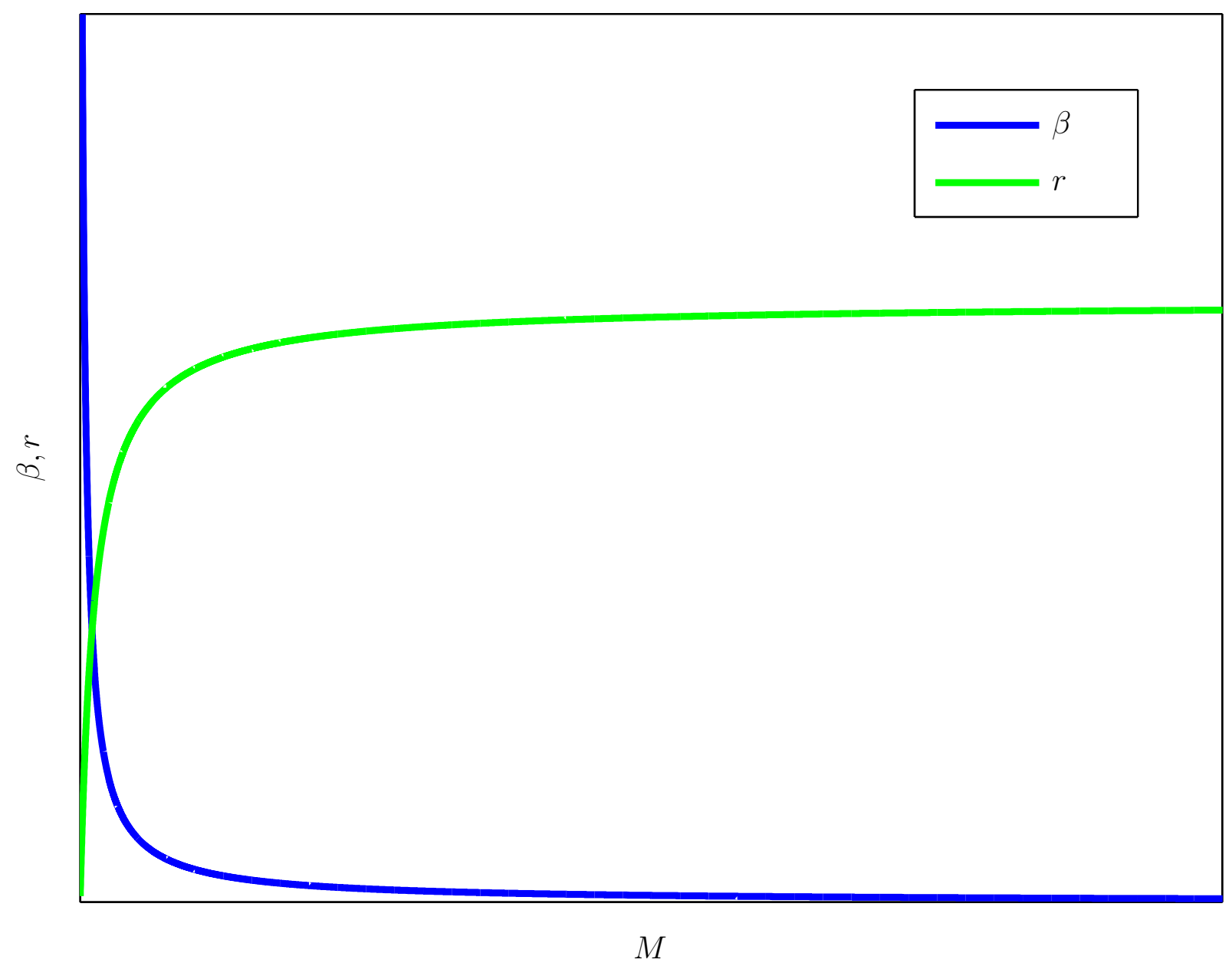

Figure 7.2. A sketch of the transmission and recovery rate functions from equations (7.5) and (7.6). The transmission rate function $\beta$, denoted by a blue line decays to zero asymptotically, while the recovery rate function $r$, denoted by a green line, grows and saturates at a maximum as income increases. 


\subsubsection{Existence of Equilibrium Solutions and Linear Stability Analysis}

We identify the following threshold parameter and use it to explore the stability of system $(7.7)$ :

$$
R_{0}^{m}=\frac{\beta_{1} \epsilon\left(M_{0}+\kappa\right)}{\left(M_{0}+\epsilon\right)\left(r_{1} M_{0}+(\gamma+\lambda)\left(M_{0}+\kappa\right)\right)} .
$$

Remark 7.2.1. The following can be deduced from the parameter $R_{0}^{m}$ :

(i) An increase in $M_{0}$, representing an improvement in economic conditions reduces $R_{0}^{m}$.

(ii) An increase in $r_{1}$, representing improved medical treatment, better nutrition, better health education, better health policies, etc., will decrease $R_{0}^{m}$.

(iii) A decrease in $\beta_{1}$ will reduce $R_{0}^{m}$.

Let $\left(I^{*}, M^{*}\right)$ be an equilibrium solution of system (7.7). Then by substituting $I=I^{*}$ and $M=M^{*}$ in system (7.7), setting the left-hand sides to zero, and solving the resulting algebraic equations, we obtain the following five equilibrium solutions:

$$
\begin{aligned}
E_{0}^{*} & =\left(I^{*}, M^{*}\right)=(0,0), \\
E_{M_{0}}^{*} & =\left(I^{*}, M^{*}\right)=\left(0, M_{0}\right), \\
E_{I}^{*} & =\left(I^{*}, M^{*}\right)=\left(\frac{\beta_{1}-(\lambda+\gamma)}{\beta_{1}-\gamma}, 0\right), \\
E_{2,3}^{*} & =\left(I^{*}, M^{*}\right)=\left(I_{i}^{*}, M_{m}^{*}\right),
\end{aligned}
$$

where the equilibrium solution $E_{I}^{*}$ exists (or is positive) only for $\beta_{1}<\gamma$ or $\beta_{1}>\lambda+\gamma$, and

$$
\begin{aligned}
I_{i}^{*} & =\frac{\beta\left(M_{m}^{*}\right)-\left(r\left(M_{m}^{*}\right)+\lambda+\gamma\right)}{\beta\left(M_{m}^{*}\right)-\gamma}, \\
M_{m}^{*} & =M_{0}\left(1-I_{i}^{*}\right), I_{i}^{*}<1 .
\end{aligned}
$$

We investigate the stability of the equilibria in (7.9) by linearizing system (7.7) about each of the equilibrium solutions. To this effect, we perturb the system about $\left(I^{*}, M^{*}\right)$ by setting $I=I^{*}+i$ and $M=M^{*}+m$, where $s$ and $m$ are small. Substituting these values into the system and expanding in a Taylor series about $\left(I^{*}, M^{*}\right)$ and retaining only linear terms in $i$ and $m$ yields the following linear system of ordinary differential equations in $i$ and $m$ : 


$$
\left(\begin{array}{c}
\frac{d i}{d t} \\
\frac{d m}{d t}
\end{array}\right)=J\left(\begin{array}{c}
i \\
m
\end{array}\right)
$$

where

$$
J=\left(\begin{array}{cc}
\frac{\beta_{1} \epsilon}{M^{*}+\epsilon}\left(1-2 I^{*}\right)-\frac{r_{1} M^{*}}{M^{*}+\kappa}-(\lambda+\gamma)+2 \gamma I^{*} & -\frac{\beta_{1} \epsilon I^{*}}{\left(M^{*}+\epsilon\right)^{2}}\left(1-I^{*}\right)-\frac{r_{1} \kappa I^{*}}{\left(M^{*}+\kappa\right)^{2}} \\
-\rho M_{0} M^{*} & -\rho\left(2 M^{*}-M_{0}\left(1-I^{*}\right)\right)
\end{array}\right)
$$

is the Jacobian matrix of the linear system. Let $\xi$ be an eigenvalue of $J$.

Now, at the trivial equilibrium solution $E_{0}^{*}=(0,0), J$ reduces to the diagonal matrix

$$
J\left(E_{0}^{*}\right)=\left(\begin{array}{cc}
\beta_{1}-(\lambda+\gamma) & 0 \\
0 & \rho M_{0}
\end{array}\right),
$$

whose eigenvalues are $\xi_{1}=\rho M_{0}>0$ and $\xi_{2}=\beta_{1}-(\lambda+\gamma)$. These eigenvalues are real and positive when $\beta_{1}>\lambda+\gamma$, and they have opposite signs when $\beta_{1}<\lambda+\gamma$. Hence, $E_{0}^{*}$ is an unstable node when $\beta_{1}>\lambda+\gamma$, and a saddle when $\beta_{1}<\lambda+\gamma$. Instability of $E_{0}^{*}$ is reasonable since it is assumed impossible to have a country with no income and no disease prevalence. Note that the condition, $\beta_{1}>\lambda+\gamma$, for which $E_{0}^{*}$ is an unstable node guarantees the existence of $E_{I}^{*}$.

At the equilibrium point $E_{M_{0}}^{*}$, the Jacobian matrix becomes:

$$
J\left(E_{M_{0}}^{*}\right)=\left(\begin{array}{cc}
\frac{\beta_{1} \epsilon}{M_{0}+\epsilon}-\frac{r_{1} M_{0}}{M_{0}+\kappa}-(\lambda+\gamma) & 0 \\
-\rho M_{0}^{2} & -\rho M_{0}
\end{array}\right)
$$

and the eigenvalues of $J\left(E_{M_{0}}^{*}\right)$ are

$$
\begin{aligned}
& \xi_{1}=-\rho M_{0}, \\
& \xi_{2}=-\frac{\left(r_{1} M_{0}+(\lambda+\gamma)\left(M_{0}+\kappa\right)\right)\left(1-R_{0}^{m}\right)}{M_{0}+\kappa}
\end{aligned}
$$


where $R_{0}^{m}$ is given by equation (7.8). Clearly, $E_{M_{0}}^{*}$ is a stable node when $R_{0}^{m} \leq 1$ and an unstable node when $R_{0}^{m}>1$. Stability of $E_{M_{0}}^{*}$ represents the situation in which the disease dies out at a certain level of income $M_{0}$.

At the equilibrium solution $E_{I}^{*}$, we have

$$
J\left(E_{I}^{*}\right)=\left(\begin{array}{cc}
\beta_{1}\left(1-2 I^{*}\right)-(\lambda+\gamma)+2 \gamma I^{*} & -\frac{\beta_{1} I^{*}}{\epsilon}\left(1-I^{*}\right)-\frac{r_{1} I^{*}}{\kappa} \\
0 & \rho M_{0}\left(1-I^{*}\right)
\end{array}\right),
$$

with eigenvalues, $\xi_{1}=\beta_{1}\left(1-2 I^{*}\right)-(\lambda+\gamma)+2 \gamma I^{*}$ and $\xi_{2}=\rho M_{0}\left(1-I^{*}\right)$, where $I^{*}$ is given by (7.9). Note from (7.9) that $I^{*}<1$. This makes sense, since $I$ is the proportion of infectious individuals. Since $I^{*}<1, \xi_{2}>0$. Therefore, $E_{I}^{*}$ is an unstable node if $\xi_{1}>0$ and a saddle if $\xi_{1}<0$. Instability of $E_{I}^{*}$ conforms with the fact that it is difficult to have a country that is stuck in a state with high disease prevalence and no income at all.

Calculating $E_{2,3}^{*}$ in closed form is difficult. Hence, studying the stability of these equilibria analytically is also difficult. We therefore resort to numerical simulations.

\subsubsection{Numerical Simulations of the Deterministic Model}

We use the following parameter values to simulate system (7.7): $\lambda=0.06, \gamma=0.02$, $\rho=0.05, \beta_{1}=40, \epsilon=15, r_{1}=13.5, \kappa=100, M_{0}=100$. The simulation results confirm as well as reinforce the analytical evaluation of the stability of the equilibrium solutions, with the trivial equilibrium $E_{0}^{*}$ and the zero income-high disease prevalence equilibrium $E_{I}^{*}$ unstable, while the high income and no disease equilibrium point $E_{M_{0}}^{*}$, is stable. In addition, the non-trivial equilibrium solution $E_{2}^{*}$, is unstable, whereas the high disease prevalence-low income equilibrium solution $E_{3}^{*}$, is stable. Note that $E_{3}^{*} \neq E_{I}^{*}$, since the equilibrium value of $M$ in $E_{3}^{*}$ is not necessarily zero as in $E_{I}^{*}$. The stable equilibrium point to which the system relaxes in the long time limit is determined strictly by the initial conditions. Trajectories that originate close to a given stable equilibrium solution relax to that equilibrium solution. Trajectories that originate to the left of, and above the unstable equilibrium $E_{2}^{*}$, converge to the high income-low disease prevalence equilibrium $E_{M_{0}}^{*}$, while trajectories that originate to the right of, and below $E_{2}^{*}$, converge to $E_{3}^{*}$. Trajectories that originate close to the unstable 
equilibrium solution $E_{I}^{*}$, converge to $E_{3}^{*}$. The stable equilibrium solution $E_{3}^{*}$, constitutes a poverty trap. The stable equilibrium $E_{M_{0}}^{*}$ is called a development equilibrium. Typical phase plots illustrating the behavior of system (7.7) are presented in Figs. 7.3-7.5.

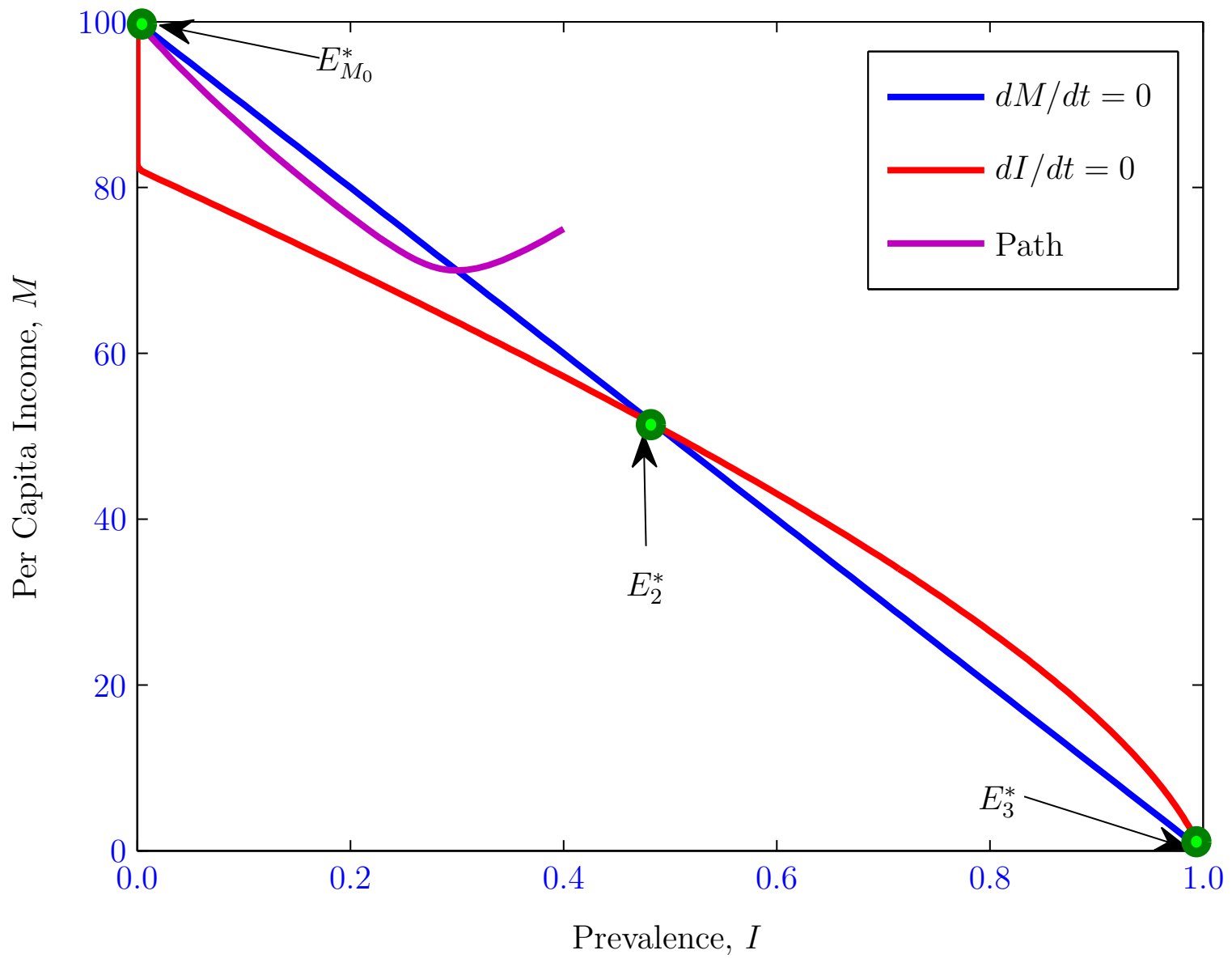

Figure 7.3. Simulation results for the deterministic model showing the path taken by a typical trajectory that starts at a particular initial point to arrive at $E_{M_{0}}^{*}$. 


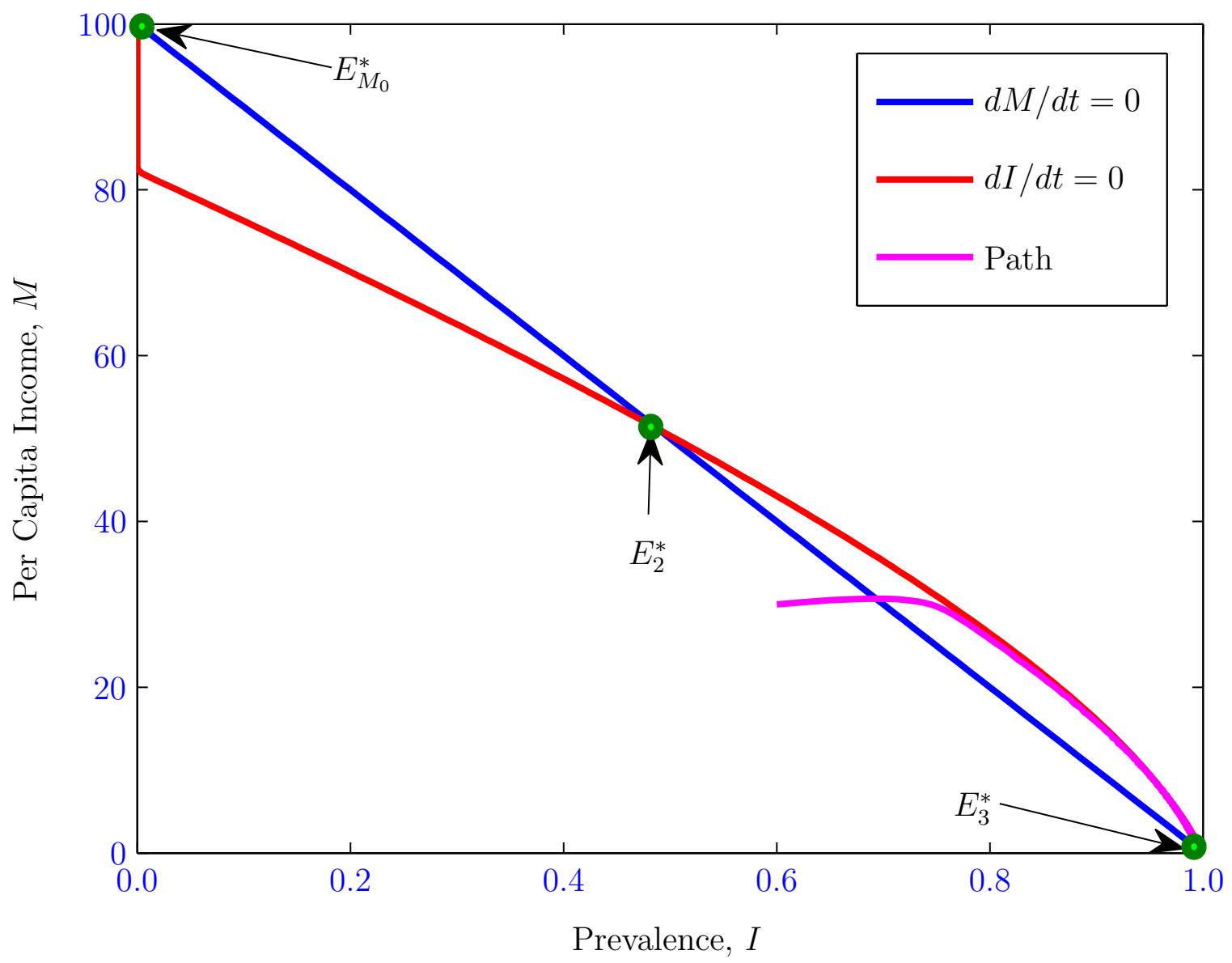

Figure 7.4. Simulation results for the deterministic model showing the path a typical trajectory that starts at an initial point takes to get to the poverty trap $E_{3}^{*}$. 


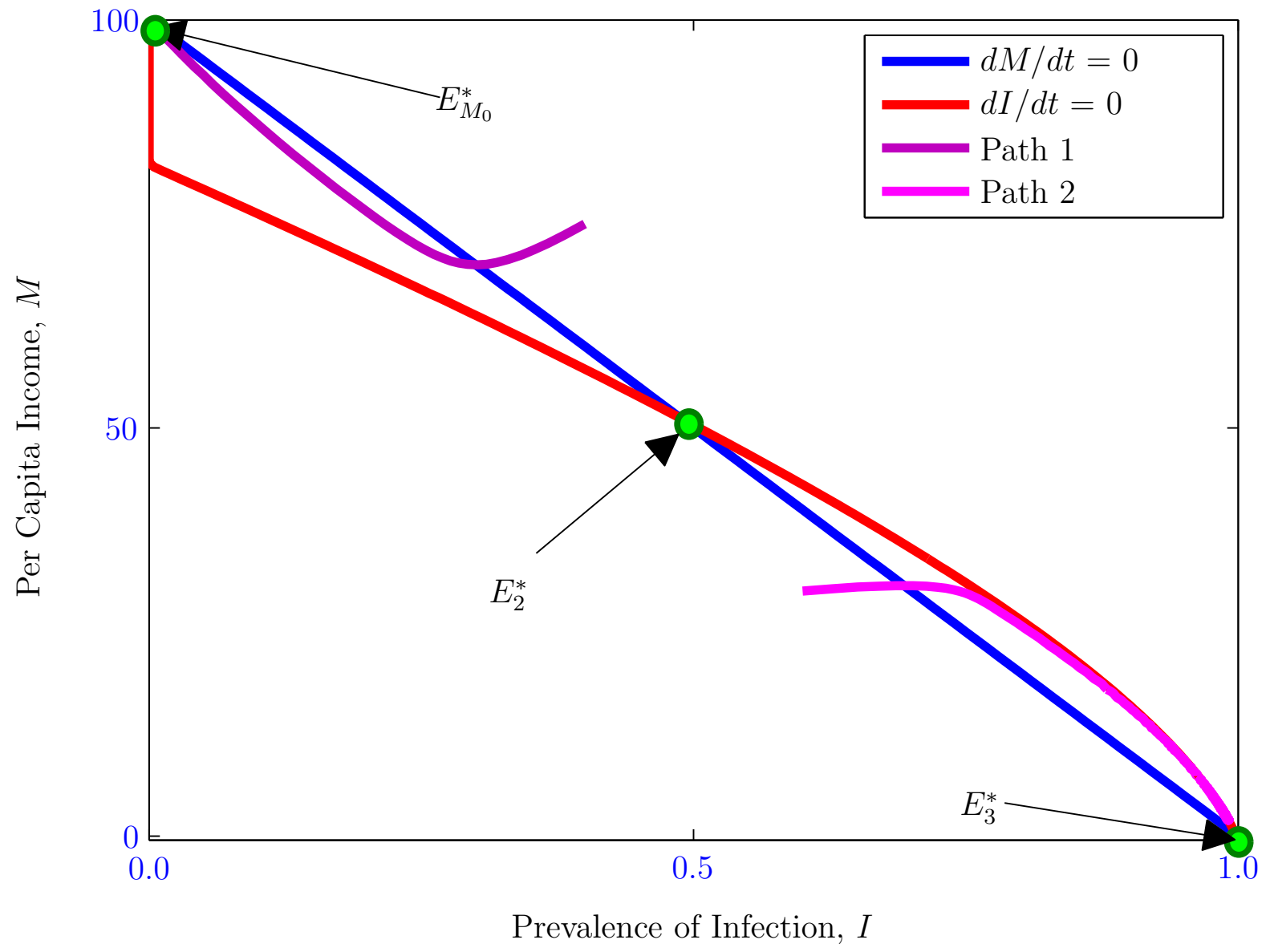

Figure 7.5. Simulation results for the deterministic model showing the paths taken by two typical trajectories that start at different initial points to arrive at the development stable equilibrium solution, $E_{M_{0}}^{*}$, and the poverty trap, $E_{3}^{*}$. The blue and the red lines represent the nullclines, $d M / d t=0$ and $d I / d t=0$, respectively, while the purple and magenta lines denote the paths taken by two typical trajectories to converge to the stable development equilibrium and the poverty trap, respectively. The parameter values used are: $\lambda=0.06, \gamma=$ $0.02, \rho=0.05, \beta_{1}=40, \epsilon=15, r_{1}=13.5, \rho=90, \kappa=100, M_{0}=100$. 
We now determine the combinations of initial values of per capita income and disease prevalence that will converge to the poverty trap over time. To achieve this, we integrate system (7.7) on a grid of initial values of per capita income and disease prevalence. The result of this simulation is illustrated in Fig. 7.6. Within the set defined by this combination, the probability that the system converges to the poverty trap is one. Outside this set, the system evolves to the development equilibrium point long run. In this case, the probability that the system will fall into the poverty trap is zero.

Although we have used a deterministic approach to explore some important features of poverty traps, modeling poverty traps deterministically has a number of limitations. For a given set of parameters, determinism implies that a country is permanently stuck in a trap of poverty and disease if its initial epidemiological and economic conditions fall within the basin of attraction of the poverty trap equilibrium, or a country is stuck in a high income and no disease state if its initial epidemiological and economic conditions are located within the basin of attraction of the development equilibrium. Determinism also indicates that a substantial initial push is required to move the state variables out of the basin of attraction of the poverty trap, or a change in the parameters that alters the existence or location of equilibria in the state space. Hence, determinism may lead to restrictive policy recommendations that require large external interventions which may not be necessary. It also provides a limited set of criteria for detecting poverty traps empirically. 


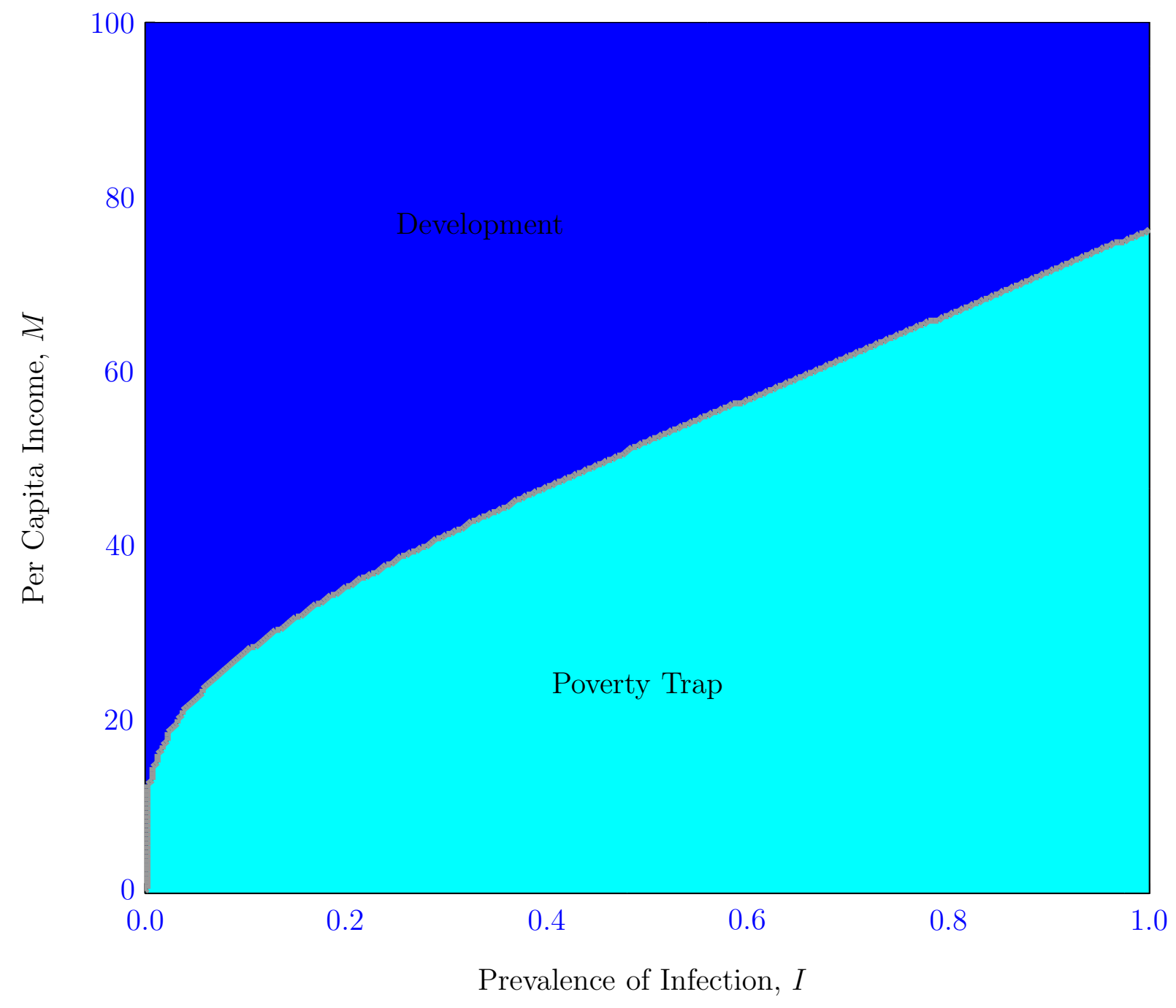

Figure 7.6. Phase plot illustrating the effect of initial conditions on determining the stable equilibrium point to which the system relaxes in the long time limit. The following parameter values are used: $\lambda=0.06, \gamma=0.02, \rho=0.05, \beta_{1}=$ $40, \epsilon=15, r_{1}=13.5, \rho=90, \kappa=100, M_{0}=100$. 


\subsection{Stochastic Model}

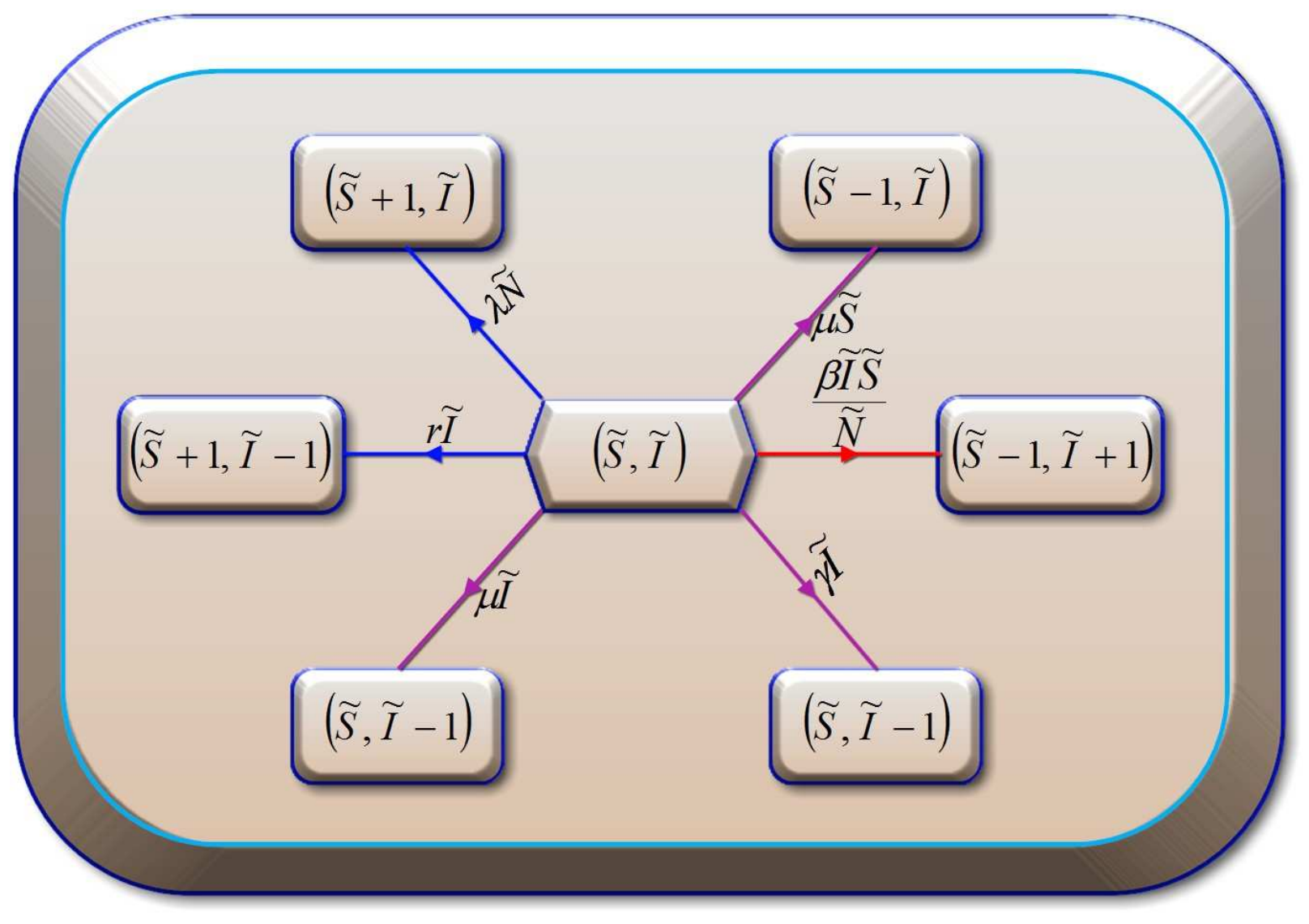

Figure 7.7. A schematic framework illustrating the states to which a typical susceptible or infectious individual can evolve. At any time, the total population is $\tilde{N}=\tilde{S}+\tilde{I}$. 
We now formulate and study a stochastic model describing the interplay between per capita income and infectious disease prevalence.

\subsubsection{Partial Stochastic Model}

Here, we study a stochastic disease model and a deterministic income model. In general, a deterministic ordinary differential equation model can give at best an approximation to a dynamical process that is by definition random. The solution to the system of ordinary differential equations described above results in the expected behavior of the system, providing no information as to the distribution of possible paths that the dynamical system can follow. In order to acquire a measure for this distribution, we numerically simulate the dynamical system using the Gillespie Algorithm. The Gillespie algorithm simulates random discrete events and the time elapsed between events. Here, the events are: birth of a susceptible individual, infection of a susceptible individual, recovery of an infectious individual, death of an infectious individual, and death of a susceptible individual. The total population that we consider as the sum of susceptible $(\mathrm{S})$ and infectious (I) individuals is not constant. Starting from a state with susceptible and infectious individuals, there are five states, $(S+1, I),(S-1, I+1),(S+1, I-1),(S-1, I)$, and $(S, I-1)$ corresponding to the five events mentioned above to which the system can evolve at different rates. See the flow chart, Fig. 7.7 and what follows for details. For notational convenience, we have dropped the wiggles on $S$ and $I$ so that $S$ and $I$ are now the number of susceptible and infectious individuals, respectively.

- The state $(S+1, I)$ represents increments to the susceptible population through births from susceptible and infectious individuals that occur at rate $\lambda$.

- The state $(S-1, I+1)$ describes movements of individuals from the susceptible class to the infectious class. These movements occur at rate $\frac{\beta I}{N}$, where $\beta$ is the contact rate between susceptible and infectious individuals. To quantify the effect of income on disease transmission, we allow $\beta$ to be a decreasing function of income.

- The state $(S+1, I-1)$ describes movements of individuals from the infectious class to the susceptible class. These are individuals who recover from the disease at rate $r$ 
to join the susceptible class. As in the deterministic model, to emphasize the effect of income on disease prevalence, we allow $r$ to be an increasing function of income.

- The state $(S-1, I)$ denotes the natural death of a susceptible individual that occur at rate $\mu$.

- The state $(S, I-1)$ represents natural and disease-induced mortalities from the infectious that occur at rates rates $\mu$ and $\gamma$, respectively.

\subsubsection{Numerical Simulations of the Partial Stochastic Model}

Using the same functional forms for $\beta$ and $r$, and the same parameter values as in the deterministic model, we simulate the above stochastic system in MATLAB. To illustrate the unique properties of the stochastic system, we start the simulations at an intermediate level of both income and disease prevalence, close to the (unstable) intermediate equilibrium point $E_{2}^{*}$ of the system. Fig. 7.8 shows the results of four different runs, with the same parameters, and the same starting point. In contrast to the deterministic case, where the system would always tend towards one of the stable steady states for all four runs, the system approaches the development equilibrium for some runs and falls into the poverty trap equilibrium for others. Hence, the stable equilibrium point to which the system relaxes in the long term is not necessarily determined by the initial condition applied to the system. Running the Gillespie algorithm multiple times from different initial conditions allows us to quantify the probability of ending up in either the development or poverty trap equilibrium. This probability was either zero or one in the deterministic case. But this is not the case with the stochastic model. As illustrated in Fig. 7.9, the probability of convergence to the poverty trap or development equilibrium is a number from zero to one. Given such a landscape plot, one can ask questions like, what is the quickest, or most likely path from the poverty trap to the development equilibrium? This is the sequence of disease-burden and income states that are likely to arise from a country moving from the poverty trap to the development equilibrium. One simple way to visualize this is to examine the plot from Fig. 7.9, and draw an arrow at each point in the direction of the neighboring point with the highest probability of ending up in the development equilibrium. By following the arrows from the bottom right 
of the plot to the top left corner of the plot, Fig. 7.10, one can trace a likely path that a population or a country can take to leave the poverty trap. The solid lines on Figs. 7.9 and 7.10 represent contour lines, suggesting that there are intermediate values of the probability of a country leaving the poverty trap to the development equilibrium. 


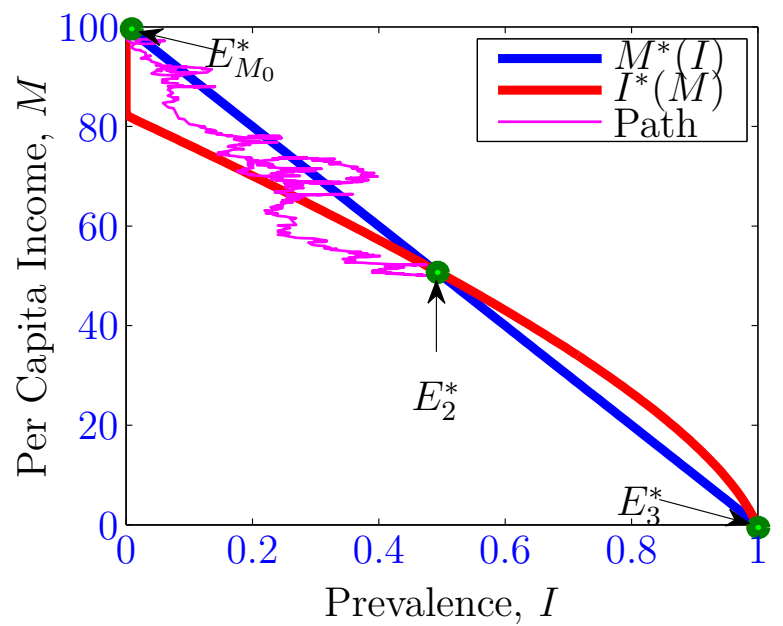

7.8.1. One possible path to the stable development equilibrium point.

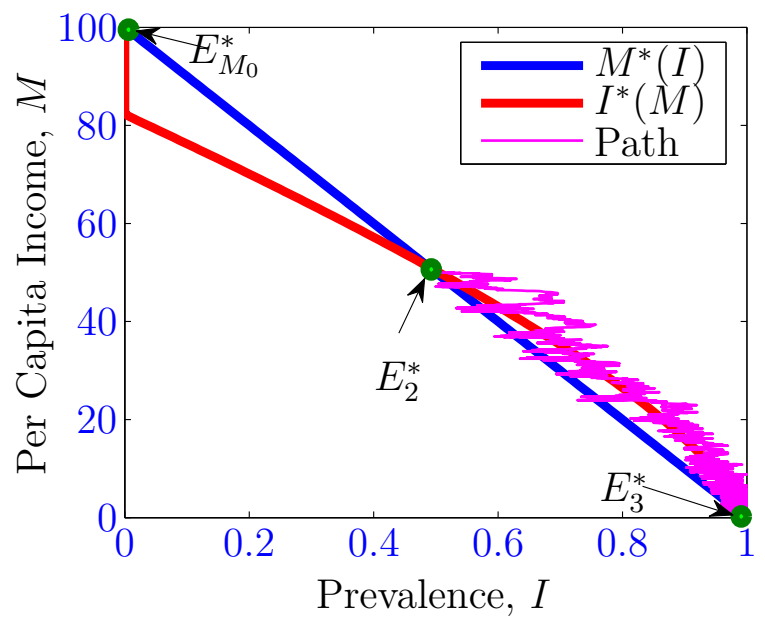

7.8.3. One possible path to the poverty trap

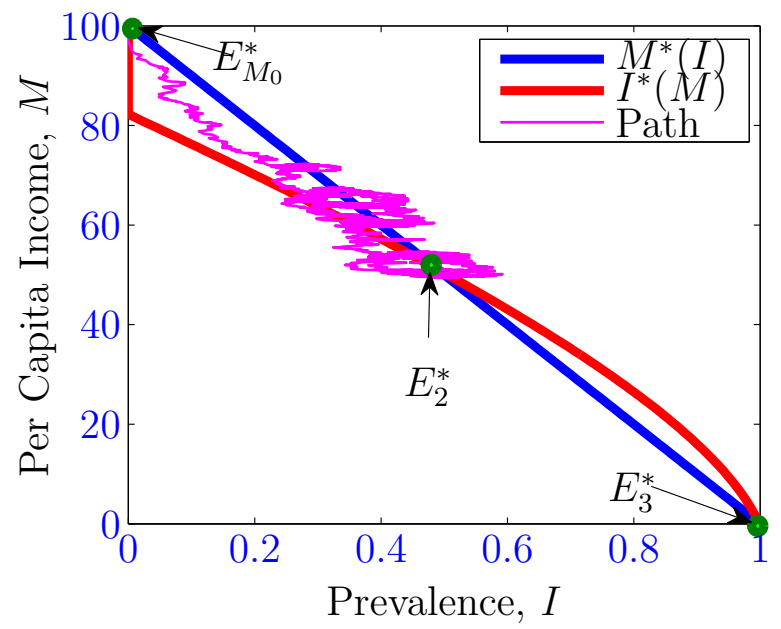

7.8.2. Second path to the stable development equilibrium point.

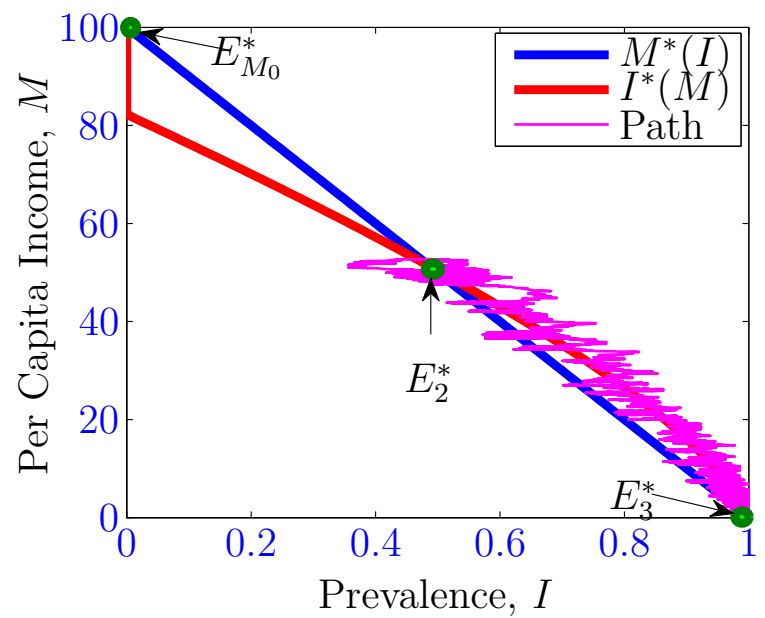

7.8.4. Second path to the poverty trap

Figure 7.8. Possible paths to the stable development and poverty trap equilibria. Notice that unlike the deterministic case, irrespective of the initial conditions, it is possible to attain any of the two stable equilibria. That is, different simulations with the same initial condition and the same parameter values can drive the system to one stable equilibrium at one time and to the other stable equilibrium at another time. 


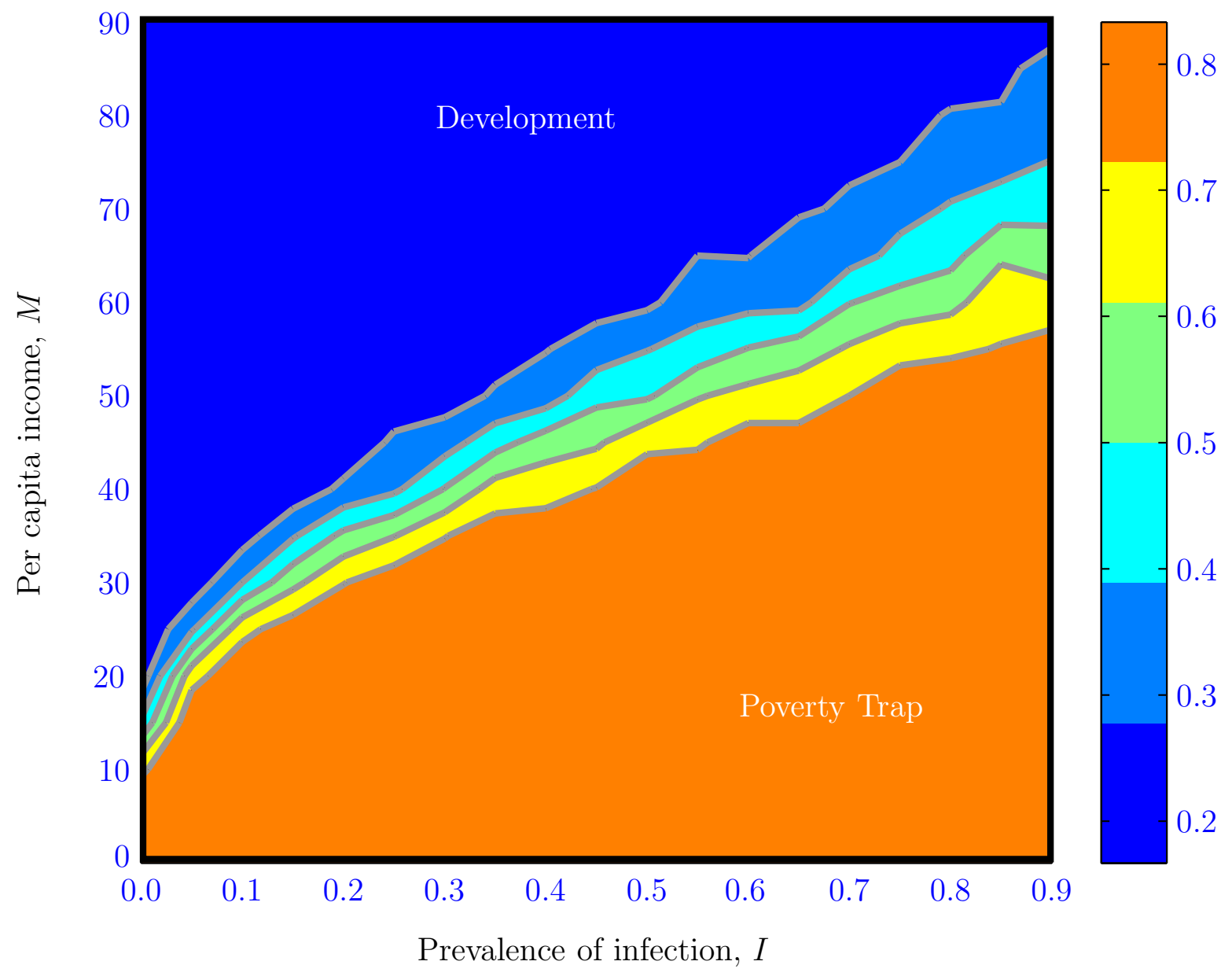

Figure 7.9. Phase plot illustrating the fact that there are intermediate values of the probability of moving from the poverty trap to the development equilibrium. 


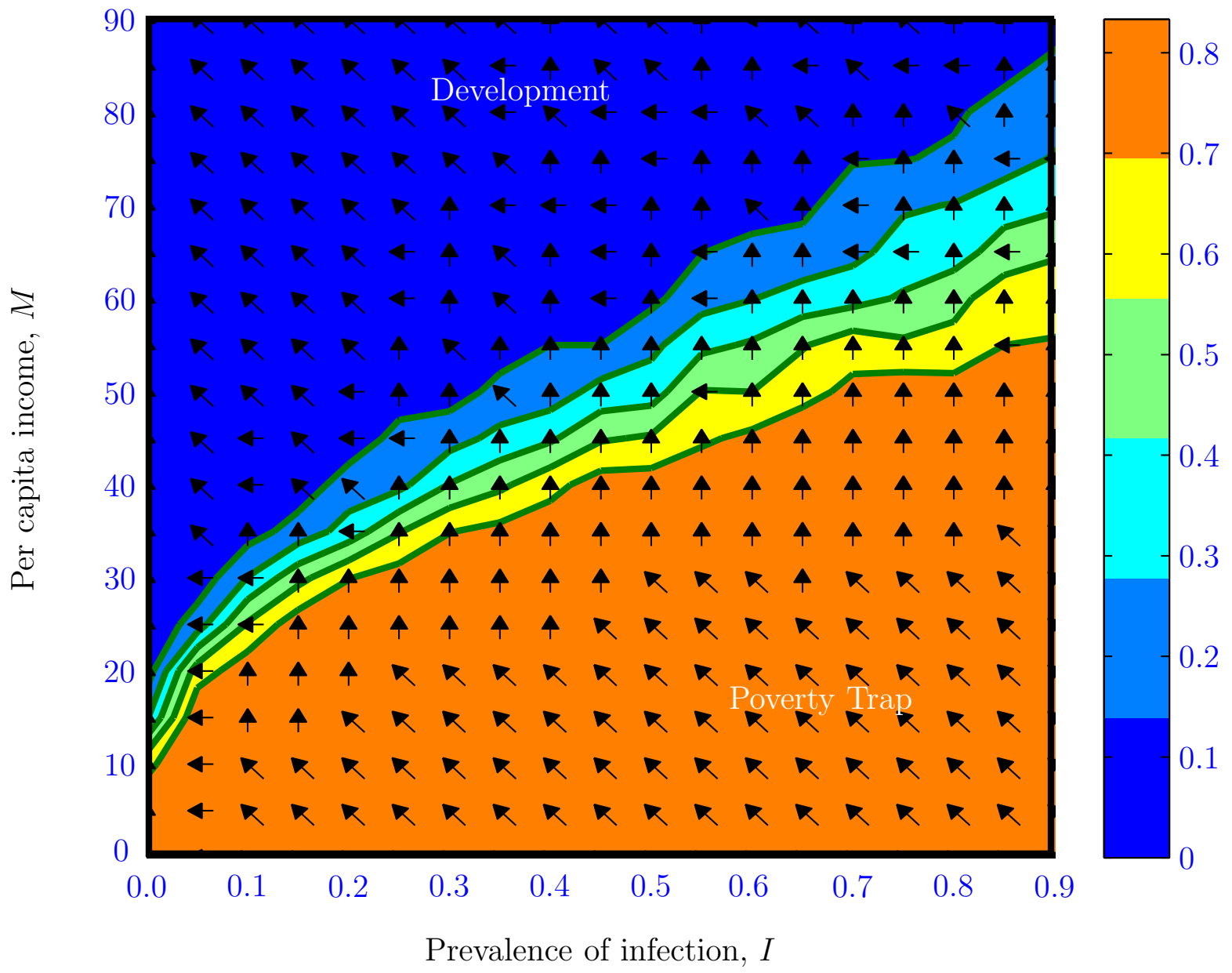

Figure 7.10. Phase plot illustrating the effect of initial conditions. The arrows indicate the quickest path to the development equilibrium, which can be interpreted as the direction in which a country should move in the prevalence-income phase plane if it wants to maximize its probability of ending up in the stable development equilibrium. 


\subsubsection{Full Stochastic Model}

For infectious disease dynamics, stochasticity is most often modeled as "demographic"; i.e., through the inherently random component of the movement between discrete states, with transitions determined by the rates in Fig. 7.7. For income, we model the stochastic process as "environmental"; i.e., through a Wiener process that includes the addition of an exogenous stochastic component. This is a standard method in economics and finance that applies naturally to poverty dynamics where incomes are often generated from highly variable agricultural productivity or through volatile earnings associated with an informal economy $[59,60]$. Because income is a continuous variable it is modeled here as a simple differential equation:

$$
d M=g(M) M d t+\sigma d W_{t}
$$

where $d W_{t}$ is a random variable with mean 0 , generated by a Wiener process, $g(M)=$ $-r\left(M-M_{0}(1-I)\right)$ is the average growth rate of per capita income, and $\sigma$ is the volatility of per capita income. Constant $\sigma$ ensures that the poor are more sensitive to swings in income.

As in Section, 7.3.1, we simulate the disease process through a Gillespie algorithm. At each event, the income is updated by numerically integrating equation (7.10) for the time elapsed. The quantity, $d W_{t}$ is approximated by a vector of normally distributed random variables with mean 0 and variance $d t$, where $d t$ is a dynamically determined step-size. Such an algorithm allows us to simulate both the disease and income processes simultaneously. As with the partial stochastic model, simulations of the full stochastic model for the same set of parameter values and initial levels of disease prevalence and income generate trajectories that can converge to the stable development equilibrium or the poverty trap equilibrium. This is illustrated in Figs. 7.11 and 7.12. On the other hand, for each point on a grid of initial disease prevalence and per capita income values, we simulate the stochastic disease-income model for 70 iterations, noting the proportion of times that convergence to the poverty trap equilibrium occurs. These proportions constitute the $z$-values in the contour plot illustrated in Fig. 7.13. Figure 7.14 is generated by drawing an arrow at each point on the grid pointing to the neighboring point that has the highest probability of convergence to the development equilibrium. The interpretation is reversed when populations fall into poverty traps. 


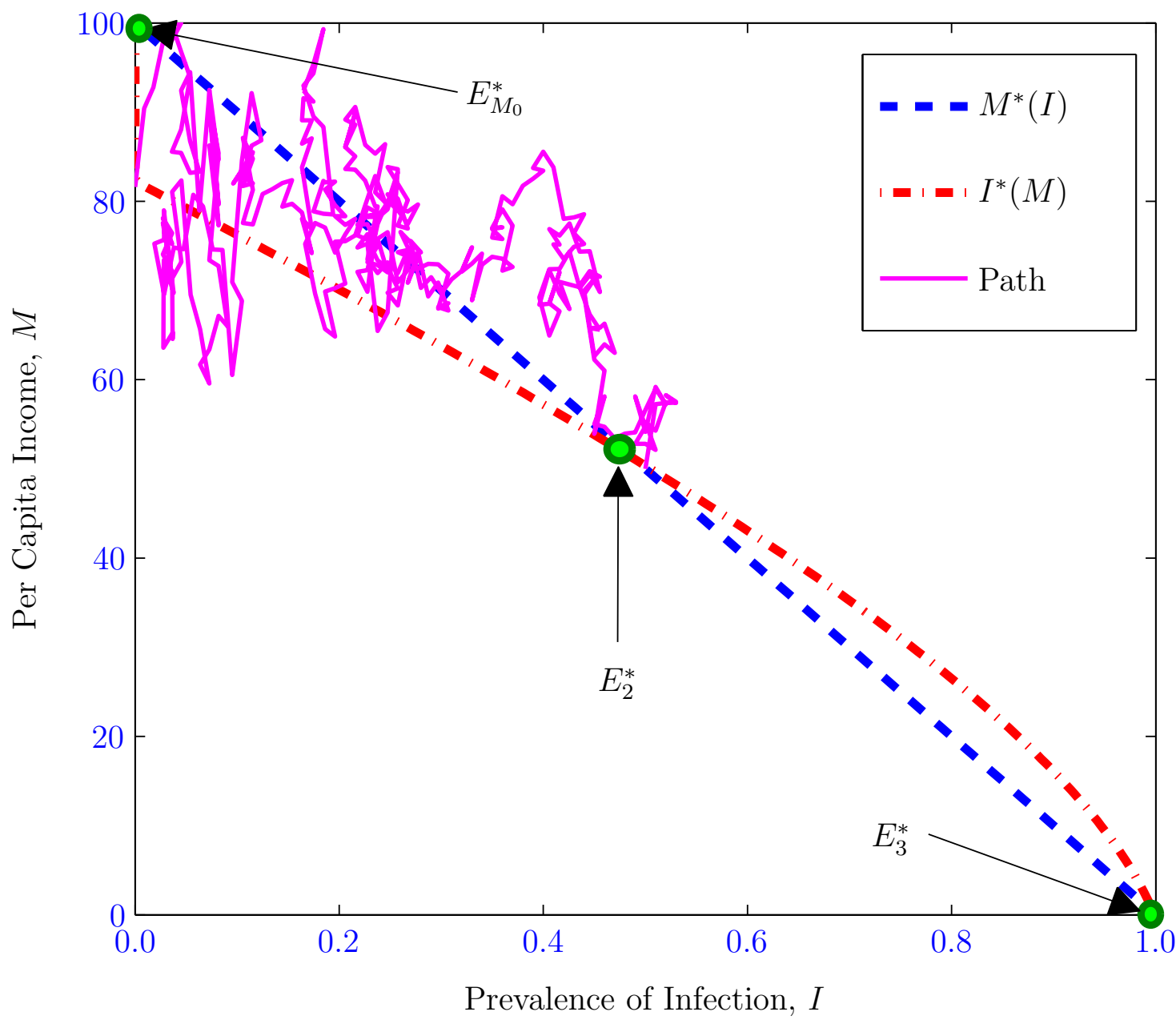

Figure 7.11. Stochastic simulations. In contrast to the deterministic case, it is possible to attain either of the two stable equilibria with the same initial conditions. For example, for the initial values $(I, M)=(0.5,50)$, the system converges to the development equilibrium during the first simulation. 


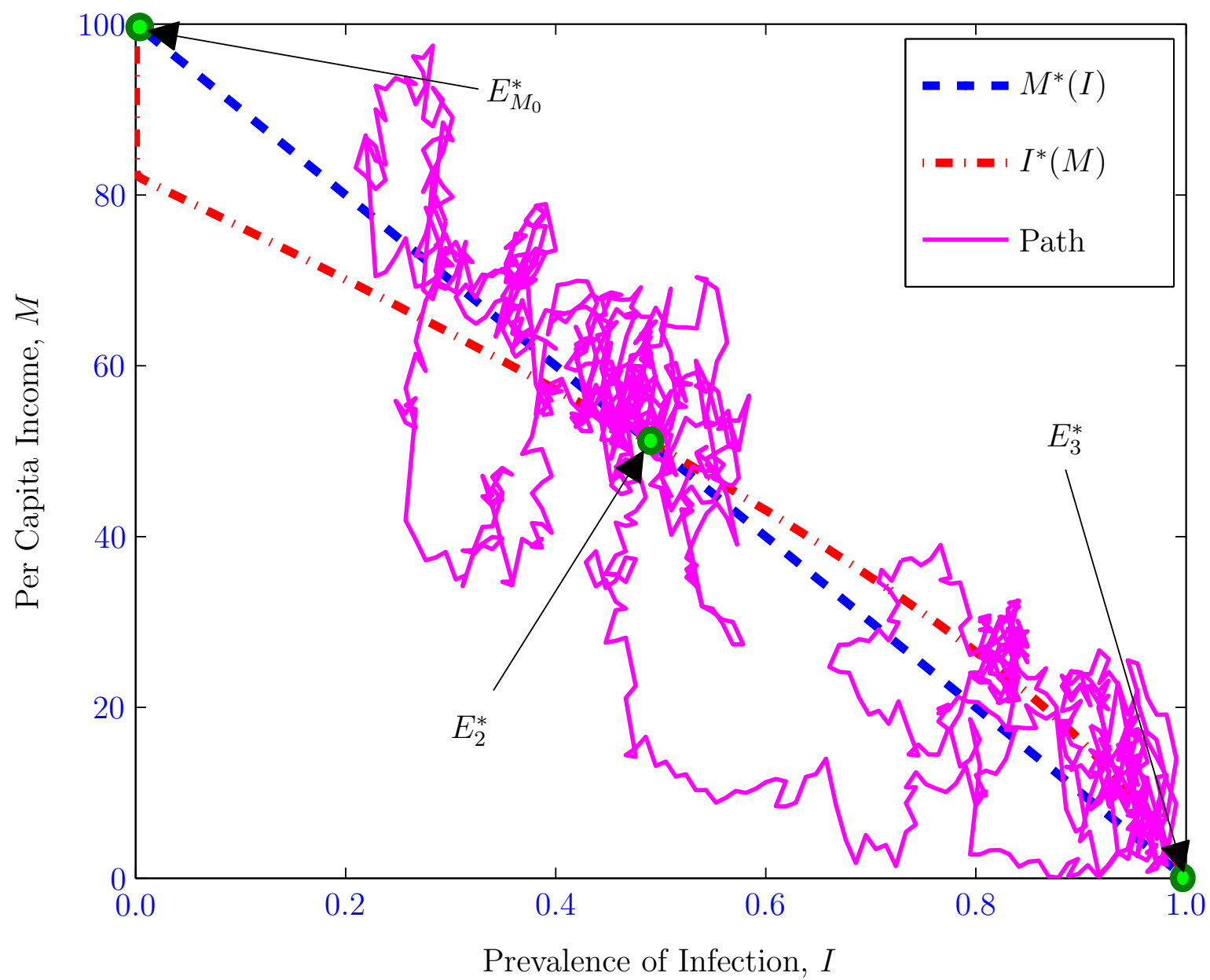

Figure 7.12. Stochastic simulations. In contrast to the deterministic case, it is possible to attain either of the two stable equilibria with the same initial conditions. For the same initial values $(I, M)=(0.5,50)$, the system converges to the poverty trap during the second simulation. 


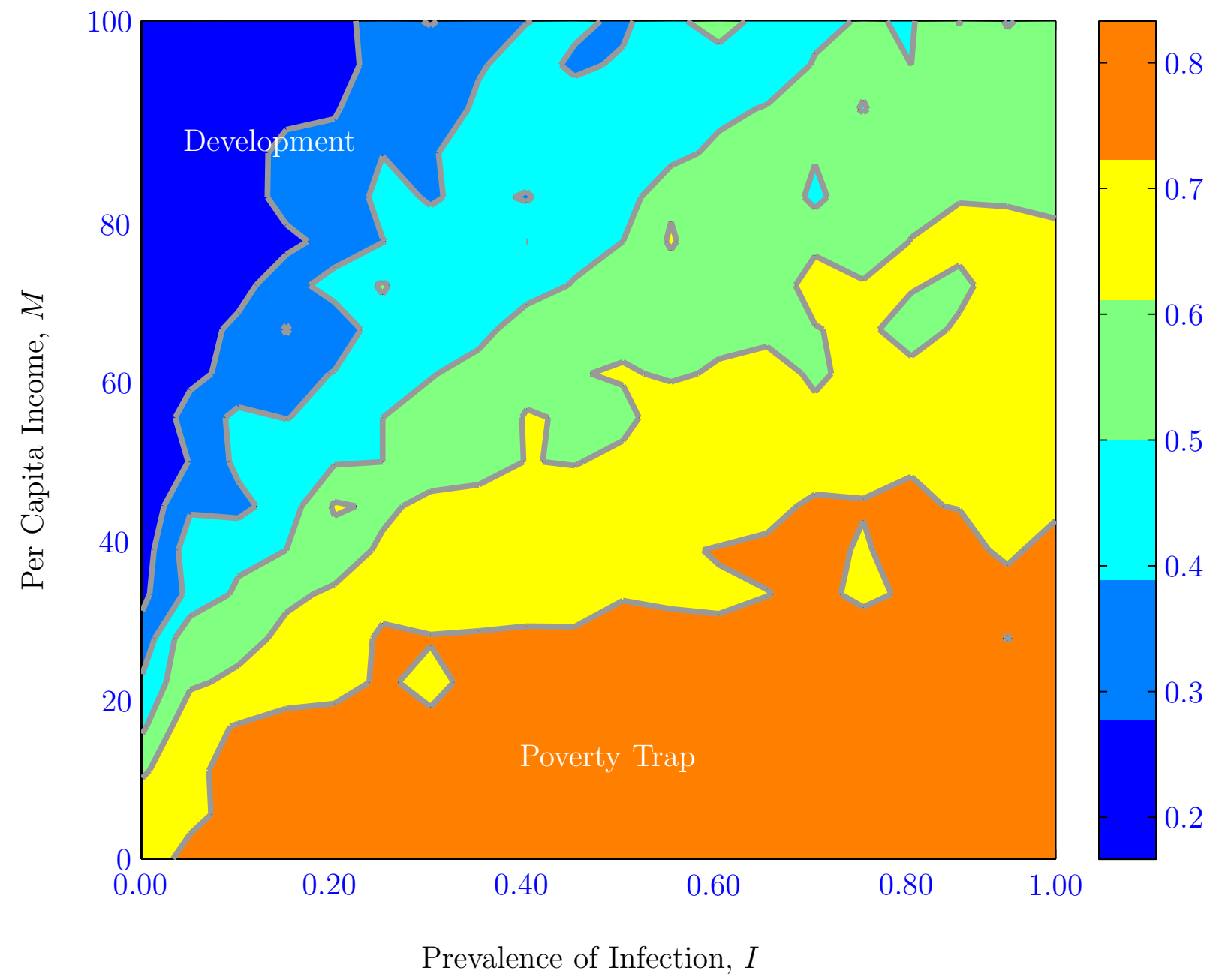

Figure 7.13. Phase plot illustrating probabilities that an initial condition will lead to landing in the poverty trap. Each solid line corresponds to a single probability of reaching either the development or poverty trap equilibrium. 


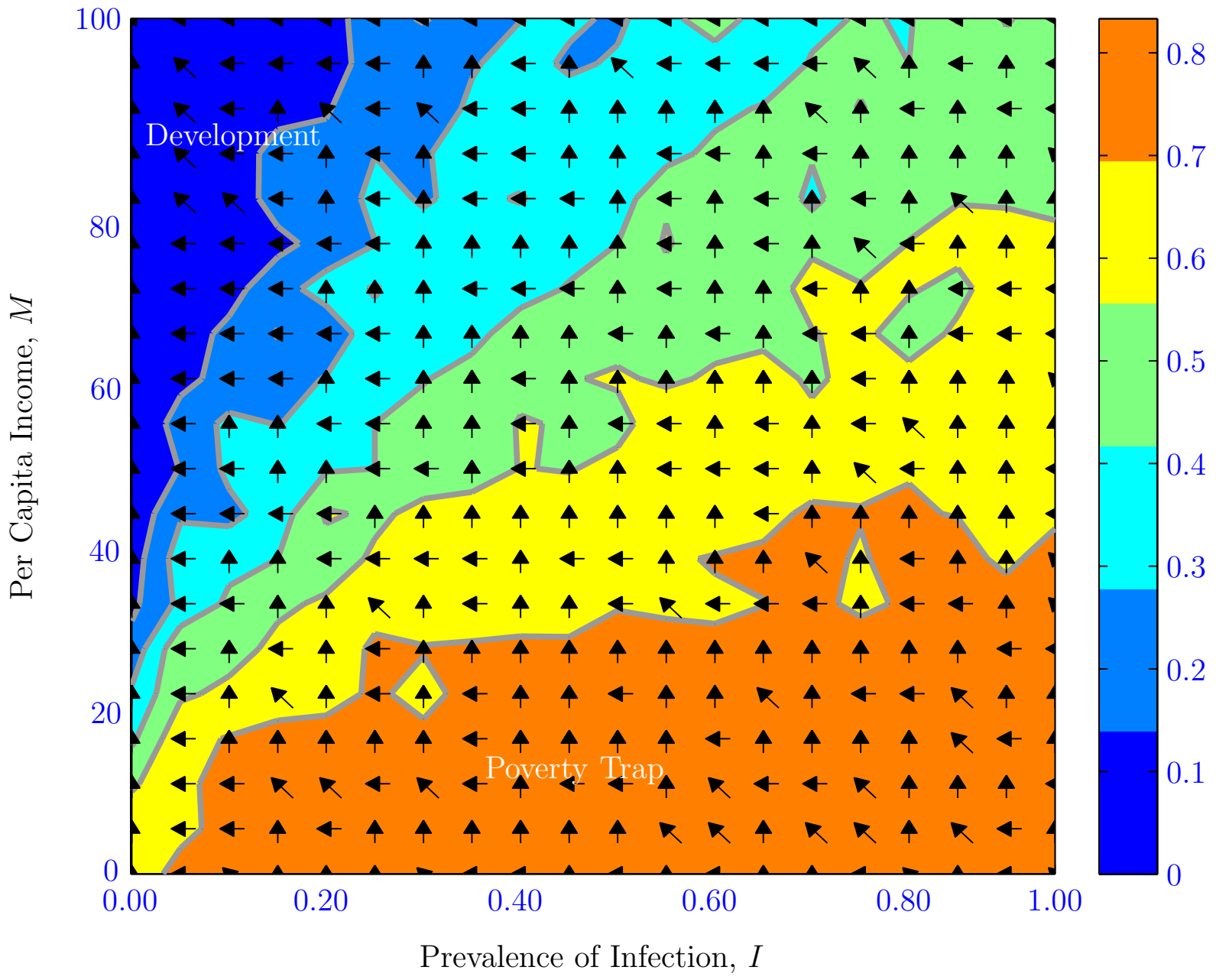

Figure 7.14. Phase plot illustrating the path to development. The arrows indicate the quickest path to the development equilibrium. Note that this path depends on the status of the level of income and disease. 


\subsection{Policy Interpretation}

As illustrated in Fig. 7.14, the level of income and disease prevalence plays a crucial role in determining the highest probability strategy of breaking a disease-driven poverty trap. Hence, a possible intervention strategy aimed at breaking disease-driven poverty traps involves effecting direct changes to the state variables, such as increasing income, reducing disease prevalence or increasing income and reducing disease prevalence simultaneously. The most efficient intervention in terms of the highest probability of leaving the poverty trap and heading towards the development equilibrium in areas of the state space where the arrows point vertically upward would be to increase income. In areas of state space where the arrows point horizontally to the left, the most efficient intervention strategy requires an improvement in health care, or a reduction in disease prevalence. Finally, in areas of state space in which the arrows point diagonally upwards and to the left, the best intervention strategy entails increasing income and reducing disease prevalence simultaneously. Such policies would be futile in a deterministic system where small interventions could change only the rate at which the system approaches its pre-destined stable equilibrium outcome.

A safety net, defined as the minimum enforced level of income and public health below which a population is not allowed to fall, constitutes another important intervention measure. Safety net interventions are modeled by incorporating an income threshold and a disease threshold and imposing conditions that prevent income from falling below the income threshold and disease prevalence from rising above the disease threshold. We then simulate the full stochastic model 500 times on a grid of initial values for disease prevalence and per capita income, with each simulation run until the development equilibrium is reached. The expected time to development equilibrium is estimated as the average of the time until the development equilibrium is reached for each of the 500 runs. The effect of safety nets is illustrated in Figs. 7.15-7.17. Development or convergence to the development equilibrium occurs even when the safety net is set within the basin of attraction of the poverty trap. Additionally, development occurs when the safety net is implemented as an economic (income safety net) and a public health (health safety net) policy simultaneously. This provides the fastest route to escape from the poverty trap. Furthermore, development is still attained 
even when the safety net is implemented only as an economic condition or only as a public health policy. Hence, safety nets can guarantee ultimate escape from a poverty trap.

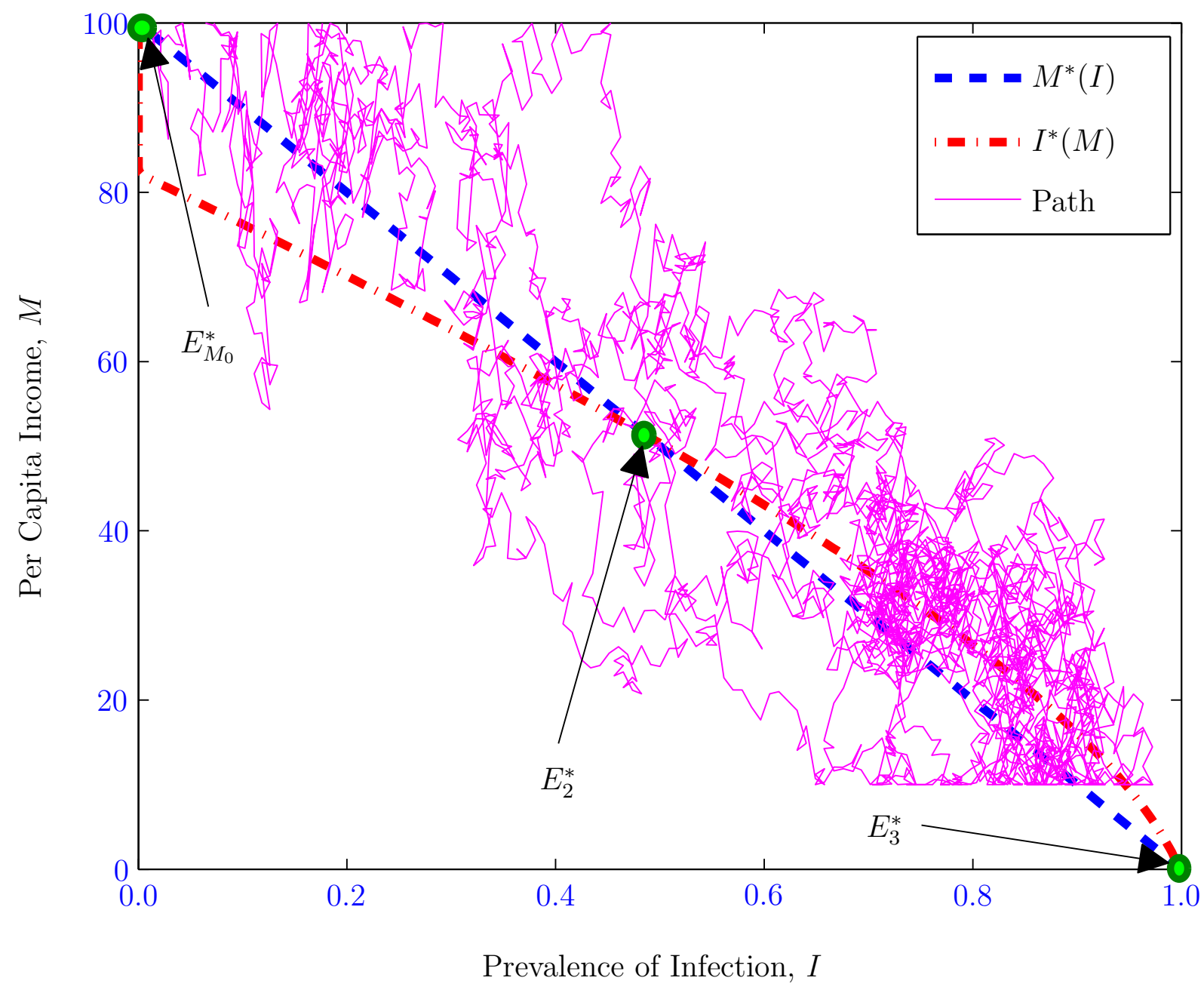

Figure 7.15. Stochastic simulation where the initial state is reinforced by a safety net. Income safety net set at $M=10$. The safety net ensures that the system is driven towards the development equilibrium even though it is set in the basin of attraction of the poverty trap. The broken blue line represents $M^{*}(I)$, the broken red line represents $I^{*}(M)$ and the magenta curve represents the path a typical trajectory follows. 


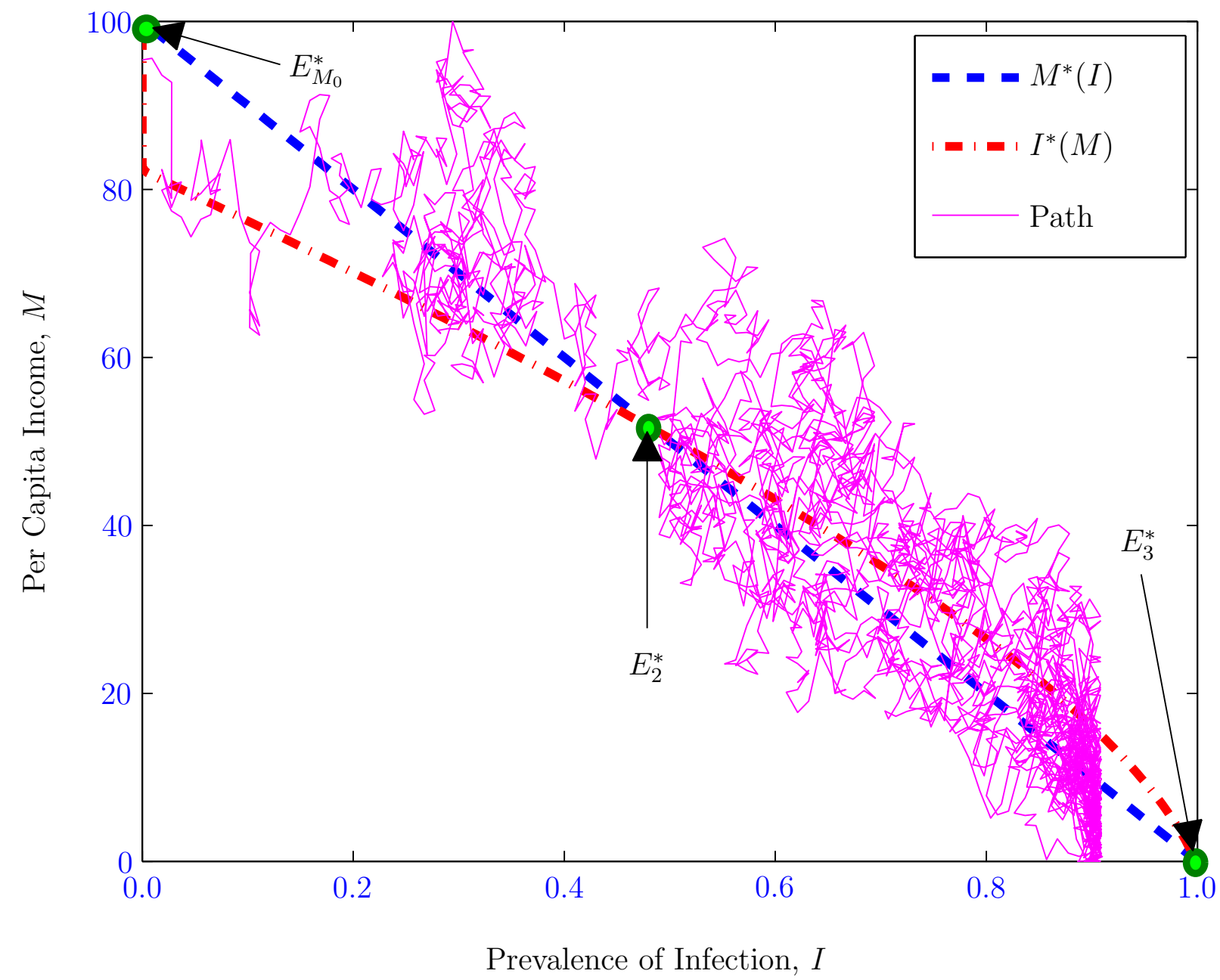

Figure 7.16. Stochastic simulation where the initial state is reinforced by a safety net. Health safety net set at $I=0.90$. The safety net ensures that the system is driven towards the development equilibrium even though it is set in the basin of attraction of the poverty trap. The broken blue line represents $M^{*}(I)$, the broken red line represents $I^{*}(M)$ and the magenta curve represents the path a typical trajectory follows. 


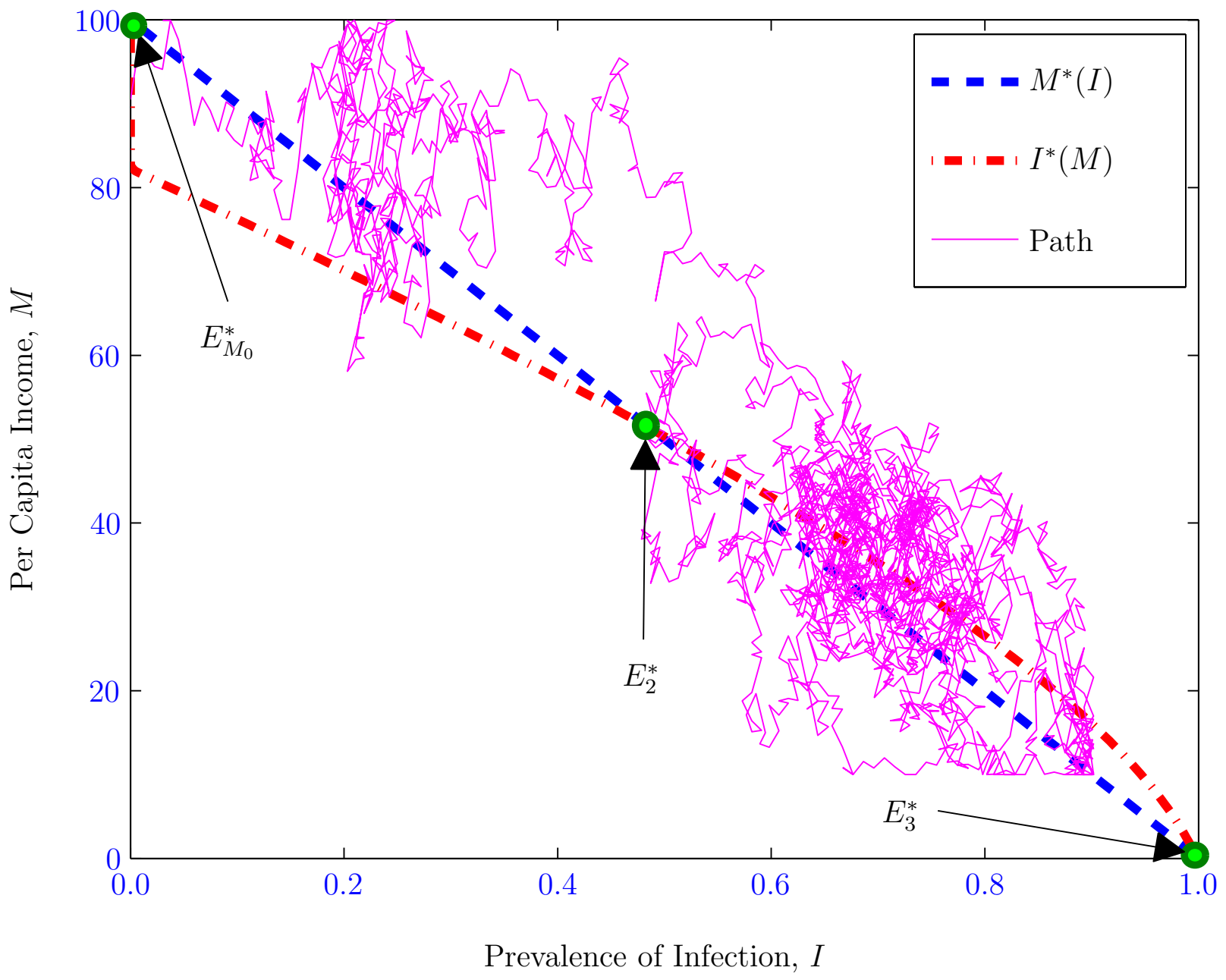

Figure 7.17. Stochastic simulation where the initial state is reinforced by a safety net. Income and health safety net, $(I, M)=(0.90,10)$. The safety net ensures that the system is driven towards the development equilibrium even though it is set in the basin of attraction of the poverty trap. The broken blue line represents $M^{*}(I)$, the broken red line represents $I^{*}(M)$ and the magenta curve represents the path a typical trajectory follows. 
The location of the safety net is important in determining the expected time to attain development. Figure. 7.18 presents the expected (i.e., mean) time it takes to reach the development equilibrium, which we define here as the "rate of development", for the full range of initial conditions when reinforced by safety nets in both health and income. The slope of the contour lines in Fig. 7.18 indicate the relative sensitivity of this rate of development to safety nets at different levels of income and disease. For example, if the slope of the contour lines in Fig. 7.18 is greater than 1, then changes in the disease safety net will have a larger impact on the rate of development than changes in the income safety net. At the extreme, a vertical contour line $($ slope $=\infty$ ) would indicate that the development process was limited only by disease, and therefore changes in a safety net in income would have no effect on the rate of development. Likewise, horizontal contours (slope $=0$ ) would signify that the rate of development is limited only by income. The proximity of the contour lines in the bottom right corner of Fig. 7.18 indicates that the rate of development is most sensitive to changes in the location of the safety net closest to the poverty trap equilibrium. 


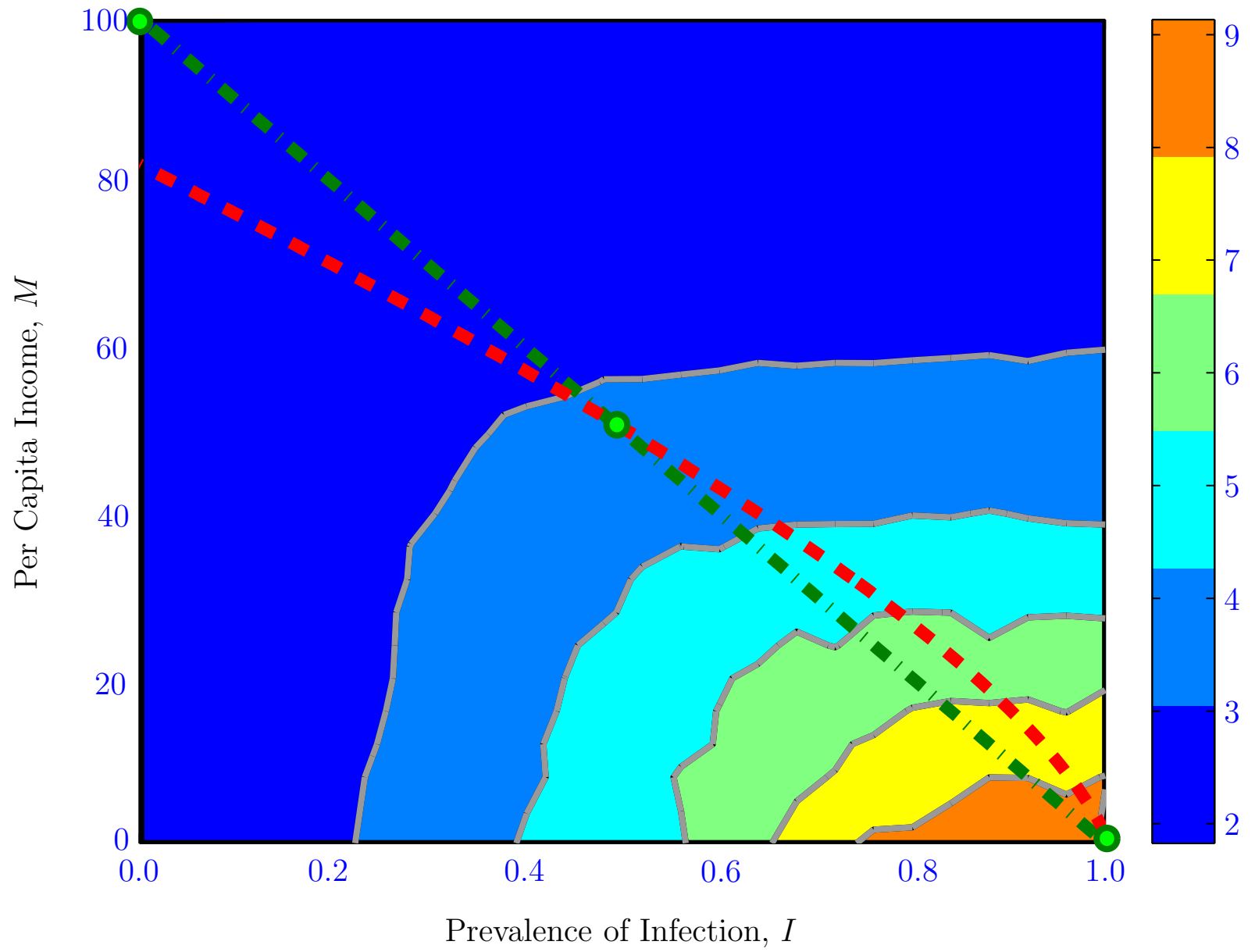

Figure 7.18. Phase plot illustrating the average time required to attain the development equilibrium from initial conditions that are reinforced by safety nets. 


\subsection{Conclusion}

We have used a simple SISM model framework to address some basic questions on the feedback between income and disease. We demonstrated that deterministic models of poverty traps have qualitatively different behavior than stochastic models, and correspond to a different, and more limited, set of policy options. Economic development in the deterministic model requires either significant changes to initial conditions or a change in the parametric structure of the system. In contrast, the stochastic model shows how external interventions in the form of safety nets can significantly improve the development trajectory of a population irrespective of knowledge of the location of the poverty trap threshold. Even minor safety nets in population health, per capita income or both can have long-term impacts on economic development. Not only can development result from safety nets that are set within the basin of attraction of the poverty trap, but the rate of development is most sensitive to changes in safety nets near the poverty trap equilibrium, where marginal improvements in population health are also likely to be the least expensive. Thus, we have shown that a safety net can guarantee ultimate escape from a poverty trap, even if it is set within the basin of attraction of the poverty trap, and even if the safety net is only in the form of a health intervention. If a population is near the poverty-trap equilibrium, the benefits of a safety net is lowest in the deterministic system and highest for the stochastic model.

The disease component of our model is generic and can be used to study a variety of human diseases such as bacterial diseases, sexually transmitted diseases, and many vectorborne diseases including malaria, which are well known for having a negative impact on economic growth. Therefore, our model can be adapted to study poverty traps for different countries-developed or developing, and for different diseases by building in factors that are specific to such countries and diseases.

In this research, we identified various optimal intervention strategies for taking populations out of disease-driven poverty traps and highlighted when it is best to apply a specific intervention measure. The research also highlights the role mathematical models can play in public policy making. In fact, based on our analysis of the stochastic model, the following optimal economic development and public health intervention questions were answered: 
(i) To help populations escape from poverty traps, is it preferable to provide health care, income/income generating resources (to enable these populations generate wealth), or both health care and income/income generating resources?

(ii) How long will it take a population in a poverty trap to attain economic development when the initial health and economic conditions are reinforced by safety nets?

Generally, the availability of good quantitative models is very important in the formulation of effective public policy. A new fundamental model such as ours can be studied in mathematical detail, and if it is found to produce good results, it can then be made more precise and used as an effective tool to guide decision makers. The relative lack of good mathematical models for understanding poverty dynamics, and the potential for this new model to explain and predict the evolution of income and poverty, makes this an exciting contribution within the field of dynamical system modeling, and on the formulation of public policy. This research provides a novel and plausible framework on which public policy can be informed by meaningful integration between economics and methodological advances in the natural sciences, epidemiology and mathematics.

Additionally, this research opens new areas for further investigation that include studying:

1. feedback between income and more realistic epidemiological models for specific diseases such as HIV, TB, malaria, childhood diseases, etc. in specific countries and

2. within-population dynamics as opposed to the previous framework whose dynamics is at the population level. This is necessary since within a given population or country, there are rich and healthy, rich and unhealthy, poor and healthy, and poor and unhealthy individuals. 


\section{References}

[1] UN Millenium Project, "Investing in Development: A Practical Plan to Achieve the Millenium Development Goals," UN Millenium Project, New York (accessed on April 20, 2011).

Online Version 164

[2] J. Sachs, End of Poverty; Economic Possibilities of Our Time (The Penguin Press, New York, NY, 2005). 164

[3] L. R. Easterly, "W. Africas Growth Tragedy: Policies and Ethnic Divisions," Quaterly Journal of Economics 112, 1203-1250 (1997).

Online Version

[4] P. Collier, "Poverty Reduction in Africa," Proceedings of the National Academy of Sciences 104, 16763-16768 (2007).

Online Version

[5] D. Bloom, D. Canning, and J. Sevilla, "Geographay and Poverty Traps," Journal of Economic Growth 8, 355-78 (2003).

Online Version 165

[6] C. B. Barrett and B. M. Swallow, "Fractal Poverty Traps," World Development 34, $1-15(2006)$.

Online Version 
[7] P. Dasgupta, "Poverty Traps: Exploring the Complexity of Causation," in J. von Braun, R. V. Hill, and R. Pandya-Lorch, eds., "The Poorest and Hungry," chapter 8, 107-188 (International Food Policy Research Institute (IFPRI), 2009).

Online Version 164

[8] S. D. Younger, "The Least Developed Countries Report 2002: Escaping the Poverty Trap," Journal of African Economies, Oxford University Press 12(4), 672-678 (2003). Online Version 164

[9] S. Bowles, S. N. Durlauf, and K. Hoff., Poverty Traps (Princeton University Press, Princeton, NJ, 2007). 165

[10] Chronic Poverty Research Centre , "Escaping Poverty Traps," The Chronic Poverty Report (2008-2009).

Online Version

[11] D. L. Gärtner and M. Gärtner, "Wage Traps as a Cause of Illiteracy, Child Labor, and Extreme Poverty," Research in Economics 65 (3), 232-242 (2011). 164

[12] S. Paternostro, "The Poverty Trap: The Dual Externality Model and its Policy Implications," World Development 25, 2071-2081 (1997).

Online Version 165

[13] C. B. Barrett and M. R. Carter, "Can’t Get Ahead for Falling Behind: New Directions for Development Policy to Escape Poverty and Relief Traps," Social Science Research Network eLibrary (2000).

[14] M. Whitehead, G. Dahlgren, and T. Evans, "Equity and Health Sector Reforms: Can Low-income Countries Escape the Medical Poverty Trap," The Lancet 358, 833-836 (2001).

Online Version

[15] D. McLeod, "How Fast did Developing Country Poverty Fall During the 1990s? Capabilities-based Tests of Rival Estimates," Economics Letters 90, 297-303 (2006). Online Version 
[16] A. Krishna, "For Reducing Poverty Faster: Target Reasons Before People," World Development 35, 1947-1960 (2007).

Online Version

[17] U. Gentilini and P. Webb, "How are We Doing on Poverty and Hunger Reduction? A New Measure of Country Performance," Food Policy 33, 521-532 (2008), food Product Composition, Consumer Health, and Public Policy.

Online Version

[18] B. H. Nordtveit, "Poverty Alleviation and Integrated Service Delivery: Literacy, Early Child Development and Health," International Journal of Educational Development 28, 405-418 (2008).

Online Version

[19] M. A. Hanjra, T. Ferede, and D. G. Gutta, "Pathways to Breaking the Poverty Trap in Ethiopia: Investments in Agricultural Water, Education, and Markets," Agricultural Water Management 96, 1596-1604 (2009).

Online Version

[20] M. A. Hanjra, T. Ferede, and D. G. Gutta, "Reducing Poverty in Sub-Saharan Africa through Investments in Water and Other Priorities," Agricultural Water Management 96, $1062-1070$ (2009).

Online Version

[21] Y. Gokan, "Poverty Traps, the Money Growth Rule, and the Stage of Financial Development," Journal of Economic Dynamics and Control 35, 1273-1287 (2011). Online Version 165

[22] D. E. Bloom and D. Canning, "The Health and Wealth of Nations," Science 287, 12071209 (2000).

Online Version 165

[23] J. L. Gallup and J. D. Sachs, "The Economic Burden of Malaria," American Journal of 
Tropical Medicine and Hygiene 85-86 (2001).

Online Version 165, 170

[24] P. Lorentzen, J. McMillan, and R. Wacziarg, "Death and Development," Journal of Economic Growth 13, 81-124 (2008).

Online Version 165

[25] P. Collier, A. Hoeffler, H. Elliot, H. Hegre, M. Reynal-Querol, and N. Sambanis, Breaking the Conflict Trap Civil War and Development Policy (Oxford University Press and World Bank, Oxford and Washington D. C., 2003). 165

[26] P. Pinstrup-Andersen and S. Shimokawa, "Do Poverty and Poor Health and Nutrition Increase the Risk of Armed Conflict Onset?" Food Policy 33, 513-520 (2008), food Product Composition, Consumer Health, and Public Policy.

Online Version 165

[27] S. Dasgupta, U. Deichmann, C. Meisner, and D. Wheeler, "Where is the Poverty Environment Nexus? Evidence from Cambodia, Lao PDR, and Vietnam," World Development 33, 617-638 (2005).

Online Version 165

[28] D. Delfino and P. J. Simmons, "Dynamics of Tuberculosis and Economic Growth," Environment and Development Economics 10, 719-7432 (2005).

Online Version 165, 171

[29] D. Bloom and D. Canning, "The Health and Poverty of Nations: From Theory to Practice," Journal of Human Development 4, 47-71 (2003).

Online Version 165

[30] C. Azariadis and J. Stachurski, "Poverty Traps," in P. Aghion and S. Durlauf, eds., "The Handbook of Economic Growth," chapter 8, 2 (Elsevier, 2005).

Online Version 165, 166

[31] P. Dasgupta, "Nutritional Status, the Capacity for Work, and Poverty Traps," Journal 
of Econometrics 77, 5-37 (1997).

Online Version

[32] A. Deaton, "Health, Inequality and Economic Development," Journal of Economic Literature 1, 113-158 (2003).

Online Version

[33] S. N. Durlauf, "A Theory of Persistent Income Inequality," Journal of Economic Growth 1, 75-93 (1996).

Online Version

[34] M. Carter and C. B. Barrett, "The Economics of Poverty Traps and Persistent Poverty: An Asset-based Approach," Journal of Development Studies 42, 178-199 (2006). Online Version 165

[35] M. Bonds, D. Keenan, P. Rohani, and J. Sachs, "Poverty Trap Formed by the Ecology of Infectious Diseases," Proceedings of the Royal Society B 277 (1685), 1185-1192 (2010).

Online Version 165, 171

[36] R. Lefever and W. Horsthemke, "Bistability in Fluctuating Environments. Implications in Tumor Immunology," Bulletin of Mathematical Biology 41, 469-490 (1979).

Online Version 166

[37] M. Samoilov, S. Plyasunov, and A. Arkin, "Stochastic Amplification and Signaling in Enzymatic Futile Cycles Through Noise-induced Bistability with Oscillations," Proceedings of the National Academy of Sciences 102, 2310-2315 (2005).

Online Version 166

[38] W. J. Den Haan, "Convergence of Stochastic Growth Models: The Importance of Understanding Why Income Levels Differ," Journal of Monetary Economics 35, 65-82 (1995).

Online Version 166 
[39] H. G. Andrewartha and L. C. Birch, The Distribution and Abundance of Animals (University of Chicago Press, Chicago, 1954). 166

[40] R. Lewontin and D. Cohen, "On Population Growth in a Randomly Varying Environment," Proceedings of the National Academy of Sciences 62, 1056-1060 (1969).

Online Version

[41] O. N. Bjørnstad and B. T. Grenfell, "Noisy Clockwork: Time Series Analysis of Population Fluctuations in Animals," Science 293, 638-643 (2001).

Online Version 166

[42] E. R. M. McCauley, R. M. Nisbet, W. W. Murdoch, A. M. de Roos, and W. S. C. Gurney, "Large Amplitude Cycles of Daphnia and its Algal Prey in Enriched Environments." Nature 402, 653-656 (1999).

Online Version

[43] C. Zimmer, "Life after Chaos," Science 284, 83-86 (1999).

Online Version

[44] B. T. Grenfell, K. Wilson, B. F. Finkenstadt, T. N. Coulson, S. Murray, S. D. Albon, J. M. Pemberton, T. H. Clutton-Brock, and M. J. Crawley, "Noise and Determinism in Synchronised Sheep Dynamics," Nature 394, 674-677 (1998).

Online Version

[45] J. M. Cushing, B. Dennis, R. A. Desharnais, and R. F. Costantino, "Moving Toward an Unstable Equilibrium: Saddle Nodes in Population Systems," Journal of Animal Ecology 67, 298-306 (1998).

Online Version

[46] T. Rouyer, J. M. Fromentin, F. Menard, B. Cazelles, K. Briand, R. Pianet, B. Planque, and N. C. Stenseth, "Complex Interplays Among Populations Dynamics, Environmental Forcing, and Exploitation in Fisheries," Proceedings of the National Academy of Sciences 105, 5420-5425 (2008).

Online Version 166 
[47] M. J. Keeling and B. T. Grenfell, "Stochastic Dynamics and a Power Law for Measles Variability," Philosophical Transactions of the Royal Society of London, B 354, 769776 (1999).

Online Version 166

[48] M. J. Keeling, P. Rohani, and B. T. Grenfell, "Seasonally Forced Disease Dynamics Explored as Switching Between Attractors," Physica D 148, 317-335 (2001). Online Version

[49] A. J. Black and A. J. McKane, "Stochasticity in Staged Models of Epidemics: Quantifying the Dynamics of Whooping Cough," Journal of the Royal Society Interface 7, 1219-1227 (2010).

Online Version

[50] P. Rohani, M. J. Keeling, and B. T. Grenfell, "The Interplay Between Determinism and Stochasticity in Childhood Diseases," American Naturalist 6, 469-481 (2002).

Online Version

[51] K. Koella and M. Pascual, "Disentangling Extrinsic from Intrinsic Factors in Disease Dynamics: A Nonlinear Time Series Approach with an Application to Cholera," American Naturalist 163, 901-913 (2004).

Online Version 166

[52] S. Gandon, M. J. Mackinnon, S. Nee, and A. F. Read, "Imperfect Vaccines and the Evolution of Parasite Virulence," Nature 414, 751-755 (2001).

Online Version 170

[53] D. L. Smith, J. Dushoff, R. Snow, and S. Hay, "The Entomological Inocolution rate of Plasmodium falciparum Infection in African Children," Nature 438, 492-495 (2005). Online Version 170

[54] T. J. N. Bailey, The Mathematical Theory of Infectious Diseases and its Application (Griffin, London, 1975), 2nd edition. 170 
[55] H. Hethcote, "Qualitative Analyses of Communicable Disease Models," Mathematical Biosciences 18, 335-356 (1976).

Online Version

[56] R. L. May and R. M. Anderson, Infectious Diseases of Humans: Dynamics and Control (Oxford University Press, Oxford, 1991).

[57] Y. Pei, S. Liu, C. Li, and L. Chen, "The Dynamics of an Impulsive Delay SI Model with Variable Coefficients," Applied Mathematical Modelling 33 (6), 2766-2776 (2009).

Online Version 170

[58] M. H. Bonds and P. Rohani, "Herd Immunity Caused Indirectly by Interactions Between the Ecology of Infectious Diseases, Demography, and Economics," Journal of the Royal Society Interface 7, 541-547 (2010).

Online Version 171

[59] D. Collins, J. Murduch, S. Rutherford, and O. Ruthven, Portfolios of the Poor (Princeton University Press, Princeton, NJ, 2009). 188

[60] C. B. Barrett and J. McPeak, "Poverty Traps and Safety Nets," in A. de Janvry and R. Kanbur, eds., "Poverty, Inequality and Development; Essays in Honor of Erik Thorbecke," chapter 8, 131-152 (Springer, 2006).

Online Version 188 


\section{Part III}

\section{Extreme Multistability in a Chemical Model \\ System}


Chapter 8

\section{Introduction to Chemical Chaos}

\subsection{Introduction to Chemical Chaos through the Autocatalator Model}

Many physical phenomena such as the weather, naturally occurring biological, physical and chemical systems, the human heartbeat, etc. have been shown to exhibit complex dynamical behaviors such as chaos [1]. Chaos is characterized by dynamical behavior that includes the emergence of strange attractors. Examples of systems that exhibit chaotic behavior include oscillatory chemical reactions. Such oscillatory systems present different periodic behaviors in response to variations in a parameter or a set of parameters in the system. Some systems exhibit period-doubling bifurcations, whereby trajectories take approximately twice as long to repeat as each bifurcation is realized. Period-doubling bifurcations culminate in chaotic behavior, whereby oscillations with infinite periods emerge. A widely studied system that exhibits such complex behavior is the Belousov-Zhabostinsky (BZ) reaction [2-4].

Oscillatory and chaotic dynamics do not require great complexity in the chemical mechanism. Chemical systems may exhibit chaotic dynamics if the chemistry includes sufficient feedback, which may also give rise to multiple steady states in a system. Chemical feedback typically occurs as a result of autocatalysis or non-isothermal reactions. Autocatalysis occurs when a product contributes in its own production while a non-isothermal reaction is one in which a change in temperature occurs. Autocatalytic reactions generally begin at slow rates, since the initial amount of the autocatalyst is small, and then speed up over time with the consumption of the reactant and production of more autocatalyst. A number of prototypical chemical reaction schemes for investigating oscillatory behavior have been pro- 
posed and analyzed. Our investigation is based on a chemical model system that has been used in many studies of chemical oscillations and chaotic dynamics, the autocatalator model [5-11]. The two-variable autocatalator system, originally introduced by Gray and Scott [12], exhibits simple oscillations [13-16], while the three-variable autocatalator model, proposed by Peng et al. [17], exhibits complex periodic and chaotic behavior. Numerical studies have been carried out to investigate the quantitative behavior of the three-variable autocatalator model (see, for example, Ref. [18]); however, fewer studies have appeared on the qualitative behavior of the model. Hence, our goal in this Chapter is to carry out a comprehensive investigation of the qualitative behavior of the three-variable autocatalator model.

The Chapter is organized as follows. In Section 8.2, we review the two-variable autocatalator model. We present the three-variable autocatalator model in Section 8.3, reproduce as well as extend previous quantitative results through numerical simulations in Section 8.3.2, present a full qualitative analysis in Section 8.3.3, and summarize our results in Section 8.4.

\subsection{The Two-variable Autocatalator Model}

The two-variable autocatalator model is possibly the simplest prototype chemical model that is capable of exhibiting oscillations. The scheme involves the conversion of a chemical precursor $A$ with constant concentration to a final product $B$ through two intermediate species, $X$ and $Y$. Let $k_{i},(i=0,1,2,3)$ be rate constants; the kinetic model is described by the following 4-step sequence of reactions:

$$
\begin{array}{rr}
A \stackrel{k_{0}}{\longrightarrow} X, & \left(R_{8.1}\right) \\
X \stackrel{k_{1}}{\longrightarrow} Y, & \left(R_{8.2}\right) \\
X+2 Y \stackrel{k_{2}}{\longrightarrow} 3 Y, & \left(R_{8.3}\right) \\
Y \stackrel{k_{3}}{\longrightarrow} B . & \left(R_{8.4}\right)
\end{array}
$$

Reaction $\left(R_{8.3}\right)$, in which the intermediate species $Y$ catalyzes its own production is the most crucial reaction of this model. This step is called the cubic autocatalysis step, which is the reason why this model is called the cubic autocatalator model. It provides a nonlinear feedback mechanism in the system that accounts for the observed oscillatory dynamics. The 
corresponding ordinary differential equation model is:

$$
\begin{aligned}
& {[\dot{X}]=k_{0} A_{0}-k_{1}[X][Y]^{2}-k_{3}[X]} \\
& {[\dot{Y}]=k_{1}[X][Y]^{2}-k_{2}[Y]+k_{3}[X]}
\end{aligned}
$$

where $A_{0}$ is the concentration of the reactant $A$. The dimensionless variables and parameters,

$$
x=\left(\frac{k_{2}}{k_{3}}\right)^{\frac{1}{2}}[X], y=\left(\frac{k_{2}}{k_{3}}\right)^{\frac{1}{2}}[Y], t=k_{3} \tau, \mu=\left(\frac{k_{2}}{k_{3}}\right) \frac{k_{0} A_{0}}{k_{2}}, \alpha=\frac{k_{1}}{k_{3}},
$$

simplify system (8.1) to

$$
\begin{gathered}
\dot{x}=\mu-x y^{2}-\alpha x, \\
\dot{y}=x y^{2}-y+\alpha x .
\end{gathered}
$$

This system has been analyzed in detail in a number of papers. Merkin et al. [13] considered the special case in which the uncatalyzed reaction $\left(R_{8.2}\right)$ is excluded from the system. They identified a stationary state $\left(x^{*}, y^{*}\right)=(1 / \mu, \mu)$ and showed that it is stable for $\mu \geq 1$. They also showed that a Hopf bifurcation occurs at $\mu=1$, and that a stable limit cycle exits for $\mu_{c}<\mu<1$, where $\mu_{c}=0.90032$. Merkin et al. [14] also studied the full system (8.2). They identified a steady state $\left(x^{*}, y^{*}\right)=\left(\mu /\left(\mu^{2}+\alpha\right), \mu\right)$, which is stable for $\alpha>0.125$. For $\alpha<0.125$, they identified two critical values of $\mu$,

$$
\mu_{0}^{c}=\sqrt{\frac{(1-2 \alpha)-\sqrt{1-8 \alpha}}{2}} \text { and } \mu_{1}^{c}=\sqrt{\frac{(1-2 \alpha)+\sqrt{1-8 \alpha}}{2}},
$$

at which Hopf bifurcations occur and showed that a stable limit cycle exists for $\mu_{0}^{c}<\mu<\mu_{1}^{c}$. Gray et al. [15] examined the effect of the inclusion of a quadratic step in the model. Forbes and Holmes [16] used analytical and numerical techniques to show that limit cycle behavior is only possible in a restricted region of the parameter space. They also found strong numerical evidence that this limit cycle is unique and stable to infinitesimal perturbations.

Figure 8.1 represents a bifurcation diagram of system (8.2) for $\alpha=0.1$ and $0.3 \leq \mu \leq 0.9$. Notice that for this value of $\alpha$, the critical values of $\mu$ at which Hopf bifurcations occur are, respectively, $\mu_{0}^{c}=0.4200$ and $\mu_{1}^{c}=0.7897$. Figures 8.2.1 and 8.2.2 are, respectively, the time series and phase plots of the system for the parameter values $\mu=0.5$ and $\alpha=0.0625$. 


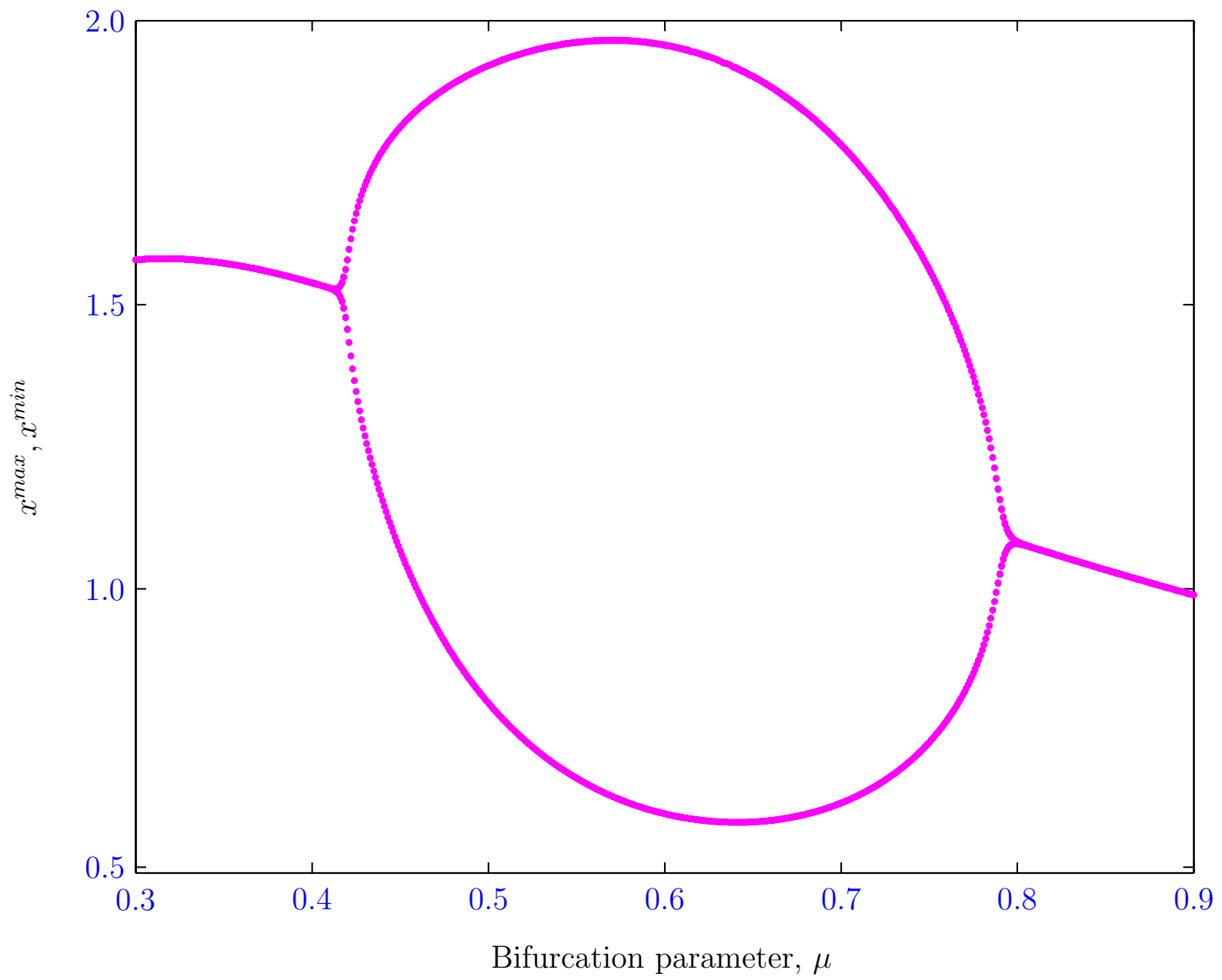

Figure 8.1. Bifurcation diagram for the two-variable autocatalator model. The maximum and minimum values of the variable $x$ are plotted against the parameter $\mu$, which serves as the bifurcation parameter. For $\alpha=0.1$, the critical values of $\mu$ at which Hopf bifurcations occur are $\mu_{0}^{c}=0.4200$ and $\mu_{1}^{c}=0.7897$. 


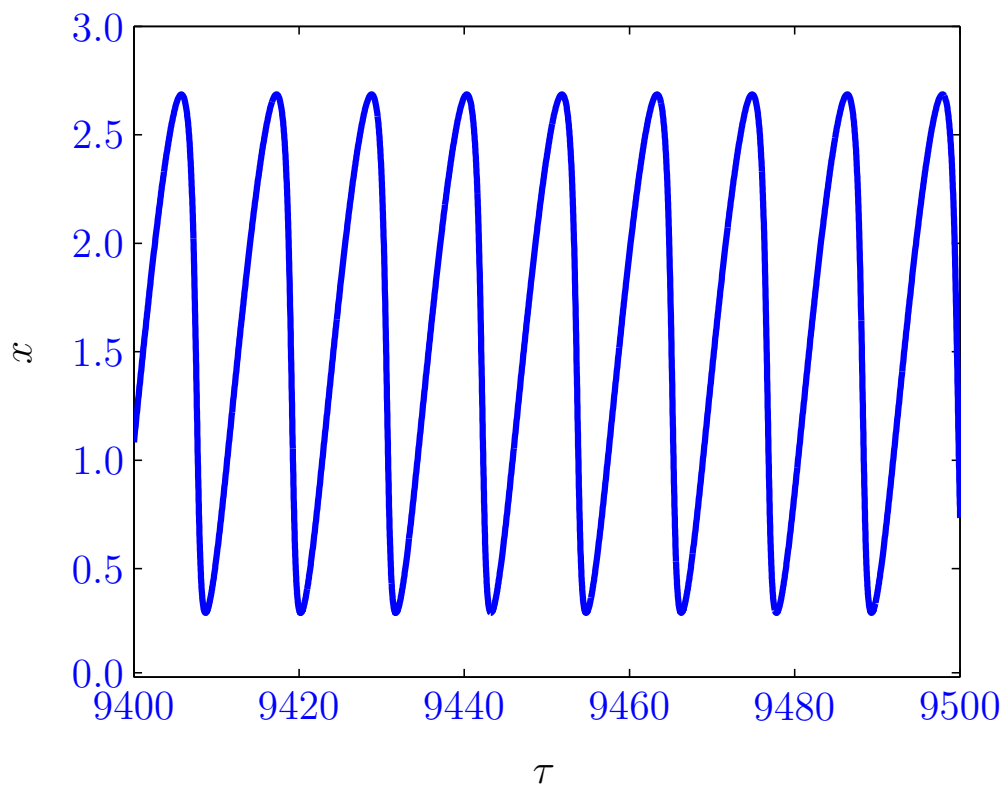

8.2.1. Time series plot showing periodic behavior.

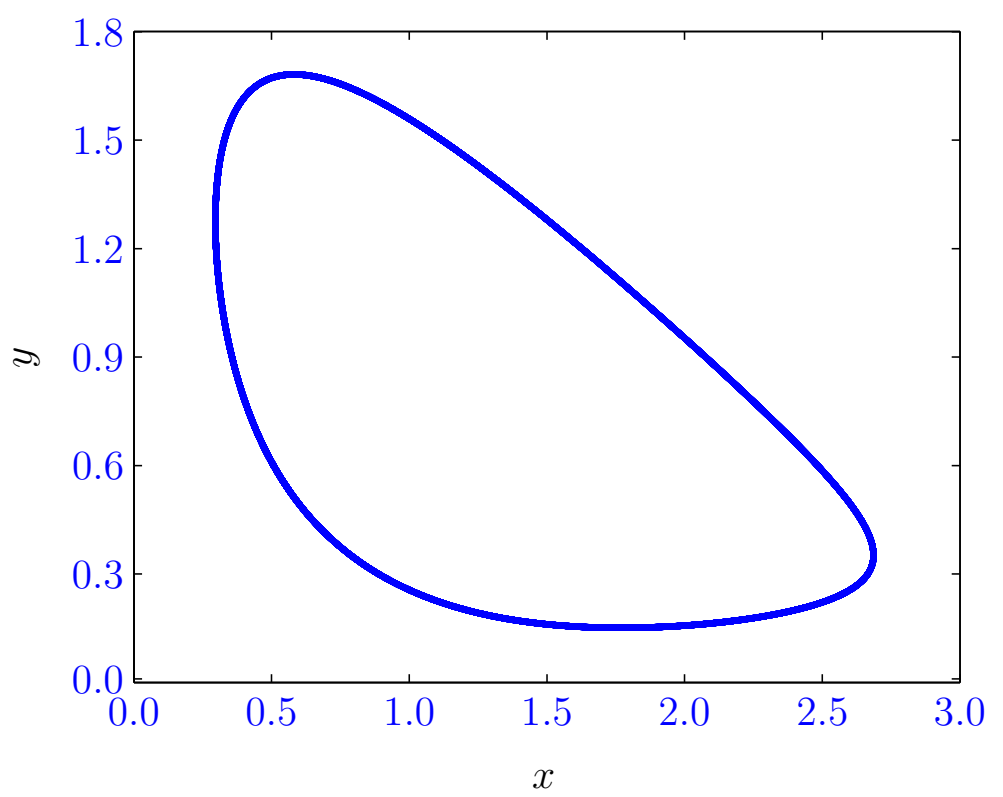

8.2.2. Phase plot showing limit cycle.

Figure 8.2. Time series and phase plots of the two-variable autocatalator model for the parameter values $\mu=0.5$ and $\alpha=0.0625$. In Fig. 8.2.1, we plot $x$ (the dimensionless concentration of the intermediate $X$ ) against time, and in Fig. 8.2.2 we plot $y$ (the dimensionless concentration of the intermediate $Y$ ) against $x$. 
Generally, the two-variable autocatalator model has one steady state, which is stable within a particular parameter regime and unstable outside this regime, with oscillatory concentrations of the intermediate species in the unstable region. The two-variable autocatalator model exhibits only steady state and oscillatory behavior. For more complex dynamical behavior such as chaos, a system with at least three variables is required.

\subsection{The Three-variable Autocatalator Model}

\subsubsection{The Model}

The original three-variable autocatalator model is derived from the two-variable model. Unlike the two-variable autocatalator model, the three-variable model incorporates a second feedback loop and, hence, is capable of exhibiting complex periodic behavior and chaos. The model reaction scheme involves the conversion of a chemical precursor $A$ with constant concentration to a final product $B$ via three intermediate species $X, Y$ and $Z$. If $k_{i}(i=0$, $1,2,3,4,5)$ are the rate constants for the reactions, the model is given by the following six reaction steps:

$$
\begin{array}{cr}
A \stackrel{k_{0}}{\longrightarrow} X, & \left(R_{8.5}\right) \\
A+Z \stackrel{k_{1}}{\longrightarrow} X+Z, & \left(R_{8.6}\right) \\
X \stackrel{k_{2}}{\longrightarrow} Y, & \left(R_{8.7}\right) \\
X+2 Y \stackrel{k_{3}}{\longrightarrow} 3 Y, & \left(R_{8.8}\right) \\
Y \stackrel{k_{4}}{\longrightarrow} Z, & \left(R_{8.9}\right) \\
Z \stackrel{k_{5}}{\longrightarrow} B . & \left(R_{8.10}\right)
\end{array}
$$

The fourth reaction, $\left(R_{8.8}\right)$, in the above system describes an autocatalytic process whereby the intermediate species $Y$ catalyzes its own production. This autocatalysis introduces a nonlinear reaction term $k_{3} X Y^{2}$, which is essential for the oscillatory behavior observed in the system. The chaotic behavior arises as a result of the introduction of a second feedback loop through the third variable $Z$ in $\left(R_{8.6}\right)$. Notice that $Z$ is produced from reaction $\left(R_{8.9}\right)$ and fed back into the system through reaction $\left(R_{8.6}\right)$ to catalyze the 
production of $X$ from $A$. Notice also that in reaction $\left(R_{8.8}\right), Y$ is an autocatalyst while in reaction $\left(R_{8.6}\right), Z$ serves as a normal catalyst.

Let $A_{0},[X],[Y]$ and $[Z]$ be the concentrations of $A, X, Y$ and $Z$, respectively. Then using standard mass action laws, which state that the instantaneous rate of change of any species is proportional to the product of the concentrations of the reacting species, we write the following system of first-order ordinary differential equations for the time evolution of the intermediate species:

$$
\begin{aligned}
{[\dot{X}] } & =k_{0} A_{0}+k_{1} A_{0}[Z]-k_{2}[X]-k_{3}[X][Y]^{2} \\
{[\dot{Y}] } & =k_{2}[X]+k_{3}[X][Y]^{2}-k_{4}[Y] \\
{[\dot{Z}] } & =k_{4}[Y]-k_{5}[Z]
\end{aligned}
$$

where the concentration of the precursor is held constant at $A_{0}$. Using the new variables

$$
x=\left(\frac{k_{3} k_{2}}{k_{4}^{2}}\right)^{\frac{1}{2}}[X], y=\left(\frac{k_{3}}{k_{2}^{2}}\right)^{\frac{1}{2}}[Y], z=\left(\frac{k_{3} k_{5}^{2}}{k_{2} k_{4}^{2}}\right)^{\frac{1}{2}}[Z], \tau=k_{2} t,
$$

and the positive dimensionless parameter groupings

$$
\mu=\left(\frac{k_{1}}{k_{5}}\right) A_{0}, \kappa=\left(\frac{k_{0} k_{5}}{k_{1} k_{4}}\right)\left(\frac{k_{3}}{k_{2}}\right)^{\frac{1}{2}}, \sigma=\left(\frac{k_{2}}{k_{4}}\right), \delta=\left(\frac{k_{2}}{k_{5}}\right),
$$

proposed by Peng et al. [18], we obtain the following scaled version of the system (8.3):

$$
\begin{aligned}
\dot{x} & =\mu(\kappa+z)-x\left(1+y^{2}\right), \\
\sigma \dot{y} & =x\left(1+y^{2}\right)-y, \\
\delta \dot{z} & =y-z .
\end{aligned}
$$

Remark 8.3.1. To arrive at the dimensionless variable and parameter groupings, we set

$$
\tau=\frac{t}{t^{0}}, x=\frac{[X]}{X^{0}}, y=\frac{[Y]}{Y^{0}} \text { and } z=\frac{[Z]}{Z^{0}}
$$

where $t^{0}, X^{0}, Y^{0}$ and $Z^{0}$ are reference variables. Substituting into system (8.3), we obtain

$$
t^{0}=\frac{1}{k_{2}}, X^{0}=\left(\frac{k_{4}^{2}}{k_{2} k_{3}}\right)^{\frac{1}{2}}, Y^{0}=\left(\frac{k_{2}^{2}}{k_{3}}\right)^{\frac{1}{2}}, Z^{0}=\left(\frac{k_{2} k_{4}^{2}}{k_{3} k_{5}^{2}}\right)^{\frac{1}{2}},
$$

and the parameter groupings given in (8.4). 
We note that $0<x, y, z<\infty$ since $x, y$ and $z$ represent dimensionless concentrations of chemical reagents. The parameters $\sigma, \delta, \mu$ and $\kappa$ are positive as well since they represent combinations of reaction rate constants. The only parameter that can vary is $\mu$, which contains the rate constants $k_{1}$ and $k_{5}$ and the fixed concentration of the precursor $A_{0}$. Hence, $\mu$ serves as the bifurcation parameter whose variations give rise to the transitions between different dynamical behaviors. The above system has been successfully used in studies on chemical chaos and controlling chemical chaos. See, for example, Refs. [18-22] for details.

\subsubsection{A Quantitative Study of the Three-variable Autocatalator Model}

In our analysis of system (8.4), we use the initial condition $\left(x_{0}, y_{0}, z_{0}\right)=(0.01,0.1,0.1)$, and reserve $\mu$ for the bifurcation parameter, allowing it to vary in the interval $[0,1)$. We also fix the other parameters in the system as follows: $\kappa=65, \sigma=5 \times 10^{-3}$ and $\delta=2 \times 10^{-2}$. For different values of $\mu$, we use a fourth order Runge-Kutta integration scheme with variable step size to integrate the system for 29,000 time steps and retain only the last 5,000 steps of the time series data in order to eliminate all transients. As $\mu$ is gradually increased, different dynamical behaviors are observed. For example, we observe stable steady state behavior for $0 \leq \mu \leq 0.015$. A Hopf bifurcation occurs at $\mu=0.016$, leading to the emergence of oscillatory behavior. The Hopf bifurcation is supercritical, since a small limit cycle emerges at the bifurcation, which grows with increasing values of $\mu$. This Hopf bifurcation is accompanied by period doubling, with period- 1 from $\mu=0.016$, period- 2 from $\mu=0.143$, period-4 from $\mu=0.153$, and chaos from $\mu=0.154$. At $\mu$ values close to the chaotic region, period-doubling occurs for very small increments in $\mu$. Further increases in $\mu$ beyond the chaotic regime leads to a reverse period-doubling sequence. A second Hopf bifurcation occurs at $\mu=0.175$ and the system eventually regains stable steady state behavior as $\mu$ is further increased. Figures 8.3 and 8.4, respectively, depict typical time series and the corresponding phase plots, while the bifurcation diagram for the system is presented in Fig. 8.5. 


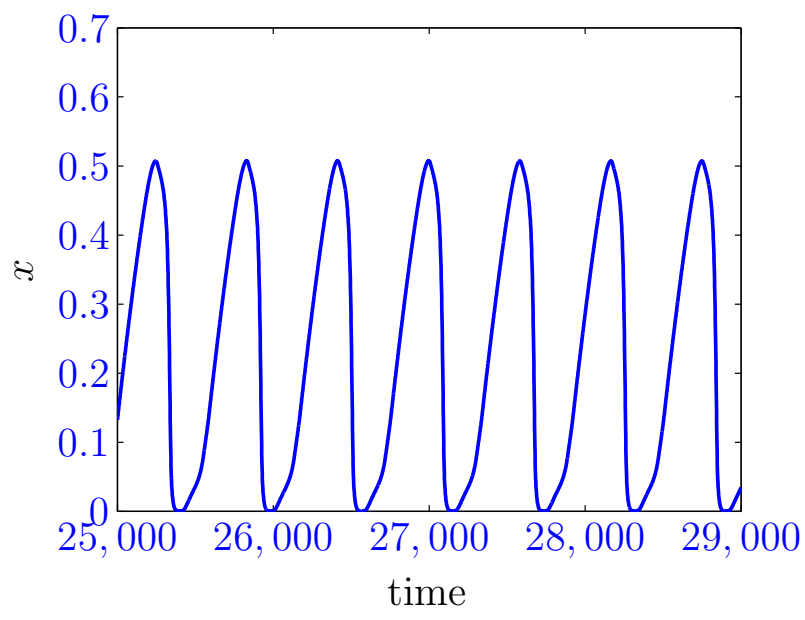

8.3.1. Time series for $\mu=0.016$ showing period-1 behavior.

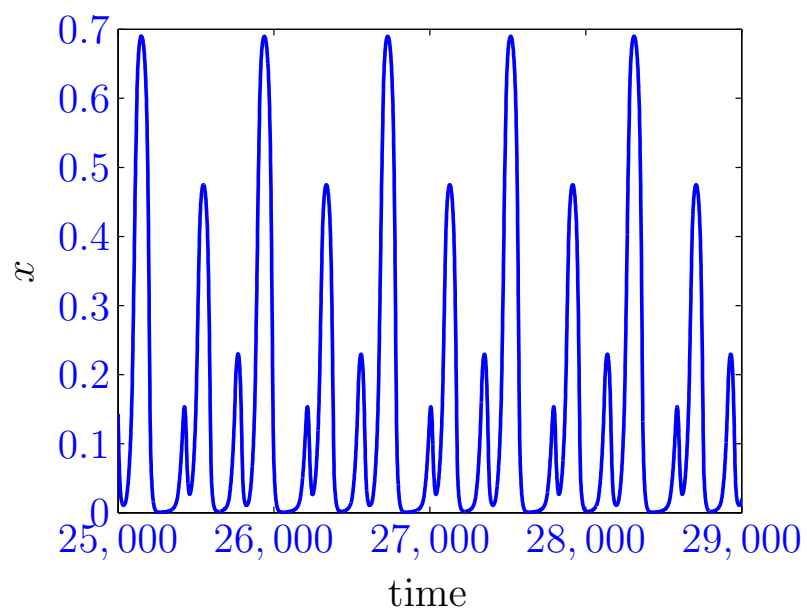

8.3.3. Time series for $\mu=0.153$ showing period-4 behavior.

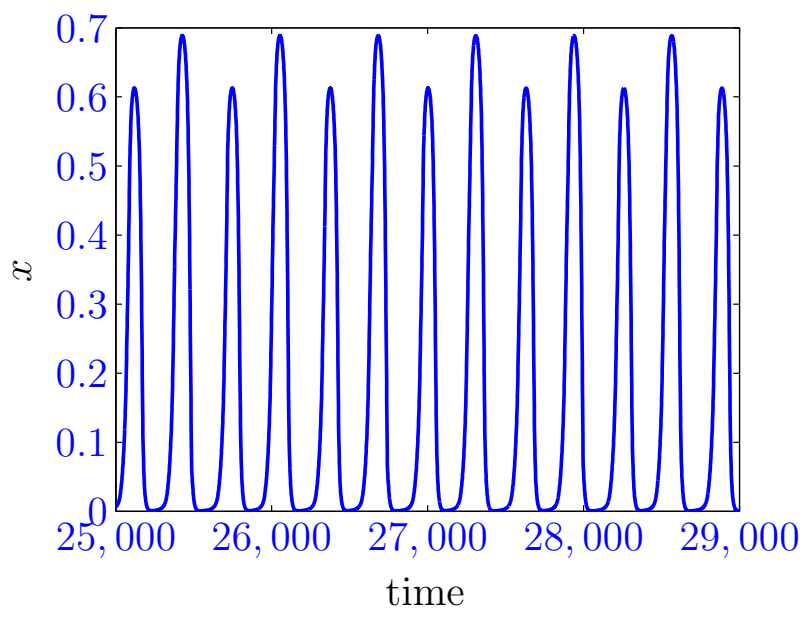

8.3.2. Time series for $\mu=0.143$ showing period-2 behavior.

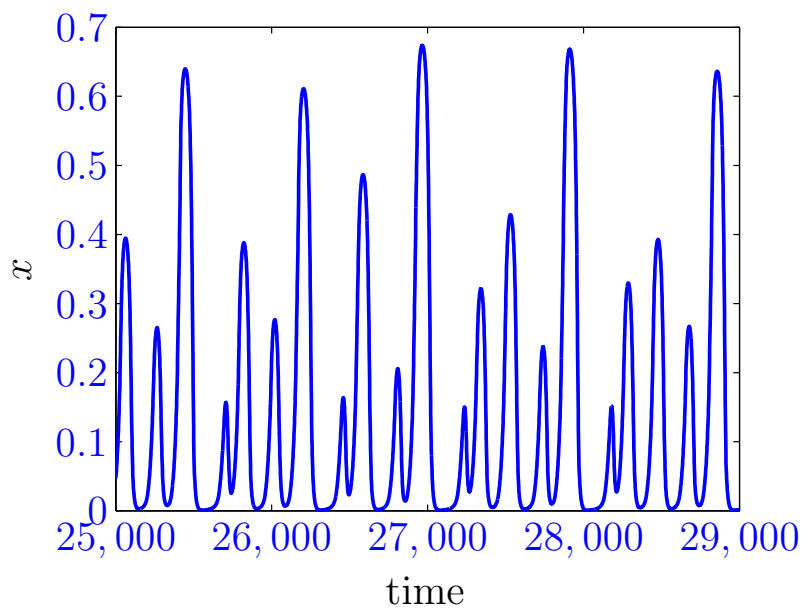

8.3.4. Time series for $\mu=0.154$ showing chaotic behavior.

Figure 8.3. Typical time series plots of the three-variable autocatalator model showing different dynamical behaviors for $\kappa=65, \sigma=5 \times 10^{-3}, \delta=2 \times 10^{-2}$ and for different values of the bifurcation parameter $\mu$. We plot $x$ (the dimensionless concentration of the intermediate product $X$ ) against time. 


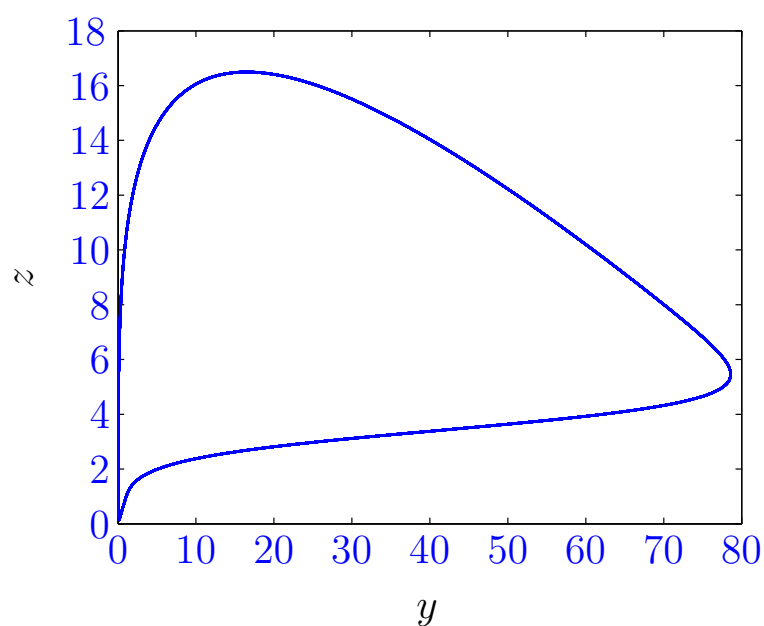

8.4.1. Phase plot for $\mu=0.016$ showing period-1 behavior.

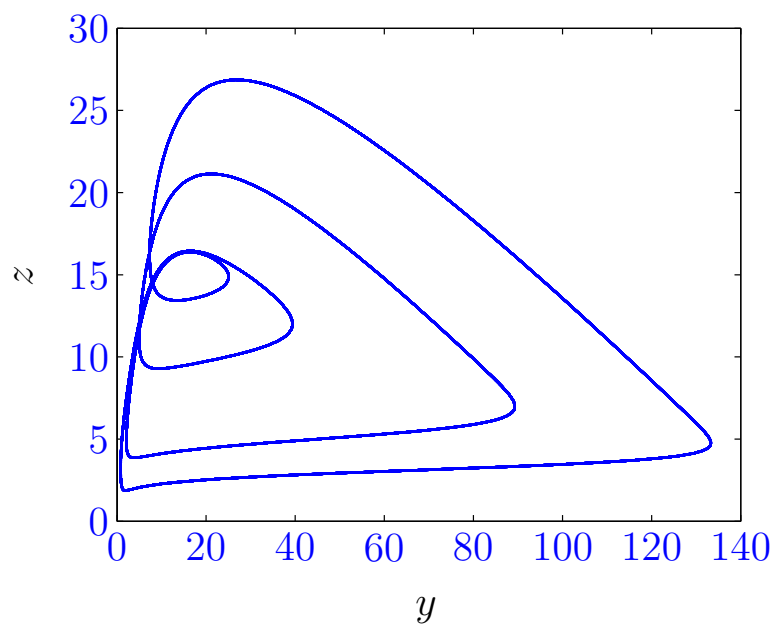

8.4.3. Phase plot for $\mu=0.153$ showing period-4 behavior.

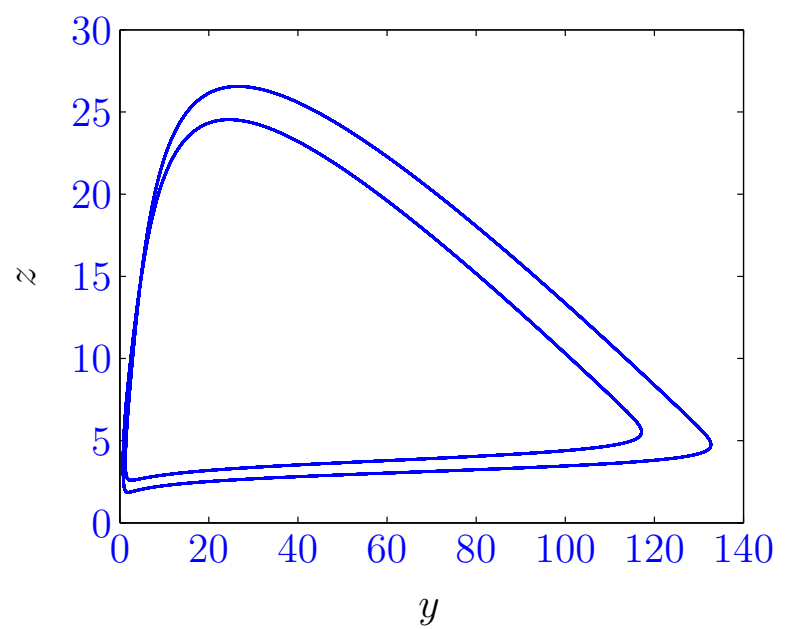

8.4.2. Phase plot for $\mu=0.143$ showing period-2 behavior.

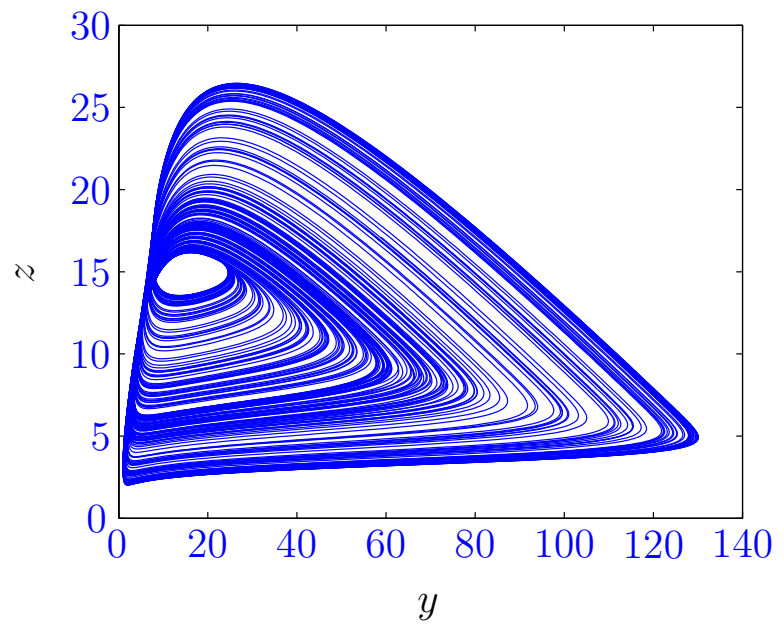

8.4.4. Phase plot for $\mu=0.154$ showing chaotic behavior.

Figure 8.4. Typical phase plots of the three-variable autocatalator model showing different periodic and chaotic attractors for $\kappa=65, \sigma=5 \times 10^{-3}, \delta=2 \times 10^{-2}$ and for different values of the bifurcation parameter $\mu$. We plot $z$ (the dimensionless concentration of the intermediate product $Z$ ) against $y$ (the dimensionless concentration of the intermediate product $Y$ ). 


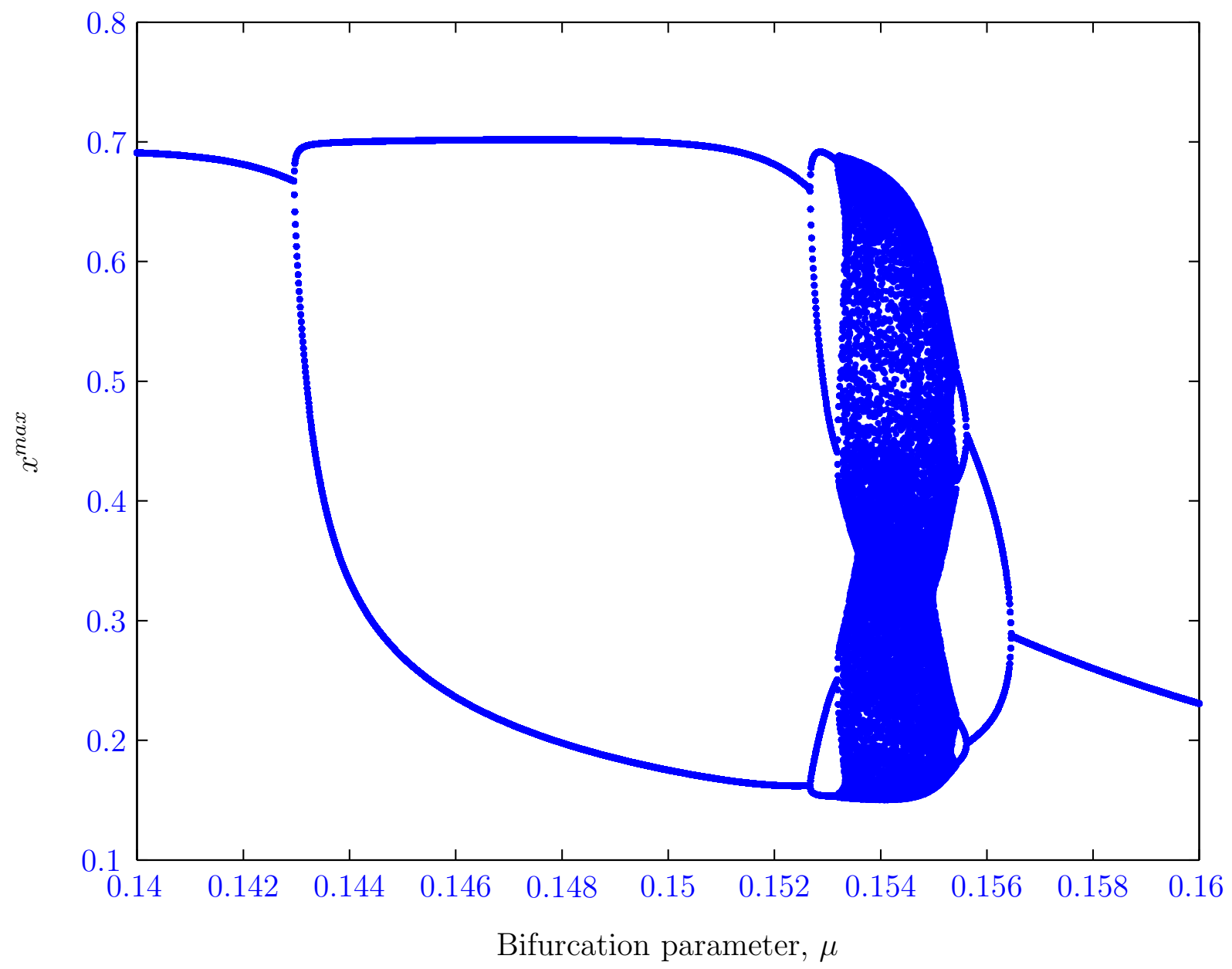

Figure 8.5. Bifurcation diagram of the three-variable autocatalator model. A plot of amplitude of $x$ against the bifurcation parameter $\mu$, showing a period-doubling sequence, chaos, and a reverse period-doubling sequence. Here, we set $\kappa=65, \sigma=5 \times 10^{-3}$, and $\delta=2 \times 10^{-2}$. 
To confirm that system (8.4) is chaotic, we computed the Lyapunov exponents of the system for the same set of parameters used in the simulations above. Lyapunov exponents provide a quantitative measure of chaotic dynamical behavior, since they measure the average exponential rate at which trajectories that originate close to each other diverge or converge over time. Chaotic behavior is governed by a positive largest Lyapunov exponent, while periodic behavior is characterized by a zero largest Lyapunov exponent. The largest Lyapunov exponent for system (8.4) within the chaotic parameter regime $(\mu=0.154)$ is $\lambda_{1}=5.28$. Positivity of the largest Lyapunov exponent indicates that any two nearby trajectories separate exponentially as $\tau \rightarrow \infty$. Hence, the system exhibits sensitive dependence on initial conditions, which results in a complete loss of information about the initial conditions in the long term [23]. To illustrate the notion of sensitive dependence on initial conditions, we vary the initial value of $x\left(x_{0}\right)$ by 0.0001 , fix the initial values of the other variables, and integrate the system for both sets of initial conditions within the chaotic regime. Here, we set $\mu=0.154$ and retain the values of the other system parameters used above. The trajectories begin close to each other due to the small difference in initial conditions but diverge over time. A plot of the Lyapunov exponents against time is presented in Fig. 8.6, while time series plots illustrating sensitive dependence on initial conditions are presented in Fig. 8.7. 


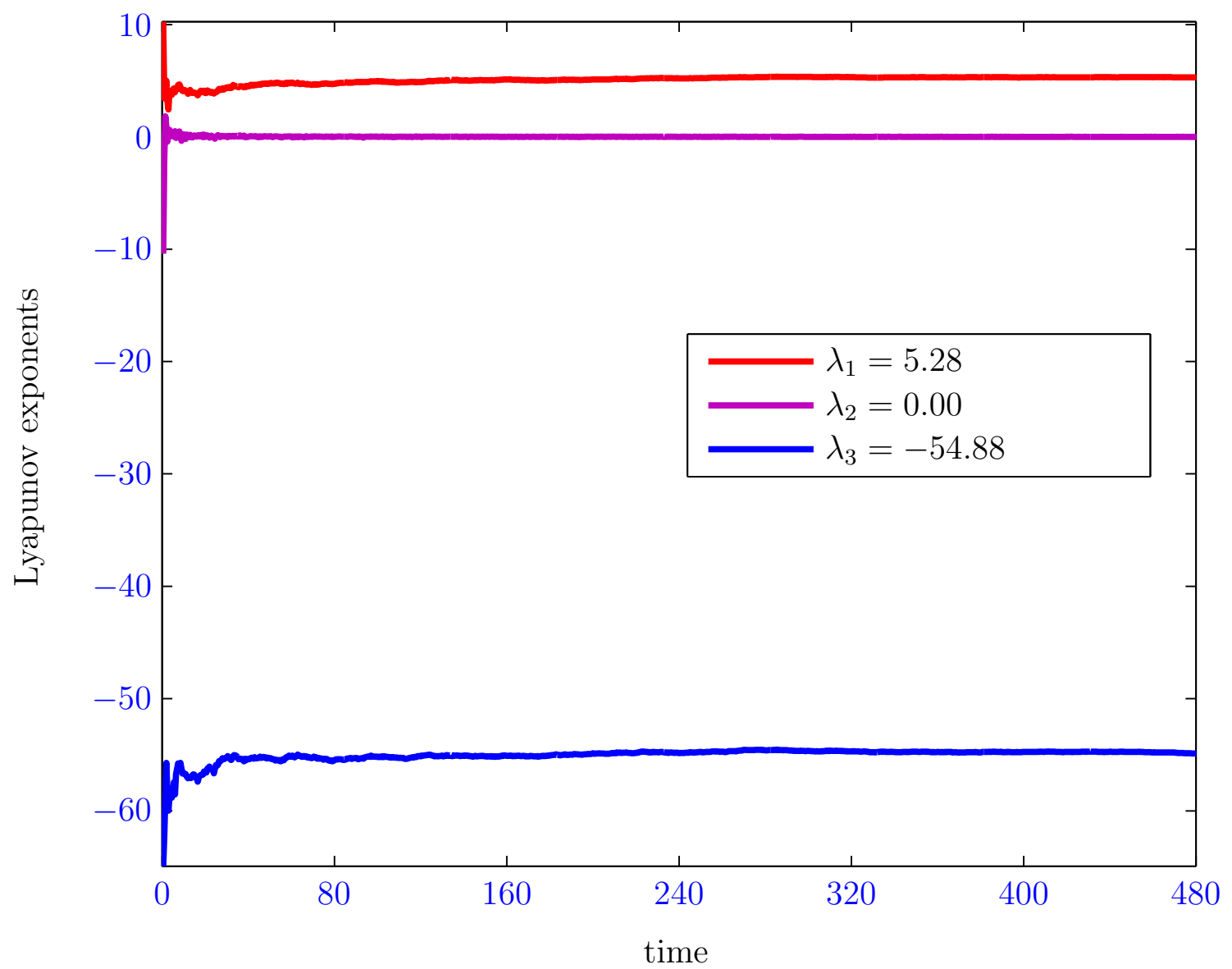

Figure 8.6. A plot of the Lyapunov exponents of system (8.4) for $\mu=0.154, \kappa=65, \sigma=$ $5 \times 10^{-3}$, and $\delta=2 \times 10^{-2}$. The largest Lyapunov exponent $\lambda_{1}$ is positive and so the system is chaotic. 


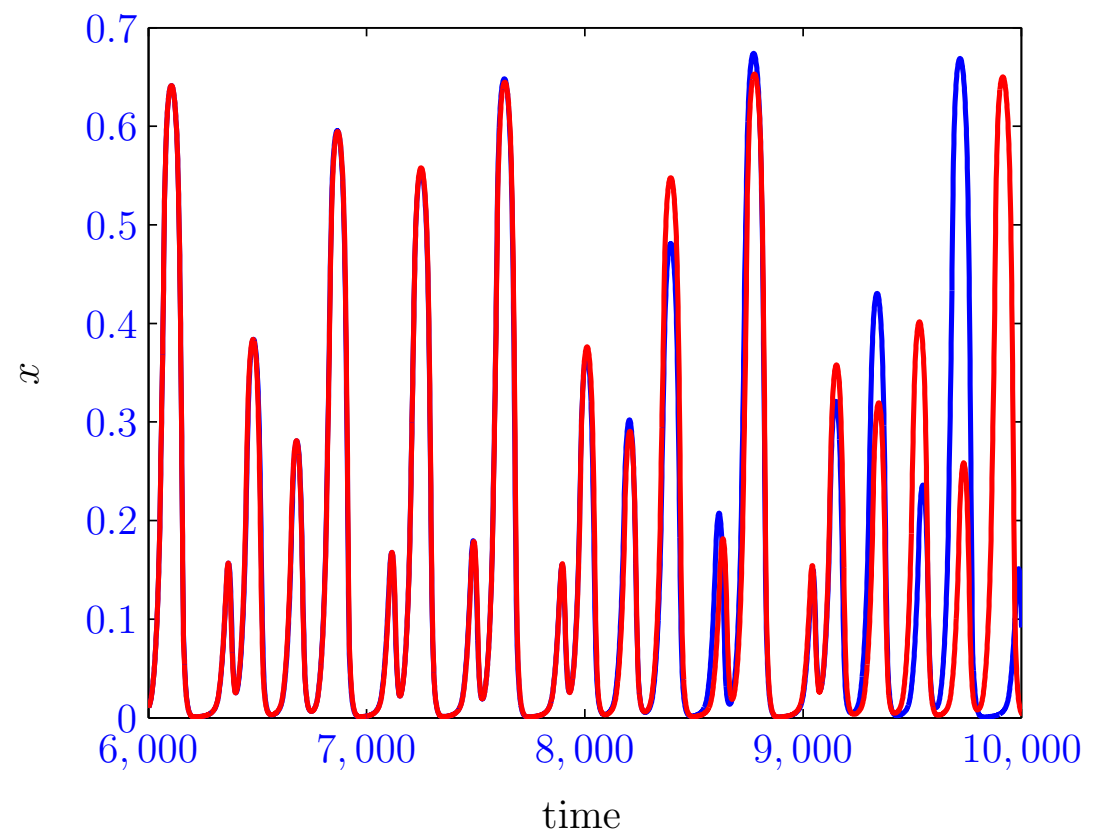

8.7.1. Small initial trajectory divergence.

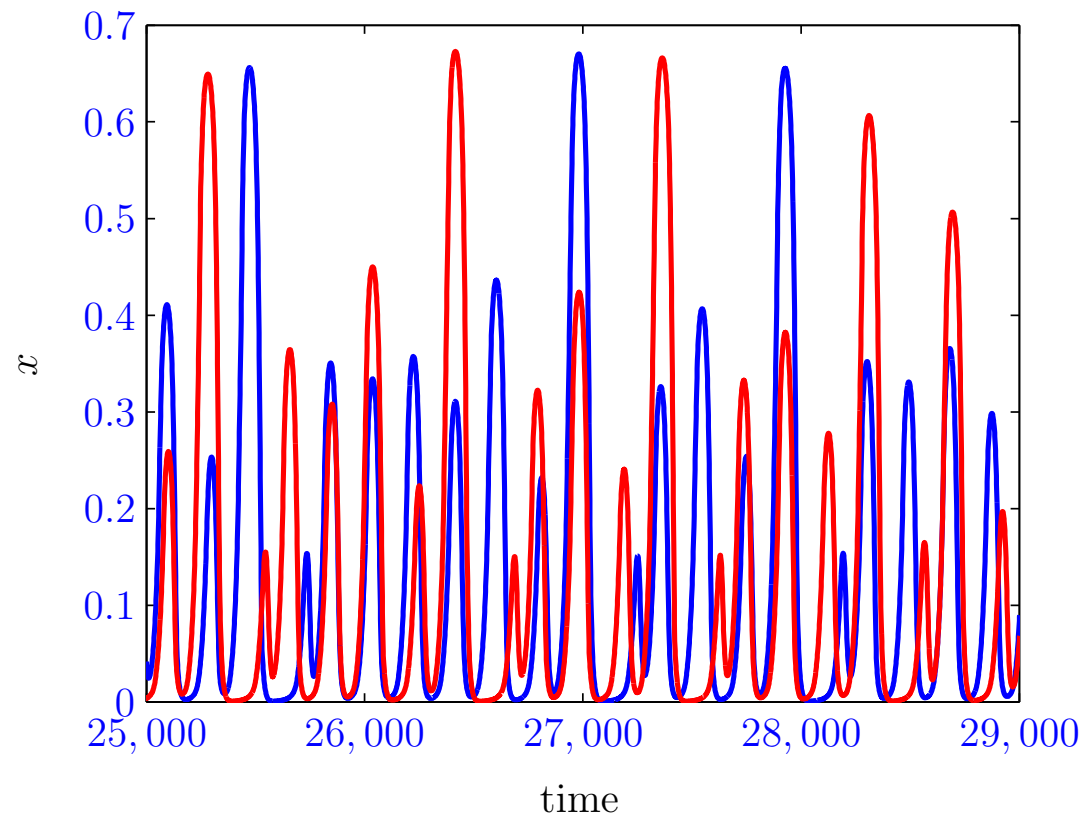

8.7.2. Large long term trajectory divergence.

Figure 8.7. Divergence of $x$ trajectories when the system exhibits chaotic dynamics for a 0.0001 difference in the initial values of $x$. The blue line represents the trajectory that starts at the smaller value of $x_{0}$, while the red line represents the trajectory that begins at the larger value of $x_{0}$. These lines almost coincide initially but drift apart over time. 
Chaotic behavior can also be characterized by one-dimensional maps. Fig. 8.8 below shows one-dimensional maps constructed from the time series of the model system within the chaotic regime. The unstable period- 1 limit cycle is depicted by the intersection of this map and the bisectrix $z_{n+1}=z_{n}$. Generally, the unstable period- $i$ where $i=1,2,4,8, \ldots$ limit cycle is given by the intersection of the plot of $z_{n+i}$ versus $z_{n}$ and the bisectrix $z_{n+i}=z_{n}$.

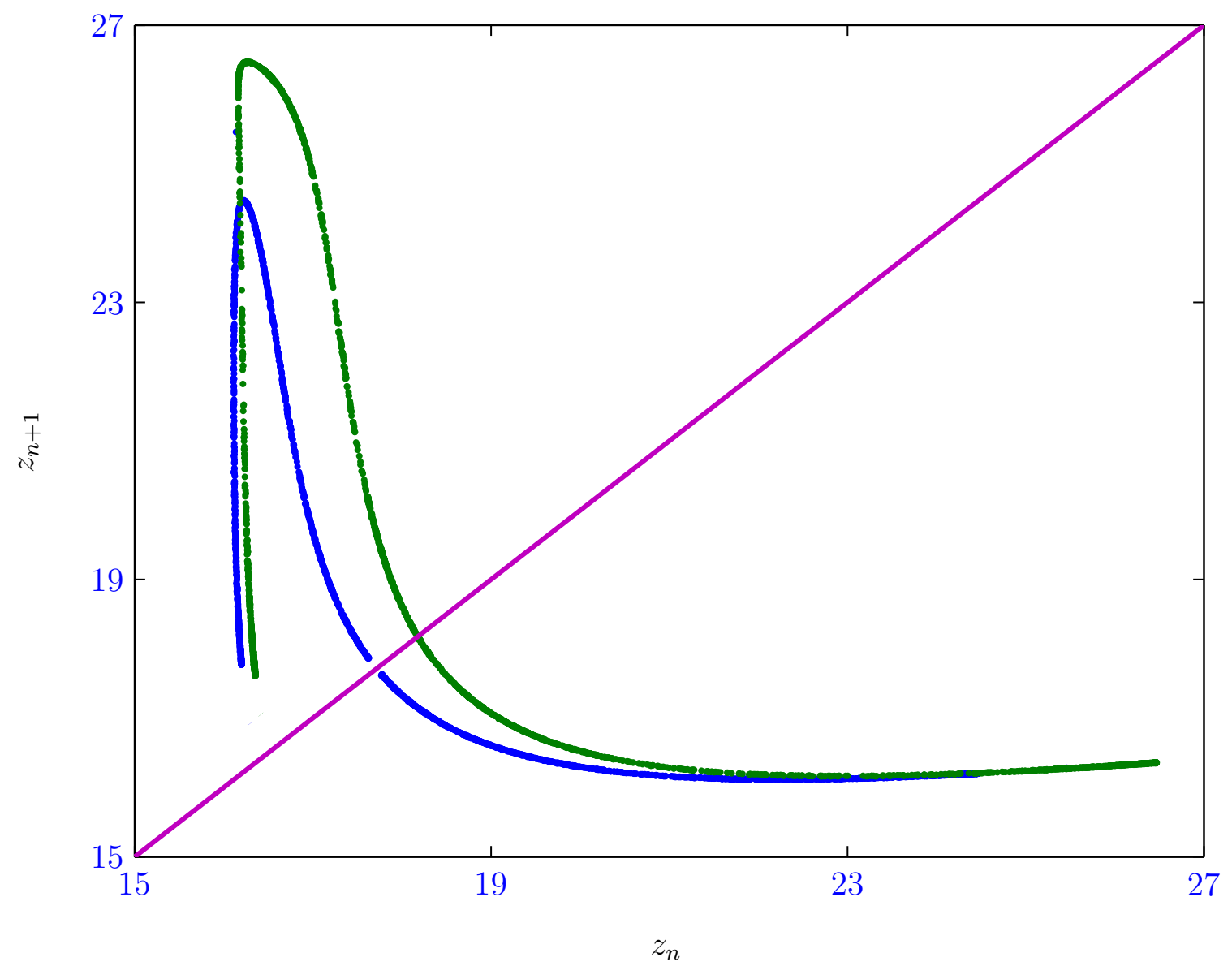

Figure 8.8. One-dimensional return maps constructed from the time series of system (8.4) within the chaotic region for $\mu=0.154$ (green) and $\mu=0.155$ (blue). The magenta line is the bisectrix $z_{n+1}=z_{n}$. 


\subsubsection{Qualitative Study of the Three-variable Autocatalator Model}

To acquire further insights into the qualitative behavior of system (8.4), we investigate the existence of steady states and perform a linear stability analysis about these steady states. We also employ analytical techniques to prove the occurrence of a Hopf bifurcation and to derive an expression for the approximate period and amplitude of the ensuing periodic solutions.

Definition 8.3.2. In the context of this work, we call a solution $(x(t), y(t), z(t)), t \in[0, \infty)$ of system (8.4) realistic if the solution is non-negative for all time.

Theorem 8.3.3. System (8.4) has a realistic equilibrium solution $S$ whenever $0 \leq \mu<1$.

Proof. Let $S=\left(x^{*}, y^{*}, z^{*}\right)$ be a steady state solution of (8.4). Then by substituting $x^{*}, y^{*}$ and $z^{*}$ in (8.4), setting the left hand sides to zero and solving the system of algebraic equations simultaneously, we obtain

$$
S=\left(x^{*}, y^{*}, z^{*}\right)=\left(\frac{\kappa \mu(1-\mu)}{(\kappa \mu)^{2}+(1-\mu)^{2}}, \frac{\kappa \mu}{1-\mu}, \frac{\kappa \mu}{1-\mu}\right) .
$$

It is now a trivial matter to see that this steady state is realistic for $0 \leq \mu<1$.

Corollary 8.3.4. The system (8.4) has a trivial steady state solution $S_{0}=(0,0,0)$ for $\mu=0$ and a non-trivial realistic steady state $S^{*}$ for $0<\mu<1$.

Proof. The proof follows from the proof of Theorem 8.3.3 by setting $\mu=0$ for $S_{0}$ and considering $0<\mu<1$ for $S$.

Remark 8.3.5. Notice that the steady state values of $x$ and $z$ above can be expressed in terms of the steady state value of $y$ as follows: $x^{*}=\frac{y^{*}}{y^{* 2}+1}$ and $z^{*}=y^{*}$.

Remark 8.3.6. Since we are concerned with chemical concentrations, only non-negative concentrations of the reactants are meaningful. All rate constants must also be positive.

Next, we present results related to the linear stability analysis of solutions to system (8.4). The linear stability of the equilibrium solutions of system (8.4) can be explored by linearizing the system about the equilibrium solution $\left(x^{*}, y^{*}, z^{*}\right)$. 
Theorem 8.3.7. Let $0 \leq \mu \leq \frac{1}{\kappa+1}$ and $\left(x^{*}, y^{*}, z^{*}\right)$ be an equilibrium solution of system (8.4). Suppose also that

$$
\lambda^{3}+P \lambda^{2}+Q \lambda+R=0
$$

is the characteristic polynomial derived from the Jacobian matrix of the linearized system, where $\lambda$ is an eigenvalue of the Jacobian matrix. Then

(i) $y^{*} \in[0,1]$,

(ii) all coefficients of (8.6) are positive,

(iii) the equilibrium solution $\left(x^{*}, y^{*}, z^{*}\right)$ is linearly stable to small perturbations for all $\gamma \in(0,1)$ and unstable for $\gamma>1$ where $\gamma=R /(P Q)$,

(iv) a Hopf bifurcation occurs at $\gamma_{c}=1$ where $\gamma_{c}$ represents a critical value of $\gamma$. Additionally, increasing $\gamma$ through $\gamma_{c}$ results in periodic solutions whose initial period can be determined as a function of the perturbation of $\gamma$ from $\gamma_{c}$.

Proof. (i) Recall that $y^{*}=\frac{\kappa \mu}{1-\mu}$, where $0 \leq \mu<1$. Clearly, $y^{*} \in[0,1]$ for $0 \leq \mu \leq \frac{1}{\kappa+1}$.

(ii) We linearize system (8.4). To this effect, we set $x=x^{*}+u, y=y^{*}+v$ and $z=z^{*}+w$, where $|u| \ll 1,|v| \ll 1$ and $|w| \ll 1$. Substituting these in (8.4) and expanding the right hand sides in a Taylor series about $\left(x^{*}, y^{*}, z^{*}\right)$ yields

$$
\begin{aligned}
\dot{u} & =\left[\mu\left(\kappa+z^{*}\right)-x^{*}\left(1+y^{* 2}\right)\right]-\left(y^{* 2}+1\right) u-\left(2 x * y^{*}\right) v+\mu w-\left(x^{*} v^{2}+2 y^{*} u v+u v^{2}\right), \\
\sigma \dot{v} & =\left[x^{*}\left(1+y^{* 2}\right)-y^{*}\right]+\left(y^{* 2}+1\right) u+\left(2 x^{*} y^{*}-1\right) v+\left(x^{*} v^{2}+2 y^{*} u v+u v^{2}\right), \\
\delta \dot{w} & =\left[v^{*}-w^{*}\right]+v-w .
\end{aligned}
$$

Recognizing the fact that each of the expressions in square brackets is zero since $\left(x^{*}, y^{*}, z^{*}\right)$ is an equilibrium solution of (8.4) and retaining only linear terms in $u, v$, and $w$ yields the following linear approximation:

$$
\begin{aligned}
\dot{u} & =-\left(y^{* 2}+1\right) u-\left(\frac{2 y^{* 2}}{y^{* 2}+1}\right) v+\mu w, \\
\sigma \dot{v} & =\left(y^{* 2}+1\right) u+\left(\frac{2 y^{* 2}}{y^{* 2}+1}-1\right) v, \\
\delta \dot{w} & =v-w,
\end{aligned}
$$


where $x^{*}=\frac{y^{*}}{y^{* 2}+1}=\frac{\kappa \mu(1-\mu)}{(\kappa \mu)^{2}+(1-\mu)^{2}}$. The Jacobian matrix of the linearized system is

$$
J=\left(\begin{array}{ccc}
-\left(y^{* 2}+1\right) & -\frac{2 y^{* 2}}{y^{* 2}+1} & \mu \\
\frac{y^{* 2}+1}{\sigma} & \frac{y^{* 2}-1}{\sigma\left(y^{* 2}+1\right)} & 0 \\
0 & \frac{1}{\delta} & -\frac{1}{\delta}
\end{array}\right) .
$$

We find linearly independent solutions to (8.8) of the form

$$
u=u_{0} e^{\lambda t}, v=v_{0} e^{\lambda t}, w=w_{0} e^{\lambda t}
$$

where $u_{0}, v_{0}$ and $w_{0}$ are constants and the eigenvalue $\lambda$ determines the growth or decay rate of solutions to the linearized system. Substituting (8.9) in (8.8) and imposing the condition for non-trivial solutions, we obtain

$$
\left|\begin{array}{ccc}
-\left(y^{* 2}+1\right)-\lambda & -\frac{2 y^{* 2}}{y^{* 2}+1} & \mu \\
\frac{y^{* 2}+1}{\sigma} & \frac{y^{* 2}-1}{\sigma\left(y^{* 2}+1\right)}-\lambda & 0 \\
0 & \frac{1}{\delta} & -\frac{1}{\delta}-\lambda
\end{array}\right|=0
$$

Expanding this determinant, we obtain the characteristic equation

$$
\left(-\left(y^{* 2}+1\right)-\lambda\right)\left(\frac{y^{* 2}-1}{\sigma\left(y^{* 2}+1\right)}-\lambda\right)\left(-\frac{1}{\delta}-\lambda\right)+\left(\frac{2 y^{* 2}}{\sigma}\right)\left(-\frac{1}{\delta}-\lambda\right)+\mu\left(\frac{y^{* 2}+1}{\sigma \delta}\right)=0,
$$

which can be written as an expanded cubic polynomial in $\lambda$ of the form given in (8.6) above, where $y^{*}$ is as calculated in the proof of Theorem 8.3.3 and

$$
\begin{aligned}
P & =\frac{\sigma\left(y^{* 2}+1\right)\left(\delta y^{* 2}+\delta+1\right)+\delta\left(1-y^{* 2}\right)}{\delta \sigma\left(y^{* 2}+1\right)} \\
Q & =\frac{\left(y^{* 2}+1\right)^{2}(\delta+\sigma)+\left(1-y^{* 2}\right)}{\delta \sigma\left(y^{* 2}+1\right)} \\
R & =\frac{\left(y^{* 2}+1\right)(1-\mu)}{\delta \sigma}
\end{aligned}
$$

Clearly, $P, Q$ and $R$ are positive by part (i) of the Theorem. Note that we discard the possibility of $y^{*} \in(-1,0)$ since this renders $y^{*}$ and consequently $z^{*}$ unrealistic.

(iii) To conclude that the equilibrium solution $\left(x^{*}, y^{*}, z^{*}\right)$ is linearly and asymptotically stable, it suffices to show that all solutions of (8.6) have negative real parts. Since 
$P>0, Q>0$ and $R>0$ (see part (ii) of the Theorem) by the Routh-Hurwitz's necessary and sufficient condition, all the solutions of (8.6) have negative real parts if $P Q-R>0$. But then $P Q-R>0$ is equivalent to

$$
p \sigma^{2}+q \sigma+r>0
$$

where

$$
\begin{aligned}
p= & \left(y^{* 2}+1\right)^{3}\left[\delta\left(y^{* 2}+1\right)+1\right], \\
q= & \left(y^{* 2}+1\right)\left[\delta^{2}\left(y^{* 2}+1\right)^{3}+\delta \mu\left(y^{* 2}+1\right)\right. \\
& \left.+2 \delta\left(y^{* 2}+1\right)\left(1-y^{* 2}\right)+\left(1-y^{* 2}\right)\right], \\
r= & \delta\left(1-y^{* 2}\right)\left[\delta\left(y^{* 2}+1\right)+\left(1-y^{* 2}\right)\right] .
\end{aligned}
$$

Hence, if inequality (8.11) is true or if $0<\gamma<1$ where $\gamma=R /(P Q)$, the equilibrium solution $\left(x^{*}, y^{*}, z^{*}\right)$ is linearly and asymptotically stable. To be more precise, $\left(x^{*}, y^{*}, z^{*}\right)$ is a stable spiral. However, if $p \sigma^{2}+q \sigma+r<0$, or if $\gamma>1$, the equilibrium solution $\left(x^{*}, y^{*}, z^{*}\right)$ is an unstable spiral since the Routh-Hurwitz condition fails.

(iv) Since the stability properties of our equilibrium solution witness a change at $\gamma_{c}=\gamma=1$, $\gamma_{c}=1$ is a critical value of $\gamma$. To acquire a better understanding of the nature of the instability, we assume that $\lambda$ depends continuously on $\gamma$. That is $\lambda=\lambda(\gamma)$ in which case equation (8.6) can be rewritten as

$$
\lambda^{3}+P \lambda^{2}+Q \lambda+\gamma P Q=0
$$

which factors to

$$
(\lambda+P)\left(\lambda^{2}+Q\right)=0
$$

at $\gamma=\gamma_{c}=1$. The solutions of (8.14) are $\lambda\left(\gamma_{c}\right)=-P$ or $\lambda\left(\gamma_{c}\right)= \pm i \sqrt{Q}$. Hence, at $\gamma_{c}=1$, we have a real and negative eigenvalue and a purely imaginary pair of eigenvalues. Thus, a Hopf bifurcation exists. 
We now perturb $\gamma$ about $\gamma_{c}$ by setting $\gamma=\gamma_{c}+\epsilon^{2} \nu$ where $|\epsilon| \ll 1$ and $\nu= \pm 1$. This yields $\lambda(\gamma)=\lambda\left(\gamma_{c}+\epsilon^{2} \nu\right)$. Expanding $\lambda$ in a Taylor series about $\gamma_{c}$ and holding onto linear terms in $\epsilon^{2} \nu$ only, we obtain

$$
\lambda(\gamma)=\lambda\left(\gamma_{c}\right)+\lambda^{\prime}\left(\gamma_{c}\right) \epsilon^{2} \nu
$$

where the prime denotes differentiation with respect to $\gamma$. Differentiating (8.13) implicitly with respect to $\gamma$ and solving for $\lambda^{\prime}$ gives

$$
\lambda^{\prime}(\gamma)=-\frac{P Q}{3 \lambda(\gamma)^{2}+2 P \lambda(\gamma)+Q} .
$$

Now,

$$
\lambda^{\prime}\left(\gamma_{c}\right)=\frac{P Q}{2\left(P^{2}+Q\right)} \pm i \frac{P^{2} \sqrt{Q}}{2\left(P^{2}+Q\right)} .
$$

Clearly, $\operatorname{Re} \lambda\left(\gamma_{c}\right)=0$ and $\operatorname{Re} \lambda^{\prime}\left(\gamma_{c}\right)=\frac{P Q}{2\left(P^{2}+Q\right)}>0$. Next,

$$
\lambda(\gamma)=\lambda\left(\gamma_{c}\right)+\lambda^{\prime}\left(\gamma_{c}\right) \epsilon^{2} \nu=\frac{P Q}{2\left(P^{2}+Q\right)} \epsilon^{2} \nu \pm i \sqrt{Q}\left(1+\frac{P^{2} \sqrt{Q}}{2\left(P^{2}+Q\right)} \epsilon^{2} \nu\right) .
$$

We have effectively demonstrated that for $0<\gamma<1$, all solutions of (8.6) have negative real parts. At $\gamma=\gamma_{c}=1$, a purely imaginary pair of eigenvalues arises and for $\gamma>1$, the real parts of the imaginary eigenvalues become positive. Thus, as $\gamma$ traverses the critical value $\gamma_{c}=1$, stable solutions of (8.4) become unstable. Associated with this loss of stability is the emergence of periodic oscillations with initial period and amplitude approximately given by

$\frac{2 \pi}{\sqrt{Q}\left(1+\frac{P^{2} \sqrt{Q}}{2\left(P^{2}+Q\right)} \epsilon^{2} \nu\right)}$ and $\exp \left(\frac{P Q}{2\left(P^{2}+Q\right)} \epsilon^{2} \nu \tau\right)$, respectively, where $\epsilon=\sqrt{\frac{\left|\gamma-\gamma_{c}\right|}{|\nu|}}$. The above completely describes a Hopf bifurcation ${ }^{1}$.

Remark 8.3.8. As illustrated by our simulations, the three-variable autocatalator model exhibits two Hopf bifurcations at two critical values of the bifurcation parameter $\mu$. These critical values can be computed from $\gamma_{c}=1$ or from the equation $p \sigma^{2}+q \sigma+r=0$.

\footnotetext{
${ }^{1} \mathrm{~A}$ good source for the statement and proof of the Hopf bifurcation Theorem is Hassard et al. [24]
} 
Corollary 8.3.9. The trivial equilibrium solution $S_{0}=(0,0,0)$ that exists when $\mu=0$ is linearly and asymptotically stable to small perturbations.

Proof. By setting $\mu=0$, the Jacobian of the linearized system above is:

$$
J=\left(\begin{array}{ccc}
-1 & 0 & \mu \\
1 / \sigma & -1 / \sigma & 0 \\
0 & 1 / \delta & -1 / \delta
\end{array}\right)
$$

and the characteristic equation (8.6) reduces to

$$
(-1-\lambda)\left(-\frac{1}{\sigma}-\lambda\right)\left(-\frac{1}{\delta}-\lambda\right)=0 .
$$

Since all three eigenvalues, $\lambda=-1,-1 / \delta,-1 / \sigma$ are negative, $(0,0,0)$ is linearly and asymptotically stable. In fact, the origin is a stable node.

Table 8.1 summarizes the solutions of (8.6), and the corresponding steady states and their stability properties for a few values of $\mu$, when $\kappa=65, \sigma=0.005, \delta=0.02$. The information in the table corresponds to that obtained from the numerical simulations.

Table 8.1. A summary of some numerical values of the equilibrium solutions, eigenvalues and stability properties of system (8.4) for $\mu=0.0,0.015,0.016,0.175,0.178$.

\begin{tabular}{||c|l|c|l|l|l||}
\hline \hline$\mu$ & \multicolumn{1}{|c|}{$\left(x^{*}, y^{*}, z^{*}\right)$} & $\lambda_{1}$ & \multicolumn{1}{|c||}{$\lambda_{2}$} & \multicolumn{1}{|c||}{$\lambda_{3}$} & Stability \\
\hline 0.000 & $(0,0,0)$ & -50 & -1 & -200 & Stable \\
\hline 0.015 & $(0.5,0.9898,0.9898)$ & -50 & $-2.010+19.79 i$ & $-2.01+19.79 i$ & Stable \\
\hline 0.016 & $(0.4992,1.0569,1.0569)$ & -50 & $4.470+20.08 i$ & $4.470-20.08 i$ & Unstable \\
\hline 0.175 & $(0.0721,13.7879,13.7879)$ & -50 & $3.380+195.40 i$ & $3.380+195.40 i$ & Unstable \\
\hline 0.178 & $(0.0707,14.0754,14.0754)$ & -50 & $-0.584+199.50 i$ & $-0.584+199.50 i$ & Stable \\
\hline \hline
\end{tabular}




\subsection{Conclusion}

We have reviewed the two-variable autocatalator model and examined the dynamical behavior of the three-variable autocatalator model. Our quantitative and qualitative studies of the three-variable autocatalator model show that for specific values of $\mu$ we can have either stable steady state behavior or unstable steady state behavior accompanied by oscillations. Our numerical studies are in line with previous studies, depicting a switch from stable steady state behavior to a period-doubling sequence through a Hopf bifurcation, chaos, a reverse period-doubling sequence and then stable steady state behavior again through a second Hopf bifurcation as the bifurcation parameter $\mu$ is gradually increased in the interval $[0,1)$. For our qualitative studies, we computed a steady state $\left(x^{*}, y^{*}, z^{*}\right)$ that exists and is nonnegative for $\mu \in[0,1)$. We also identified a threshold parameter $\gamma$ and showed that $\left(x^{*}, y^{*}, z^{*}\right)$ is stable for $\gamma \in(0,1)$, unstable for $\gamma \in(1, \infty)$ and that a Hopf bifurcation occurs at $\gamma=1$. It is also important to mention that both quantitative and qualitative investigations indicate that the three-variable autocataltor model possesses a unique attractor for all values considered of the bifurcation parameter $\mu$. That is, all initial conditions converge to a specific attractor for each given value of $\mu$. This indicates that there is no sign of multistability corresponding to coexisting attractors for any parameter set in the system studied here. Multistability was reported by Petrov et al. [5] for other parameter values. 


\section{References}

[1] J. Gleick, Chaos (Viking Penguin, New York, 1987). 210

[2] G. L. Baker and J. P. Gollub, Chaotic Dynamics: An Introduction (Cambridge University Press, Cambridge, England, 1990). 210

[3] G. Rowland, Non-linear Phenomena in Science and Engineering (Ellis Horwood, Chichester, England, 1990).

[4] R. Kapral and K. Showalter, Chemical Waves and Patterns (Kluwer Academic Press, Dordrecht, the Netherlands, 1995). 210

[5] V. Petrov, S. Scott, and K. Showalter, "Mixed-mode Oscillations in Chemical Systems," Journal of Chemical Physics 97, 6191-6198 (1992).

Online Version 211, 231

[6] P. Coveney and A. Chaudry, "Quantifying Chaos and Transient Chaos in Nonlinear Chemically Reacting Systems," Journal of Chemical Physics 97, 7448-7458 (1992). Online Version

[7] A. Milik, P. Szmolyan, H. Loffelmann, and E. Groller, "Geometry of mixed-mode Oscillations in the 3-D Autocatalator," International Journal of Bifurcation Chaos 8, 505-519 (1998).

Online Version

[8] I. Z. Kiss and V. Gaspar, "Controlling Chaos with Artificial Neural Network: Numerical 
Studies and Experiments," The Journal of Physical Chemistry A 104, 8033-8037 (2000). Online Version

[9] Y. Li, X. Liao, C. Li, T. Huang, and D. Yang, "Impulsive Synchronization and Parameter Mismatch of the Three-variable Autocatalator Model," Physics Letters A 366, 52-60 (2007).

Online Version

[10] C. Piccardi, "Parameter Estimation for Systems with Peak-to-peak Dynamics," International Journal of Bifurcation Chaos 18, 745-753 (2008).

Online Version

[11] J. Guckenheimer, "Singular Hopf Bifurcation in Systems with Two Slow Variables," SIAM Journal on Applied Dynamical Systems 7, 1355-1377 (2008).

Online Version 211

[12] P. Gray and S. K. Scott, "Autocatalytic Reactions in the Isothermal Continuous Stirred Tank Reactor: Isolas and Other Forms of Multistability," Chemical Engineering Science 39, 29-43 (1983).

Online Version 211

[13] J. H. Merkin, D. J. Needham, and S. K. Scott, "On the Creation, Growth and Extinction of Oscillatory Solutions for a Simple Pooled Chemical Reaction Scheme," SIAM Journal on Applied Mathematics 47, 1040-1060 (1987).

Online Version 211, 212

[14] J. H. Merkin, D. J. Needham, and S. K. Scott, "On the Structural Stability of a Simple Pooled Chemical System," Journal of Engineering Mathematics 21, 115-127 (1987). Online Version 212

[15] B. F. Gray, M. J. Roberts, and J. H. Merkin, "The Cubic Autocatalator: The Influence of the Quadratic Autocatalytic and the Uncatalysed Reactions," Journal of Engineering Mathematics 22, 267-284 (1988).

Online Version 212 
[16] L. K. Forbes and C. A. Holmes, "Limit Cycle Behavior in a Model for Chemical Reaction: The Cubic Autocatalator," Journal of Engineering Mathematics 24, 179-189 (1990).

Online Version 211, 212

[17] B. Peng, S. K. Scott, and K. Showalter, "Period Doubling and Chaos in a Three-variable Autocatalator," The Journal of Physical Chemistry 94, 5243-5246 (1990).

Online Version 211

[18] B. Peng, V. Petrov, and K. Showalter, "Controlling Chemical Chaos," Journal of Physical Chemistry 95, 4957-4959 (1991).

Online Version 211, 216, 217

[19] S. K. Scott, Chemical Chaos (Oxford University Press, New York, 1991).

[20] S. K. Scott, Oscillations, Waves, and Chaos in Chemical Kinetics (Oxford University Press Inc., New York, 1994).

[21] P. Gray and S. K. Scott, Chemical Oscillations and Instabilities. Non-linear Chemical Kinetics (Oxford University Press, New York, 1994).

[22] V. Petrov, B. Peng, and K. Showalter, "A Map-based Algorithm for Controlling Lowdimensional Chaos," Journal of Chemical Physics 96, 7506-7513 (1992).

Online Version 217

[23] D. Ruelle, "Sensitive Dependence on Initial Conditions and Turbulent Behavior of Dynamical Systems," Annals of the New York Academy of Sciences 317, 408-416 (1979). Online Version 221

[24] B. D. Hassard, N. D. Kazarinoff, and Y. N. Wan, Theory and Application of Hopf Bifurcations (Cambridge University Press, Cambridge, 1981). 229 
$\overline{\text { Chapter }} 9$

\section{Extreme Multistability in the Autocatalator Model}

\subsection{Introduction}

Nonlinear dynamical systems in general and dissipative systems in particular possess a rich variety of interesting long-term dynamical behaviors, ranging from stationary points (such as nodes, spirals, saddles and combinations of these) to limit cycles or periodic and quasi-periodic oscillations to chaotic behavior. The asymptotic behavior of such systems is characterized by a finite number of attractors, typically one in most cases. However, some systems exhibit more than one attractor, and the number of attractors typically changes as a system parameter is varied. Over the years, most of the research devoted to dynamical systems theory has focused on systems having only one or a few coexisting attractors for a given set of system parameters. But there are many systems in nature, especially in biology and physics that exhibit a multitude of coexisting attractors [1-3]. Simple instances of multistability include the existence of more than one stable steady state and the case of a subcritical Hopf bifurcation. Examples of multistable systems include systems from laser and semiconductor physics [4-7], chemistry [8, 9], neuroscience [10] and population dynamics [11]. Multistability is a rather common phenomenon, found in different classes of systems such as weakly dissipative systems $[12,13]$, coupled systems [14-16] and systems with delays [17-19]. See, for example, Refs. [2, 3] for a general exposition on multistable systems.

Many studies on dynamical systems in general and dynamical chaos in particular have been centered around fixing a set of initial conditions and varying a parameter or a set of parameters in the system. This might give rise to new qualitative dynamical behavior of the 
system for some values of the control parameter(s) (see, for example, Refs. [20-24]). Through this approach, various bifurcations and transitions to chaos have been identified and studied, and the control parameter(s) can be used to characterize the behavior of dynamical systems [25, 26]. However, studies by H. Sun et al. [27] based on two coupled Lorenz systems, in which all parameters in the coupled system were held fixed and only the initial conditions were varied, revealed unusual and interesting dynamical behavior for a purely dissipative system. Generally, small perturbations of a dissipative system from an asymptotically stable attractor eventually decay over time and the system finds itself at the same attractor. Consequently, the solutions of dissipative systems for a given set of parameters exhibit the same dynamical behavior in the long run irrespective of the choice of initial conditions. In contrast to most other dissipative systems, which would relax back to the original stable state when the initial conditions are varied, variation of initial conditions caused the coupled Lorenz system to evolve to completely different states. This study suggests a new class of nonlinear dynamical systems in which the asymptotic behavior is determined by the initial conditions. Perturbations or changes in initial conditions push these dynamical systems to evolve to completely new attractors with different statistical properties. Consequently, such systems may exhibit an infinite number of asymptotic attractors, some stationary, some periodic and some chaotic. To address the notions of multistability and synchronization, we refer to this behavior as extreme multistability. Extreme multistability should not be confused with the famous sensitive dependence on initial conditions of chaotic systems. Rather, it is a form of multistability, since for the same parameter or set of parameter values we can have completely different attractors depending on the initial conditions. Extreme multistability is governed by an interplay between multistability and synchronization. However, extreme multistability is different from known examples of multistability in that we have an infinite number of attractors, whereas multistability involves the coexistence of a finite number of attractors. The complexity of the dynamics in extreme multistability is visualized by plotting the long-term attractors obtained in simulations versus the initial condition. Surprisingly, these plots resemble bifurcation diagrams, though the initial condition can not be regarded as a bifurcation parameter. Moreover, all known transitions between the different attractors can be observed by simply varying the starting point for one of the state variables. The 
associated dynamics of some coupled and high order systems can be studied through appropriate reduced systems in which the quantitative behavior may be different from that of the original system but the qualitative properties are essentially equivalent.

Chemical systems are among the most studied examples of dynamical systems exhibiting complex dynamics, chaos and pattern formation [28-30]. The dynamics leading to extreme multistability might account for reported irreproducibility of behavior in the chloratethiosulfate reaction [31] and in the chlorite-iodide reaction [32]. In these two reactions, the dynamical behavior varies from experiment to experiment under the same set of experimental conditions. The inability to reproduce the dynamical behavior for the same set of conditions, despite care to ensure reproducibility, suggests that the inherent dynamics of these systems is playing some role. In a modified three-variable autocatalator model, Wang et al. [33] showed that this system can possess infinitely many coexisting attractors when a "buffer step" is included in the chemical kinetics, where two reactants are produced and consumed in the same processes, a mechanism similar to that proposed by Epstein and co-workers for the chlorate-thiosulfate and chlorite-iodide reactions [31, 32].

In this Chapter we explore the dynamics of a coupled chemical model system possessing an infinite number of coexisting attractors. We show how this coupling scheme yields extreme multistability. We also demonstrate that the phenomenon of multistability is associated with generalized synchronization of the two coupled subsystems as well as the emergence of a conserved quantity. The conserved quantity is determined by a conservation law in which all intermediate chemical reactants are weighted by their reaction time constants and is therefore given by the initial concentrations of the intermediates. We show that the state space is divided into submanifolds, each of which is characterized by a certain value of the conserved quantity as well as a certain type of attractor. Since the conserved quantity can take any real value, we obtain infinitely many attractors.

This Chapter is organized as follows: We begin by coupling two three-variable autocatalator systems into a six-variable system in Section 9.2 and then present a non-dimensionalization of the coupled system in Section 9.3. We show that the extended system exhibits extreme multistability in Section 9.4. In Section 9.5, we show that the dynamics of this system is characterized by a conserved quantity that can be used to reduce the system to a five- 
variable system. We study the robustness of the phenomenon of extreme multistability with respect to a slight mismatch of the parameters of the subsystems (making the subsystems non-identical) in Section 9.6, and end the Chapter with concluding remarks in Section 9.7.

\subsection{Coupled Autocatalator Model}

The dynamical behavior becomes more complex when two three-variable autocatalator models are coupled in a particular way. To achieve this coupling, we start with two threevariable autocatalator subsystems with variables $X_{1}, Y_{1}, Z_{1}$ and $X_{2}, Y_{2}, Z_{2}$, respectively. As shown below, the coupling is realized through $Z_{2}$ in reaction $\left(R_{9.12}\right)$ and indirectly through $Z_{1}$ in reaction $\left(R_{9.22}\right)$, since the reactant $E$ is produced from $Z_{1}$ in reaction $\left(R_{9.16}\right)$. The chemical model obtained applying this coupling follows:

$$
\begin{gathered}
A \stackrel{k_{0}}{\longrightarrow} X_{1}, \\
A+Z_{2} \stackrel{k_{1}}{\longrightarrow} X_{1}+Z_{2}, \\
X_{1} \stackrel{k_{2}}{\longrightarrow} Y_{1}, \\
X_{1}+2 Y_{1} \stackrel{k_{3}}{\longrightarrow} 3 Y_{1}, \\
Y_{1} \stackrel{k_{4}}{\longrightarrow} Z_{1}, \\
Z_{1} \stackrel{k_{5}}{\longrightarrow} E, \\
A \stackrel{k_{0}}{\longrightarrow} X_{2}, \\
A+Z_{2} \stackrel{k_{1}}{\longrightarrow} X_{2}+Z_{2}, \\
X_{2} \stackrel{k_{2}}{\longrightarrow} Y_{2}, \\
X_{2}+2 Y_{2} \stackrel{k_{3}}{\longrightarrow} 3 Y_{2}, \\
Y_{2} \stackrel{k_{4}}{\longrightarrow} Z_{2}, \\
E+Z_{2} \stackrel{k_{5}^{\prime}}{\longrightarrow} F .
\end{gathered}
$$

Let $A_{0},\left[X_{1}\right],\left[Y_{1}\right]\left[Z_{1}\right],\left[X_{2}\right],\left[Y_{2}\right]$ and $\left[Z_{2}\right]$ be the concentrations of $A, X_{1}, Y_{1} Z_{1}, X_{2}, Y_{2}$ and $Z_{2}$ respectively and let $k_{5}^{\prime} \gg k_{5}$. Then using the law of mass action, which states that the effect of the instantaneous rate of change of any species is proportional to the product of 
the concentrations of the reacting species, we formulate the following mathematical model:

$$
\begin{aligned}
{\left[\dot{X}_{1}\right] } & =k_{0} A_{0}+k_{1} A_{0}\left[Z_{2}\right]-k_{2}\left[X_{1}\right]-k_{3}\left[X_{1}\right]\left[Y_{1}\right]^{2}, \\
{\left[\dot{Y}_{1}\right] } & =k_{2}\left[X_{1}\right]+k_{3}\left[X_{1}\right]\left[Y_{1}\right]^{2}-k_{4}\left[Y_{1}\right], \\
{\left[\dot{Z}_{1}\right] } & =k_{4}\left[Y_{1}\right]-k_{5}\left[Z_{1}\right], \\
{\left[\dot{X}_{2}\right] } & =k_{0} A_{0}+k_{1} A_{0}\left[Z_{2}\right]-k_{2}\left[X_{2}\right]-k_{3}\left[X_{2}\right]\left[Y_{2}\right]^{2}, \\
{\left[\dot{Y}_{2}\right] } & =k_{2}\left[X_{2}\right]+k_{3}\left[X_{2}\right]\left[Y_{2}\right]^{2}-k_{4}\left[Y_{2}\right], \\
{\left[\dot{Z}_{2}\right] } & =k_{4}\left[Y_{2}\right]-k_{5}\left[Z_{1}\right] .
\end{aligned}
$$

\subsection{Nondimensionalization}

Nondimensionalization reduces the number of parameters in the system as well as leaves us with dimensionless quantities that facilitate analysis, and we therefore rescale system (9.1). To this effect, we set

$$
\tau=\frac{t}{t^{*}}, x_{1}=\frac{\left[X_{1}\right]}{x_{1}^{*}}, y_{1}=\frac{\left[Y_{1}\right]}{y_{1}^{*}}, z_{1}=\frac{\left[Z_{1}\right]}{z_{1}^{*}}, x_{2}=\frac{\left[X_{2}\right]}{x_{1}^{*}}, y_{2}=\frac{\left[Y_{2}\right]}{y_{1}^{*}}, \text { and } z_{2}=\frac{\left[Z_{2}\right]}{z_{1}^{*}},
$$

where $t^{*}, x_{1}^{*}, y_{1}^{*}$, and $z_{1}^{*}$ are reference values with the same units as $t,\left[X_{1}\right],\left[Y_{1}\right],\left[Z_{1}\right]$, respectively. Note that $x_{1}^{*}, y_{1}^{*}$ and $z_{1}^{*}$ also has the same units as $\left[X_{2}\right],\left[Y_{2}\right]$ and $\left[Z_{2}\right]$, respectively. Now,

$$
\tau=\frac{t}{t^{*}} \Rightarrow \frac{d}{d t}=\frac{d}{d \tau} \frac{d \tau}{d t}=\frac{1}{t^{*}} \frac{d}{d \tau}
$$

and

$$
u_{i}=\frac{\left[U_{i}\right]}{u_{1}^{*}} \Rightarrow\left[\dot{U}_{i}\right]=\frac{d U_{i}}{d t}=\frac{u_{1}^{*}}{t^{*}} \frac{d u_{i}}{d \tau}=\frac{u_{1}^{*}}{t^{*}} \dot{u}_{i}
$$

where the dot on $u_{i}$ denotes differentiation with respect to $\tau$ and $u$ represents the variables $x_{i}, y_{i}, z_{i}, i=1,2$. Substituting these in (9.1), we obtain 


$$
\begin{aligned}
& \frac{x_{1}^{*}}{t^{*}} \dot{x}_{1}=k_{0} A_{0}+k_{1} A_{0} z_{1}^{*} z_{2}-k_{2} x_{1}^{*} x_{1}-k_{3} x_{1}^{*} x_{1} y_{1}^{* 2} y_{1}^{2}, \\
& \frac{y_{1}^{*}}{t^{*}} \dot{y}_{1}=k_{2} x_{1}^{*} x_{1}+k_{3} x_{1}^{*} x_{1} y_{1}^{* 2} y_{1}^{2}-k_{4} y_{1}^{*} y_{1}, \\
& \frac{z_{1}^{*}}{t^{*}} \dot{z}_{1}=k_{4} y_{1}^{*} y_{1}-k_{5} z_{1}^{*} z_{1}, \\
& \frac{x_{1}^{*}}{t^{*}} \dot{x}_{2}=k_{0} A_{0}+k_{1} A_{0} z_{1}^{*} z_{2}-k_{2} x_{1}^{*} x_{2}-k_{3} x_{1}^{*} x_{2} y_{1}^{* 2} y_{2}^{2}, \\
& \frac{y_{1}^{*}}{t^{*}} \dot{y}_{2}=k_{2} x_{1}^{*} x_{2}+k_{3} x_{1}^{*} x_{2} y_{1}^{* 2} y_{2}^{2}-k_{4} y_{1}^{*} y_{2}, \\
& \frac{z_{1}^{*}}{t^{*}} \dot{z}_{2}=k_{4} y_{1}^{*} y_{2}-k_{5} z_{1}^{*} z_{1} .
\end{aligned}
$$

Dividing each equation by the coefficient of the differential on the left-hand side, we have

$$
\begin{aligned}
& \dot{x}_{1}=\frac{k_{0} A_{0} t^{*}}{x_{1}^{*}}+\frac{k_{1} A_{0} z_{1}^{*} t^{*}}{x_{1}^{*}} z_{2}-k_{2} t^{*} x_{1}-k_{3} x_{1}^{*} y_{1}^{* 2} t^{*} x_{1} y_{1}^{2}, \\
& \dot{y}_{1}=\frac{k_{2} x_{1}^{*} t^{*}}{y_{1}^{*}} x_{1}+k_{3} x_{1}^{*} y_{1}^{*} t^{*} x_{1} y_{1}^{2}-k_{4} t^{*} y_{1}, \\
& \dot{z}_{1}=\frac{k_{4} y_{1}^{*} t^{*}}{z_{1}^{*}} y_{1}-k_{5} t^{*} z_{1}, \\
& \dot{x}_{2}=\frac{k_{0} A_{0} t^{*}}{x_{1}^{*}}+\frac{k_{1} A_{0} z_{1}^{*} t^{*}}{x_{1}^{*}} z_{2}-k_{2} t^{*} x_{2}-k_{3} x_{1}^{*} y_{1}^{* 2} t^{*} x_{2} y_{2}^{2}, \\
& \dot{y}_{2}=\frac{k_{2} x_{1}^{*} t^{*}}{y_{1}^{*}} x_{2}+k_{3} x_{1}^{*} y_{1}^{*} t^{*} x_{2} y_{2}^{2}-k_{4} t^{*} y_{2}, \\
& \dot{z}_{2}=\frac{k_{4} y_{1}^{*} t^{*}}{z_{1}^{*}} y_{2}-k_{5} t^{*} z_{1} .
\end{aligned}
$$

By setting $t^{*}=1 / k_{2}$ and $y_{1}^{*}=\left(k_{2} / k_{3}\right)^{\frac{1}{2}}$ into system (9.3) and simplifying, we obtain

$$
\begin{aligned}
\dot{x}_{1} & =\frac{k_{0} A_{0}}{k_{2} x_{1}^{*}}+\frac{k_{1} A_{0} z_{1}^{*}}{k_{2} x_{1}^{*}} z_{2}-x_{1}\left(1+y_{1}^{2}\right), \\
\frac{k_{2}}{k_{4}} \dot{y}_{1} & =\frac{\left(k_{2} k_{3}\right)^{\frac{1}{2}} x_{1}^{*}}{k_{4}} x_{1}\left(1+y_{1}^{2}\right)-y_{1}, \\
\frac{k_{2}}{k_{5}} \dot{z}_{1} & =\frac{k_{4} y_{1}^{*}}{k_{5} z_{1}^{*}} y_{1}-z_{1}, \\
\dot{x}_{2} & =\frac{k_{0} A_{0}}{k_{2} x_{1}^{*}}+\frac{k_{1} A_{0} z_{1}^{*}}{k_{2} x_{1}^{*}} z_{2}-x_{2}\left(1+y_{2}^{2}\right), \\
\frac{k_{2}}{k_{4}} \dot{y}_{2} & =\frac{\left(k_{2} k_{3}\right)^{\frac{1}{2}} x_{1}^{*}}{k_{4}} x_{2}\left(1+y_{2}^{2}\right)-y_{2}, \\
\frac{k_{2}}{k_{5}} \dot{z}_{2} & =\frac{k_{4} y_{1}^{*}}{k_{5} z_{1}^{*}} y_{2}-z_{1} .
\end{aligned}
$$


Next, by setting

$$
x_{1}^{*}=\frac{k_{4}}{\left(k_{2} k_{3}\right)^{\frac{1}{2}}} \text { and } z_{1}^{*}=\frac{k_{4}}{k_{5}}\left(\frac{k_{2}}{k_{3}}\right)^{\frac{1}{2}}
$$

in system (9.4) and simplifying, we have

$$
\begin{aligned}
\dot{x}_{1} & =\frac{k_{1} A_{0}}{k_{5}}\left(\left(\frac{k_{0} k_{5}}{k_{1} k_{4}}\right)\left(\frac{k_{3}}{k_{2}}\right)^{\frac{1}{2}}+z_{2}\right)-x_{1}\left(1+y_{1}^{2}\right), \\
\frac{k_{2}}{k_{4}} \dot{y}_{1} & =x_{1}\left(1+y_{1}^{2}\right)-y_{1}, \\
\frac{k_{2}}{k_{5}} \dot{z}_{1} & =y_{1}-z_{1}, \\
\dot{x}_{2} & =\frac{k_{1} A_{0}}{k_{5}}\left(\left(\frac{k_{0} k_{5}}{k_{1} k_{4}}\right)\left(\frac{k_{3}}{k_{2}}\right)^{\frac{1}{2}}+z_{2}\right)-x_{2}\left(1+y_{2}^{2}\right), \\
\frac{k_{2}}{k_{4}} \dot{y}_{2} & =x_{2}\left(1+y_{2}^{2}\right)-y_{2}, \\
\frac{k_{2}}{k_{5}} \dot{z}_{2} & =y_{2}-z_{1} .
\end{aligned}
$$

Finally, on setting

$$
\mu=\frac{k_{1}}{k_{5}} A_{0}, \kappa=\left(\frac{k_{0} k_{5}}{k_{1} k_{4}}\right)\left(\frac{k_{3}}{k_{2}}\right)^{\frac{1}{2}}, \sigma=\frac{k_{2}}{k_{4}} \text { and } \delta=\frac{k_{2}}{k_{5}}
$$

in system (9.5), we obtain a nondimensionalized version of system (9.1). In summary, the dimensionless variable and positive parameter groupings

$$
\begin{gathered}
\tau=k_{2} t, x_{1}=\frac{\left(k_{1} k_{3}\right)^{\frac{1}{2}}}{k_{4}}\left[X_{1}\right], y_{1}=\left(\frac{k_{3}}{k_{2}}\right)^{\frac{1}{2}}\left[Y_{1}\right], z_{1}=\left(\frac{k_{5}}{k_{4}}\right)\left(\frac{k_{3}}{k_{2}}\right)^{\frac{1}{2}}\left[Z_{1}\right], \\
x_{2}=\frac{\left(k_{1} k_{3}\right)^{\frac{1}{2}}}{k_{4}}\left[X_{2}\right], y_{2}=\left(\frac{k_{3}}{k_{2}}\right)^{\frac{1}{2}}\left[Y_{2}\right], z_{2}=\left(\frac{k_{5}}{k_{4}}\right)\left(\frac{k_{3}}{k_{2}}\right)^{\frac{1}{2}}\left[Z_{2}\right], \\
\mu=\frac{k_{1} A_{0}}{k_{5}}, \kappa=\left(\frac{k_{0} k_{5}}{k_{1} k_{4}}\right)\left(\frac{k_{3}}{k_{2}}\right)^{\frac{1}{2}}, \sigma=\frac{k_{2}}{k_{4}} \text { and } \delta=\frac{k_{2}}{k_{5}}
\end{gathered}
$$

simplify the coupled six-variable system (9.1) to the following dimensionless system: 


$$
\begin{aligned}
\dot{x}_{1} & =\mu\left(\kappa+z_{2}\right)-x_{1}\left(1+y_{1}^{2}\right) \\
\sigma \dot{y}_{1} & =x_{1}-y_{1}+x_{1} y_{1}^{2} \\
\delta \dot{z}_{1} & =y_{1}-z_{1} \\
\dot{x}_{2} & =\mu\left(\kappa+z_{2}\right)-x_{2}\left(1+y_{2}^{2}\right) \\
\sigma \dot{y}_{2} & =x_{2}-y_{2}+x_{2} y_{2}^{2}, \\
\delta \dot{z}_{2} & =y_{2}-z_{1},
\end{aligned}
$$

where $0<x_{1}, y_{1}, z_{1}, x_{2}, y_{2}, z_{2}<\infty$ since $x_{1}, y_{1}, z_{1}, x_{2}, y_{2}$ and $z_{2}$ are the dimensionless chemical concentrations. The parameter $\mu>0$, since it is related to the constant concentration of the reactant $A$ and rate constants. All the other parameters in system (9.6) are also positive since they are dimensionless rate constant groupings. The corresponding coupling for this nondimensional system is achieved by replacing $z_{1}$ in the first equation of system (9.6) by $z_{2}$ and $z_{2}$ in the sixth equation of system (9.6) by $z_{1}$.

\subsection{Model Analysis}

Theorem 9.4.1 (Existence of equilibria). Let $\kappa>0, \mu>0, y_{2}^{*}>0$ and $y_{2}^{*} \geq \kappa \mu$ where $y_{2}^{*}$ is the equilibrium value of $y_{2}$. Then system (9.6) has a non-negative equilibrium solution $E^{*}$ that is expressible in terms of $y_{2}^{*}$. Furthermore, this equilibrium solution is nontrivial and positive when $y_{2}^{*}>\kappa \mu$ and reduces to

$$
E^{*}=\left(\frac{\kappa \mu}{1+(\kappa \mu)^{2}}, \kappa \mu, \kappa \mu, \frac{\kappa \mu}{1+(\kappa \mu)^{2}}, \kappa \mu, 0\right)
$$

when $y_{2}^{*}=\kappa \mu$.

Proof. Let $E^{*}=\left(x_{1}^{*}, y_{1}^{*}, z_{1}^{*}, x_{2}^{*}, y_{2}^{*}, z_{2}^{*}\right)$ be an equilibrium solution of system (9.6). Then substituting $E^{*}$ in (9.6), setting the right hand sides of the equations to zero and solving the ensuing system of algebraic equations for $x_{1}^{*}, y_{1}^{*}, z_{1}^{*}, x_{2}^{*}, y_{2}^{*}$ and $z_{2}^{*}$, we obtain

$$
E^{*}=\left(x_{1}^{*}, y_{1}^{*}, z_{1}^{*}, x_{2}^{*}, y_{2}^{*}, z_{2}^{*}\right)=\left(\frac{y_{2}^{*}}{1+y_{2}^{* 2}}, y_{2}^{*}, y_{2}^{*}, \frac{y_{2}^{*}}{1+y_{2}^{* 2}}, y_{2}^{*}, \frac{y_{2}^{*}-\kappa \mu}{\mu}\right)
$$


It is now easy to see that each term in (9.7) is non-negative when $\kappa>0, \mu>0, y_{2}^{*}>$ 0 and $y_{2}^{*} \geq \kappa \mu$, since the last term $z_{2}^{*}$ would either be zero or strictly positive.

It also follows from (9.7) that $E^{*}$ is nontrivial and positive when $y_{2}^{*}>\kappa \mu$ and that

$$
E^{*}=\left(\frac{\kappa \mu}{1+(\kappa \mu)^{2}}, \kappa \mu, \kappa \mu, \frac{\kappa \mu}{1+(\kappa \mu)^{2}}, \kappa \mu, 0\right)
$$

when $y_{2}^{*}=\kappa \mu$.

The algebra of the linear stability analysis of this system is very complex, and we therefore resort to numerical simulations to investigate the stability and dynamical behavior of the system. While the dynamics of a single subsystem possesses a unique attractor for any given set of parameters and for all initial conditions, as shown in Chapter 8, we now observe a multitude of coexisting attractors. To be more specific, the coupled system exhibits extreme multistability, i.e., there exist infinitely many attractors for a given set of parameters.

To demonstrate this behavior, we fix the parameters in such a way that the uncoupled three-variable autocatalator exhibits a simple periodic solution $(\mu=0.157, \kappa=65, \sigma=$ $5 \times 10^{-3}, \delta=2 \times 10^{-2}$, which is reached from any initial condition in the three-dimensional state space. Keep in mind that only positive initial conditions make sense, since we are dealing with chemical entities that correspond to concentrations of reactants. Let us now check to which final state the dynamics of the coupled autocatalator systems converge. To this end, we fix five of the six initial conditions, $\left(0.01,0.1,0.1,0.0, y_{02}, 0.0\right)$ and vary the initial concentration of $y_{2}$ within the interval $4.0 \leq y_{02} \leq 8.0$. To plot the long-term dynamical behavior depicted in Fig. 9.3, we integrate system (9.6) using a fourth-order Runge-Kutta method following the scheme of Dormand and Prince with variable step size control (see, for example, Ref. [34]). The computations were sufficiently long for asymptotic behavior to be exhibited, and the simulation time was mostly determined by the parameter regime under consideration. In particular, the simulations were carried out for at least 50,000 time steps, and only the last one-tenth of each time series was used. Identical behavior was found in simulations with one-half and three-quarters as many time steps, showing that the behavior is asymptotic. We also employed higher-order Runge-Kutta methods (7th-8th order) to verify the accuracy of our results. As shall be seen in Section 9.5, system (9.6) can be reduced to 
a simpler system using a conserved quantity. This reduced system yields exactly the same results, which is a strong check of the validity of the numerical integration.

We find a great variety of dynamics, stretching from simple periodic motion for large values of $y_{02}$ to oscillations of different periods for intermediate values to chaotic behavior for low values of $y_{02}$. Period-doubling is found at $y_{02}=7.145(T \rightarrow 2 T), y_{02}=5.515(2 T \rightarrow 4 T)$, $y_{02}=5.165(4 T \rightarrow 8 T)$, and $y_{02}=5.095(8 T \rightarrow 16 T)$. Chaotic behavior immersed with periodic windows is evident as well. Typical time series and phase plots depicting periodic behaviors of different periods and chaotic behavior of this coupled system are presented in Figs. 9.1 and 9.2, respectively. Figure 9.3 shows the maximum values of the amplitude of $x_{1}$, corresponding to a Poincaré section, so that a simple oscillation with a period $T$ appears in the plot as a fixed point. We obtain a picture, which looks like a complete bifurcation diagram; however, we do not change a bifurcation parameter but the initial condition, and therefore it only resembles a bifurcation diagram. 


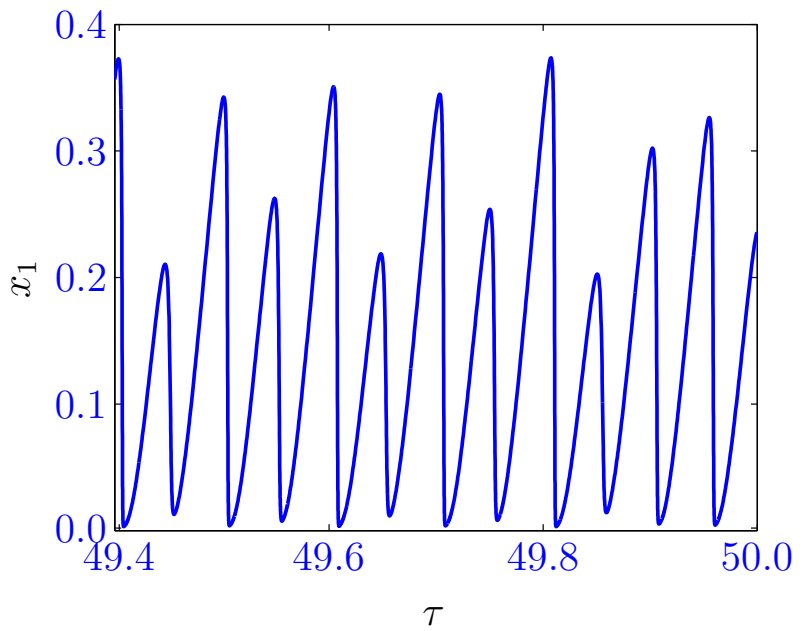

9.1.1. Chaotic behavior at $y_{02}=4.0$.

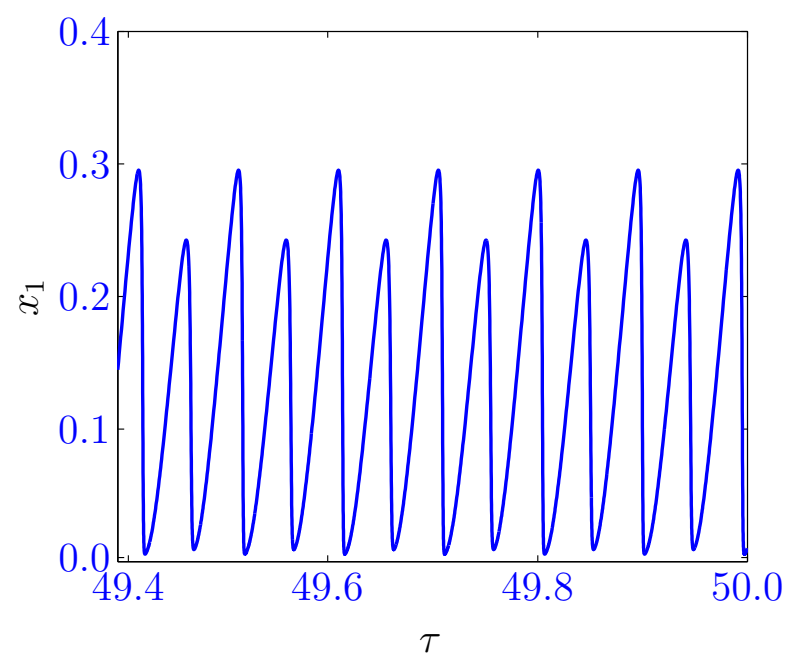

9.1.3. Period-2 behavior at $y_{02}=6.5$.

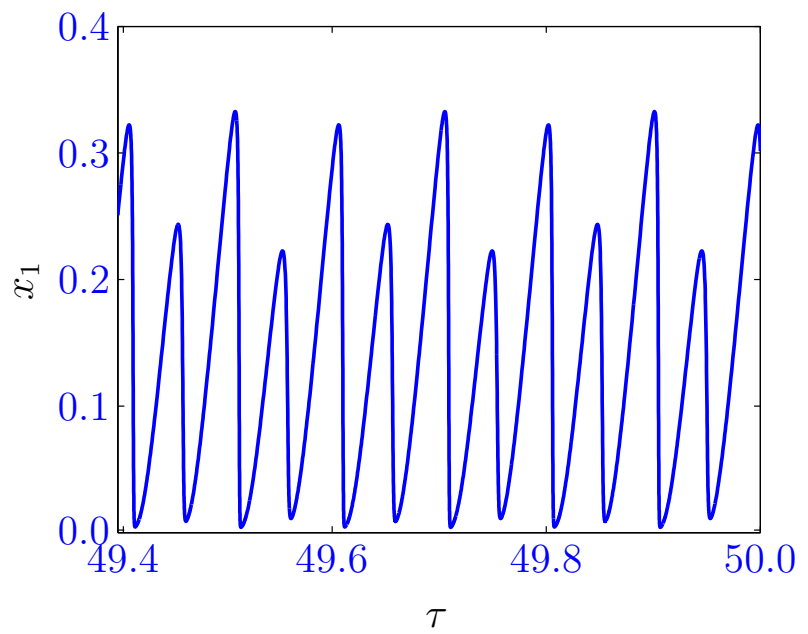

9.1.2. Period-2 behavior at $y_{02}=5.2$

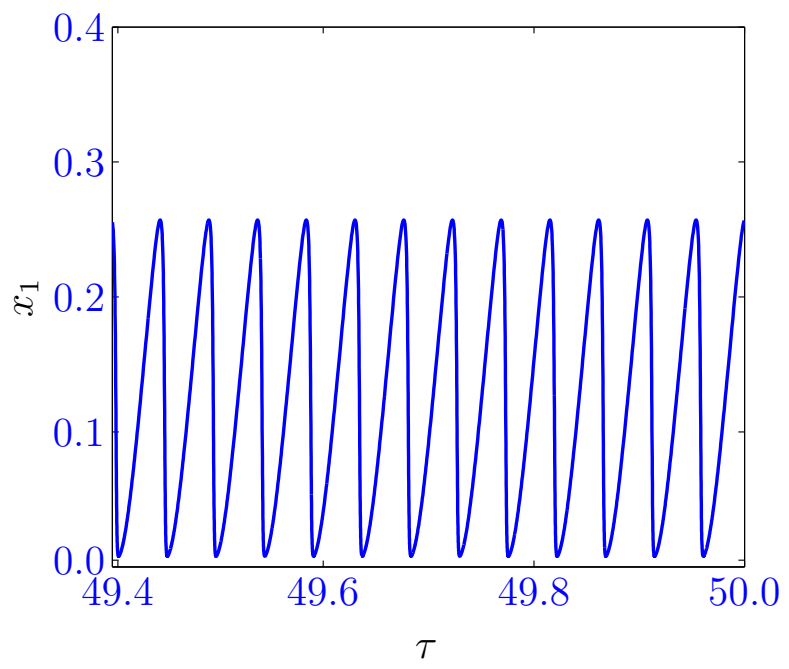

9.1.4. Period-1 behavior at $y_{02}=8.0$.

Figure 9.1. Time series plots of the coupled autocatalator model for $\kappa=65, \mu=$ $0.157, \sigma=5 \times 10^{-3}, \delta=2 \times 10^{-2}$ showing different dynamical behaviors for different values $y_{02}$. We plot $x_{1}$ (the dimensionless concentration of the intermediate product $X_{1}$ ) against dimensionless time $\tau$. 


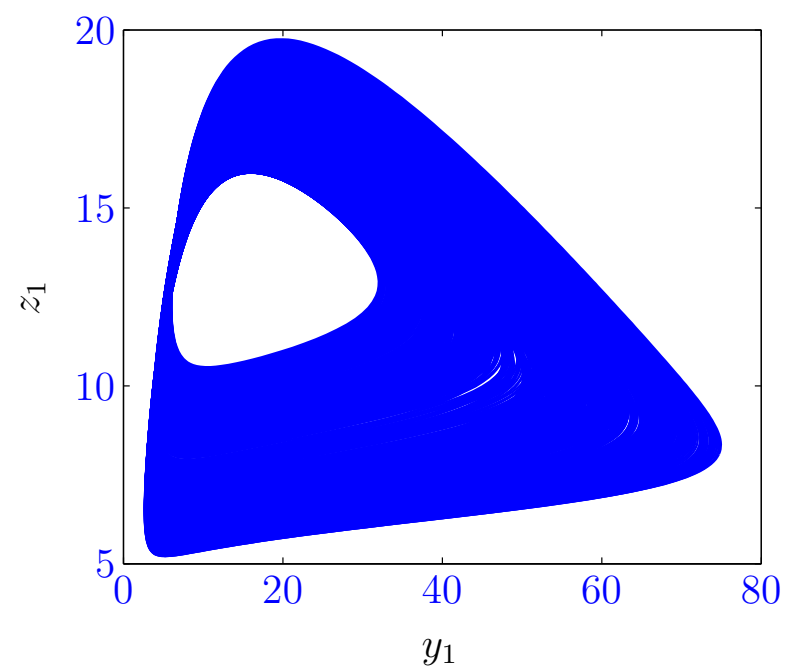

9.2.1. Chaotic attractor at $y_{02}=4.0$.

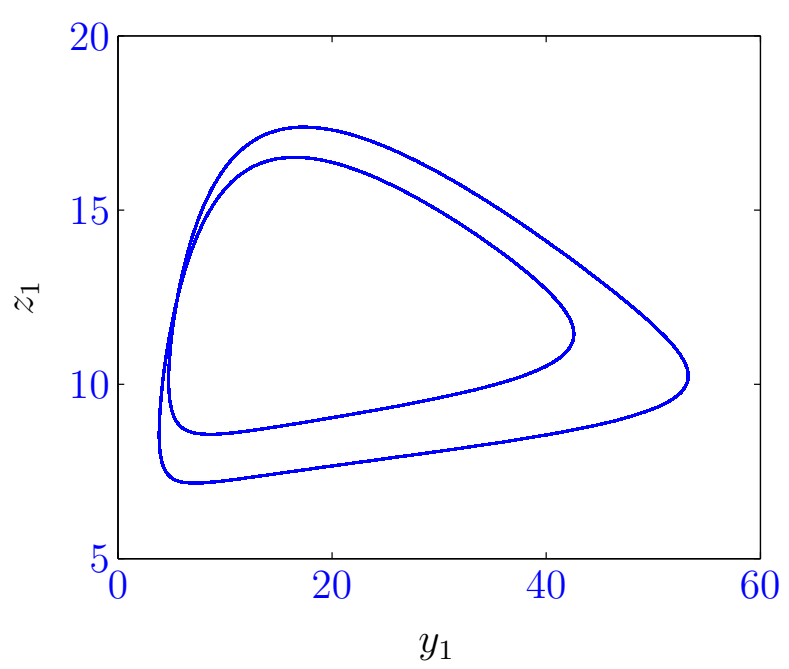

9.2.3. Period-2 attractor at $y_{02}=6.5$.

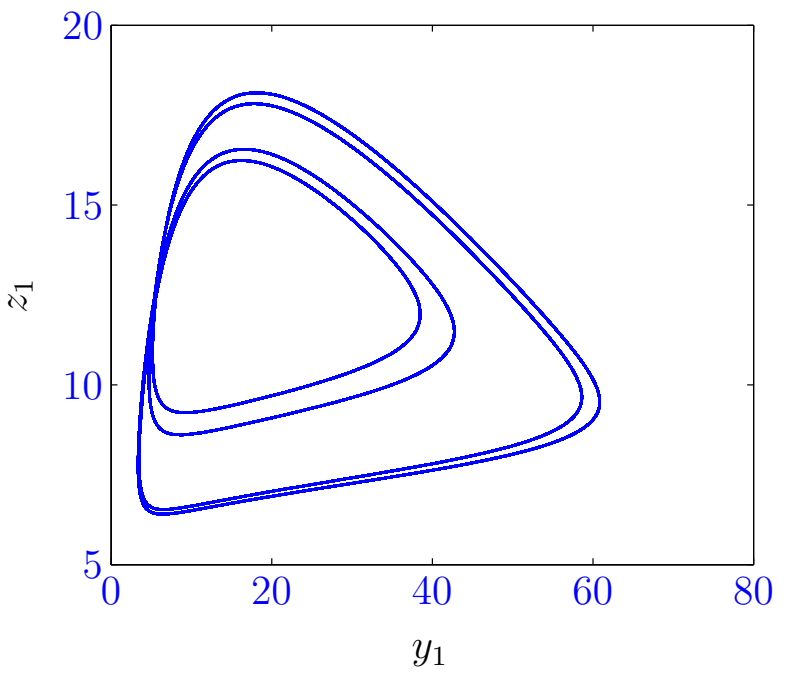

9.2.2. Period-2 attractor at $y_{02}=5.2$.

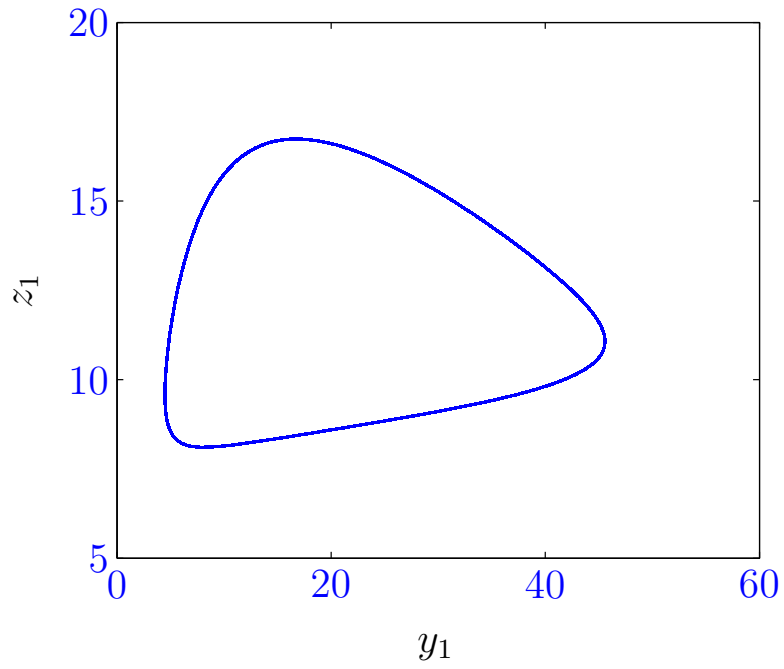

9.2.4. Period-1 limit cycle at $y_{02}=8.0$.

Figure 9.2. Phase plots of the coupled six-variable autocatalator model for $\kappa=65, \mu=$ $0.157, \sigma=5 \times 10^{-3}, \delta=2 \times 10^{-2}$ showing different dynamical attractors for different values of $y_{02}$. We plot $z_{1}$ (the dimensionless concentration of the species $Z_{1}$ ) against $y_{1}$ (the dimensionless concentration of the species $Y_{1}$ ). 


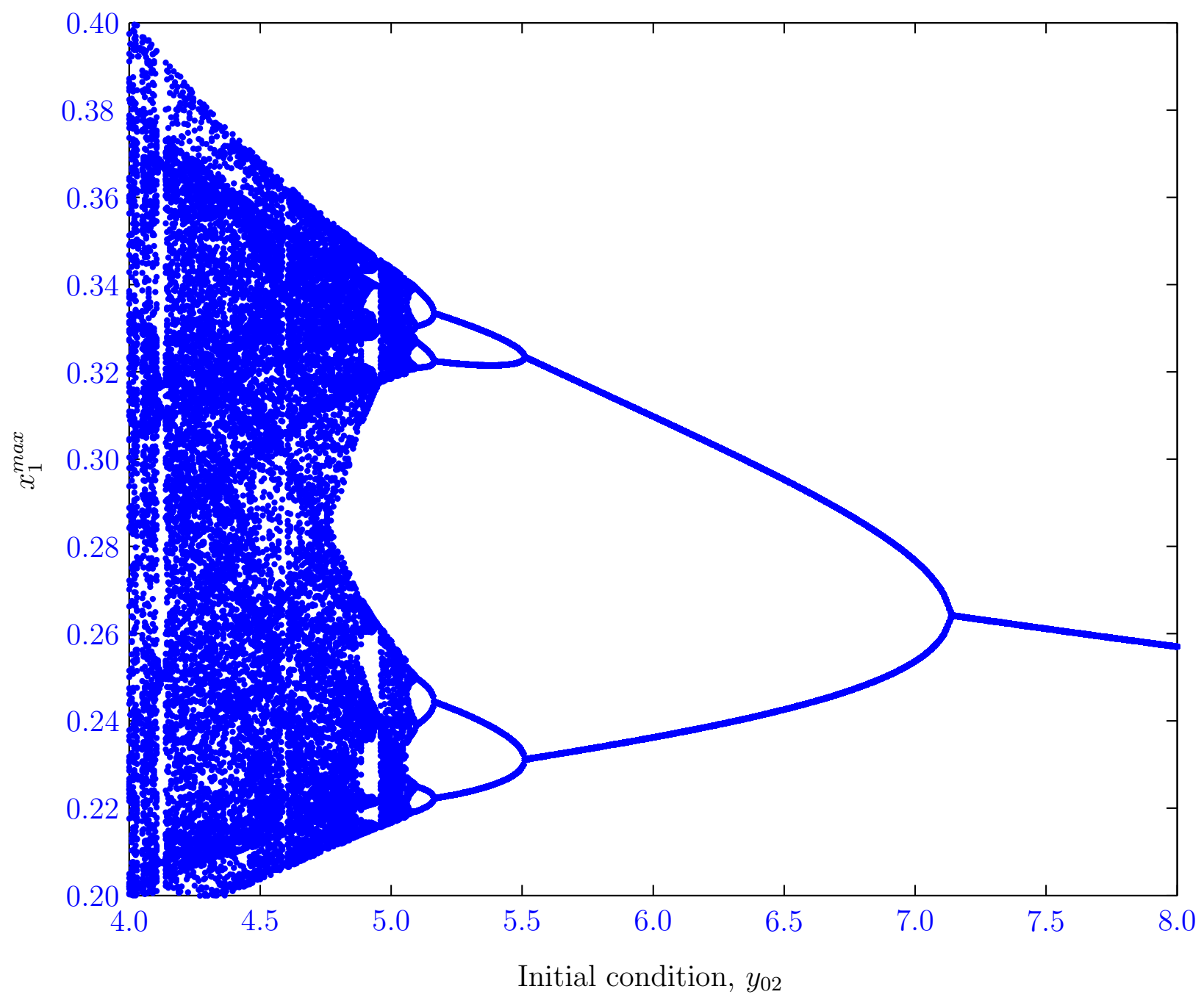

Figure 9.3. Long term dynamics of the coupled six-variable autocatalator model. A plot of the maximum amplitude of $x_{1}$ against the initial condition $y_{02}$ showing a reverse period-doubling sequence. 
Considering the quantitative behavior of the system, one could argue that the number of attractors is finite because there are entire intervals of $y_{02}$ leading to an oscillation of a certain period, say, period 2T. However for each initial condition within this interval the location of the $2 T$ periodic orbit is slightly different. This means that for any pair of nearby initial conditions the corresponding final attractors are never identical. In that sense we obtain an infinite number of different attractors in state space, so that each initial condition converges to a different attractor. Moreover, taking into account the qualitative behavior of the system, we can also argue that there is an infinite number of different coexisting attractors, since the period-doubling cascade is complete, with bifurcations between period-1, period-2, period-4, period-8, period-16, etc. up to period-infinity.

This behavior of extreme multistability in coupled systems is quite surprising since the uncoupled system possesses a unique attractor. In Ref. [27] it was shown that this special type of dynamics is related to the emergence of generalized synchronization between the two subsystems. In case of the two coupled Lorenz systems considered in Ref. [27], two of the variables are fully synchronized $\left(y_{1}=y_{2}\right.$ and $\left.z_{1}=z_{2}\right)$ while the difference between one of the variables takes a constant value $c$ in the long-term limit $\left(c=x_{1}-x_{2}\right)$. Which value of $c$ is reached depends on the initial condition. For the two coupled autocatalator systems described by Eqs. (9.6), we do not find such a simple kind of generalized synchronization, as shown in Figs. 9.4-9.6. There is seemingly no simple synchronization scheme visible, but as we will show later, we obtain a more complicated form of generalized synchronization. 


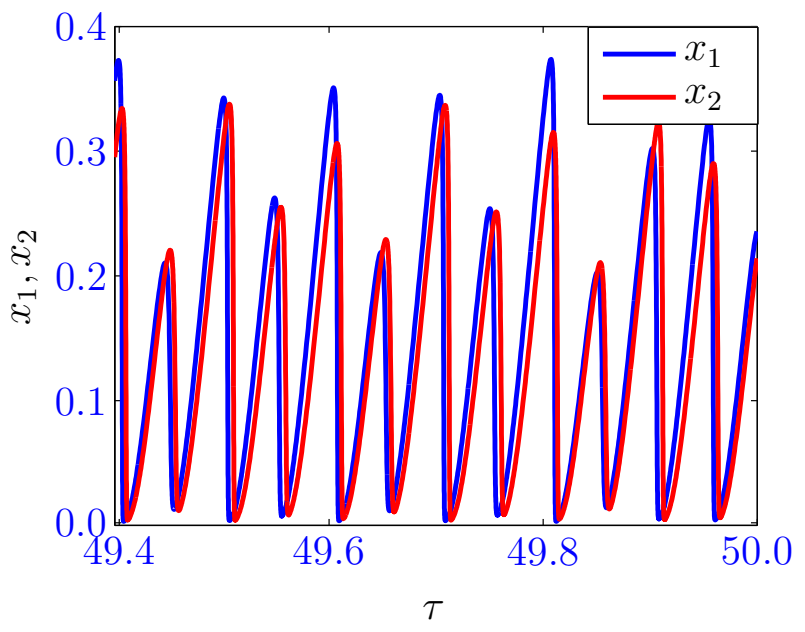

9.4.1. $y_{02}=4.0$, chaotic regime.

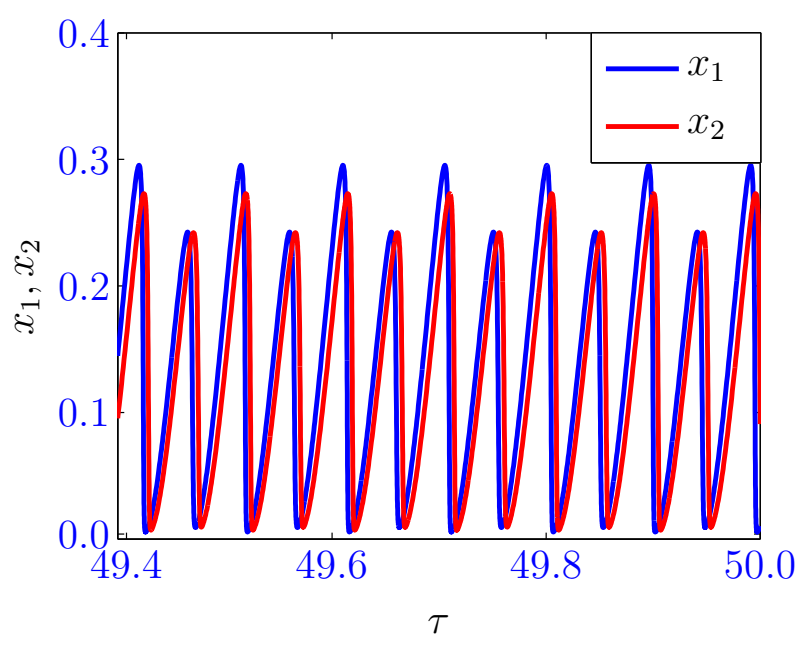

9.4.3. $y_{02}=6.5$, period- 2 regime.

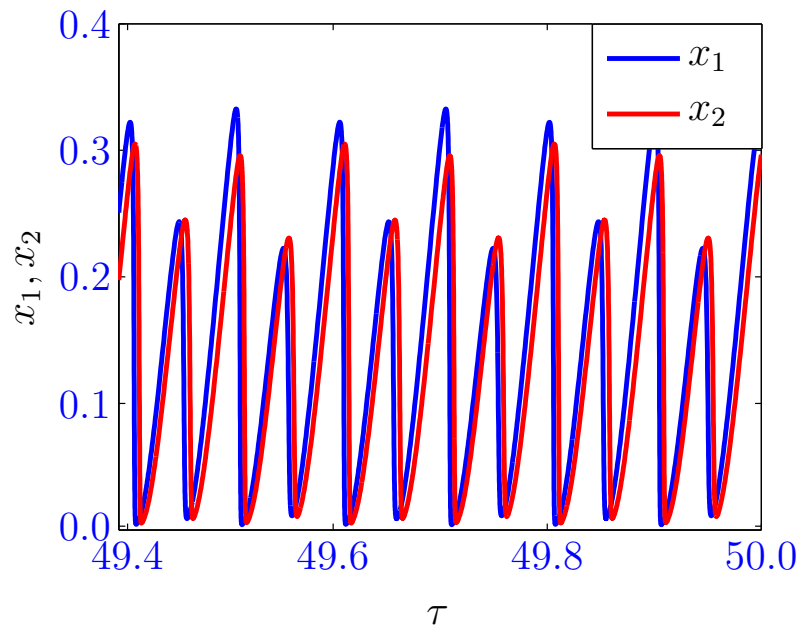

9.4.2. $y_{02}=5.2$, period-4 regime.

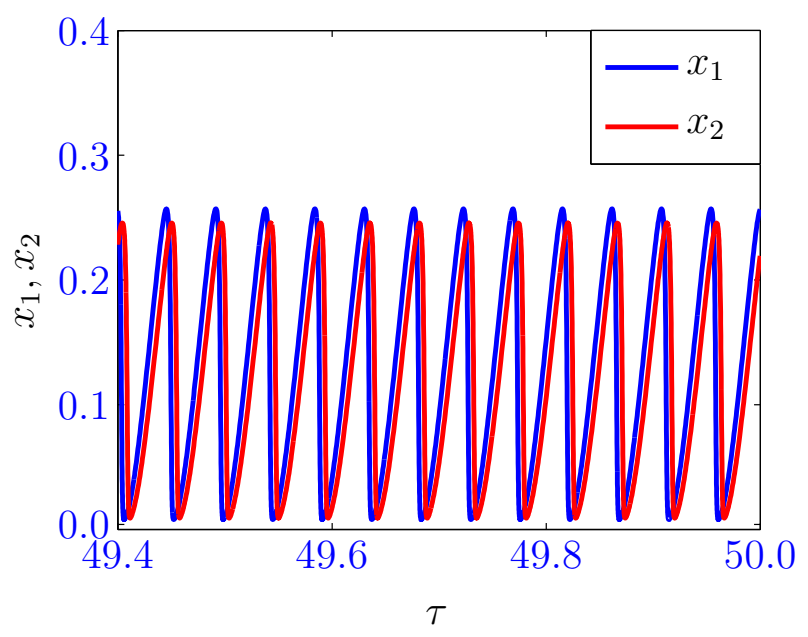

9.4.4. $y_{02}=8$, period-1 regime.

Figure 9.4. Time series plots illustrating the difference between $x_{1}$ and $x_{2}$ within the chaotic, period-4, period-2 and period-1 regimes. 


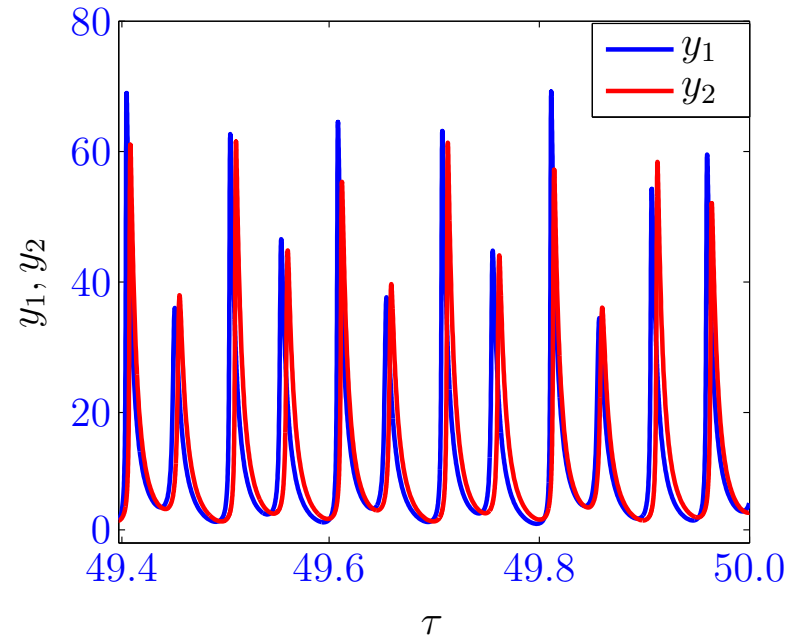

9.5.1. $y_{02}=4.0$, chaotic regime.

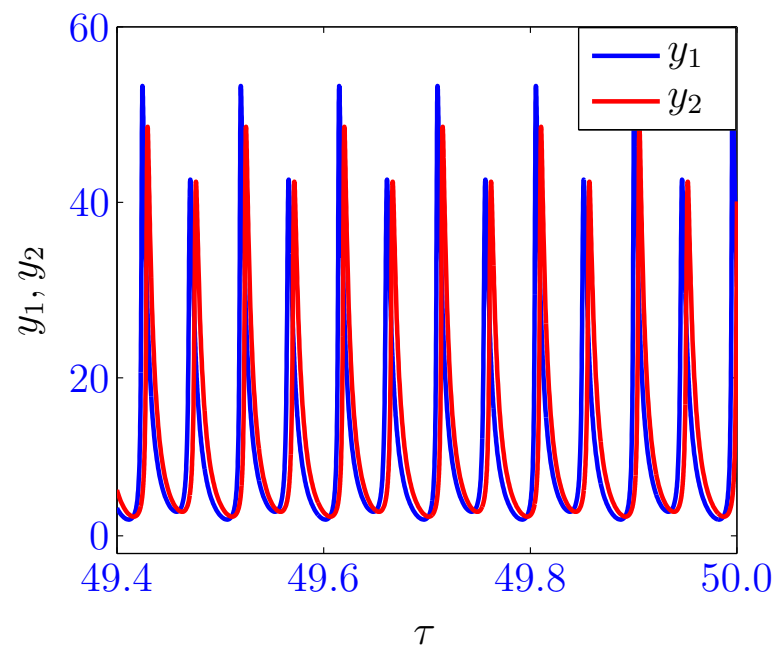

9.5.3. $y_{02}=6.5$, period- 2 regime.

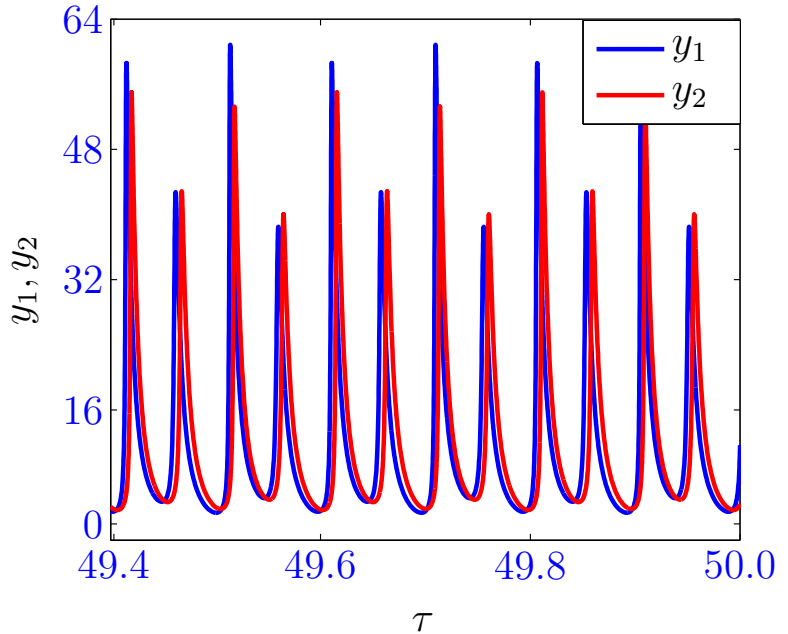

9.5.2. $y_{02}=5.2$, period-4 regime.

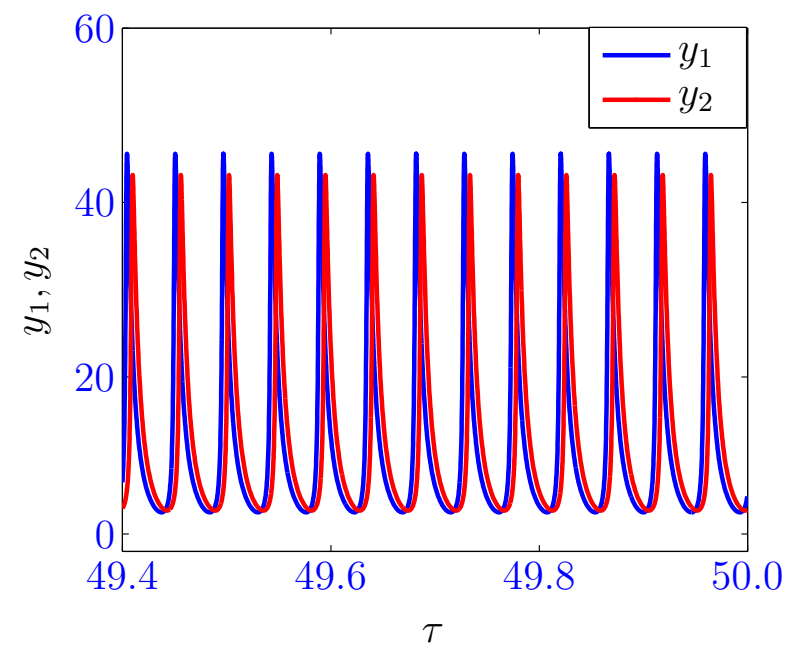

9.5.4. $y_{02}=8$, period-1 regime.

Figure 9.5. Time series plots illustrating the difference between $y_{1}$ and $y_{2}$ within the chaotic, period-4, period-2 and period-1 regimes. 


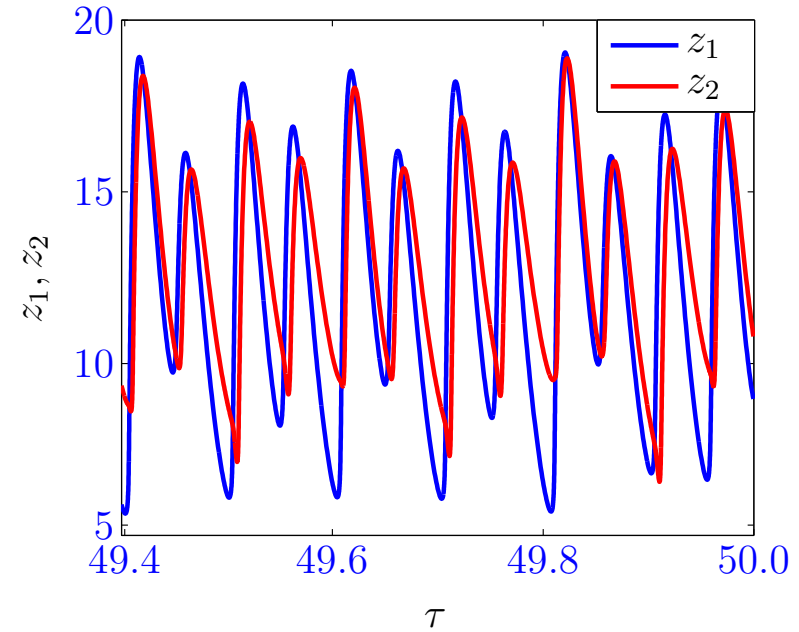

9.6.1. $y_{02}=4.0$, chaotic regime.

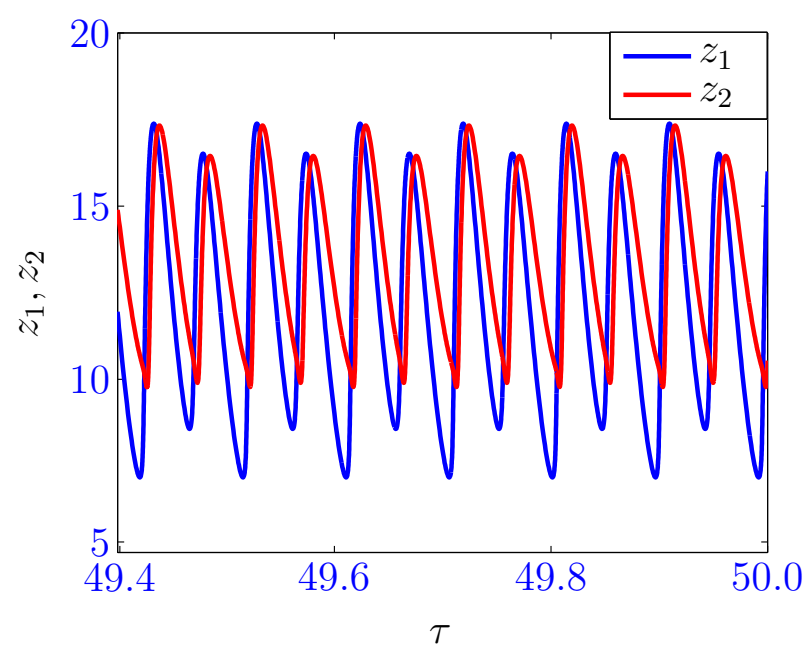

9.6.3. $y_{02}=6.5$, period- 2 regime.

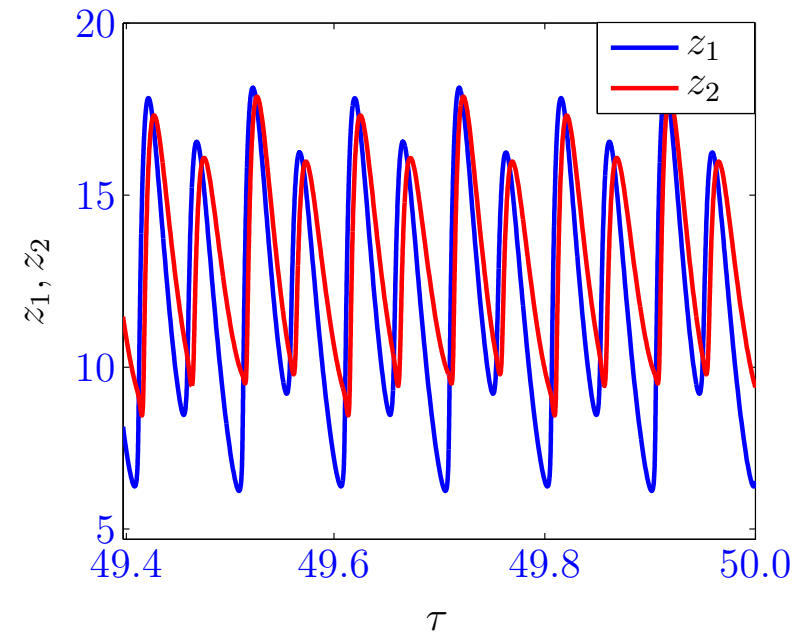

9.6.2. $y_{02}=5.2$, period- 4 regime.

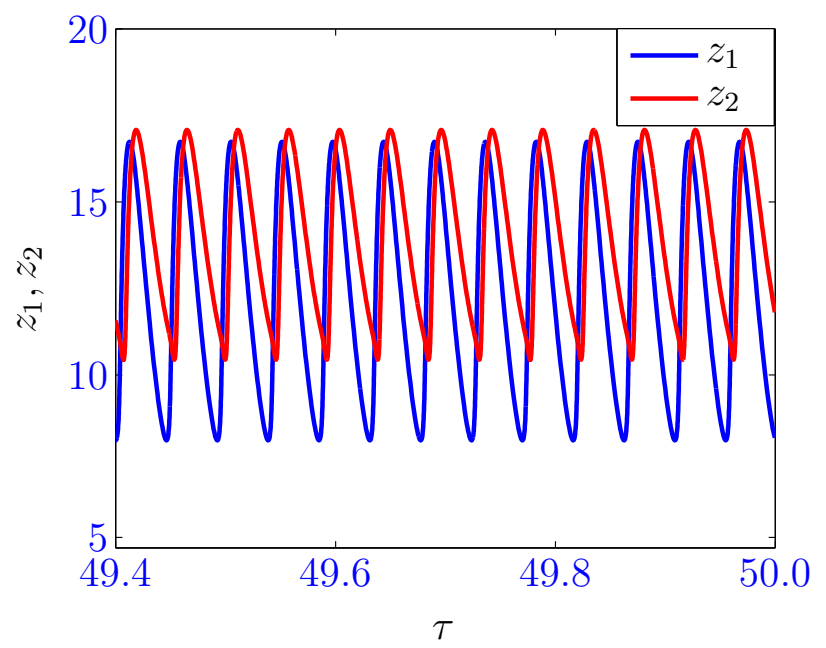

9.6.4. $y_{02}=8$, period-1 regime.

Figure 9.6. Time series plots illustrating the difference between $z_{1}$ and $z_{2}$ within the chaotic, period-4, period-2 and period-1 regimes. 


\subsection{Conserved Quantity}

To explain the appearance of extreme multistability we note that the coupling scheme implies the emergence of a conserved quantity. This quantity, which we call $C$ with $d C / d t=0$, corresponds to the sum of the weighted differences of the concentrations of all corresponding intermediate species. The weighting is provided by ratios of the reaction time constants.

Lemma 9.5.1 (Conserved quantity). There is a conserved quantity,

$$
C=x_{2}+\sigma y_{2}+\delta z_{2}-\left(x_{1}+\sigma y_{1}+\delta z_{1}\right)
$$

associated with system (9.6) that satisfies $d C / d t=0$.

Proof. By setting $e_{1}=x_{2}-x_{1}, e_{2}=y_{2}-y_{1}$ and $e_{3}=z_{2}-z_{1}$ in Eqs. (9.6) and simplifying, we obtain the following three-variable system of differences:

$$
\begin{aligned}
\dot{e}_{1} & =-x_{2}\left(1+y_{2}^{2}\right)+x_{1}\left(1+y_{1}^{2}\right), \\
\sigma \dot{e}_{2} & =x_{2}\left(1+y_{2}^{2}\right)-x_{1}\left(1+y_{1}^{2}\right)-y_{2}+y_{1}, \\
\delta \dot{e}_{3} & =y_{2}-y_{1} .
\end{aligned}
$$

Adding Eqs. (9.9)-(9.11), we obtain

$$
\dot{e}_{1}+\sigma \dot{e}_{2}+\delta \dot{e}_{3}=0 \text { or }\left(\dot{x}_{2}-\dot{x}_{1}\right)+\sigma\left(\dot{y}_{2}-\dot{y}_{1}\right)+\delta\left(\dot{z}_{2}-\dot{z}_{1}\right)=0 \text {. }
$$

On integrating Eq. (9.12), we obtain

$$
e_{1}+\sigma e_{2}+\delta e_{3}=\text { constant or }\left(x_{2}-x_{1}\right)+\sigma\left(y_{2}-y_{1}\right)+\delta\left(z_{2}-z_{1}\right)=\text { constant. }
$$

By setting the constant to $C$ and rearranging, we obtain the desired conserved quantity. We show that $d C / d t=0$ is always fulfilled by setting $C=e_{1}+\sigma e_{2}+\delta e_{3}$ in equation (9.12).

The value of $C$ is given by the initial condition $C=x_{02}+\sigma y_{02}+\delta z_{02}-\left(x_{01}+\sigma y_{01}+\delta z_{01}\right)$ and defines a complicated manifold in state space on which the dynamics takes place. Based on the existence of the conserved quantity $C$ we can illustrate extreme multistability with the following schematic views depicted in Figs. 9.7 and 9.8. The whole state space is densely filled with manifolds (hypersurfaces), each associated with a value of $C$. In each of these 
manifolds there exists at least one attractor. Since $C$ can take any real value, the state space is "sliced" into infinitely many such manifolds, each of them containing another long-term dynamics. Changing the initial condition corresponds to a change of the manifold in which the dynamics takes place.

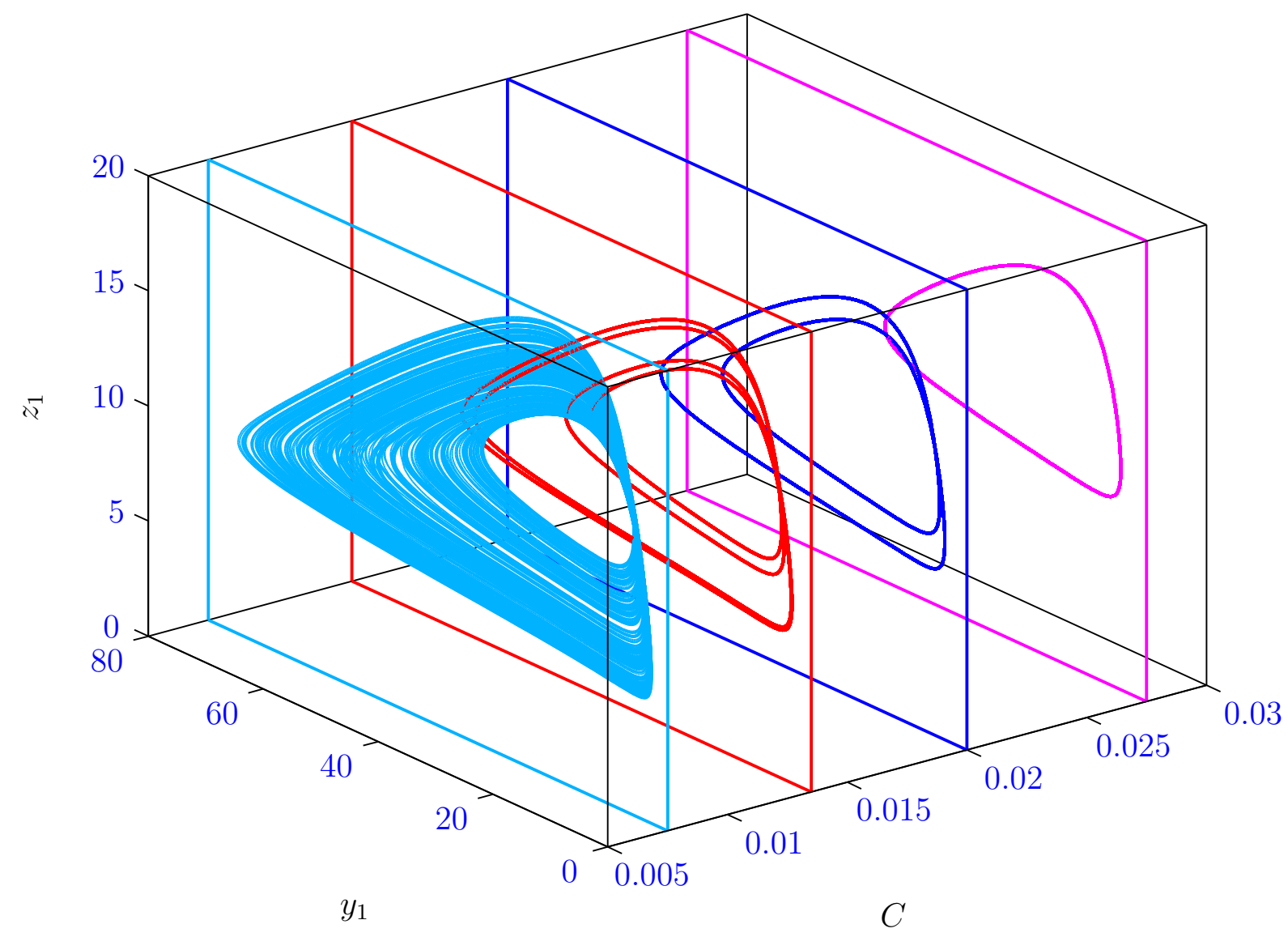

Figure 9.7. A sampling of the synchronization manifolds as a function of the conserved quantity $C$. Chaotic (light blue), period-4 (red), period-2 (dark blue), period1 (magenta) behavior is exhibited for $C=0.0075,0.0135,0.020$, and 0.0275 . 


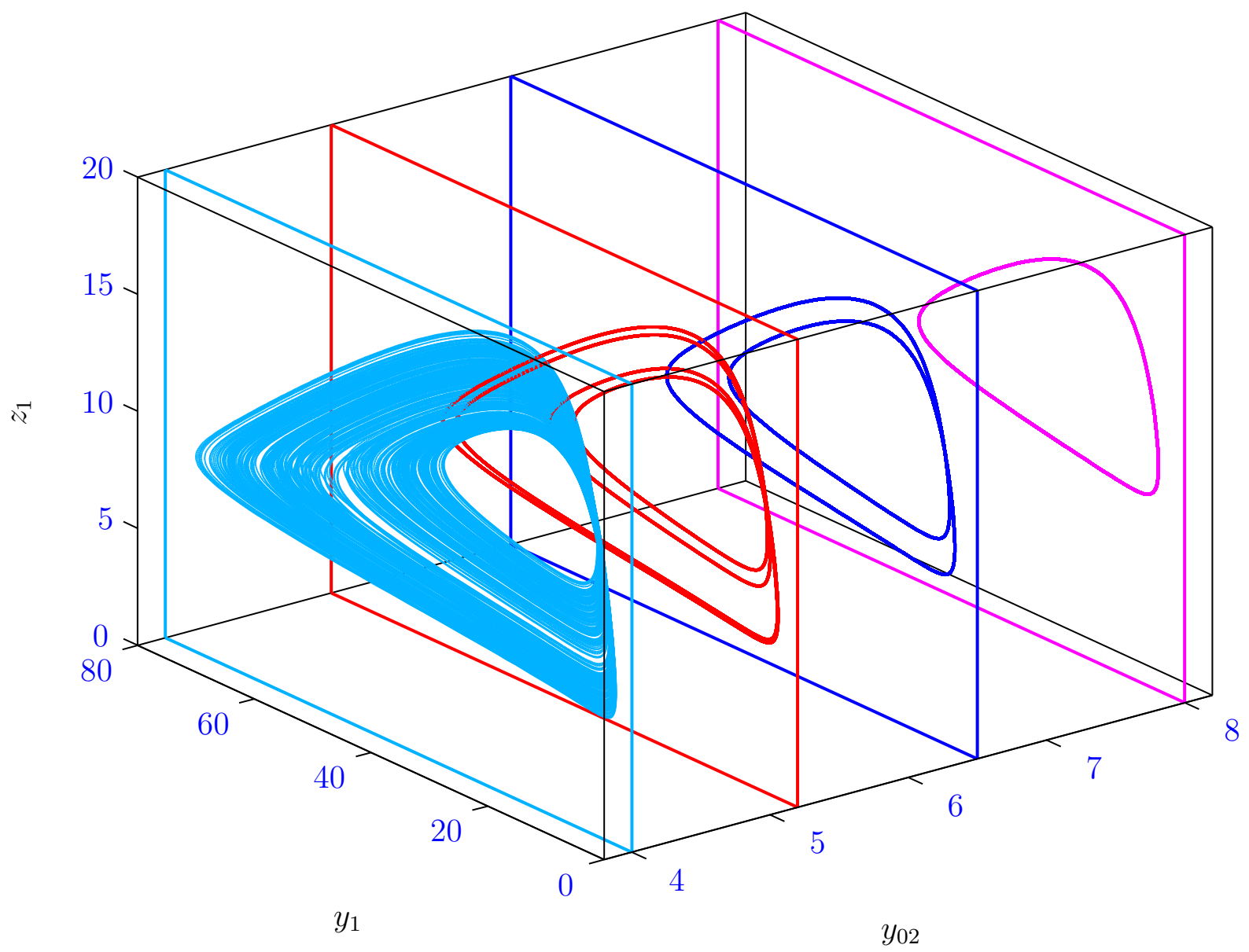

Figure 9.8. A sampling of the synchronization manifolds as a function of the initial condition $y_{02}$. Due to the existence of the conserved quantity $C$, the state space is divided into submanifolds corresponding to different values of $C$. In each of these submanifolds another long-term dynamics can be found. Chaotic (light blue), period-4 (red), period-2 (dark blue), period-1 (magenta) behavior is exhibited for $y_{02}=4,5.2,6.5$ and 8 respectively. In terms of the conserved quantity $C$, chaotic, period-4, period-2 and period-1 behavior is exhibited for $C=0.0075,0.0135,0.020$, and 0.0275 respectively. 
The existence of a conserved quantity allows us to reduce the dimension of the dynamical system describing the two coupled autocatalators by one. From Eq. (9.8),

$$
z_{2}=\left(C+x_{1}+\sigma y_{1}+\delta z_{1}-\left(x_{2}+\sigma y_{2}\right)\right) / \delta
$$

Substituting $z_{2}$ in Eqs. (9.6) leads to

$$
\begin{aligned}
\dot{x}_{1} & =\mu\left(\kappa+\left(x_{1}+\sigma y_{1}+\delta z_{1}+C-x_{2}-\sigma y_{2}\right) / \delta\right)-x_{1}\left(1+y_{1}^{2}\right), \\
\sigma \dot{y}_{1} & =x_{1}-y_{1}+x_{1} y_{1}^{2}, \\
\delta \dot{z}_{1} & =y_{1}-z_{1}, \\
\dot{x_{2}} & =\mu\left(\kappa+\left(x_{1}+\sigma y_{1}+\delta y_{1}-C-x_{2}-\sigma y_{2}\right) / \delta\right)-x_{2}\left(1+y_{2}^{2}\right), \\
\sigma \dot{y_{2}} & =x_{2}-y_{2}+x_{2} y_{2}^{2},
\end{aligned}
$$

From this rewriting it becomes obvious that the conserved quantity $C$, and hence the initial condition $y_{02}$, can be indeed regarded as a bifurcation parameter in the reduced system (9.13). We see that Fig. 9.3 can be interpreted as a bifurcation diagram in the mathematical sense, exhibiting many known dynamical transitions. Now, using the conserved quantity $C$ as a bifurcation parameter for system (9.13) and fixing all the system parameters and initial conditions as for the full system, we obtain dynamical behavior that is qualitatively identical to that of the full system. We observe chaotic behavior for small values of $C$ and reverse period-doubling for larger values of $C$. There is a switch from period-16 to period-8 at $C=0.01298$, period-8 to period- 4 at $C=0.01334$, period- 4 to period-2 at $C=0.01508$ and period-2 to period-1 at $C=0.2326$. Figure 9.9 illustrates the behavior of system (9.13). 


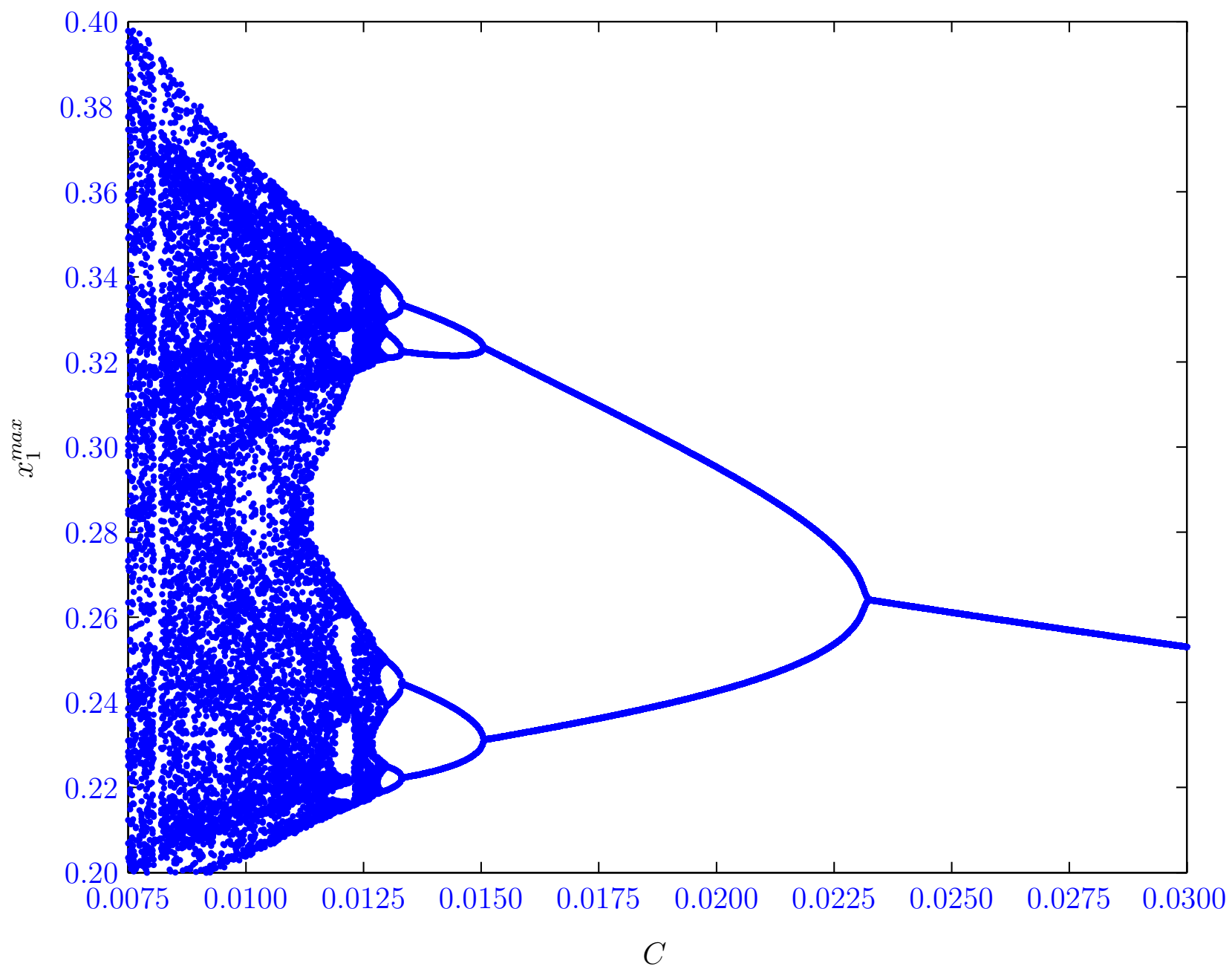

Figure 9.9. A bifurcation diagram of the reduced autocatalator model showing a reverse period-doubling sequence. Here, we are plotting the maximum value of $x_{1}$ (the dimensionless concentration of the intermediate species $X_{1}$ ) against the conserved quantity $C$ for $\mu=157, \kappa=65, \sigma=5 \times 10^{-3}$ and $\delta=2 \times 10^{-2}$. 
The existence of a conserved quantity is also apparent when computing Lyapunov exponents to characterize chaotic behavior in system (9.6). To verify this, we computed the Lyapunov exponents both as functions of time and the initial conditions. Computations of Lyapunov exponents as a function of time yield a positive largest Lyapunov exponent, $\lambda_{1}=5.08$, two zero Lyapunov exponents, $\lambda_{2}=\lambda_{3}=0.00$, and three negative Lyapunov exponents, $\lambda_{4}=-21.19, \lambda_{5}=-27.00, \lambda_{6}=-16.75$, as shown in Fig. 9.10. As discussed in Chapters 1 and 8, a positive largest Lyapunov exponent indicates that solution trajectories that originate close to each other drift apart exponentially as time become infinite.

For computations of Lyapunov exponents as a function of the initial condition $y_{02}$, we also obtain two zero Lyapunov exponents, where the first zero is the usual one corresponding to the Lyapunov exponent along the trajectory, while the second zero indicates the existence of a conserved quantity. We also have a positive largest Lyapunov exponent within the chaotic region. The Lyapunov exponents are negative within the periodic regions, except for the zero values. A plot of the largest three Lyapunov exponents is presented in Fig. 9.11. 


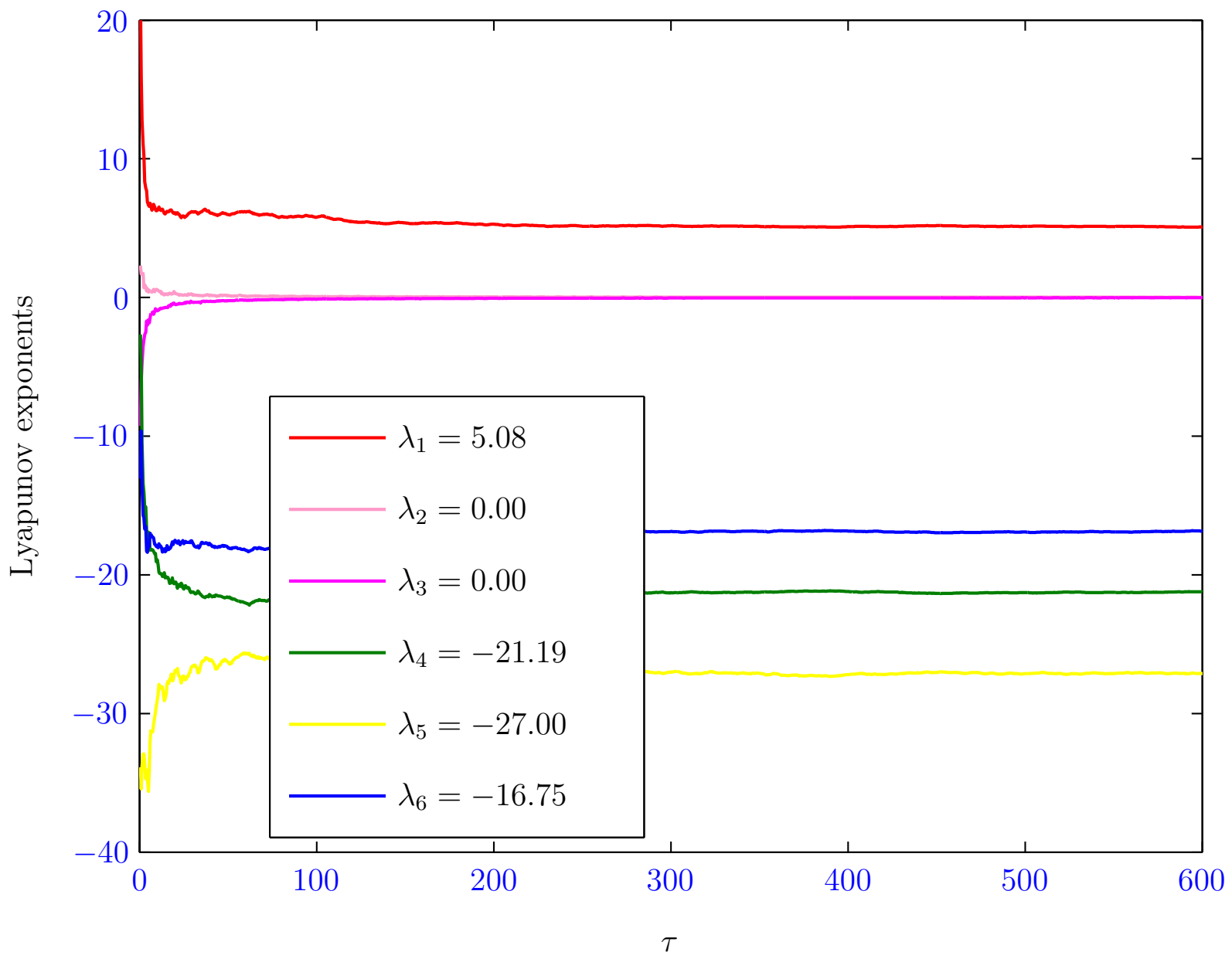

Figure 9.10. Dynamics of Lyapunov exponents of the coupled six-variable autocatalator model versus time. The largest Lyapunov exponent is positive while two of the Lyapunov exponents are zero. One is the usual zero Lyapunov exponent while the second is a result of the conserved quantity. 


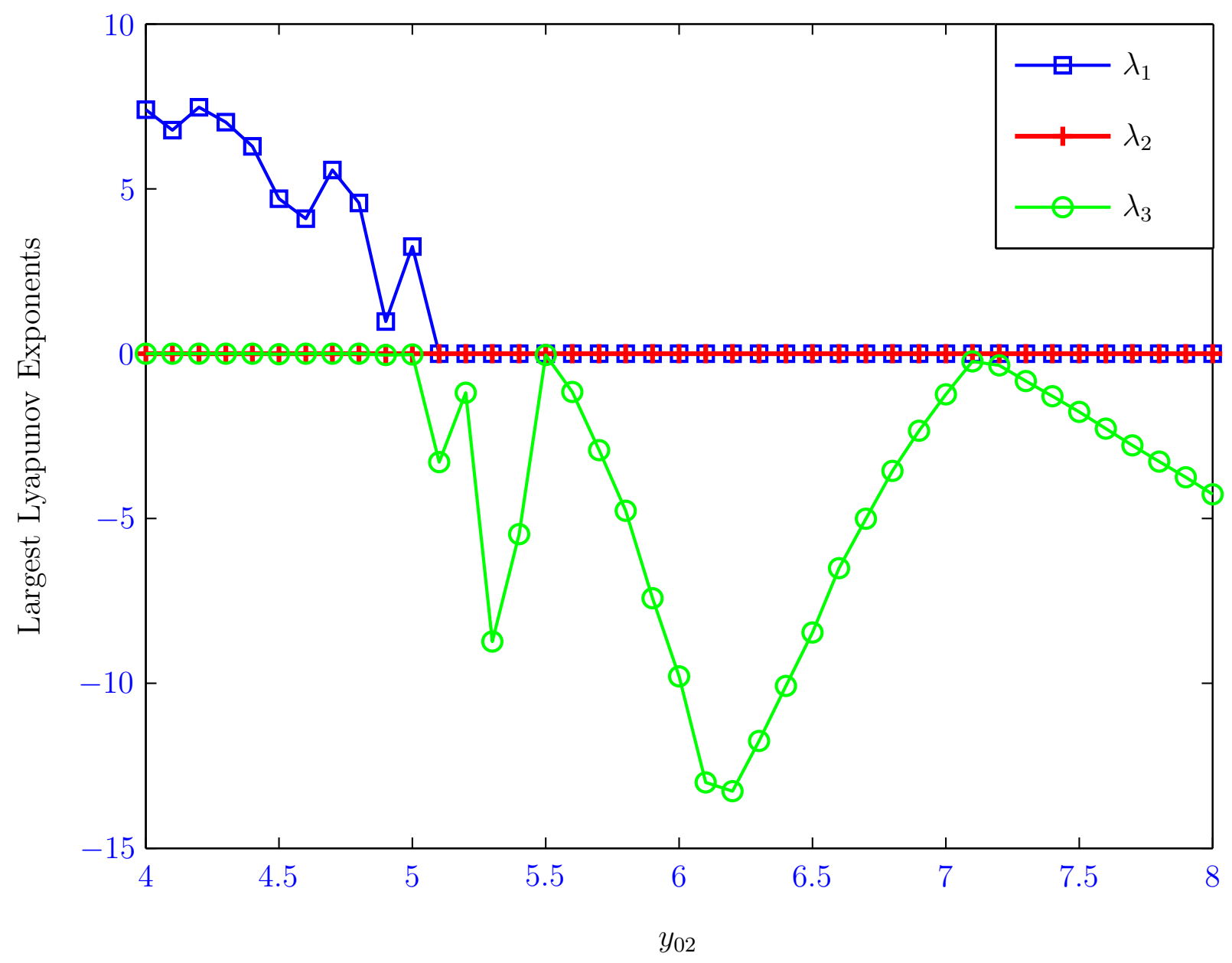

Figure 9.11. Dynamics of the three largest Lyapunov exponents of the coupled sixvariable autocatalator model (9.6) versus the initial condition $y_{02}$. 
We now discuss the relation between the dynamics of the two coupled oscillators. As already mentioned and shown in Figs. 9.4-9.6, the two three-variable oscillators synchronize with a certain time lag so that the phase retains a fixed difference, while the amplitude difference varies. Hence, there is no amplitude synchronization. In Figs. 9.4-9.6, we observe lag synchronization in the periodic and chaotic cases. Lag synchronization generally emerges while varying the coupling strength between two oscillators [35]; however, such a change is not possible in this system, where the coupling is realized by chemical reactions. With this restriction in mind, we note that the coupled autocatalators exhibit a type of generalized synchronization with features much like those seen in chaotic synchronization.

The concept of basins of attraction, the sets of initial conditions that converge to the same attractor, of coexisting attractors is very important when studying multistable systems. To discuss this concept in the case of extreme multistability, we first note that the conserved quantity $C$ is defined in equation $(9.8)$ by the initial values $x_{01}, y_{01}, z_{01}, x_{02}, y_{02}$ and $z_{02}$. Therefore, $C$ can be realized through many different initial conditions. This is because $C$ is determined solely by sums and differences and different combinations of $x_{i}, y_{i}, z_{i},(i=$ $1,2)$ give rise to the same value of $C$. Nonetheless, the question remains whether all these combinations of initial conditions leading to the same value of $C$ also lead to the same attractor. To address this question, we fixed four of the initial values, say, $x_{01}, z_{01}, x_{02}$ and $z_{02}$ and varied only two corresponding initial values, say, $y_{01}$ and $y_{02}$ in such a way that the difference between them is always the same. This way, we obtained new initial conditions in $y_{01}$ and $y_{02}$ while holding the value of $C$ constant. We then spanned the bifurcation diagram, considering the chaotic, period-8, period-4, period-2 and period- 1 regimes, and then repeated the entire process so that different initial conditions for the other pairs $\left(x_{01}, x_{02}\right)$ and $\left(z_{01}, z_{02}\right)$ and combinations of all three pairs were also explored. In any specific region of the bifurcation diagram, we always obtained the same attractor for all the different initial conditions leading to the same value of $C$. The different initial condition combinations therefore lead to the same attractor for any specific value of $C$. Thus, each of the infinite coexisting attractors of system (9.6) is associated with its own basin of attraction, which is the set of initial conditions that satisfy the conservation equation (9.8). The basin of attraction of each attractor is therefore the manifold determined by the particular value of 
the conserved quantity. However, these manifolds or slices in state space are dense, so that in each arbitrarily close neighborhood of each manifold there is another manifold leading to another attractor. That is, in each arbitrarily close neighborhood of an attractor there are points belonging to another manifold and, hence, to another basin of attraction. As a consequence, all attractors here are weak attractors in the Milnor sense [36].

Finally, another essential novelty in this work is the discovery of a new class of dynamical systems that manifests features of both dissipative and conservative systems. As described above, the system is dissipative because any set of initial conditions that satisfies the conservation relation, for a particular value of the conserved quantity, will relax to the associated attractor. For example, there are an infinite number of initial conditions that satisfy equation (9.8) for the value of $\mathrm{C}$ that gives rise to a period-1 orbit. We note that the limit cycle is stable only to perturbations that satisfy (9.8), while perturbations that do not satisfy (9.8) will move the system to another attractor. On the other hand, due to the existence of a conserved quantity, the dynamics becomes similar to the dynamics of Hamiltonian systems, where the marginally stable invariant set reached in the long-term limit depends on the value of the energy of the system. However, it is important to note that in contrast to

the Hamiltonian case, the coupled autocatalator system is dissipative, and the invariant sets approached as time goes to infinity are attractors.

\subsection{Nonidentical coupled systems}

Another issue that needs to be addressed is the chemical plausibility of the reactions. So far we have assumed that the two subsystems coupled together are identical. That is, they are described with the same set of parameters, and the corresponding reactions in both subsystems therefore have the same rate constants. This is a very strong assumption, since it is difficult to find identical systems in nature apart from such cases as optical isomers. Naturally occurring subsystems would usually have at least some small mismatch in the parameters, arising, for example, from different isotopes. The question therefore arises whether extreme multistability is a robust feature occurring when there is a small mismatch in the parameters. For the chemical systems considered here, a mismatch in the parameters can 
be interpreted as a mismatch in the rate constants for the reactions.

We now demonstrate that extreme multistability is sustained for small parameter mismatches. The mismatch values are obtained by considering the various dimensionless parameter groupings $(\kappa, \mu, \sigma$ and $\delta)$ and scaling each of them by 0 to $0.5 \%$ of their values accordingly to reflect the effect of the rate constants lumped into the parameter. First we note that the bifurcation diagrams shift with changing parameters in such a way that the period-doubling cascade occurs over a narrower range. Consequently, for rather large parameter mismatches, only period-1 and chaotic solutions survive, as higher periods disappear as the mismatch is increased. To demonstrate that extreme multistability is still possible, we have examined the long-term dynamics obtained with parameter mismatches ranging from $0.01 \%$ to $0.5 \%$, while closely examining $0.01 \%, 0.05 \%, 0.1 \%, 0.2 \%$, and $0.5 \%$ parameter mismatches. Figures 9.12 and 9.13 show the bifurcation sequences generated with $0.01 \%$ and $0.05 \%$ parameter mismatches, and Fig. 9.15 shows some time series and attractor plots derived from a $0.5 \%$ parameter mismatch. Our investigation indicates that the number of qualitatively different attractors is still infinite in the case of very small parameter mismatch. However, for larger parameter mismatches, say for $0.1 \%$ and higher, the number of qualitatively different attractors no longer tends to infinity because the period-doubling bifurcations are no longer resolved. Therefore, we conclude that extreme multistability is not dependent on the coupled systems being identical, but it is more likely to occur when systems are identical or are almost identical. 


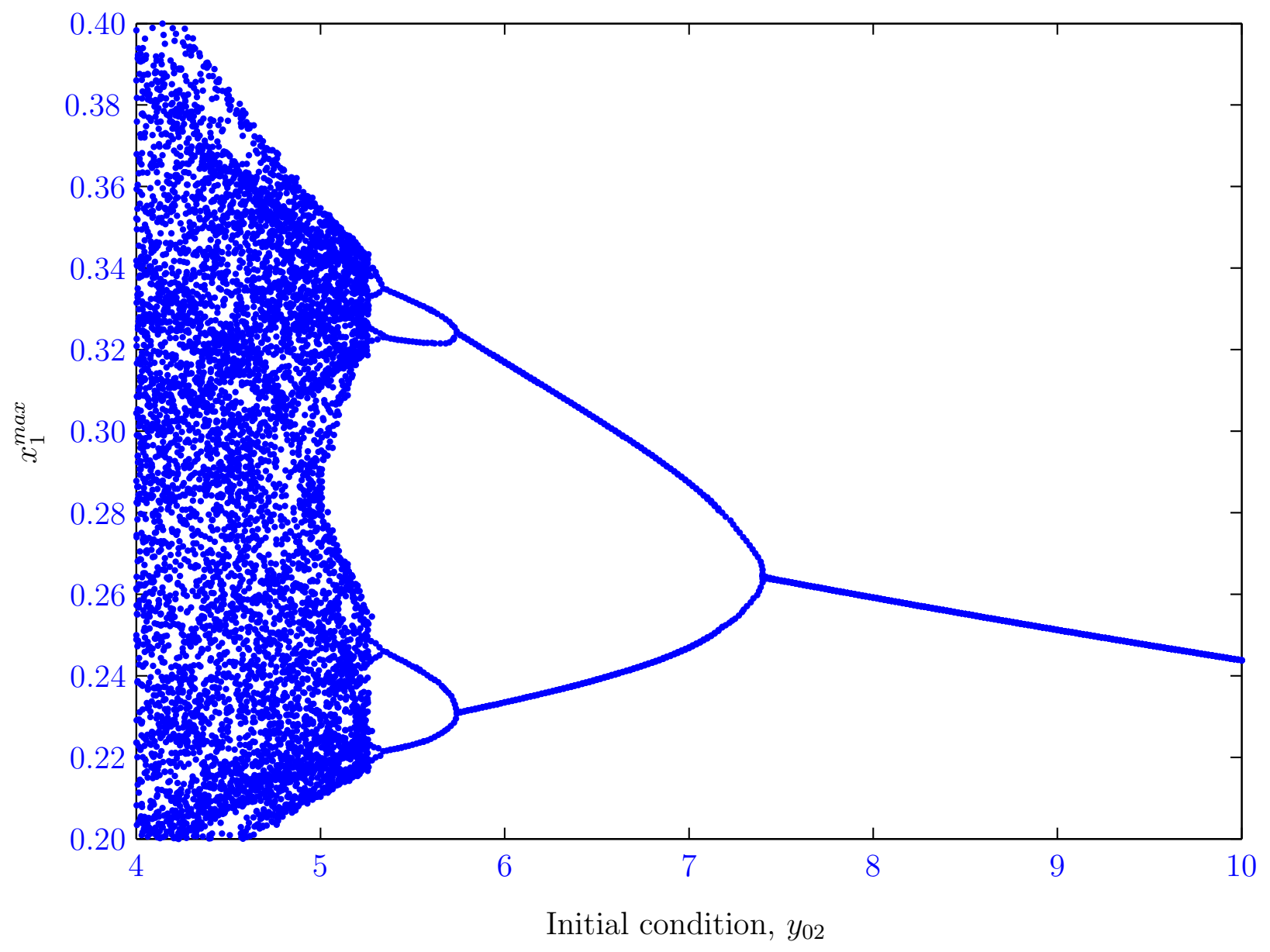

Figure 9.12. Long-term dynamics for two coupled autocatalator systems with $0.01 \%$ parameter mismatch vs. initial condition $y_{02}$. 


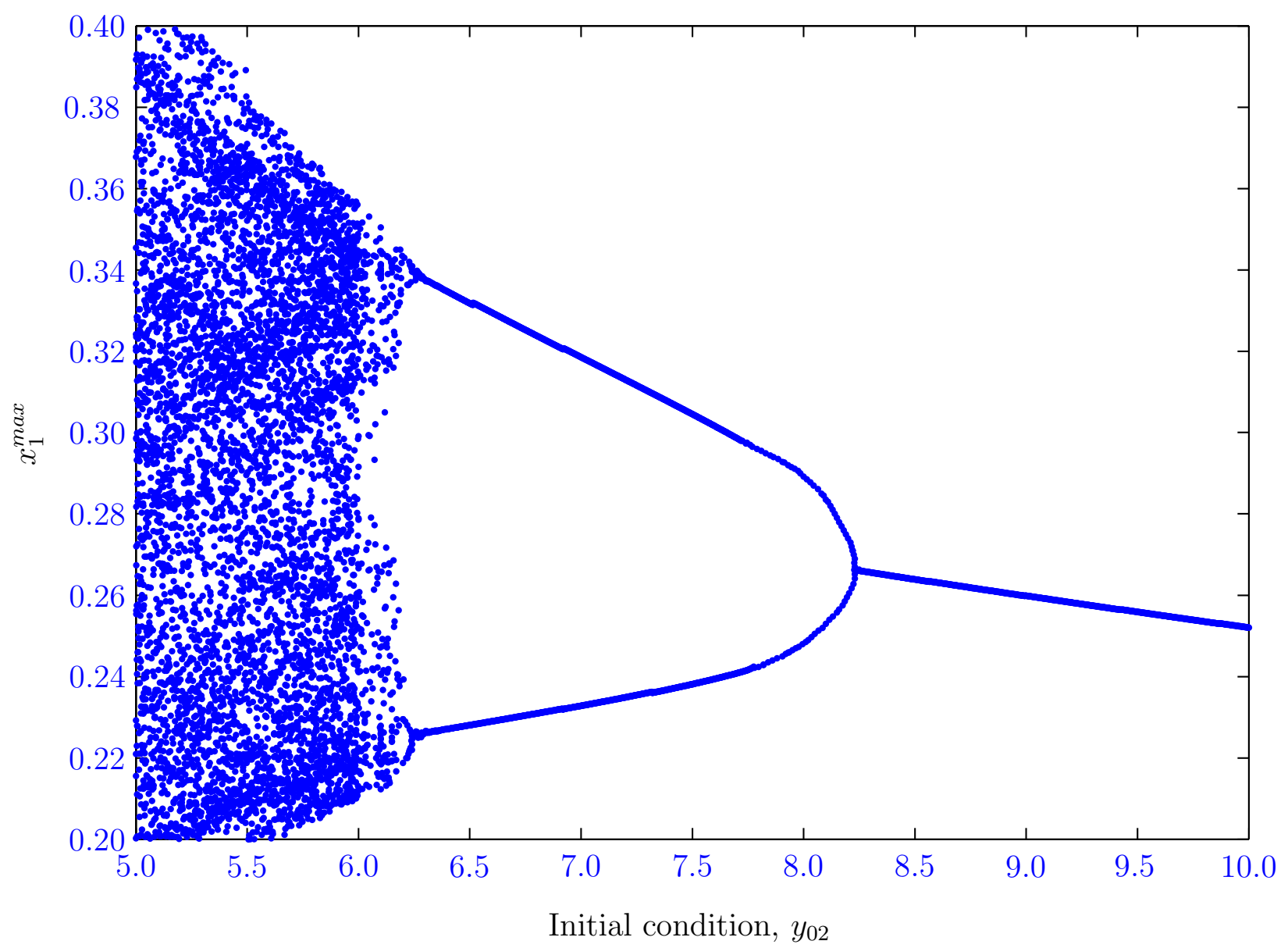

Figure 9.13. Long-term dynamics for two coupled autocatalator systems with $0.05 \%$ parameter mismatch vs. initial condition $y_{02}$.

Table 9.1 lists values of $y_{02}$ for period- 8 to period- 4 , period- 4 to period- 2 and period- 2 to period-1 transition for $0 \%, 0.01 \%$ and $0.05 \%$ parameter mismatches.

Table 9.1. Values of $y_{02}$ for period-doubling transitions with increasing mismatches.

\begin{tabular}{||c|c|c|c||}
\hline \hline Transition & $0 \%$ mismatch & $0.01 \%$ mismatch & $0.05 \%$ mismatch \\
\hline Period-8 to period-4 & 5.165 & 5.34 & 6.2 \\
\hline Period-4 to period-2 & 5.51 & 5.735 & 6.23 \\
\hline Period-2 to period-1 & 7.14 & 7.4 & 8.23 \\
\hline \hline
\end{tabular}




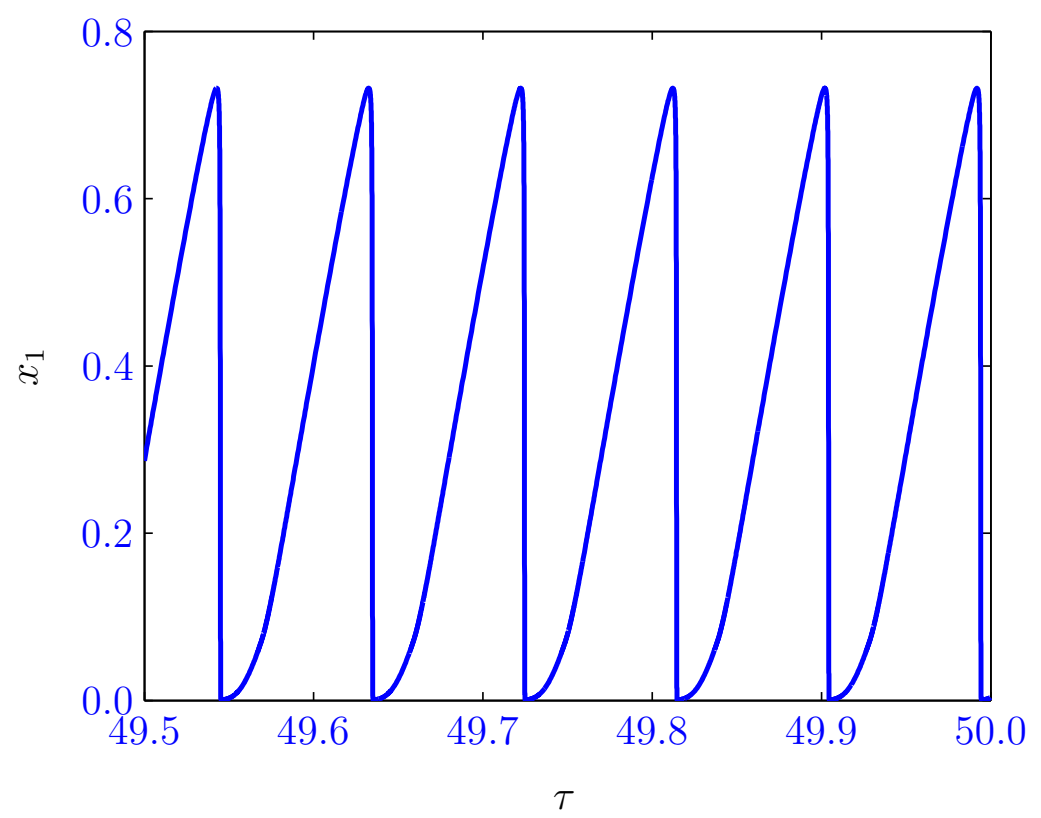

9.14.1. $y_{02}=25$, period-1 regime.

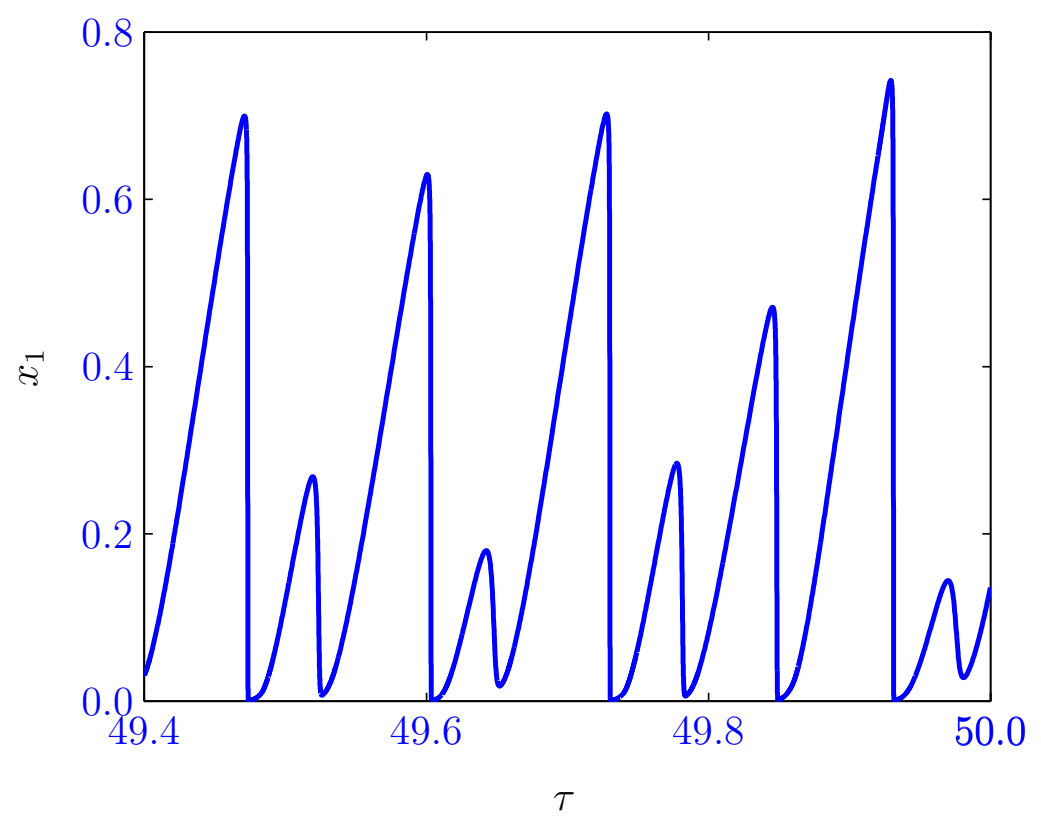

9.14.2. $y_{02}=20$, chaotic regime.

Figure 9.14. Plots for a $0.5 \%$ parameter mismatch. Only the chaotic and period-1 regimes are prominent. Squeezing of the bifurcation diagram renders it difficult to distinguish the period-doubling sequence from chaos especially for periods higher than $2 T$. 


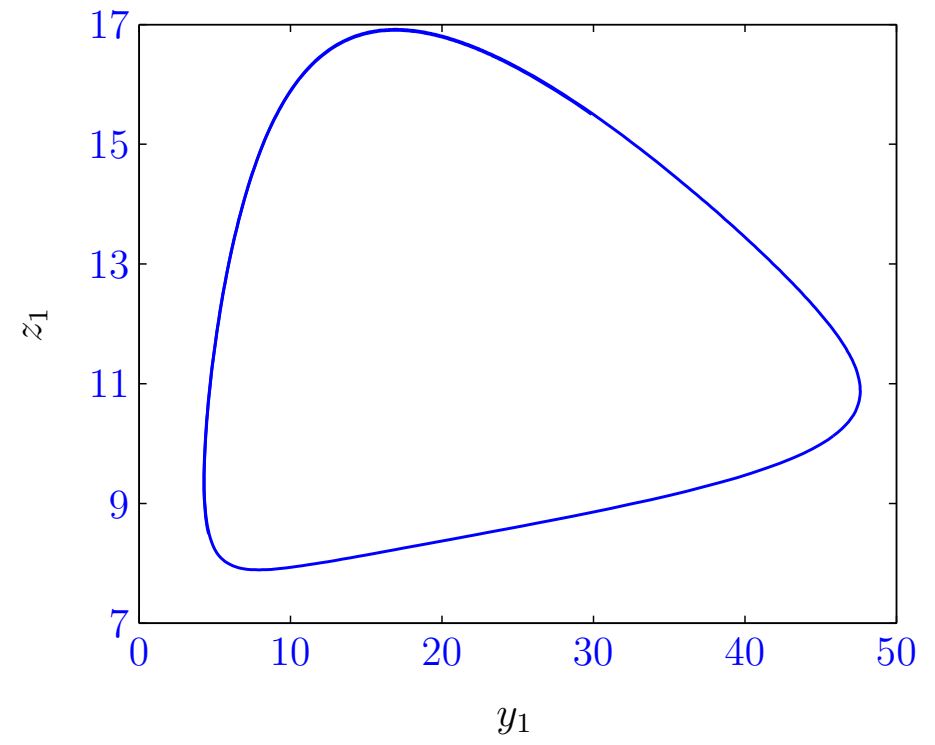

9.15.1. $y_{02}=25$ : Limit cycle.

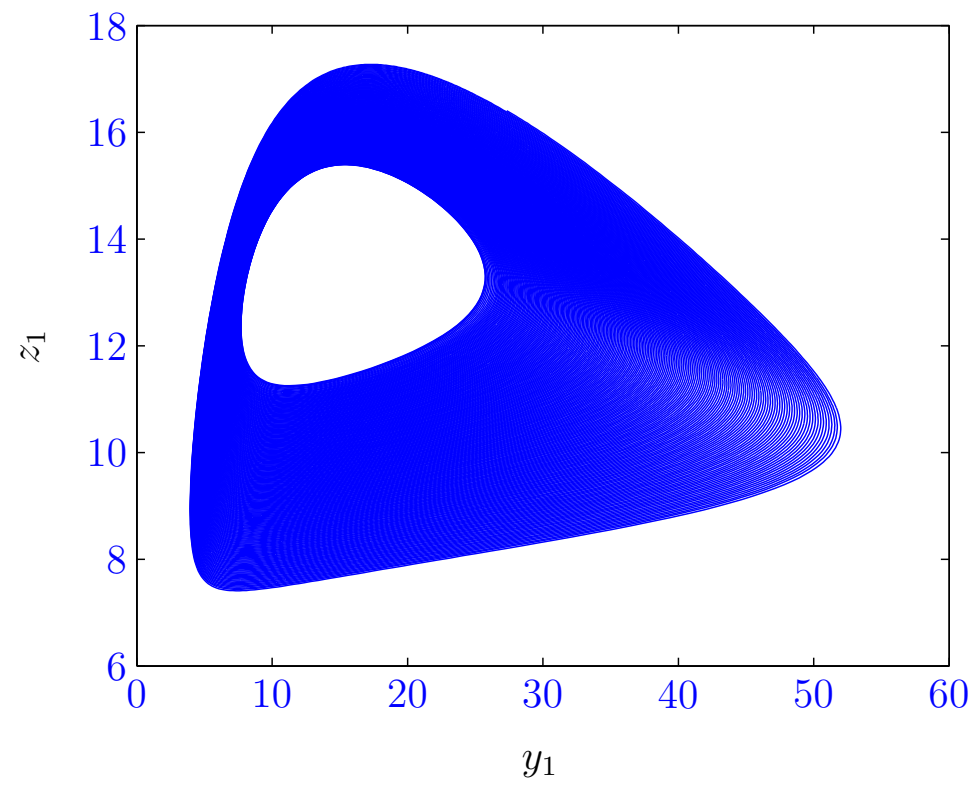

9.15.2. $y_{02}=20$ : Chaotic behavior.

Figure 9.15. Plots for a $0.5 \%$ parameter mismatch. Only the chaotic and period-1 regimes are prominent. Squeezing of the bifurcation diagram renders it difficult to distinguish the period-doubling sequence from chaos especially for periods higher than $2 T$. 
Fig. 9.16 below illustrates the narrowing of the reverse period-doubling sequence. Here, we plot selected values of the initial condition $y_{02}$ within the period-1, period-2, and chaotic regimes against the corresponding parameter mismatch.

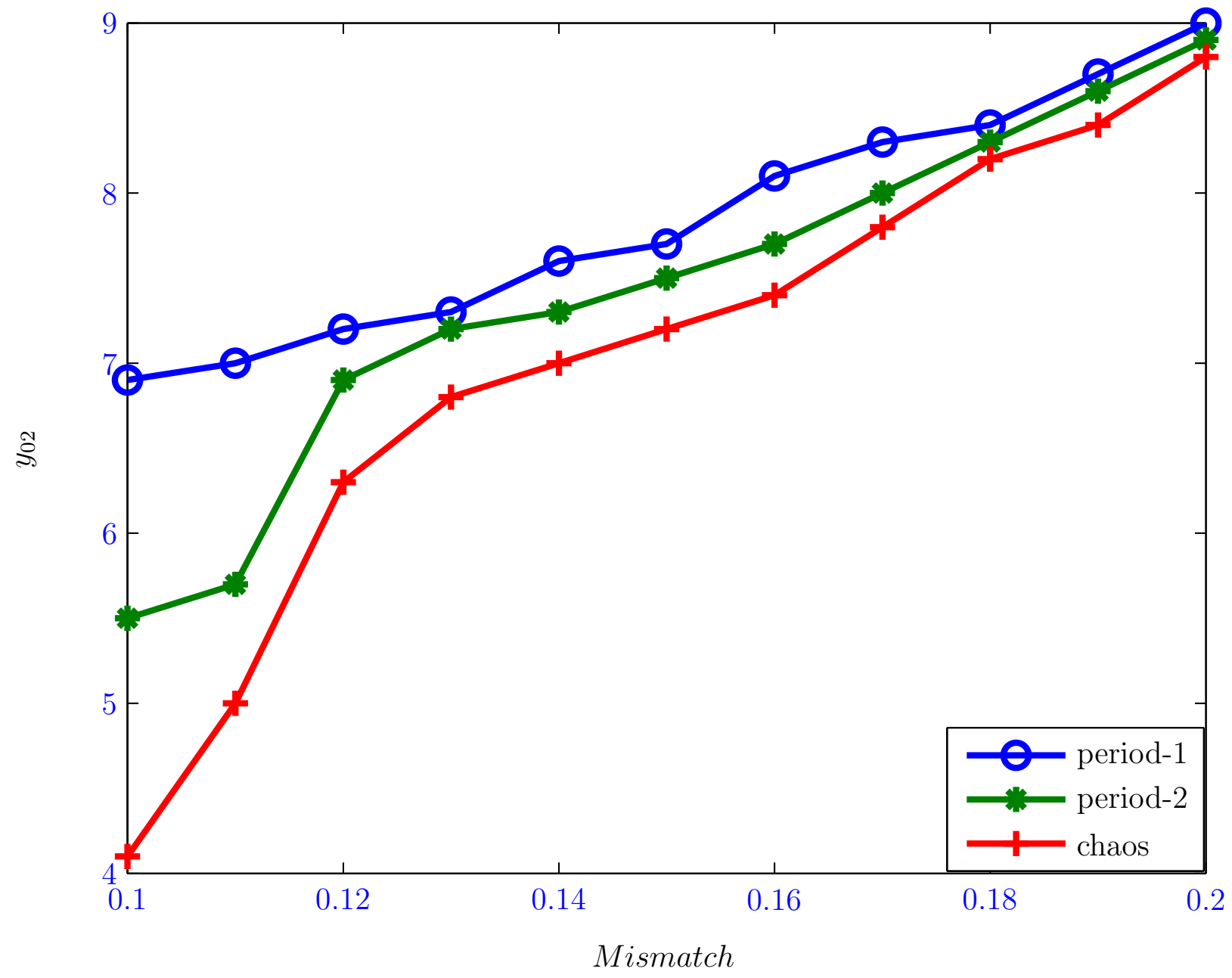

Figure 9.16. A plot of values of $y_{02}$ against corresponding parameter mismatches within the period-1, period-2 and chaotic regimes illustrating the narrowing of the reverse period-doubling sequence as the parameter mismatch is increased. 


\subsection{Conclusion}

Our investigation shows that depending on the choice of initial conditions, an infinite number of different attractors can be attained, including fixed points, periodic orbits and chaotic orbits. This phenomenon is not exhibited with a single uncoupled three-variable autocatalator model. The phenomenon of extreme multistability is closely linked to the emergence of a conserved quantity that is determined by the initial conditions. This conserved quantity determines a complex manifold in state space in which the dynamics occur. An infinite number of attractors arise, since this conserved quantity can serve as a bifurcation parameter in a reduced system that gives rise to chaos in an infinite period-doubling cascade. Hence, the system contains orbits of all periods. Our investigations also indicate that extreme multistability has a close association with the emergence of generalized synchronization between the two autocatalator subsystems coupled together.

We have demonstrated that the phenomenon of extreme multistability emerges in a coupled six-variable system obtained by coupling two identical or slightly different three-variable systems. The fact that we are able to vary corresponding parameters of the three-variable systems by at least small amounts indicates extreme multistability is robust. We shall show in the next chapter, how this coupling scheme is not unique. 


\section{References}

[1] B. Goswami and A. Pisarchik, "Controlling Multistability by Small Perturbations," International Journal of Bifurcation and Chaos 18, 1645-1673 (2008).

Online Version 235

[2] U. Feudel, "Complex Dynamics in Multistable Systems," International Journal of Bifurcation and Chaos 18 (6), 1607-1626 (2008).

Online Version 235

[3] V. Balzani, A. Credi, and M. Venturi, Molecular Devices and Machines (Wiley-VCH Verlag GmbH and Co. KGaA, 2008), second edition. 235

[4] F. Arecchi, R. Meucci, G. Puccioni, and J. Tredicce, "Experimental Evidence of Subharmonic Turbulence in a Q-switched Gas Laser," Physical Review Letters 49, 1217-1220 (1982).

Online Version 235

[5] C. Masoller, "Noise-induced Resonance in Delayed Feedback Systems," Physical Review Letters 88, 034102-1-4 (2002).

Online Version

[6] J. Kastrup, H. Grahn, K. Ploog, F. Prengel, A. Wacker, and E. Schöll, "Multistability of the Current-voltage Characteristics in Doped GaAs-AlAs Superlattices," Applied Physics Letters 65, 1808-1810 (1994).

Online Version 
[7] G. Schwarz, C. Lehmann, and E. Schöll, "Self-organized Symmetry-breaking Current Filamentation and Multistability in Corbino Disks," Physical Review B 61, 1019410200 (2000).

Online Version 235

[8] N. Ganapathisubramanian and K. Showalter, "Bistability, Mushrooms, and Isolas," Journal of Chemical Physics 80, 4177-4184 (1984).

Online Version 235

[9] P. Marmillot, M. Kaufman, and J.-F. Hervagault, "Multiple Steady States and Dissipative Structures in a Circular and Linear Array of Three Cells: Numerical and Experimental Approaches," Journal of Chemical Physics 95, 1206-1214 (1991).

Online Version 235

[10] J. Foss, A. Longtin, B. Mensour, and J. Milton, "Multistability and Delayed Recurrent Loops," Physical Review Letters 76, 708-711 (1996).

Online Version 235

[11] J. Huisman and F. Weissing, "Fundamental Unpredictability in Multispecies Competition," The American Naturalist 157, 488-494 (2001).

Online Version 235

[12] U. Feudel, C. Grebogi, B. R. Hunt, and J. A. Yorke, "A Map with More than 100 Coexisting Low-period Periodic Attractors," Physical Review E 54, 71-81 (1996). Online Version 235

[13] B. Goswami, "Multiple Attractors in the Self-similar Bifurcation-structure," Rivista del Nuovo Cimento 23, 1-115 (2005).

Online Version 235

[14] K. Kaneko, Theory and Applications of Coupled Map Lattices (Wiley, New York, 1993). 235

[15] A. Pikovsky, O. Popovych, and Y. Maistrenko, "Resolving Clusters in Chaotic Ensembles of Globally Coupled Identical Oscillators," Physical Review Letters 87, 044102-1-4 
(2001).

Online Version

[16] V. Astakhov, A. Shabunin, W. Uhm, and S. Kim, "Multistabiliaty Formation and Synchronization Loss in Coupled Hénon Maps: Two Sides of the Single Bifurcational Mechanism," Physical Review E 63, 56212-1-8 (2001).

Online Version 235

[17] A. Balanov, N. Janson, and E. Schöll, "Delayed Feedback Control of Chaos: Bifurcation Analysis," Physical Review E 71, 016222-1-9 (2005).

Online Version 235

[18] C. Masoller and N. Abraham, "Stability and Dynamical Properties of the Coexisting Attractors of an External-cavity," Physical Review A 57, 1313-1322 (1998).

Online Version

[19] B. Krauskopf, "Semiconductor Lasers as Dynamical Systems," in F. Dumortier, D. Roose, and A. Vanderbauwhede, eds., "Trends in Dynamical Systems," 33-45 (Handelingen van de Koninklijke Vlaamse Academie van Belgie, 2006). 235

[20] C. Sparrow, The Lorenz Equations: Bifurcations, Chaos, and Strange Attractors (Springer, New York, 1982). 236

[21] B. Peng, S. K. Scott, and K. Showalter, "Period Doubling and Chaos in a Three-variable Autocatalator," The Journal of Physical Chemistry 94, 5243-5246 (1990). Online Version

[22] A. Hastings and T. Powell, "Chaos in a Three-species Food Chain," Ecology 72 (3), 896-903 (1991).

Online Version

[23] J. M. González-Miranda, "Complex Bifurcations Structures in the Hindmarsh-Rose Neuron Model," International Journal of Bifurcation and Chaos 17 (9), 3071-3083 (2007).

Online Version 
[24] H. R. Dullin and S. Schmidt, "Extended Phase Diagram of the Lorenz Model," International Journal of Bifurcation and Chaos 17 (9), 3013-3033 (2007).

Online Version 236

[25] J. Guckenheimer and P. Holmes, Nonlinear Oscillations, Dynamical systems, and Bifurcations of Vector Fields (Springer Verlag, Berlin, Heidelberg, New York, 1986). 236

[26] E. Ott, Chaos in Dynamical Systems (Cambridge University Press, Cambridge, 1992). 236

[27] H. Sun, S. Scott, and K. Showalter, "Uncertain Destination Dynamics," Physical Review E 60, 3876-3880 (1999).

Online Version 236, 248

[28] S. K. Scott, Oscillations, Waves, and Chaos in Chemical Kinetics (Oxford University Press Inc., New York, 1994). 237

[29] P. Gray and S. K. Scott, Chemical Oscillations and Instabilities. Non-linear Chemical Kinetics (Oxford University Press, New York, 1994).

[30] S. K. Scott, Chemical Chaos (Oxford University Press, New York, 1991). 237

[31] M. Orban and I. Epstein, "Systematic Desgin of Chemical Oscillators .13. Complex Periodic and Aperiodic Oscillation in the Chlorite Thiosulfate Reaction," Journal of Chemical Physics 86, 3907-3910 (1982).

Online Version 237

[32] I. Nagypal and I. Epstein, "Systematic Design of Chemical Oscillators. 37. Fluctuations and Stirring Rate Effects in the Chlorite Thiosulfate Reaction," Journal of Chemical Physics 90, 6285-6292 (1986).

Online Version 237

[33] J. Wang, H. Sun, S. K. Scott, and K. Showalter, "Uncertain Dynamics in Nonlinear Chemical Reactions," Physical Chemistry Chemical Physics 5, 5444-5447 (2003). Online Version 237 
[34] E. Hairer and G. Wanner, Solving Ordinary Differential Equations II: Stiff and Differential-Algebraic Problems. (Springer-Verlag, 1996), 2nd edition. 243

[35] A. Pikovsky, M. Rosenblum, and J. Kurths, Synchronisation (Cambridge University Press, Cambridge, 2000). 260

[36] J. Milnor, "On the Concept of Attractor," Communications in Mathematical Physics 99, 177-195 (1985).

Online Version 261 
$\overline{\text { Chapter }} 10$

\section{Robustness of Extreme Multistability}

\subsection{Introduction}

In Chapter 9, we demonstrated that extreme multistability emerges in a coupled sixvariable system obtained by coupling two three-variable autocatalator systems in a special way. To show that the coupling technique is not unique, we examine three more coupling schemes, each of which gives rise to extreme multistability. We show that, in contrast to system (9.6), the conserved quantities associated with extreme multistability for the first two coupling schemes are no longer contained in the equations but arise in a non-trivial way as a result of the dynamics in the long-term limit. The conserved quantities of these two models are similar to that in the system studied by Sun et al. [1]. The conserved quantity for the last coupling scheme is similar to that of system (9.6).

This Chapter is organized as follows: In Section 10.2, we use a different scheme to couple two three-variable autocatalator systems, and show that the coupled system exhibits extreme multistability. We also show that the dynamics of the coupled system can be studied through a reduced system that differs from the original three-variable system only by a constant, the conserved quantity. We examine the dynamical behavior associated with two more coupling schemes in Sections 10.3 and 10.4, respectively, and conclude the chapter in Section 10.5. 


\subsection{Second Coupling Scheme}

\subsubsection{The Model}

The second model involves the conversion of a chemical precursor $A$ to a final product $E$ through six intermediate species $X_{i}, Y_{i}$ and $Z_{i}(i=1,2)$. This occurs in two subsystems with an intermediate species from each subsystem involved in the other subsystem, comparable to model $\left(R_{9.11}\right)-\left(R_{9.22}\right)$. In the course of the conversion, two intermediate species $C$ and $D$ are formed that do not take part in the dynamics. The final product $E$ does not participate in any reaction. We therefore neglect the time evolution of $C, D$ and $E$ in deriving system (10.1). Let $k_{i}(i=0,1,2,3,4,5), k_{4}^{\prime}$ and $k_{4}^{\prime \prime}$ be rate constants. Then the chemical representation of our model is as follows:

$$
\begin{array}{rr}
A \stackrel{k_{0}}{\longrightarrow} X_{1}, & \left(R_{10.23}\right) \\
A+Z_{1} \stackrel{k_{1}}{\longrightarrow} X_{1}+Z_{1}, & \left(R_{10.24}\right) \\
X_{1} \stackrel{k_{2}}{\longrightarrow} Y_{1}, & \left(R_{10.25}\right) \\
X_{1}+2 Y_{1} \stackrel{k_{3}}{\longrightarrow} 3 Y_{1}, & \left(R_{10.26}\right) \\
B+Y_{1} \stackrel{k_{4}}{\longrightarrow} C, & \left(R_{10.27}\right) \\
B+Y_{2} \stackrel{k_{4}^{\prime}}{\longrightarrow} Z_{1}+Z_{2}+Y_{2}, & \left(R_{10.28}\right) \\
Z_{1} \stackrel{k_{5}}{\longrightarrow} E, & \left(R_{10.30}\right) \\
A^{A} \stackrel{k_{0}}{\longrightarrow} X_{2}, & \left(R_{10.31}\right) \\
A+Z_{2} \stackrel{k_{1}}{\longrightarrow} X_{2}+Z_{2}, & \left(R_{10.32}\right) \\
X_{2} \stackrel{k_{2}}{\longrightarrow} Y_{2}, & \left(R_{10.33}\right) \\
X_{2}+2 Y_{1} \stackrel{k_{3}}{\longrightarrow} Y_{2}+2 Y_{1}, & \left(R_{10.34}\right) \\
C+Y_{2} \stackrel{k_{4}^{\prime \prime}}{\longrightarrow} D, & \left(R_{10.35}\right) \\
Z_{2} \stackrel{k_{5}}{\longrightarrow} E . &
\end{array}
$$

We keep the concentrations of $A$ and $B$ constant at $A_{0}$ and $B_{0}$. Additionally, we assume that $k_{4}^{\prime \prime} \gg k_{4}^{\prime} B_{0}=k_{4}$. Reaction $\left(R_{10.26}\right)$ describes an autocatalytic process in which $Y_{1}$ catalyzes its own production. Since $Y_{1}$ is produced through autocatalysis in reaction $\left(R_{10.26}\right)$ 
and is used to produce $Y_{2}$ in reaction $\left(R_{10.33}\right)$, we can also imagine an indirect autocatalysis occurring in the fourth equation of the second subsystem $\left(R_{10.33}\right)$. In contrast to each of the individual three-variable systems, each of which has simple feedback loops, there are more complex feedback loops in the coupled system. For example, $Z_{1}$ and $Z_{2}$ are produced in reaction $\left(R_{10.28}\right)$ and fed back into the system through reactions $\left(R_{10.24}\right)$ and $\left(R_{10.31}\right)$, respectively, to catalyze the production of $X_{1}$ from $A$, and the production of $X_{2}$ from $A$ respectively. Note that while $B$ is constant with the value $B_{0}, C$ is generated in reaction $\left(R_{10.27}\right)$ of the first subsystem and rapidly consumed in reaction $\left(R_{10.34}\right)$ of the second subsystem. Therefore, the rate of reaction $\left(R_{10.34}\right)$ is determined by the rate of reaction $\left(R_{10.27}\right)$. Consequently, we use $k_{4} Y_{1}=k_{4}^{\prime} B_{0} Y_{1}$ instead of $k_{4}^{\prime \prime} C Y_{2}$ in the corresponding mathematical model. This contributes to the coupling of the two subsystems. The first subsystem is also coupled to the second subsystem through $Y_{2}$ in reaction $\left(R_{10.28}\right)$, while the second subsystem is also coupled to the first system through $Y_{1}$ in reaction $\left(R_{10.33}\right)$.

Let $A_{0},\left[X_{1}\right],\left[Y_{1}\right],\left[Z_{1}\right],\left[X_{2}\right],\left[Y_{2}\right]$ and $\left[Z_{2}\right]$ be the concentrations of $A, X_{1}, Y_{1}, Z_{1}, X_{2}, Y_{2}$ and $Z_{2}$ respectively. Then using the law of mass action we write the following system of first-order ordinary differential equations for the above model:

$$
\begin{aligned}
{\left[\dot{X}_{1}\right] } & =k_{0} A_{0}+k_{1} A_{0}\left[Z_{1}\right]-k_{2}\left[X_{1}\right]-k_{3}\left[X_{1}\right]\left[Y_{1}\right]^{2}, \\
{\left[\dot{Y}_{1}\right] } & =k_{2}\left[X_{1}\right]+k_{3}\left[X_{1}\right]\left[Y_{1}\right]^{2}-k_{4}\left[Y_{1}\right], \\
{\left[\dot{Z}_{1}\right] } & =k_{4}\left[Y_{2}\right]-k_{5}\left[Z_{1}\right], \\
{\left[\dot{X}_{2}\right] } & =k_{0} A_{0}+k_{1} A_{0}\left[Z_{2}\right]-k_{2}\left[X_{2}\right]-k_{3}\left[X_{2}\right]\left[Y_{1}\right]^{2}, \\
{\left[\dot{Y}_{2}\right] } & =k_{2}\left[X_{2}\right]+k_{3}\left[X_{2}\right]\left[Y_{1}\right]^{2}-k_{4}\left[Y_{1}\right], \\
{\left[\dot{Z}_{2}\right] } & =k_{4}\left[Y_{2}\right]-k_{5}\left[Z_{2}\right] .
\end{aligned}
$$

Using a scaling procedure similar to that of Chapter 9, we obtain the following dimensionless variable and parameter groupings:

$$
\begin{aligned}
\tau & =k_{2} t, x_{1}=\frac{\left(k_{1} k_{3}\right)^{\frac{1}{2}}}{k_{4}}\left[X_{1}\right], y_{1}=\left(\frac{k_{3}}{k_{2}}\right)^{\frac{1}{2}}\left[Y_{1}\right], z_{1}=\left(\frac{k_{5}}{k_{4}}\right)\left(\frac{k_{3}}{k_{2}}\right)^{\frac{1}{2}}\left[Z_{1}\right], \\
x_{2} & =\frac{\left(k_{1} k_{3}\right)^{\frac{1}{2}}}{k_{4}}\left[X_{2}\right], y_{2}=\left(\frac{k_{3}}{k_{2}}\right)^{\frac{1}{2}}\left[Y_{2}\right], z_{2}=\left(\frac{k_{5}}{k_{4}}\right)\left(\frac{k_{3}}{k_{2}}\right)^{\frac{1}{2}}\left[Z_{2}\right], \\
\mu & =\frac{k_{1} A_{0}}{k_{5}}, \kappa=\left(\frac{k_{0} k_{5}}{k_{1} k_{4}}\right)\left(\frac{k_{3}}{k_{2}}\right)^{\frac{1}{2}}, \sigma=\frac{k_{2}}{k_{4}} \text { and } \delta=\frac{k_{2}}{k_{5}},
\end{aligned}
$$


which reduce system (10.1) to

$$
\begin{aligned}
\dot{x_{1}} & =\mu\left(\kappa+z_{1}\right)-x_{1}\left(1+y_{1}^{2}\right), \\
\sigma \dot{y_{1}} & =x_{1}\left(1+y_{1}^{2}\right)-y_{1}, \\
\delta \dot{z_{1}} & =y_{2}-z_{1}, \\
\dot{x_{2}} & =\mu\left(\kappa+z_{2}\right)-x_{2}\left(1+y_{1}^{2}\right), \\
\sigma \dot{y_{2}} & =x_{2}\left(1+y_{1}^{2}\right)-y_{1}, \\
\delta \dot{z_{2}} & =y_{2}-z_{2} .
\end{aligned}
$$

\subsubsection{Model Analysis}

System (10.2) has a nonnegative equilibrium solution,

$$
\left(x_{1}^{*}, y_{1}^{*}, z_{1}^{*}, x_{2}^{*}, y_{2}^{*}, z_{2}^{*}\right)=\left(\frac{y_{1}^{*}}{y_{1}^{* 2}+1}, y_{1}^{*}, \frac{y_{1}^{*}-\kappa \mu}{\mu}, \frac{y_{1}^{*}}{y_{1}^{* 2}+1}, \frac{y_{1}^{*}-\kappa \mu}{\mu}, \frac{y_{1}^{*}-\kappa \mu}{\mu}\right),
$$

for $y_{1}^{*} \geq \kappa \mu, \kappa>0$ and $\mu>0$. Notice that when $y_{1}^{*}>\kappa \mu$, this equilibrium solution is positive and when $y_{1}^{*}=\kappa \mu$, it reduces to

$$
\left(x_{1}^{*}, y_{1}^{*}, z_{1}^{*}, x_{2}^{*}, y_{2}^{*}, z_{2}^{*}\right)=\left(\frac{\kappa \mu}{1+(\kappa \mu)^{2}}, y_{1}^{*}, 0, \frac{\kappa \mu}{1+(\kappa \mu)^{2}}, 0,0\right) .
$$

We use numerical simulations to investigate the dynamical behavior of the system. Throughout this chapter, we set $\kappa=65, \sigma=5 \times 10^{-3}$ and $\delta=2 \times 10^{-2}$. In Section 10.2.2.1, we allow $\mu$ to vary in the interval $[0,1)$, and in Section 10.2 .2 .2 , we set $\mu=0.145$.

\subsubsection{Dynamical Behavior for Varying $\mu$}

We study the coupled system with $\mu$ serving as a bifurcation parameter. Using the initial conditions $\left(x_{01}, y_{01}, z_{01}, x_{02}, y_{02}, z_{02}\right)=(0.5,0.9898,0.9896,0.5,0.9898,0.9896)$, which is the steady state to which the system relaxes with initial conditions $\left(x_{01}, y_{01}, z_{01}, x_{02}, y_{02}, z_{02}\right)$ $=(0.01,0.1,0.1,0.0,0.0,0.0)$, we integrate system $(10.2)$ for different values of $\mu$, retaining only the last tenth of the total number of time steps in order to eliminate any transients. To ensure that this represents the asymptotic behavior of the system, we verified that the behavior of our solutions for the last quarter, fifth and tenth of the total number of time 
steps was the same. In each case, we found the behavior to be asymptotic. The qualitative behavior of the system for different values of $\mu$ is identical to that of the three-variable system. As $\mu$ is increased, the solutions switch from stable to chaotic through a perioddoubling cascade. This is followed by period halving, which takes the system back to stable steady state behavior. For example, for $0 \leq \mu \leq 0.015$, we have steady state solutions, which switch to period-1 through a Hopf bifurcation at $\mu=0.016$. As $\mu$ is further increased, there is a switch from period-1 to period- 2 at $\mu=0.143$, a switch from period-2 to period-4 at $\mu=0.1527$, a switch from period- 4 to period- 8 at $\mu=0.1532$ and chaos at $\mu=0.154$. As $\mu$ is increased beyond the chaotic region, period halving occurs, and the system finally returns to stable a steady state through a second Hopf bifurcation at $\mu=0.175$. A bifurcation diagram for the system showing different periodic and chaotic behaviors is presented in Fig. 10.1. 


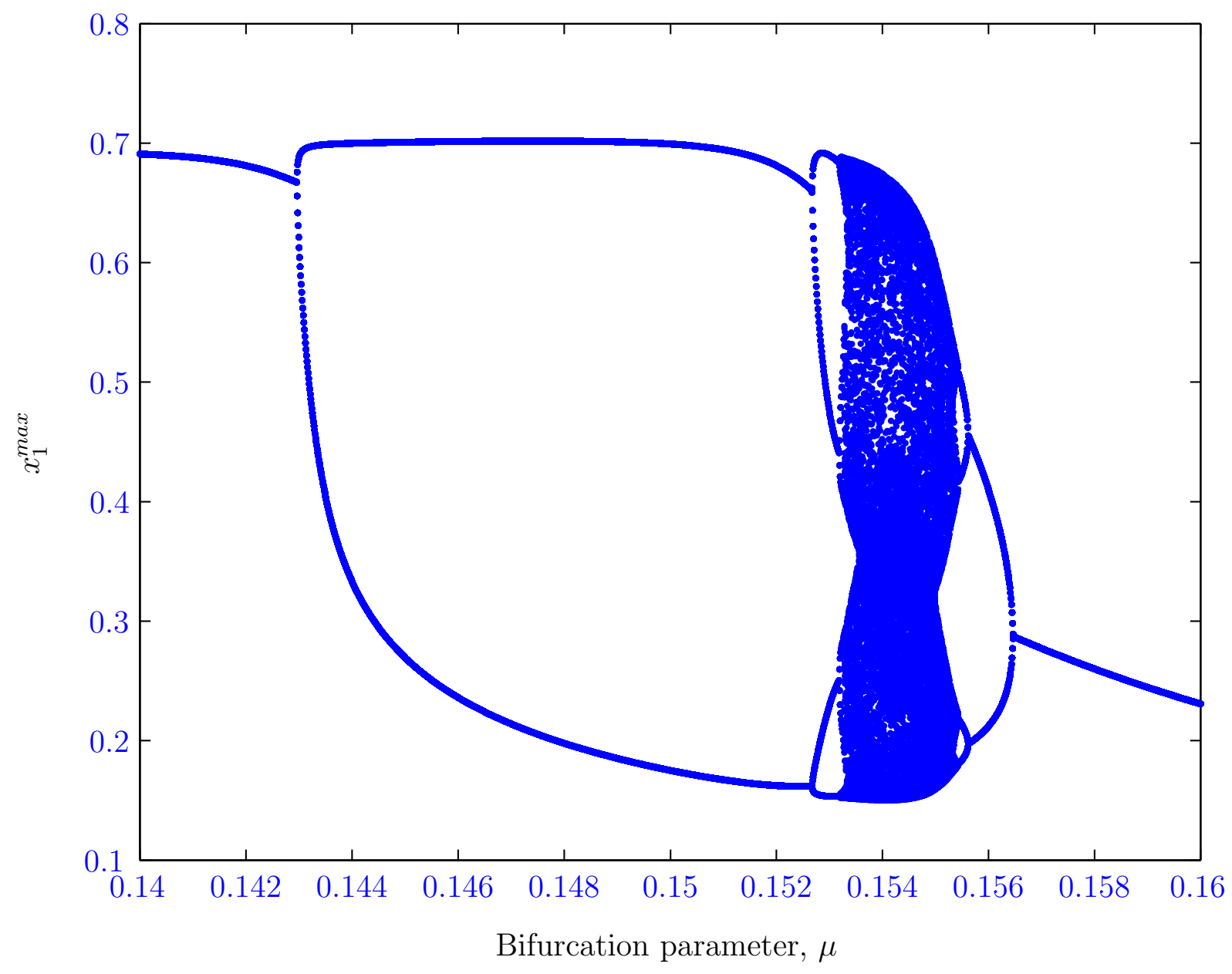

Figure 10.1. Long-term dynamics of the coupled six-variable autocatalator model depicting chaotic behavior, period-doubling and reverse period-doubling sequences. Here, we plot the amplitude of $x_{1}$ against the bifurcation parameter $\mu$. As $\mu$ is increased beyond the region where chaotic behavior was first observed, windows of parameter values where periodic solutions exist can be identified. Hence, an aperiodic parameter regime is sandwiched between periodic parameter regimes. 


\subsubsection{Illustration of Extreme Multistability}

To explore the phenomenon of extreme multistability, we set $\left(x_{01}, y_{01}, z_{01}, x_{02}, y_{02}, z_{02}\right)$ $=\left(0.01,0.1,0.1,0, y_{02}, 0\right)$, where $y_{02}$ is allowed to vary from 0 to 10 . We also set $\mu=0.145$, which corresponds to a solution of period $2 T$ for a single uncoupled three-variable autocatalator, and integrate the system for different values of $y_{02}$. As $y_{02}$ is gradually increased from 0 through 10, we observe stable steady state behavior and a transition to chaos through perioddoubling. As $y_{02}$ is increased beyond the chaotic region, a reverse period doubling sequence that takes the system back to stable steady state behavior occurs. For example, solutions switch from period- 1 to period- 2 when $y_{02}=0.955$, period- 2 to period- 4 when $y_{02}=6.525$, period- 4 to period- 8 when $y_{02}=6.835$, etc. Hence, this coupled system also exhibits the phenomenon of extreme multistability as illustrated in Fig. 10.2. Corresponding time series and phase plots are shown on Figs. 10.3 and 10.4 respectively. 


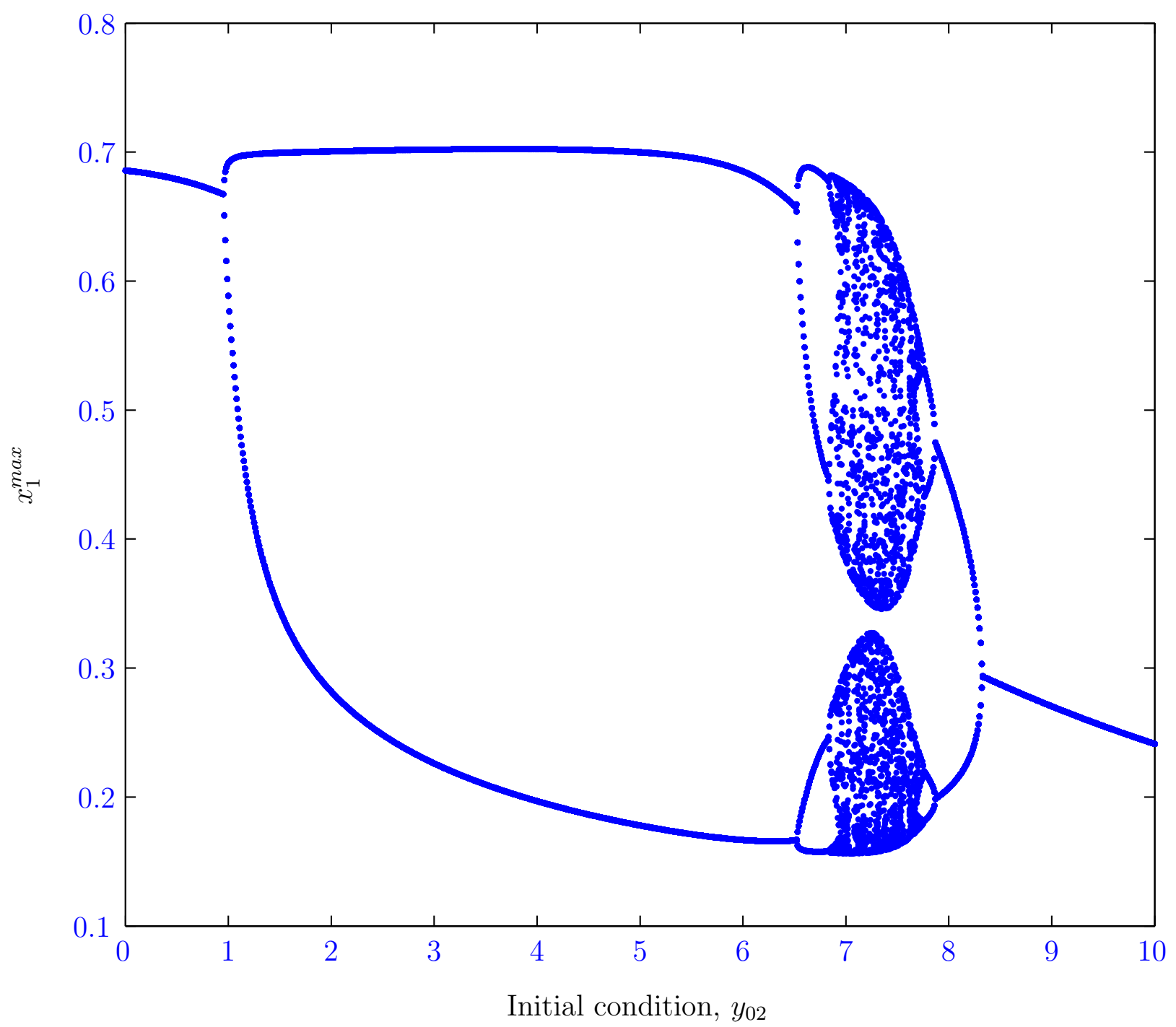

Figure 10.2. Long-term dynamics of the coupled six-variable autocatalator model depicting chaotic behavior as well as period doubling phenomena. The amplitude of $x_{1}$ is plotted on the ordinate axis while the initial condition $y_{02}$ is plotted on the abscissa. A period doubling sequence is identified for values of $y_{02}$ prior to the chaotic region. For values of $y_{02}$ beyond the region where chaotic behavior is observed, periodic solutions reappear. Hence, an aperiodic solution regime is sandwiched between two periodic solution regimes. 


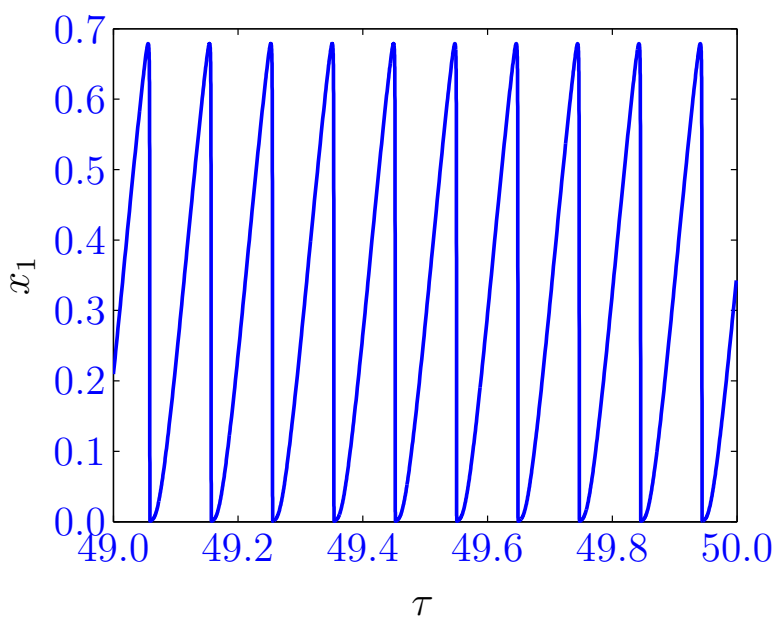

10.3.1. $y_{02}=0.5$ : Period -1

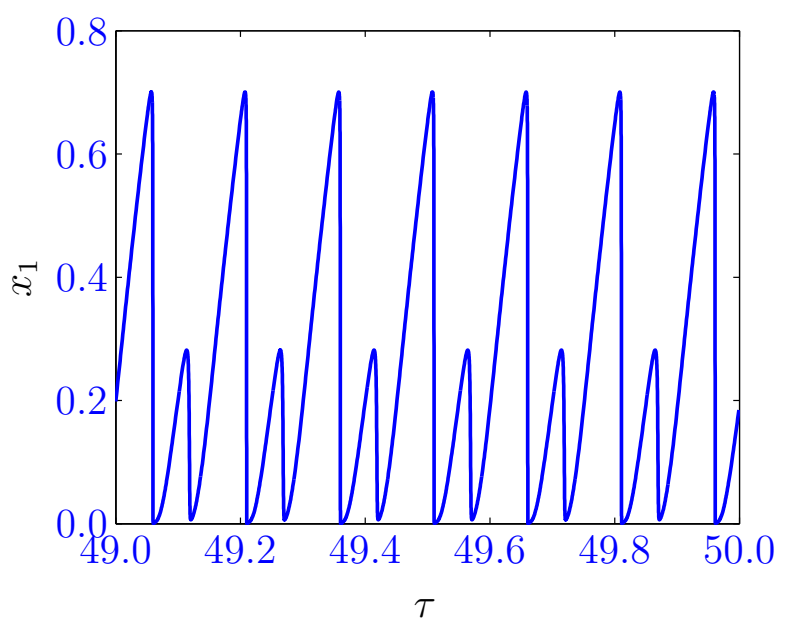

10.3.2. $y_{02}=2.0$ : Period -2

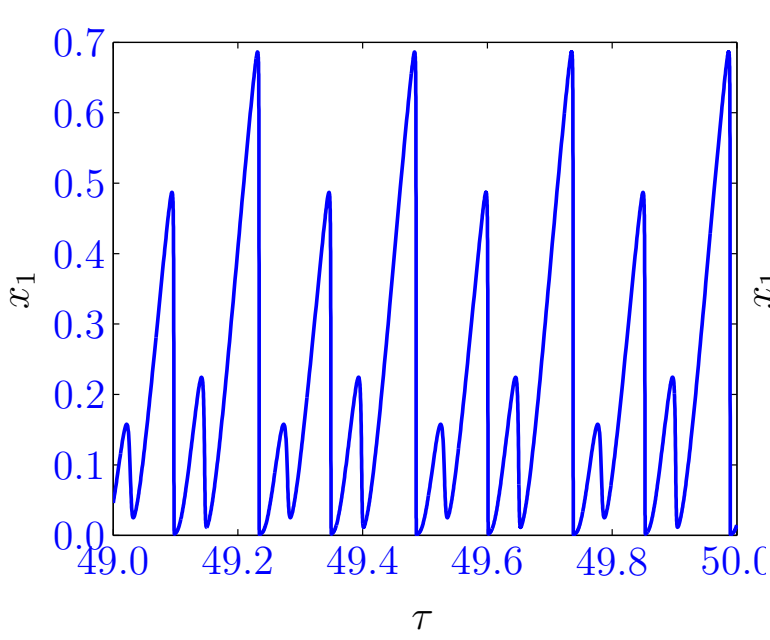

10.3.3. $y_{02}=6.7$ : Period- 4

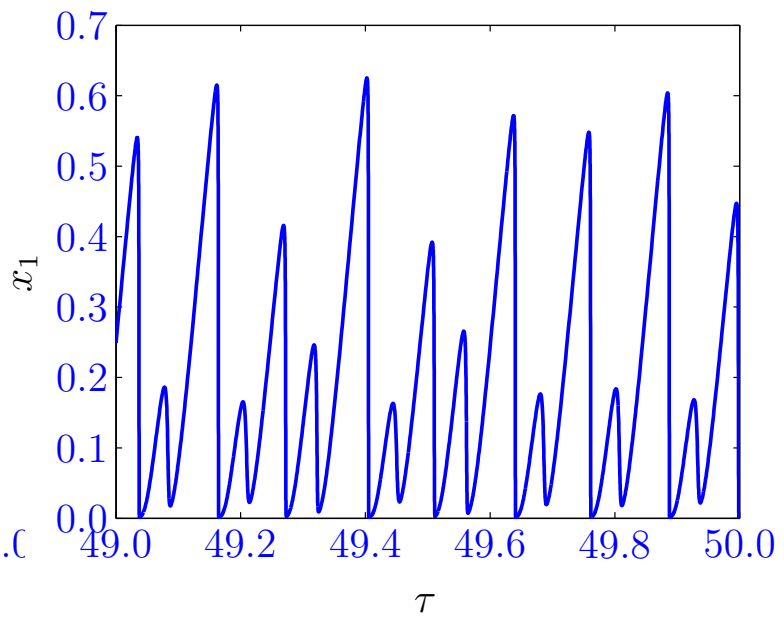

10.3.4. $y_{02}=7.325$ : Chaotic behavior

Figure 10.3. Typical plots of system (10.2) for $\kappa=65, \mu=0.145, \sigma=5 \times 10^{-3}, \delta=$ $2 \times 10^{-2}$ showing different dynamical behaviors for different values of $y_{02}$. 


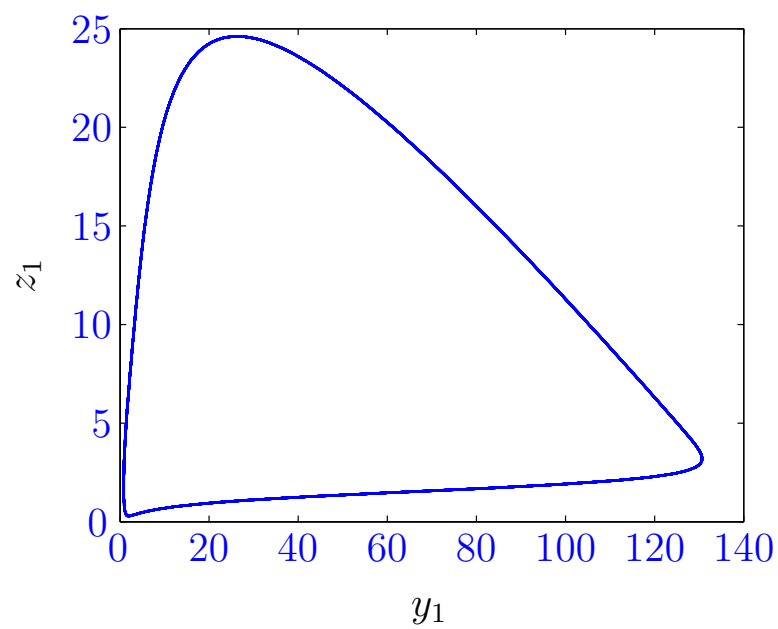

10.4.1. $y_{02}=0.5:$ Period-1

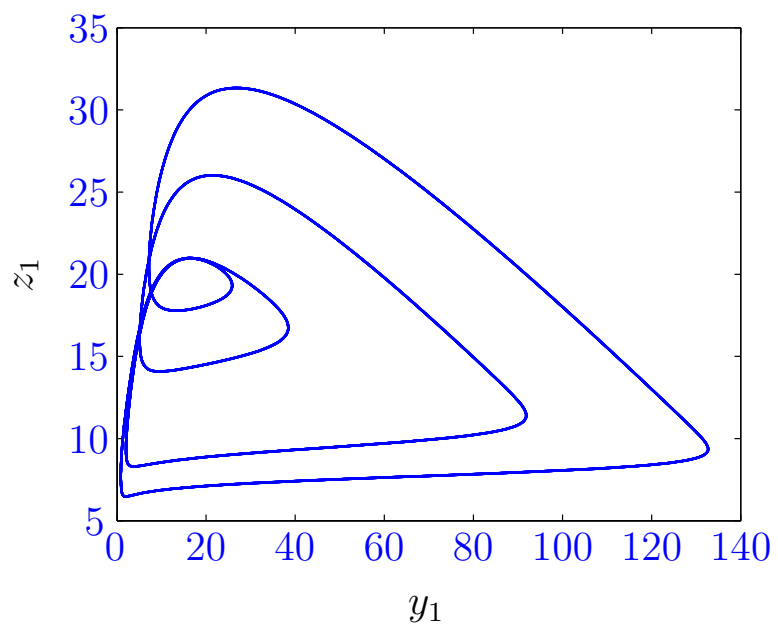

10.4.3. $y_{02}=6.7:$ Period -4

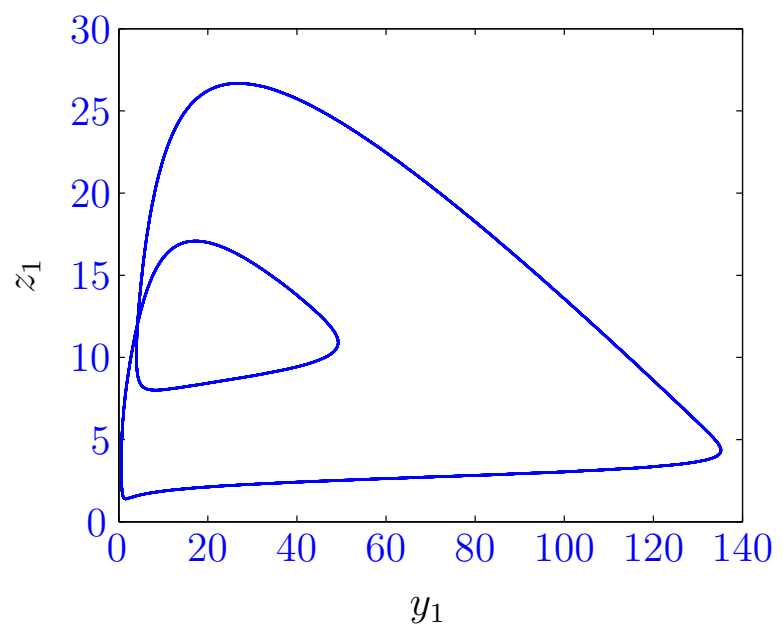

10.4.2. $y_{02}=2.0:$ Period-2

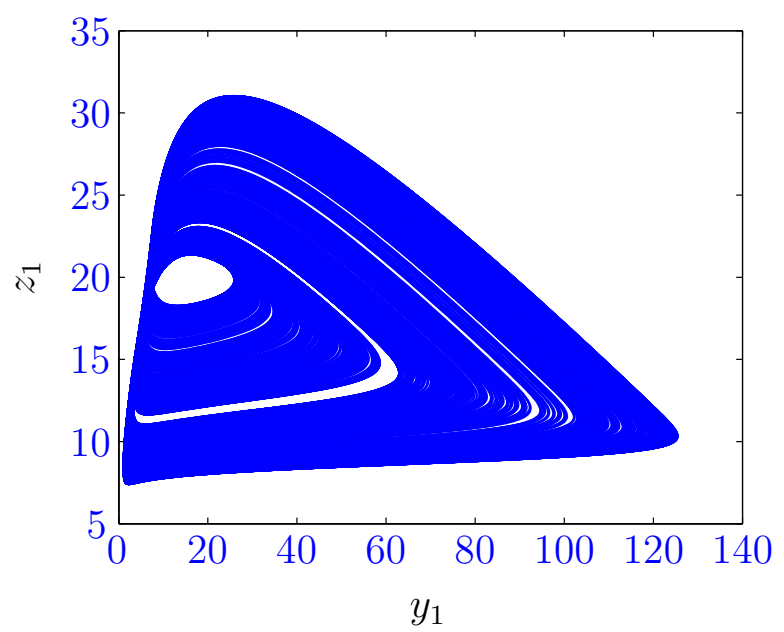

10.4.4. $y_{02}=7.325$ : Chaotic behavior

Figure 10.4. Typical phase plots of system (10.2) for $\kappa=65, \mu=0.145, \sigma=5 \times 10^{-3}, \delta=$ $2 \times 10^{-2}$ showing different dynamical behaviors for different values of $y_{02}$. We plot $z_{1}$ against $y_{1}$. 


\subsubsection{Reduced System}

We now show that the dynamics of system (10.2) can be studied through a reduced system. In contrast to the first coupled autocatalator system studied in Chapter 9, the new coupling gives rise to simple generalized synchronization between the two subsystems, in which two pairs of variables, $\left(x_{1}, x_{2}\right)$ and $\left(z_{1}, z_{2}\right)$, synchronize completely, while the variables in the third pair, $\left(y_{1}, y_{2}\right)$, differ by a constant, $c=y_{2}-y_{1}$. This type of synchronization is also associated with the emergence of a conserved quantity that characterizes the synchronization manifold. Compared to system (9.6), the conserved quantity $c$ appears as a result of the dynamics, which takes its final value only in the long-term limit as $\tau \rightarrow \infty$. To justify this statement, we introduce the new variables $e_{i}, i=1,2,3$, which are appropriate for characterizing synchronization [2], since they can be interpreted as deviations from complete synchronization:

$$
e_{1}=x_{2}-x_{1}, e_{2}=y_{2}-y_{1}, e_{3}=z_{2}-z_{1}
$$

By subtracting each of the first three equations of system (10.2) from the corresponding equation in the last three equations of system (10.2), simplifying and applying the new variable definitions (10.3), we obtain the following equations for the deviations $e_{i}$ :

$$
\begin{aligned}
\dot{e_{1}} & =\mu e_{3}-\left(1+x_{2}^{2}\right) e_{1}, \\
\sigma \dot{e_{2}} & =\left(1+x_{2}^{2}\right) e_{1}, \\
\delta \dot{e_{3}} & =-e_{3} .
\end{aligned}
$$

Theorem 10.2.1 (Existence and stability of steady state solutions). System (10.4) exhibits a single steady state solution, $\left(e_{1}^{*}, e_{2}^{*}, e_{3}^{*}\right)=\left(0, e_{2}^{*}, 0\right)$, where $e_{2}^{*}$ is a constant that depends on the initial conditions. Moreover, this steady state solution is asymptotically stable.

Proof. By setting the right-hand side of (10.4) to zero and solving the ensuing system of algebraic equations for $e_{1}^{*}, e_{2}^{*}$ and $e_{3}^{*}$, where $e_{i}^{*}, i=1,2,3$ represents the steady state value of $e_{i}$, we obtain $\left(e_{1}^{*}, e_{2}^{*}, e_{3}^{*}\right)=\left(0, e_{2}^{*}, 0\right)$. This proves the first part of the Theorem.

We now determine the stability of $\left(0, e_{2}^{*}, 0\right)$ by computing a Lyapunov function for system (10.4). Notice that all the terms on the right-hand side of system (10.4) are linear in $e_{1}$ and $e_{3}$ and that these terms are equally independent of $e_{2}$. Consequently, a good Lyapunov 
function candidate would be a function that consists of a combination of terms in $e_{1}, e_{3}$ and $e_{1} e_{3}$, with the total power of each term even. We consider the following candidate:

$$
v\left(e_{1}, e_{2}, e_{3}\right)=a e_{1}^{2}+b e_{1} e_{3}+c e_{3}^{2},
$$

where $a, b$ and $c$ with $4 a c>b^{2}$ are constants to be determined. Let $\gamma=1+x_{2}^{2}$, then $\gamma>0, \forall x$

$\dot{v}\left(e_{1}, e_{2}, e_{3}\right)=\frac{\partial v}{\partial e_{1}} \dot{e_{1}}+\frac{\partial v}{\partial e_{2}} \dot{e_{2}}+\frac{\partial v}{\partial e_{3}} \dot{e_{3}}=\left(2 \mu a-b\left(\gamma+\frac{1}{\delta}\right)\right) e_{1} e_{3}-2 a \gamma e_{1}^{2}-\left(\frac{2 c}{\delta}-\mu b\right) e_{3}^{2}$.

Now, for $\dot{v}<0$, we must have:

$$
2 \mu a-b\left(\gamma+\frac{1}{\delta}\right)=0, a>0, \text { and } \frac{2 c}{\delta}-\mu b>0
$$

That is, we must choose $a, b$ and $c$ such that all three conditions in (10.5) are satisfied. We satisfy the third condition by setting $c=1$ and $b=\frac{1}{\delta \mu}$. This gives $\frac{2 c}{\delta}-\mu b=\frac{1}{\delta}>0$ since $\delta>0$. Next, from the equation $2 \mu a-b\left(\gamma+\frac{1}{\delta}\right)=0$, we obtain $a=\frac{\gamma \delta+1}{2(\delta \mu)^{2}}>0$ since $\gamma>0$, and $\delta>0$. Thus, the conditions in (10.5) are satisfied if we choose

$$
a=\frac{\gamma \delta+1}{2(\delta \mu)^{2}}, b=\frac{1}{\delta \mu} \text { and } c=1
$$

Notice that with these values of $a, b$ and $c$,

$$
a>0, b>0, c>0 \text { and } 4 a c-b^{2}=\frac{2\left(1+x_{2}^{2}\right) \delta+1}{(\delta \mu)^{2}}>0 .
$$

Thus, $v\left(e_{1}, e_{2}, e_{3}\right)>0$ for $\left(e_{1}, e_{2}, e_{3}\right) \neq\left(0, e_{2}^{*}, 0\right)$ and $v\left(e_{1}, e_{2}, e_{3}\right)=0$ only at $\left(e_{1}, e_{2}, e_{3}\right)=$ $\left(0, e_{2}^{*}, 0\right)$. The function,

$$
v\left(e_{1}, e_{2}, e_{3}\right)=\frac{\left(1+x_{2}^{2}\right) \delta+1}{2(\delta \mu)^{2}} e_{1}^{2}+\frac{1}{\delta \mu} e_{1} e_{3}+e_{3}^{2}
$$

satisfies the following conditions:

- $v$ is continuous and has continuous first partial derivatives,

- $v>0$ for all $\left(e_{1}, e_{2}, e_{3}\right)$ and $v=0$ only for $\left(e_{1}, e_{2}, e_{3}\right)=\left(e_{1}^{*}, e_{2}^{*}, e_{3}^{*}\right)=\left(0, e_{2}^{*}, 0\right)$,

- $\dot{v}=-\left(\frac{\gamma(\gamma \delta+1)}{(\delta \mu)^{2}} e_{1}^{2}+\frac{1}{\delta} e_{3}^{2}\right)<0$ for all $\left(e_{1}, e_{2}, e_{3}\right)$ and $\dot{v}=0$ only for $\left(e_{1}, e_{2}, e_{3}\right)=\left(0, e_{2}^{*}, 0\right)$. 
Hence, $v$ is a Lyapunov function for $(10.4)$ and $\left(0, e_{2}^{*}, 0\right)$ is asymptotically stable [3].

We have shown in Theorem 10.2.1 that the steady state $\left(0, e_{2}^{*}, 0\right)$ is asymptotically stable. Hence, any solution of (10.4) converges to this steady state over time, and any perturbation of the system from this steady state decays to zero asymptotically. Consequently, the pairs of variables $\left(x_{1}, x_{2}\right)$ and $\left(z_{1}, z_{2}\right)$ whose differences constitute $e_{1}$ and $e_{3}$, respectively, are completely synchronized in the long-term limit, and since $e_{1} \rightarrow 0$ as $\tau \rightarrow \infty$, the second equation of (10.4) implies that $e_{2}=y_{2}-y_{1} \rightarrow c$, where $c$ is a constant that depends on the initial conditions of the full system (10.2). To see the dependence of $c$ on the initial conditions of the full system, we note that in the long term, the second equation of (10.4) assumes the form $\dot{y}_{2}-\dot{y}_{1} \rightarrow 0$, since $e_{1} \rightarrow 0$ as $\tau \rightarrow \infty$. But the general solution of the differential equation $\dot{y}_{2}-\dot{y}_{1}=0$ is $y_{2}-y_{1}=c$, where $c$ is a constant that depends on the initial values of $y_{1}$ and $y_{2}$. Therefore, $y_{2}-y_{1} \rightarrow c$ or $y_{2} \rightarrow y_{1}+c$ as $\tau \rightarrow \infty$. The constant $c$ is a conserved quantity, which, in contrast to the conserved quantity computed for model (9.6), is not given directly by the initial conditions but evolves to its final value on approaching the asymptotic state and, hence, depends on the initial conditions in a nontrivial way. The value of $c$ determines the synchronization manifold on which the long-term dynamics takes place. A schematic representation like that depicted in Fig. 9.7 holds only as $\tau \rightarrow \infty$. In the long-term limit, the state space is divided into infinitely many synchronization manifolds, each corresponding to a particular value of $c$, as shown in Fig. 10.5. There exists at least one attractor in each synchronization manifold. 


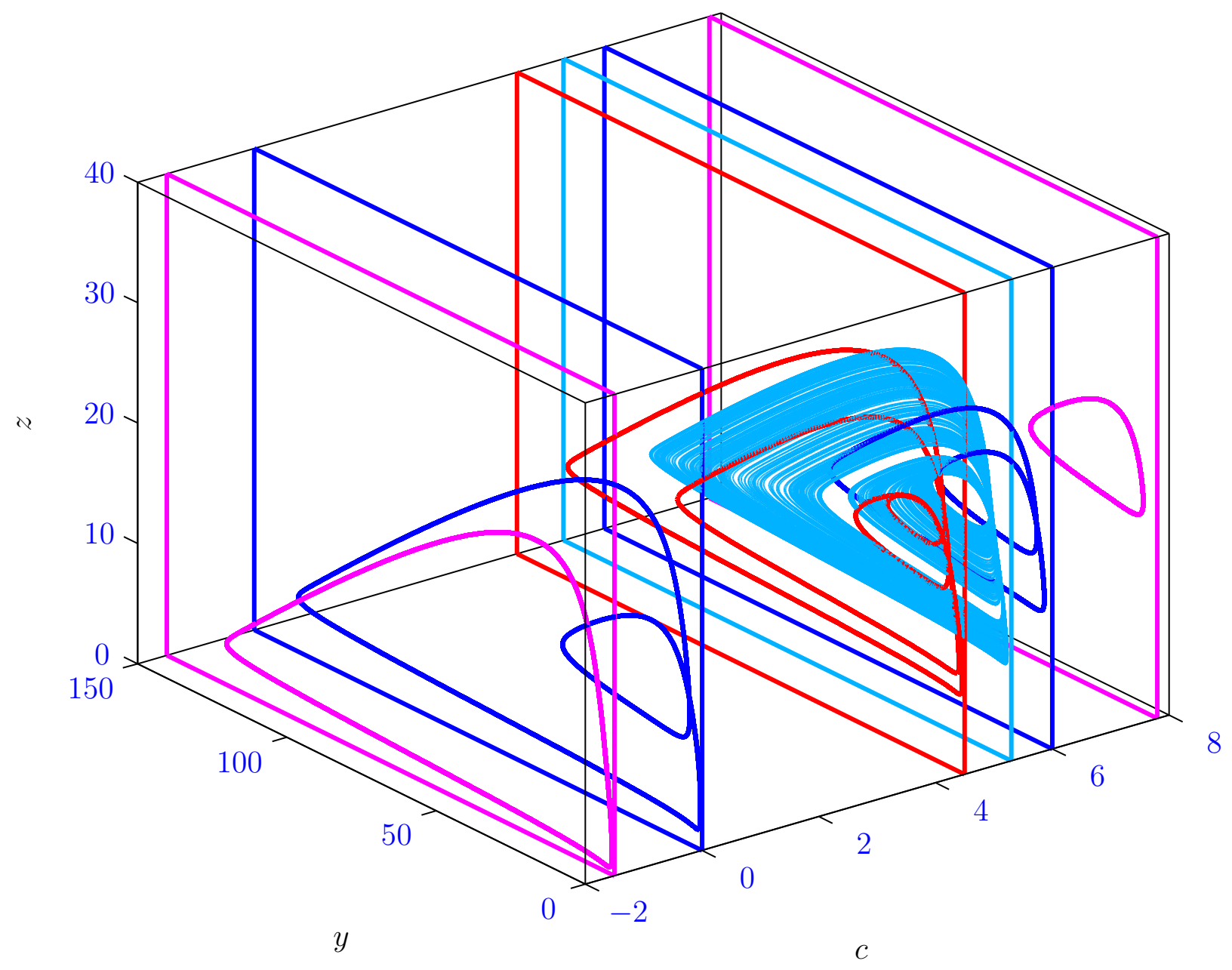

Figure 10.5. A sampling of the synchronization manifolds as a function of the conserved quantity $c$. Chaotic (light blue), period-4 (red), period-2 (dark blue), period-1 (magenta) behavior is exhibited for $c=5.3,4.5,0.0$, and 6.0, and -1.5 , and 7.8 . 
The initial condition is not necessarily contained in the synchronization manifold. A new value of $c$ is obtained for each new set of initial conditions, establishing a constant difference between the remaining pair of variables $\left(y_{1}, y_{2}\right)$. Therefore, we can substitute $y_{2}=y_{1}+c$ in the third equation of the first subsystem in the asymptotic state and obtain a reduced system, which can be further used to explore extreme multistability for $\tau \rightarrow \infty$ :

$$
\begin{aligned}
\dot{x_{1}} & =\mu\left(\kappa+z_{1}\right)-x_{1}\left(1+y_{1}^{2}\right), \\
\sigma \dot{y_{1}} & =x_{1}\left(1+y_{1}^{2}\right)-y_{1}, \\
\delta \dot{z_{1}} & =\left(y_{1}+c\right)-z_{1},
\end{aligned}
$$

The only difference between this system and the original three-variable autocatalator system arises from the introduction of an additional parameter $c$, which depends on the initial conditions of the full system (10.2) in a complex manner. Our goal is to use $c$ as a bifurcation parameter to study the dynamical behavior of system (10.6), and show that the reduced system can be used to explore the dynamics of the full system, (10.2). Using the same parameter values as in Section 10.2.2.2, we first integrate system (10.2) for different initial conditions as we did in Section 10.2.2.2 and use the last values of $y_{1}$ and $y_{2}$ to compute $c$ according to the formula $c=y_{2}-y_{1}$. Notice that using the last values of these variables ensures that all transients are eliminated and asymptotic dynamical behavior is attained. With these values of $c$ and the initial condition $(0.01,0.1,0.1)$, we integrate the reduced system using a Runge-Kutta scheme with variable step size. A transition from simple to complex dynamical behavior is observed as $c$ is gradually increased. Solutions of the system switch from period- 1 to period-2 at $c=-1.208$, from period- 2 to period- 4 at $c=4.362$, from period-4 to period- 8 at $c=4.672$, etc. The period doubling sequence leads to chaos that is followed by a period halving sequence that culminates in a stable steady state. Typical time evolution and phase plots of the solutions of the reduced system (10.6) for different values of $c$ are presented in Fig. 10.6, while a bifurcation sequence depicting the dynamics of the system as a function of the conserved quantity $c$ is illustrated in Fig. 10.7. 


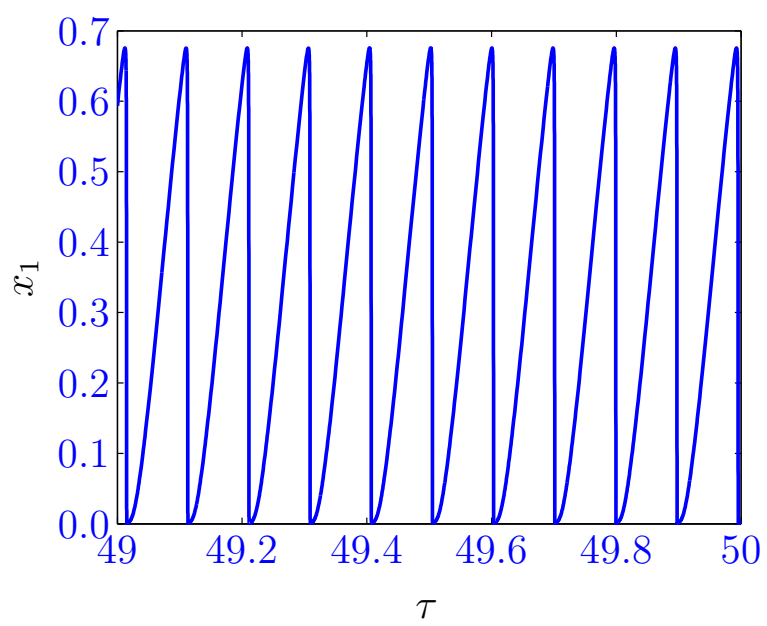

10.6.1. Period-1 behavior at $c=-1.5$.

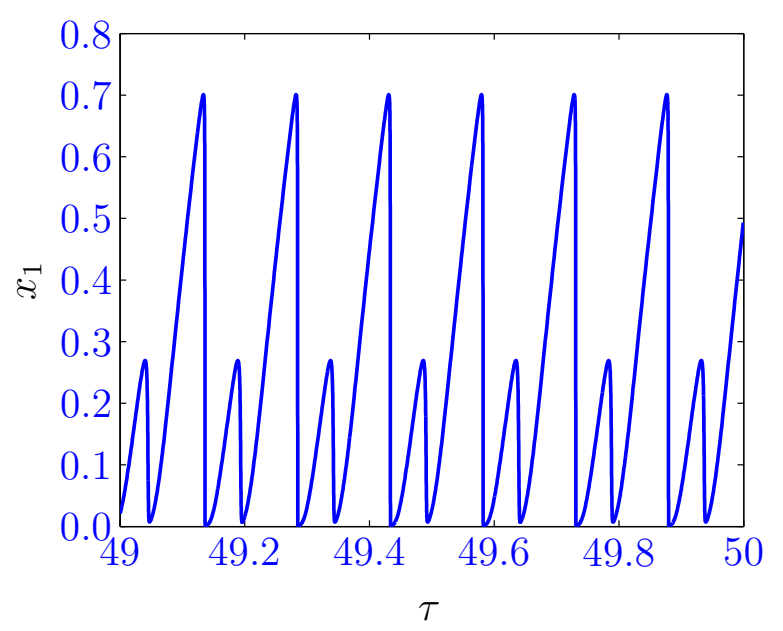

10.6.3. Period-2 behavior at $c=0.0$.

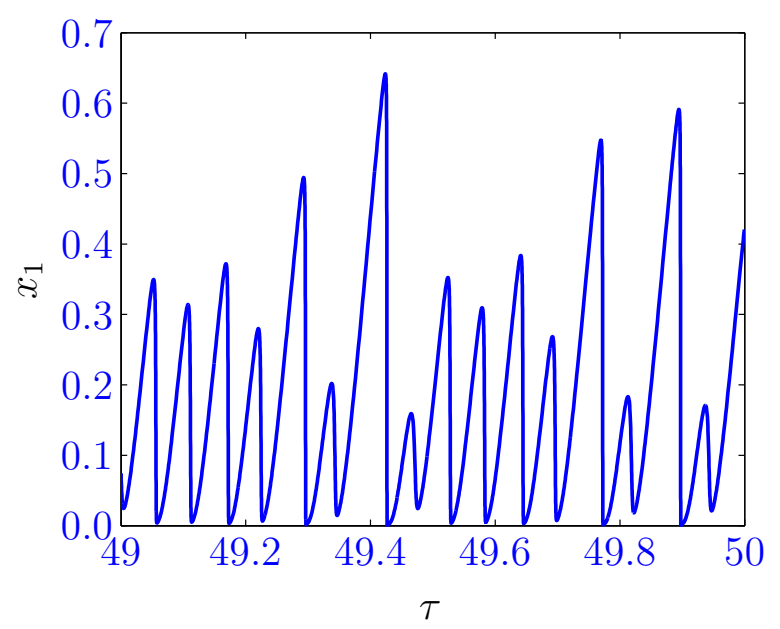

10.6.5. Chaotic behavior at $c=5.2$.

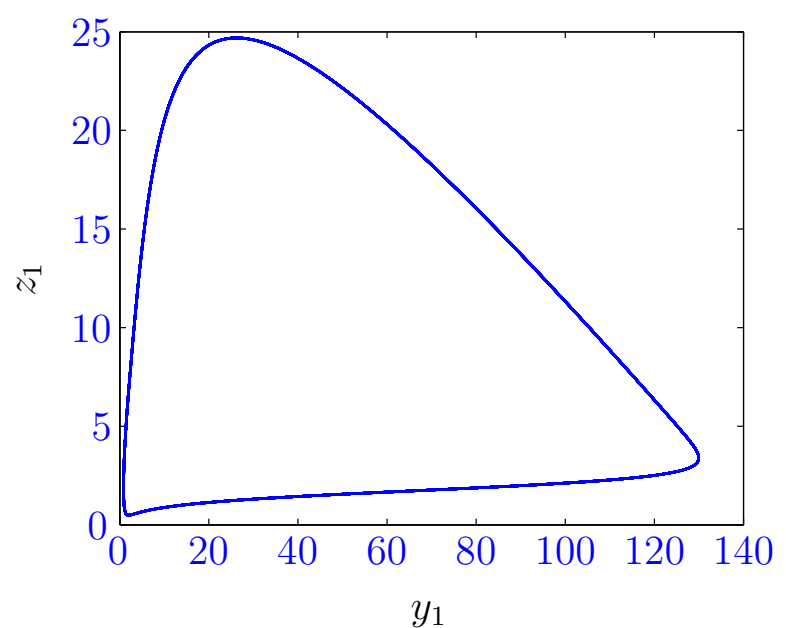

10.6.2. Period-1 behavior at $c=-1.5$.

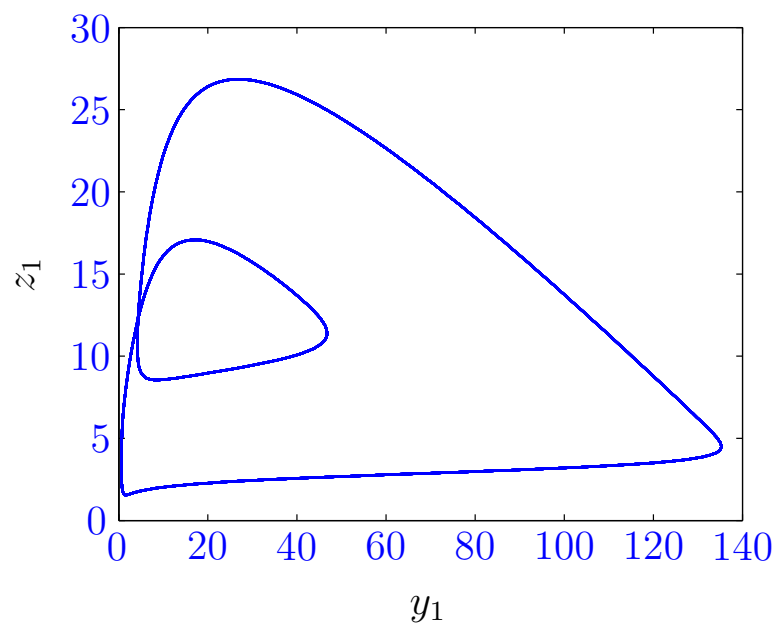

10.6.4. Period-2 behavior at $c=0.0$.

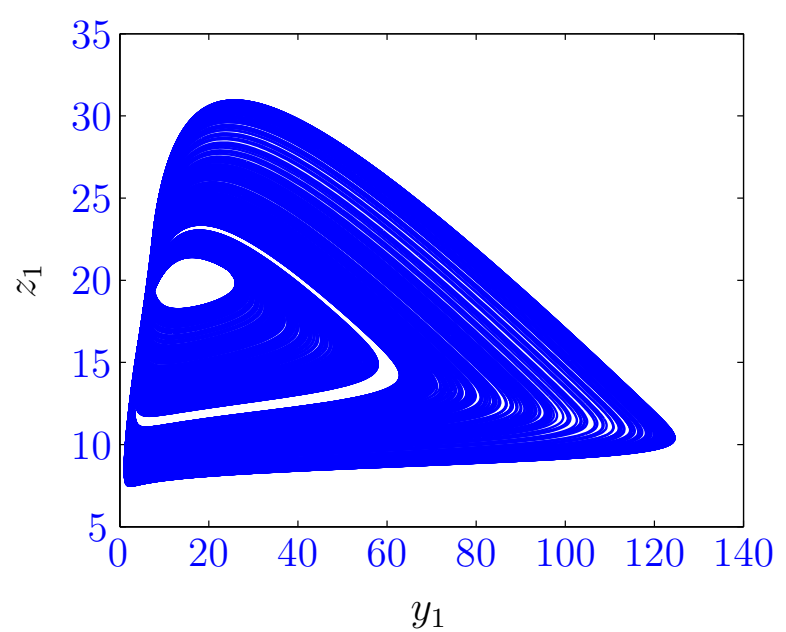

10.6.6. Chaotic behavior at $c=5.2$.

Figure 10.6. Typical time evolution and phase plots of the reduced system (10.6). 


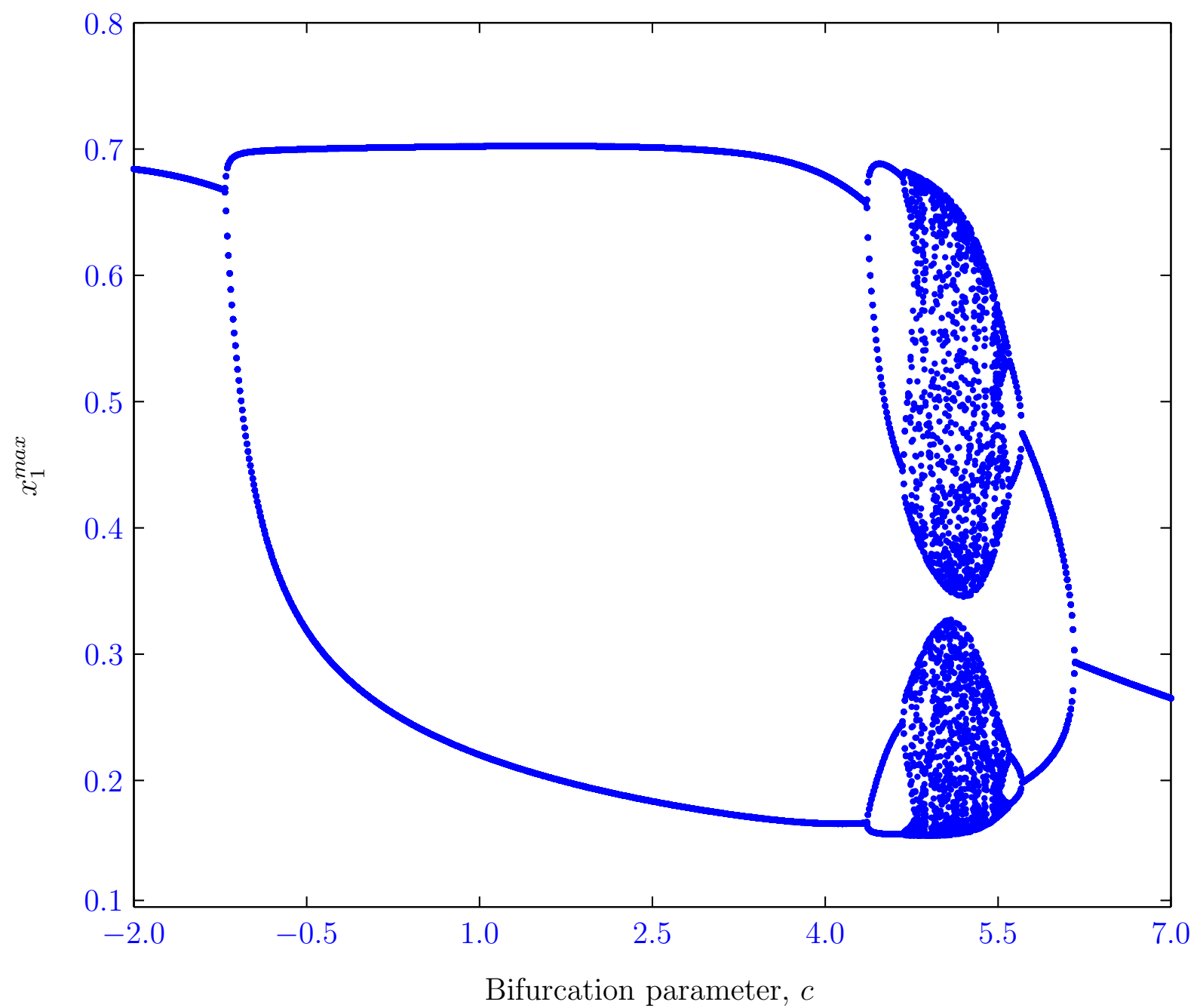

Figure 10.7. Bifurcation diagram of the reduced autocatalator model (10.6). A plot of the maximum amplitude of $x_{1}$ against $c$. 
The parameter, $c$, characterizes a synchronization manifold in state space describing generalized synchronization, where two pairs of variables in the two subsystems are fully synchronized while the variables in the third pair are separated from each other by a constant c. The equivalence between the dynamical behavior of the full six-dimensional model (10.2) as a function of $y_{02}$ and the reduced three-dimensional system (10.6) as a function of $c$ can be observed by comparing the bifurcation diagram in Fig. 10.7 and the initial condition diagram in Fig. 10.2. The two diagrams are in complete correspondence. Figure 10.2 represents the long-term dynamical behavior for different initial conditions, which resembles a bifurcation diagram, while Fig. 10.7 is a bifurcation diagram in the mathematical sense, since $c$ is a bifurcation parameter for the reduced system (10.6).

Figure 10.8 depicts typical time series plots for the full six-dimensional system in the long-term state. It highlights the nontrivial dependence of the final value of the conserved quantity $c$ on the initial conditions. Figure 10.9 illustrates complete synchronization of the $x_{i}$ and $z_{i},(\mathrm{i}=1,2)$ variables. For all of the plots, $y_{01}=0.1$. This indicates that the initial difference $y_{02}-y_{01}$ is not equal to the final value of the conserved quantity $c=y_{2}-y_{1}$. 


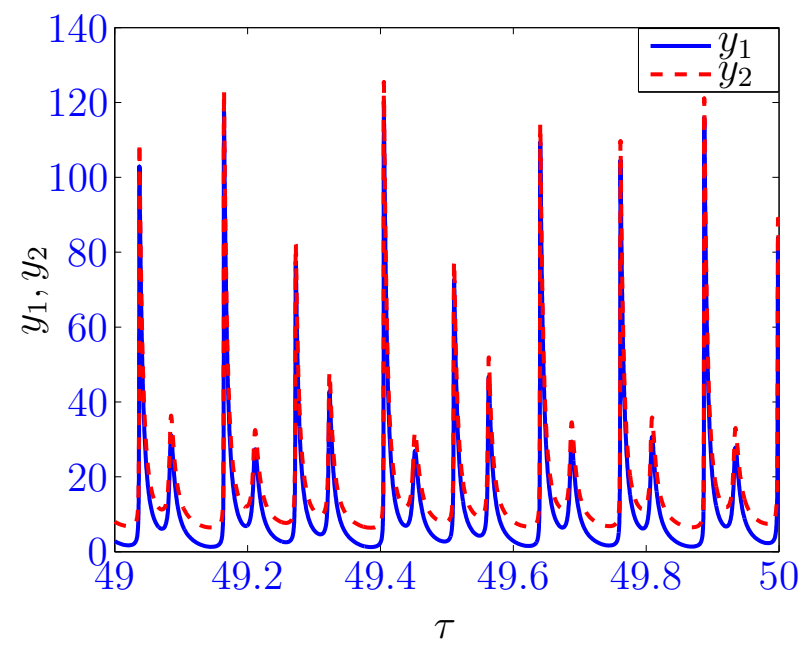

10.8.1. Chaotic regime: $y_{02}=7.325, c=$ $5.1670, y_{02}-y_{01}=7.225$.

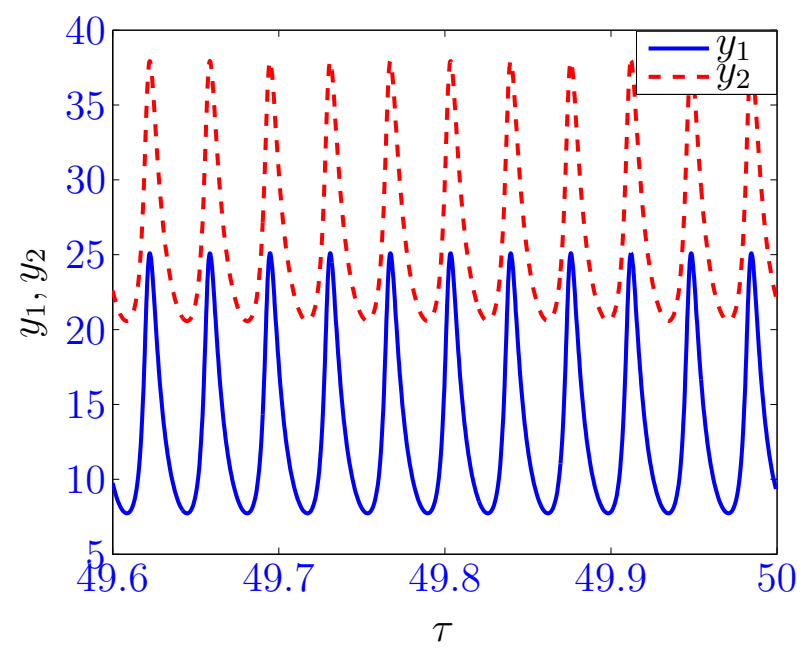

10.8.3. Period-1 regime: $y_{02}=15, c=$ $12.842, y_{02}-y_{01}=14.9$.

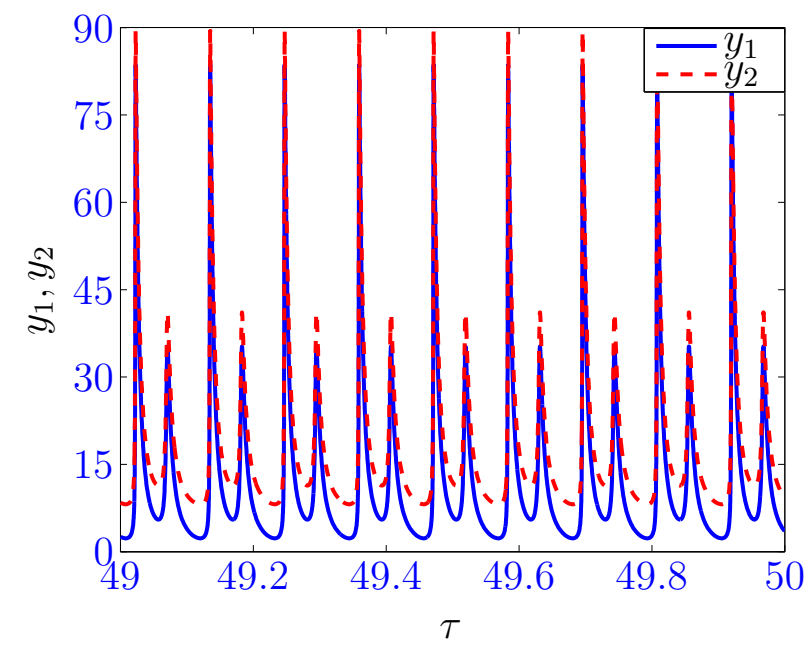

10.8.2. Period-2 regime: $y_{02}=8.0, c=$ $5.842, y_{02}-y_{01}=7.9$.

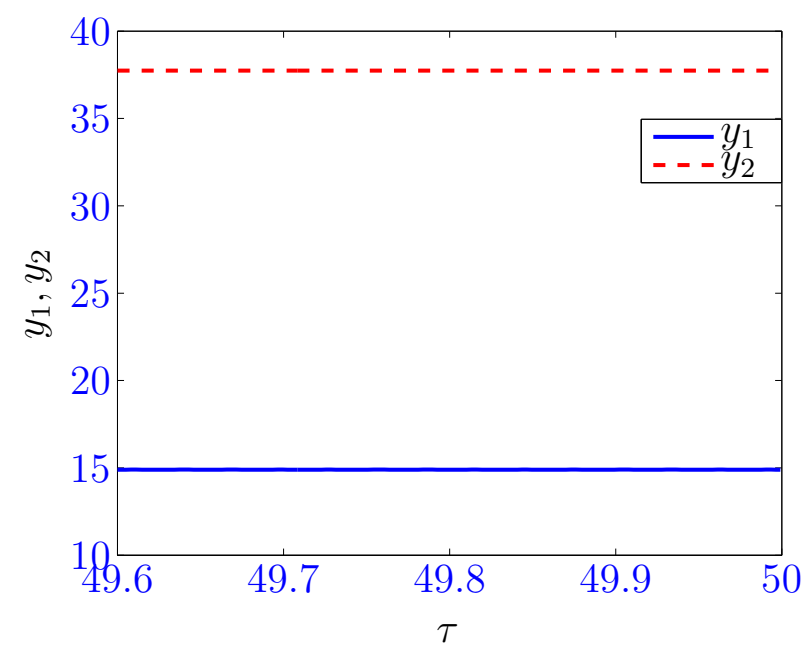

10.8.4. Stable steady state regime: $y_{02}=$ $25, c=22.842, y_{02}-y_{01}=24.9$.

Figure 10.8. Time series plots illustrating the constant difference between $y_{1}$ and $y_{2}$ within the chaotic, period-2, period-1 and stable steady state regimes. 


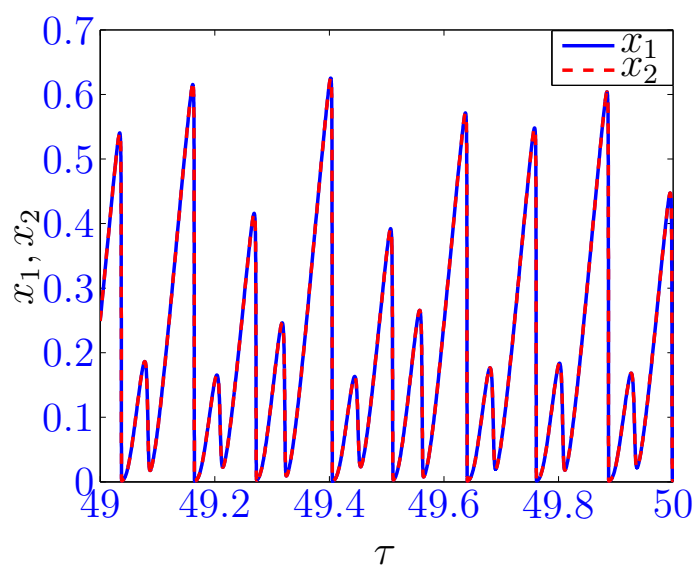

10.9.1. Chaotic regime: $y_{02}=$ 7.33 .

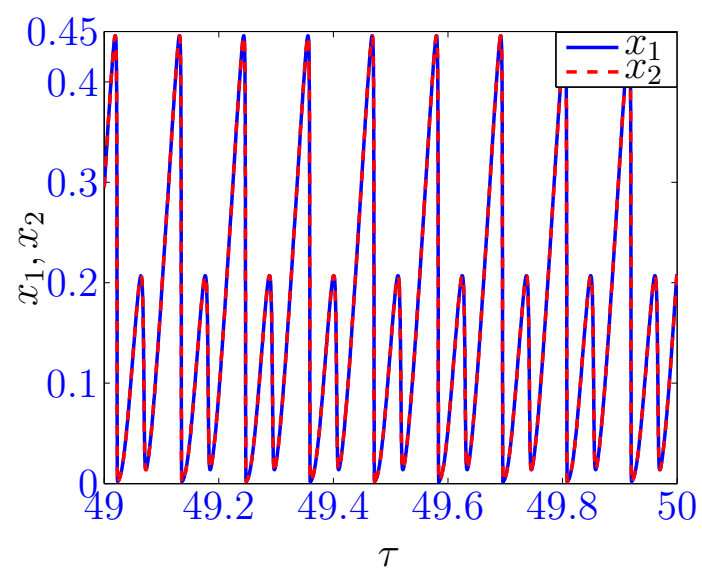

10.9.3. Period-2 regime: $y_{02}=8$.

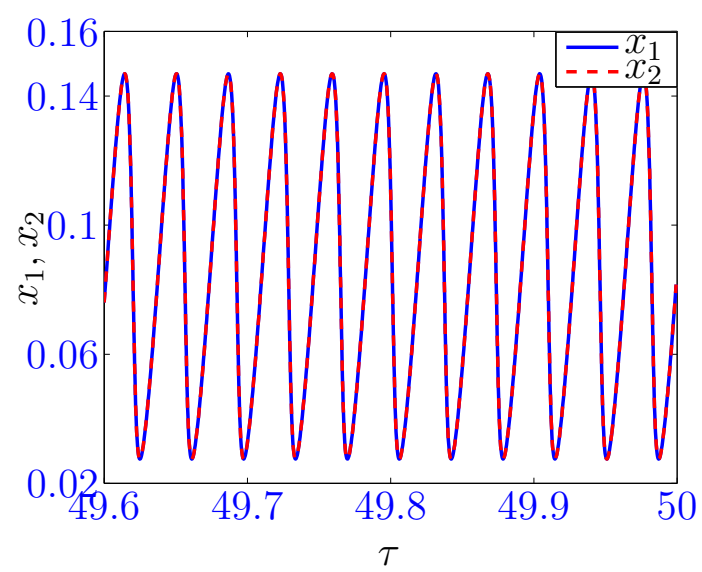

10.9.5. Period-1 regime: $y_{02}=15$.

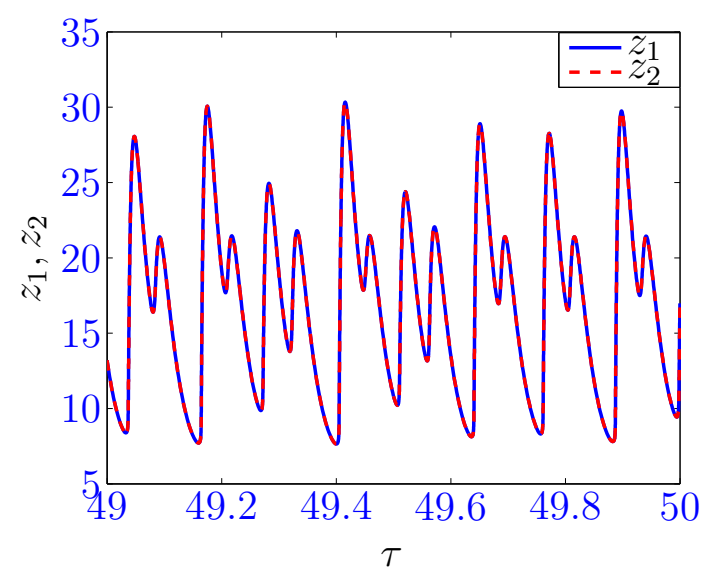

10.9.2. Chaotic regime: $y_{02}=$ 7.33 .

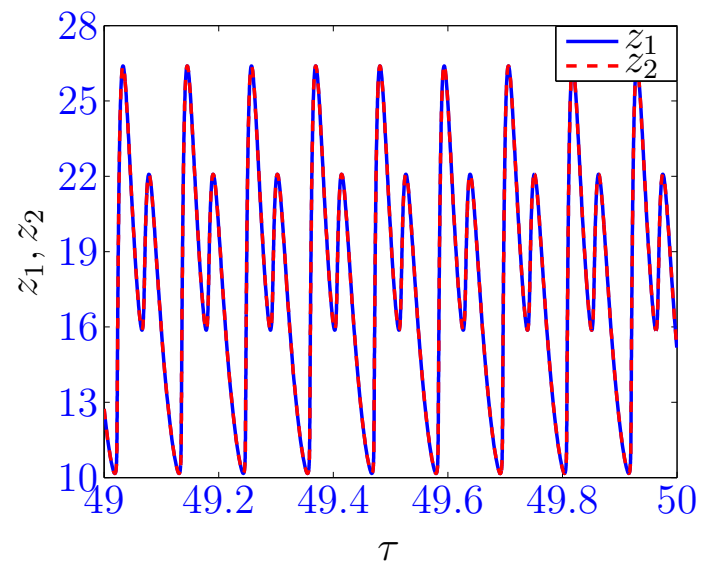

10.9.4. Period-2 regime: $y_{02}=8$.

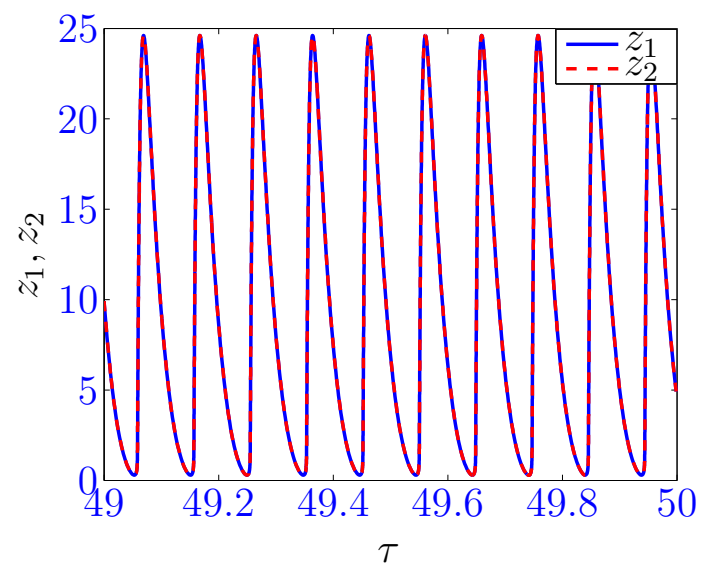

10.9.6. Period-1 regime: $y_{02}=15$.

Figure 10.9. Time series plots illustrating complete synchronization of the variables $x_{1}$ and $x_{2}$ and $z_{1}$ and $z_{2}$ within the chaotic, period- 2 and period- 1 regions. 
Computing the Lyapunov exponents and plotting them as a function of $y_{02}$ reveals a similar behavior to that observed in Chapter 9. Due to the existence of a conserved quantity, there are two zero Lyapunov exponents for all initial conditions (Fig. 10.10).

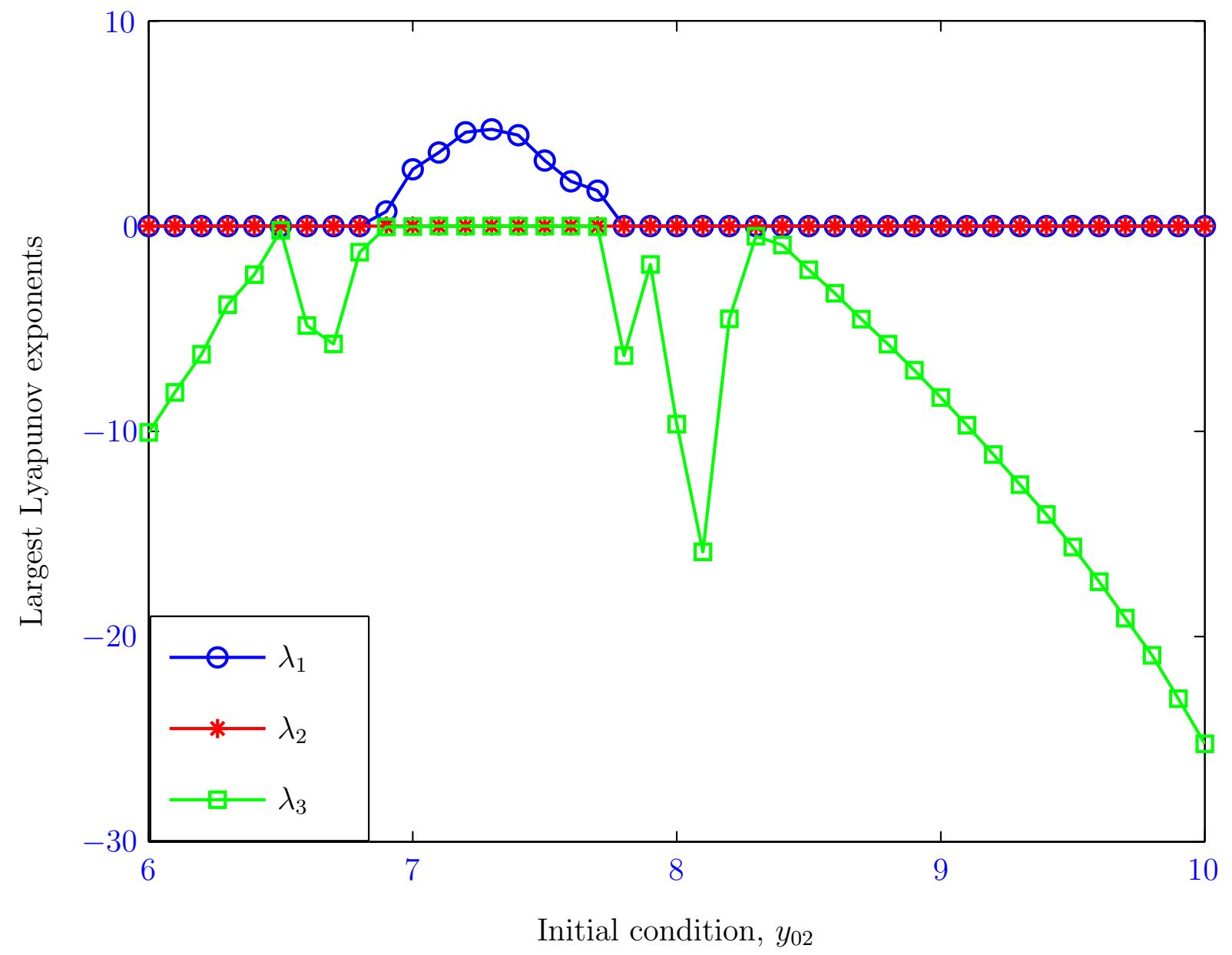

Figure 10.10. The three largest Lyapunov exponents of the coupled six-variable autocatalator model (10.2) vs. the initial condition $y_{02}$. 


\subsection{Third Coupling Scheme}

The third coupling scheme is accomplished through $Y_{1}$ in reaction $\left(R_{10.47}\right)$ of the second subsystem, and through $Y_{2}$ and $Z_{2}$ in reactions $\left(R_{10.39}\right)$ and $\left(R_{10.41}\right)$, respectively, of the first subsystem. We assume that the formation of the intermediate species is accompanied by the formation of side products $B, C, D$ and $P$, whose time evolution we omit. We also assume that the reaction between $D$ and $Y_{2}$ is so fast that its rate is determined by the rate of formation of $D$. This permits us to use $k_{4} Y_{1}$ instead of $k_{4}^{\prime} D Y_{2}$ as the removal term in the fifth equation of the corresponding mathematical system. This coupling yields the model

$$
\begin{array}{cc}
A \stackrel{k_{0}}{\longrightarrow} X_{1}, & \left(R_{10.36}\right) \\
A+Z_{1} \stackrel{k_{1}}{\longrightarrow} X_{1}+Z_{1}, & \left(R_{10.37}\right) \\
X_{1} \stackrel{k_{2}}{\longrightarrow} Y_{1}, & \left(R_{10.38}\right) \\
X_{1}+2 Y_{2} \stackrel{k_{3}}{\longrightarrow} B, & \left(R_{10.39}\right) \\
X_{1}+2 Y_{1} \stackrel{k_{3}}{\longrightarrow} X_{1}+3 Y_{1}, & \left(R_{10.40}\right) \\
Y_{1} \stackrel{k_{4}}{\longrightarrow} D+Z_{1}+Z_{2}, & \left(R_{10.41}\right) \\
Z_{1} \stackrel{k_{5}}{\longrightarrow} E, & \left(R_{10.42}\right) \\
\left.A_{10.43}\right) & \left(R_{10.44}\right) \\
A+Z_{2} \stackrel{k_{1}}{\longrightarrow} X_{2}, & \left(R_{10.45}\right) \\
X_{2} \stackrel{k_{2}}{\longrightarrow} Y_{2}, & \left(R_{10.46}\right) \\
X_{2}+2 Y_{2} \stackrel{k_{3}}{\longrightarrow} C, & \left(R_{10.47}\right) \\
X_{2}+2 Y_{1} \stackrel{k_{3}}{\longrightarrow} X_{2}+2 Y_{1}+Y_{2}, & \left(R_{10.48}\right) \\
D+Y_{2} \stackrel{k_{4}^{\prime}}{\longrightarrow} P, & \left(R_{10.49}\right) \\
Z_{2} \stackrel{k_{5}}{\longrightarrow} E, & \\
& \\
\hline
\end{array}
$$


where $k_{4}^{\prime} \gg k_{4}$. The corresponding mathematical model is:

$$
\begin{aligned}
{\left[\dot{X}_{1}\right] } & =k_{0} A_{0}+k_{1} A_{0}\left[Z_{1}\right]-k_{2}\left[X_{1}\right]-k_{3}\left[X_{1}\right]\left[Y_{2}\right]^{2}, \\
{\left[\dot{Y}_{1}\right] } & =k_{2}\left[X_{1}\right]+k_{3}\left[X_{1}\right]\left[Y_{1}\right]^{2}-k_{4}\left[Y_{1}\right], \\
{\left[\dot{Z}_{1}\right] } & =k_{4}\left[Y_{1}\right]-k_{5}\left[Z_{1}\right], \\
{\left[\dot{X}_{2}\right] } & =k_{0} A_{0}+k_{1} A_{0}\left[Z_{2}\right]-k_{2}\left[X_{2}\right]-k_{3}\left[X_{2}\right]\left[Y_{2}\right]^{2}, \\
{\left[\dot{Y}_{2}\right] } & =k_{2}\left[X_{2}\right]+k_{3}\left[X_{2}\right]\left[Y_{1}\right]^{2}-k_{4}\left[Y_{1}\right], \\
{\left[\dot{Z}_{2}\right] } & =k_{4}\left[Y_{1}\right]-k_{5}\left[Z_{2}\right] .
\end{aligned}
$$

Using a nondimensionalization procedure similar to that of Chapter 9, we obtain:

$$
\begin{aligned}
\dot{x_{1}} & =\mu\left(\kappa+z_{1}\right)-x_{1}\left(1+y_{2}^{2}\right), \\
\sigma \dot{y_{1}} & =x_{1}-y_{1}+x_{1} y_{1}^{2}, \\
\delta \dot{z_{1}} & =y_{1}-z_{1}, \\
\dot{x_{2}} & =\mu\left(\kappa+z_{2}\right)-x_{2}\left(1+y_{2}^{2}\right), \\
\sigma \dot{y_{2}} & =x_{2}-y_{1}+x_{2} y_{1}^{2}, \\
\delta \dot{z_{2}} & =y_{1}-z_{2} .
\end{aligned}
$$

We use the parameters $\kappa=65, \sigma=5 \times 10^{-3}$ and $\delta=2 \times 10^{-2}$. The bifurcation parameter is set to $\mu=0.157$ which corresponds to a solution of period-1 for a single uncoupled three-variable model. System (10.8) exhibits the phenomenon of extreme multistability as illustrated in Fig. 10.11. We also identify a conserved quantity, $c$ associated with this system. This quantity is similar to that of system (10.2) and is also given by $y_{2}-y_{1}=c$ in the long term limit. Substituting $y_{2}=y_{1}+c$ in the first equation of system (10.8) in the asymptotic state, we obtain the following reduced system which can also be used to further explore the dynamics of the full system (10.8) as $\tau \rightarrow \infty$ :

$$
\begin{aligned}
\dot{x_{1}} & =\mu\left(\kappa+z_{1}\right)-x_{1}\left(1+\left(y_{1}+c\right)^{2}\right), \\
\sigma \dot{y_{1}} & =x_{1}-y_{1}+x_{1} y_{1}^{2} \\
\delta \dot{z_{1}} & =y_{1}-z_{1} .
\end{aligned}
$$




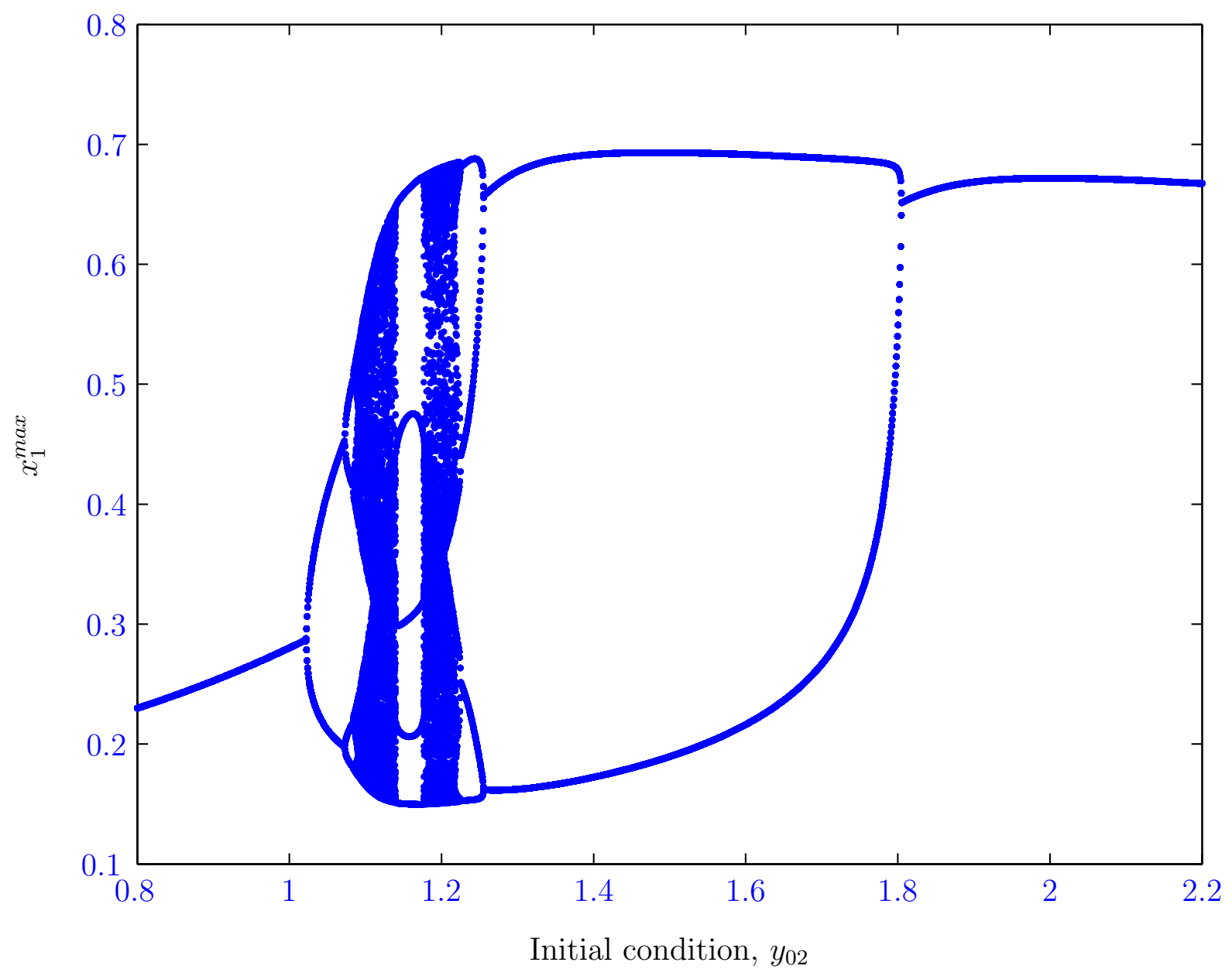

Figure 10.11. Long term dynamics of the coupled six-variable autocatalator system (10.8) showing periodic and chaotic behavior.

\subsection{Fourth Coupling Scheme}

The fourth coupling is achieved through $Z_{1}$ in reaction $\left(R_{10.57}\right)$ of the second subsystem, and indirectly through $Z_{2}$ in reaction $\left(R_{10.55}\right)$ of the first subsystem. The coupled chemical 
model is:

$$
\begin{array}{cc}
A \stackrel{k_{0}}{\longrightarrow} X_{1}, & \left(R_{10.50}\right) \\
A+Z_{1} \stackrel{k_{1}}{\longrightarrow} X_{1}+Z_{1}, & \left(R_{10.51}\right) \\
X_{1} \stackrel{k_{2}}{\longrightarrow} Y_{1}, & \left(R_{10.52}\right) \\
X_{1}+2 Y_{1} \stackrel{k_{3}}{\longrightarrow} 3 Y_{1}, & \left(R_{10.53}\right) \\
Y_{1} \stackrel{k_{4}}{\longrightarrow} Z_{1}, & \left(R_{10.54}\right) \\
E+Z_{1} \stackrel{k_{5}^{\prime}}{\longrightarrow} F, & \left(R_{10.55}\right) \\
& \\
A \stackrel{k_{0}}{\longrightarrow} X_{2}, & \left(R_{10.56}\right) \\
A+Z_{1} \stackrel{k_{1}}{\longrightarrow} X_{2}+Z_{1}, & \left(R_{10.57}\right) \\
X_{2} \stackrel{k_{2}}{\longrightarrow} Y_{2}, & \left(R_{10.58}\right) \\
X_{2}+2 Y_{2} \stackrel{k_{3}}{\longrightarrow} 3 Y_{2}, & \left(R_{10.59}\right) \\
Y_{2} \stackrel{k_{4}}{\longrightarrow} Z_{2}, & \left(R_{10.60}\right) \\
Z_{2} \stackrel{k_{5}}{\longrightarrow} E, & \left(R_{10.61}\right)
\end{array}
$$

where $k_{5}^{\prime} \gg k_{5}$. The corresponding mathematical model is:

$$
\begin{aligned}
{\left[\dot{X}_{1}\right] } & =k_{0} A_{0}+k_{1} A_{0}\left[Z_{1}\right]-k_{2}\left[X_{1}\right]-k_{3}\left[X_{1}\right]\left[Y_{1}\right]^{2}, \\
{\left[\dot{Y}_{1}\right] } & =k_{2}\left[X_{1}\right]+k_{3}\left[X_{1}\right]\left[Y_{1}\right]^{2}-k_{4}\left[Y_{1}\right], \\
{\left[\dot{Z}_{1}\right] } & =k_{4}\left[Y_{1}\right]-k_{5}\left[Z_{2}\right], \\
{\left[\dot{X}_{2}\right] } & =k_{0} A_{0}+k_{1} A_{0}\left[Z_{1}\right]-k_{2}\left[X_{2}\right]-k_{3}\left[X_{2}\right]\left[Y_{2}\right]^{2}, \\
{\left[\dot{Y}_{2}\right] } & =k_{2}\left[X_{2}\right]+k_{3}\left[X_{2}\right]\left[Y_{2}\right]^{2}-k_{4}\left[Y_{2}\right], \\
{\left[\dot{Z}_{2}\right] } & =k_{4}\left[Y_{2}\right]-k_{5}\left[Z_{2}\right] .
\end{aligned}
$$

Using a similar scaling as that of the previous sections, we obtain the following system: 


$$
\begin{aligned}
\dot{x_{1}} & =\mu\left(\kappa+z_{1}\right)-x_{1}\left(1+y_{1}^{2}\right), \\
\sigma \dot{y_{1}} & =x_{1}-y_{1}+x_{1} y_{1}^{2}, \\
\delta \dot{z_{1}} & =y_{1}-z_{2}, \\
\dot{x_{2}} & =\mu\left(\kappa+z_{1}\right)-x_{2}\left(1+y_{2}^{2}\right), \\
\sigma \dot{y_{2}} & =x_{2}-y_{2}+x_{2} y_{2}^{2}, \\
\delta \dot{z_{2}} & =y_{2}-z_{2} .
\end{aligned}
$$

System (10.11) also exhibits extreme multistability, as illustrated in Fig. 10.12. As in system (9.6) of Chapter 9, the conserved quantity is the same expression:

$$
C=x_{2}+\sigma y_{2}+\delta z_{2}-\left(x_{1}+\sigma y_{1}+\delta z_{1}\right)
$$

By setting $z_{2}=\left(C+x_{1}+\sigma y_{1}+\delta z_{1}-\left(x_{2}+\sigma y_{2}\right)\right) / \delta$ in system (10.11), we obtain the following reduced five-variable system that can be used to explore the dynamics of system (10.11) when the conserved quantity $C$ is used as the bifurcation parameter:

$$
\begin{aligned}
\dot{x_{1}} & =\mu\left(\kappa+z_{1}\right)-x_{1}\left(1+y_{1}^{2}\right), \\
\sigma \dot{y_{1}} & =x_{1}-y_{1}+x_{1} y_{1}^{2} \\
\delta \dot{z_{1}} & =y_{1}-\left(C+x_{1}+\sigma y_{1}+\delta z_{1}-\left(x_{2}+\sigma y_{2}\right)\right) / \delta \\
\dot{x_{2}} & =\mu\left(\kappa+z_{1}\right)-x_{2}\left(1+y_{2}^{2}\right), \\
\sigma \dot{y_{2}} & =x_{2}-y_{2}+x_{2} y_{2}^{2} .
\end{aligned}
$$




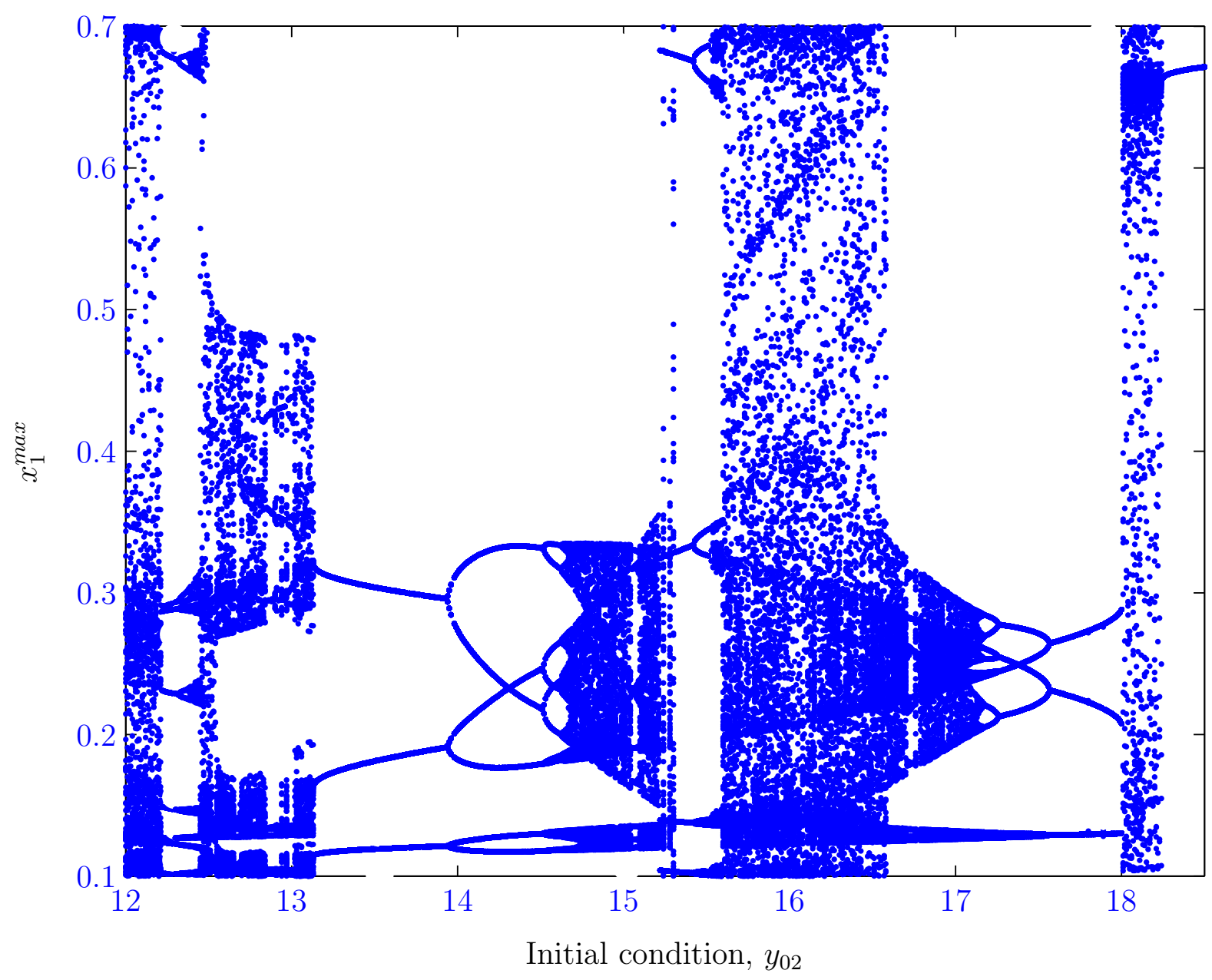

Figure 10.12. Long term dynamics of the six-variable autocatalator model (10.11) showing period doubling and chaotic behavior. 


\subsection{Brief Synopsis and Conclusion}

We have demonstrated that two three-variable autocatalator models coupled in four different ways can exhibit extreme multistability. Therefore, the coupling technique leading to extreme multistability is not unique. We also illustrated that the dynamics of each of the coupled system is associated with a emergence of a conserved quantity. However, the conserved quantity for two of the coupled systems, (10.2) and (10.8) does not depend on the initial conditions of the full coupled system in a trivial way as that for systems (9.6) and (10.11). The conserved quantity for systems (10.2) and (10.8) emerges only from the dynamics in the long-term limit. The long-term dynamics of these two systems can be studied through reduced three-variable systems, each of which differs from the original three-variable system only by a constant $c$ that arises as $\tau \rightarrow \infty$. These two systems exhibit generalized synchronization in which two pairs of variables are completely synchronized and the third pair has variables that are separated by a constant. The robustness of extreme multistability with respect to a mismatch in the parameters of the uncoupled three-variable subsystems can also be verified for each of the three coupled systems studied here. 


\section{References}

[1] H. Sun, S. Scott, and K. Showalter, "Uncertain Destination Dynamics," Physical Review E 60, 3876-3880 (1999).

Online Version 274

[2] L. Pecora and T. Carroll, "Synchronization in Chaotic Systems," Physical Review Letters 64, 821-824 (1990).

Online Version 284

[3] D. W. Jordan and P. Smith, Nonlinear Ordinary Differential Equations. An Introduction to Dynamical Systems (Oxford University Press, oxford, UK, 1999), 3rd edition. 286 
Chapter 11

\section{Generalization of Extreme Multistability}

\subsection{Introduction}

In this chapter, we summarize the results of our studies on extreme multistability and outline some evidence in support of the fact that the special coupling technique leading to extreme multistability gives rise to a new class of dynamical systems that exhibits characteristics of both dissipative and conservative systems. We also use our results to conclude that extreme multistability is a general concept.

\subsection{Overview and Generalization}

We have demonstrated the phenomenon of extreme multistability using four different coupling schemes of a chemical model system. For each of these couplings, we showed that the asymptotic behavior of the coupled system exhibits an infinite number of attractors, some stationary, some periodic and some chaotic. This situation was not possible with each of the two three-variable subsystems prior to the coupling. We explained the phenomenon of extreme multistability by illustrating that it is associated with the emergence of a conserved quantity. This conserved quantity can appear due to a special choice of the coupling scheme leading to a coupled system whose variables are no longer independent. In this case, the conserved quantity is determined directly by the initial conditions of the coupled system. This is the case for systems (9.6) and (10.11). On the other hand, other coupling options may lead to conserved quantities that emerge from the dynamics in the long-term limit. This 
case is more complex and intriguing since the conserved quantity is not given but appears only when the trajectory reaches the attractor. Such conserved quantities were found in the coupled six-variable autocatalator systems (10.2) and (10.8). The dynamics of these coupled systems are governed by two properties of extreme multistability:

1. The dynamics takes place on a manifold in state space that is determined by the value of the conserved quantity and contains at least one attractor. Hence in the limit $\tau \rightarrow \infty$, extreme multistability can be interpreted in terms of a division of the state space into infinitely many manifolds on which the dynamics takes place.

2. Extreme multistability is associated with the appearance of generalized synchronization between the coupled subsystems.

The emergence of a conserved quantity in the long-term limit allows a model reduction of the coupled systems, (10.2) and (10.8). Since the dynamics for $\tau \rightarrow \infty$ takes place on a hypersurface in state space determined by the value of the conserved quantity, each of these systems can be reduced to a new three-variable system that differs from the original threevariable system only by a constant, the conserved quantity. The conserved quantity serves as a bifurcation parameter in the classical sense for each of the reduced three-variable models. This model reduction confirms the existence of an infinite number of attractors since the variation of the new bifurcation parameter, the conserved quantity, gives rise to an infinite cascade of period-doubling in transition to chaos. Extreme multistability therefore contains periodic orbits of all periods. In terms of the original system variables, the analysis of systems (10.2) and (10.8), and the corresponding reduced systems indicate that small differences in the concentrations of the intermediate species $Y_{1}$ and $Y_{2}$ may not only alter the dynamic behavior of the system quantitatively but also qualitatively. The variables $x_{1}$ and $x_{2}$ relax to the same value, and $z_{1}$ and $z_{2}$ also relax to the same value. However, the variables $y_{1}$ and $y_{2}$ relax such that $y_{2}-y_{1}=c$. Consequently, the plots of $y_{1}$ and $y_{2}$ are identical except for a shift by the factor of $c$. Note that the variables $y_{1}$ and $y_{2}$ exhibit phase synchronization. The analysis for systems (9.6) and (10.11), in which the associated conserved quantities emerge as well as depend on the initial conditions in a simple way is slightly different. There is no complete synchronization between any of the variable pairs. Thus, the conserved quantity 
can be used to reduce each of these two systems by only a single variable. The conserved quantity can also serve as a bifurcation parameter in the mathematical sense.

The coupled six-dimensional chemical systems studied here exhibit both characteristics of dissipative and conservative dynamics. Common examples of conservative systems are the Lotka model [1] and the undamped simple pendulum system briefly examined in Chapter 1. The main difference between the coupled autocatalator systems and purely conservative systems resides in the fact that the former also possesses dissipative components, since the system relaxes to its asymptotic state, whereas there is no relaxation to an attractor in the latter. Due to the existence of a conserved quantity, the dynamics of the coupled autocatalator systems has features in common with Hamiltonian systems. However, the four systems studied here are dissipative and, hence, possess attractors in contrast to Hamiltonian systems, which exhibit marginally stable orbits. Conserved quantities in Hamiltonian systems, like energy, have fixed values from the very beginning. This is also true with systems (9.6) and (10.11). However, the conserved quantity for each of the systems in (10.2) and (10.8) emerges during the time evolution of the system and its value is fixed only as $\tau \rightarrow \infty$. Almost all perturbations of the coupled systems lead to changes in the value of the conserved quantity and consequently to a change in the long-term dynamics. The exception, of course, is a perturbation in the values of the variables that preserve the conserved quantity, for example, a perturbation that changes $y_{1}$ and $y_{2}$ such that the conserved quantity remains the same. The set of initial conditions or perturbations that leave a particular conserved quantity unchanged constitutes the basin of attraction of the attractor defined by the value of that conserved quantity. Hence, each of the attractors has its own basin of attraction.

In each of the coupled systems, the rate constants for corresponding reactions in the subsystems are the same. To illustrate that extreme multistability can arise in a wider range of systems, we addressed the chemical plausibility of the reactions in the coupled systems. We demonstrated that it is possible to vary the rate constants for corresponding reactions by at least a small amount (less than 1\%) without losing extreme multistability. For other systems such as the coupled Lorenz systems studied in [2], extreme multistability is lost for parameter mismatches that exceed $0.1 \%$ [3]. Thus, we conclude that extreme multistability is more likely to occur when almost identical subsystems are coupled. 
Noise is inevitable in naturally occurring systems. Hence, it is necessary to address the robustness of extreme multistability against noise. For multistable systems possessing a large number of coexisting attractors it has been shown that noisy dynamics can be viewed as a combination of two phases of motion, where the first phase is characterized by a motion around the attractor and the second phase corresponds to a jump from one attractor to another [4]. Therefore, the duration of these two phases of motion is irregular and the overall dynamics appears as a hopping process between different attractors. For systems exhibiting extreme multistability, we also observe this hopping as noise of a certain strength drives the system to move from one synchronization manifold to another. However, it is important to note that this hopping can only be observed for small noise strengths, since strong noise leads to a diffusion process in state space.

The four different coupling schemes of the autocatalator model, each leading to extreme multistability, demonstrates that the special coupling technique is not unique. In an attempt to generalize the approach and the phenomenon of extreme multistability, we tried to find a generic prescription for constructing models that exhibit infinitely many attractors. Different factors including symmetry and asymmetry, coupling both subsystems through a single variable, coupling both subsystems through two or more variables, selecting coupling variables from any of the equations involved in the system, etc. have been considered. Taking these factors into account, we developed a number of different couplings for the autocatalator system. Some of these coupled models exhibit extreme multistability and some do not. Our investigations revealed that symmetry and asymmetry do not appear to play a major rule in the occurrence of extreme multistability. We also discovered that all coupling attempts through a single variable failed to exhibit extreme multistability. Hence, the coupling should involve at least two variables.

Apart from the coupling, we may also try to generalize the notion of extreme multistability by considering the nature of the original subsystems. Here, we conjecture that a requirement for extreme multistability is chaos or chaotic subsystems. This is definitely a requirement for an infinite number of qualitatively different attractors, since the perioddoubling cascade to chaos is the main characteristic that leads to this feature in all systems known to exhibit extreme multistability. We could not find extreme multistability in coupled 
two-variable oscillatory systems of ordinary differential equations.

In addition to the Lorenz and the autocatalator systems, we also demonstrated the occurrence of extreme multistability in biological and ecological models such as the Hindmarsh-

Rose neuron model [5, 6] and the ratio-dependent food chain model [7]. We are thus reasonably convinced that given a system that exhibits chaotic behavior we can find extreme multistability by appropriately coupling two such systems. In contrast to the autotacalator system in which we could easily verify that the couplings make sense physically, it is more difficult to verify whether the coupled Lorenz and biological systems are physically plausible.

\subsection{Conclusion}

Extreme multistability is an unusual kind of multistability characterized by the coexistence of an infinite number of attractors. It appears when two identical or almost identical systems are coupled in a special way. The special coupling gives rise to a new class of dynamical systems that exhibits characteristics of both dissipative and conservative dynamical systems. Extreme multistability is closely associated with generalized synchronization and the emergence of a conserved quantity. 


\section{References}

[1] A. J. Lotka, Elements of Physical Biology (Williams and Wilkins Co., Baltimore, MD, 1924). 305

[2] H. Sun, S. Scott, and K. Showalter, "Uncertain Destination Dynamics," Physical Review E 60, 3876-3880 (1999).

Online Version 305

[3] U. Feudel, "Complex Dynamics in Multistable Systems," International Journal of Bifurcation and Chaos 18 (6), 1607-1626 (2008). 305

[4] U. Feudel and C. Grebogi, "Multistability and the Control of Complexity," Chaos 7, 597-604 (1997). 306

[5] J. L. Hindmarsh and R. M. Rose, "A Model for Neural Bursting Using Three Coupled First Order Differential Equations," Proceedings of the Royal Society London, Series B 221 (1222), 87-102 (1984).

Online Version 307

[6] J. M. González-Miranda, "Complex Bifurcations Structures in the Hindmarsh-Rose Neuron Model," International Journal of Bifurcation and Chaos 17 (9), 3071-3083 (2007). 307

[7] S. B. Hsu, T. W. Hwang, and Y. Kuang, "A Ratio-dependent Food Chain Model and its Applications to Biological Control," Mathematical Biosciences 181, 55-83 (2003). Online Version 307 
Appendices 
$\overline{\text { Appendix }} \mathrm{A}$

\section{Principle of Linear Stability}

A linear stability analysis provides information about the local stability of equilibrium solutions to a dynamical system described by an autonomous system of ordinary differential equations. The principle of linear stability can be used to determine the equilibrium solution $x^{*}$ of the nonlinear autonomous system of ordinary differential equations $\dot{x}=f(x)$ and its stability from the stability of the zero solution of the corresponding linear system $\tilde{x}=J \tilde{x}$, where $J$ is the Jacobian matrix of the linear system and $\tilde{x}$ is a perturbation of the variable $x$ from $x^{*}$. We briefly examine the principle of linear stability below.

Let us begin by considering the following autonomous system of differential equations:

$$
\dot{x}=f(x),
$$

where $f \in \mathbb{R}^{n}$ is a continuously differentiable function of $x$ and $x \in \mathbb{R}^{n}$ is a continuous function of $t$ with $t \in \mathbb{R}$. Suppose $x^{*}$ is an equilibrium solution of (A.1); then we can study the linear stability of $x^{*}$ by perturbing the system about $x^{*}$. To achieve this, we set

$$
\tilde{x}=x-x^{*} \text { or } x=x^{*}+\tilde{x}
$$

where $\|\tilde{x}\| \ll 1$ in (A.1) and expand in a Taylor series about $x^{*}$. This results in the system

$$
\dot{\tilde{x}}=f\left(x^{*}\right)+J \tilde{x}+H O T
$$

where HOT represents higher order terms in $\tilde{x}$. The entries $a_{i j}$ of the $n \times n$ Jacobian matrix $J$ can be determined according to the formula $a_{i j}=\frac{\partial f_{i}\left(x^{*}\right)}{\partial x_{j}}, i, j=1,2,3, \ldots, n$. Now, since $x^{*}$ is an equilibrium solution of (A.1), $f\left(x^{*}\right)=0$, and since $\|\tilde{x}\| \ll 1$, we can re-write system 
(A.2) while disregarding $f\left(x^{*}\right)$ and HOT as follows:

$$
\dot{\tilde{x}}=J \tilde{x} .
$$

In expanded form, $J$ can be written as

$$
J=\left(\begin{array}{cccc}
\frac{\partial f_{1}}{\partial x_{1}} & \frac{\partial f_{1}}{\partial x_{2}} & \cdots & \frac{\partial f_{1}}{\partial x_{n}} \\
\frac{\partial f_{2}}{\partial x_{1}} & \frac{\partial f_{2}}{\partial x_{2}} & \cdots & \frac{\partial f_{2}}{\partial x_{n}} \\
\vdots & \vdots & \ddots & \vdots \\
\frac{\partial f_{n}}{\partial x_{1}} & \frac{\partial f_{n}}{\partial x_{2}} & \cdots & \frac{\partial f_{n}}{\partial x_{n}}
\end{array}\right)
$$

Next, we seek linearly independent solutions of this linear system of the form $\tilde{x}=v e^{\lambda t}$, where $\lambda$ is an eigenvalue that measures the temporal response of the perturbation and $v \neq 0$ is the corresponding eigenvector that spans the null space of the system (A.3). Substituting this exponential solution in (A.3) and simplifying yields the new system

$$
\left(J-\lambda I_{n}\right) v=0, v \neq 0,
$$

where $I_{n}$ is the $n \times n$ identity matrix. From linear algebra, it is obvious that (A.4) has non-trivial solutions only when $\left|J-\lambda I_{n}\right|=0$ or

$$
\lambda^{n}+a_{1} \lambda^{n-1}+a_{2} \lambda^{n-2}+---+a_{n-2} \lambda^{2}+a_{n-1} \lambda+a_{n}=0,
$$

where the coefficients $a_{i}, i=1,2,3, \cdots, n$ are real constants. Equation (A.5) is an $n$-degree polynomial equation in $\lambda$, called a characteristic equation. Note that with these values of $\lambda$, the corresponding eigenvectors can be found from (A.4). Once we have values for $\lambda$ and the corresponding eigenvectors, the solution of system (A.3) can be expressed in the form

$$
\tilde{x}(t)=\sum_{i=1}^{n} k_{i} v_{i} e^{\lambda t}
$$

if all eigenvalues are distinct, and in the form

$$
\tilde{x}(t)=\sum_{i=1}^{m} k_{i} v_{m} t^{m-1} e^{\lambda_{m} t}+\sum_{i=m+1}^{n} k_{i} v_{i} e^{\lambda t},
$$


if $\lambda_{m}$ is a repeated eigenvalue of multiplicity $m$. In either case, the $k_{i}$ 's are constants whose values can be computed if initial conditions are specified.

Without necessarily determining the explicit solution of the system (A.3), it is possible to establish the local stability of solutions to system (A.3) and consequently system (A.1) as long as we are capable of determining the eigenvalues of (A.3). To this end, equilibrium solutions to (A.3), and consequently to (A.1), are linearly and asymptotically stable if all eigenvalues of (A.3) have strictly negative real parts, stable if the eigenvalues have nonpositive real parts, and unstable if at least one eigenvalue has a positive real part. If $\lambda$ is complex, we can have damped oscillations when the real part of $\lambda$ is negative, growing oscillations when the real part of $\lambda$ is positive, and sustained periodic oscillations when the real part of $\lambda$ is zero; that is, when $\lambda$ is purely imaginary. The system is neutrally stable when $\lambda$ is purely imaginary. Neutral stability is fragile to perturbations. This is because small perturbations can destabilize the system or cause it to become attractive. 
$\overline{\text { Appendix }} \mathrm{B}$

\section{Libnitz Rule, Descartes' Rule of Signs, and Routh-Hurwitz Criterion}

\section{B.1 Libnitz Rule}

The Libnitz Rule [1-4] is important in differentiating definite integrals whose limits are differentiable functions. It states that,

$$
\frac{d}{d t} \int_{u(t)}^{v(t)} p(s, t) d s=\int_{u(t)}^{v(t)} \frac{\partial p(s, t)}{\partial t} d s+p(v(t)) v^{\prime}(t)-p(u(t)) u^{\prime}(t),
$$

where the primes on $u$ and $v$ denote differentiation with respect to $t$.

Descartes' rule of signs $[5,6]$ and the Routh-Hurwitz criterion $[7,8]$ play an important role in determining the stability of linear systems of autonomous ordinary differential equations.

\section{B.2 Descartes' Rule of Signs}

Given the sequence $\left\{a_{i}\right\}_{i=1}^{n}$ formed from the coefficients of the $n$-degree polynomial equation (A.5), and given that $m$ is the number of sign switches from one non-zero coefficient of equation (A.5) to the next, then Descartes' rule of signs states that the number of real positive roots of equation (A.5) is either $m$ or $m-k$, where $k$ is a positive even number. By repeating the rule with $\lambda$ replaced by $-\lambda$, the number of negative real roots of (A.5) can be determined. Note that although Descartes' rule of signs predicts the number of positive roots of a polynomial, it does not provide any information about the values of the roots. 


\section{B.3 Routh-Hurwitz Criterion}

The Routh-Hurwitz stability criterion provides necessary and sufficient conditions for all the roots of a polynomial equation to be negative if they are real or have negative real parts if they are complex. We now state the Routh-Hurwitz criterion.

Consider the $n$-degree characteristic polynomial equation (A.5), and the following Hurwitz matrices constructed from the coefficients of the polynomial:

$$
\begin{gathered}
H_{1}=\left(a_{1}\right), H_{2}=\left(\begin{array}{cc}
a_{1} & 1 \\
a_{3} & a_{2}
\end{array}\right), H_{3}=\left(\begin{array}{ccc}
a_{1} & 1 & 0 \\
a_{3} & a_{2} & a_{1} \\
a_{5} & a_{4} & a_{3}
\end{array}\right), H_{4}=\left(\begin{array}{cccc}
a_{1} & 1 & 0 & 0 \\
a_{3} & a_{2} & a_{1} & 1 \\
a_{5} & a_{4} & a_{3} & a_{2} \\
a_{7} & a_{5} & a_{4} & a_{3}
\end{array}\right), \cdots, \\
H_{n}=\left(\begin{array}{ccccccc}
a_{1} & 1 & 0 & 0 & 0 & \cdots & 0 \\
a_{3} & a_{2} & 1 & 0 & 0 & \cdots & 0 \\
a_{5} & a_{4} & a_{3} & a_{2} & 1 & \cdots & 0 \\
\vdots & \vdots & \vdots & \vdots & \vdots & \vdots & \vdots \\
0 & 0 & 0 & 0 & 0 & \cdots & a_{n}
\end{array}\right) .
\end{gathered}
$$

Suppose $a_{i}>0$, where $i=1,2,3, \cdots, n$. Then $\left|H_{i}\right|>0, i=1,2,3, \cdots, n$, where the vertical bars to the left and right of the Hurwitz matrix $H_{i}$ denote, "determinant of", is a necessary and sufficient condition for all values of $\lambda$; that is, all the roots of the characteristic equation (A.5) to have negative real parts.

To construct the matrix $H_{n}$, the coefficients $a_{1}, a_{2}, a_{3}, \cdots, a_{n}$ are arranged along the leading diagonal in ascending order of subscript from the top left corner of the matrix to the bottom right corner. The columns consist of coefficients with either odd or even subscripts only, arranged in ascending order from top to bottom. Note that the entries of the columns alternate between coefficients with odd and even subscripts. For example, the first column consist of coefficients with odd subscripts, the second column consists of coefficients with even subscripts, the third column consists of elements with odd subscripts, etc. Entries with subscripts less than 0 or bigger than $n$ are set to 0 . We now state the Routh-Hurwitz 
stability criterion for the first few values of $n$, say, for $n=2,3,4,5$.

All values of $\lambda$ that satisfy the characteristic equation (A.5) have negative real parts (or lie within the left half plane) if and only if:

- for $n=2$ :

$$
\left|H_{2}\right|=\left|\begin{array}{cc}
a_{1} & 1 \\
0 & a_{2}
\end{array}\right|=a_{1} a_{2}>0
$$

or $a_{1}>0$ and $a_{2}>0$.

- for $n=3$ :

$$
\left|H_{3}\right|=\left|\begin{array}{ccc}
a_{1} & 1 & 0 \\
a_{3} & a_{2} & a_{1} \\
0 & 0 & a_{3}
\end{array}\right|=a_{3}\left(a_{1} a_{2}-a_{3}\right)>0
$$

or $a_{1}>0, a_{2}>0, a_{3}>0$ and $a_{1} a_{2}>a_{3}$.

- for $n=4$ :

$$
\left|H_{4}\right|=\left|\begin{array}{cccc}
a_{1} & 1 & 0 & 0 \\
a_{3} & a_{2} & a_{1} & 1 \\
0 & a_{4} & a_{3} & a_{2} \\
0 & 0 & 0 & a_{4}
\end{array}\right|=a_{4}\left(a_{1} a_{2} a_{3}-a_{3}^{2}-a_{1}^{2} a_{4}\right)>0
$$

or $a_{1}>0, a_{2}>0, a_{3}>0, a_{4}>0$ and $a_{1} a_{2} a_{3}>a_{3}^{2}+a_{1}^{2} a_{4}$.

- for $n=5$ :

$$
\left|H_{5}\right|=\left|\begin{array}{ccccc}
a_{1} & 1 & 0 & 0 & 0 \\
a_{3} & a_{2} & a_{1} & 1 & 0 \\
a_{5} & a_{4} & a_{3} & a_{2} & 1 \\
0 & 0 & a_{5} & a_{4} & a_{3} \\
0 & 0 & 0 & 0 & a_{5}
\end{array}\right|>0
$$

or $a_{1}>0, a_{2}>0, a_{3}>0, a_{4}>0, a_{5}>0, a_{1} a_{2} a_{3}>a_{3}^{2}+a_{1}^{2} a_{4}$ and $\left(a_{1} a_{4}-a_{5}\right)\left(a_{1} a_{2} a_{3}-\right.$ $\left.a_{3}^{2}-a_{1}^{2} a_{4}\right)>a_{5}\left(a_{1} a_{2}-a_{3}\right)^{2}+a_{1} a_{5}^{2}$. 


\section{References}

[1] H. Flanders, "Differentiation Under the Integral Sign," The American Mathematical Monthly 80, 615-627 (1973).

Online Version 313

[2] W. Kaplan, Integrals Depending on a Parameter-Leibnitz's Rule, Advanced Calculus (Addison-Wesley, Reading, MA, 1992), 4th edition.

[3] O. Hijab, Introduction to Calculus and Classical Analysis (Springer-Verlag, New York, 1997).

[4] G. Boros and V. Moll, Irresistible Integrals: Symbolics, Analysis and Experiments in the Evaluation of Integrals (Cambridge University Press, Cambridge, England, 2004). 313

[5] P. Henrici, The Rule of Descartes(in Applied and Computational Complex Analysis): Power Series-Integration-Conformal Mapping-Location of Zeros, volume 1 (Wiley, New York, 1988). 313

[6] B. Anderson, J. Jackson, and M. Sitharam, "Descartes' Rule of Signs Revisited," American Mathematical Monthly 105 (5), 447-451 (1998). 313

[7] F. R. Gantmacher, "Applications of the Theory of Matrices," Interscience 641, 1-8 (1959). 313

[8] I. S. Gradshteyn and I. M. Ryzhik, Routh-Hurwitz Theorem (in Tables of Integrals, Series, and Products) (Academic Press, San Diego, CA, 2000), 6th edition. 313 
$\overline{\text { Appendix }} \mathrm{C}$

\section{Existence and Uniqueness Theorem for}

\section{Solutions to Systems of First-order Ordinary}

\section{Differential Equations}

We state a Theorem that guarantees the existence and uniqueness of solutions to a system of first-order ordinary differential equations. We reproduce the Theorem from Ref. [1].

Theorem C.0.1 (Existence and Uniqueness). For the nth order system

$$
\dot{x}=f(x, t)
$$

suppose that $f$ is continuous and $\partial f_{j} / \partial x_{i}, i, j=1,2, \cdots, n$ are continuous for $x \in \mathscr{D}, t \in I$, where $\mathscr{D}$ is a domain and $I$ is an open interval. Then if $x_{0} \in \mathscr{D}$ and $t_{0} \in I$, there exists a solution $x^{*}(t)$, defined uniquely in some neighbourhood of $\left(x_{0}, t_{0}\right)$, which satisfies $x^{*}\left(t_{0}\right)=x_{0}$.

See Refs. [2-4] for an elaborate exploration of existence and uniqueness theorems for solutions to systems of first-order ordinary differential equations. 


\section{References}

[1] D. W. Jordan and P. Smith, Nonlinear Ordinary Differential Equations. An Introduction to Dynamical Systems (Oxford University Press, oxford, UK, 1999), 3rd edition. 317

[2] F. Brauer and J. Nohel, The Qualitative Theory of Ordinary Differential Equations: An Introduction (Dover Publications, New York, 1989). 317

[3] J. K. Hale, Ordinary Differential Equations (Dover Publications, USA, 2009).

[4] P. D. Ritger and N. J. Rose, Differential Equations with Applications (Dover Publications, USA, 2010), unabridged edition. 317 
$\overline{\text { Appendix }} \mathrm{D}$

\section{Lyapunov Functions and Lyapunov Exponents}

\section{D.1 Lyapunov Functions}

A positive definite scalar-valued function $V$ for which $\dot{V}$ is negative semi-definite or negative definite with respect to a dynamical system described by a system or ordinary differential equations constitutes a Lyapunov function for the system. That is, a function $v$ is called a Lyapunov function for a regular autonomous system of ordinary differential equations $\dot{x}=f(x)$, where $x, x^{*}, f \in \mathbb{R}^{n}$ are vectors and $x^{*}$ is an equilibrium solution of the system if it satisfies the following properties:

1. $v$ is continuous and has continuous first partial derivatives,

2. $v>0$ for all $x$ and $v=0$ only for $x=0$,

3. $\dot{v}<0$ for all $x$ and $\dot{v}=0$ only for $x=x^{*}$.

Lyapunov functions provide a sufficient but not necessary condition for assessing the stability of equilibria to dynamical systems described by systems of ordinary differential equations without necessarily solving the systems explicitly. In order to show that an equilibrium solution $x^{*}$ of a regular autonomous system of ordinary differential equations is asymptotically stable, it suffices to construct a Lyapunov function $v=v(x)$ for the system. Lyapunov functions are not unique and they can also be used for establishing global stability. Even though Lyapunov functions play a crucial role in establishing the stability of equilibrium solutions of dynamical systems, there is, however, no general or fixed approach for constructing 
them [1]. Generally, one has to approach this through trial-and-error. However, there are some documented methods for computing Lyapunov functions for certain classes of systems. For example, Ref. [2] outlines a method for computing Lyapunov functions for a linearized two dimensional system of ordinary differential equations and shows that the same function is also a Lyapunov function for the corresponding two dimensional nonlinear system. In some cases, the system at hand may furnish us with a clue on how to compute its Lyapunov function. As an example, we construct a Lyapunov function for the following system:

$$
\begin{aligned}
\dot{x_{1}} & =\mu x_{3}-\gamma x_{1}, \\
\sigma \dot{x_{2}} & =\gamma x_{1}, \\
\delta \dot{x_{3}} & =-x_{3},
\end{aligned}
$$

All of the terms on the right-hand side of system (D.1) are linear in $x_{1}$ and $x_{3}$, while $\delta, \gamma, \mu$ and $\sigma$ are positive constants. These terms are equally independent of $x_{2}$. Consequently, good Lyapunov function candidates would be functions that consist of a combination of terms in $x_{1}, x_{3}$, and $x_{1} x_{3}$, with the total power of each term even. For example,

- $v\left(x_{1}, x_{2}, x_{3}\right)=a x_{1}^{2}+b x_{3}^{2}$, where $a$ and $b$ are positive coefficients to be determined,

- $v\left(x_{1}, x_{2}, x_{3}\right)=a x_{1}^{4}+b x_{3}^{4}$, where $a$ and $b$ are positive coefficients to be determined,

- $v\left(x_{1}, x_{2}, x_{3}\right)=a x_{1}^{2}+b x_{1} x_{3}+c x_{3}^{2}$, where $a, b$ and $c$ with $4 a c-b^{2}>0$ are constants.

The third candidate $v\left(x_{1}, x_{2}, x_{3}\right)=a x_{1}^{2}+b x_{1} x_{3}+c x_{3}^{2}$, where $a, b$ and $c$ with $4 a c>b^{2}$ are constants is the best choice for system (D.1). Let $v_{y}$ denote differentiation with respect to $y$, where $y=x_{1}, x_{2}, x_{3}$ and a dot on $v, x_{1}, x_{2}$ and $x_{3}$ denote time derivatives. Then by differentiating $v$ with respect to time $t$, we obtain the following:

$$
\begin{aligned}
\dot{v}\left(x_{1}, x_{2}, x_{3}\right) & =v_{x_{1}} \dot{x}_{1}+v_{x_{2}} \dot{x}_{2}+v_{x_{1}} \dot{x}_{3} \\
& =\left(2 a x_{1}+b x_{3}\right) \dot{x_{1}}+(0) \dot{x_{2}}+\left(b x_{1}+2 c x_{3}\right) \dot{x_{3}} \\
& =\left(2 a x_{1}+b x_{3}\right)\left(\mu x_{3}-\gamma x_{1}\right)+(0)\left(\frac{\gamma}{\sigma} x_{1}\right)+\left(b x_{1}+2 c x_{3}\right)\left(-\frac{1}{\delta} x_{3}\right) \\
& =\left(\frac{2 a \mu \delta-b(\gamma \delta+1)}{\delta}\right) x_{1} x_{3}-2 a \gamma x_{1}^{2}-\left(\frac{2 c-b \mu \delta}{\delta}\right) x_{3}^{2} .
\end{aligned}
$$

Now, for $\dot{v}<0$, we must have 


$$
\begin{aligned}
& -\frac{2 a \mu \delta-b(\gamma \delta+1)}{\delta}=0 \\
& \text { - } a>0, \text { and } \\
& \text { - } \frac{2 c-b \mu \delta}{\delta}>0
\end{aligned}
$$

That is, we must choose $a, b$ and $c$ such that the three conditions above are satisfied. To this effect, we set $c=1$ and $b=1 /(\delta \mu)$. This gives $(2 c-b \mu \delta) / \delta=1 / \delta>0$ since $\delta>0$. Next, we determine $a$ from the equation $(2 a \mu \delta-b(\gamma \delta+1)) / \delta=0$. The result is $a=(\gamma \delta+1) /\left(2(\delta \mu)^{2}\right)>0$ since $\gamma>0, \forall x$ and $\delta>0$. Thus, the above conditions are satisfied if we choose $a=$ $(\gamma \delta+1) /\left(2(\delta \mu)^{2}\right)>0, b=1 /(\delta \mu)$ and $c=1$. Notice that $4 a c-b^{2}=(2 \gamma \delta+1) /(\delta \mu)^{2}>0$ and that $v$ satisfies conditions (1)-(3) above. Therefore, the following function is a Lyapunov function for system (D.1):

$$
v\left(x_{1}, x_{2}, x_{3}\right)=\frac{\gamma \delta+1}{2(\delta \mu)^{2}} x_{1}^{2}+\frac{1}{\delta \mu} x_{1} x_{3}+x_{3}^{2} .
$$

See the proof of Theorem 3.3.7 for an example on how to compute Lyapunov functions for epidemiological models and Refs. [3-5] for more on Lyapunov functions.

\section{D.2 The Largest Lyapunov Exponent}

Largest Lyapunov exponents provide a quantitative measure of chaotic behavior of dynamical systems. They measure the average exponential rate at which trajectories that originate close to each other either diverge from or converge to each other over time. Chaotic behavior is governed by a positive largest Lyapunov exponent, while periodic behavior is characterized by a zero largest Lyapunov exponent. Positivity of the largest Lyapunov exponent indicates that any two nearby trajectories separate exponentially in the long term. In this case, the system exhibits sensitive dependence on initial conditions. This results to a complete loss of information about the initial data in the long term [6]. Negative Lyapunov exponents are common among asymptotically stable dissipative systems and denote stable equilibrium points or stable limit cycles.

Consider the following autonomous system of ordinary differential equations:

$$
\dot{x}=f(x),
$$


where $x, f \in \mathbb{R}^{n}$. Let $x\left(t_{0}\right)=x_{0}$ be the vector of initial values of $x$, then Theorem C.0.1 provides conditions under which system (D.2) can have a unique solution, say, $x(t)=F(x, t)$. However, our interested here resides in the long-term asymptotic behavior of $d F$; that is, the differential of the solution $F$. We perturb the system about a reference trajectory, say $x^{*}$ by setting $x=x^{*}+u$, where $x^{*}, u \in \mathbb{R}^{n}$ and $\|u\| \ll\left\|x^{*}\right\|$. Substituting in system (D.2), expanding in a Taylor series about $x^{*}$, and retaining only linear terms in $u$ yields the following linear system:

$$
\dot{u}=\left(\begin{array}{cccc}
\frac{\partial f_{1}}{\partial x_{1}} & \frac{\partial f_{1}}{\partial x_{2}} & \cdots & \frac{\partial f_{1}}{\partial x_{n}} \\
\frac{\partial f_{2}}{\partial x_{1}} & \frac{\partial f_{2}}{\partial x_{2}} & \cdots & \frac{\partial f_{2}}{\partial x_{n}} \\
\vdots & \vdots & \ddots & \vdots \\
\frac{\partial f_{n}}{\partial x_{1}} & \frac{\partial f_{n}}{\partial x_{2}} & \cdots & \frac{\partial f_{n}}{\partial x_{n}}
\end{array}\right)_{x=x^{*}} u .
$$

Let $u\left(t_{0}^{*}\right)=u_{0}$ be the initial conditions of the linearized system (D.3), then the system has a unique solution $\mathrm{u}(\mathrm{t})$ that can be expressed in terms of the solution of the original system as follows:

$$
u(t)=F\left(t, t_{0}^{*}\right) u_{0}
$$

where $F\left(t, t_{0}^{*}\right)$ is a matrix obtained by linearizing the solution of system (D.2). If

$$
\lim _{t \rightarrow \infty} \frac{1}{t} \ln \left\|F\left(t, t^{*}\right)\right\|<\infty
$$

then the largest Lyapunov exponent of the system is given by

$$
\lambda_{L}=\lim _{t \rightarrow \infty} \frac{1}{t} \ln \left(\frac{\|u(t)\|}{\left\|u_{0}\right\|}\right) .
$$

See [7] for details on how to calculate the largest Lyapunov exponents. 


\section{References}

[1] H. Khalil, Nonlinear Systems (Prentice Hall, Englewood Cliffs, NJ, 2002), 3rd edition. 320

[2] D. W. Jordan and P. Smith, Nonlinear Ordinary Differential Equations. An Introduction to Dynamical Systems (Oxford University Press, oxford, UK, 1999), 3rd edition. 320

[3] F. Brauer and J. A. Nohel, The Qualitative Theory of Ordinary Differential Equations: An Introduction (Dover, New York, 1989). 321

[4] D. Zwillinger, Liapunov Functions: In Handbook of Differential Equations (Academic Press, Boston, MA, 1997), 3rd edition.

[5] M. Malisoff and F. Mazenc, Constructions of Strict Lyapunov Functions, Communications and Control Engineering (Springer-Verlag, London, 2009). 321

[6] D. Ruelle, "Sensitive Dependence on Initial Conditions and Turbulent Behavior of Dynamical Systems," Annals of the New York Academy of Sciences 317, 408-416 (1979). Online Version 321

[7] S. Rugonyi and K.-J. Bathe, "An Evaluation of the Lyapunov Characteristic Exponent of Chaotic Continuous Systems," International Journal of Numerical Methods in Engineering 56, 145-163 (2003).

Online Version 322 
Appendix E

\section{LaSalle's Invariance Principle}

LaSalle's invariance principle is an extension of Lyapunov's method and it is useful in proving global asymptotic stability for continuous and discrete dynamical systems. It uses the notions of limit sets and invariance to define Lyapunov functions in a less restrictive manner. For example, LaSalle's invariance principle eliminates the strict requirement that a Lyapunov function $V$ for a continuous dynamical system that is described by an autonomous system of ordinary differential equations must have a negative definite time derivative for the system to be asymptotically stable.

Definition E.0.1 (Invariant set). A set $\Psi$ is said to be invariant with respect to the system of ordinary differential equations $\dot{x}=f(x)$, if all trajectories $x(t)$ that originate from the set do not leave the set $\Psi$ at any time.

Theorem E.0.2 (LaSalle's invariance principle). Suppose the set $\Psi \subset D$ is compact and positively invariant with respect to the system of ordinary differential equations $\dot{x}=f(x)$. Suppose further that the function $V$ defined by $V: D \rightarrow \mathbb{R}$ is continuously differentiable and

that $\dot{V} \leq 0$ on $\Psi$. If $E=\{x \in \Psi: \dot{V}(x)=0\}$ and $E^{*}$ is the largest invariant set in $E$, then every solution of the system of ordinary differential equations $\dot{x}=f(x)$ that begins in the set $\Psi$ approaches $E^{*}$ as time $t$ approaches infinity.

See Refs. [1-6] for more on LaSalle's invariance principle. 


\section{References}

[1] J. P. LaSalle, "Some Extensions of Liapunov's Second Method," IRE Transactions on Circuit Theory CT-7, 520-527 (1960). 324

[2] N. N. Krasovskii, Problems of the Theory of Stability of Motion (Stanford University Press, Stanford, CA, 1963).

[3] J. P. LaSalle, The Stability of Dynamical Systems (SIAM, Philadelphia, PA, 1976).

[4] G. Chen, J. Zhou, and S. Celikovsky, "On LaSalle's Invariance Principle and its Application to Robust Synchronization of General Vector Liénard Equations," Automatic Control, IEEE 869-874, 50 (6) (2005).

Online Version

[5] H. Shim and J. H. Seo, "Improving LaSalle's Invariance Principle using Geometric Clues," SICE-ICASE, International Joint Conference 5253-5255 (2007).

Online Version

[6] F. Mazenc and M. Malisoff, "Strict Lyapunov Function Constructions Under LaSalle Conditions With an Application to Lotka-Volterra Systems," Automatic Control, IEEE Transactions on 55, 841-854 (2010).

Online Version 324 
$\overline{\text { Appendix }} \mathrm{F}$

\section{Third Hands-On Research in Complex Systems Advanced Study Institute: University of Buea, Cameroon, August 2-13, 2010}

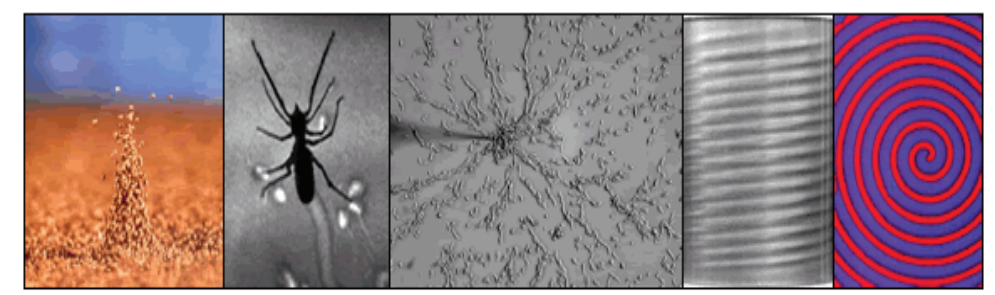

HANDS-DN RESEARCH IN COMPLEX SYSTEMS SCHOOL http: //www . handsonresearch.org/

This Appendix consists of teaching material I prepared for the Third Hands-On Research in Complex Systems Advanced Study Institute that took place at the University of Buea, Cameroon from August 2-13, 2010. The audience was composed of outstanding graduate students and young faculty selected from developing countries. The hands-on sessions ran for three hours each day. My session received much attention and participants requested extended three-hour sessions almost on a daily basis. The Directors of the Institute were Professors Rajarshi Roy (University of Maryland), Kenneth Showalter (West Virginia University), and Harry L. Swinney (University of Texas) and the local organizers were Dr. Josepha Foba and Dr. Gideon Ngwa (University of Buea, Cameroon). See the next page for the sponsors of the Institute, and the website http://www.handsonresearch.org/for more information on the Institute and for information on past and future Hands-On Schools. 


\section{Hands-On Research School in Cameroon (2010):}

participants received full travel \& subsistence support

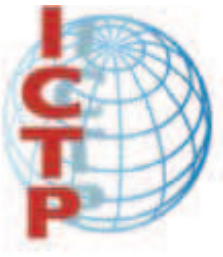

ICTP/UNESCO through the International Centre for Theoretical Physics

(Trieste, Italy)
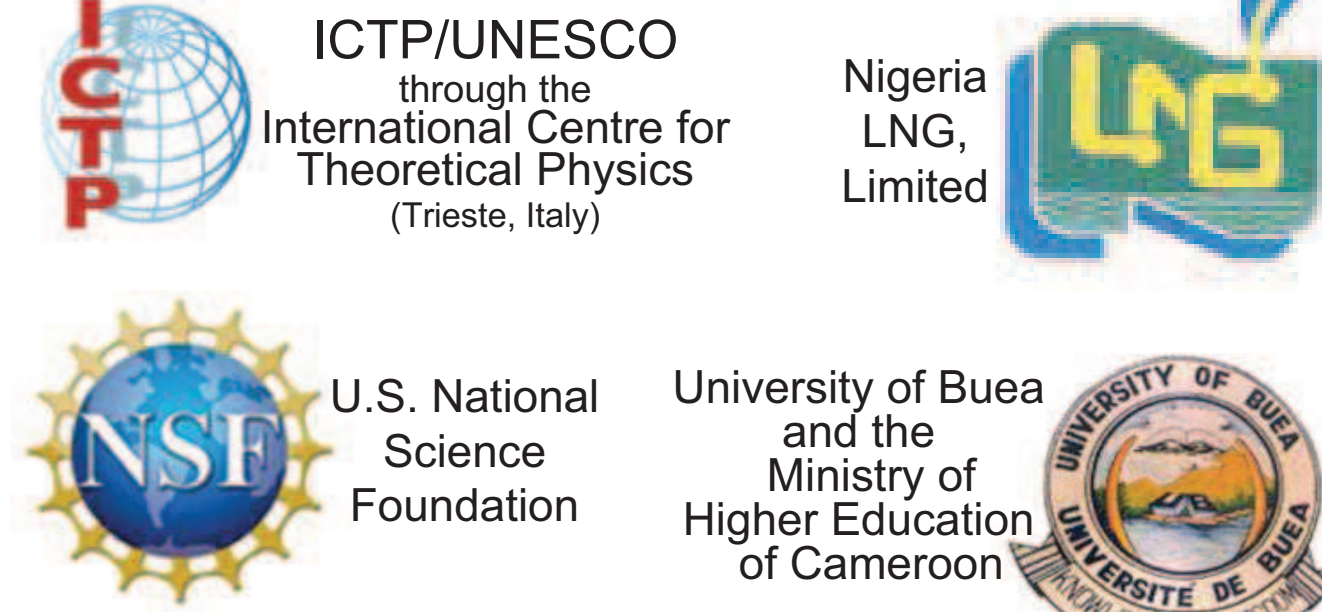

University of Buea and the Ministry of Higher Education of Cameroon

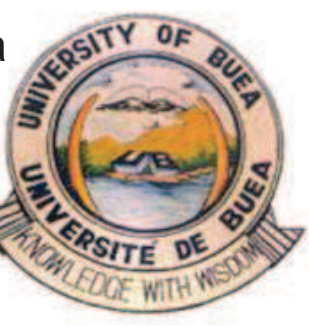

http://www.handsonresearch.org/pdf/2010-handsonresearch-story.pdf

\section{Communicable Diseases Session}

Dr Gideon A. Ngwa (University of Buea, Cameroon), assisted by Calistus N. Ngonghala and Professor Kenneth Showalter (West Virginia University), Dr. Fidelis Cho-Ngwa (University of Buea, Cameroon) and Henry Dilonga (University of Buea, Cameroon)

\section{F.1 Session Description Summary}

Communicable diseases are carried by microorganisms and are diseases that can be communicated between persons. Examples of communicable diseases include malaria, HIV/AIDS, ringworm, and intestinal worms (Ascaries, Taenia, etc.). These diseases are called communicable because the infection can be "communicated" just as information can be communicated from one person to another. A human-to-human communicable disease could be passed through blood, mucus, uterine fluid, breast milk, semen, saliva, or breath. Question: 
Can money harbor pathogens and thus serve as a communicable disease vector? It is well known that money is handled by persons of varying health and hygienic standards and also stored under varying environmental and personal hygienic conditions. Thus the chances of pathogens being present on money and persisting through multiple handling are high.

\section{F.1.1 Theoretical and Experimental Part}

In the first part of the session, participants will have the opportunity to carry out simple microscopic examinations to establish the existence of microorganisms (and the possible types) that can persist on the surfaces of money (coins and notes). In the second part, participants will learn how to develop simple mathematical models for indirectly transmitted diseases of humans. In the third part, participants will have the opportunity to formulate the hypotheses and mechanisms that may be useful in proposing mathematical models to assess the efficacy of money as a vector for communicating diseases between humans and then using MATLAB to analyze these models.

\section{F.1.2 Simulation Introduction}

Participants will explore the existence and stability of equilibrium solutions to an SIR disease model with and without vital dynamics using standard analytical techniques. MATLAB codes for an SIR disease model will be provided: The first two codes are for a deterministic SIR model with and without births and deaths (or vital dynamics). The third code will be for a stochastic version of an SIR disease model. The fourth code will be a deterministic SIR infectious disease model with a periodic contact rate. Participants will vary the contact rate to observe different dynamical behaviors, ranging from a stable steady state to a period-doubling cascade. A C code for an SEIRS model for endemic malaria from Ref. [1], which incorporates a spatial component, will be examined. Participants will use the built-in C compiler in MATLAB to compile and execute this code. They will then vary system parameters and diffusion coefficients to observe their effects on disease prevalence and the wave front of an infection process propagating through the community. 


\section{F.2 Detailed Session Description}

\section{F.2.1 Deterministic SIR Model}

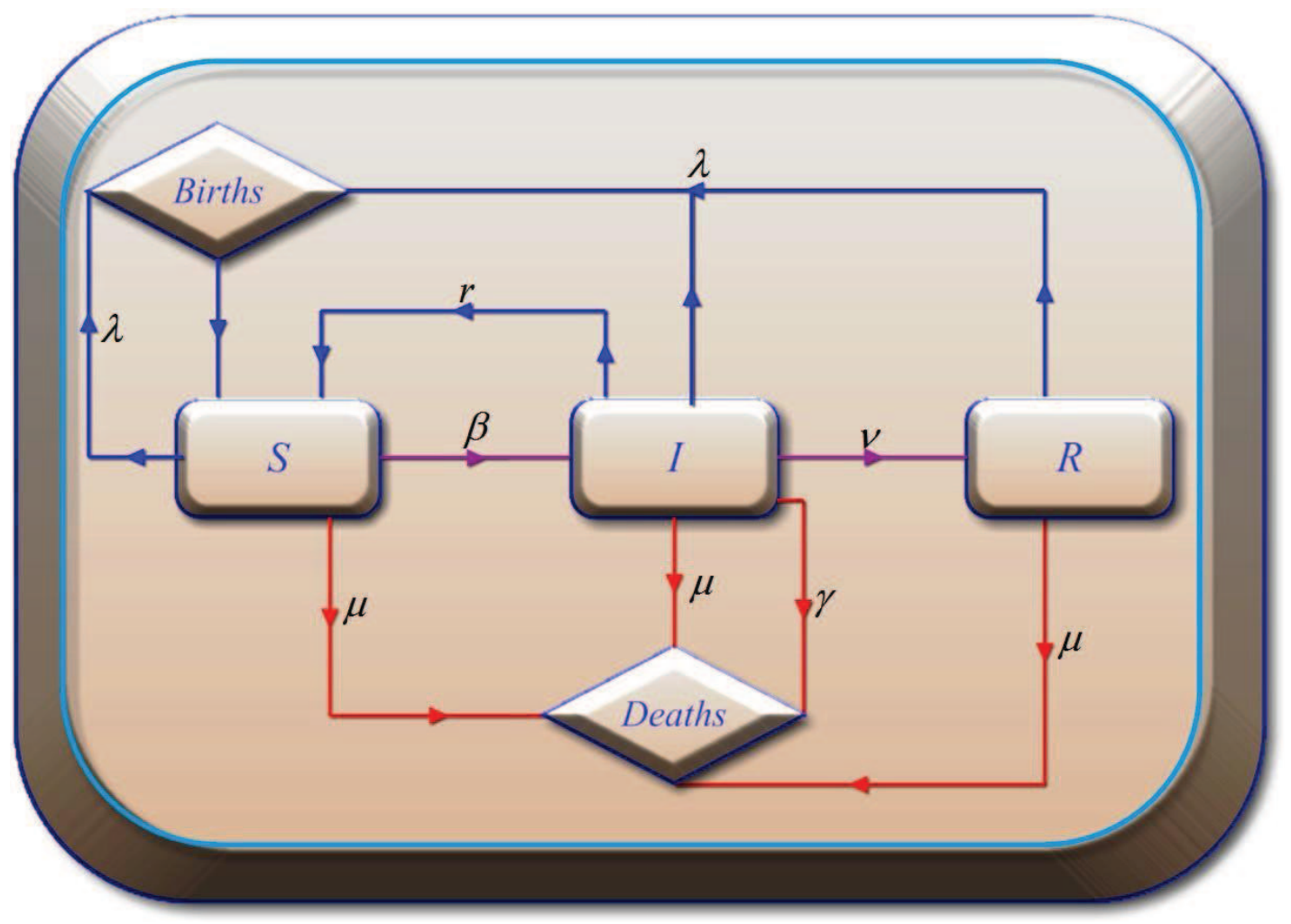

Figure F.1. A schematic framework illustrating the flow of individuals between the susceptible, infectious and immune classes. At any time, the total population is $N=S+I+R$.

Deterministic models are described by ordinary or partial differential equations. Predictions of system behavior can be obtained from the solutions of the system if such solutions can be computed explicitly, or via a stability analysis, if closed form solutions can not be found. These models are preferred when the population size under consideration is large.

To obtain the classical SIR model we simulate here, the total population is divided into susceptible individuals (those who have not contracted the disease), infectious individuals (those who have contracted the disease and can transmit it to others), and removed or immune individuals. The model is described by the following system of differential equations: 


$$
\begin{aligned}
\frac{d S}{d t} & =\mu N-\beta S I-\mu S \\
\frac{d I}{d t} & =\beta S I-(\mu+\nu) I \\
\frac{d R}{d t} & =\nu I-\mu R
\end{aligned}
$$

where $\mu$ is the birth/natural death rate, $\beta$ is the contact rate, and $\nu$ is the removal rate. The basic reproduction number is

$$
R_{0}=\frac{\beta N}{\mu+\nu}
$$

The threshold parameter, $R_{0}$ determines the existence and stability of equilibrium solutions

to the system. When $R_{0} \leq 1$, there exists a stable disease free equilibrium, representing the situation in which the disease dies out, and when $R_{0}>1$, there exists a stable endemic equilibrium, indicating that the disease establishes itself in the community.

\section{F.2.1.1 Exercise}

1. Compute the equilibrium solutions of system (F.1).

2. Verify that the basic reproduction number $R_{0}$ presented above is correct.

3. Determine the linear stability of the equilibrium solutions computed above.

4. Verify whether the equilibrium solutions are globally asymptotically stable or not.

\section{F.2.1.2 Simulating the Deterministic Model with no Vital Dynamics}

By no vital dynamics, we are referring to the situation in which there are no births and deaths; i.e., $\mu=0$. The MATLAB code SIRNoVitalDyn.m is provided for the simulations.

1. Run the code by typing SIRNoVitalDyn in the command prompt and hitting "return".

2. Vary the parameters and observe their effects on disease prevalence. 


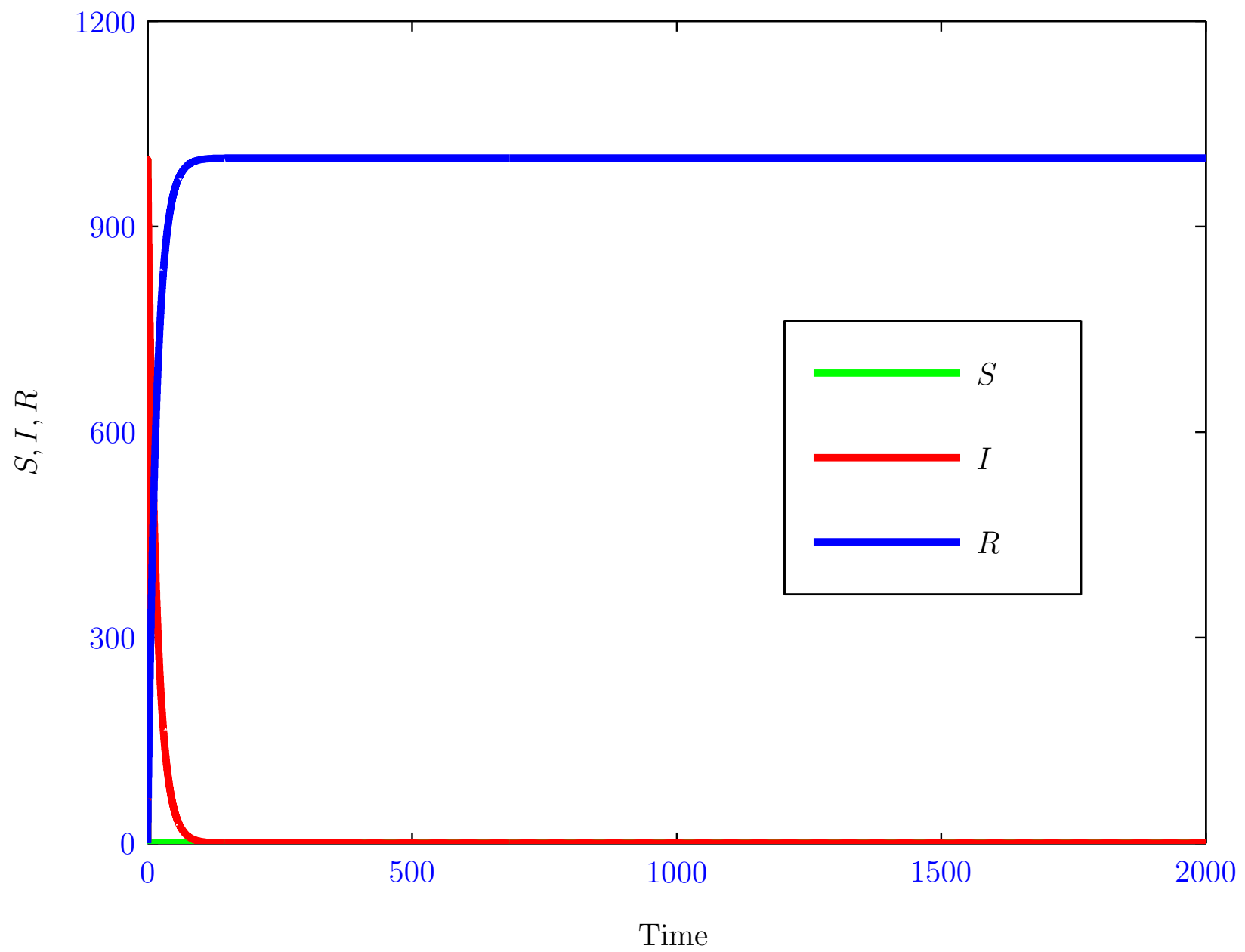

Figure F.2. Simulation results with $\mu=0$.

\section{F.2.1.3 Simulating the Deterministic Model with Vital Dynamics}

We now simulate the entire system, taking into consideration births and deaths. That is, we consider the situation in which $\mu \neq 0$. To this effect, the MATLAB code SIRVitalDyn.m is provided. See Fig. F.3 for a sample time series and phase plot.

\section{Exercise}

1. Vary the parameters to determine their effects on both $R_{0}$ and disease prevalence.

2. Verify the linear stability results for the cases $R_{0} \leq 1$ and $R_{0}>1$. 


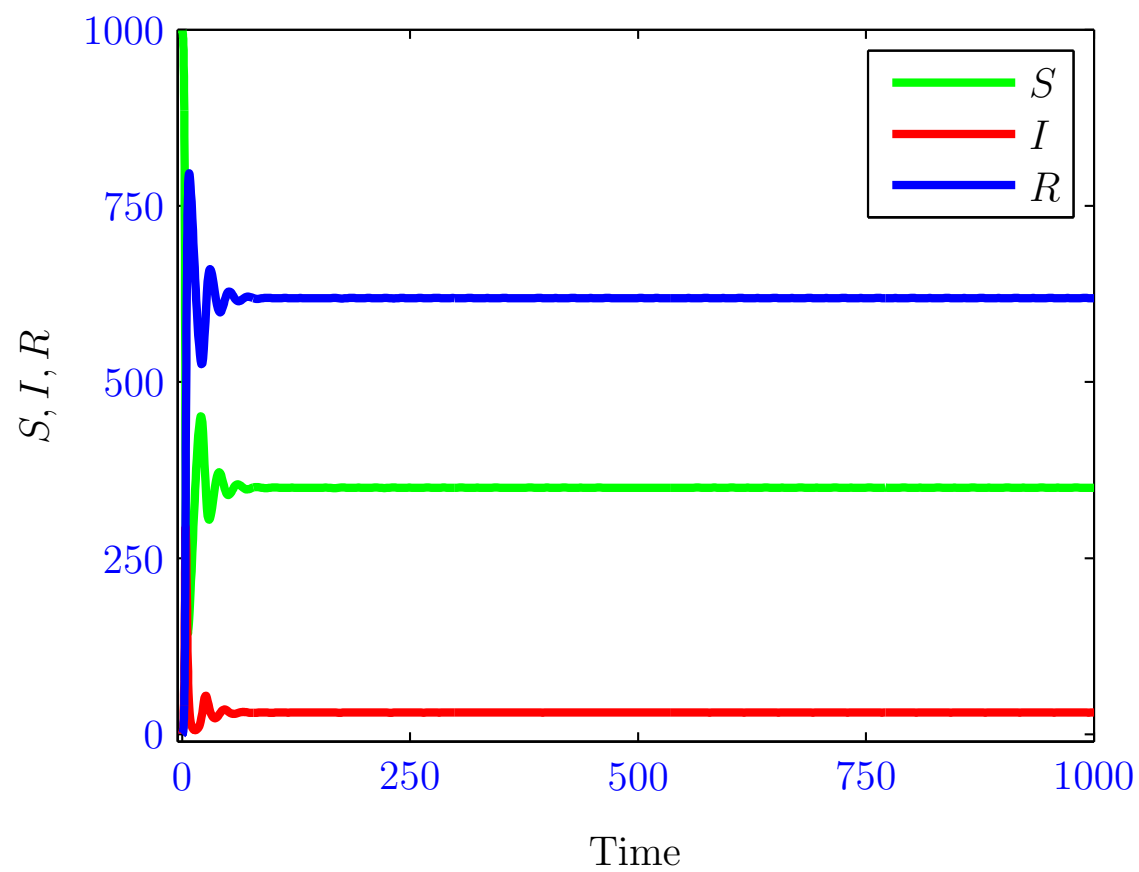

F.3.1. Time series

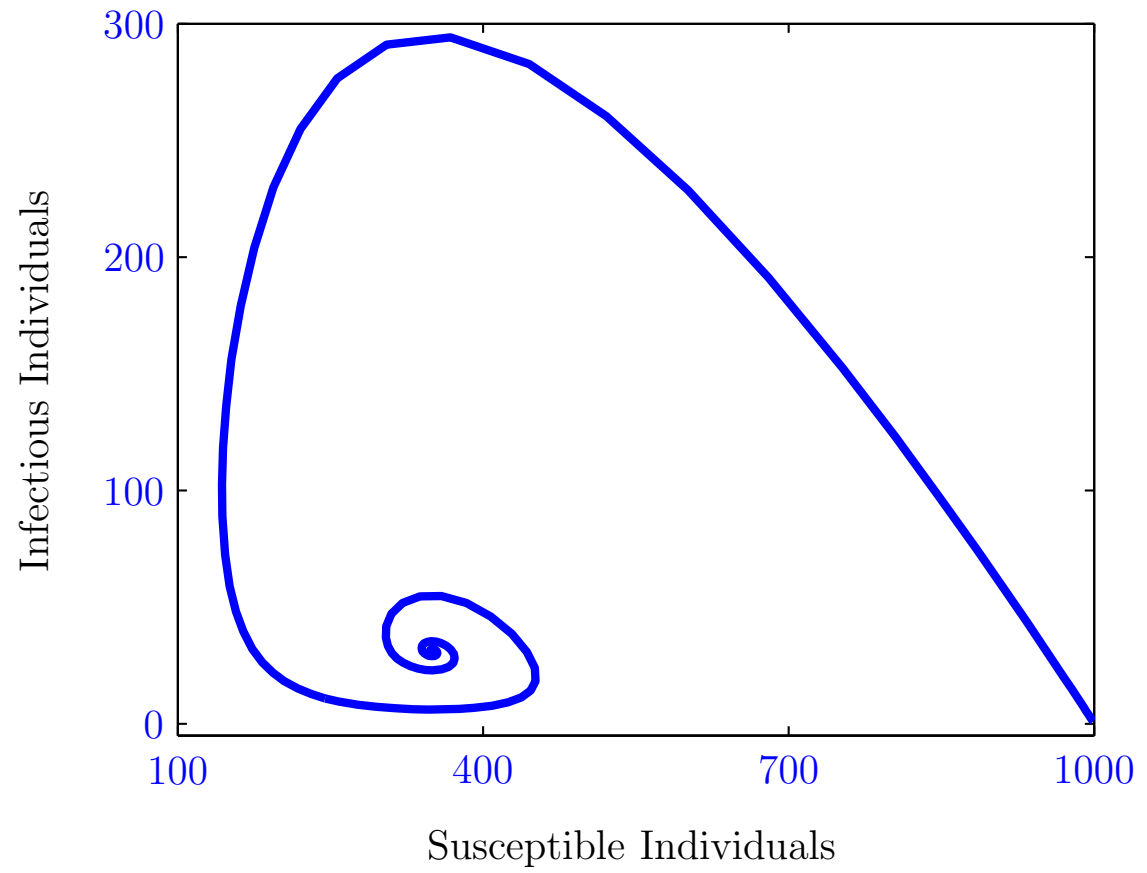

F.3.2. Phase plot

Figure F.3. Solution of system with vital dynamics 


\section{F.2.2 SIR Model with Periodic Contact Rate}

\section{F.2.2.1 Background}

The prevalence of some diseases vary with seasonality and so the dynamics may exhibit oscillations. For instance, the prevalence of contagious diseases of children of school age is usually higher during the school term than during holidays. The dynamics of some communicable diseases of humans such as malaria vary with seasonality. This is due to variations in the mosquito population dynamics. One way of capturing such oscillations is by incorporating periodic contact rates as in Ref. [2]. See Refs. [3, 4] for other techniques of capturing periodicity in epidemiological models. Here, we re-examine the SIR model from Section F.2.1. As in Ref. [2], we consider a periodic contact rate given by $\beta(t)=\beta_{0}\left(1+\beta_{1} \cos 2 \pi t\right)$ where $B_{0}$ and $\beta_{1}$ are positive constants.

\section{F.2.2.2 Simulations}

The MATLAB script, SIRPeriodic.m is provided. SIRPeriodic.m integrates the system using a fourth-order Runge Kutta method and produces both a time series and a phase plot of the results. This script can be run by typing "SIRPeriodic" at the MATLAB command prompt and hitting "return". A preliminary run with the parameters $\mu=0.02, \nu=100, \beta_{0}=$ $1800, \beta_{1} \in\{0.0,0.02,0.08\}$ and initial conditions $S_{0}=0.0556$ and $I_{0}=0.001$ provides the output plotted in Figs. F.4 and F.5. 


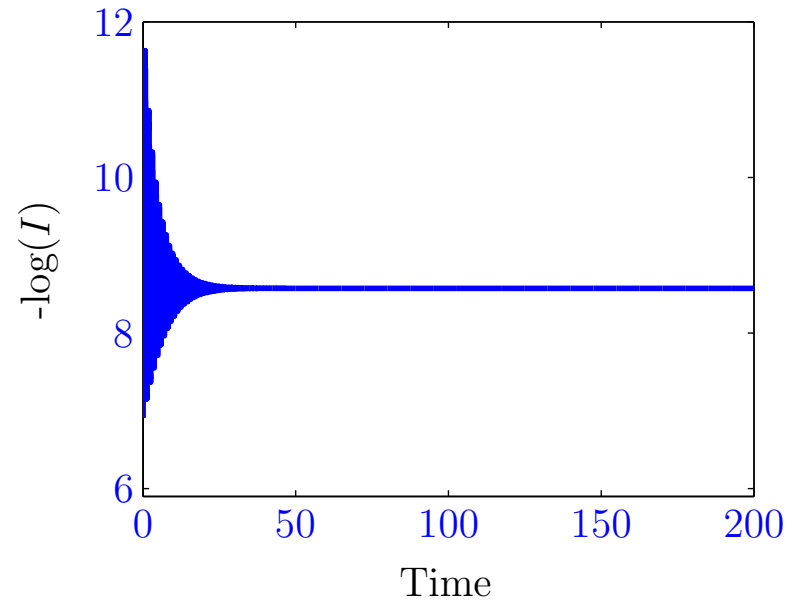

F.4.1. Stable equilibrium solution.

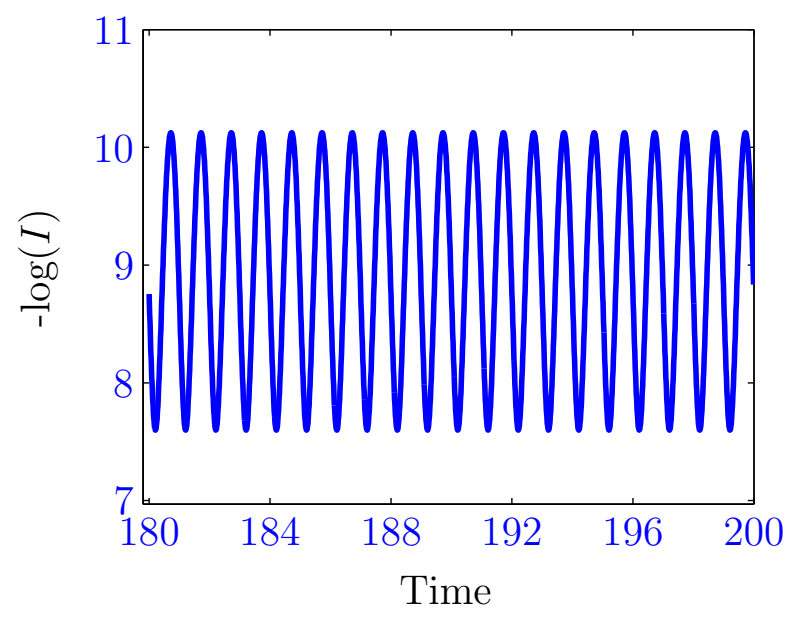

F.4.3. Time series showing period-1.

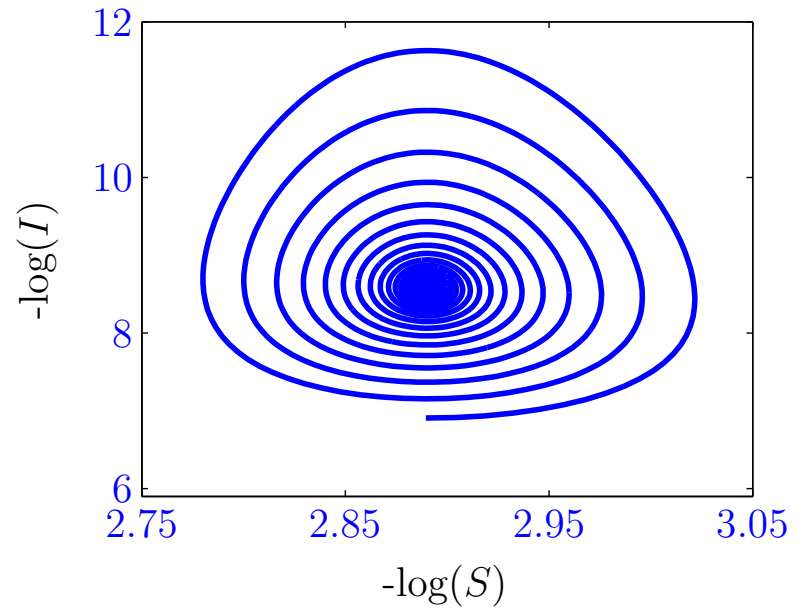

F.4.2. Stable spiral.

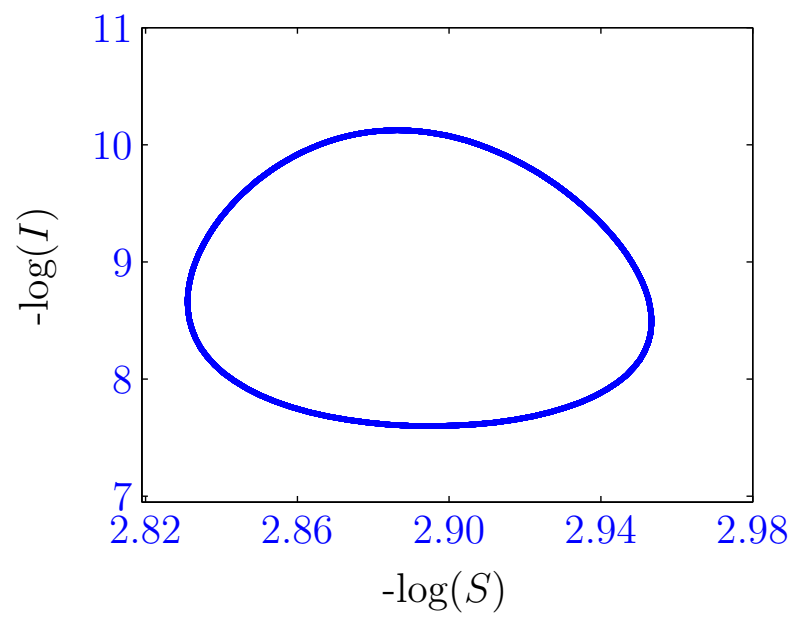

F.4.4. Limit cycle.

Figure F.4. Typical dynamical behaviors for different values of $\beta_{1}$. Stable spiral when $\beta_{1}=0.0$ and period-1 behavior when $\beta_{1}=0.02$. 


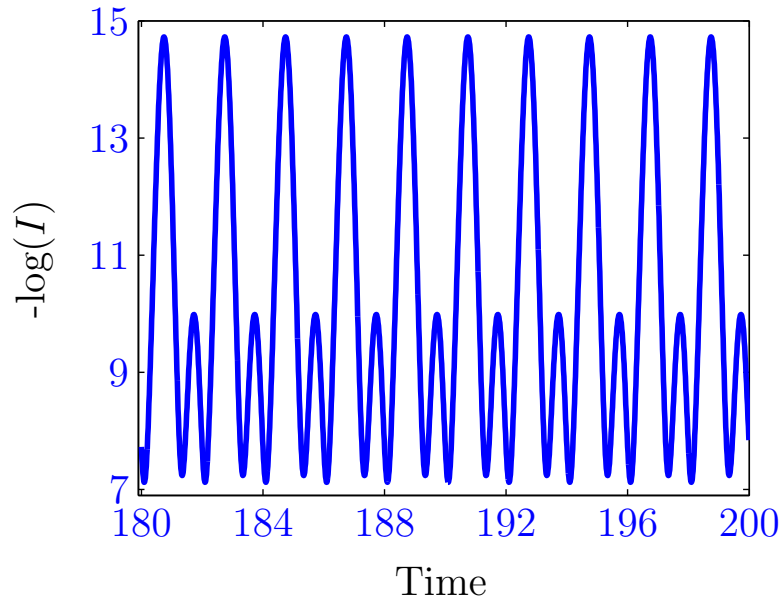

F.5.1. Time series showing period-2.

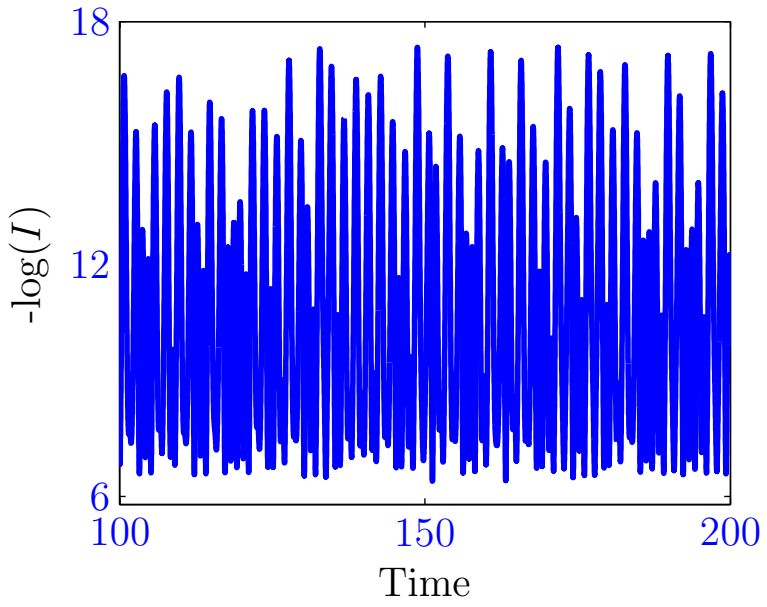

F.5.3. Time series showing chaos.

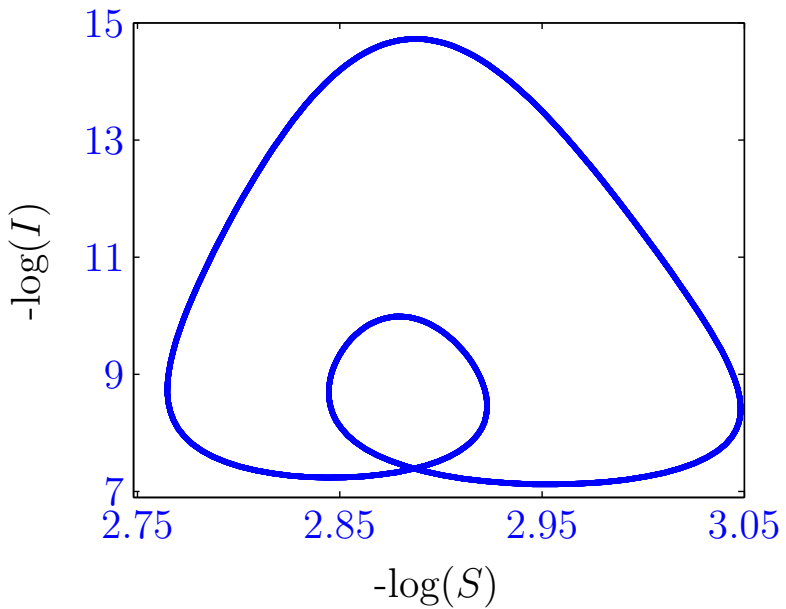

F.5.2. Phase plot showing Period-2.

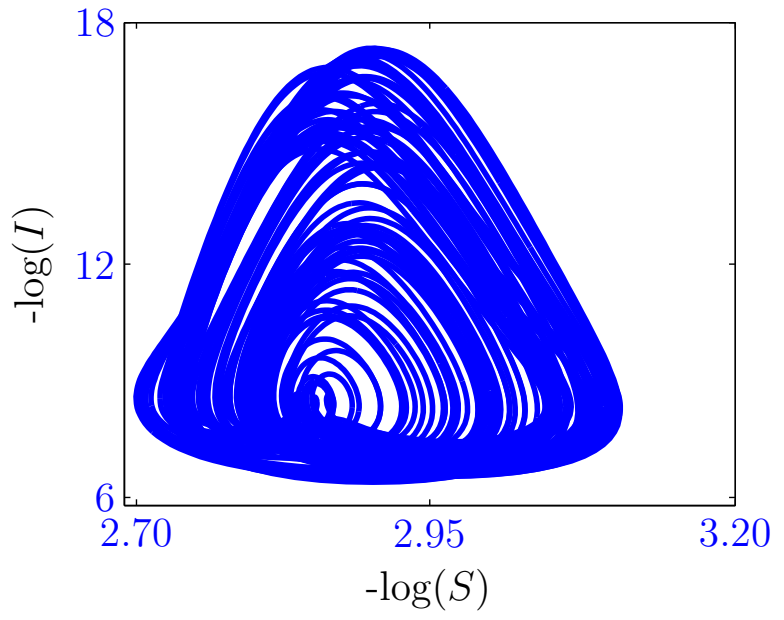

F.5.4. Chaotic attractor.

Figure F.5. Typical dynamical behaviors for different values of $\beta_{1}$. Period-2 oscillations for $\beta_{1}=0.08$ and chaotic behavior for $\beta_{1}=0.095$. 


\section{F.2.2.3 Exercise}

1. Fix all system parameters and vary only the contact rate by varying the parameter $\beta_{1}$ to observe different dynamical behaviors, ranging from stable steady state behavior through a period-doubling cascade.

2. Fix the contact rate and vary the other system parameters.

3. Determine the parameter regimes for which $R_{0}<1, R_{0}=1$ and $R_{0}>1$, and investigate the consequences on disease prevalence.

\section{F.2.3 Stochastic SIR Model}

\section{F.2.3.1 Model Description}

We now consider a stochastic version of the deterministic SIR model above. As in deterministic models, variables are also used to denote states in stochastic models. However, the transition rates between these states are probabilities instead of simple reaction or flow rates as in deterministic models. In general, a deterministic ordinary differential equation model can only give an approximation of the corresponding stochastic model. Stochastic models are usually computationally more expensive than deterministic models; however, they are more appropriate when the population size under consideration is small. With the same set of initial data, stochastic models can yield different outcomes due to fluctuations. 


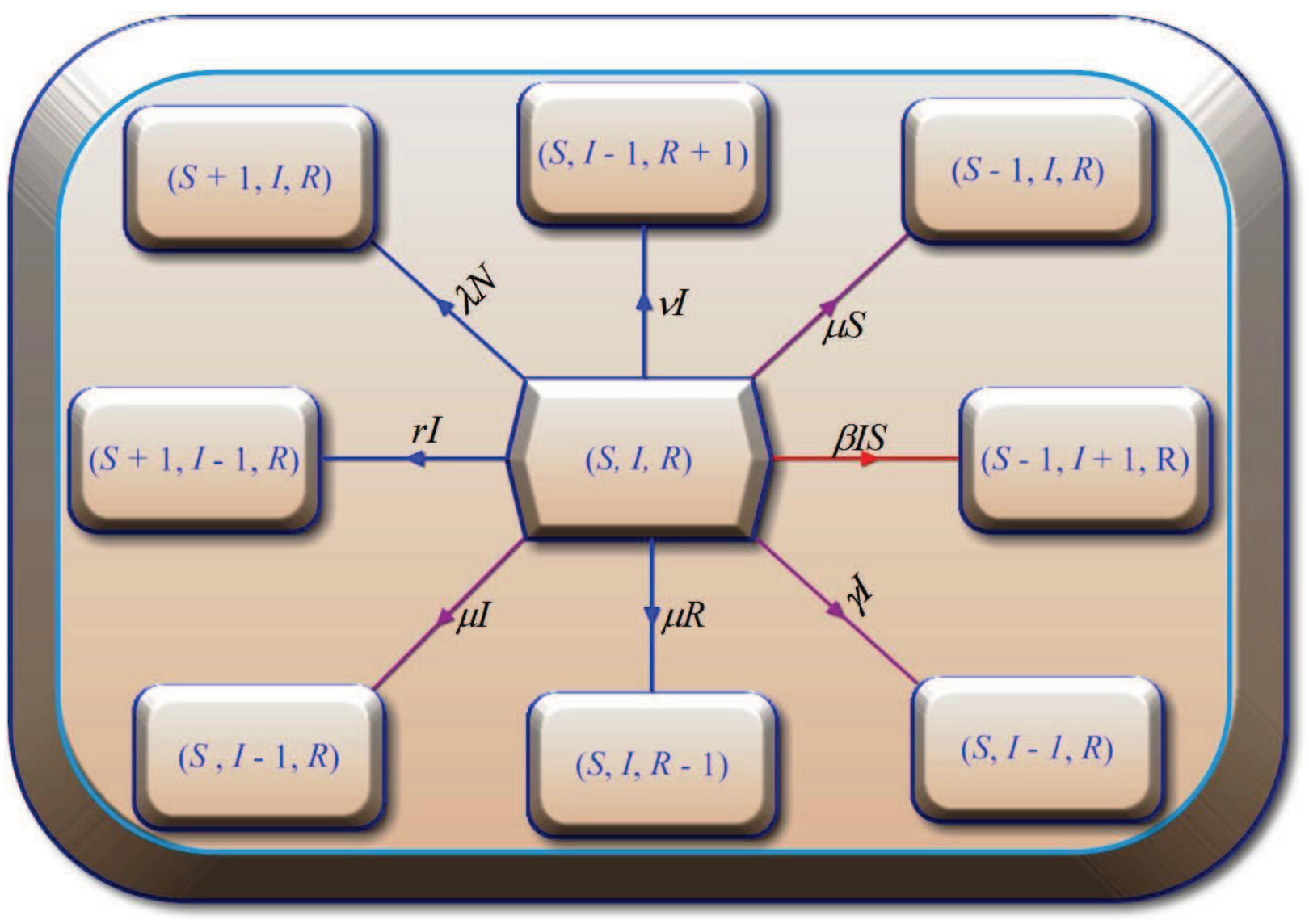

Figure F.6. A schematic framework illustrating the states to which individuals can evolve. The labels along the arrows are the rates.

The flow chart in Fig. F.6 represents a conceptual framework of the stochastic model. Starting from a state with susceptible, infectious and immune individuals, there are eight states, $(S+1, I, R),(S-1, I+1, R),(S+1, I-1, R),(S-1, I, R),(S, I-1, R),(S, I-1, R)$, $(S, I-1, R+1)$, and $(S, I, R-1)$ to which the system can evolve at different rates. Notice that the state $(S, I-1, R)$ is repeated since an infectious individual can either die naturally or as a result of the disease. Also notice that the ratio of the rates to the sum of the rates are effectively the probabilities for the events: birth, infection, recovery, natural death of a susceptible individual, natural death of an infectious individual, disease-induced death of an infectious individual, immunity and natural death of an immune individual along the arrows. That is, the transition probabilities or the probability that a specific event occurs is the ratio of the rate at which the event occurs to the sum of all the rates. 
Table F.1. Summary of events and transition probabilities. The sum of the rates is given by $\Gamma=\lambda N+\beta S I+r I+\mu S+\mu I+\gamma S+\mu R+\nu I$.

\begin{tabular}{||l|l|c|c||}
\hline \hline Event & State & Rate & Probability \\
\hline \hline Birth & $S+1, I, R$ & $\lambda N$ & $\lambda N / \Gamma$ \\
\hline Infection & $S-1, I+1, R$ & $\beta S I$ & $\beta S I / \Gamma$ \\
\hline Recovery & $S+1, I-1, R$ & $r I$ & $r I / \Gamma$ \\
\hline Natural death of a susceptible individual & $S-1, I, R$ & $\mu S$ & $\mu S / \Gamma$ \\
\hline Natural death of an infectious individual & $S, I-1, R$ & $\mu I$ & $\mu I / \Gamma$ \\
\hline Disease-induced death of an infectious individual & $S, I-1, R$ & $\gamma S$ & $\gamma S / \Gamma$ \\
\hline Natural death of an immune individual & $S, I, R-1$ & $\mu R$ & $\mu R / \Gamma$ \\
\hline Immunity & $S, I-1, R+1$ & $\nu I$ & $\nu I / \Gamma$ \\
\hline \hline
\end{tabular}

\section{F.2.3.2 Simulation of the Stochastic Model}

We numerically simulate the model using the Gillespie Algorithm ${ }^{1}$ [7-13]. Figure F.7 depicts the results for four simulations. Notice that, unlike the deterministic model that gives the same result for the same set of parameters and initial conditions irrespective of the number of runs, the outcomes of the stochastic model can be different for different runs with the same initial conditions and parameters. The MATLAB script StochasticSIR.m is used and can be run by typing "StochasticSIR" at the command prompt and hitting "return".

\footnotetext{
${ }^{1}$ The Gillespie algorithm simulates random discrete events and the time elapsed between such events. The crux of the algorithm resides on drawing two numbers at each step - one to identify the time that will elapse before the next event occurs and the other to select which event occurs next. The algorithm proceeds in the following simple steps (c.f. Refs. $[5,6])$ :

(i) Initialization of system variables, parameters, and random number generator.

(ii) Determination of the propensity functions and their sum.

(iii) Selection of the next event and the time interval through the aid of randomly generated numbers.

(iv) Updating the time step and state variables.

(v) Return to to step (ii) if the allocated final time has not been attained or if the variables are not zero.
} 


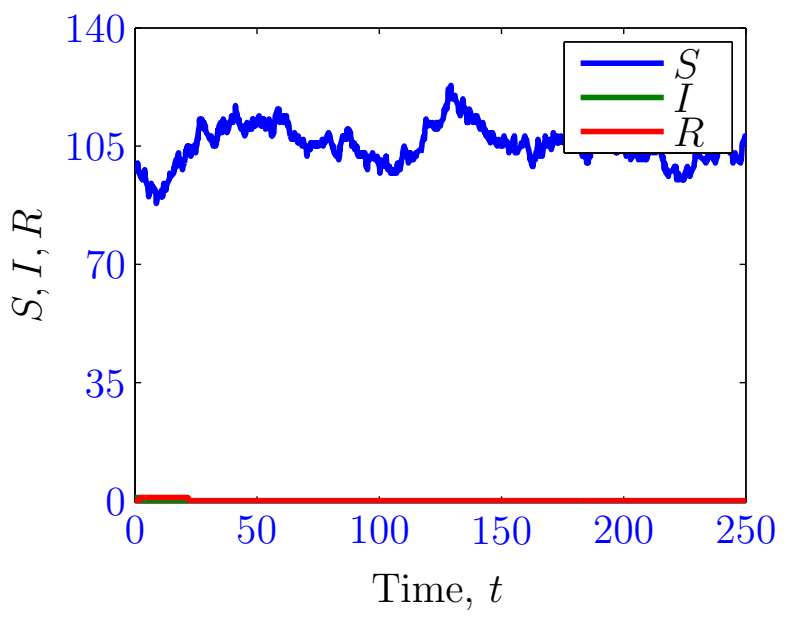

F.7.1. Run number 1 . The single infectious individual dies leaving a disease-free community.

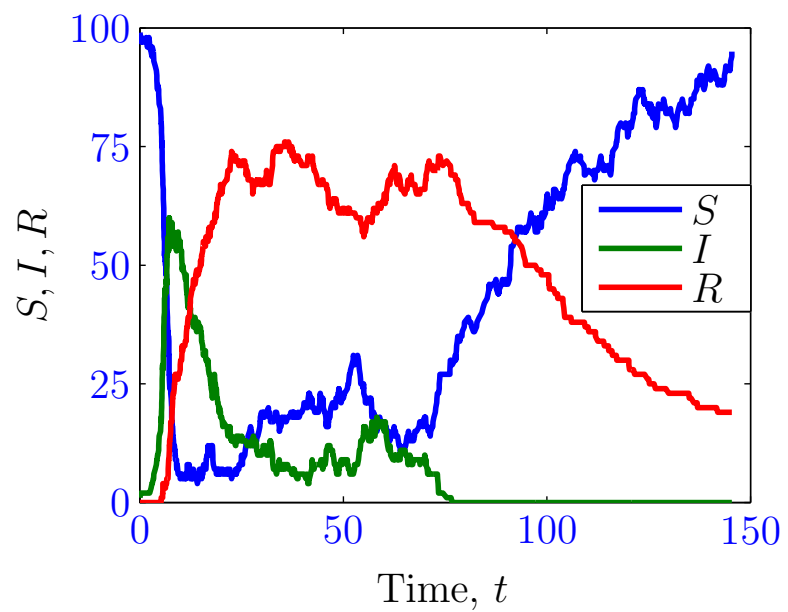

F.7.3. Run number 3. Disease dies out.

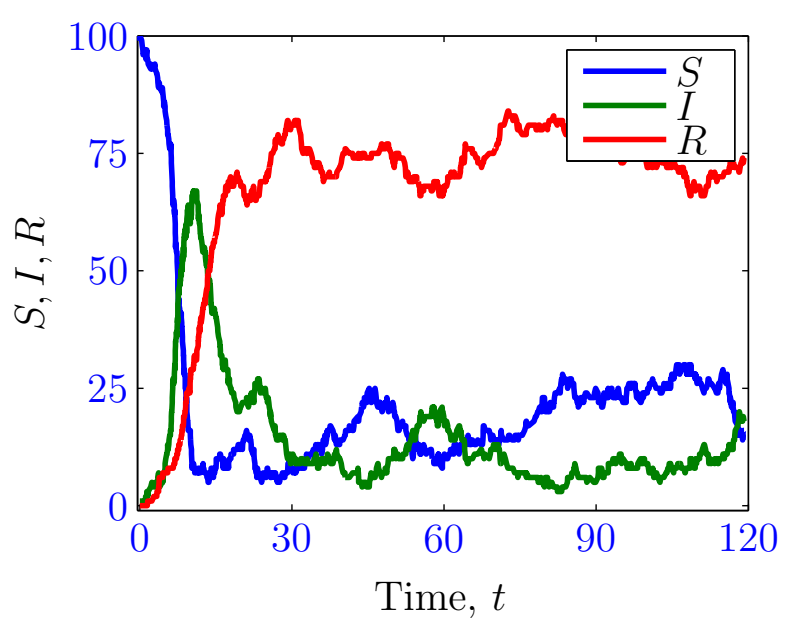

F.7.2. Run number 2. Disease persists in the community.

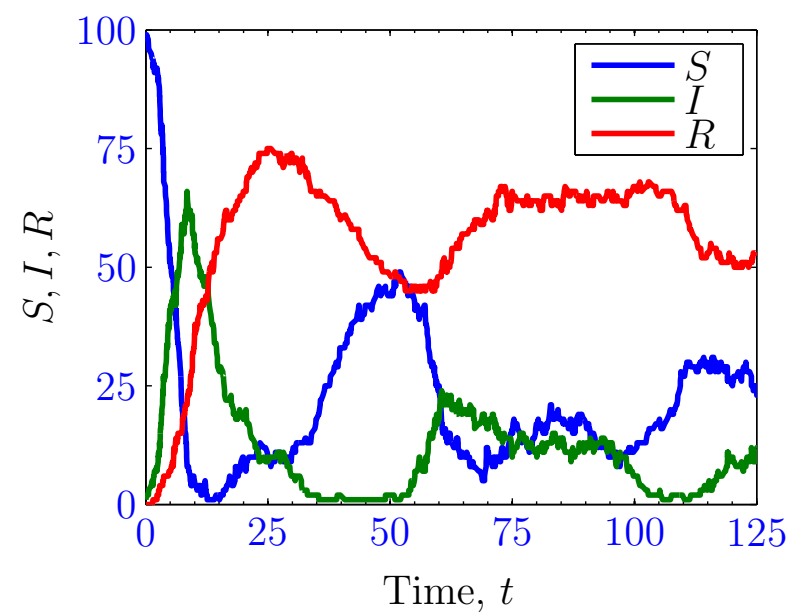

F.7.4. Run number 4 .

Figure F.7. Plots for different runs with the same parameter values, initial conditions and the same noise seed showing different behaviors. The parameter values are $\beta=0.03, \nu=0.05, \gamma=0.01, r=0.1, \mu=0.02$. We consider a total population of 100 and assume that 99 of these individuals are susceptible initially, 1 is infectious and none is immune. 


\section{F.2.3.3 Exercise}

1. Run the system a few of times with the given parameter values and initial populations.

2. Vary the initial and the total populations. Increase $N$ up to 1000 .

3. Vary the parameters and observe the effect on the system.

4. Vary the length of time for which the code runs.

5. The model is set up such that infectious individuals can recover from the disease and rejoin the susceptible class directly without acquiring immunity. Assume the disease confers temporary immunity so that immune individuals eventually lose their immunity and join the susceptible class. This transforms the model into an SIRS model. Modify the script StochasticSIR.m to incorporate this assumption and repeat the exercises.

\section{F.2.4 Spatiotemporal SEIRS Malaria Model}

\section{F.2.4.1 Background}

In this Section we explore the dynamics of a spatiotemporal SEIRS model for endemic malaria. Our model is obtained by introducing diffusion into the vector population of the ODE model developed and studied in Ngwa and Shu [1]. This model is described by the following nondimensional system of partial differential equations:

$$
\begin{aligned}
& \frac{\partial u}{\partial \tau}=\lambda(1-u)+\beta R+r w+\gamma w u-\xi u z \\
& \frac{\partial w}{\partial \tau}=\nu(1-u-R)+\gamma w^{2}-(r+\alpha+\gamma+\lambda+\nu) w \\
& \frac{\partial R}{\partial \tau}=\alpha w-\gamma w R-(\beta+\lambda) R, \\
& \frac{\partial s}{\partial \tau}=D_{s}\left(\frac{\partial^{2} s}{\partial x^{2}}+\frac{\partial^{2} s}{\partial y^{2}}\right)+a(1-s)-b s w-c s R, \\
& \frac{\partial z}{\partial \tau}=D_{z}\left(\frac{\partial^{2} z}{\partial x^{2}}+\frac{\partial^{2} z}{\partial y^{2}}\right)+e(1-s)-(a+e) z,
\end{aligned}
$$

where $u$ is the proportion of susceptible humans, $w$ is the proportion of infectious humans, $R$ is the proportion of immune humans, $s$ is the proportion of susceptible vectors, and $z$ is the proportion of infectious vectors. $D_{s}$ and $D_{z}$ are, respectively, the diffusion coefficients of the 
fractions of susceptible and infectious vectors, while $\gamma, \xi, \alpha, r, \beta, \lambda, \nu, a, b$ and $c$ are positive dimensionless parameter groupings. It is worth noting that the dimensionless dependent variables $u, w, R, s$ and $z$ are functions of the spatial variables $x$ and $y$ and the temporal variable $\tau$. The original system from Ref. [1] is a seven-variable model. However, since the total human and vector populations for the model reformulated in terms of proportions are given by $u+v+w+R=1$ and $s+q+z=1$, respectively, where $v$ and $q$ are the proportions of exposed humans and vectors, the substitutions $v=1-(u+w+R)$ and $q=1-(s+z)$ make it possible to eliminate the equations for the proportions of exposed individuals in each population, thereby leaving us with system (F.3).

The basic reproduction number for the corresponding ODE system was computed in Ref. [1] and it is given by

$$
R_{0}=\frac{\xi e \nu(\alpha c+b(\beta+\lambda))}{a(a+e)(\beta+\lambda)(\lambda+\nu)(\alpha+r+\gamma+\lambda)} .
$$

It was shown that when $R_{0} \leq 1$, there exists a disease free equilibrium solution $(1,0,0,1,0)$ that is locally and asymptotically stable, and when $R_{0}>1$, there exists a unique endemic equilibrium $\left(u^{*}, w^{*}, R^{*}, s^{*}, z^{*}\right)$ that is locally and asymptotically stable.

\section{F.2.4.2 Simulations}

First, the MATLAB m-file, ODESEIRS.m, required to solve the system presented in [1] is provided. ODESEIRS.m solves the system and plots the results. See Fig. F.8 for the results of one run with initial conditions $(1.0,0.0,0.0,0.9,0.1)$ and the parameters in Table F.2.

Second, the code Malaria.c written in C together with the MATLAB function SEIR.m are provided. Malaria.c integrates system (F.3) above and it can be opened as well as edited using the MATLAB editor. This code can be compiled as well as executed within MATLAB using the built-in C compiler. This process requires the use of MEX, a utility within MATLAB that facilitates calling of $\mathrm{C}, \mathrm{C}++$ or Fortran codes in MATLAB. The code is compiled into a MEX-file that is executable in MATLAB just like any built-in MATLAB function. See the Mathworks support page for details [14]. To compile Malaria.c within MATLAB, type "mex Malaria.c" at the MATLAB command prompt and hit "return". This compiles the code and generates a mex file called Malaria. Simply type "Malaria" and 
hit "return" at the MATLAB command prompt to execute.

The function SEIR.m plots solutions within some chosen cells and can be evoked by typing "SEIR" at the MATLAB command prompt. The function makemovie.m produces a propagating wave showing how the infection spreads within the community. This function can be run simply by typing "makemovie" at the command prompt and hitting return.

For our preliminary simulation, we use a grid size of $10 \times 10$ and begin with completely susceptible human and vector populations in each of the cells and then introduce some infectious vectors only in cell $(1,1)$. We also use the parameter values presented in Table F.2 which, apart from the last two parameters, are obtained from Ref. [1]. See the referenced paper for a discussion on the dimensional and physical significance of these parameters.

Table F.2. Parameter values for the SEIRS malaria model.

\begin{tabular}{||c|c|l|l|l|l||}
\hline \hline Parameter & Value 1 & Value 2 & Value 3 & Value 4 & Value 5 \\
\hline \hline$\gamma$ & 0.00100 & & & & \\
\hline$\xi$ & 4.06600 & & & & \\
\hline $\mathrm{r}$ & 0.30000 & & & & \\
\hline$\beta$ & 0.20000 & & & & \\
\hline$\lambda$ & 0.00184 & & & & \\
\hline$\nu$ & 2.00000 & & & & \\
\hline$a$ & 1.00200 & & & & \\
\hline$b$ & 10.0000 & & & & \\
\hline$c$ & 1.00000 & & & & \\
\hline$e$ & 2.40000 & & & & \\
\hline$D_{s}$ & 0.00001 & & & & \\
\hline$D_{z}$ & 0.00001 & & & & \\
\hline \hline
\end{tabular}




\section{F.2.4.3 Exercise}

1. Using ODESEIRSMain.m, vary the parameter values to determine their effects on disease prevalence. Fill in your parameter values in the spaces provided in Table F.2.

2. Compile and execute Malaria.c with the above parameters, switching off diffusion the first and second time. Plot the results from different sample cells.

3. Vary the system parameters in Malaria.c to determine their effects on disease prevalence. Your target should be arriving at a realistic parameter regime for which there exists a stable disease-free equilibrium and another for which there exists a stable endemic equilibrium.

4. Vary the diffusion coefficients in Malaria.c and observe how the disease spreads within the population. What happens when the diffusion coefficient is too small? What happens when the diffusion coefficient is too large? What happens when the diffusion coefficient is neither too large nor too small?

5. Using Malaria.c, vary the cell(s) with infectious vectors and the proportion of infectious vectors in each cell.

6. Vary the grid size, the time step, and the length of time the code is run.

7. Formulate and simulate the stochastic version of the SEIRS model (F.3) above. Assume $D_{s}=D_{z}=0$. 


\section{F.2.4.4 Some Results with the Parameter Values in Table F.2}

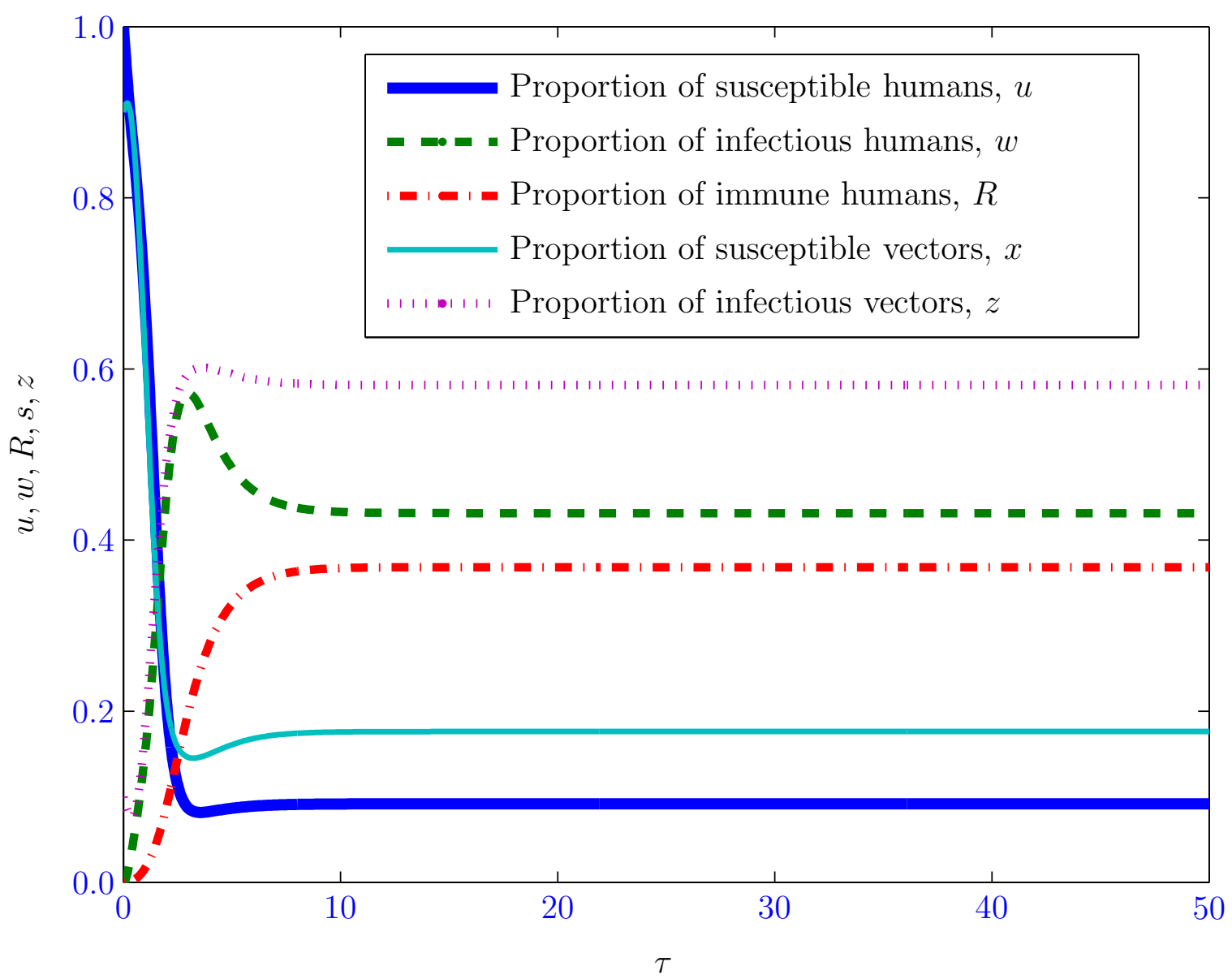

Figure F.8. Simulation results for the ode model using ODESEIRSMain.m. 


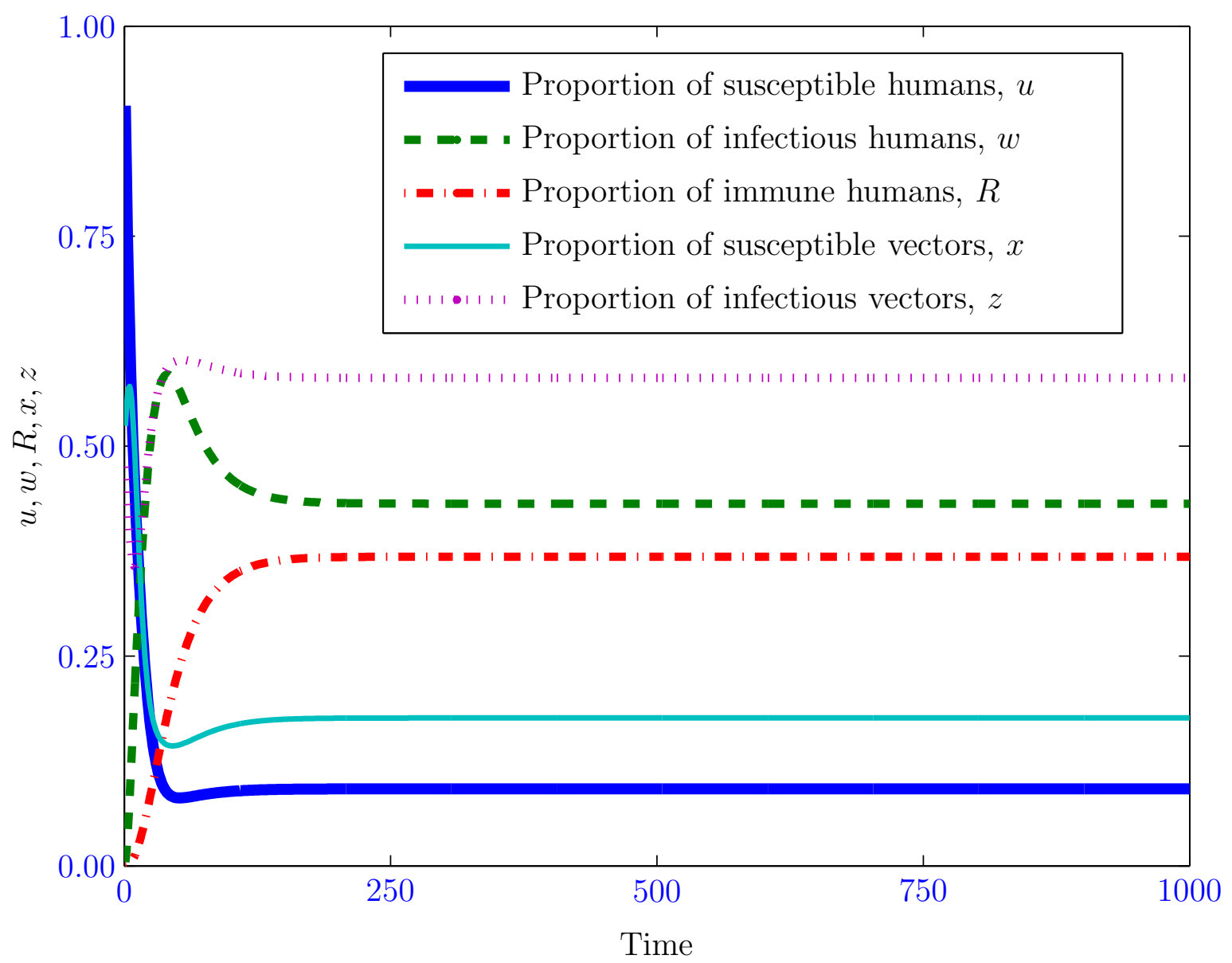

Figure F.9. Solution of the ode model in cell $(1,1)$. All variables converge to the unique endemic equilibrium $(0.0918,0.4315,0.3683,0.1762,0.5811)$ as $t \rightarrow \infty$. $R_{0}=104.895$ and the corresponding equilibrium values of the fractions of exposed humans and vectors are 0.1084 and 0.2427 , respectively. 


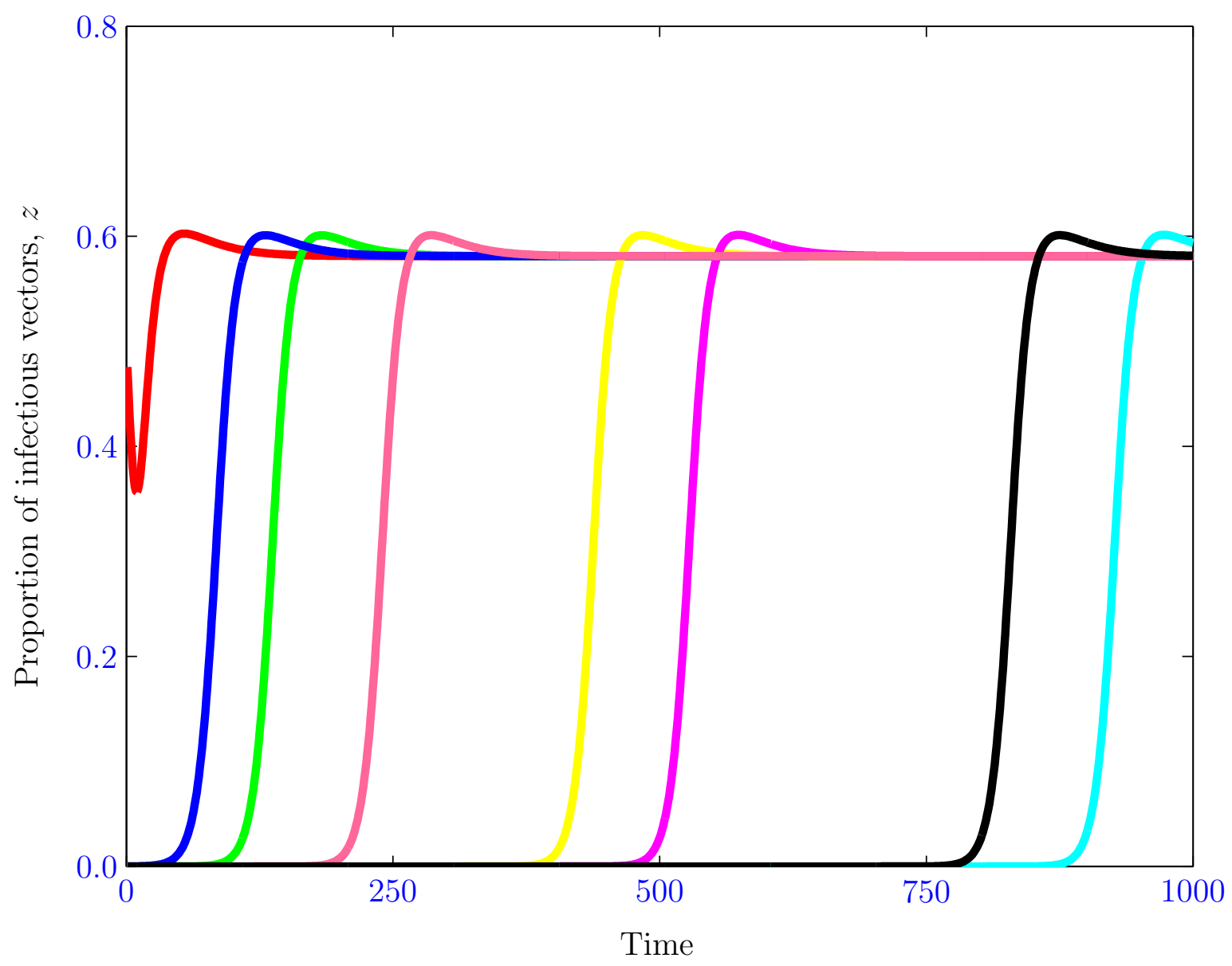

Figure F.10. Plots of some solutions within cells $(1,1)$-red, (2,1)-blue, $(2,2)$-green, $(3,3)$ pink, (5,5)-yellow, (1, 10)-magenta, (9,9)-black and (10, 10)-cyan, depicting the spread of the infection. 


\section{References}

[1] G. A. Ngwa and W. S. Shu, "A Mathematical Model for Endemic Malaria with Variable Human and Mosquito Populations," Mathematical and Computer Modelling 32, 747763 (2000).

Online Version 328, 340, 341, 342

[2] J. L. Aron and I. B. Schwartz, "Seasonality and Period-doubling Bifurcations in an Epidemic Model," Journal of Theoretical Biology 110, 665-679 (1984). 333

[3] G. A. Ngwa, C. N. Ngonghala, and N. B. S. Wilson, "A Model for Endemic Malaria with Delay and Variable Populations," Jounal of the Cameroon Academy of Sciences 1 (3), 169-186 (2001). 333

[4] H. W. Hethcote and S. A. Levin, Periodicity in Epidemiological Models, in Applied Mathematical Ecology (Springer, New York, 1989).

Online Version 333

[5] D. Gillespie, "A General Method for Numerically Simulating the Stochastic Time Evolution of Coupled Chemical Reactions," Journal of Computational Physics 22, 403-434 (1976).

Online Version 338

[6] H. Li and L. R. Petzold, "Stochastic Simulation of Biochemical Systems on the Graphics Processing Unit," Bioinformatics 00, 1-5 (2005).

Online Version 338 
[7] D. T. Gillespie, "Exact Stochastic Simulation of Coupled Chemical Reactions," The Journal of Physical Chemistry 81, 2340-2361 (1977).

Online Version 338

[8] M. A. Gibson and J. Bruck, "Efficient Exact Stochastic Simulation of Chemical Systems with Many Species and Many Channels," The Journal of Physical Chemistry A 104, 1876-1889 (2000).

Online Version

[9] D. T. Gillespie, "Approximate Accelerated Stochastic Simulation of Chemically Reacting Systems," Journal of Chemical Physics 115 (4), 1716-1733 (2001).

Online Version

[10] Y. Cao, H. Li, and L. Petzold, "Efficient Formulation of the Stochastic Simulation Algorithm for Chemically Reacting Systems," Journal of Chemical Physics 121 (9), 4059-4067 (2004).

Online Version

[11] M. Pineda-Krch, "Gillespie SSA: Implementing the Stochastic Simulation Agorithm in R," Journal of Statistical Software 25 (12), 1-18 (2008).

Online Version

[12] H. Li, Y. Cao, L. Petzold, and D. Gillespie, "Algorithms and Software for Stochastic Simulation of Biochemical Reacting Systems," Biotechnology Progress 24, 56-61 (2008). Online Version

[13] R. Ramaswamy, N. González-Segredo, and I. F. Sbalzarin, "A New Class of Highly Efficient Exact Stochastic Simulation Algorithms for Chemical Reaction Networks," The Journal of Chemical Physics 130, 244104-1-13 (2009).

Online Version 338

[14] The MathWorks Product Support.

Online Version 341 
$\overline{\text { Appendix }} \mathrm{G}$

\section{Latex Files, MATLAB Codes, and Figures}

See the accompanying DVD and external hard-drive for all the codes and figures. The latex files and the figures used to generate this dissertation are indexed as in Table G.1, while the codes used for generating the figures and the original figures are indexed as in Table G.2.

\section{G.1 Latex Code}

Table G.1. Latex Code and Figures

\begin{tabular}{||c|l|l||}
\hline \hline S/N & Folder/File & Description \\
\hline 01 & CNN_Main & All required files \\
\hline 02 & CNN_DissMain & Main code \\
\hline 04 & CNN_Ref & Reference files \\
\hline 05 & CNN_Chapters & Chapter codes \\
\hline 06 & CNN_Appendix & Appendix codes \\
\hline 07 & CNN_Part1Figs & Part 1 Figures \\
\hline 08 & CNN_Part2Figs & Part 2 Figures \\
\hline 09 & CNN_Part3Figs & Part 3 Figures \\
\hline 10 & CNN_HandsFigs & Hands-on Figures \\
\hline 11 & mynewstyle3.bst & Bibtex style \\
\hline 12 & sty & Fancy Chapters \\
\hline 13 & SecStyle & Section Style \\
\hline \hline
\end{tabular}

\section{G.2 MATLAB Codes}

Table G.2. MATLAB Codes and Figures

\begin{tabular}{||c|l|c||}
\hline \hline S/N & \multicolumn{1}{|c|}{ Folder } & Chapter \\
\hline 14 & Simple Pendulum & 1 \\
\hline 15 & Classical Malaria Model & 3 \\
\hline 16 & Disease Free Model & 5 \\
\hline 17 & Disease Model & 5 \\
\hline 18 & Determin Poverty Traps & 7 \\
\hline 19 & Stochastic Poverty Traps & 7 \\
\hline 20 & Intro to Chemical Chaos & 8 \\
\hline 21 & Extreme Mult. Model 1 & 9 \\
\hline 22 & Extreme Mult. Model 2 & 10 \\
\hline 24 & Extreme Mult. Model 3 & 10 \\
\hline 25 & Extreme Mult, Model 4 & 10 \\
\hline 26 & Buea Hands-on & F \\
\hline \hline
\end{tabular}

John $\mathrm{H}$. Hagen

Digitally signed by John $\mathrm{H}$. Hagen $\mathrm{DN}: \mathrm{cn}=J$ ohn $\mathrm{H}$. Hagen, $\mathrm{o}=$ West 